\title{
Angewandte
}

Supporting Information

(C) Wiley-VCH 2015

69451 Weinheim, Germany

Iron-Catalyzed 1,2-Addition of Perfluoroalkyl Iodides to Alkynes and Alkenes**

Tao Xu, Chi Wai Cheung, and Xile Hu*

anie_201402511_sm_miscellaneous_information.pdf 


\section{General Considerations.}

All commercially available compounds were used as received. ${ }^{1} \mathrm{H}$ and ${ }^{13} \mathrm{C}$ spectra were recorded on a Bruker Avance 400 spectrometer, and $\mathrm{CDCl}_{3}$ was purchased from Aldrich. The chemical shifts $(\delta)$ are given in parts per million relative to internal standard TMS (0 ppm for $\left.{ }^{1} \mathrm{H}\right), \mathrm{CDCl}_{3}\left(77.0 \mathrm{ppm}\right.$ for $\left.{ }^{13} \mathrm{C}\right)$. Solvents were purified using a two-column solid-state purification system (Innovative Technology, NJ, USA) and transferred to the glove box without exposure to air. Unless otherwise noted, all other reagents and starting materials were purchased from commercial sources and used without further purification.

\section{Screening Results.}

In a $4 \mathrm{~mL}$ sealed glass vial, 1-Octyne (1a), $\mathrm{C}_{4} \mathrm{~F}_{9} \mathrm{I}$ (1.2-1.5 equiv.) and base were dissolved in dry solvent under $\mathrm{N}_{2}$ following the condition listed in the table below. The mixture was stirred at $60^{\circ} \mathrm{C}$ in the dark for $18-24 \mathrm{~h}$. The reaction was quenched by aq. $\mathrm{HCl}$ solution and diluted with diethyl ether. Decane $(60 \mathrm{ul})$ was added to the organic layer as an internal standard and the solution was analyzed by GC to give the yield.

\subsection{Screening different metal catalysts.}

\section{Table S1}

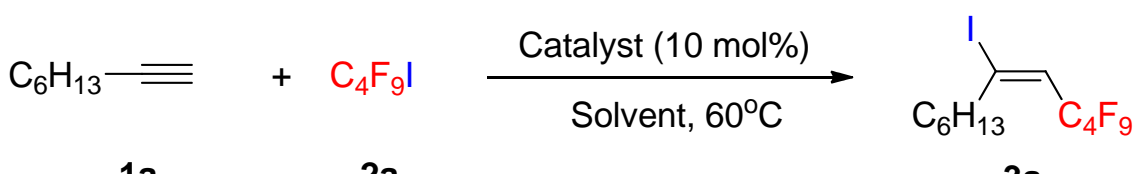

$1 a \quad 2 a$

\begin{tabular}{c|c|c|c|c|c|c|c}
\hline Entry & Catalyst & co-Cat. & Base & Solvent & o-TMEDA & Conversion & Yield \\
\hline 1 & --- & --- & $\mathrm{Cs}_{2} \mathrm{CO}_{3}$ & Dioxane & --- & $74 \%$ & $51 \%$ \\
2 & $\mathrm{Cul}$ & --- & $\mathrm{Cs}_{2} \mathrm{CO}_{3}$ & Dioxane & --- & $89 \%$ & $49 \%$ \\
3 & $\mathrm{CoBr}$ & --- & $\mathrm{Cs}_{2} \mathrm{CO}_{3}$ & Dioxane & 2 equiv. & $67 \%$ & $30 \%$ \\
4 & $\mathrm{Cu}(\mathrm{OTf})_{2}$ & --- & $\mathrm{Cs}_{2} \mathrm{CO}_{3}$ & Dioxane & 2 equiv. & $77 \%$ & $23 \%$ \\
5 & $\mathrm{NiCl}_{2 .} \mathrm{dme}$ & $\mathrm{Cul}$ & $\mathrm{Cs}_{2} \mathrm{CO}_{3}$ & Dioxane & --- & $98 \%$ & $61 \%$ \\
6 & $\mathrm{NiCl}_{2 .} \mathrm{dme}$ & $\mathrm{Cul}$ & $\mathrm{Cs}_{2} \mathrm{CO}_{3}$ & THF & --- & $77 \%$ & $27 \%$ \\
7 & $\mathrm{NiCl}_{2 .} \mathrm{dme}$ & $\mathrm{Cul}$ & ${ }^{t} \mathrm{BuOK}_{2}$ & Dioxane & --- & $97 \%$ & 0 \\
8 & $\mathrm{NiCl}_{2 .} \mathrm{dme}$ & --- & $\mathrm{Cs}_{2} \mathrm{CO}_{3}$ & Dioxane & 2 equiv. & $68 \%$ & $30 \%$ \\
9 & $\mathrm{FeBr}_{2}$ & $\mathrm{Cul}$ & $\mathrm{Cs}_{2} \mathrm{CO}_{3}$ & Dioxane & --- & $94 \%$ & $63 \%$ \\
10 & $\mathrm{FeBr}_{2}$ & --- & $\mathrm{Cs}_{2} \mathrm{CO}_{3}$ & Dioxane & --- & $96 \%$ & $68 \%$ \\
11 & $\mathrm{FeBr}_{2}$ & --- & $\mathrm{Cs}_{2} \mathrm{CO}_{3}$ & Dioxane & 2 equiv. & $65 \%$ & $31 \%$ \\
\hline
\end{tabular}

Condition: $1 \mathbf{a}(0.5 \mathrm{mmol}), \mathbf{2 a}(1.2$ equiv.), Catalyst (10 mol\%), co-Catalyst (10 mol\%),

Base (1.5 equiv.), o-TMEDA (2 equiv.), Solvent $(2 \mathrm{~mL}), 60^{\circ} \mathrm{C}, 18-24 \mathrm{~h}$.

o-TMEDA = bis[2-(N,N-dimethylaminoethyl)]ether 


\subsection{Screening concentration.}

Table S2

\begin{tabular}{|c|c|c|c|c|c|}
\hline \multicolumn{2}{|c|}{$\mathrm{C}_{6} \mathrm{H}_{13}=$} & $+\mathrm{C}_{4} \mathrm{~F}_{9} \mathrm{I}$ & $\frac{\text { cat. } \mathrm{FeBr}_{2} \text { (Loadin }}{\text { Dioxane, } 60^{\circ} \mathrm{C}}$ & 1 & $\mathrm{C}_{4} \mathrm{~F}_{9}$ \\
\hline & $1 a$ & $2 a$ & & \multicolumn{2}{|c|}{$3 a$} \\
\hline Entry & Loading & Alkyne (Con.) & Base (equiv.) & Conversion & Yield \\
\hline 1 & $5 \%$ & $0.25 \mathrm{M}$ & $\mathrm{Cs}_{2} \mathrm{CO}_{3}(0.8)$ & $99 \%$ & $95 \%(87 \%)$ \\
\hline 2 & $5 \%$ & $0.25 \mathrm{M}$ & --- & 0 & 0 \\
\hline 3 & --- & $0.25 \mathrm{M}$ & --- & 0 & 0 \\
\hline 4 & --- & $0.25 \mathrm{M}$ & $\mathrm{Cs}_{2} \mathrm{CO}_{3}(2.0)$ & $97 \%$ & $63 \%$ \\
\hline 5 & --- & $0.25 \mathrm{M}$ & $\mathrm{Cs}_{2} \mathrm{CO}_{3}(0.2)$ & $58 \%$ & $47 \%$ \\
\hline 6 & --- & $0.25 \mathrm{M}$ & $\mathrm{CsCl}(0.2)$ & 0 & 0 \\
\hline 7 & --- & $0.25 \mathrm{M}$ & $\mathrm{K}_{2} \mathrm{CO}_{3}(0.2)$ & 0 & 0 \\
\hline 8 & --- & $0.25 \mathrm{M}$ & DABCO (0.2) & 0 & 0 \\
\hline 9 & --- & $0.25 \mathrm{M}$ & $\mathrm{Cs}_{2} \mathrm{CO}_{3}(0.2)$ & $43 \%$ & $17 \%$ \\
\hline 10 & --- & $0.50 \mathrm{M}$ & $\mathrm{Cs}_{2} \mathrm{CO}_{3}(0.2)$ & $81 \%$ & $79 \%$ \\
\hline 11 & --- & $1.00 \mathrm{M}$ & $\mathrm{Cs}_{2} \mathrm{CO}_{3}(0.2)$ & $91 \%$ & $90 \%$ \\
\hline 12 & --- & $1.00 \mathrm{M}$ & $\mathrm{Cs}_{2} \mathrm{CO}_{3}(0.25)$ & $96 \%$ & $95 \%(87 \%)$ \\
\hline
\end{tabular}

Condition: 1a (1.0 mmol), 2a (1.5 equiv.), Catalyst (5 mol\%), Base (1.5 equiv.), Dioxane, $60^{\circ} \mathrm{C}, 18-24 \mathrm{~h}$.

\section{General Procedure for Experiments.}

\subsection{General procedure for addition to alkyne.}

$\mathrm{Cs}_{2} \mathrm{CO}_{3}$-catalyed reaction:

In a $4 \mathrm{~mL}$ sealed glass vial, $\mathrm{Cs}_{2} \mathrm{CO}_{3}(81.5 \mathrm{mg}, 0.25 \mathrm{mmol}, 0.25$ equiv. $)$, alkyne (1.0 mmol) or alkene $(1.0 \mathrm{mmol})$, Perfluoroalkyl idodide (1.5 mmol, 1.5equiv.) were dissolved in dry dioxane $(1 \mathrm{~mL})$ under $\mathrm{N}_{2}$. The mixture was stirred at $60^{\circ} \mathrm{C}$ in the dark for $18-24 \mathrm{~h}$, and the reaction was quenched by aq. $\mathrm{HCl}$ solution and extracted with diethyl ether. The organic layer was combined and concentrated under vacuum and the residue was purified by column chromatography on silica gel with a gradient eluant of Hexane and ethyl acetate affording the product.

$\mathrm{FeBr}_{2}$-catalyed reaction:

In a $4 \mathrm{~mL}$ sealed glass vial, $\mathrm{FeBr}_{2}\left(10.8 \mathrm{mg}, 0.05 \mathrm{mmol}, 0.1\right.$ equiv.), $\mathrm{Cs}_{2} \mathrm{CO}_{3}$ (130.4 mg, $0.4 \mathrm{mmol}$, 0.8equiv. $)$, alkyne or alkene $(0.5 \mathrm{mmol})$, Perfluoroalkyl idodide $(0.75$ mmol, 1.5equiv.) were dissolved in dry dioxane $(2 \mathrm{~mL})$ under $\mathrm{N}_{2}$. The mixture was stirred at $60^{\circ} \mathrm{C}$ in the dark for $18-24 \mathrm{~h}$, and the reaction was quenched by aq. $\mathrm{HCl}$ solution and extracted with diethyl ether. The organic layer was combined and concentrated under vacuum and the residue was purified by column chromatography 
on silica gel with a gradient eluant of Hexane and ethyl acetate affording the product.

For $\mathrm{CF}_{3} \mathrm{I}$, firstly, $\mathrm{CF}_{3} \mathrm{I}$ (1.5mmol, 3equiv.) was dissolved in the dioxane $(2 \mathrm{~mL})$ at room temperature, then this solvent was added to the mixture of $\mathrm{FeBr}_{2}(10.8 \mathrm{mg}$, $0.05 \mathrm{mmol}, 0.1$ equiv.), $\mathrm{Cs}_{2} \mathrm{CO}_{3}$ (130.4 mg, $0.4 \mathrm{mmol}, 0.8$ equiv.), alkyne or alkene (0.5 mmol) in a $4 \mathrm{~mL}$ sealed glass vial under $\mathrm{N}_{2}$. The mixture was stirred at $60^{\circ} \mathrm{C}$ in the dark for $18-24 \mathrm{~h}$, and the reaction was quenched by aq. $\mathrm{HCl}$ solution and extracted with diethyl ether. The organic layer was combined and concentrated under vacuum and the residue was purified by column chromatography on silica gel with a gradient eluant of hexane and ethyl acetate affording the product.

\subsection{Procedures for coupling reactions.}

In a $4 \mathrm{~mL}$ sealed glass vial, $\mathrm{Fe}(\mathrm{acac})_{3}(13.1 \mathrm{mg}, 0.0373 \mathrm{mmol}, 0.5$ equiv. $)$ and compound 4a $(27.0 \mathrm{mg}, 0.0746 \mathrm{mmol})$ were dissolved in dry THF $(1 \mathrm{~mL})$ and NMP (200 uL) under $\mathrm{N}_{2}$. Then ${ }^{n} \mathrm{C}_{5} \mathrm{H}_{9} \mathrm{MgBr}(75 \mathrm{uL}, 2.0 \mathrm{M}, 0.15 \mathrm{mmol}, 2.0$ equiv.) was added The mixture was stirred at $-30^{\circ} \mathrm{C}$ for $1 \mathrm{~h}$, and the reaction was quenched by aq. $\mathrm{NH}_{4} \mathrm{Cl}$ solution and extracted with diethyl ether. The organic layer was combined and concentrated under vacuum and the residue was purified by column chromatography on silica gel with a gradient eluant of hexane affording the product 6a $(15.8 \mathrm{mg}$, $69 \%)$.

In a $4 \mathrm{~mL}$ sealed glass vial, $\mathrm{PdCl}_{2}\left(\mathrm{PPh}_{3}\right)_{2}$ (7.0 mg, $0.01 \mathrm{mmol}, 0.1$ equiv.), $\mathrm{K}_{2} \mathrm{CO}_{3}(3.1$ $\mathrm{mg}, 0.022 \mathrm{mmol}, 0.22$ equiv.), $\mathrm{PhB}(\mathrm{OH})_{2}(36.6 \mathrm{mg}, 0.3 \mathrm{mmol}$, 3equiv.) and compound 3n (57.0 mg, $0.1 \mathrm{mmol})$ were dissolved in Toluene $(1 \mathrm{~mL})$ and $\mathrm{H}_{2} \mathrm{O}(200 \mathrm{uL})$ under $\mathrm{N}_{2}$. The mixture was stirred at $80^{\circ} \mathrm{C}$ for $16 \mathrm{~h}$. After that, another $\mathrm{K}_{2} \mathrm{CO}_{3}(3.0 \mathrm{mg}, 0.022$ mmol, 0.22equiv.) and $\mathrm{PhB}(\mathrm{OH})_{2}(36.6 \mathrm{mg}, 0.3 \mathrm{mmol}$, 3equiv.) were added and the reaction was stirred at $80^{\circ} \mathrm{C}$ for additional $16 \mathrm{~h}$ followed by quenched with water and extracted with diethyl ether. The organic layer was combined and concentrated under vacuum and the residue was purified by column chromatography on silica gel with a gradient eluant of hexane and ethyl acetate affording the product $6 \mathbf{b}(45.3 \mathrm{mg}, 86 \%)$.

In a $4 \mathrm{~mL}$ sealed glass vial, $4 \mathrm{l}(58.0 \mathrm{mg}, 0.126 \mathrm{mmol}), \mathrm{Pd}\left(\mathrm{PPh}_{3}\right)_{4}(9.3 \mathrm{mg}, 0.008$ mmol, 0.063equiv.), $\mathrm{K}_{2} \mathrm{CO}_{3} \quad(43.5 \quad \mathrm{mg}, 0.315 \quad \mathrm{mmol}$, 2.5equiv.), $E-\mathrm{C}_{6} \mathrm{H}_{13}-\mathrm{CH}=\mathrm{CH}-\mathrm{B}(\mathrm{OH})_{2}(49.1 \mathrm{mg}, 0.315 \mathrm{mmol}, 2.5$ equiv. $)$ were dissolved in THF (1 $\mathrm{mL}$ ) under $\mathrm{N}_{2}$. The mixture was stirred at $65^{\circ} \mathrm{C}$ for $18 \mathrm{~h}$. The reaction was filtered by fast column and washed with diethyl ether. The organic layer was combined and concentrated under vacuum and the residue was purified by column chromatography on silica gel with a gradient eluant of Hexane and ethyl acetate affording the product 6c (42.2 mg, 75\%).

In a $4 \mathrm{~mL}$ sealed glass vial, 4 h $(56.0 \mathrm{mg}, 0.142 \mathrm{mmol}), \mathrm{Pd}\left(\mathrm{PPh}_{3}\right)_{4}(16.4 \mathrm{mg}, 0.014$ mmol, 0.1 equiv.), CuI (5.4 mg, 0.028 mmol, 0.2equiv.), 1-Octyne (35.8 mg, 0.325 mmol, 2.3equiv.) were dissolved in TEA $(1 \mathrm{~mL})$ under $\mathrm{N}_{2}$. The mixture was stirred at 
$50^{\circ} \mathrm{C}$ for $16 \mathrm{~h}$. The reaction was filtered by fast column and washed with diethyl ether. The organic layer was combined and concentrated under vacuum and the residue was purified by column chromatography on silica gel with a gradient eluant of hexane and ethyl acetate affording the product $\mathbf{6 d}(49.2 \mathrm{mg}, 92 \%)$.

In a $4 \mathrm{~mL}$ sealed glass vial, $\mathrm{CuI}$ ( $4.7 \mathrm{mg}, 0.024 \mathrm{mmol}, 0.2$ equiv.), 1,10-Phenanthroline (4.4 mg, 0.024 mmol, 0.2equiv.), $\mathrm{PPh}_{3}$ (13.1 mg, 0.050 mmol, 0.4equiv.), $\mathrm{K}_{3} \mathrm{PO}_{4}$ (44.0 $\mathrm{mg}, 0.207 \mathrm{mmol}$, 1.7equiv.) and compound $3 \mathbf{j}$ (59.0 $\mathrm{mg}, 0.124 \mathrm{mmol})$ were dissolved in Toluene $(1 \mathrm{~mL})$ under $\mathrm{N}_{2}$, then $\mathrm{PhSH}(26.0 \mathrm{mg}, 0.236 \mathrm{mmol}, 1.9$ equiv.) was added. The mixture was stirred at $110^{\circ} \mathrm{C}$ for $16 \mathrm{~h}$. The reaction was quenched with aq. $\mathrm{HCl}$ and extracted with diethyl ether. The organic layer was combined and concentrated under vacuum and the residue was purified by column chromatography on silica gel with a gradient eluant of hexane and ethyl acetate affording the product $6 \mathbf{e}(45.9 \mathrm{mg}$, $81 \%)$.

In a $4 \mathrm{~mL}$ sealed glass vial, $\mathrm{FeBr}_{2}(7.1 \mathrm{mg}, 0.01 \mathrm{mmol}, 0.1$ equiv. $)$ and compound $5 \mathbf{e}$ (60.0 mg, $0.174 \mathrm{mmol})$ were dissolved in NMP $(0.73 \mathrm{~mL})$ under $\mathrm{N}_{2}$, then the relevant alkyne Gridge reagent $(0.55 \mathrm{~mL}, 0.5 \mathrm{M}, 0.275 \mathrm{mmol}, 1.6$ equiv. $)$ was added. The mixture was stirred at room temperature for $16 \mathrm{~h}$. The reaction was quenched with aq. $\mathrm{HCl}$ and extracted with diethyl ether. The organic layer was combined and concentrated under vacuum and the residue was purified by column chromatography on silica gel with a gradient eluant of hexane and ethyl acetate affording the product 6f (36.1 mg, 50\%).

\subsection{General procedure for the synthesis of the substrates}

These substrateswere synthesized according to the literature by Mitsunobu reaction ${ }^{\mathrm{S} 1}$.<smiles>C#CCCCOc1ccc(C(=O)c2ccccc2)cc1</smiles>

${ }^{1} \mathrm{H}$ NMR (400 MHz, $\left.\mathrm{CDCl}_{3}\right) \delta 7.82(\mathrm{~d}, J=8.4 \mathrm{~Hz}, 2 \mathrm{H}), 7.75(\mathrm{~d}, J=7.6 \mathrm{~Hz}, 2 \mathrm{H}), 7.56$ (t, $J=7.6 \mathrm{~Hz}, 1 \mathrm{H}), 7.47(\mathrm{t}, J=7.6 \mathrm{~Hz}, 2 \mathrm{H}), 6.96(\mathrm{~d}, J=8.4 \mathrm{~Hz}, 2 \mathrm{H}), 4.16(\mathrm{t}, J=6.4$ $\mathrm{Hz}, 2 \mathrm{H}), 2.43$ (td, $J=6.8,2.8 \mathrm{~Hz}, 2 \mathrm{H}), 2.04$ (tt, $J=6.8,6.4 \mathrm{~Hz}, 2 \mathrm{H}), 1.99$ (t, $J=2.8$ $\mathrm{Hz}, 1 \mathrm{H}) ;{ }^{13} \mathrm{C}$ NMR $\left(100 \mathrm{MHz}, \mathrm{CDCl}_{3}\right) \delta 195.5,162.5,138.3,132.5,131.8,130.2$, $129.7,128.2,114.0,83.1,69.1,66.3,28.0,15.1 ; \mathrm{HRMS}: \mathrm{m} / \mathrm{z}(\mathrm{ESI})$ calculated $[\mathrm{M}+\mathrm{H}]^{+}$: 265.1229, measured: 265.1238 .

\footnotetext{
S1 a) X. Chen, J. Thomas, P. Gangopadhyay, R. A. Norwood, N. Peyghambarian, D. V. McGrath, J. Am. Chem. Soc.2009, 131, 13840-13843. b) R. R. Frey, C. K. Wada, et. al, Bio. \& Med. Chem. Lett. 2002, 12, 3443-3447.
} 


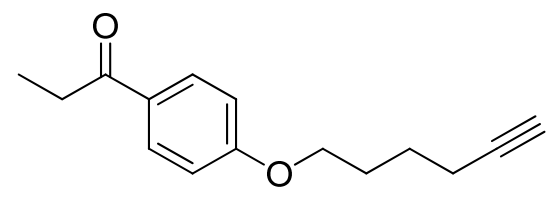

${ }^{1} \mathrm{H}$ NMR $\left(400 \mathrm{MHz}, \mathrm{CDCl}_{3}\right) \delta 7.94(\mathrm{~d}, J=8.8 \mathrm{~Hz}, 2 \mathrm{H}), 6.92(\mathrm{~d}, J=8.8 \mathrm{~Hz}, 2 \mathrm{H}), 4.05$ (t, $J=6.4 \mathrm{~Hz}, 2 \mathrm{H}), 2.96(\mathrm{q}, J=7.2 \mathrm{~Hz}, 2 \mathrm{H}), 2.37-2.25(\mathrm{~m}, 2 \mathrm{H}), 2.07-1.88(\mathrm{~m}, 3 \mathrm{H})$, $1.81-1.68(\mathrm{~m}, 2 \mathrm{H}), 1.21(\mathrm{t}, J=7.2 \mathrm{~Hz}, 3 \mathrm{H}) ;{ }^{13} \mathrm{C} \mathrm{NMR}\left(100 \mathrm{MHz}, \mathrm{CDCl}_{3}\right) \delta 199.5$, $162.7,130.2,129.8,114.0,83.9,68.7,67.4,31.3,28.0,24.9,18.1,8.4 ; \mathrm{HRMS}: \mathrm{m} / \mathrm{z}$ (ESI) calculated $[\mathrm{M}+\mathrm{Ag}]^{+}: 337.0358$, measured: 337.0357.

These substrateswere synthesized from the acyl chloride and alcohol with pyridine or TEA as base in DCM at room temperature according to the literature ${ }^{\mathrm{S} 2}$.<smiles>C#CCCCCOC(=O)CCCCCCC</smiles>

${ }^{1} \mathrm{H}$ NMR $\left(400 \mathrm{MHz}, \mathrm{CDCl}_{3}\right) \delta 4.09(\mathrm{t}, J=6.4 \mathrm{~Hz}, 2 \mathrm{H}), 2.37-2.18(\mathrm{~m}, 4 \mathrm{H}), 2.01-1.93$ $(\mathrm{m}, 1 \mathrm{H}), 1.82-1.71(\mathrm{~m}, 2 \mathrm{H}), 1.67-1.55(\mathrm{~m}, 4 \mathrm{H}), 1.39-1.15(\mathrm{~m}, 16 \mathrm{H}), 0.88(\mathrm{t}, J=6.8 \mathrm{~Hz}$, $3 \mathrm{H}) ;{ }^{13} \mathrm{C}$ NMR $\left(100 \mathrm{MHz}, \mathrm{CDCl}_{3}\right) \delta 173.0,83.8,68.6,63.6,34.3,31.9,29.5,29.4$, 29.3, 29.2, 29.1 , 29.0, 27.6, 24.9, 24.8, 22.6, 18.0, 14.1; HRMS: m/z (ESI) calculated $[\mathrm{M}+\mathrm{Ag}]^{+}:$387.1453, measured: 387.1434 .<smiles>C#CCCCOC(=O)c1ccc(Cl)cc1</smiles>

${ }^{1} \mathrm{H}$ NMR $\left(400 \mathrm{MHz}, \mathrm{CDCl}_{3}\right) \delta 7.96(\mathrm{~d}, \mathrm{~J}=8.0 \mathrm{~Hz}, 2 \mathrm{H}), 7.39(\mathrm{~d}, \mathrm{~J}=8.0 \mathrm{~Hz}, 2 \mathrm{H}), 4.23$ $(\mathrm{t}, \mathrm{J}=6.0 \mathrm{~Hz}, 2 \mathrm{H}), 2.41-2.32(\mathrm{~m}, 2 \mathrm{H}), 2.04-1.93(\mathrm{~m}, 3 \mathrm{H}) ;{ }^{13} \mathrm{C}$ NMR (100 MHz, $\left.\mathrm{CDCl}_{3}\right) \delta 165.4,139.2,130.8,128.5,128.6,82.8,69.1,63.6,27.5,15.2 ; \mathrm{HRMS}: \mathrm{m} / \mathrm{z}$ (ESI) calculated $[\mathrm{M}+\mathrm{Ag}]^{+}: 328.9499$, measured: 328.9495.<smiles>C#CCCOC(=O)c1ccc(C#N)cc1</smiles>

${ }^{1} \mathrm{H}$ NMR $\left(400 \mathrm{MHz}, \mathrm{CDCl}_{3}\right) \delta 8.16(\mathrm{~d}, \mathrm{~J}=8.8 \mathrm{~Hz}, 2 \mathrm{H}), 7.76(\mathrm{~d}, \mathrm{~J}=8.8 \mathrm{~Hz}, 2 \mathrm{H}), 4.47$

[S²] S. Peddibhotla, Y. Dang, J. O. Liu, D. Romo, J. Am. Chem. Soc.2007, 129, 12222-12231. 
$(\mathrm{t}, J=6.4 \mathrm{~Hz}, 2 \mathrm{H}), 2.69(\mathrm{td}, J=6.4,2.8 \mathrm{~Hz}, 2 \mathrm{H}), 2.05(\mathrm{t}, J=2.8 \mathrm{~Hz}, 1 \mathrm{H}) ;{ }^{13} \mathrm{C} \mathrm{NMR}$ $\left(100 \mathrm{MHz}, \mathrm{CDCl}_{3}\right) \delta 164.6,133.7,132.2,130.1,117.9,116.5,79.6,70.2,63.2,19.0$; HRMS: m/z (ESI) calculated [M+Ag] $]^{+}$305.9684, measured: 305.9682.<smiles>C#CCCCCCOC(=O)c1ccc(Cl)cc1</smiles>

${ }^{1} \mathrm{H}$ NMR (400 MHz, CDCl 3$) \delta 7.97(\mathrm{~d}, \mathrm{~J}=8.4 \mathrm{~Hz}, 2 \mathrm{H}), 7.40(\mathrm{~d}, \mathrm{~J}=8.4 \mathrm{~Hz}, 2 \mathrm{H}), 4.32$ (t, $J=6.4 \mathrm{~Hz}, 2 \mathrm{H}), 2.23(\mathrm{td}, J=6.8,2.4 \mathrm{~Hz}, 2 \mathrm{H}), 1.97$ (t, $J=2.4 \mathrm{~Hz}, 1 \mathrm{H}), 1.78$ (tt, $J=$ 7.2, $6.8 \mathrm{~Hz}, 2 \mathrm{H}), 1.65-1.50(\mathrm{~m}, 4 \mathrm{H}) ;{ }^{13} \mathrm{C} \mathrm{NMR}\left(100 \mathrm{MHz}, \mathrm{CDCl}_{3}\right) \delta 165.6,139.1$, 130.8, 128.7, 128.5, 84.0, 68.4, 64.9, 28.0, 27.9, 25.0, 18.2; HRMS: m/z (ESI) calculated $[\mathrm{M}+\mathrm{Ag}]^{+}:$356.9811, measured: 356.9796 .

\subsection{The characterization of the new products}

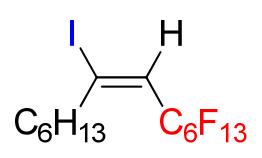

${ }^{1} \mathrm{H}$ NMR (400 MHz, $\left.\mathrm{CDCl}_{3}\right) \delta 6.32(\mathrm{~d}, J=14.4 \mathrm{~Hz}, 1 \mathrm{H}), 2.69-2.56(\mathrm{~m}, 2 \mathrm{H})$, 1.65-1.49 (m, 2H), 1.41-1.21 (m, 6H), 0.95-0.83 (m, 3H); ${ }^{13} \mathrm{C}$ NMR (100 MHz, $\left.\mathrm{CDCl}_{3}\right) \delta 126.6(\mathrm{t}, J=243.0 \mathrm{~Hz}), 126.1(\mathrm{t}, J=5.7 \mathrm{~Hz}), 120-100(\mathrm{~m}), 41.2,31.5,30.1$, 28.2, 22.5, 13.9; ${ }^{19} \mathrm{~F}$ NMR (376 MHz, $\left.\mathrm{CDCl}_{3}\right) \delta-81.2(\mathrm{t}, J=9.8 \mathrm{~Hz}, 3 \mathrm{~F}),-105.6(\mathrm{t}, J$ $=12.0 \mathrm{~Hz}, 2 \mathrm{~F}),-121.9(\mathrm{~m}, 2 \mathrm{~F}),-123.1(\mathrm{~m}, 2 \mathrm{~F}),-123.5$ (m, 2F), -126.4 (m, 2F); HRMS: $\mathrm{m} / \mathrm{z}$ (APCI) calculated [M] $]^{+}: 555.9933$, measured: 555.9920 .

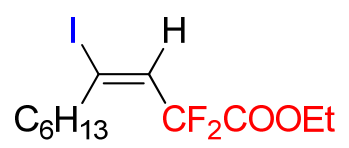

${ }^{1} \mathrm{H}$ NMR (400 MHz, CDCl 3 ) $\delta 6.40(\mathrm{t}, J=12.8 \mathrm{~Hz}, 1 \mathrm{H}), 4.40-4.27(\mathrm{~m}, 2 \mathrm{H}), 2.63-2.54$ $(\mathrm{m}, 2 \mathrm{H}), 1.62-1.47(\mathrm{~m}, 2 \mathrm{H}), 1.46-1.21(\mathrm{~m}, 9 \mathrm{H}), 0.95-0.84(\mathrm{~m}, 3 \mathrm{H}) ;{ }^{13} \mathrm{C}$ NMR $(100$ $\left.\mathrm{MHz}, \mathrm{CDCl}_{3}\right) \delta 163.2(\mathrm{t}, J=34.2 \mathrm{~Hz}), 131.2(\mathrm{t}, J=26.8 \mathrm{~Hz}), 119.6(\mathrm{t}, J=7.4 \mathrm{~Hz})$, $111.5(\mathrm{t}, J=251.0 \mathrm{~Hz}), 63.3,40.7,31.5,29.8,28.0,22.5,14.0,13.9 ;{ }^{19} \mathrm{~F}$ NMR (376 $\left.\mathrm{MHz}, \mathrm{CDCl}_{3}\right) \delta-97.7(\mathrm{~s})$; HRMS: $\mathrm{m} / \mathrm{z}$ (ESI) calculated $[\mathrm{M}+\mathrm{Na}]^{+}: 383.0296$, measured: 383.0286 .

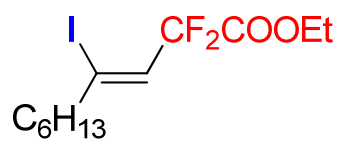


${ }^{1} \mathrm{H}$ NMR $\left(400 \mathrm{MHz}, \mathrm{CDCl}_{3}\right) \delta 6.37(\mathrm{t}, J=11.6 \mathrm{~Hz}, 1 \mathrm{H}), 4.41-4.31(\mathrm{~m}, 2 \mathrm{H}), 2.59(\mathrm{t}, J$ $=7.2 \mathrm{~Hz}, 2 \mathrm{H}), 1.61-1.50(\mathrm{~m}, 2 \mathrm{H}), 1.37(\mathrm{t}, J=7.2 \mathrm{~Hz}, 3 \mathrm{H}), 1.33-1.24(\mathrm{~m}, 6 \mathrm{H}), 0.89(\mathrm{t}$, $J=6.8 \mathrm{~Hz}, 3 \mathrm{H}) ;{ }^{13} \mathrm{C} \mathrm{NMR}\left(100 \mathrm{MHz}, \mathrm{CDCl}_{3}\right) \delta 162.6(\mathrm{t}, J=34.2 \mathrm{~Hz}), 128.1(\mathrm{t}, J=$ $29.5 \mathrm{~Hz}), 115.0$ (t, $J=10.0 \mathrm{~Hz}), 112.1(\mathrm{t}, J=240.9 \mathrm{~Hz}), 63.2,46.7,31.4,28.9,27.6$, 22.5, 14.0, 13.9; ${ }^{19} \mathrm{~F}$ NMR (376 MHz, $\left.\mathrm{CDCl}_{3}\right) \delta-97.9(\mathrm{~s}) ; \mathrm{HRMS}: \mathrm{m} / \mathrm{z}$ (ESI) calculated $[\mathrm{M}+\mathrm{Na}]^{+}:$383.0296, measured: 383.0291 .<smiles>O=C(c1ccccc1)c1ccc(OCCC/C(I)=C\C(F)(F)F)cc1</smiles>

${ }^{1} \mathrm{H}$ NMR (400 MHz, $\left.\mathrm{CDCl}_{3}\right) \delta 7.82(\mathrm{~d}, J=8.4 \mathrm{~Hz}, 2 \mathrm{H}), 7.75(\mathrm{~d}, J=8.0 \mathrm{~Hz}, 2 \mathrm{H}), 7.57$ $(\mathrm{t}, J=6.8 \mathrm{~Hz}, 1 \mathrm{H}), 7.48(\mathrm{t}, J=6.8 \mathrm{~Hz}, 2 \mathrm{H}), 6.95(\mathrm{~d}, J=8.4 \mathrm{~Hz}, 2 \mathrm{H}), 6.40(\mathrm{t}, J=14.6$ $\mathrm{Hz}, 1 \mathrm{H}), 4.08(\mathrm{t}, J=6.4 \mathrm{~Hz}, 2 \mathrm{H}), 2.94-2.83(\mathrm{~m}, 2 \mathrm{H}), 2.18-2.05(\mathrm{~m}, 2 \mathrm{H}) ;{ }^{13} \mathrm{C} \mathrm{NMR}$ $\left(100 \mathrm{MHz}, \mathrm{CDCl}_{3}\right) \delta 195.4,162.2,138.2,132.5,131.8,130.2,129.7,128.1,127.3(\mathrm{t}, J$ $=23.8 \mathrm{~Hz}), 120.9(\mathrm{t}, J=6.0 \mathrm{~Hz}), 113.9,120-100(\mathrm{~m}), 66.1,37.8,29.5 ;{ }^{19} \mathrm{~F} \mathrm{NMR}(376$ $\left.\mathrm{MHz}, \mathrm{CDCl}_{3}\right) \delta-81.0(\mathrm{t}, J=9.4 \mathrm{~Hz}, 3 \mathrm{~F}),-105.8(\mathrm{t}, J=12.0 \mathrm{~Hz}, 2 \mathrm{~F}),-124.1(\mathrm{~m}, 2 \mathrm{~F})$, -125.8 (m, 2F); HRMS: m/z (ESI) calculated [M+H] $]^{+}$611.0129, measured: 611.0131. $Z$ product: ${ }^{19} \mathrm{~F}$ NMR $\left(376 \mathrm{MHz}, \mathrm{CDCl}_{3}\right) \delta-81.0(\mathrm{~m}, 3 \mathrm{~F}),-108.9(\mathrm{t}, J=12.4 \mathrm{~Hz}, 2 \mathrm{~F})$, $-123.8(\mathrm{~m}, 2 \mathrm{~F}),-125.7(\mathrm{~m}, 2 \mathrm{~F})$; HRMS: m/z (ESI) calculated $[\mathrm{M}+\mathrm{H}]^{+}: 611.0129$, measured: 611.0126 .<smiles>FC(F)(F)/C=C(/I)CCNCOC1CCCCO1</smiles>

${ }^{1} \mathrm{H}$ NMR $\left(400 \mathrm{MHz}, \mathrm{CDCl}_{3}\right) \delta 6.34(\mathrm{t}, J=14.4 \mathrm{~Hz}, 1 \mathrm{H}), 4.63-4.57(\mathrm{~m}, 1 \mathrm{H}), 3.93-3.84$ (m, $1 \mathrm{H}), 3.77(\mathrm{tt}, J=10.0,6.4 \mathrm{~Hz}, 1 \mathrm{H}), 3.56-3.49(\mathrm{~m}, 1 \mathrm{H}), 3.44(\mathrm{tt}, J=10.0,6.4 \mathrm{~Hz}$, $1 \mathrm{H}), 2.67(\mathrm{t}, J=7.2 \mathrm{~Hz}, 2 \mathrm{H}), 1.90-1.80(\mathrm{~m}, 1 \mathrm{H}), 1.78-1.70(\mathrm{~m}, 1 \mathrm{H}), 1.69-1.51(\mathrm{~m}$, 8H), 1.49-1.38 (m, 2H); ${ }^{13} \mathrm{C}$ NMR $\left(100 \mathrm{MHz}, \mathrm{CDCl}_{3}\right) \delta 126.4(\mathrm{t}, J=23.8 \mathrm{~Hz}), 122.8$ (t, $J=6.4 \mathrm{~Hz}), 120-100(\mathrm{~m}), 98.8,67.2,62.2,41.1,30.7,29.8,29.4,25.4,25.2,19.5$; ${ }^{19} \mathrm{~F}$ NMR $\left(376 \mathrm{MHz}, \mathrm{CDCl}_{3}\right) \delta-81.0(\mathrm{t}, J=9.4 \mathrm{~Hz}, 3 \mathrm{~F}),-105.6(\mathrm{t}, J=12.0 \mathrm{~Hz}, 2 \mathrm{~F})$, $-124.2(\mathrm{~m}, 2 \mathrm{~F}),-125.8(\mathrm{~m}, 2 \mathrm{~F})$; HRMS: $\mathrm{m} / \mathrm{z}(\mathrm{ESI})$ calculated $[\mathrm{M}+\mathrm{Na}]^{+}: 565.0262$, measured: 565.0263 .<smiles>FC(F)(F)C=C(I)CCCOC1CCCCO1</smiles>

${ }^{1} \mathrm{H}$ NMR $\left(400 \mathrm{MHz}, \mathrm{CDCl}_{3}\right) \delta 6.24(\mathrm{t}, J=12.8 \mathrm{~Hz}, 1 \mathrm{H}), 4.63-4.50(\mathrm{~m}, 1 \mathrm{H}), 3.91-3.81$ 
(m, 1H), 3.80-3.70 (m, 1H), 3.55-3.46 (m, 1H), 3.43-3.35 (m, 1H), 2.76-2.63 (m, 2H), 1.80-1.33 (m, 12H); ${ }^{13} \mathrm{C}$ NMR $\left(100 \mathrm{MHz}, \mathrm{CDCl}_{3}\right) \delta 121.6(\mathrm{t}, J=23.5 \mathrm{~Hz}), 116.5(\mathrm{t}, J$ $=6.1 \mathrm{~Hz}), 120-100(\mathrm{~m}), 98.9,67.2,62.4,48.3,30.7,29.3,28.9,25.4,24.8,19.6 ;{ }^{19} \mathrm{~F}$ NMR $\left(376 \mathrm{MHz}, \mathrm{CDCl}_{3}\right) \delta-81.0(\mathrm{t}, J=10.1 \mathrm{~Hz}, 3 \mathrm{~F}),-108.7(\mathrm{t}, J=12.4 \mathrm{~Hz}, 2 \mathrm{~F})$, $-123.9(\mathrm{~m}, 2 \mathrm{~F}),-125.8(\mathrm{~m}, 2 \mathrm{~F})$; HRMS: $\mathrm{m} / \mathrm{z}(\mathrm{ESI})$ calculated $[\mathrm{M}+\mathrm{Na}]^{+}: 565.0262$, measured: 565.0247.

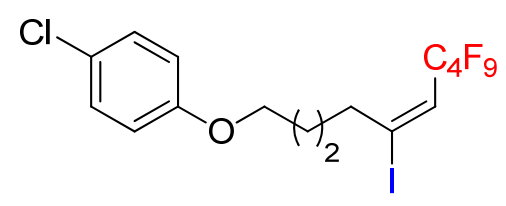

${ }^{1} \mathrm{H}$ NMR (400 MHz, $\left.\mathrm{CDCl}_{3}\right) \delta$ 7.28-7.19 (m, 2H), 6.87-6.77 (m, 2H), $6.36(\mathrm{t}, J=14.0$ $\mathrm{Hz}, 1 \mathrm{H}), 4.00-3.89(\mathrm{~m}, 2 \mathrm{H}), 2.78-2.64(\mathrm{~m}, 2 \mathrm{H}), 1.87-1.70(\mathrm{~m}, 4 \mathrm{H}) ;{ }^{13} \mathrm{C}$ NMR $(100$ $\left.\mathrm{MHz}, \mathrm{CDCl}_{3}\right) \delta 157.5,129.3,126.9(\mathrm{t}, J=23.5 \mathrm{~Hz}), 125.5,122.2(\mathrm{t}, J=6.4 \mathrm{~Hz})$, 115.7, 120-100 (m), 67.6, 40.6, 27.8, 26.6; $\left.{ }^{19} \mathrm{~F} \mathrm{NMR} \mathrm{(376} \mathrm{MHz,} \mathrm{CDCl}_{3}\right) \delta-81.0(\mathrm{t}, J$ $=9.4 \mathrm{~Hz}, 3 \mathrm{~F}),-105.5(\mathrm{t}, J=12.4 \mathrm{~Hz}, 2 \mathrm{~F}),-124.2(\mathrm{~m}, 2 \mathrm{~F}),-125.8(\mathrm{~m}, 2 \mathrm{~F}) ; \mathrm{HRMS}: \mathrm{m} / \mathrm{z}$ (APCI) calculated $[\mathrm{M}]^{+}:$553.9556, measured: 553.9550.

$Z$ product: ${ }^{19} \mathrm{~F}$ NMR $\left(376 \mathrm{MHz}, \mathrm{CDCl}_{3}\right) \delta-81.0(\mathrm{t}, J=9.4 \mathrm{~Hz}, 3 \mathrm{~F}),-108.8(\mathrm{t}, J=12.4$ Hz, 2F), -123.8 (m, 2F), -125.8 (m, 2F); LRMS: m/z (EI) 427 (M-I), 299, 128 (100), $127,111$.

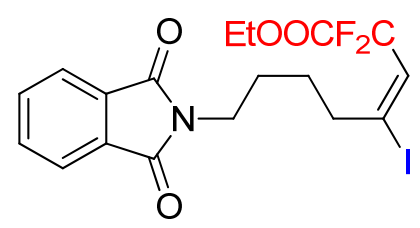

${ }^{1} \mathrm{H}$ NMR (400 MHz, $\left.\mathrm{CDCl}_{3}\right) \delta$ 7.89-7.78 (m, 2H), 7.74-7.65 (m, 2H), $6.38(\mathrm{t}, J=12.8$ $\mathrm{Hz}, 1 \mathrm{H}), 4.28$ (q, $J=7.2 \mathrm{~Hz}, 2 \mathrm{H}), 3.67$ (t, $J=6.4 \mathrm{~Hz}, 2 \mathrm{H}), 2.69-2.56(\mathrm{~m}, 2 \mathrm{H})$, $1.72-1.51(\mathrm{~m}, 4 \mathrm{H}), 1.31(\mathrm{t}, J=7.2 \mathrm{~Hz}, 3 \mathrm{H}) ;{ }^{13} \mathrm{C} \mathrm{NMR}\left(100 \mathrm{MHz}, \mathrm{CDCl}_{3}\right) \delta 168.3$, $163.1(\mathrm{t}, J=34.2 \mathrm{~Hz}), 133.9,132.1,131.8(\mathrm{t}, J=26.8 \mathrm{~Hz}), 123.2,118.4(\mathrm{t}, J=7.4 \mathrm{~Hz})$, $111.5(\mathrm{t}, J=251.0 \mathrm{~Hz}), 63.4,39.9,37.6,27.2,27.1,13.9 ;{ }^{19} \mathrm{~F} \mathrm{NMR}\left(376 \mathrm{MHz}, \mathrm{CDCl}_{3}\right)$ $\delta$-97.7(s); HRMS: m/z (ESI) calculated $[\mathrm{M}+\mathrm{H}]^{+}: 478.0327$, measured: 478.0329.

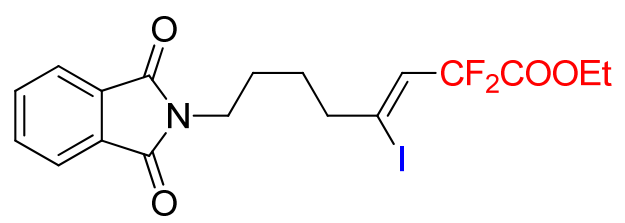

${ }^{1} \mathrm{H}$ NMR $\left(400 \mathrm{MHz}, \mathrm{CDCl}_{3}\right) \delta 8.02-7.80(\mathrm{~m}, 2 \mathrm{H}), 7.78-7.58(\mathrm{~m}, 2 \mathrm{H}), 6.41(\mathrm{t}, J=11.2$ $\mathrm{Hz}, 1 \mathrm{H}), 4.35$ (q, $J=7.2 \mathrm{~Hz}, 2 \mathrm{H}), 3.82-3.63(\mathrm{~m}, 2 \mathrm{H}), 2.76-2.55(\mathrm{~m}, 2 \mathrm{H}), 1.76-1.56(\mathrm{~m}$, $4 \mathrm{H}), 1.36(\mathrm{t}, J=7.2 \mathrm{~Hz}, 3 \mathrm{H}) ;{ }^{13} \mathrm{C} \mathrm{NMR}\left(100 \mathrm{MHz}, \mathrm{CDCl}_{3}\right) \delta 168.3,162.6,134.0$, 132.0, $128.8(\mathrm{t}, J=29.5 \mathrm{~Hz}), 123.2,113.8(\mathrm{t}, J=9.7 \mathrm{~Hz}), 111.9(\mathrm{t}, J=247.0 \mathrm{~Hz}), 63.3$, 45.9, 37.3, 26.9, 26.1, 13.9; ${ }^{19} \mathrm{~F}$ NMR (376 MHz, $\left.\mathrm{CDCl}_{3}\right) \delta-98.0(\mathrm{~s}) ; \mathrm{HRMS}: \mathrm{m} / \mathrm{z}$ (ESI) 
calculated $[\mathrm{M}+\mathrm{Na}]^{+}:$500.0146, measured: 500.0086 .<smiles>CSc1ccc(OCCC/C(I)=C\C(F)(F)F)cc1</smiles>

${ }^{1} \mathrm{H}$ NMR (400 MHz, $\left.\mathrm{CDCl}_{3}\right) \delta 7.26(\mathrm{~d}, J=8.8 \mathrm{~Hz}, 2 \mathrm{H}), 6.83(\mathrm{~d}, J=8.8 \mathrm{~Hz}, 2 \mathrm{H}), 6.38$ (t, $J=14.0 \mathrm{~Hz}, 1 \mathrm{H}), 3.97(\mathrm{t}, J=6.0 \mathrm{~Hz}, 2 \mathrm{H}), 2.86(\mathrm{t}, J=7.2 \mathrm{~Hz}, 2 \mathrm{H}), 2.45(\mathrm{~s}, 3 \mathrm{H})$, 2.12-1.99 (m, 2H); ${ }^{13} \mathrm{C}$ NMR $\left(100 \mathrm{MHz}, \mathrm{CDCl}_{3}\right) \delta 157.2,130.0,129.0,127.2(\mathrm{t}, \mathrm{J}=$ $24.0 \mathrm{~Hz}), 121.2,115.1,120-100(\mathrm{~m}), 66.1,38.0,29.7,18.0 ;{ }^{19} \mathrm{~F}$ NMR (376 MHz, $\left.\mathrm{CDCl}_{3}\right) \delta-81.0(\mathrm{t}, J=9.8 \mathrm{~Hz}, 3 \mathrm{~F}),-105.8(\mathrm{t}, J=13.2 \mathrm{~Hz}, 2 \mathrm{~F}),-124.1(\mathrm{~m}, 2 \mathrm{~F}),-125.8$ (m, 2F); LRMS: m/z (EI) 425 (M-I), 413, 285, 140 (100), 125, 117, 97, 77.

$Z$ product: ${ }^{19} \mathrm{~F}$ NMR $\left(376 \mathrm{MHz}, \mathrm{CDCl}_{3}\right) \delta-81.0(\mathrm{t}, J=9.4 \mathrm{~Hz}, 3 \mathrm{~F}),-108.9(\mathrm{t}, J=12.4$ $\mathrm{Hz}, 2 \mathrm{~F}),-123.8(\mathrm{~m}, 2 \mathrm{~F}),-125.8(\mathrm{~m}, 2 \mathrm{~F})$; HRMS: $\mathrm{m} / \mathrm{z}(\mathrm{ESI})$ calculated $[\mathrm{M}+\mathrm{K}]^{+}$: 590.9303, measured: 590.9391.

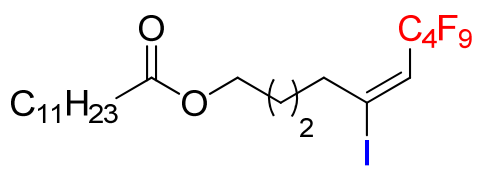

${ }^{1} \mathrm{H}$ NMR $\left(400 \mathrm{MHz}, \mathrm{CDCl}_{3}\right) \delta 6.36(\mathrm{t}, J=14.4 \mathrm{~Hz}, 1 \mathrm{H}), 4.17-4.02(\mathrm{~m}, 2 \mathrm{H}), 2.77-2.59$ $(\mathrm{m}, 2 \mathrm{H}), 2.37-2.25(\mathrm{~m}, 2 \mathrm{H}), 1.73-1.58(\mathrm{~m}, 6 \mathrm{H}), 1.34-1.20(\mathrm{~m}, 16 \mathrm{H}), 0.92-0.83(\mathrm{~m}$, $3 \mathrm{H}) ;{ }^{13} \mathrm{C} \mathrm{NMR}\left(100 \mathrm{MHz}, \mathrm{CDCl}_{3}\right) \delta 173.8,126.9(\mathrm{t}, J=23.5 \mathrm{~Hz}), 121.9,120-100(\mathrm{~m})$, 63.5, 40.5, 34.3, 31.9, 29.6, 29.5, 29.4, 29.3, 29.2, 29.1, 27.3, 26.5, 25.0, 22.6, 14.0; ${ }^{19} \mathrm{~F}$ NMR (376 MHz, $\left.\mathrm{CDCl}_{3}\right) \delta-81.0(\mathrm{~m}, 3 \mathrm{~F}),-105.6(\mathrm{~m}, 2 \mathrm{~F}),-124.2(\mathrm{~m}, 2 \mathrm{~F}),-125.8$ (m, 2F); HRMS: m/z (ESI) calculated $[\mathrm{M}+\mathrm{H}]^{+}: 627.1382$, measured: 627.1381.

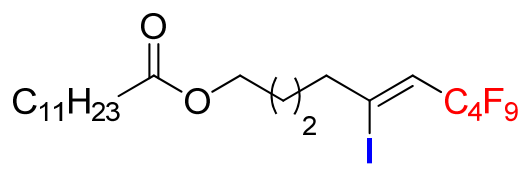

${ }^{1} \mathrm{H}$ NMR (400 MHz, CDCl 3$) \delta 6.27(\mathrm{t}, J=13.2 \mathrm{~Hz}, 1 \mathrm{H}), 4.09(\mathrm{t}, J=6.0 \mathrm{~Hz}, 2 \mathrm{H})$, 2.77-2.66 (m, 2H), $2.30(\mathrm{t}, J=7.6 \mathrm{~Hz}, 2 \mathrm{H}), 1.70-1.57(\mathrm{~m}, 6 \mathrm{H}), 1.32-1.21(\mathrm{~m}, 16 \mathrm{H})$, $0.88(\mathrm{t}, J=6.8 \mathrm{~Hz}, 3 \mathrm{H}) ;{ }^{13} \mathrm{C} \mathrm{NMR}\left(100 \mathrm{MHz}, \mathrm{CDCl}_{3}\right) \delta 173.9,122.1(\mathrm{t}, J=23.5 \mathrm{~Hz})$, 115.9, 120-100 (m), 63.5, 47.8, 34.3, 31.9, 29.7, 29.6, 29.4, 29.3, 29.2, 29.1, 27.0, 25.6, 25.0, 22.7, 14.1; ${ }^{19} \mathrm{~F}$ NMR (376 MHz, $\left.\mathrm{CDCl}_{3}\right) \delta-81.0(\mathrm{~m}, 3 \mathrm{~F}),-108.8(\mathrm{~m}, 2 \mathrm{~F})$, -123.8 (m, 2F), -125.8 (m, 2F); HRMS: m/z (ESI) calculated [M+Na] ${ }^{+}: 649.1201$, measured: 649.1210 .<smiles>CCOC(=O)C(F)=CC(I)=CCCOc1ccc(Cl)cc1</smiles> 
${ }^{1} \mathrm{H}$ NMR (400 MHz, $\left.\mathrm{CDCl}_{3}\right) \delta 7.22(\mathrm{~d}, J=8.8 \mathrm{~Hz}, 2 \mathrm{H}), 6.82(\mathrm{~d}, J=8.8 \mathrm{~Hz}, 2 \mathrm{H}), 6.44$ (t, $J=12.8 \mathrm{~Hz}, 1 \mathrm{H}), 4.33$ (q, $J=7.2 \mathrm{~Hz}, 2 \mathrm{H}), 3.97-3.89$ (m, 2H), 2.69 (t, $J=6.4 \mathrm{~Hz}$, $2 \mathrm{H}), 1.83-1.67(\mathrm{~m}, 4 \mathrm{H}), 1.35(\mathrm{t}, J=7.2 \mathrm{~Hz}, 3 \mathrm{H}) ;{ }^{13} \mathrm{C} \mathrm{NMR}\left(100 \mathrm{MHz}, \mathrm{CDCl}_{3}\right)$ $\delta 157.5,131.7(\mathrm{t}, J=26.8 \mathrm{~Hz}), 129.2,125.4,118.7,115.7,115.6,111.5(\mathrm{t}, J=255.0$ $\mathrm{Hz}), 67.6,63.4,40.2,27.7,26.4,13.9 ;{ }^{19} \mathrm{~F} \mathrm{NMR}\left(376 \mathrm{MHz}, \mathrm{CDCl}_{3}\right) \delta-97.7$ (s); HRMS: m/z (ESI) calculated $[\mathrm{M}+\mathrm{H}]^{+}:$480.9855, measured: 480.9862.<smiles>CCOC(=O)[C-]C=C(I)CCCOc1ccc(Cl)cc1</smiles>

${ }^{1} \mathrm{H}$ NMR (400 MHz, $\left.\mathrm{CDCl}_{3}\right)$ 8 7.29-7.14 (m, 2H), 6.87-6.70 (m, 2H), $6.42(\mathrm{t}, J=11.6$ $\mathrm{Hz}, 1 \mathrm{H}), 4.42-4.24(\mathrm{~m}, 2 \mathrm{H}), 3.99-3.83(\mathrm{~m}, 2 \mathrm{H}), 2.72-2.56(\mathrm{~m}, 2 \mathrm{H}), 1.83-1.67(\mathrm{~m}, 4 \mathrm{H})$, $1.42-1.30(\mathrm{~m}, 3 \mathrm{H}) ;{ }^{13} \mathrm{C} \mathrm{NMR}\left(100 \mathrm{MHz}, \mathrm{CDCl}_{3}\right) \delta 157.4,129.3,128.7(\mathrm{t}, J=28.7 \mathrm{~Hz})$, 125.5, 115.7, 114.4, 114,1 (t, $J=9.4 \mathrm{~Hz}), 112.0(\mathrm{t}, J=246.4 \mathrm{~Hz}), 67.6,63.3,46.3$, 27.5, 25.6, 13.9; ${ }^{19} \mathrm{~F}$ NMR (376 MHz, $\left.\mathrm{CDCl}_{3}\right) \delta-98.0$ (s); HRMS: m/z (ESI) calculated $[\mathrm{M}+\mathrm{Na}]^{+}: 480.9855$, measured: 480.9850 .<smiles>CCC(=O)c1ccc(OCCC(I)=CC(F)(F)F)cc1</smiles>

${ }^{1} \mathrm{H}$ NMR (400 MHz, $\left.\mathrm{CDCl}_{3}\right) \delta 7.94(\mathrm{~d}, J=8.8 \mathrm{~Hz}, 2 \mathrm{H}), 6.92(\mathrm{~d}, J=8.8 \mathrm{~Hz}, 2 \mathrm{H}), 6.37$ (t, $J=14.4 \mathrm{~Hz}, 1 \mathrm{H}), 4.05(\mathrm{t}, J=6.0 \mathrm{~Hz}, 2 \mathrm{H}), 2.96$ (q, $J=7.2 \mathrm{~Hz}, 2 \mathrm{H}), 2.79-2.69$ (m, $2 \mathrm{H}), 1.93-1.74(\mathrm{~m}, 4 \mathrm{H}), 1.21(\mathrm{t}, J=7.2 \mathrm{~Hz}, 3 \mathrm{H}) ;{ }^{13} \mathrm{C} \mathrm{NMR}\left(100 \mathrm{MHz}, \mathrm{CDCl}_{3}\right)$ $\delta 199.3,162.6,130.1,129.9,126.8(\mathrm{t}, J=23.5 \mathrm{~Hz}), 122.0,114.0,120-100(\mathrm{~m}), 67.4$, 40.5, 31.3, 27.7, 26.5, 8.3; ${ }^{19} \mathrm{~F}$ NMR $\left(376 \mathrm{MHz}, \mathrm{CDCl}_{3}\right) \delta-81.2(\mathrm{~m}, 3 \mathrm{~F}),-105.5(\mathrm{t}, J=$ $11.7 \mathrm{~Hz}, 2 \mathrm{~F}),-124.2(\mathrm{~m}, 2 \mathrm{~F}),-125.9(\mathrm{~m}, 2 \mathrm{~F})$; HRMS: m/z (ESI) calculated $[\mathrm{M}+\mathrm{H}]^{+}$: 577.0286, measured: 577.0280.<smiles>CCC(=O)c1ccc(OCC(I)=CC(F)(F)F)cc1</smiles>

${ }^{1} \mathrm{H}$ NMR $\left(400 \mathrm{MHz}, \mathrm{CDCl}_{3}\right) \delta 7.95(\mathrm{~d}, J=8.8 \mathrm{~Hz}, 2 \mathrm{H}), 6.92(\mathrm{~d}, J=8.8 \mathrm{~Hz}, 2 \mathrm{H}), 6.30$ (t, $J=12.8 \mathrm{~Hz}, 1 \mathrm{H}), 4.15-4.00(\mathrm{~m}, 2 \mathrm{H}), 2.96(\mathrm{q}, J=7.2 \mathrm{~Hz}, 2 \mathrm{H}), 2.84-2.71(\mathrm{~m}, 2 \mathrm{H})$, 1.90-1.77 (m, 4H), $\left.1.21(\mathrm{t}, J=7.2 \mathrm{~Hz}, 3 \mathrm{H}) ;{ }^{13} \mathrm{C} \mathrm{NMR} \mathrm{(100} \mathrm{MHz,} \mathrm{CDCl}_{3}\right) \delta 199.5$, $162.6,130.2,130.0,122.1(\mathrm{t}, J=23.5 \mathrm{~Hz}), 115.9(\mathrm{t}, J=6.0 \mathrm{~Hz}), 114.1,120-100(\mathrm{~m})$, 67.5, 47.9, 31.4, 27.5, 25.8, 8.4; ${ }^{19} \mathrm{~F}$ NMR $\left(376 \mathrm{MHz} \mathrm{CDCl}_{3}\right) \delta-81.0(\mathrm{t}, J=9.4 \mathrm{~Hz}$, 
3F), -108.8 (t, $J=11.7 \mathrm{~Hz}, 2 \mathrm{~F}),-123.8(\mathrm{~m}, 2 \mathrm{~F}),-125.8(\mathrm{~m}, 2 \mathrm{~F}) ; \mathrm{HRMS}: \mathrm{m} / \mathrm{z}$ (ESI) calculated $[\mathrm{M}+\mathrm{H}]^{+}:$577.0286, measured: 577.0223 .<smiles>O=C(OCCNC(I)=CC(F)(F)F)c1ccc(Cl)cc1</smiles>

${ }^{1} \mathrm{H}$ NMR (400 MHz, $\left.\mathrm{CDCl}_{3}\right) \delta 7.96(\mathrm{~d}, J=8.8 \mathrm{~Hz}, 2 \mathrm{H}), 7.42(\mathrm{~d}, J=8.8 \mathrm{~Hz}, 2 \mathrm{H}), 6.39$ $(\mathrm{t}, J=14.4 \mathrm{~Hz}, 1 \mathrm{H}), 4.36(\mathrm{t}, J=6.0 \mathrm{~Hz}, 2 \mathrm{H}), 2.85(\mathrm{t}, J=7.6 \mathrm{~Hz}, 2 \mathrm{H}), 2.14-2.02(\mathrm{~m}$, $2 \mathrm{H}) ;{ }^{13} \mathrm{C} \mathrm{NMR}\left(100 \mathrm{MHz}, \mathrm{CDCl}_{3}\right) \delta 165.5,139.5,130.9,128.7,128.4,127.3(\mathrm{t}, J=$ $23.8 \mathrm{~Hz}), 120.7(\mathrm{t}, J=6.7 \mathrm{~Hz}), 120-100(\mathrm{~m}), 63.4,38.0,29.2 ;{ }^{19} \mathrm{~F}$ NMR (376 MHz, $\left.\mathrm{CDCl}_{3}\right) \delta-81.1(\mathrm{t}, J=9.4 \mathrm{~Hz}, 3 \mathrm{~F}),-105.8(\mathrm{t}, J=14.3 \mathrm{~Hz}, 2 \mathrm{~F}),-124.1(\mathrm{~m}, 2 \mathrm{~F}),-125.9$ (m, 2F); HRMS: m/z (ESI) calculated [M+Na] $:$ 590.9246, measured: 590.9293.

$Z$ product: ${ }^{19} \mathrm{~F}$ NMR $\left(376 \mathrm{MHz}, \mathrm{CDCl}_{3}\right) \delta-81.0(\mathrm{t}, J=9.4 \mathrm{~Hz}, 3 \mathrm{~F}),-108.9(\mathrm{t}, J=12.4$ $\mathrm{Hz}, 2 \mathrm{~F}),-123.8$ (m, 2F), -125.8 (m, 2F); LRMS: m/z (EI) 441 (M-I), 285, 157, 139 (100), 127, 111.<smiles>CCOC(=O)C(F)(F)/C=C(/I)CCCOc1ccc(SC)cc1</smiles>

${ }^{1} \mathrm{H}$ NMR $\left(400 \mathrm{MHz}, \mathrm{CDCl}_{3}\right) \delta 7.26(\mathrm{~d}, J=8.0 \mathrm{~Hz}, 2 \mathrm{H}), 6.84(\mathrm{~d}, J=8.0 \mathrm{~Hz}, 2 \mathrm{H}), 6.46$ $(\mathrm{t}, J=13.2 \mathrm{~Hz}, 1 \mathrm{H}), 4.30(\mathrm{q}, J=7.2 \mathrm{~Hz}, 2 \mathrm{H}), 3.96(\mathrm{t}, J=6.4 \mathrm{~Hz}, 2 \mathrm{H}), 2.83(\mathrm{t}, J=7.2$ $\mathrm{Hz}, 2 \mathrm{H}), 2.44(\mathrm{~s}, 3 \mathrm{H}), 2.10-1.98(\mathrm{~m}, 2 \mathrm{H}), 1.34(\mathrm{t}, J=7.2 \mathrm{~Hz}, 3 \mathrm{H}) ;{ }^{13} \mathrm{C}$ NMR $(100$ $\left.\mathrm{MHz}, \mathrm{CDCl}_{3}\right) \delta 157.2,131.9(\mathrm{t}, J=26.8 \mathrm{~Hz}), 129.9,128.7,117.7(\mathrm{t}, J=7.4 \mathrm{~Hz})$, 115.1, 114.0, $111.4(\mathrm{t}, J=252.3 \mathrm{~Hz}), 66.2,63.4,37.5,29.4,17.9,13.8 ;{ }^{19} \mathrm{~F}$ NMR (376 $\left.\mathrm{MHz}, \mathrm{CDCl}_{3}\right) \delta-97.7$ (s); HRMS: m/z (ESI) calculated [M+Na] $]^{+}$478.9966, measured: 478.9955 .<smiles>CCOC(=O)C(F)(F)C=C(I)CCCOc1ccc(SC)cc1</smiles>

${ }^{1} \mathrm{H}$ NMR (400 MHz, $\left.\mathrm{CDCl}_{3}\right) \delta 7.26(\mathrm{~d}, J=8.8 \mathrm{~Hz}, 2 \mathrm{H}), 6.84(\mathrm{~d}, J=8.8 \mathrm{~Hz}, 2 \mathrm{H}), 6.44$ (t, $J=11.6 \mathrm{~Hz}, 1 \mathrm{H}), 4.34(\mathrm{q}, J=7.2 \mathrm{~Hz}, 2 \mathrm{H}), 3.94(\mathrm{t}, J=6.0 \mathrm{~Hz}, 2 \mathrm{H}), 2.81(\mathrm{t}, J=6.4$ $\mathrm{Hz}, 2 \mathrm{H}), 2.44(\mathrm{~s}, 3 \mathrm{H}), 2.11-1.97(\mathrm{~m}, 2 \mathrm{H}), 1.35(\mathrm{t}, J=7.2 \mathrm{~Hz}, 3 \mathrm{H}) ;{ }^{13} \mathrm{C}$ NMR $(100$ $\left.\mathrm{MHz}, \mathrm{CDCl}_{3}\right) \delta 157.2,130.0,129.1(\mathrm{t}, J=29.5 \mathrm{~Hz}), 129.0,116.4,115.1,113.1,111.9$ $(\mathrm{t}, J=245.4 \mathrm{~Hz}), 65.6,63.3,43.2,28.5,17.9,13.9 ;{ }^{19} \mathrm{~F} \mathrm{NMR}\left(376 \mathrm{MHz}, \mathrm{CDCl}_{3}\right) \delta$ -98.1(s); HRMS: m/z (ESI) calculated [M+Na] $]^{+}$478.9966, measured: 478.9972. 
<smiles>N#Cc1ccc(C(=O)OCC/C(I)=C\C(F)(F)F)cc1</smiles>

${ }^{1} \mathrm{H}$ NMR (400 MHz, $\left.\mathrm{CDCl}_{3}\right) \delta 8.13(\mathrm{~d}, J=8.0 \mathrm{~Hz}, 2 \mathrm{H}), 7.75(\mathrm{~d}, J=8.0 \mathrm{~Hz}, 2 \mathrm{H}), 6.54$ $(\mathrm{t}, J=14.0 \mathrm{~Hz}, 1 \mathrm{H}), 4.58(\mathrm{t}, J=6.0 \mathrm{~Hz}, 2 \mathrm{H}), 3.17(\mathrm{~d}, J=6.0 \mathrm{~Hz}, 2 \mathrm{H}) ;{ }^{13} \mathrm{C} \mathrm{NMR}(100$ $\left.\mathrm{MHz}, \mathrm{CDCl}_{3}\right) \delta 164.5,133.4,132.2,130.1,129.3(\mathrm{t}, J=23.5 \mathrm{~Hz}), 117.8,116.6,115.7$ $(\mathrm{t}, J=5.6 \mathrm{~Hz}), 120-100(\mathrm{~m}), 63.8,39.7 ;{ }^{19} \mathrm{~F} \mathrm{NMR}\left(376 \mathrm{MHz}, \mathrm{CDCl}_{3}\right) \delta-81.1(\mathrm{t}, J=$ $9.4 \mathrm{~Hz}, 3 \mathrm{~F}),-105.5(\mathrm{t}, J=12.4 \mathrm{~Hz}, 2 \mathrm{~F}),-124.1(\mathrm{~m}, 2 \mathrm{~F}),-125.8(\mathrm{~m}, 2 \mathrm{~F}) ; \mathrm{HRMS}: \mathrm{m} / \mathrm{z}$ (ESI) calculated $[\mathrm{M}+\mathrm{Na}]^{+}: 567.9432$, measured: 567.9432 .

$Z$ product: ${ }^{19} \mathrm{~F}$ NMR $\left(376 \mathrm{MHz}, \mathrm{CDCl}_{3}\right) \delta-81.0(\mathrm{t}, J=9.4 \mathrm{~Hz}, 3 \mathrm{~F}),-109.4(\mathrm{t}, J=12.4$ Hz, 2F), -123.8 (m, 2F), -125.8 (m, 2F); LRMS: m/z (EI) 418 (M-I), 130 (100), 127 , $102,69$.

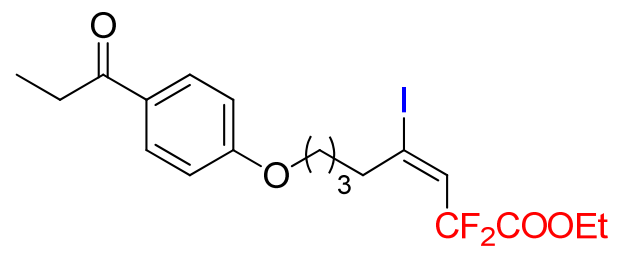

${ }^{1} \mathrm{H}$ NMR (400 MHz, CDCl 3$) \delta 7.94(\mathrm{~d}, J=8.4 \mathrm{~Hz}, 2 \mathrm{H}), 6.92(\mathrm{~d}, J=8.4 \mathrm{~Hz}, 2 \mathrm{H}), 6.45$ (t, $J=13.2 \mathrm{~Hz}, 1 \mathrm{H}), 4.33(\mathrm{q}, J=7.2 \mathrm{~Hz}, 2 \mathrm{H}), 4.04(\mathrm{t}, J=6.0 \mathrm{~Hz}, 2 \mathrm{H}), 2.95$ (q, $J=7.2$ $\mathrm{Hz}, 2 \mathrm{H}), 2.71(\mathrm{t}, J=6.4 \mathrm{~Hz}, 2 \mathrm{H}), 1.89-1.70(\mathrm{~m}, 4 \mathrm{H}), 1.35(\mathrm{t}, J=7.2 \mathrm{~Hz}, 3 \mathrm{H}), 1.21(\mathrm{t}$, $J=7.2 \mathrm{~Hz}, 3 \mathrm{H}) ;{ }^{13} \mathrm{C} \mathrm{NMR}\left(100 \mathrm{MHz}, \mathrm{CDCl}_{3}\right) \delta 199.2,163.0(\mathrm{t}, J=34.2 \mathrm{~Hz}), 162.6$, $131.7(\mathrm{t}, J=26.8 \mathrm{~Hz}), 130.0,129.8,118.5(\mathrm{t}, J=7.8 \mathrm{~Hz}), 114.0,111.4(\mathrm{t}, J=251.0 \mathrm{~Hz})$, 67.4, 63.3, 40.0, 31.2, 27.6, 26.2, 13.8, 8.3; ${ }^{19} \mathrm{~F}$ NMR (376 MHz, $\left.\mathrm{CDCl}_{3}\right) \delta-97.6(\mathrm{~s})$; HRMS: m/z (ESI) calculated $[\mathrm{M}+\mathrm{H}]^{+}:$481.0688, measured: 481.0696.

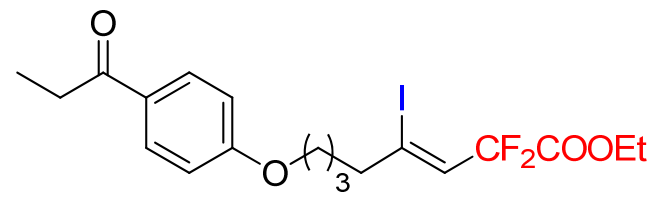

${ }^{1} \mathrm{H}$ NMR (400 MHz, CDCl 3$) \delta 7.95(\mathrm{~d}, J=8.4 \mathrm{~Hz}, 2 \mathrm{H}), 6.92(\mathrm{~d}, J=8.4 \mathrm{~Hz}, 2 \mathrm{H}), 6.43$ (t, $J=11.6 \mathrm{~Hz}, 1 \mathrm{H}), 4.36$ (q, $J=7.2 \mathrm{~Hz}, 2 \mathrm{H}), 4.10-4.00(\mathrm{~m}, 2 \mathrm{H}), 2.96$ (q, $J=7.2 \mathrm{~Hz}$, $2 \mathrm{H}), 2.74-2.64(\mathrm{~m}, 2 \mathrm{H}), 1.87-1.72(\mathrm{~m}, 4 \mathrm{H}), 1.37(\mathrm{t}, J=7.2 \mathrm{~Hz}, 3 \mathrm{H}), 1.22(\mathrm{t}, J=7.2$ $\mathrm{Hz}, 3 \mathrm{H}) ;{ }^{13} \mathrm{C} \mathrm{NMR}\left(100 \mathrm{MHz}, \mathrm{CDCl}_{3}\right) \delta 199.4,162.6,162.5(\mathrm{t}, J=34.2 \mathrm{~Hz}), 130.2$, 130.0, 128.7 (t, $J=28.9 \mathrm{~Hz}), 114.1,114.0,112.0(\mathrm{t}, J=246.3 \mathrm{~Hz}), 67.5,63.3,46.2$, $31.4,27.4,25.5,13.9,8.4 ;{ }^{19} \mathrm{~F}$ NMR $\left(376 \mathrm{MHz}, \mathrm{CDCl}_{3}\right) \delta-98.0$ (s); HRMS: m/z (ESI) calculated $[\mathrm{M}+\mathrm{H}]^{+}:$481.0688, measured: 481.0699 . 


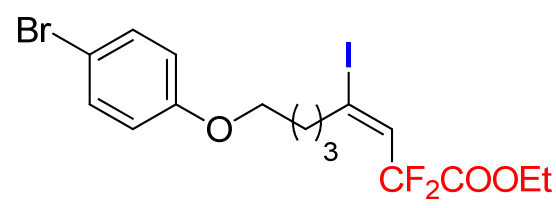

${ }^{1} \mathrm{H}$ NMR (400 MHz, $\left.\mathrm{CDCl}_{3}\right) \delta 7.36(\mathrm{~d}, J=8.8 \mathrm{~Hz}, 2 \mathrm{H}), 6.78(\mathrm{~d}, J=8.8 \mathrm{~Hz}, 2 \mathrm{H}), 6.44$ (t, $J=13.2 \mathrm{~Hz}, 1 \mathrm{H}), 4.33$ (q, $J=7.2 \mathrm{~Hz}, 2 \mathrm{H}), 3.93$ (t, $J=6.4 \mathrm{~Hz}, 2 \mathrm{H}), 2.69$ (q, $J=6.4$ $\mathrm{Hz}, 2 \mathrm{H}), 1.85-1.66(\mathrm{~m}, 4 \mathrm{H}), 1.35(\mathrm{t}, J=7.2 \mathrm{~Hz}, 3 \mathrm{H}), 1.21(\mathrm{t}, J=7.2 \mathrm{~Hz}, 3 \mathrm{H}) ;{ }^{13} \mathrm{C}$ NMR $\left(100 \mathrm{MHz}, \mathrm{CDCl}_{3}\right) \delta 163.0(\mathrm{t}, J=33.8 \mathrm{~Hz}), 157.9,132.1,131.7(\mathrm{t}, J=26.8 \mathrm{~Hz})$, $118.7(\mathrm{t}, J=7.4 \mathrm{~Hz}), 116.2,112.6,111.4(\mathrm{t}, J=250.5 \mathrm{~Hz}), 67.5,63.4,40.1,27.7,26.3$, 13.8; ${ }^{19} \mathrm{~F}$ NMR (376 MHz, $\mathrm{CDCl}_{3}$ ) $\delta$-97.6 (s); HRMS: m/z (ESI) calculated $[\mathrm{M}+\mathrm{Na}]^{+}$: 524.9350, measured: 524.9343 .

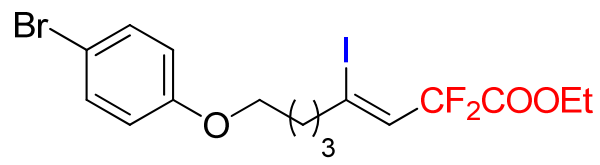

${ }^{1} \mathrm{H}$ NMR $\left(400 \mathrm{MHz}, \mathrm{CDCl}_{3}\right) \delta 7.37(\mathrm{~d}, J=8.4 \mathrm{~Hz}, 2 \mathrm{H}), 6.77(\mathrm{~d}, J=8.4 \mathrm{~Hz}, 2 \mathrm{H}), 6.43$ (t, $J=11.2 \mathrm{~Hz}, 1 \mathrm{H}), 4.36$ (q, $J=7.2 \mathrm{~Hz}, 2 \mathrm{H}), 3.99-3.86(\mathrm{~m}, 2 \mathrm{H}), 2.75-2.59(\mathrm{~m}, 2 \mathrm{H})$, 1.83-1.69 (m, 4H), $1.37(\mathrm{t}, J=7.2 \mathrm{~Hz}, 3 \mathrm{H}), 1.21(\mathrm{t}, J=7.2 \mathrm{~Hz}, 3 \mathrm{H}) ;{ }^{13} \mathrm{C}$ NMR $(100$ $\left.\mathrm{MHz}, \mathrm{CDCl}_{3}\right) \delta 163.2,157.9,132.2,128.7(\mathrm{t}, J=29.1 \mathrm{~Hz}), 116.2,114.1(\mathrm{t}, J=9.8 \mathrm{~Hz})$, $112.8,112.0$ (t, $J=243.9 \mathrm{~Hz}), 67.5,63.3,46.3,27.5,25.6,13.9 ;{ }^{19} \mathrm{~F}$ NMR $(376 \mathrm{MHz}$, $\left.\mathrm{CDCl}_{3}\right) \delta-98.0(\mathrm{~s}) ; \mathrm{HRMS}: \mathrm{m} / \mathrm{z}(\mathrm{ESI})$ calculated $[\mathrm{M}+\mathrm{Na}]^{+}: 524.9350$, measured: 524.9324 .

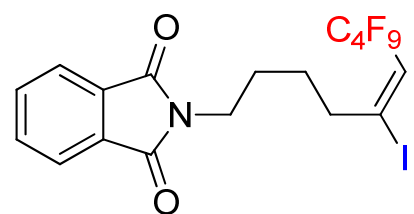

${ }^{1} \mathrm{H}$ NMR (400 MHz, $\left.\mathrm{CDCl}_{3}\right) \delta$ 7.90-7.83 (m, 2H), 7.77-7.70 (m, 2H), $6.34(\mathrm{t}, J=14.4$ $\mathrm{Hz}, 1 \mathrm{H}), 3.72(\mathrm{t}, J=7.2 \mathrm{~Hz}, 2 \mathrm{H}), 2.77-2.63(\mathrm{~m}, 2 \mathrm{H}), 1.80-1.60(\mathrm{~m}, 4 \mathrm{H}) ;{ }^{13} \mathrm{C}$ NMR $\left(100 \mathrm{MHz}, \mathrm{CDCl}_{3}\right) \delta 168.2,133.8,132.0,126.9(\mathrm{t}, J=23.4 \mathrm{~Hz}), 123.1,121.8$, 120-100 (m), 40.2, 37.4, 27.1, 27.0; ${ }^{19} \mathrm{~F}$ NMR $\left(376 \mathrm{MHz}, \mathrm{CDCl}_{3}\right) \delta-81.2(\mathrm{t}, J=9.0 \mathrm{~Hz}$, 3F), $-105.6(\mathrm{t}, J=12.0 \mathrm{~Hz}, 2 \mathrm{~F}),-124.2(\mathrm{~m}, 2 \mathrm{~F}),-125.9$ (m, 2F); HRMS: $\mathrm{m} / \mathrm{z}$ (ESI) calculated $[\mathrm{M}+\mathrm{Na}]^{+}: 595.9745$, measured: 595.9846 .

$Z$ product: ${ }^{19} \mathrm{~F}$ NMR $\left(376 \mathrm{MHz}, \mathrm{CDCl}_{3}\right) \delta-81.0(\mathrm{~m}, 3 \mathrm{~F}),-108.8(\mathrm{~m}, 2 \mathrm{~F}),-123.8(\mathrm{~m}$, $2 \mathrm{~F}),-125.8(\mathrm{~m}, 2 \mathrm{~F}) ; \mathrm{HRMS}: \mathrm{m} / \mathrm{z}(\mathrm{ESI})$ calculated $[\mathrm{M}+\mathrm{Na}]^{+}: 595.9745$, measured: 595.9846 .<smiles>CCCCCCCCCCCC(=O)OCCNCC(I)=CCOC(=O)OCC</smiles> 
${ }^{1} \mathrm{H}$ NMR $\left(400 \mathrm{MHz}, \mathrm{CDCl}_{3}\right) \delta 6.43(\mathrm{t}, J=13.2 \mathrm{~Hz}, 1 \mathrm{H}), 4.34(\mathrm{q}, J=7.2 \mathrm{~Hz}, 2 \mathrm{H})$, 4.16-4.04 (m, 2H), 2.75-2.60 (m, 2H), $2.31(\mathrm{t}, J=7.2 \mathrm{~Hz}, 2 \mathrm{H}), 1.70-1.56(\mathrm{~m}, 6 \mathrm{H})$, $1.42-1.19(\mathrm{~m}, 19 \mathrm{H}), 0.88(\mathrm{t}, J=6.4 \mathrm{~Hz}, 3 \mathrm{H}) ;{ }^{13} \mathrm{C} \mathrm{NMR}\left(100 \mathrm{MHz}, \mathrm{CDCl}_{3}\right) \delta 173.7$, 163.0, 131.7 (t, $J=26.9 \mathrm{~Hz}), 118.5$ (t, $J=7.3 \mathrm{~Hz}), 111.4$ (t, $J=251.0 \mathrm{~Hz}), 63.6,63.3$, 40.1, 34.2, 31.8, 29.6, 29.5, 29.4, 29.3, 29.2, 29.1, 27.2, 26.2, 24.9, 22.6, 14.0, 13.8; ${ }^{19} \mathrm{~F}$ NMR $\left(376 \mathrm{MHz}, \mathrm{CDCl}_{3}\right) \delta-97.7(\mathrm{~s})$; HRMS: $\mathrm{m} / \mathrm{z}$ (ESI) calculated $[\mathrm{M}+\mathrm{H}]^{+}$: 531.1783, measured: 531.1777 .

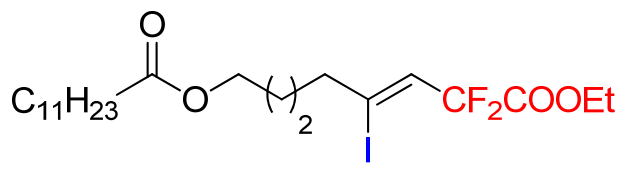

${ }^{1} \mathrm{H}$ NMR $\left(400 \mathrm{MHz}, \mathrm{CDCl}_{3}\right) \delta 6.41(\mathrm{t}, J=11.6 \mathrm{~Hz}, 1 \mathrm{H}), 4.36(\mathrm{q}, J=7.2 \mathrm{~Hz}, 2 \mathrm{H})$, 4.14-4.04 (m, 2H), 2.68-2.59 (m, 2H), $2.30(\mathrm{t}, J=7.2 \mathrm{~Hz}, 2 \mathrm{H}), 1.70-1.57(\mathrm{~m}, 6 \mathrm{H})$, $1.41-1.19(\mathrm{~m}, 19 \mathrm{H}), 0.88(\mathrm{t}, J=6.8 \mathrm{~Hz}, 3 \mathrm{H}) ;{ }^{13} \mathrm{C}$ NMR $\left(100 \mathrm{MHz}, \mathrm{CDCl}_{3}\right) \delta 173.9$, $161.8,128.7$ (t, $J=28.8 \mathrm{~Hz}), 114.0(\mathrm{t}, J=6.2 \mathrm{~Hz}), 112.0$ (t, $J=246.3 \mathrm{~Hz}), 63.5,63.3$, 46.1, 34.3, 31.9, 29.6, 29.5, 29.4, 29.3, 29.2, 29.1, 27.0, 25.4, 25.0, 22.7, 14.1, 13.9; ${ }^{19} \mathrm{~F}$ NMR $\left(376 \mathrm{MHz}, \mathrm{CDCl}_{3}\right) \delta-98.0(\mathrm{~s})$; HRMS: $\mathrm{m} / \mathrm{z}$ (ESI) calculated $[\mathrm{M}+\mathrm{H}]^{+}$: 531.1783, measured: 531.1776 .<smiles>O=C(OCCNCC(I)=CC(F)(F)F)c1ccc(Cl)cc1</smiles>

${ }^{1} \mathrm{H}$ NMR (400 MHz, CDCl 3 ) $\delta 7.97(\mathrm{~d}, J=8.4 \mathrm{~Hz}, 2 \mathrm{H}), 7.41(\mathrm{~d}, J=8.4 \mathrm{~Hz}, 2 \mathrm{H}), 6.35$ (t, $J=14.4 \mathrm{~Hz}, 1 \mathrm{H}), 4.33$ (t, $J=6.4 \mathrm{~Hz}, 2 \mathrm{H}), 2.67(\mathrm{q}, J=7.2 \mathrm{~Hz}, 2 \mathrm{H}), 1.85-1.76(\mathrm{~m}$, $2 \mathrm{H}), 1.72-1.62(\mathrm{~m}, 2 \mathrm{H}), 1.54-1.44(\mathrm{~m}, 2 \mathrm{H}) ;{ }^{13} \mathrm{C}$ NMR $\left(100 \mathrm{MHz}, \mathrm{CDCl}_{3}\right) \delta 165.7$, $139.3,130.9,128.8,128.7,126.9(\mathrm{t}, J=24.2 \mathrm{~Hz}), 122.2,120-100(\mathrm{~m}), 64.9,40.8,29.6$, 28.4, 24.8; ${ }^{19} \mathrm{~F}$ NMR $\left(376 \mathrm{MHz}, \mathrm{CDCl}_{3}\right) \delta-68.0(\mathrm{t}, J=13.9 \mathrm{~Hz}, 2 \mathrm{~F}),-105.3(\mathrm{t}, J=$ $13.5 \mathrm{~Hz}, 2 \mathrm{~F}),-120.1$ (m, 2F), -121.3 (m, 2F), -121.5 (m, 2F), -123.2 (m, 2F); LRMS: $\mathrm{m} / \mathrm{z}(\mathrm{EI})$ 429, 409, 157, 139 (100), 127, 111.<smiles>CC(=CC(F)(F)F)CCCOC(=O)c1ccc(Cl)cc1</smiles>

${ }^{1} \mathrm{H}$ NMR (400 MHz, CDCl 3 ) $\delta 7.97(\mathrm{~d}, J=8.4 \mathrm{~Hz}, 2 \mathrm{H}), 7.41(\mathrm{~d}, J=8.4 \mathrm{~Hz}, 2 \mathrm{H}), 6.26$ (t, $J=13.2 \mathrm{~Hz}, 1 \mathrm{H}), 4.33$ (t, $J=6.4 \mathrm{~Hz}, 2 \mathrm{H}), 2.70(\mathrm{q}, J=7.2 \mathrm{~Hz}, 2 \mathrm{H}), 1.85-1.76(\mathrm{~m}$, 2H), 1.73-1.63 (m, 2H), 1.51-1.41 (m, 2H); ${ }^{13} \mathrm{C}$ NMR (100 MHz, $\left.\mathrm{CDCl}_{3}\right) \delta$ 165.7, 139.4, 130.9, 128.7, 128.6, 122.0 (t, $J=23.5 \mathrm{~Hz}), 116.0$ (t, $J=6.0 \mathrm{~Hz}), 120-100(\mathrm{~m})$, 64.8, 48.1, 28.7, 28.3, 24.5; ${ }^{19} \mathrm{~F}$ NMR $\left(376 \mathrm{MHz}, \mathrm{CDCl}_{3}\right) \delta-68.0(\mathrm{t}, J=13.9 \mathrm{~Hz}, 2 \mathrm{~F})$, $-108.5(\mathrm{t}, J=13.5 \mathrm{~Hz}, 2 \mathrm{~F}),-120.1(\mathrm{~m}, 2 \mathrm{~F}),-121.2(\mathrm{~m}, 2 \mathrm{~F}),-121.4(\mathrm{~m}, 2 \mathrm{~F}),-122.8(\mathrm{~m}$, 
2F); LRMS: m/z (EI) 429, 409, 207, 157, 139 (100), 127, 111.<smiles>CCOC(=O)C(F)(C(=O)OCC)C(C)=C(I)CC</smiles>

${ }^{1} \mathrm{H}$ NMR (400 MHz, $\left.\mathrm{CDCl}_{3}\right)$ 8 4.44-4.26 (m, 2H), 2.76-2.61 (m, 2H), 2.52-2.37 (m, $2 \mathrm{H}), 1.59-1.43(\mathrm{~m}, 4 \mathrm{H}), 1.40-1.25(\mathrm{~m}, 7 \mathrm{H}), 1.01-0.84(\mathrm{~m}, 6 \mathrm{H}) ;{ }^{13} \mathrm{C}$ NMR $(100 \mathrm{MHz}$, $\left.\mathrm{CDCl}_{3}\right) \delta 163.6(\mathrm{t}, J=34.9 \mathrm{~Hz}), 136.5(\mathrm{t}, J=22.5 \mathrm{~Hz}), 119.5(\mathrm{t}, J=6.1 \mathrm{~Hz}), 111.9(\mathrm{t}, J$ $=254.3 \mathrm{~Hz}), 63.1,42.5,39.0,32.6,30.1,22.6,21.7,13.9,13.8,13.7 ;{ }^{19} \mathrm{~F}$ NMR $(376$ $\left.\mathrm{MHz}, \mathrm{CDCl}_{3}\right) \delta-96.6(\mathrm{~s}) ; \mathrm{HRMS}: \mathrm{m} / \mathrm{z}(\mathrm{ESI})$ calculated $[\mathrm{M}+\mathrm{Na}]^{+}: 411.0608$, measured: 411.0595 .

$Z$ product: ${ }^{19} \mathrm{~F}$ NMR $\left(376 \mathrm{MHz}, \mathrm{CDCl}_{3}\right) \delta-98.0$ (s); LRMS: m/z (EI) 261 (M-I), 233, 157, 127 (100), 111, 91, 55.<smiles>FC(F)(F)/C=C(/I)CCOCc1ccc(Br)cc1</smiles>

${ }^{1} \mathrm{H}$ NMR (400 MHz, $\left.\mathrm{CDCl}_{3}\right) \delta 7.37(\mathrm{~d}, J=8.8 \mathrm{~Hz}, 2 \mathrm{H}), 6.78(\mathrm{~d}, J=8.8 \mathrm{~Hz}, 2 \mathrm{H}), 6.44$ (q, $J=7.6 \mathrm{~Hz}, 1 \mathrm{H}), 3.94(\mathrm{t}, J=7.2 \mathrm{~Hz}, 2 \mathrm{H}), 2.69$ (t, $J=7.6 \mathrm{~Hz}, 2 \mathrm{H}), 1.86-1.70$ (m, $4 \mathrm{H}) ;{ }^{13} \mathrm{C}$ NMR $\left(100 \mathrm{MHz}, \mathrm{CDCl}_{3}\right) \delta 158.0,132.2,129.5$ (q, $\left.J=34.2 \mathrm{~Hz}\right), 121.8(\mathrm{q}, J$ $=272.5 \mathrm{~Hz}), 120.1(\mathrm{t}, J=6.0 \mathrm{~Hz}), 116.2,112.8,67.5,40.2,27.7,26.2 ;{ }^{19} \mathrm{~F} \mathrm{NMR}(376$ $\left.\mathrm{MHz}, \mathrm{CDCl}_{3}\right) \delta-57.7$ (s); HRMS: m/z (APCI) calculated $[\mathrm{M}]^{+}:$447.9147, measured: 447.9141.<smiles>O=C(OCCC(I)=CC(F)(F)F)c1ccc(Cl)cc1</smiles>

${ }^{1} \mathrm{H}$ NMR (400 MHz, CDCl 3$) \delta 7.97(\mathrm{~d}, J=8.4 \mathrm{~Hz}, 2 \mathrm{H}), 7.43(\mathrm{~d}, J=8.4 \mathrm{~Hz}, 2 \mathrm{H}), 6.46$ $(\mathrm{q}, J=7.6 \mathrm{~Hz}, 1 \mathrm{H}), 4.35(\mathrm{t}, J=6.0 \mathrm{~Hz}, 2 \mathrm{H}), 2.83(\mathrm{t}, J=7.2 \mathrm{~Hz}, 2 \mathrm{H}), 2.07(\mathrm{tt}, J=7.2$, $6.0 \mathrm{~Hz}, 2 \mathrm{H}) ;{ }^{13} \mathrm{C}$ NMR $\left(100 \mathrm{MHz}, \mathrm{CDCl}_{3}\right) \delta 165.5,139.4,130.9,129.9$ (q, $J=34.2$ $\mathrm{Hz}), 128.7,128.4,121.6(\mathrm{q}, J=272.5 \mathrm{~Hz}), 118.5(\mathrm{q}, J=6.1 \mathrm{~Hz}), 63.3,37.5,28.7 ;{ }^{19} \mathrm{~F}$ NMR (376 MHz, $\left.\mathrm{CDCl}_{3}\right) \delta-57.9(\mathrm{~s})$; HRMS: m/z (APPI) calculated $[\mathrm{M}+\mathrm{H}]^{+}$: 418.9523, measured: 418.9520 .<smiles>O=C(OCCNC(I)=CC(F)(F)F)c1ccc(Cl)cc1</smiles> 
${ }^{1} \mathrm{H}$ NMR (400 MHz, $\left.\mathrm{CDCl}_{3}\right) \delta 7.97(\mathrm{~d}, J=8.0 \mathrm{~Hz}, 2 \mathrm{H}), 7.43(\mathrm{~d}, J=8.0 \mathrm{~Hz}, 2 \mathrm{H}), 6.38$ $(\mathrm{q}, J=6.4 \mathrm{~Hz}, 1 \mathrm{H}), 4.35(\mathrm{t}, J=7.2 \mathrm{~Hz}, 2 \mathrm{H}), 2.79(\mathrm{t}, J=8.4 \mathrm{~Hz}, 2 \mathrm{H}), 2.08(\mathrm{tt}, J=8.4$, $7.2 \mathrm{~Hz}, 2 \mathrm{H}) ;{ }^{13} \mathrm{C}$ NMR $\left(100 \mathrm{MHz}, \mathrm{CDCl}_{3}\right) \delta 165.5,139.6,131.0,128.8,128.4,125.9$ $(\mathrm{q}, J=35.5 \mathrm{~Hz}), 125.7,121.4(\mathrm{q}, J=254.8 \mathrm{~Hz}), 63.2,43.6,29.7 ;{ }^{19} \mathrm{~F}$ NMR $(376 \mathrm{MHz}$, $\left.\mathrm{CDCl}_{3}\right) \delta-60.2$ (s); LRMS: m/z (EI) 291 (M-I), 157, 139 (100), 127, 111, 75.<smiles>O=C1c2ccccc2C(=O)N1CCCC/C(I)=C\C(F)(F)F</smiles>

${ }^{1} \mathrm{H}$ NMR (400 MHz, $\left.\mathrm{CDCl}_{3}\right) \delta$ 7.98-7.80 (m, 2H), 7.78-7.62 (m, 2H), 6.41 (q, J= 7.2 $\mathrm{Hz}, 1 \mathrm{H}), 3.82-3.56(\mathrm{~m}, 2 \mathrm{H}), 2.75-2.52(\mathrm{~m}, 2 \mathrm{H}), 1.82-1.47(\mathrm{~m}, 4 \mathrm{H}) ;{ }^{13} \mathrm{C}$ NMR $(100$ $\left.\mathrm{MHz}, \mathrm{CDCl}_{3}\right) \delta 168.3,133.9,132.0,129.5(\mathrm{q}, J=34.2 \mathrm{~Hz}), 123.2,121.6(\mathrm{q}, J=247.7$ $\mathrm{Hz}), 119.8$ (q, $J=6.0 \mathrm{~Hz}), 39.8,37.4,27.1,26.8 ;{ }^{19} \mathrm{~F} \mathrm{NMR}\left(376 \mathrm{MHz}, \mathrm{CDCl}_{3}\right) \delta-57.7$ (s); HRMS: $\mathrm{m} / \mathrm{z}$ (ESI) calculated $[\mathrm{M}+\mathrm{H}]^{+}:$424.0021, measured: 424.0022.<smiles>O=C1c2ccccc2C(=O)N1CCCC/C(I)=C/C(F)(F)F</smiles>

${ }^{1} \mathrm{H}$ NMR (400 MHz, $\left.\mathrm{CDCl}_{3}\right) \delta$ 7.92-7.81 (m, 2H), 7.78-7.68 (m, 2H), 6.34 (q, J=7.2 $\mathrm{Hz}, 1 \mathrm{H}), 3.72(\mathrm{t}, J=6.4 \mathrm{~Hz}, 2 \mathrm{H}), 2.66(\mathrm{t}, J=6.4 \mathrm{~Hz}, 2 \mathrm{H}), 1.75-1.57(\mathrm{~m}, 4 \mathrm{H}) ;{ }^{13} \mathrm{C}$ $\operatorname{NMR}\left(100 \mathrm{MHz}, \mathrm{CDCl}_{3}\right) \delta 168.4,134.0,132.0,125.4(\mathrm{q}, J=35.6 \mathrm{~Hz}), 123.3,121.2(\mathrm{q}$, $J=269.1 \mathrm{~Hz}), 115.0(\mathrm{q}, J=6.0 \mathrm{~Hz}), 46.0,37.3,27.0,26.1 ;{ }^{19} \mathrm{~F}$ NMR $(376 \mathrm{MHz}$, $\left.\mathrm{CDCl}_{3}\right) \delta-60.1(\mathrm{~s})$; HRMS: $\mathrm{m} / \mathrm{z}$ (ESI) calculated $[\mathrm{M}+\mathrm{H}]^{+}:$424.0021, measured: 424.0027.<smiles>FC(F)(F)/C=C(/I)CNCCOC1CCCCO1</smiles>

${ }^{1} \mathrm{H}$ NMR $\left(400 \mathrm{MHz}, \mathrm{CDCl}_{3}\right) \delta 6.39(\mathrm{q}, J=7.6 \mathrm{~Hz}, 1 \mathrm{H}), 4.59(\mathrm{dd}, J=4.4,2.4 \mathrm{~Hz}, 1 \mathrm{H})$, 3.93-3.82 (m, 1H), 3.75 (dt, $J=9.6,6.4 \mathrm{~Hz}, 1 \mathrm{H}), 3.56-3.46(\mathrm{~m}, 1 \mathrm{H}), 3.39$ (dt, $J=9.6$, $6.4 \mathrm{~Hz}, 1 \mathrm{H}), 2.62(\mathrm{t}, J=7.2 \mathrm{~Hz}, 2 \mathrm{H}), 1.88-1.78(\mathrm{~m}, 1 \mathrm{H}), 1.77-1.68(\mathrm{~m}, 1 \mathrm{H}), 1.66-1.50$ $(\mathrm{m}, 8 \mathrm{H}), 1.46-1.37(\mathrm{~m}, 2 \mathrm{H}) ;{ }^{13} \mathrm{C} \mathrm{NMR}\left(100 \mathrm{MHz}, \mathrm{CDCl}_{3}\right) \delta 129.0(\mathrm{q}, J=34.3 \mathrm{~Hz})$, $121.8(\mathrm{q}, J=272.5 \mathrm{~Hz}), 120.6(\mathrm{q}, J=6.0 \mathrm{~Hz}), 98.8,67.2,62.2,40.7,30.7,29.4,29.3$, 25.4, 25.1, 19.6; ${ }^{19} \mathrm{~F}$ NMR (376 MHz, $\left.\mathrm{CDCl}_{3}\right) \delta-57.8(\mathrm{~s}) ; \mathrm{HRMS}: \mathrm{m} / \mathrm{z}$ (ESI) calculated $[\mathrm{M}+\mathrm{Na}]^{+}:$415.0358, measured: 415.0353 . 
<smiles>FC(F)(F)/C=C(/I)CCCOC1CCCCO1</smiles>

${ }^{1} \mathrm{H}$ NMR $\left(400 \mathrm{MHz}, \mathrm{CDCl}_{3}\right) \delta 6.30(\mathrm{q}, J=7.2 \mathrm{~Hz}, 1 \mathrm{H}), 4.58(\mathrm{dd}, J=4.4,2.0 \mathrm{~Hz}, 1 \mathrm{H})$, 3.91-3.82 (m, 1H), $3.75(\mathrm{dt}, J=9.6,6.8 \mathrm{~Hz}, 1 \mathrm{H}), 3.56-3.46(\mathrm{~m}, 1 \mathrm{H}), 3.39$ (dt, $J=9.6$, $6.4 \mathrm{~Hz}, 1 \mathrm{H}), 2.62(\mathrm{t}, J=7.2 \mathrm{~Hz}, 2 \mathrm{H}), 1.88-1.77(\mathrm{~m}, 1 \mathrm{H}), 1.76-1.68(\mathrm{~m}, 1 \mathrm{H}), 1.66-1.49$ $(\mathrm{m}, 8 \mathrm{H}), 1.43-1.36(\mathrm{~m}, 2 \mathrm{H}) ;{ }^{13} \mathrm{C}$ NMR $\left(100 \mathrm{MHz}, \mathrm{CDCl}_{3}\right) \delta 124.9(\mathrm{q}, J=35.6 \mathrm{~Hz})$, 121.4 (q, $J=269.1 \mathrm{~Hz}), 115.9,98.9,67.2,62.4,46.8,30.7,29.3,28.8,25.4,24.8,19.7$; ${ }^{19} \mathrm{~F}$ NMR $\left(376 \mathrm{MHz}, \mathrm{CDCl}_{3}\right) \delta-60.0(\mathrm{~s})$; HRMS: m/z (ESI) calculated $[\mathrm{M}+\mathrm{Na}]^{+}$: 415.0358, measured: 415.0349 .<smiles>N#Cc1ccc(C(=O)OCC/C(I)=C\C(F)(F)F)cc1</smiles>

${ }^{1} \mathrm{H}$ NMR (400 MHz, $\left.\mathrm{CDCl}_{3}\right) \delta 8.13(\mathrm{~d}, J=8.0 \mathrm{~Hz}, 2 \mathrm{H}), 7.76(\mathrm{~d}, J=8.0 \mathrm{~Hz}, 2 \mathrm{H}), 6.59$ $(\mathrm{q}, J=7.6 \mathrm{~Hz}, 1 \mathrm{H}), 4.56(\mathrm{t}, J=7.2 \mathrm{~Hz}, 2 \mathrm{H}), 3.14(\mathrm{t}, J=7.2 \mathrm{~Hz}, 2 \mathrm{H}) ;{ }^{13} \mathrm{C}$ NMR $(100$ $\left.\mathrm{MHz}, \mathrm{CDCl}_{3}\right) \delta 164.5,133.4,132.2,131.8(\mathrm{q}, J=34.2 \mathrm{~Hz}), 130.1,121.6(\mathrm{q}, J=272.4$ $\mathrm{Hz}), 117.8,116.5,113.7(\mathrm{q}, J=6.0 \mathrm{~Hz}), 63.4,39.5 ;{ }^{19} \mathrm{~F} \mathrm{NMR}\left(376 \mathrm{MHz}, \mathrm{CDCl}_{3}\right) \delta$ -57.7 (s); LRMS: m/z (EI) 268 (M-I), 130 (100), 127, 121, 102, 75.

$Z$ product: ${ }^{19} \mathrm{~F}$ NMR $\left(376 \mathrm{MHz}, \mathrm{CDCl}_{3}\right) \delta-60.6$ (s); LRMS: m/z (EI) 268 (M-I), 130 (100), 127, 121, 102, 75.<smiles>CSc1ccc(OCCC/C(I)=C\C(F)(F)F)cc1</smiles>

${ }^{1} \mathrm{H}$ NMR (400 MHz, $\left.\mathrm{CDCl}_{3}\right) \delta 7.26(\mathrm{~d}, J=8.4 \mathrm{~Hz}, 2 \mathrm{H}), 6.83(\mathrm{~d}, J=8.4 \mathrm{~Hz}, 2 \mathrm{H}), 6.45$ (q, $J=7.2 \mathrm{~Hz}, 1 \mathrm{H}), 3.96(\mathrm{t}, J=6.0 \mathrm{~Hz}, 2 \mathrm{H}), 2.84(\mathrm{t}, J=6.4 \mathrm{~Hz}, 2 \mathrm{H}), 2.44(\mathrm{~s}, 3 \mathrm{H})$, 2.14-2.01 (m, 2H); ${ }^{13} \mathrm{C}$ NMR (100 MHz, $\left.\mathrm{CDCl}_{3}\right) \delta 157.2,130.0,129.8(\mathrm{q}, J=34.3$ $\mathrm{Hz}), 129.0,121.8$ (q, $J=272.5 \mathrm{~Hz}), 119.1$ (q, $J=6.1 \mathrm{~Hz}), 115.1,66.0,37.5,29.3$, $17.9 ;{ }^{19} \mathrm{~F}$ NMR $\left(376 \mathrm{MHz}, \mathrm{CDCl}_{3}\right) \delta-57.9(\mathrm{~s}) ; \mathrm{HRMS}: \mathrm{m} / \mathrm{z}(\mathrm{ESI})$ calculated $[\mathrm{M}+\mathrm{K}]^{+}$: 440.9399, measured: 440.9635 .

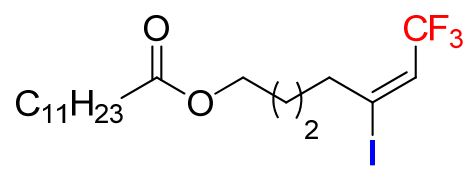

${ }^{1} \mathrm{H}$ NMR (400 MHz, $\left.\mathrm{CDCl}_{3}\right) \delta 6.43(\mathrm{q}, J=7.2 \mathrm{~Hz}, 1 \mathrm{H}), 4.17-4.00(\mathrm{~m}, 2 \mathrm{H}), 2.71-2.58$ $(\mathrm{m}, 2 \mathrm{H}), 2.36-2.27(\mathrm{~m}, 2 \mathrm{H}), 1.71-1.57(\mathrm{~m}, 6 \mathrm{H}), 1.37-1.18(\mathrm{~m}, 16 \mathrm{H}), 0.92-0.83(\mathrm{~m}$, 
$3 \mathrm{H}) ;{ }^{13} \mathrm{C} \mathrm{NMR}\left(100 \mathrm{MHz}, \mathrm{CDCl}_{3}\right) \delta 173.7,129.5(\mathrm{q}, J=34.2 \mathrm{~Hz}), 121.8(\mathrm{q}, J=$ $271.8 \mathrm{~Hz}), 119.8(\mathrm{q}, J=6.0 \mathrm{~Hz}), 63.5,40.1,34.2,31.9,29.6,29.5,29.4,29.3,29.2$, 29.1, 27.2, 26.0, 24.9, 22.6, 14.0; ${ }^{19} \mathrm{~F}$ NMR $\left(376 \mathrm{MHz}, \mathrm{CDCl}_{3}\right) \delta-57.8$ (s); HRMS: $\mathrm{m} / \mathrm{z}(\mathrm{ESI})$ calculated $[\mathrm{M}+\mathrm{H}]^{+}: 477.1477$, measured: 477.1490 .<smiles>CCCCCCCCCCCCCCOC(=O)OCCC(I)=CC(F)(F)F</smiles>

${ }^{1} \mathrm{H}$ NMR (400 MHz, $\left.\mathrm{CDCl}_{3}\right) \delta 6.33$ (q, $\left.J=7.2 \mathrm{~Hz}, 1 \mathrm{H}\right), 4.17-3.99(\mathrm{~m}, 2 \mathrm{H}), 2.75-2.54$ (m, 2H), 2.38-2.23 (m, 2H), 1.72-1.52 (m, 6H), 1.38-1.13 (m, 16H), 0.95-0.80 (m, $3 \mathrm{H}) ;{ }^{13} \mathrm{C} \mathrm{NMR}\left(100 \mathrm{MHz}, \mathrm{CDCl}_{3}\right) \delta 173.9,125.3(\mathrm{q}, J=35.6 \mathrm{~Hz}), 121.2$ (q, $J=269.7$ $\mathrm{Hz}), 115.2(\mathrm{q}, J=5.6 \mathrm{~Hz}), 63.5,46.3,34.3,31.9,29.7,29.6,29.5,29.3,29.2,29.1$, 27.1, 25.5, 25.0, 22.7, 14.1; ${ }^{19} \mathrm{~F}$ NMR (376 MHz, $\left.\mathrm{CDCl}_{3}\right) \delta-60.1$ (s); HRMS: m/z (ESI) calculated $[\mathrm{M}+\mathrm{Na}]^{+}: 499.1297$, measured: 499.1287.<smiles>O=C(OCCCC(I)=CC(F)(F)F)c1ccc(Cl)cc1</smiles>

${ }^{1} \mathrm{H}$ NMR (400 MHz, CDCl 3$) \delta 7.97(\mathrm{~d}, J=7.6 \mathrm{~Hz}, 2 \mathrm{H}), 7.42(\mathrm{~d}, J=7.6 \mathrm{~Hz}, 2 \mathrm{H}), 6.41$ $(\mathrm{q}, J=7.6 \mathrm{~Hz}, 1 \mathrm{H}), 4.32(\mathrm{t}, J=6.4 \mathrm{~Hz}, 2 \mathrm{H}), 2.64(\mathrm{t}, J=4.4 \mathrm{~Hz}, 2 \mathrm{H}), 1.86-1.76(\mathrm{~m}$, 2H), 1.70-1.61 (m, 2H), 1.53-1.44 (m, 2H); $\left.{ }^{13} \mathrm{C} \mathrm{NMR} \mathrm{(100} \mathrm{MHz,} \mathrm{CDCl}_{3}\right) \delta 165.7$, $139.3,130.9,129.3$ (q, $J=34.2 \mathrm{~Hz}), 128.8,128.7,121.8$ (q, $J=272.5 \mathrm{~Hz}), 120.2$ (q, $J$ $=6.1 \mathrm{~Hz}), 64.9,40.4,29.2,28.3,24.7 ;{ }^{19} \mathrm{~F} \mathrm{NMR}\left(376 \mathrm{MHz}, \mathrm{CDCl}_{3}\right) \delta-57.7(\mathrm{~s})$; HRMS: m/z (APPI) calculated $[\mathrm{M}+\mathrm{H}]^{+}:$446.9836, measured: 446.9830.<smiles>O=C(OCCCC(I)=CC(F)(F)F)c1ccc(Cl)cc1</smiles>

${ }^{1} \mathrm{H}$ NMR (400 MHz, CDCl 3$) \delta 7.97(\mathrm{~d}, J=8.0 \mathrm{~Hz}, 2 \mathrm{H}), 7.42(\mathrm{~d}, J=8.0 \mathrm{~Hz}, 2 \mathrm{H}), 6.31$ (q, $J=7.2 \mathrm{~Hz}, 1 \mathrm{H}), 4.33(\mathrm{t}, J=6.4 \mathrm{~Hz}, 2 \mathrm{H}), 2.64(\mathrm{t}, J=6.0 \mathrm{~Hz}, 2 \mathrm{H}), 1.85-1.75(\mathrm{~m}$, $2 \mathrm{H}), 1.71-1.63(\mathrm{~m}, 2 \mathrm{H}), 1.50-1.41(\mathrm{~m}, 2 \mathrm{H}) ;{ }^{13} \mathrm{C} \mathrm{NMR}\left(100 \mathrm{MHz}, \mathrm{CDCl}_{3}\right) \delta 165.7$, $139.4,130.9,128.9,128.8,125.2(\mathrm{q}, J=35.5 \mathrm{~Hz}), 121.3(\mathrm{q}, J=269.8 \mathrm{~Hz}), 115.5$ (q, $J$ $=6.0 \mathrm{~Hz}), 64.8,46.4,28.6,28.3,24.5 ;{ }^{19} \mathrm{~F} \mathrm{NMR}\left(376 \mathrm{MHz}, \mathrm{CDCl}_{3}\right) \delta-60.1(\mathrm{~s})$; HRMS: m/z (APPI) calculated $[\mathrm{M}+\mathrm{H}]^{+}:$446.9836, measured: 446.9840. 


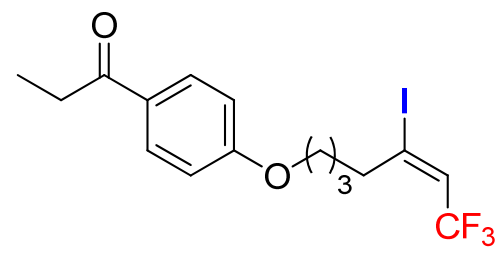

${ }^{1} \mathrm{H}$ NMR (400 MHz, CDCl 3 ) $\delta 7.94(\mathrm{~d}, J=8.0 \mathrm{~Hz}, 2 \mathrm{H}), 6.92(\mathrm{~d}, J=8.0 \mathrm{~Hz}, 2 \mathrm{H}), 6.44$ $(\mathrm{q}, J=7.2 \mathrm{~Hz}, 1 \mathrm{H}), 4.15-3.96(\mathrm{~m}, 2 \mathrm{H}), 3.06-2.87(\mathrm{~m}, 2 \mathrm{H}), 2.80-2.63(\mathrm{~m}, 2 \mathrm{H})$, 1.94-1.70 (m, 4H), 1.34-1.13 (m, 3H); ${ }^{13} \mathrm{C}$ NMR (100 MHz, $\left.\mathrm{CDCl}_{3}\right) \delta$ 199.5, 162.6, $130.2,130.1,129.6(\mathrm{q}, J=34.2 \mathrm{~Hz}), 124.5(\mathrm{q}, J=273.2 \mathrm{~Hz}), 119.9$ (q, $J=6.1 \mathrm{~Hz})$, 111.1, 67.4, 40.1, 31.4, 27.6, 26.1, 8.4; $\left.{ }^{19} \mathrm{~F} \mathrm{NMR} \mathrm{(376} \mathrm{MHz,} \mathrm{CDCl}_{3}\right) \delta-57.7$ (s); HRMS: $\mathrm{m} / \mathrm{z}$ (ESI) calculated $[\mathrm{M}+\mathrm{H}]^{+}:$427.0382, measured: 427.0385 .

$Z$ product: ${ }^{19} \mathrm{~F}$ NMR $\left(376 \mathrm{MHz}, \mathrm{CDCl}_{3}\right) \delta-60.1(\mathrm{~s})$; HRMS: $\mathrm{m} / \mathrm{z}$ (ESI) calculated $[\mathrm{M}+\mathrm{H}]^{+}:$427.0382, measured: 427.0399 .<smiles>O=C(c1ccccc1)c1ccc(OCCC/C(I)=C\C(F)(F)F)cc1</smiles>

${ }^{1} \mathrm{H}$ NMR $\left(400 \mathrm{MHz}, \mathrm{CDCl}_{3}\right) \delta 7.83(\mathrm{~d}, J=8.8 \mathrm{~Hz}, 2 \mathrm{H}), 7.76(\mathrm{~d}, J=7.6 \mathrm{~Hz}, 2 \mathrm{H}), 7.57$ (t, $J=7.6 \mathrm{~Hz}, 1 \mathrm{H}), 7.48$ (dd, $J=7.6,7.6 \mathrm{~Hz}, 2 \mathrm{H}), 6.95$ (d, $J=8.8 \mathrm{~Hz}, 2 \mathrm{H}), 6.47$ (q, $J$ $=7.6 \mathrm{~Hz}, 1 \mathrm{H}), 4.07(\mathrm{t}, J=6.0 \mathrm{~Hz}, 2 \mathrm{H}), 2.87(\mathrm{t}, J=7.2 \mathrm{~Hz}, 2 \mathrm{H}), 2.12(\mathrm{tt}, J=7.2,6.0$ $\mathrm{Hz}, 2 \mathrm{H}) ;{ }^{13} \mathrm{C}$ NMR $\left(100 \mathrm{MHz}, \mathrm{CDCl}_{3}\right) \delta 195.5,162.3,138.2,132.5,131.9,130.2$, $130.0(\mathrm{q}, J=34.2 \mathrm{~Hz}), 129.7,128.2,121.8(\mathrm{q}, J=272.5 \mathrm{~Hz}), 118.8(\mathrm{q}, J=5.3 \mathrm{~Hz})$, 113.9, 66.0, 37.4, 29.0; ${ }^{19} \mathrm{~F}$ NMR (376 MHz, $\left.\mathrm{CDCl}_{3}\right) \delta-57.9$ (s); HRMS: m/z (ESI) calculated $[\mathrm{M}+\mathrm{H}]^{+}:$461.0226, measured: 461.0229 .<smiles>CCOC(=O)C(F)(F)CC(I)CCOc1ccc(Br)cc1</smiles>

${ }^{1} \mathrm{H}$ NMR (400 MHz, $\left.\mathrm{CDCl}_{3}\right) \delta 7.40(\mathrm{~d}, J=8.0 \mathrm{~Hz}, 2 \mathrm{H}), 6.81(\mathrm{~d}, J=8.0 \mathrm{~Hz}, 2 \mathrm{H})$, 4.54-4.44 (m, 1H), 4.38 (q, $J=7.2 \mathrm{~Hz}, 2 \mathrm{H}), 4.18-4.02(\mathrm{~m}, 2 \mathrm{H}), 3.13-2.96(\mathrm{~m}, 1 \mathrm{H})$, 2.95-2.78 (m, 1H), 2.37-2.13 (m, 2H), $1.40(\mathrm{t}, J=7.2 \mathrm{~Hz}, 3 \mathrm{H}) ;{ }^{13} \mathrm{C}$ NMR (100 MHz, $\left.\mathrm{CDCl}_{3}\right) \delta 163.2(\mathrm{t}, J=32.2 \mathrm{~Hz}), 157.5,132.2,116.3,115.0(\mathrm{q}, J=251.6 \mathrm{~Hz}), 113.1$, 67.6, 63.2, $45.3(\mathrm{q}, J=23.1 \mathrm{~Hz}), 39.3,18.4,13.8 ;{ }^{19} \mathrm{~F}$ NMR $\left(376 \mathrm{MHz}, \mathrm{CDCl}_{3}\right) \delta$ $-101.8(\mathrm{~d}, J=264.0 \mathrm{~Hz}, 1 \mathrm{~F}),-106.3(\mathrm{~d}, J=264.0 \mathrm{~Hz}, 1 \mathrm{~F}) ; \mathrm{HRMS}: \mathrm{m} / \mathrm{z}$ (ESI) calculated $[\mathrm{M}+\mathrm{Na}]^{+}:$498.9193, measured: 498.9215. 
<smiles>O=C(OCC(I)CCC(F)(F)Cl)c1ccc(Cl)cc1</smiles>

${ }^{1} \mathrm{H}$ NMR (400 MHz, $\left.\mathrm{CDCl}_{3}\right) \delta 7.98(\mathrm{~d}, J=8.8 \mathrm{~Hz}, 2 \mathrm{H}), 7.41(\mathrm{~d}, J=8.8 \mathrm{~Hz}, 2 \mathrm{H})$, 4.40-4.27 (m, 3H), 3.05-2.70 (m, 2H), 1.97-1.68 (m, 5H), $1.65-1.53(\mathrm{~m}, 1 \mathrm{H}) ;{ }^{13} \mathrm{C}$ NMR $\left(100 \mathrm{MHz}, \mathrm{CDCl}_{3}\right) \delta 165.7,139.4,130.9,128.7,128.6,120-100$ (m), 64.6, 41.6 $(\mathrm{t}, J=20.8 \mathrm{~Hz}), 39.6,27.6,26.2,20.3 ;{ }^{19} \mathrm{~F} \mathrm{NMR}\left(376 \mathrm{MHz}, \mathrm{CDCl}_{3}\right) \delta-68.0(\mathrm{t}, J=$ $13.5 \mathrm{~Hz}, 2 \mathrm{~F}),-111.5(\mathrm{dt}, J=269.6,14.7 \mathrm{~Hz}, 1 \mathrm{~F}),-114.6(\mathrm{dt}, J=269.6,12.8 \mathrm{~Hz}, 1 \mathrm{~F})$, -120.1 (m, 2F), -121.3 (m, 2F), -121.6 (m, 2F), -123.6 (m, 2F); LRMS: m/z (EI) 417, 157, 139 (100), 127, 111, 81.<smiles>COc1ccc(CC(I)CC(F)(F)F)cc1</smiles>

${ }^{1} \mathrm{H}$ NMR $\left(400 \mathrm{MHz}, \mathrm{CDCl}_{3}\right) \delta 7.11(\mathrm{~d}, J=8.4 \mathrm{~Hz}, 2 \mathrm{H}), 6.87(\mathrm{~d}, J=8.4 \mathrm{~Hz}, 2 \mathrm{H})$, 4.39-4.24 (m, 1H), 3.81 (s,3H), 3.25-3.12 (m, 2H), 2.93-2,74 (m, 2H); ${ }^{13} \mathrm{C}$ NMR (100 $\left.\mathrm{MHz} \mathrm{CDCl}_{3}\right) \delta 158.7,130.6,130.0,125.6(\mathrm{q}, J=276.5 \mathrm{~Hz}), 113.9,55.2,45.8,43.5(\mathrm{q}$, $J=28.2 \mathrm{~Hz}), 21.1 ;{ }^{19} \mathrm{~F}$ NMR (376 MHz, $\left.\mathrm{CDCl}_{3}\right) \delta-63.6$ (s); LRMS: m/z (EI) 344 (M), 217 (M-I, 100), 134, 127, 121, 91, 77.<smiles>CCC(=O)c1ccc(OCC(I)CC(F)(F)F)cc1</smiles>

${ }^{1} \mathrm{H}$ NMR $\left(400 \mathrm{MHz}, \mathrm{CDCl}_{3}\right) \delta 7.95(\mathrm{~d}, J=8.4 \mathrm{~Hz}, 2 \mathrm{H}), 6.82(\mathrm{~d}, J=8.4 \mathrm{~Hz}, 2 \mathrm{H})$, 4.29-4.18 (m, 1H), 4.05 (t, $J=6.0 \mathrm{~Hz}, 2 \mathrm{H}), 3.02-2.73(\mathrm{~m}, 4 \mathrm{H}), 1,96-1,56(\mathrm{~m}, 6 \mathrm{H})$, $1.21(\mathrm{t}, J=7.2 \mathrm{~Hz}, 3 \mathrm{H}) ;{ }^{13} \mathrm{C} \mathrm{NMR}\left(100 \mathrm{MHz}, \mathrm{CDCl}_{3}\right) \delta 199.4,162.6,130.2,129.9$, $125.5(\mathrm{q}, J=277.2 \mathrm{~Hz}), 114.0,67.6,44.8$ (q, $J=28.2 \mathrm{~Hz}), 39.2,31.3,28.0,26.2,21.2$, 8.4; ${ }^{19} \mathrm{~F}$ NMR (376 MHz, $\left.\mathrm{CDCl}_{3}\right) \delta-63.9$ (s); HRMS: m/z (ESI) calculated $[\mathrm{M}+\mathrm{H}]^{+}$: 429.0538, measured: 429.0548 .<smiles>O=C(OCCC(I)CC(F)(F)F)c1ccc(Cl)cc1</smiles>

${ }^{1} \mathrm{H}$ NMR (400 MHz, CDCl 3$) \delta 7.98(\mathrm{~d}, J=8.8 \mathrm{~Hz}, 2 \mathrm{H}), 7.42(\mathrm{~d}, J=8.8 \mathrm{~Hz}, 2 \mathrm{H})$, 4.42-4.30 (m, 2H), 4.28-4.17 (m, 1H), 3.05-2.72 (m, 2H), 1,95-1,52 (m, 6H); ${ }^{13} \mathrm{C}$ NMR $\left(100 \mathrm{MHz}, \mathrm{CDCl}_{3}\right) \delta 165.7,139.3,130.9,128.7,125.5(\mathrm{q}, J=277.1 \mathrm{~Hz}), 114.9$, 64.6, 44.9 (q, $J=28.2 \mathrm{~Hz}), 39.0,27.6,26.1,21.1 ;{ }^{19} \mathrm{~F}$ NMR $\left(376 \mathrm{MHz}, \mathrm{CDCl}_{3}\right) \delta$ 
-63.9 (s); HRMS: m/z (ESI) calculated [M+Na] $]^{+}$456.9655, measured: 456.9665.<smiles>CC(C)(C)C(I)C(C)(c1ccccc1)C(F)(F)F</smiles>

${ }^{1} \mathrm{H}$ NMR $\left(400 \mathrm{MHz}, \mathrm{CDCl}_{3}\right) \delta[4.36(\mathrm{~d}, J=11.2 \mathrm{~Hz}), 4.31-4.24](1 \mathrm{H}),[2.54-2.42(\mathrm{~m})]$ $(0.5 \mathrm{H}),[2.08-1.90(\mathrm{~m})](1 \mathrm{H}),[1.87-1.62(\mathrm{~m})](2.5 \mathrm{H}),[1.61-1.43(\mathrm{~m})](3 \mathrm{H}),[1,42-1,21$ $(\mathrm{m})](14 \mathrm{H}),[0.96-0.84(\mathrm{~m})](6 \mathrm{H}) ;{ }^{13} \mathrm{C} \mathrm{NMR}\left(100 \mathrm{MHz}, \mathrm{CDCl}_{3}\right) \delta[126.7$ (q, $J=$ $280.1 \mathrm{~Hz}), 126.4(\mathrm{q}, J=281.5 \mathrm{~Hz})],[51.0(\mathrm{q}, J=23.2 \mathrm{~Hz}), 48.6(\mathrm{q}, J=24.5 \mathrm{~Hz})],[38.9$, 38.9], [35.7, 35.7], [32.8 (q, $J=2.4 \mathrm{~Hz}), 31.1(\mathrm{q}, J=3.1 \mathrm{~Hz})$ ], [31.6, 31.5], [30.5, 30.5], [29.8, 29.8], [29.3, 29.3], [29.3, 29.1], [28.2, 28.2], [27.8, 27.7], [26.7, 26.7], [22.6, 22.5], [14.0, 14.0]; ${ }^{19} \mathrm{~F}$ NMR $\left(376 \mathrm{MHz}, \mathrm{CDCl}_{3}\right) \delta[-65.4(\mathrm{~s}),-67.1$ (s)]; LRMS: $\mathrm{m} / \mathrm{z}$ (EI) 265 (M-I), 127, 85, 71, 57 (100).

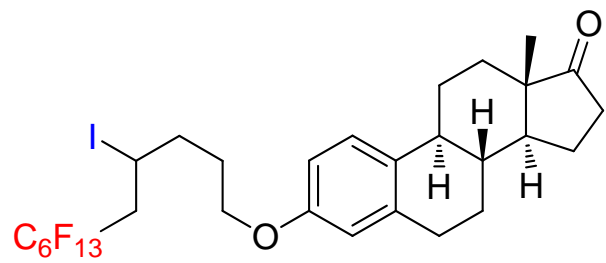

${ }^{1} \mathrm{H}$ NMR $\left(400 \mathrm{MHz}, \mathrm{CDCl}_{3}\right) \delta 7.20(\mathrm{~d}, J=8.8 \mathrm{~Hz}, 1 \mathrm{H}), 6.70(\mathrm{dd}, J=8.8,2.8 \mathrm{~Hz}, 1 \mathrm{H})$, $6.63(\mathrm{~d}, J=2.8 \mathrm{~Hz}, 1 \mathrm{H}), 4.45-4.34(\mathrm{~m}, 1 \mathrm{H}), 4.04-3.94(\mathrm{~m}, 2 \mathrm{H}), 3.06-2.73(\mathrm{~m}, 4 \mathrm{H})$, $2.50(\mathrm{dd}, J=18.4,8.8 \mathrm{~Hz}, 1 \mathrm{H}), 2.43-2.36(\mathrm{~m}, 1 \mathrm{H}), 2.30-2.21(\mathrm{~m}, 1 \mathrm{H}), 2.20-1.83(\mathrm{~m}$, 8H), 1.69-1.36 (m, 1H), $0.91(\mathrm{~s}, 3 \mathrm{H}) ;{ }^{13} \mathrm{C} \mathrm{NMR}\left(100 \mathrm{MHz}, \mathrm{CDCl}_{3}\right) \delta$ 156.7, 137.6, 132.1, 126.2, 114.4, 111.9, 66.3, 50.3, 47.8, 43.9, 41.5 (t, $J=20.8 \mathrm{~Hz}$ ), 38.2, 37.0, $35.7,31.5,29.5,26.4,25.8,21.4,19.9,13.7 ;{ }^{19} \mathrm{~F} \mathrm{NMR}\left(376 \mathrm{MHz}, \mathrm{CDCl}_{3}\right) \delta-80.8(\mathrm{t}, J$ $=9.4 \mathrm{~Hz}, 3 \mathrm{~F}),-111.7(\mathrm{dm}, J=269.4 \mathrm{~Hz}, 1 \mathrm{~F}),-114.4(\mathrm{dt}, J=269.4,13.9 \mathrm{~Hz}, 1 \mathrm{~F})$, -121.7 (m, 2F), -122.8 (m, 2F), -123.6 (m, 2F), -126.1 (m, 2F); HRMS: m/z (ESI) calculated $[\mathrm{M}+\mathrm{H}]^{+}:$785.1161, measured: 785.1270 .

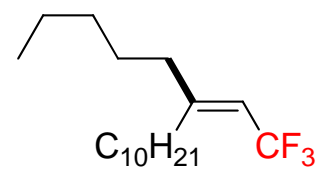

${ }^{1} \mathrm{H}$ NMR(400 MHz, $\left.\mathrm{CDCl}_{3}\right) \delta 5.37(\mathrm{q}, J=8.8 \mathrm{~Hz}, 1 \mathrm{H}), 2.22(\mathrm{td}, J=8.0,1.2 \mathrm{~Hz}, 2 \mathrm{H})$, 2.11-2.04 (m, 2H), 1.48-1.36 (m, 4H), 1.35-1.20 (m, 18H), 0.94-0.83 (m, 6H); ${ }^{13} \mathrm{C}$ NMR (100 MHz, $\left.\mathrm{CDCl}_{3}\right) \delta 156.2,123.8(\mathrm{q}, J=269.1 \mathrm{~Hz}), 113.6(\mathrm{q}, J=32.9 \mathrm{~Hz})$, 36.3, 31.9, 31.5, 31.4, 29.7, 29.6, 29.5, 29.4, 29.3, 28.2, 27.1, 22.7, 22.5, 14.1, 14.0; ${ }^{19} \mathrm{~F} \mathrm{NMR}\left(376 \mathrm{MHz}, \mathrm{CDCl}_{3}\right) \delta-56.4$ (s); LRMS: m/z (EI) 306 (M), 180, 165, 126, 97 , $83,69,56(100)$. 


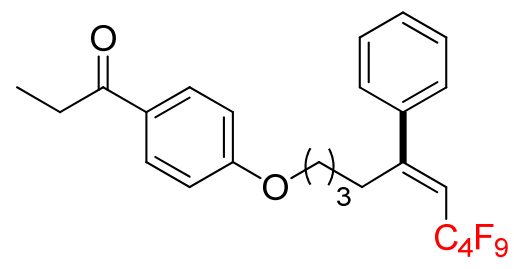

${ }^{1} \mathrm{H}$ NMR (400 MHz, $\left.\mathrm{CDCl}_{3}\right) \delta 7.91(\mathrm{~d}, J=8.0 \mathrm{~Hz}, 2 \mathrm{H}), 7.45-7.30(\mathrm{~m}, 5 \mathrm{H}), 6.84(\mathrm{~d}, J$ $=8.0 \mathrm{~Hz}, 2 \mathrm{H}), 5.67(\mathrm{t}, J=15.6 \mathrm{~Hz}, 1 \mathrm{H}), 3.94(\mathrm{t}, J=5.2 \mathrm{~Hz}, 2 \mathrm{H}), 3.00-2.90(\mathrm{~m}, 2 \mathrm{H})$, 2.87-2.77 (m, 2H), 1.86-1.75 (m, 2H), 1.59-1.47 (m, 2H), $1.21(\mathrm{t}, J=6.0 \mathrm{~Hz}, 3 \mathrm{H}) ;{ }^{13} \mathrm{C}$ NMR $\left(100 \mathrm{MHz}, \mathrm{CDCl}_{3}\right) \delta 199.5,162.6,156.2,140.4,130.1,129.8,129.0,128.7$, 126.6, $114.2(\mathrm{t}, J=22.1 \mathrm{~Hz}), 114.0,120-100(\mathrm{~m}), 67.5,31.3,30.8,28.7,24.9,8.4 ;{ }^{19} \mathrm{~F}$ NMR (376 MHz, $\left.\mathrm{CDCl}_{3}\right) \delta-81.0(\mathrm{~m}, 3 \mathrm{~F}),-105.0(\mathrm{~m}, 2 \mathrm{~F}),-124.2(\mathrm{~m}, 2 \mathrm{~F}),-125.6(\mathrm{~m}$, 2F); HRMS: $\mathrm{m} / \mathrm{z}$ (ESI) calculated $[\mathrm{M}+\mathrm{H}]^{+}: 527.1633$, measured: 527.1630.<smiles>CCC=C/C(=C/C(F)(F)F)CCOc1ccc(C(=O)c2ccccc2)cc1</smiles>

${ }^{1} \mathrm{H}$ NMR $\left(400 \mathrm{MHz}, \mathrm{CDCl}_{3}\right) \delta 7.82(\mathrm{~d}, J=8.8 \mathrm{~Hz}, 2 \mathrm{H}), 7.75(\mathrm{~d}, J=7.6 \mathrm{~Hz}, 2 \mathrm{H}), 7.56$ (t, $J=7.6 \mathrm{~Hz}, 1 \mathrm{H}), 7.47(\mathrm{t}, J=7.6 \mathrm{~Hz}, 2 \mathrm{H}), 6.95(\mathrm{~d}, J=8.8 \mathrm{~Hz}, 2 \mathrm{H}), 6.09$ (dt, $J=$ $16.0,6.8 \mathrm{~Hz}, 1 \mathrm{H}), 5.96(\mathrm{~d}, J=16.0 \mathrm{~Hz}, 1 \mathrm{H}), 5.52(\mathrm{q}, J=8.8 \mathrm{~Hz}, 1 \mathrm{H}), 4.06(\mathrm{t}, J=6.0$ $\mathrm{Hz}, 2 \mathrm{H}), 6.63(\mathrm{td}, J=8.0,1.2 \mathrm{~Hz}, 2 \mathrm{H}), 2.13(\mathrm{td}, J=7.2,6.8 \mathrm{~Hz}, 2 \mathrm{H}), 2.05-1.95(\mathrm{~m}$, $2 \mathrm{H}), 1.44-1.34(\mathrm{~m}, 2 \mathrm{H}), 1.32-1.21(\mathrm{~m}, 6 \mathrm{H}), 0.88(\mathrm{t}, J=7.2 \mathrm{~Hz}, 3 \mathrm{H}) ;{ }^{13} \mathrm{C}$ NMR $(100$ $\left.\mathrm{MHz}, \mathrm{CDCl}_{3}\right) \delta 195.5,162.5,149.4(\mathrm{q}, J=5.4 \mathrm{~Hz}), 138.3,136.6,132.6,131.9,130.1$, 129.7, 128.2, 123.9 (q, $J=268.4 \mathrm{~Hz}), 116.0$ (q, $J=32.9 \mathrm{~Hz}), 113.9,67.4,32.9$, 31.6, 28.9, 28.8, 24.3, 22.5, 14.0; ${ }^{19} \mathrm{~F}$ NMR (376 MHz, $\left.\mathrm{CDCl}_{3}\right) \delta-56.3(\mathrm{~s}) ; \mathrm{HRMS}: \mathrm{m} / \mathrm{z}$ (ESI) calculated $[\mathrm{M}+\mathrm{H}]^{+}:$445.2354, measured: 445.2346 .<smiles>CCCCCCCCC(=CCCOC(=O)c1ccc(C#N)cc1)C=C(C(F)(F)F)C(F)(F)F</smiles>

${ }^{1} \mathrm{H}$ NMR (400 MHz, $\left.\mathrm{CDCl}_{3}\right) \delta 8.13(\mathrm{~d}, J=8.4 \mathrm{~Hz}, 2 \mathrm{H}), 7.74(\mathrm{~d}, J=8.4 \mathrm{~Hz}, 2 \mathrm{H}), 5.95$ $(\mathrm{q}, J=8.4 \mathrm{~Hz}, 1 \mathrm{H}), 4.58(\mathrm{t}, J=6.4 \mathrm{~Hz}, 2 \mathrm{H}), 2.82(\mathrm{t}, J=6.4 \mathrm{~Hz}, 2 \mathrm{H}), 2.30$ (t, $J=7.2$ $\mathrm{Hz}, 2 \mathrm{H}), 1.15(\mathrm{tt}, J=7.6,6.8 \mathrm{~Hz}, 2 \mathrm{H}), 1.40-1.32(\mathrm{~m}, 2 \mathrm{H}), 1.31-1.22(\mathrm{~m}, 4 \mathrm{H}), 0.88(\mathrm{t}$, $J=7.2 \mathrm{~Hz}, 3 \mathrm{H}) ;{ }^{13} \mathrm{C} \mathrm{NMR}\left(100 \mathrm{MHz}, \mathrm{CDCl}_{3}\right) \delta 164.7,133.8,132.8(\mathrm{q}, J=5.4 \mathrm{~Hz})$, 132.1, 130.1, 123.9 (q, $J=33.6 \mathrm{~Hz}), 122.7$ (q, $J=268.4 \mathrm{~Hz}), 117.9,116.4,96.8,78.5$, 63.0, 31.6, 31.2, 28.5, 28.2, 22.4, 19.3, 13.9; $\left.{ }^{19} \mathrm{~F} \mathrm{NMR} \mathrm{(376} \mathrm{MHz,} \mathrm{CDCl}_{3}\right) \delta-56.8(\mathrm{~s})$;

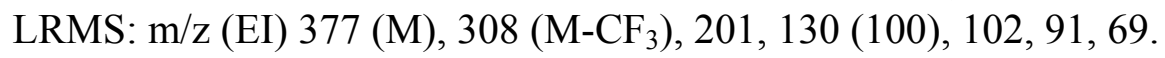




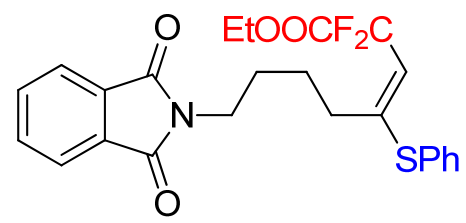

${ }^{1} \mathrm{H} \mathrm{NMR}\left(400 \mathrm{MHz}, \mathrm{CDCl}_{3}\right) \delta 7.85(\mathrm{dd}, J=5.2,2.8 \mathrm{~Hz}, 2 \mathrm{H}), 7.71(\mathrm{dd}, J=5.2,2.8 \mathrm{~Hz}$, 2H), 7.47-7.42 (m, 2H), 7.41-7.36 (m, 3H), $5.06(\mathrm{t}, J=14.0 \mathrm{~Hz}, 1 \mathrm{H}), 4.25(\mathrm{q}, J=7.2$ $\mathrm{Hz}, 2 \mathrm{H}), 3.70$ (t, $J=6.4 \mathrm{~Hz}, 2 \mathrm{H}), 2.52(\mathrm{t}, J=6.4 \mathrm{~Hz}, 2 \mathrm{H}), 1.78-1.64(\mathrm{~m}, 4 \mathrm{H}), 1.28$ (t, $J=7.2 \mathrm{~Hz}, 3 \mathrm{H}) ;{ }^{13} \mathrm{C} \mathrm{NMR}\left(100 \mathrm{MHz}, \mathrm{CDCl}_{3}\right) \delta 168.3,153.8,135.1,133.8,132.3$, 132.1, 130.1, 129.7, 129.5, 123.2, 113.5 (t, $J=26.9 \mathrm{~Hz}), 112.5$ (t, $J=248.3 \mathrm{~Hz}), 62.9$, 37.6, 31.9, 28.1, 26.6, 13.9; ${ }^{19} \mathrm{~F}$ NMR (376 MHz, $\left.\mathrm{CDCl}_{3}\right) \delta-96.3$ (s); HRMS: m/z (ESI) calculated $[\mathrm{M}+\mathrm{Na}]^{+}: 482.1214$, measured: 482.1211.

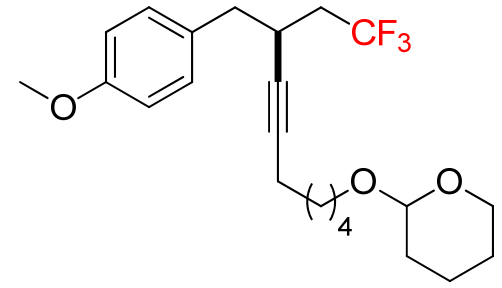

${ }^{1} \mathrm{H} \mathrm{NMR}\left(400 \mathrm{MHz}, \mathrm{CDCl}_{3}\right) \delta 7.13(\mathrm{~d}, J=8.4 \mathrm{~Hz}, 2 \mathrm{H}), 6.84(\mathrm{~d}, J=8.4 \mathrm{~Hz}, 2 \mathrm{H}), 4.57$ $(\mathrm{dd}, J=4.4,2.4 \mathrm{~Hz}, 1 \mathrm{H}), 3.86$ (ddd, $J=10.8,7.2,3.2 \mathrm{~Hz}, 1 \mathrm{H}), 3.79$ (s, 3H), 3.73 (dtd, $J=9.6,6.8,1.2 \mathrm{~Hz}, 1 \mathrm{H}), 3.54-3.46(\mathrm{~m}, 1 \mathrm{H}), 3.38$ (dt, $J=9.6,6.4 \mathrm{~Hz}, 1 \mathrm{H}), 2.94-2.84$ $(\mathrm{m}, 1 \mathrm{H}), 2.76(\mathrm{dd}, J=6.8,4.8 \mathrm{~Hz}, 2 \mathrm{H}), 2.24-2.09(\mathrm{~m}, 4 \mathrm{H}), 1.88-1.77(\mathrm{~m}, 1 \mathrm{H})$, 1.75-1.67 (m, 1H), 1.64-1.37 (m, 10H); $\left.{ }^{13} \mathrm{C} \mathrm{NMR} \mathrm{(100} \mathrm{MHz,} \mathrm{CDCl}_{3}\right) \delta 158.3,130.3$, $130.2,126.3(\mathrm{q}, J=275.8 \mathrm{~Hz}), 113.6,98.8,83.2,80.2,67.4,62.3,55.2,40.3,38.2$ (q, $J=27.5 \mathrm{~Hz}), 30.7,29.2,28.6,27.8,25.5,25.4,19.6,18.6 ;{ }^{19} \mathrm{~F}$ NMR $(376 \mathrm{MHz}$, $\left.\mathrm{CDCl}_{3}\right) \delta-63.9(\mathrm{~s}) ; \mathrm{HRMS}: \mathrm{m} / \mathrm{z}$ (ESI) calculated $[\mathrm{M}+\mathrm{Na}]^{+}:$435.2123, measured: 435.2132 .

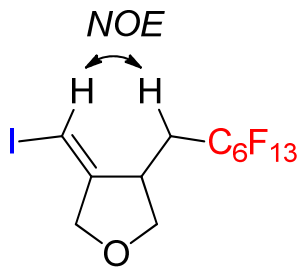

${ }^{1} \mathrm{H} \mathrm{NMR}\left(400 \mathrm{MHz}, \mathrm{CDCl}_{3}\right) \delta 6.13(\mathrm{ddd}, J=2.8,2.8,2.0 \mathrm{~Hz}, 1 \mathrm{H}), 4.37-4.19(\mathrm{~m}, 3 \mathrm{H})$, $3.75(\mathrm{dd}, J=8.8,7.2 \mathrm{~Hz}, 1 \mathrm{H}), 3.16-3.06(\mathrm{~m}, 1 \mathrm{H}), 2.50-2.31(\mathrm{~m}, 1 \mathrm{H}), 2.30-2.12(\mathrm{~m}$, $1 \mathrm{H}) ;{ }^{13} \mathrm{C}$ NMR $\left(100 \mathrm{MHz}, \mathrm{CDCl}_{3}\right) \delta 152.8,120-100(\mathrm{~m}), 75.6,74.8(\mathrm{~d}, J=4.0 \mathrm{~Hz})$, 68.8, 39.4, $32.9(\mathrm{t}, J=21.5 \mathrm{~Hz}) ;{ }^{19} \mathrm{~F}$ NMR $\left(376 \mathrm{MHz}, \mathrm{CDCl}_{3}\right) \delta-80.8(\mathrm{t}, J=10.1 \mathrm{~Hz}$, $3 \mathrm{~F}),-112.1(\mathrm{dm}, J=270.0 \mathrm{~Hz}, 1 \mathrm{~F}),-113.9(\mathrm{dm}, J=270.0 \mathrm{~Hz}, 1 \mathrm{~F}),-121.7(\mathrm{~m}, 2 \mathrm{~F})$, $-122.8(\mathrm{~m}, 2 \mathrm{~F}),-123.4(\mathrm{~m}, 2 \mathrm{~F}),-126.1(\mathrm{~m}, 2 \mathrm{~F})$; HRMS: $\mathrm{m} / \mathrm{z}(\mathrm{ESI})$ calculated $[\mathrm{M}+\mathrm{Na}]^{+}$: 
415.0368, measured: 415.0363 .

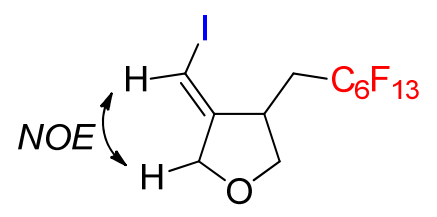

${ }^{1} \mathrm{H} \mathrm{NMR}\left(400 \mathrm{MHz}, \mathrm{CDCl}_{3}\right) \delta 6.19(\mathrm{ddd}, J=2.0,2.0,2.0 \mathrm{~Hz}, 1 \mathrm{H}), 4.36(\mathrm{dt}, J=13.2$, $1.6 \mathrm{~Hz}, 1 \mathrm{H}), 4.23(\mathrm{dd}, J=13.2,2.0 \mathrm{~Hz}, 1 \mathrm{H}), 4.06(\mathrm{dd}, J=9.2,2.0 \mathrm{~Hz}, 1 \mathrm{H}), 3.96(\mathrm{dd}$, $J=9.2,5.6 \mathrm{~Hz}, 1 \mathrm{H}), 3.23-3.14(\mathrm{~m}, 1 \mathrm{H}), 2.68-2.50(\mathrm{~m}, 1 \mathrm{H}), 2.34-2.15(\mathrm{~m}, 1 \mathrm{H}) ;{ }^{13} \mathrm{C}$ NMR (100 MHz, $\left.\mathrm{CDCl}_{3}\right) \delta 152.8,120-100(\mathrm{~m}), 72.7$ (d, $\left.J=4.7 \mathrm{~Hz}\right), 71.4,69.7,41.1$, $31.7(\mathrm{t}, J=20.5 \mathrm{~Hz}) ;{ }^{19} \mathrm{~F}$ NMR $\left(376 \mathrm{MHz}, \mathrm{CDCl}_{3}\right) \delta-80.8(\mathrm{bs}, 3 \mathrm{~F}),-112.7(\mathrm{dt}, J=$ 270.7, 23.3 Hz, 1F), -114.0 (dt, $J=270.7,24.1 \mathrm{~Hz}, 1 \mathrm{~F}),-121.7$ (bs, 2F), -122.8 (bs, 2F), -123.6 (bs, 2F), -126.1 (bs, 2F); HRMS: m/z (APPI) calculated [M-I] ${ }^{+}: 415.0368$, measured: 415.0361 .

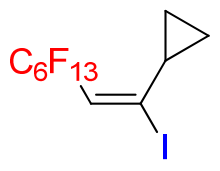

${ }^{1} \mathrm{H}$ NMR(400 MHz, $\left.\mathrm{CDCl}_{3}\right) \delta 6.39(\mathrm{t}, J=14.8 \mathrm{~Hz}, 1 \mathrm{H}), 1.57-1.46(\mathrm{~m}, 1 \mathrm{H}), 0.89-0.79$ $(\mathrm{m}, 4 \mathrm{H}) ;{ }^{13} \mathrm{C} \mathrm{NMR}\left(100 \mathrm{MHz}, \mathrm{CDCl}_{3}\right) \delta 129.9,125.6(\mathrm{t}, J=3.5 \mathrm{~Hz}), 120-100(\mathrm{~m})$, $17.9,11.1 ;{ }^{19} \mathrm{~F}$ NMR $\left(376 \mathrm{MHz}, \mathrm{CDCl}_{3}\right) \delta-80.8(\mathrm{t}, J=9.4 \mathrm{~Hz}, 3 \mathrm{~F}),-104.5(\mathrm{t}, J=12.4$ $\mathrm{Hz}, 2 \mathrm{~F}),-121.7$ (m, 2F), -122.8 (m, 2F), -123.2 (m, 2F), -126.1 (m, 2F); LRMS: m/z (EI) 385 (M-I, 100), 365, 196, 166, 146, 127, 115, 97, 69.

$Z$ product: ${ }^{19} \mathrm{~F}$ NMR $\left(376 \mathrm{MHz}, \mathrm{CDCl}_{3}\right) \delta-80.8(\mathrm{t}, J=8.3 \mathrm{~Hz}, 3 \mathrm{~F}),-107.2(\mathrm{t}, J=12.4$ $\mathrm{Hz}, 2 \mathrm{~F}),-121.6$ (m, 2F), -122.8 (m, 4F), -126.1 (m, 2F); LRMS: m/z (EI) 385 (M-I, 100), 365, 196, 166, 146, 127, 115, 97, 69.

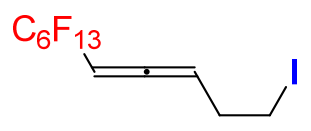

${ }^{1} \mathrm{H} \mathrm{NMR}\left(400 \mathrm{MHz}, \mathrm{CDCl}_{3}\right) \delta$ 5.81-5.70 (m, 1H), 5.54-5.42 (m, 1H), $3.21(\mathrm{td}, J=7.2$, $1.6 \mathrm{~Hz}, 2 \mathrm{H}), 2.70$ (tdd, $J=7.2,6.8,2.8 \mathrm{~Hz}, 2 \mathrm{H}) ;{ }^{13} \mathrm{C} \mathrm{NMR}\left(100 \mathrm{MHz}, \mathrm{CDCl}_{3}\right) \delta 206.7$ $(\mathrm{t}, J=7.8 \mathrm{~Hz}), 120-100(\mathrm{~m}), 97.7,85.7(\mathrm{t}, J=28.2 \mathrm{~Hz}), 31.5,2.0 ;{ }^{19} \mathrm{~F}$ NMR $(376$ $\left.\mathrm{MHz} \mathrm{CDCl}_{3}\right) \delta-80.8(\mathrm{~m}, 3 \mathrm{~F}),-108.2(\mathrm{~m}, 2 \mathrm{~F}),-121.6(\mathrm{~m}, 2 \mathrm{~F}),-122.9(\mathrm{~m}, 2 \mathrm{~F}),-123.2$ (m, 2F), -126.1 (m, 2F); LRMS: m/z (EI) 385 (M-I, 100), 365, 345, 295, 196, 166, $155,146,127,115,97,69$. 

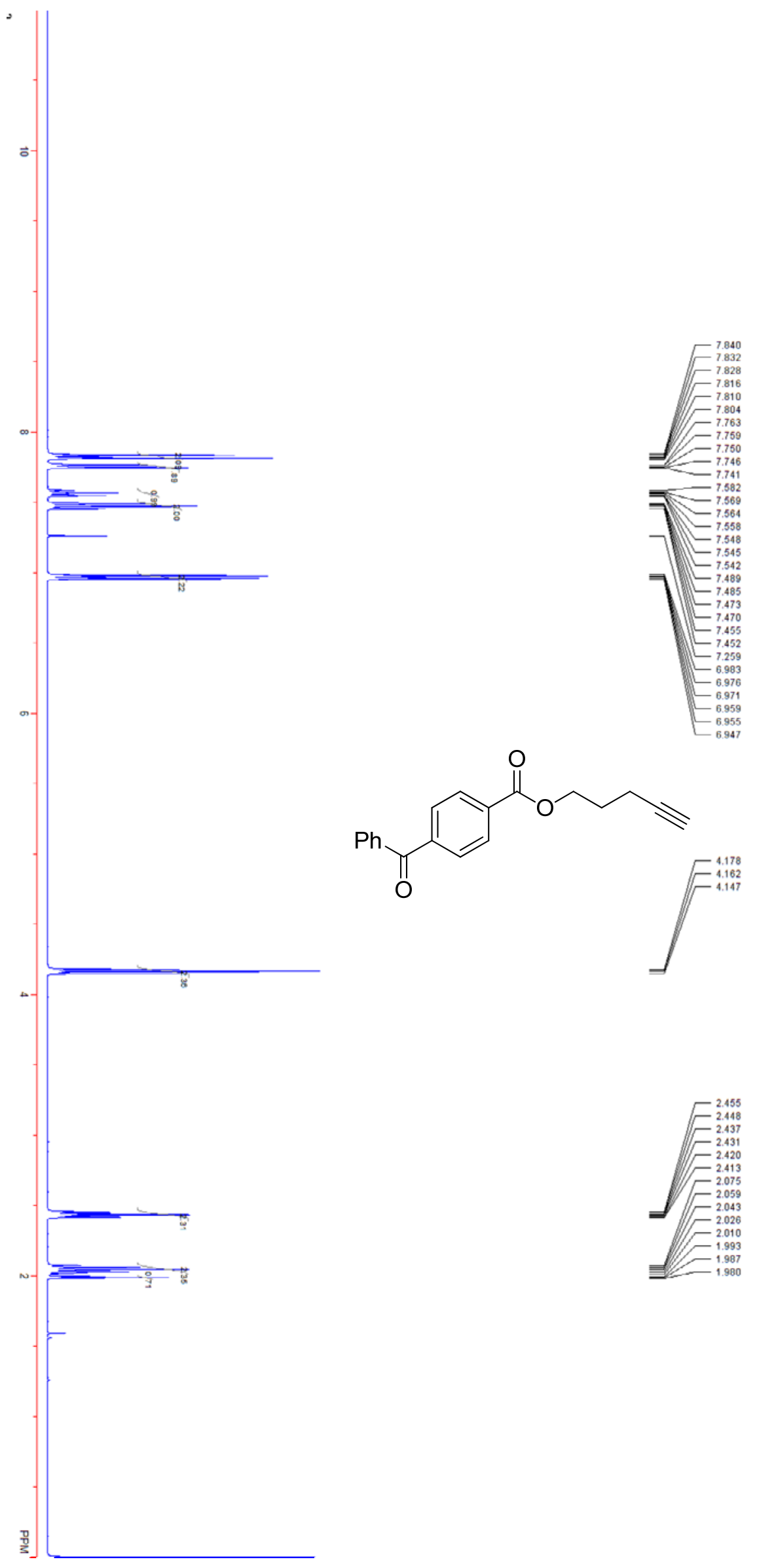

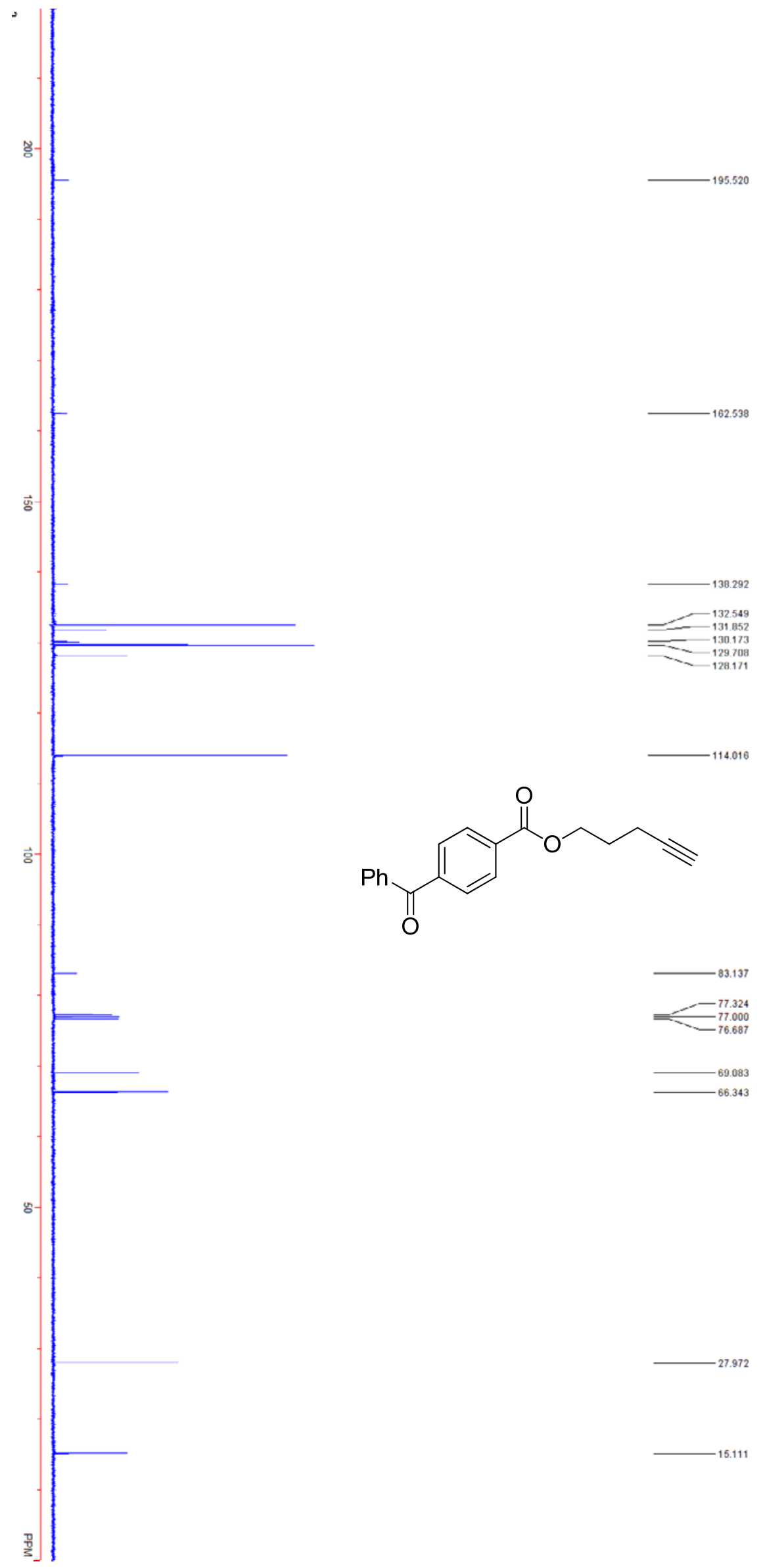<smiles>C#CCCCOC(=O)c1ccc(C(=O)c2ccccc2)cc1</smiles>

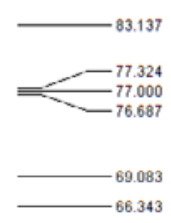




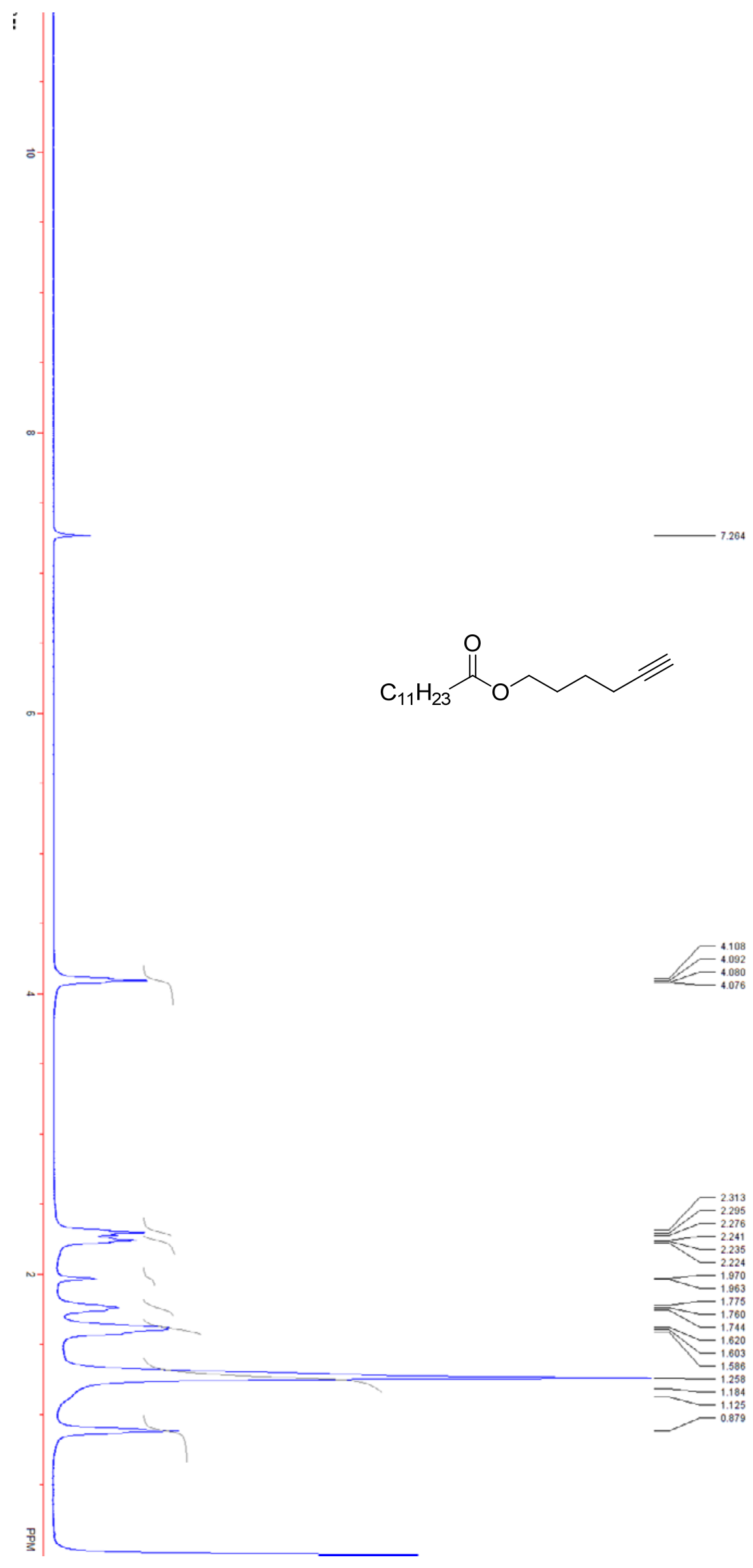




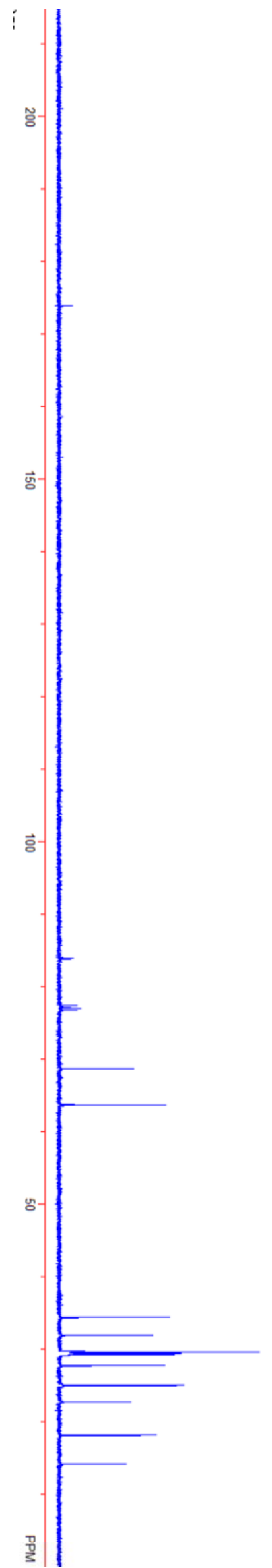<smiles>C#CCCCCOC(=O)[14CH2][14CH2]</smiles>
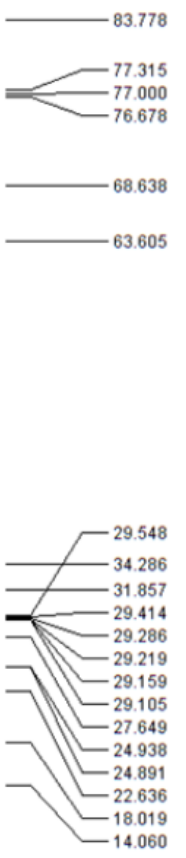


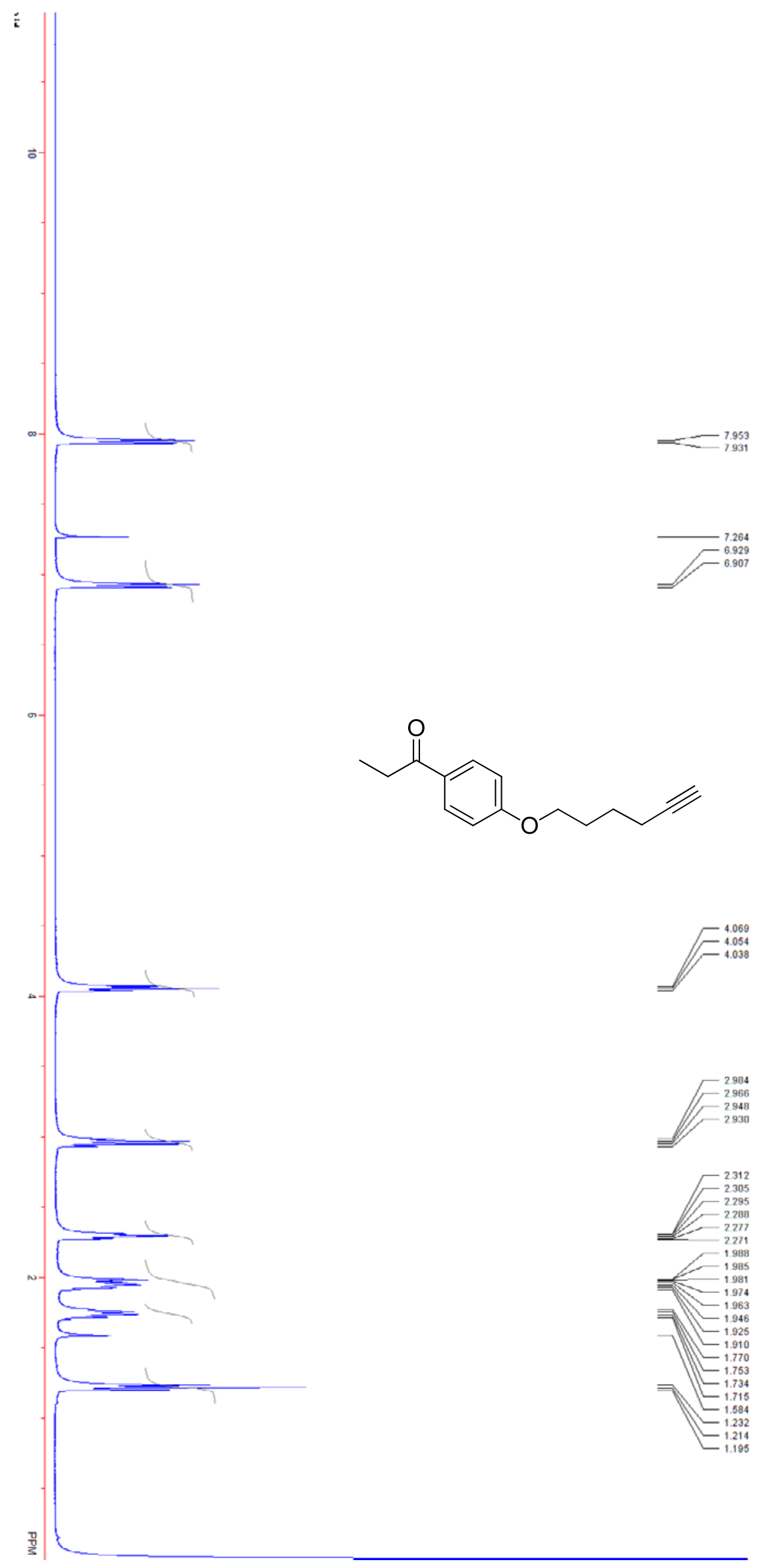



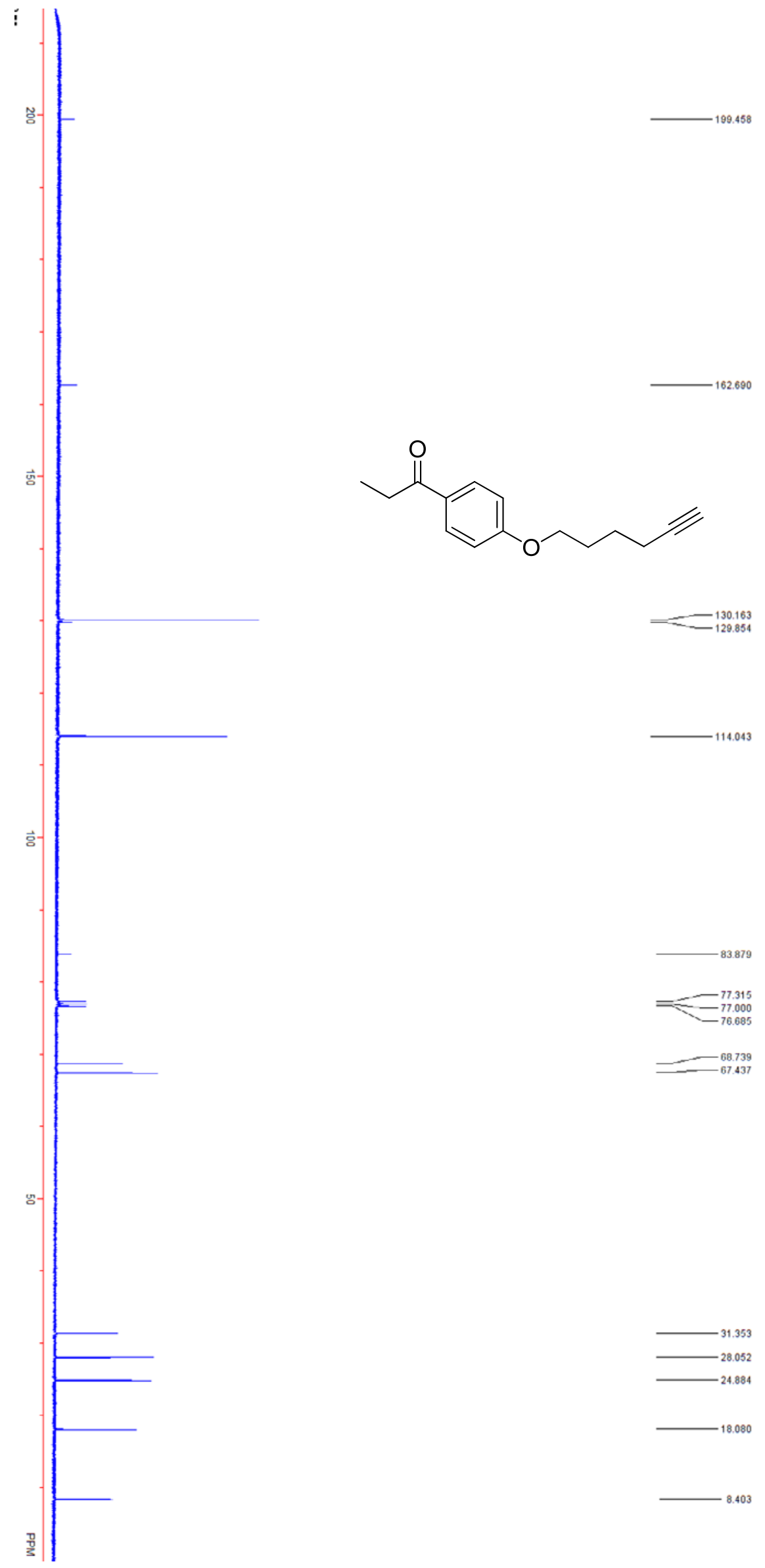

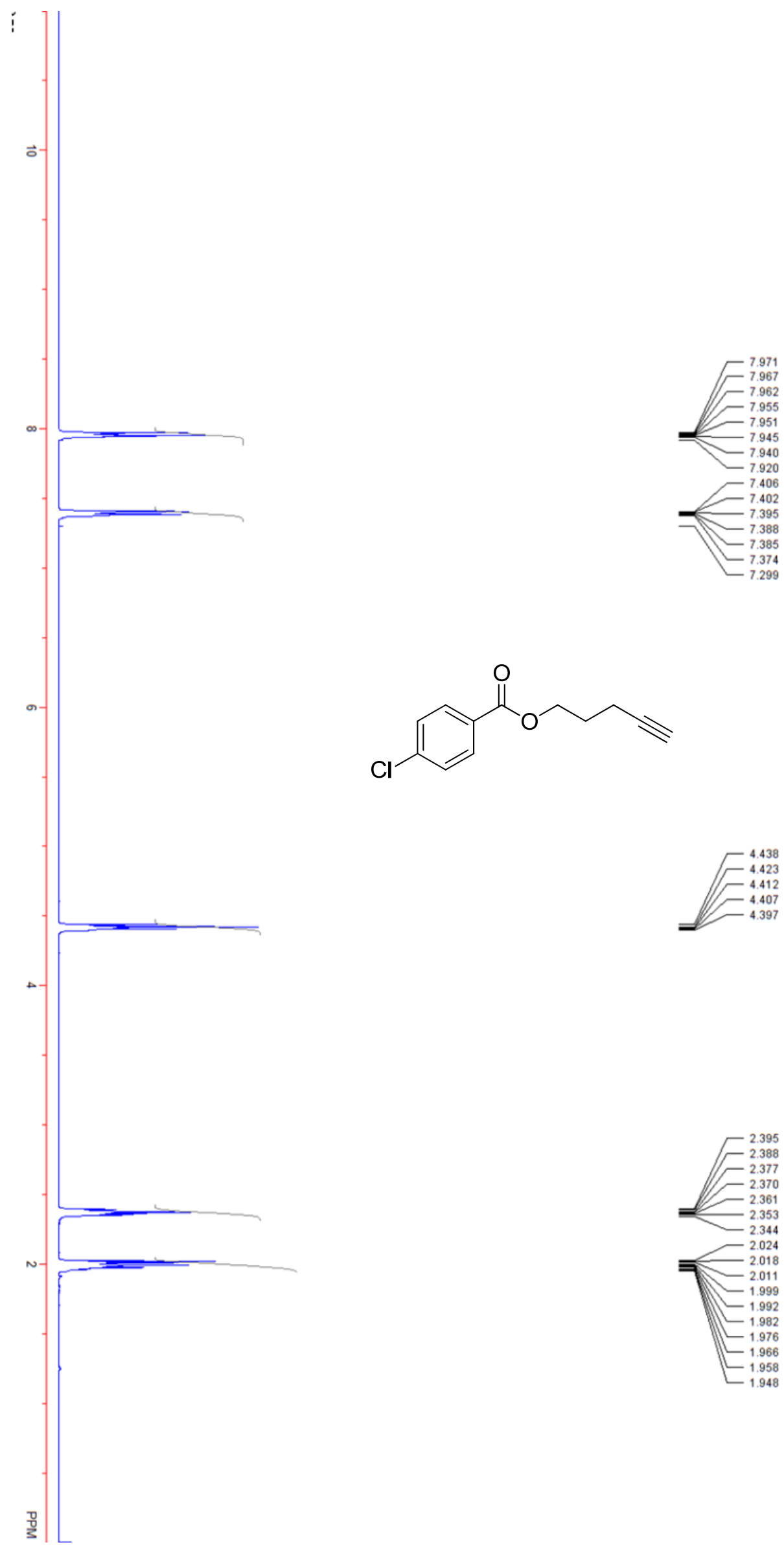<smiles>C#CCCCOC(=O)c1ccc(Cl)cc1</smiles>
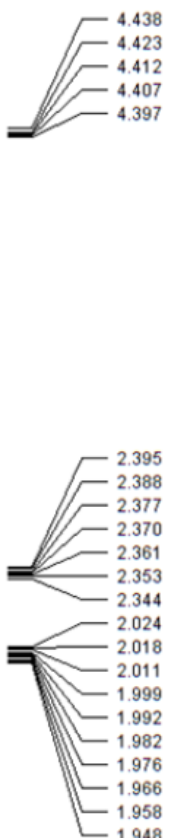


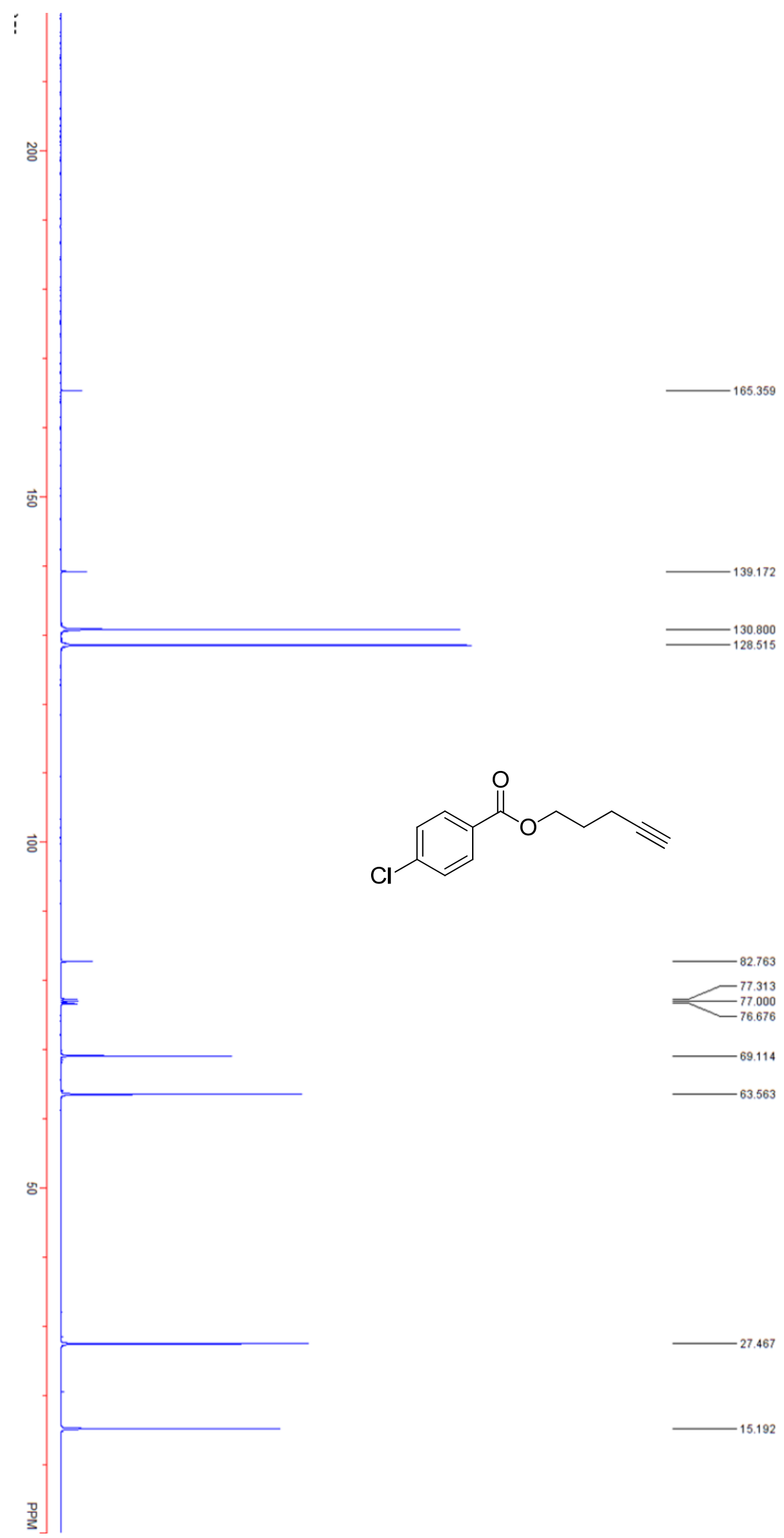




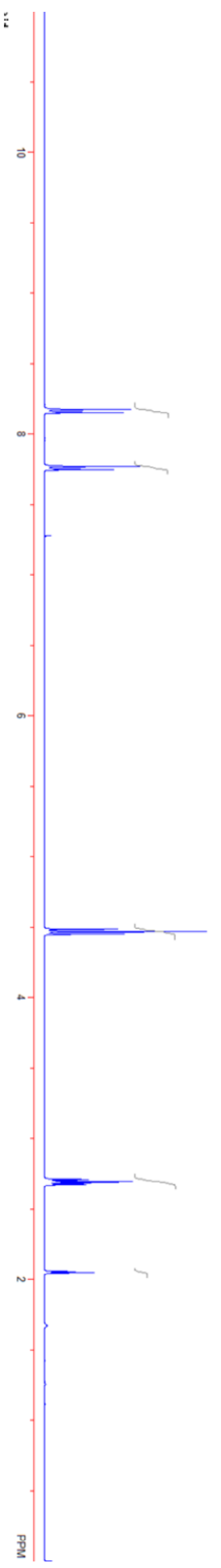<smiles>C#CCCOC(=O)c1ccc(C#N)cc1</smiles>

$\overbrace{}^{4.486}$

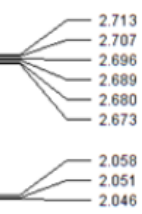



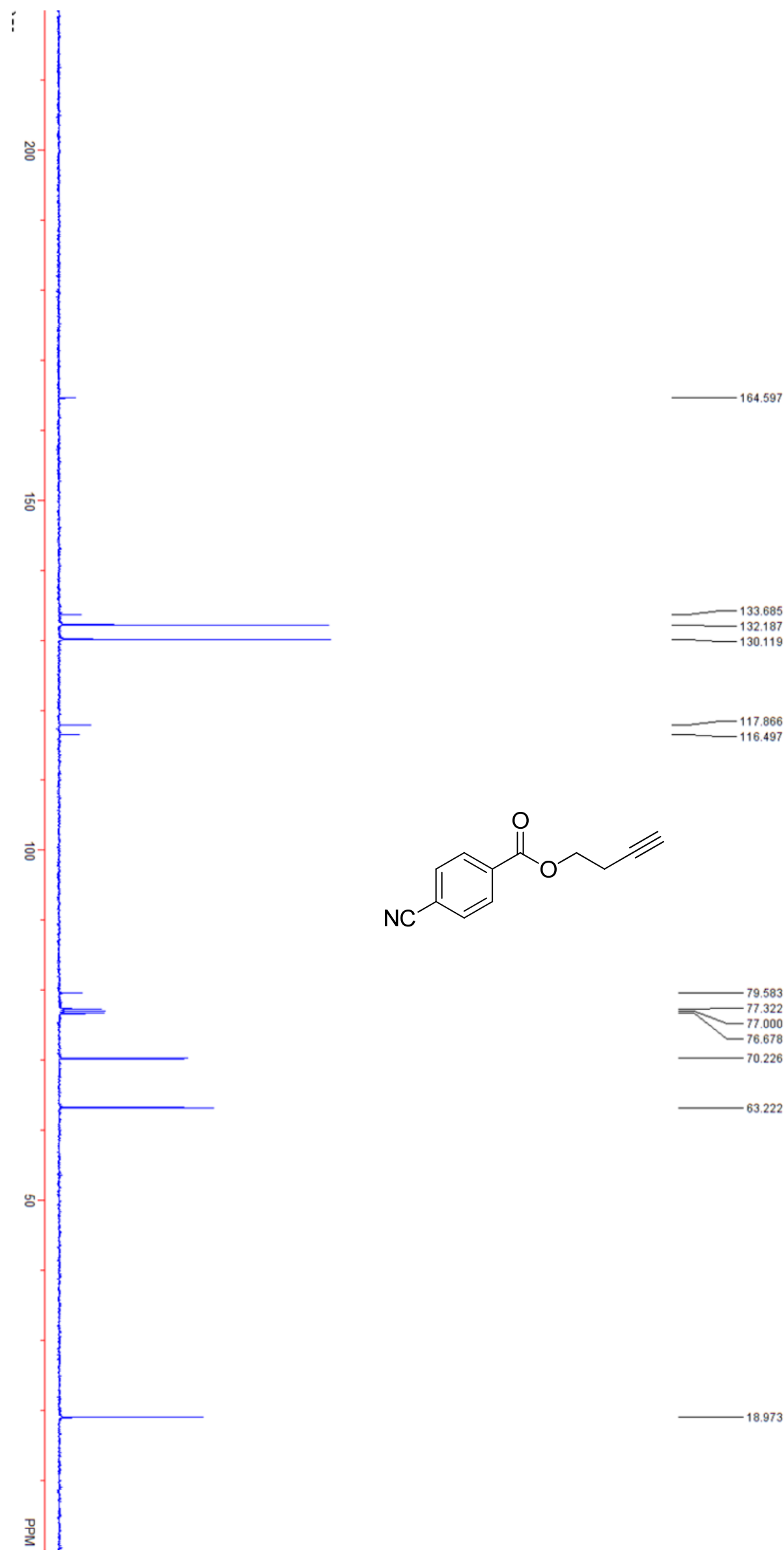

117.866
116.497<smiles>C#CCCOC(=O)c1ccc(C#N)cc1</smiles>
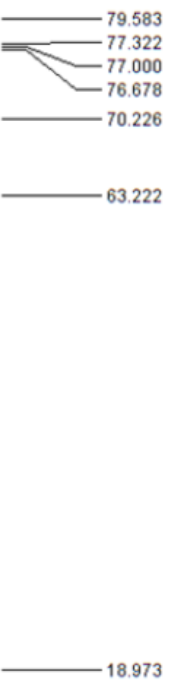


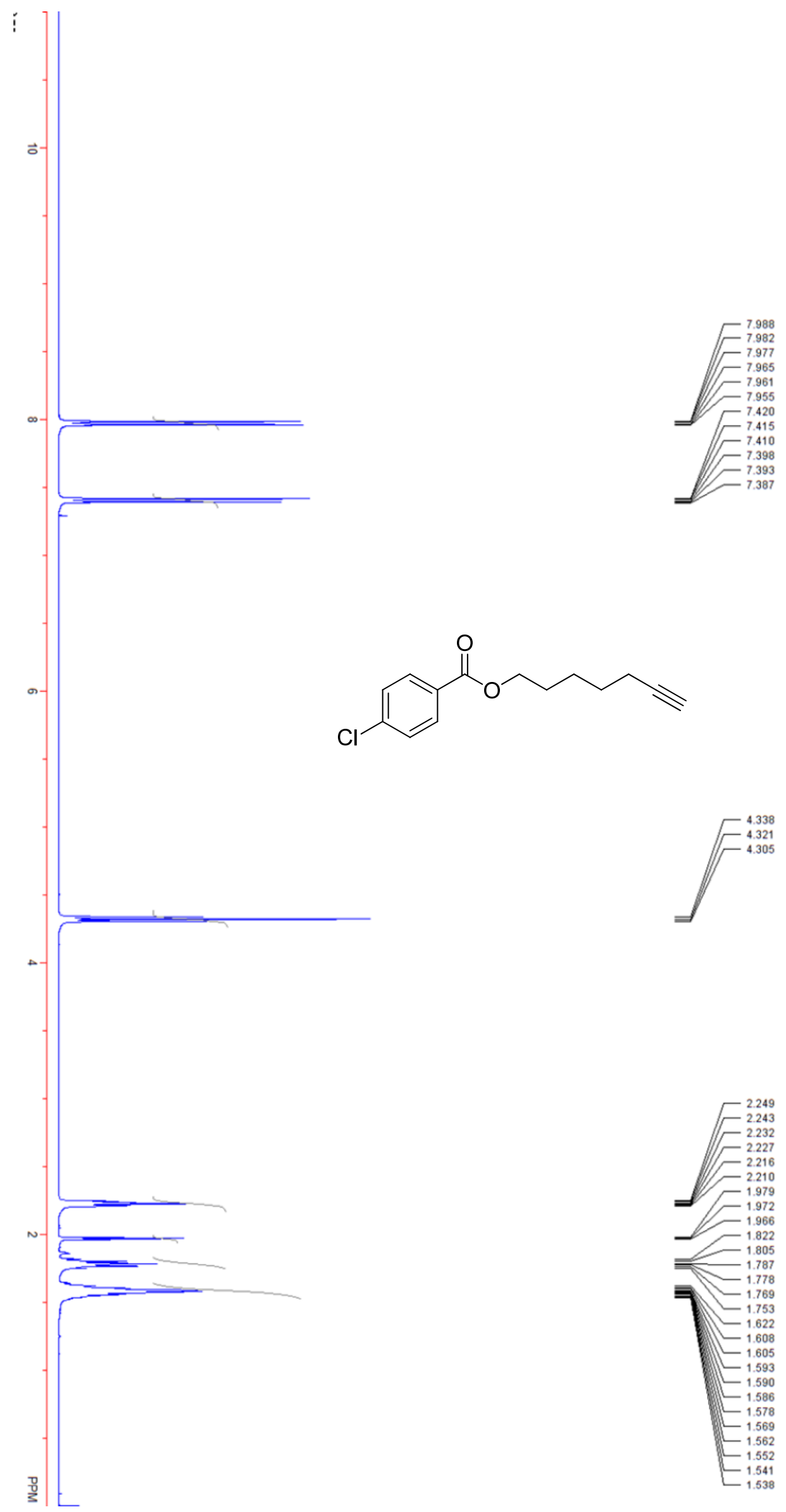




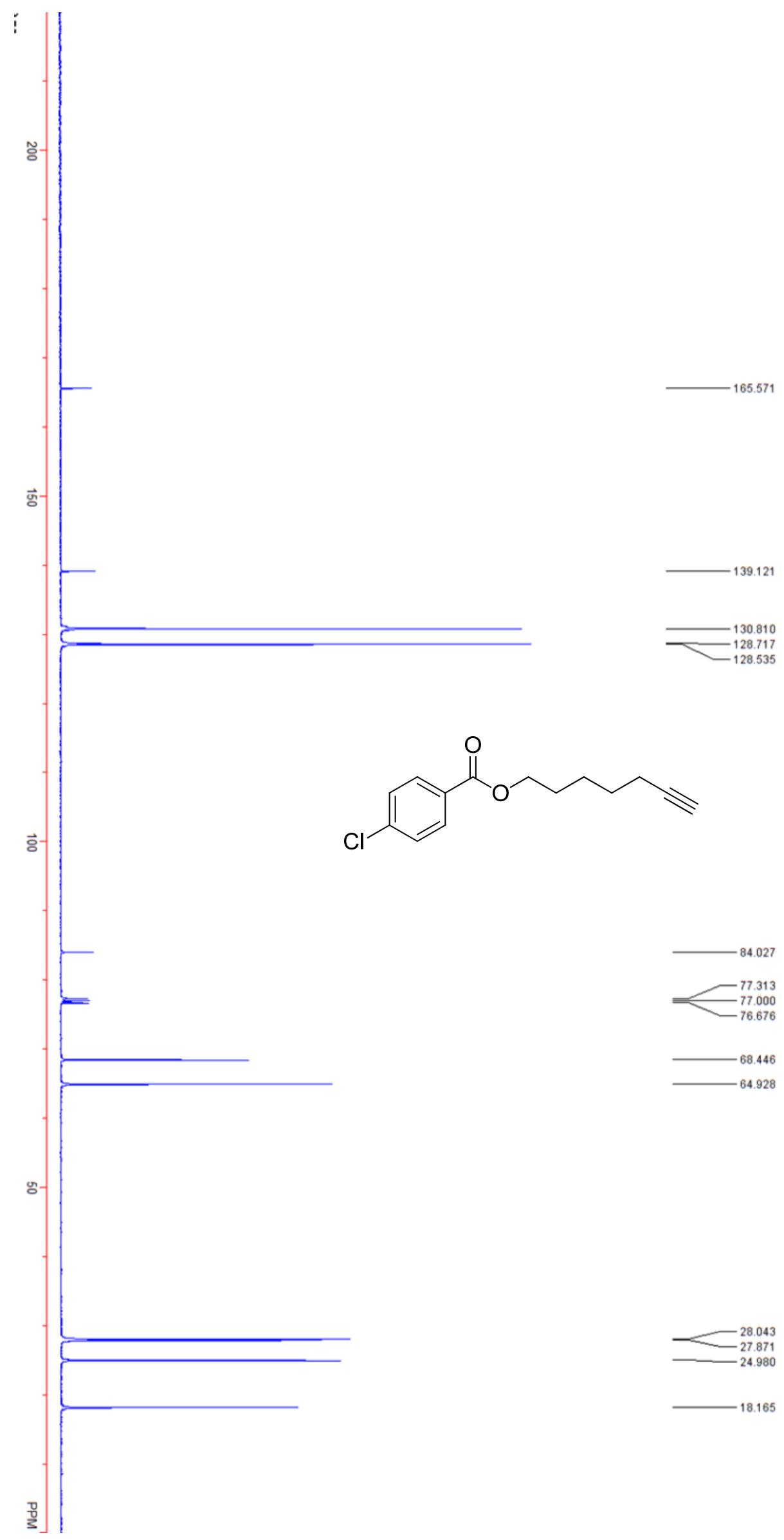




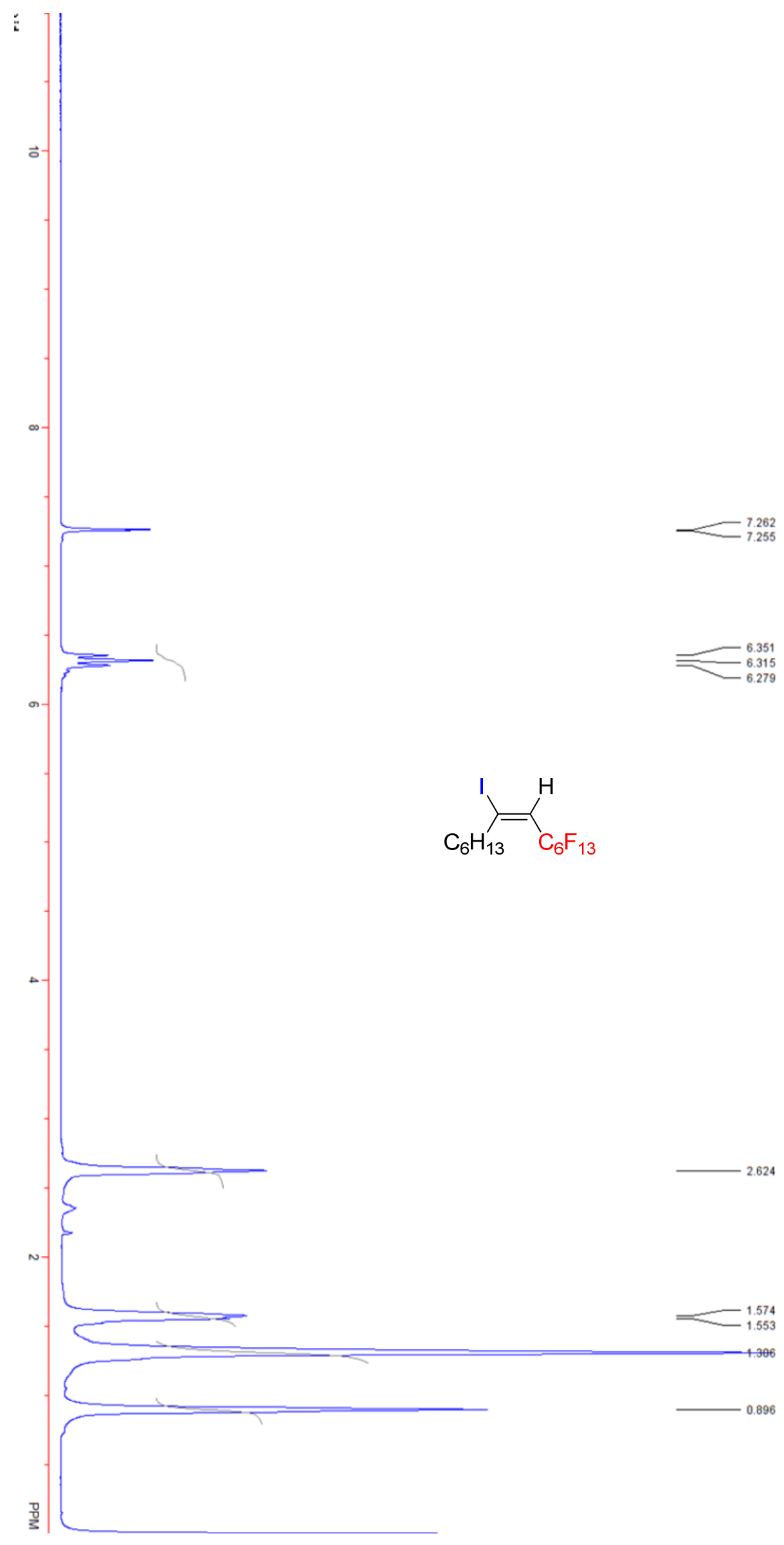



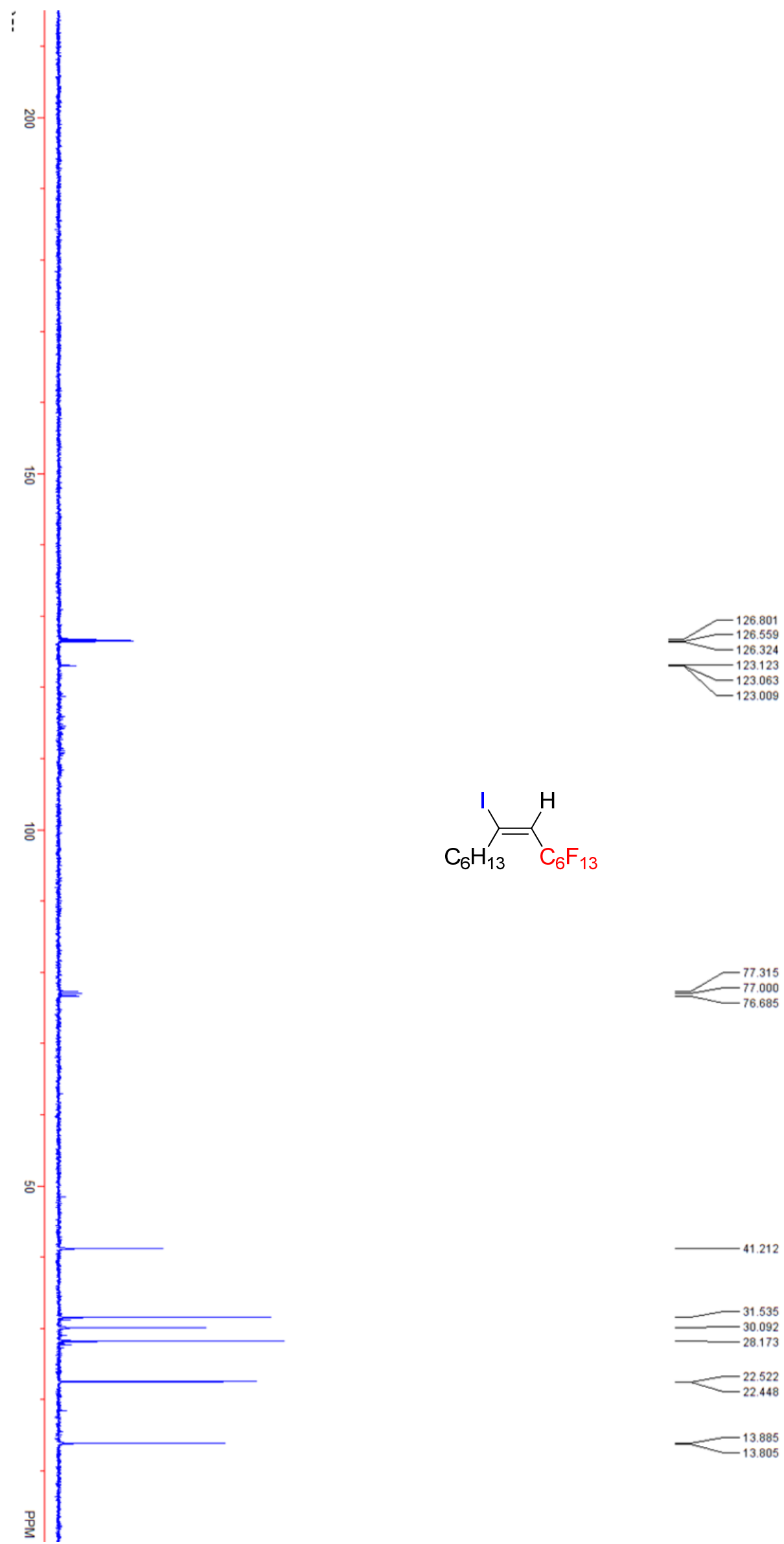

77.000
-76.685
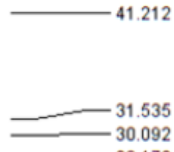

.
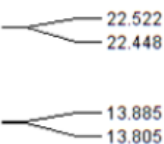


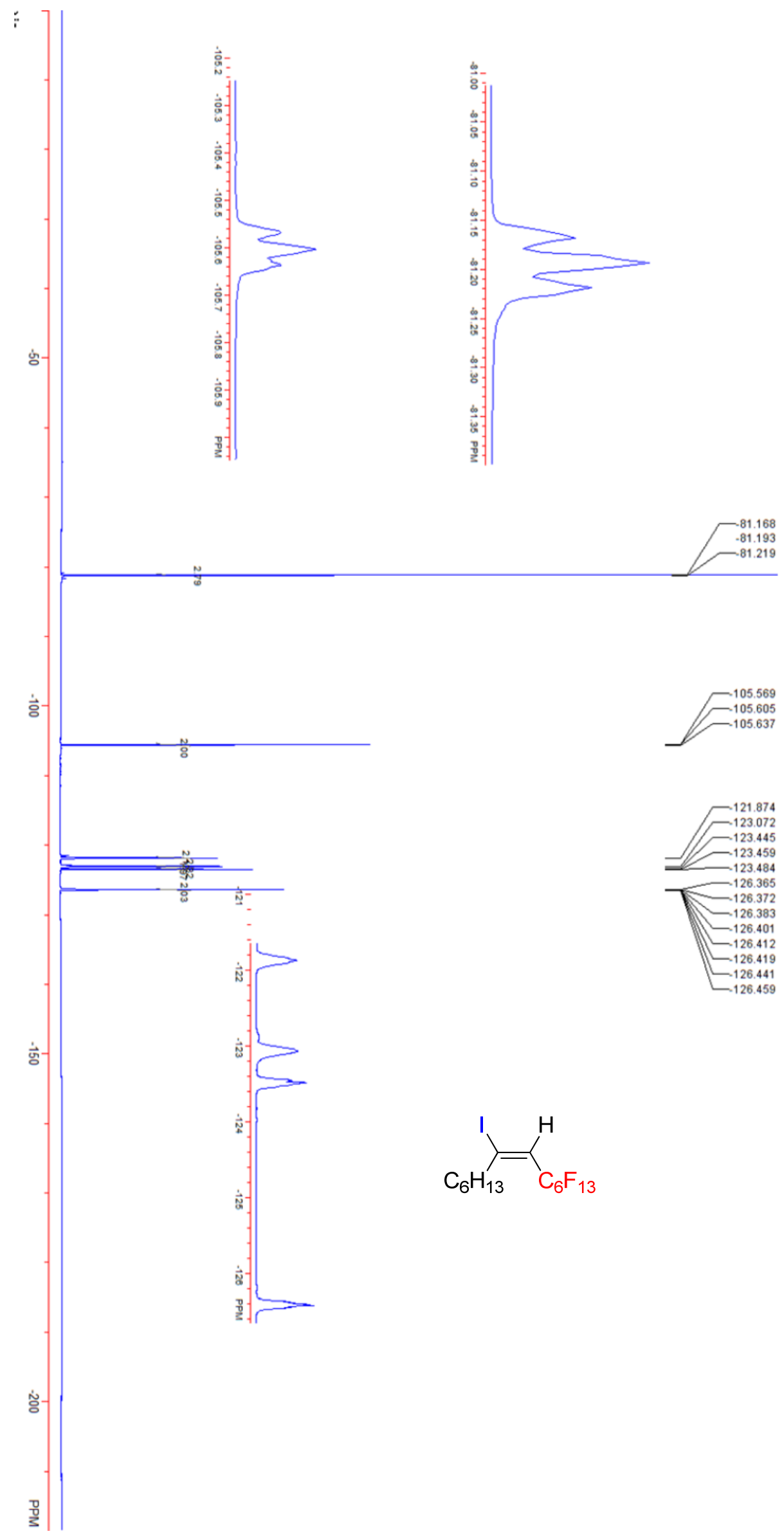




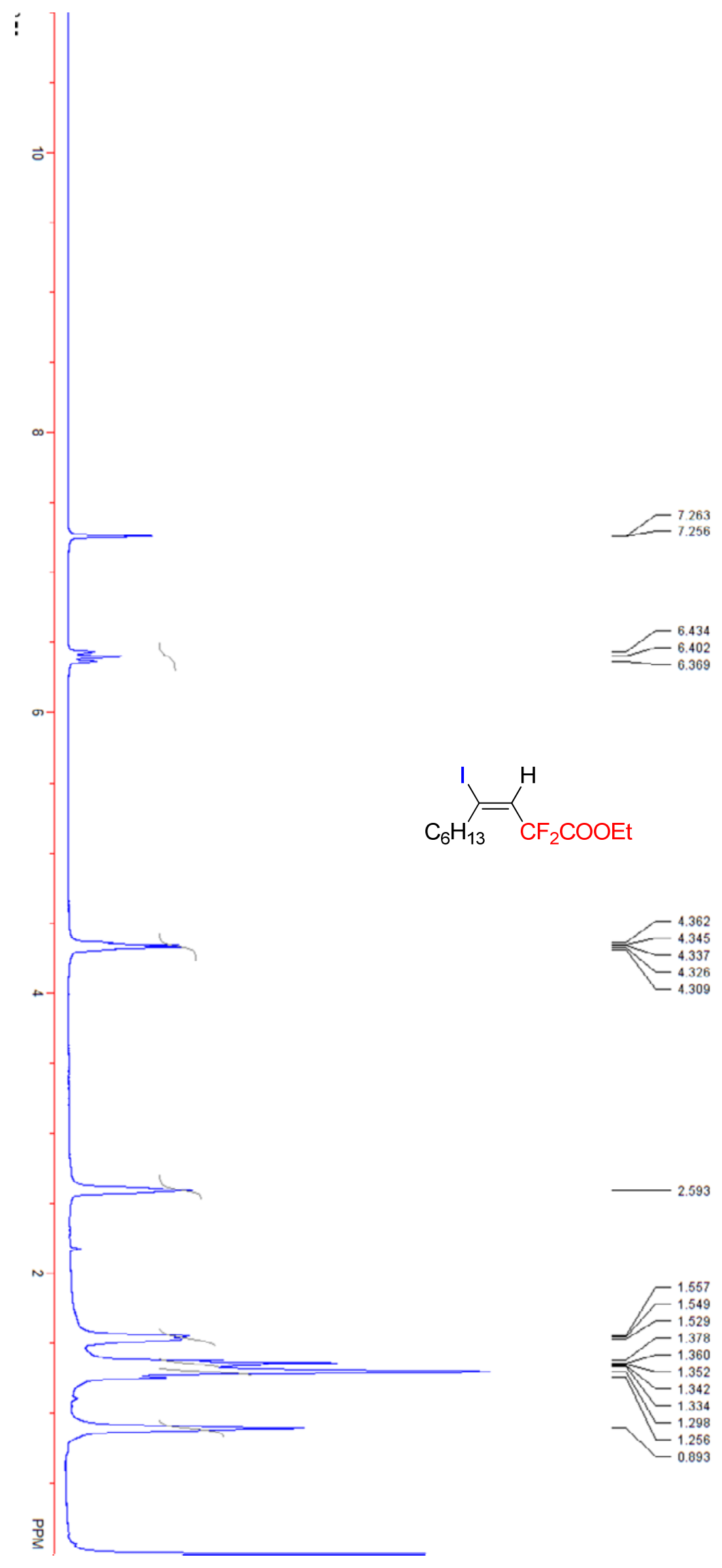



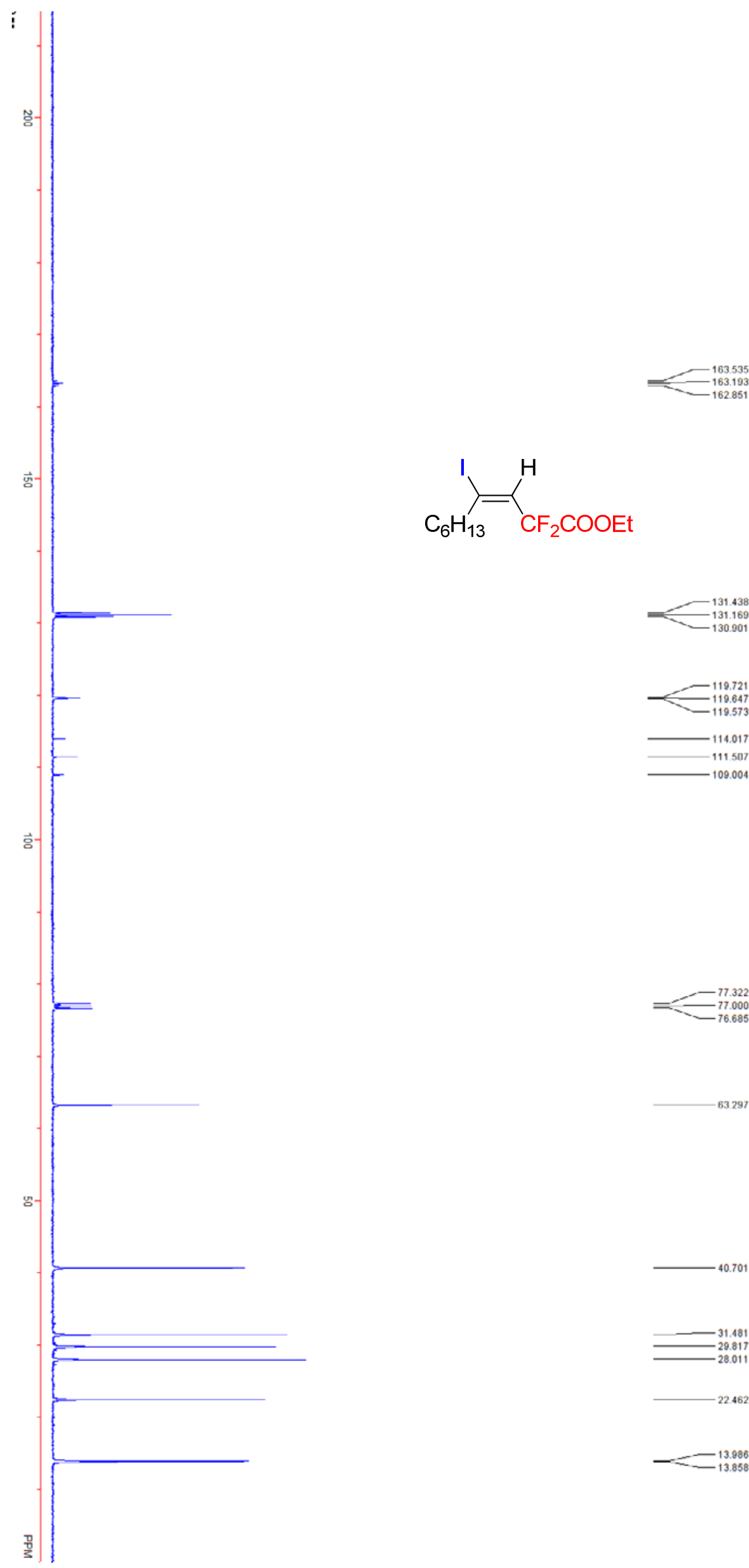

$\mathrm{C}_{6} \mathrm{H}_{13} \quad \mathrm{CF}_{2} \mathrm{COOEt}$
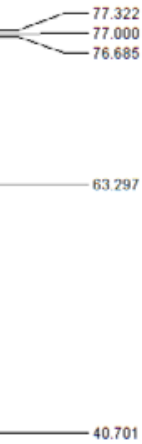

$\begin{array}{r}31.481 \\ -{ }^{2} .817 \\ \hline\end{array}$

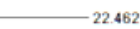

$\longrightarrow=\square_{13.858}^{13.966}$ 


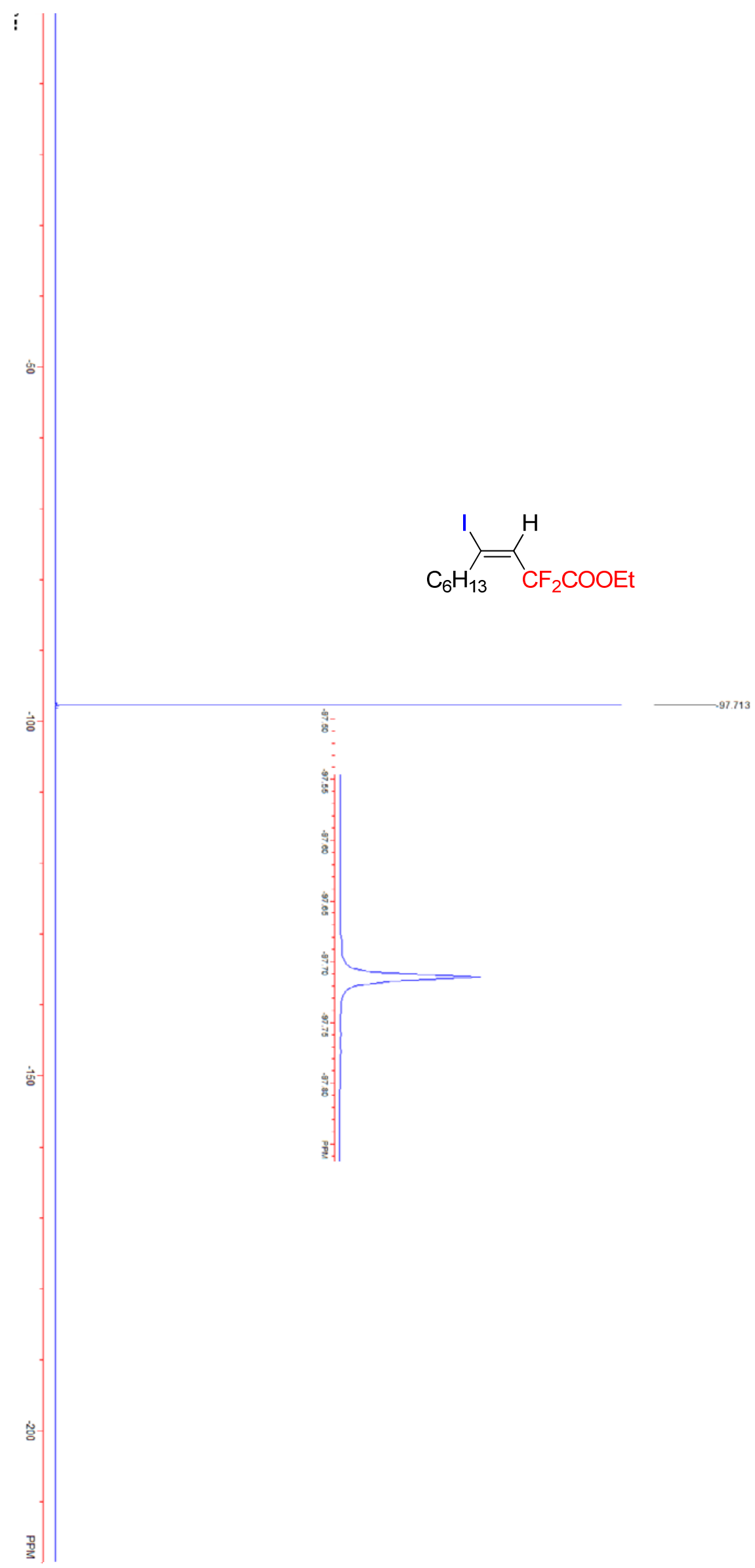




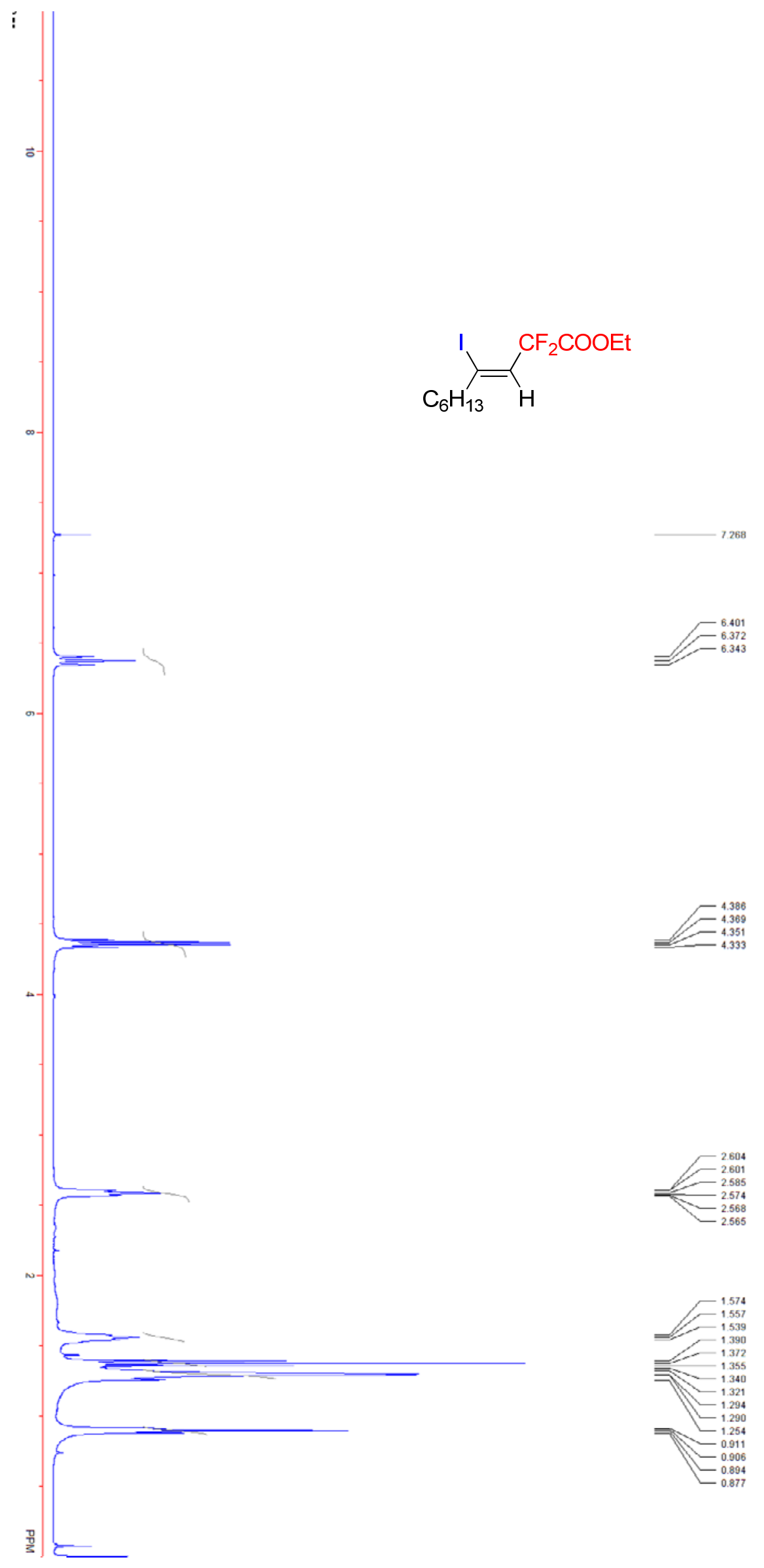



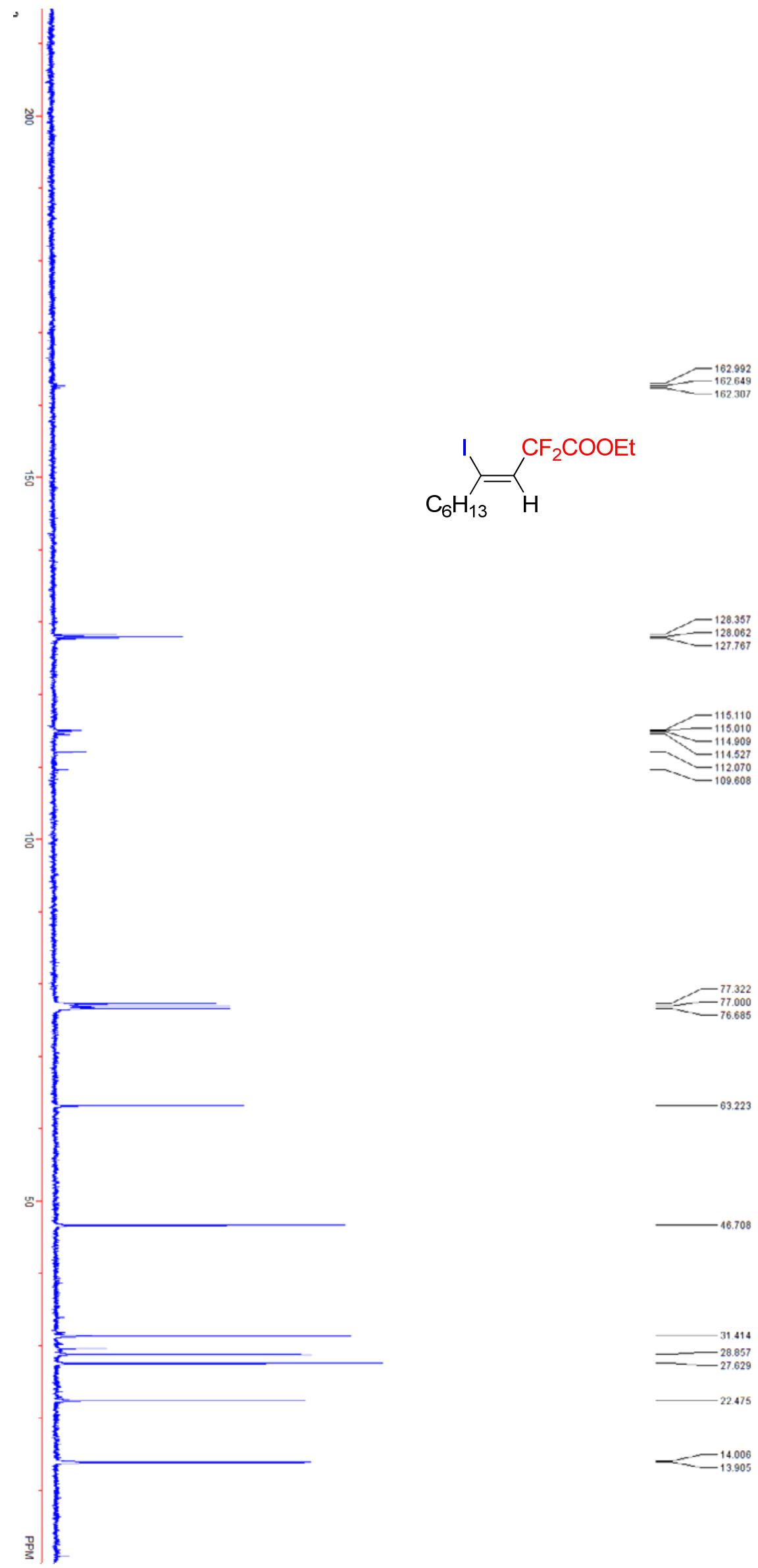


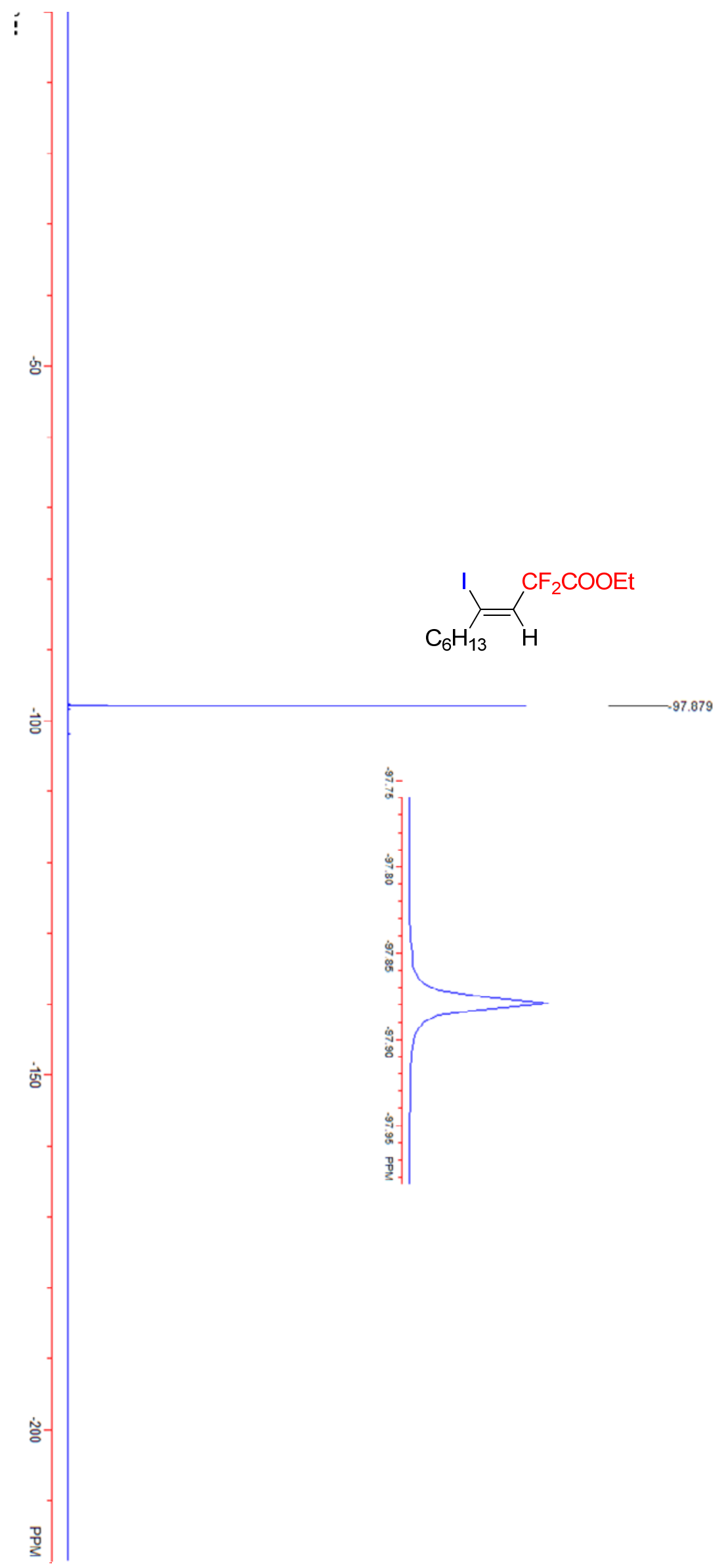




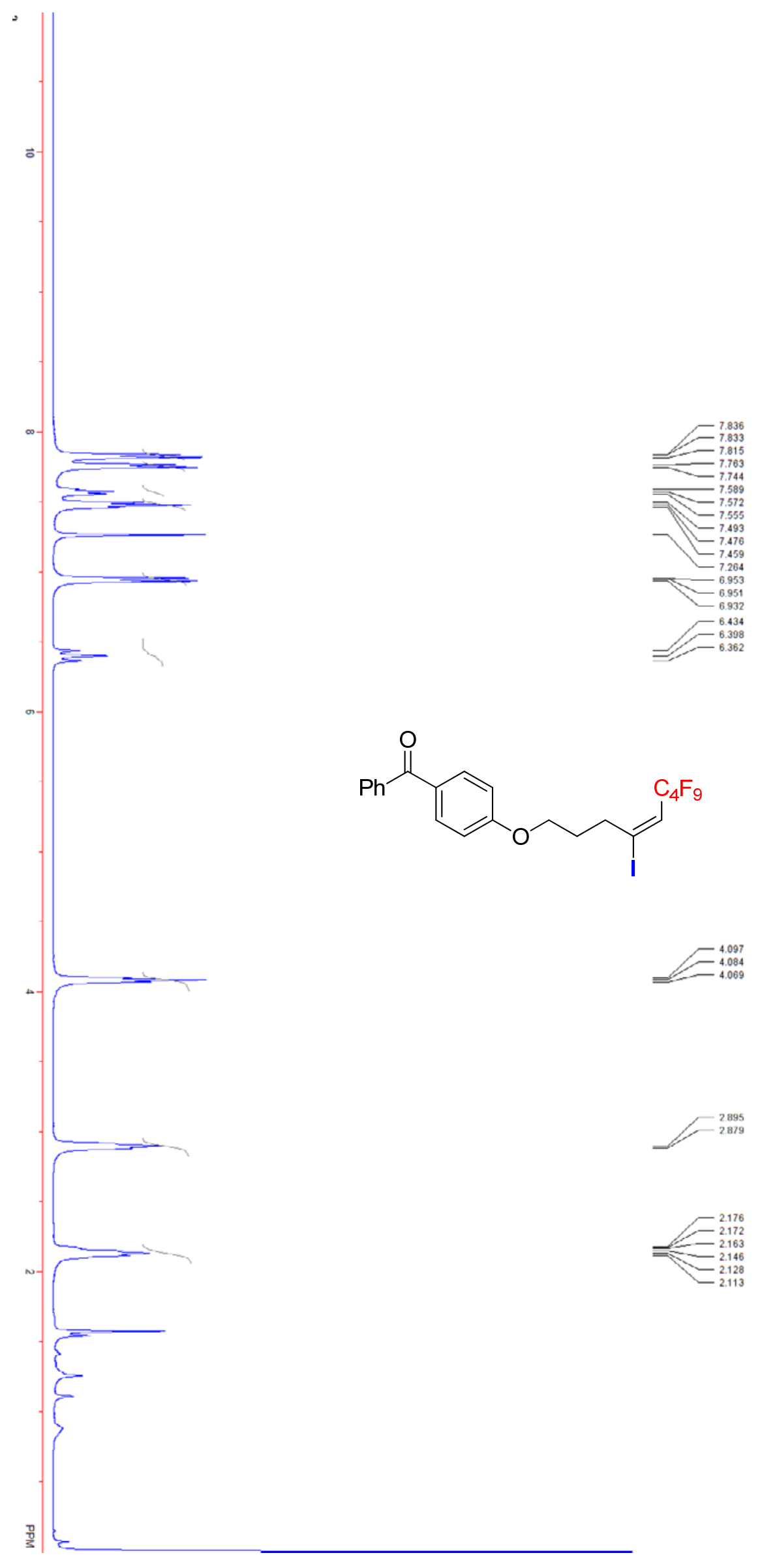




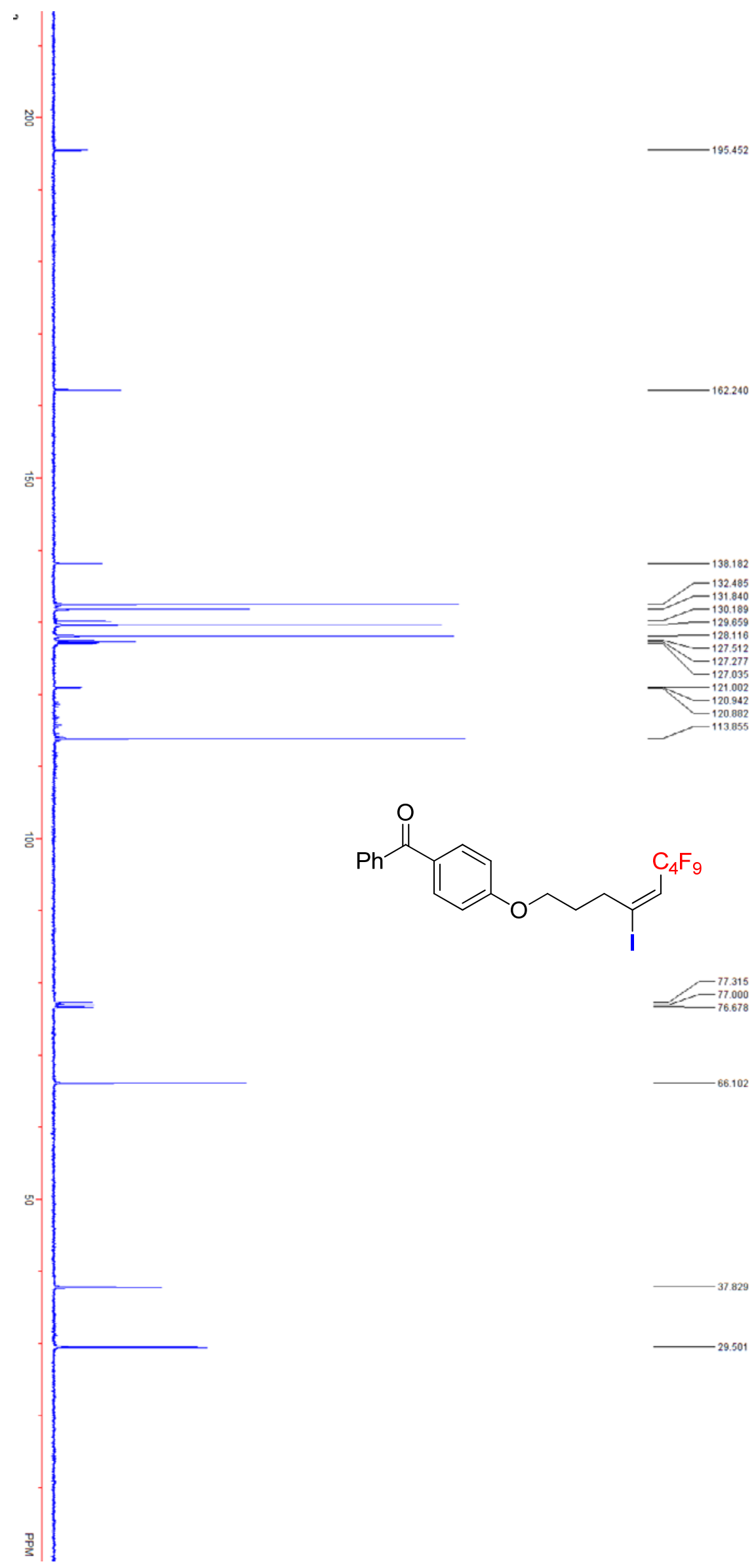




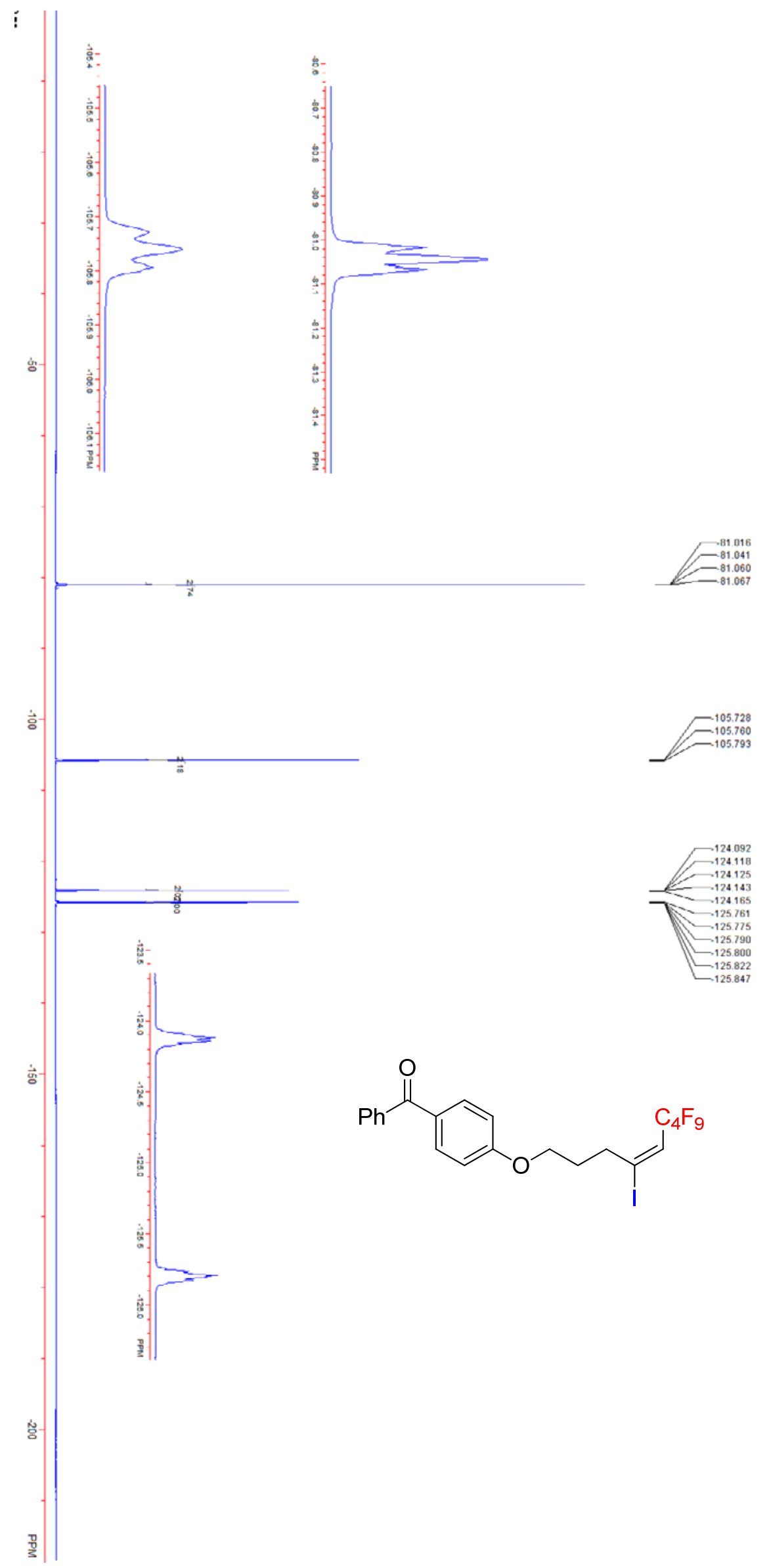




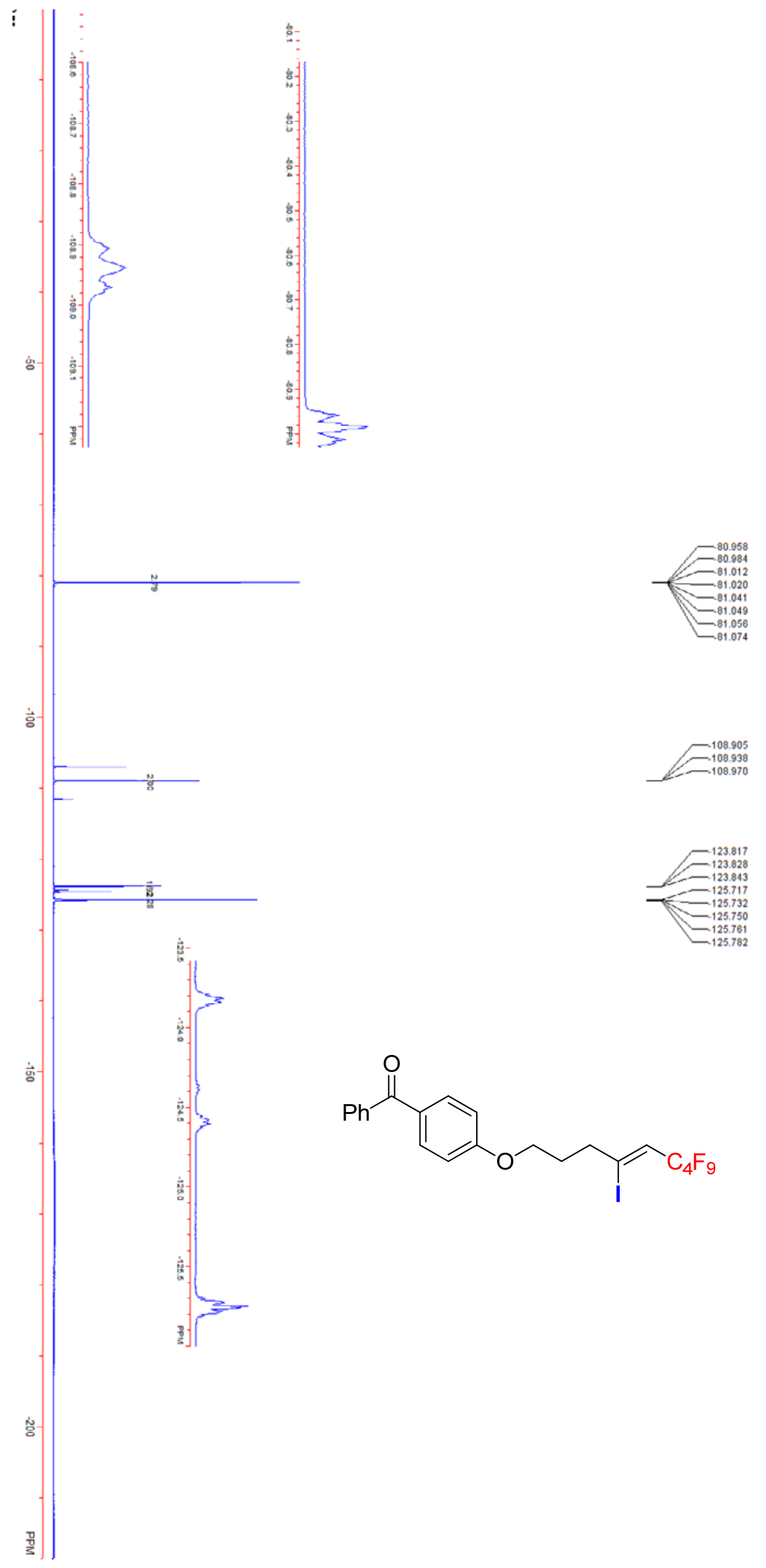




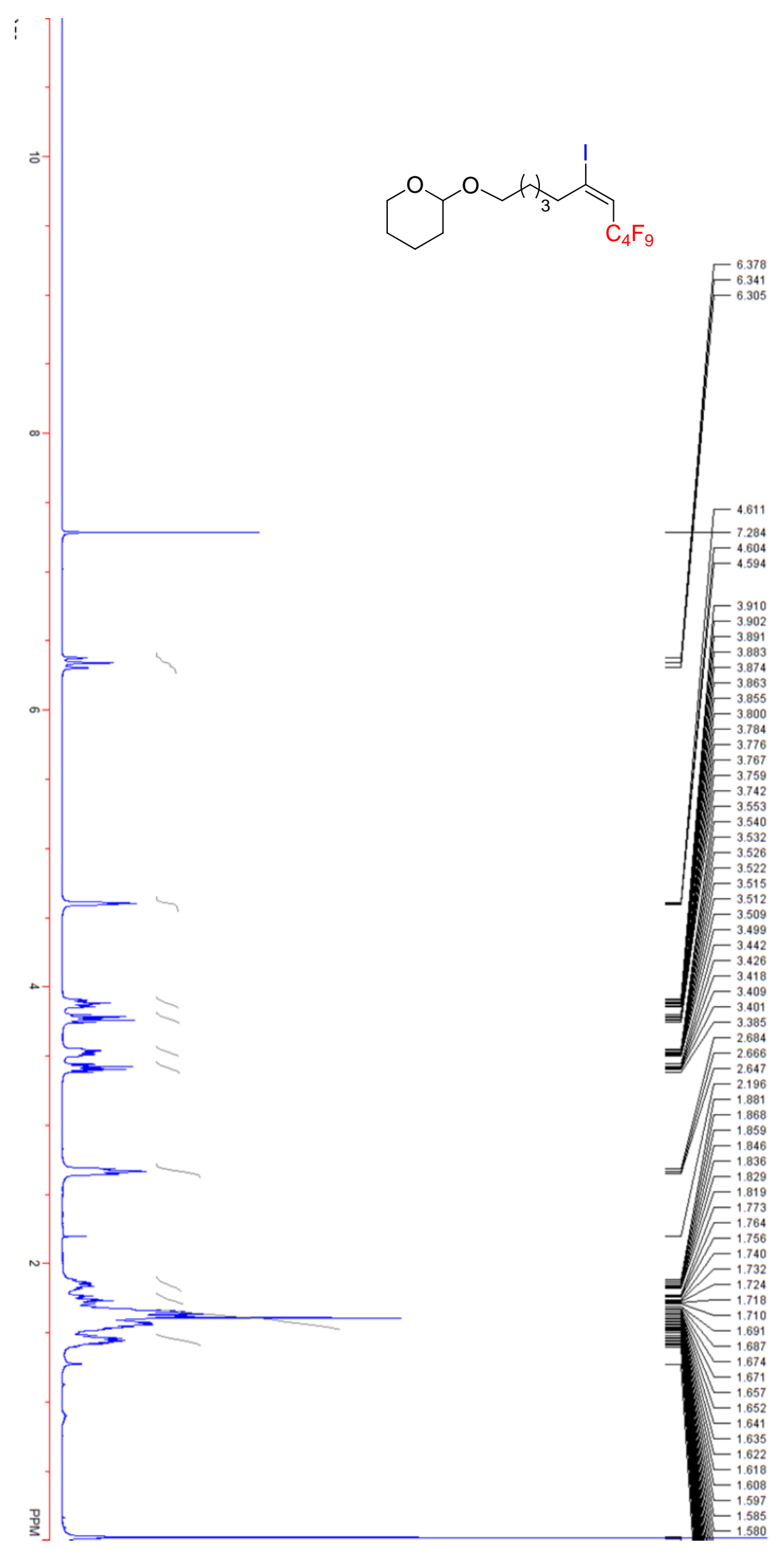

S51 

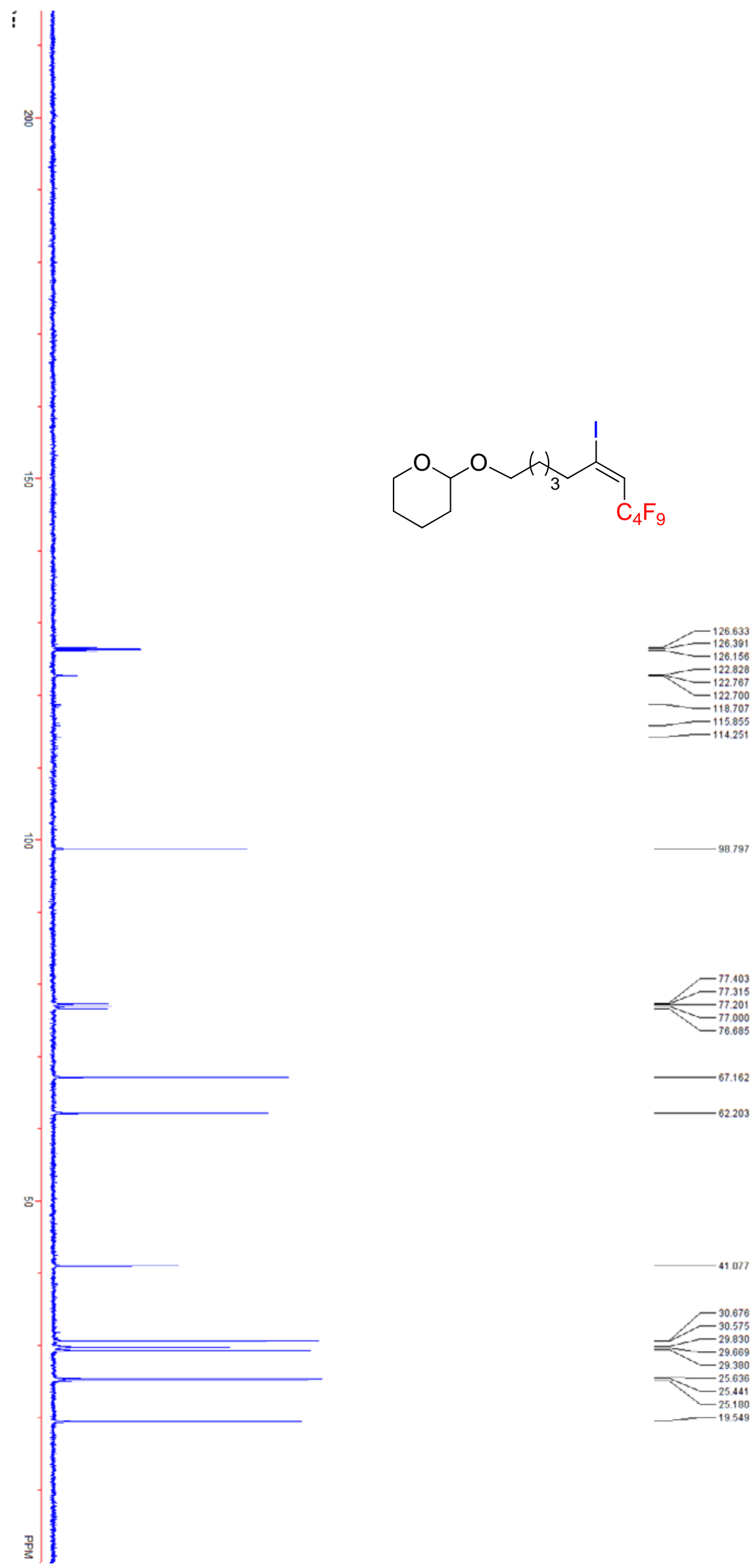


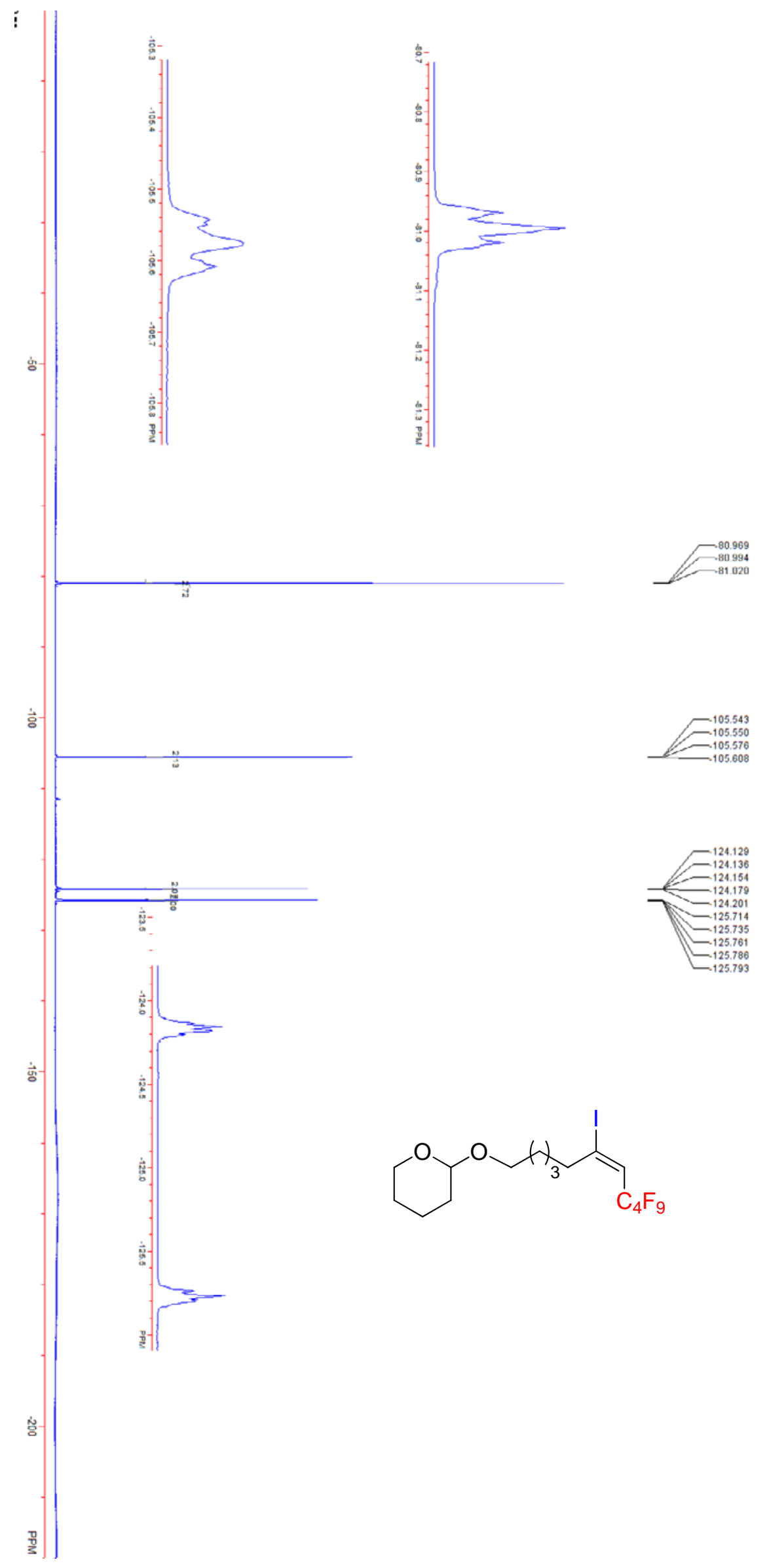




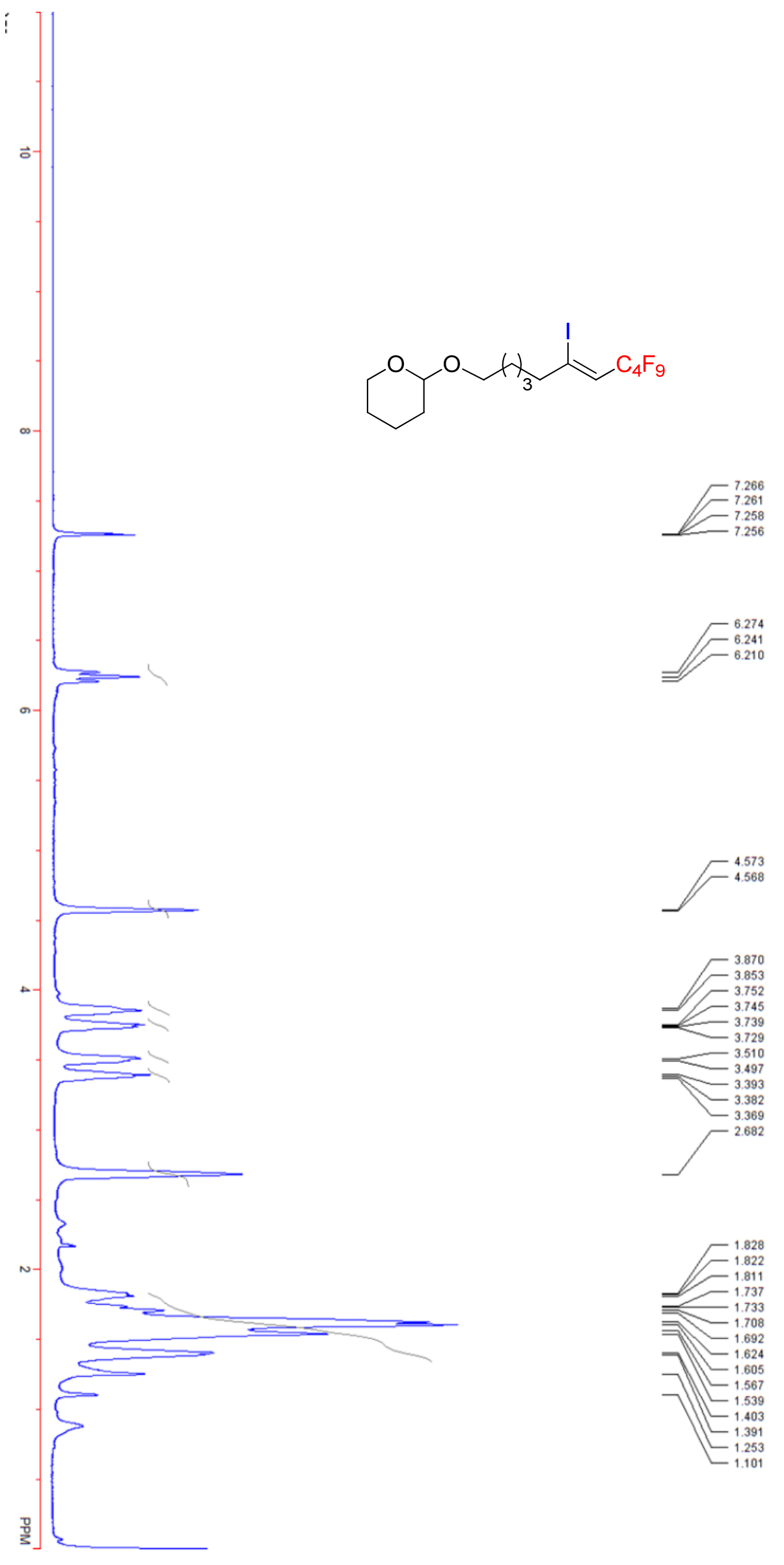




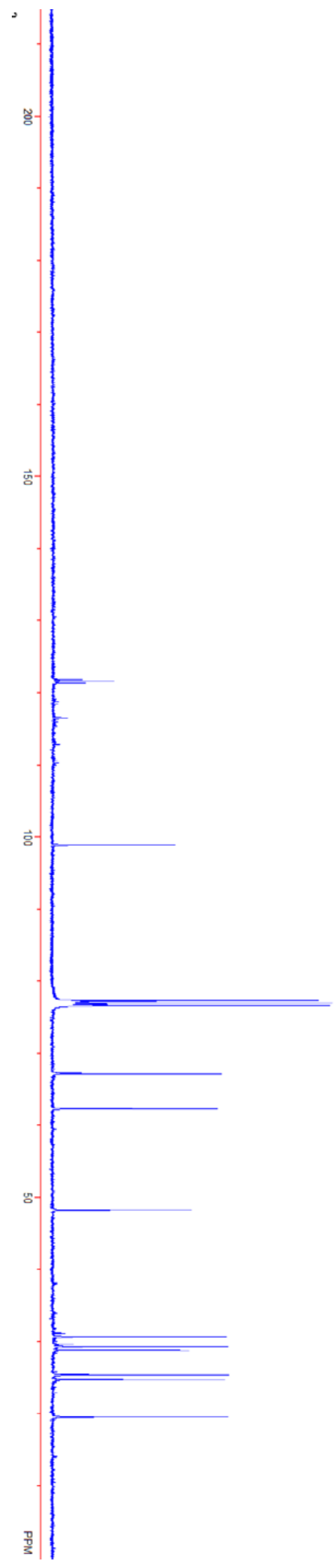

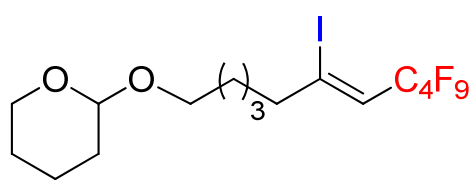
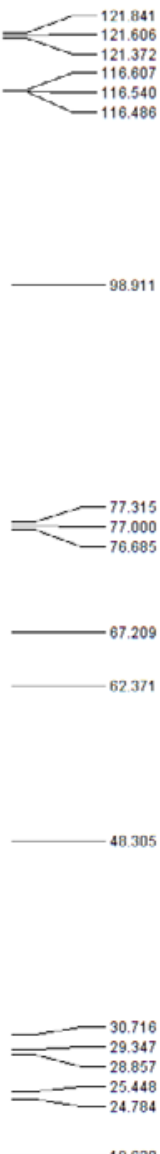


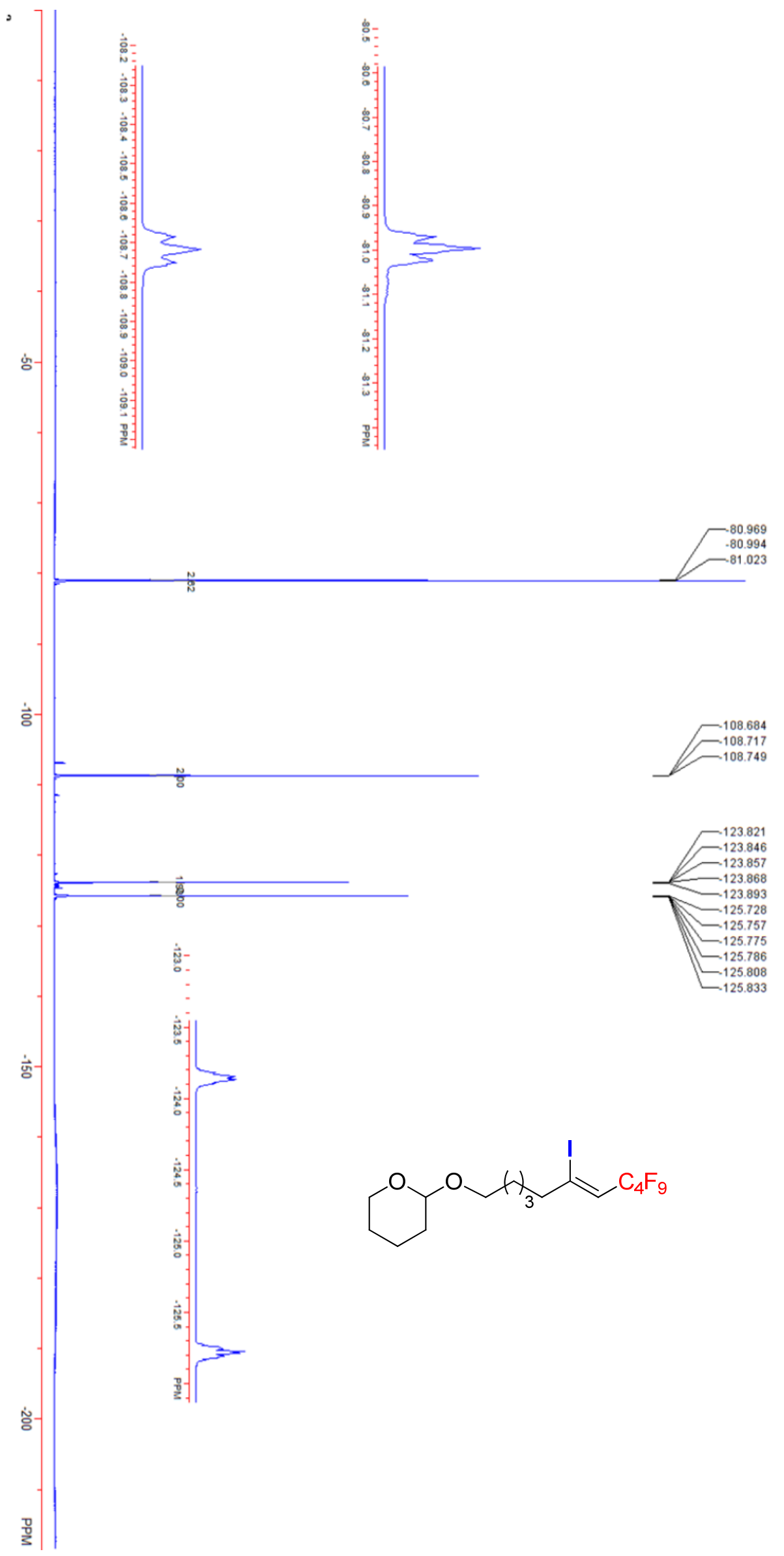




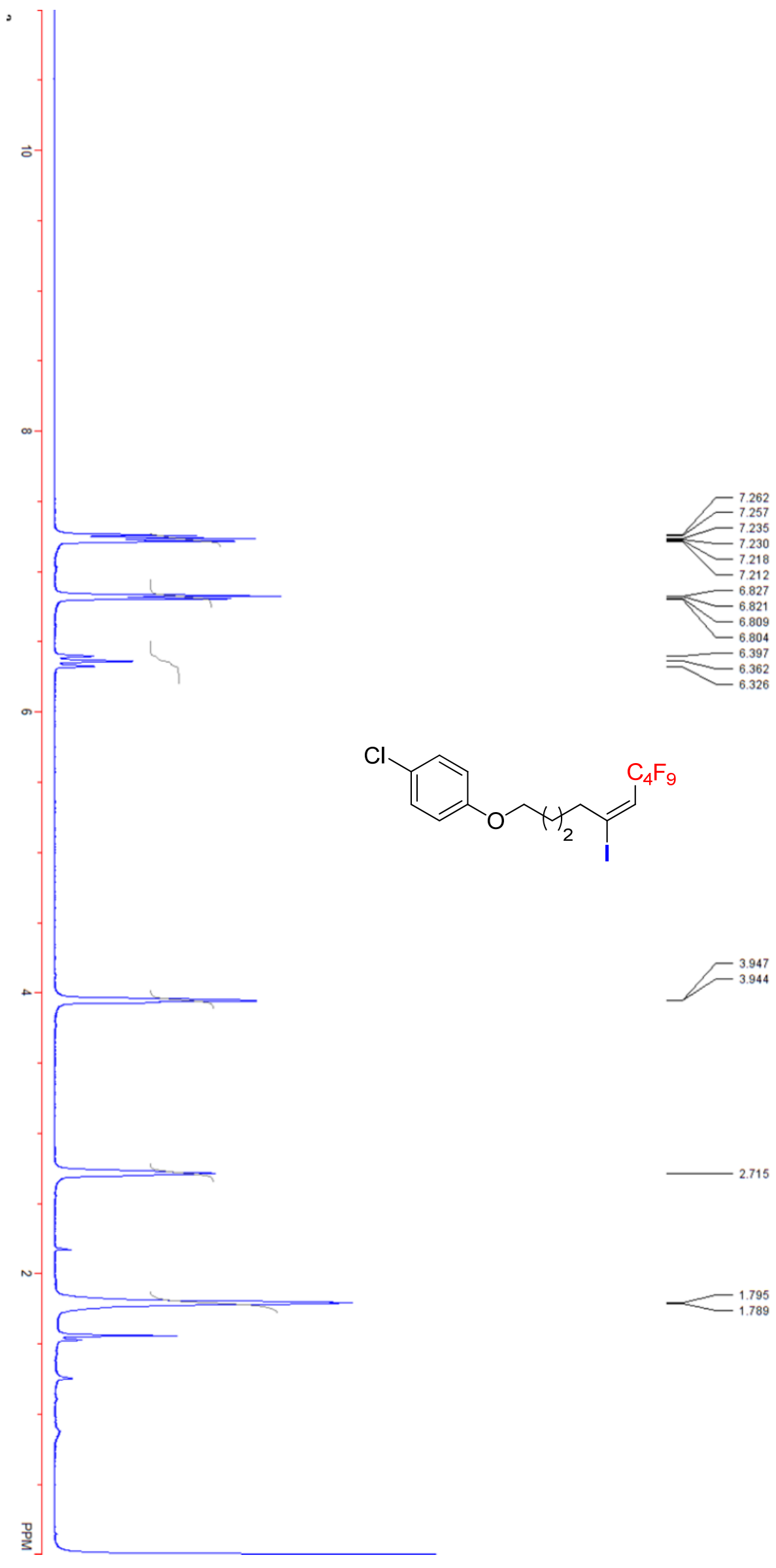

S57 


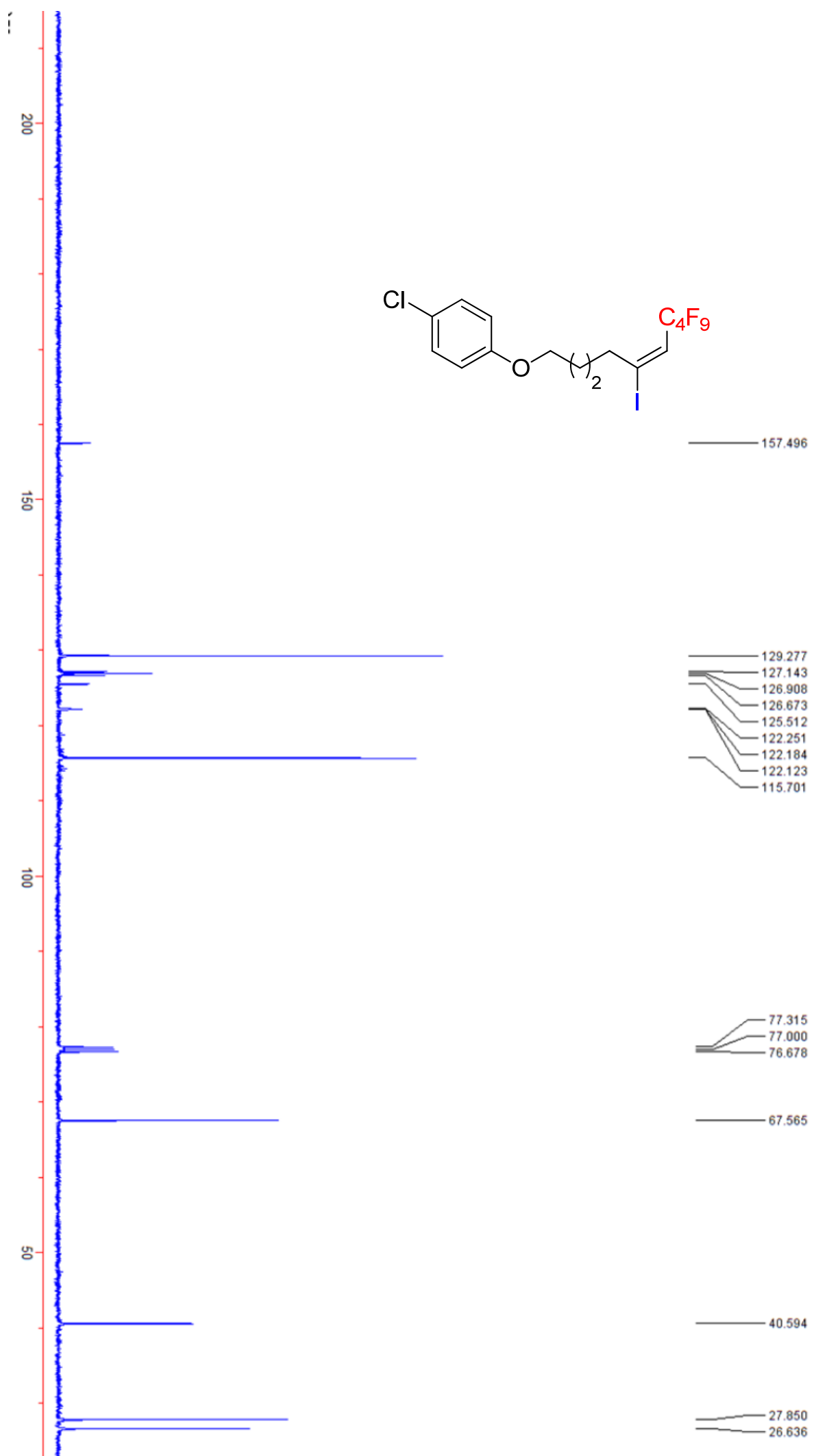

S58 


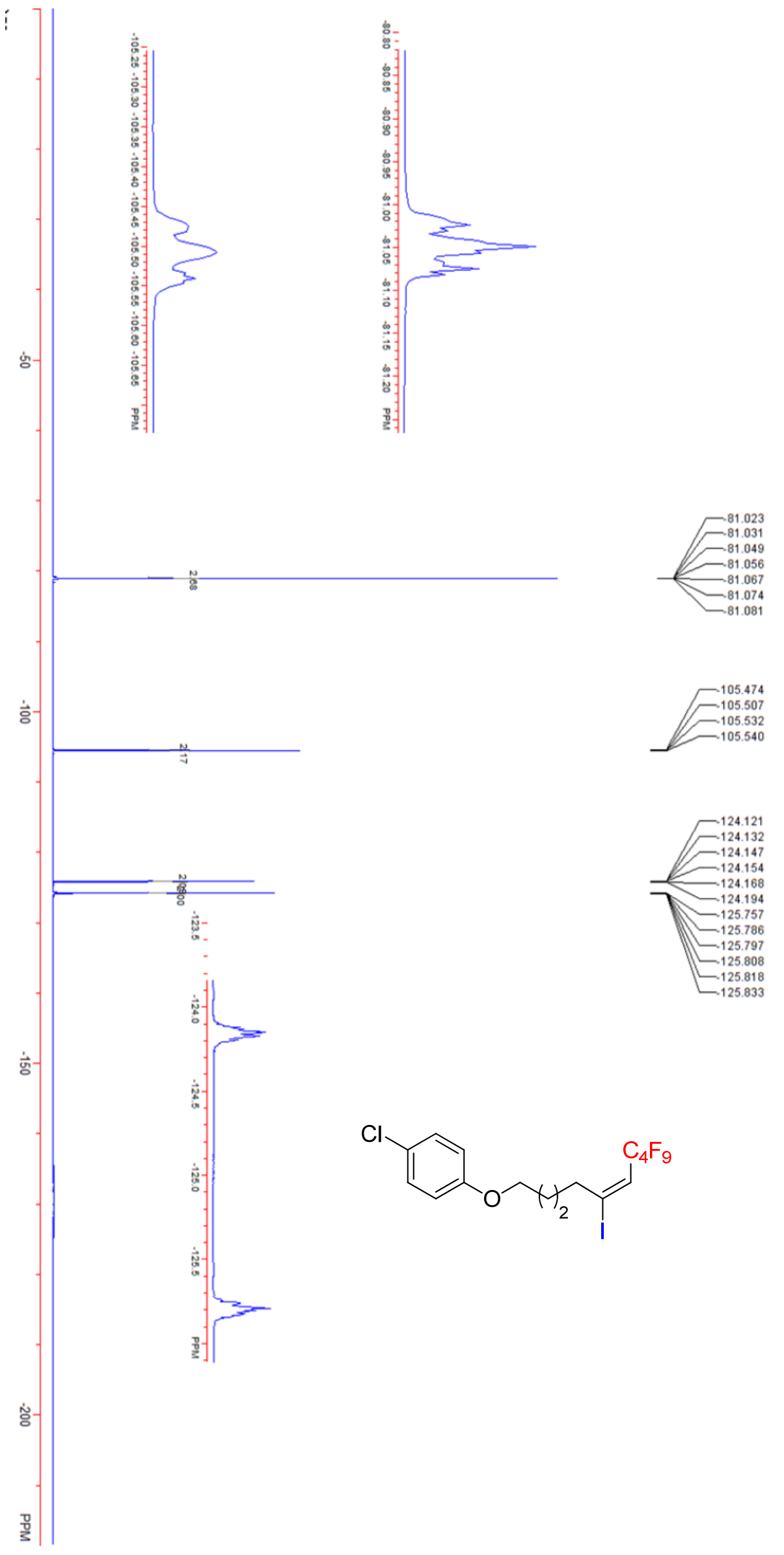




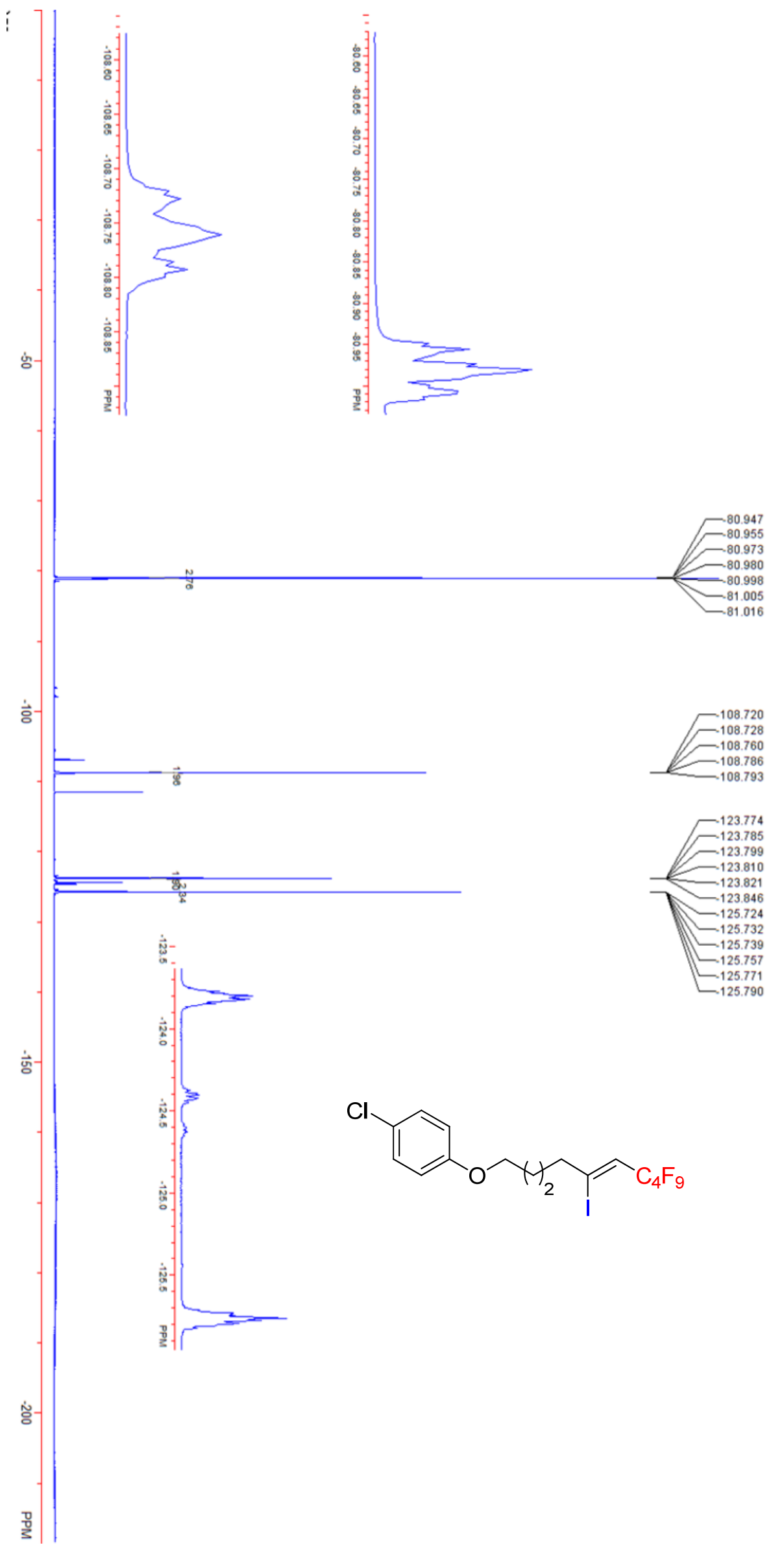



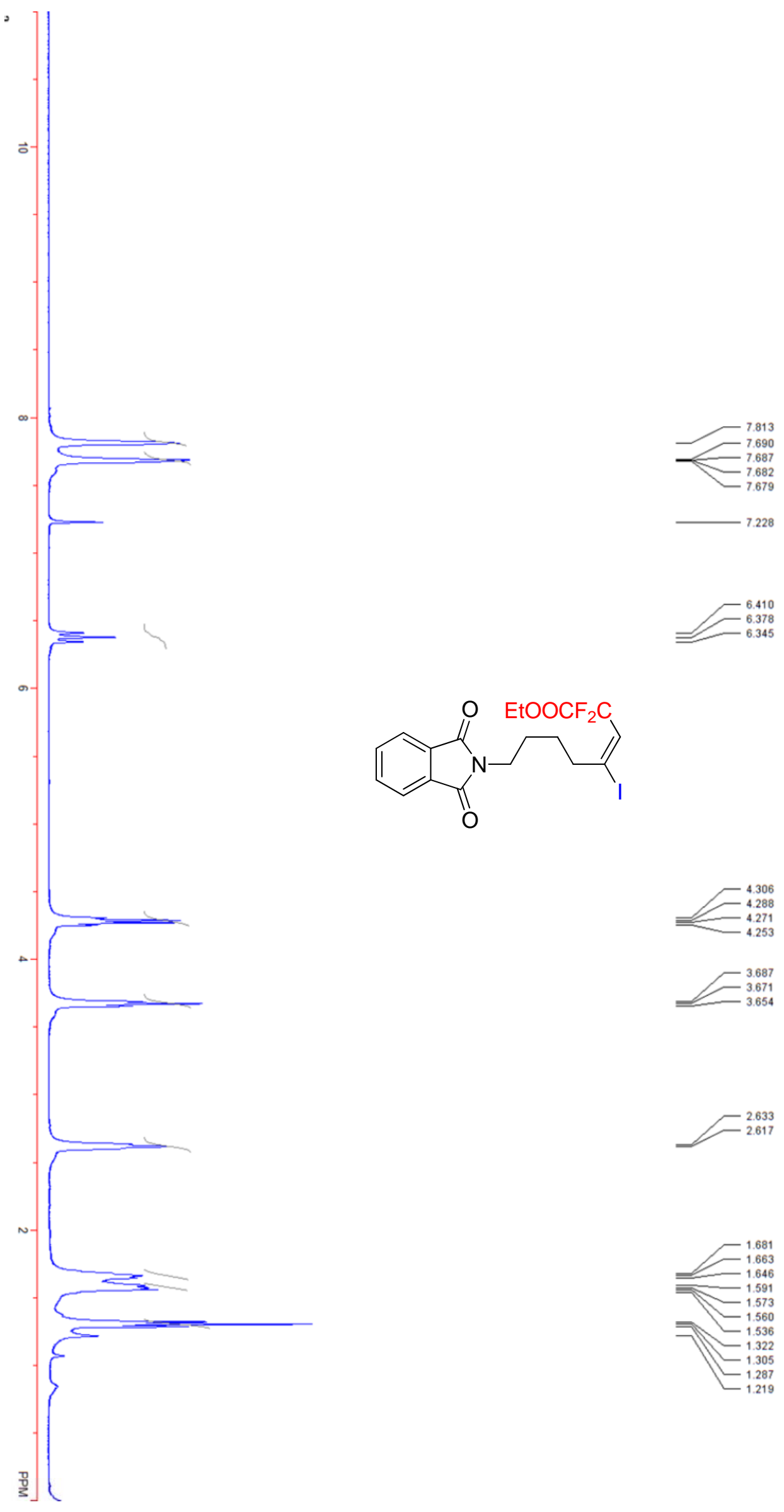


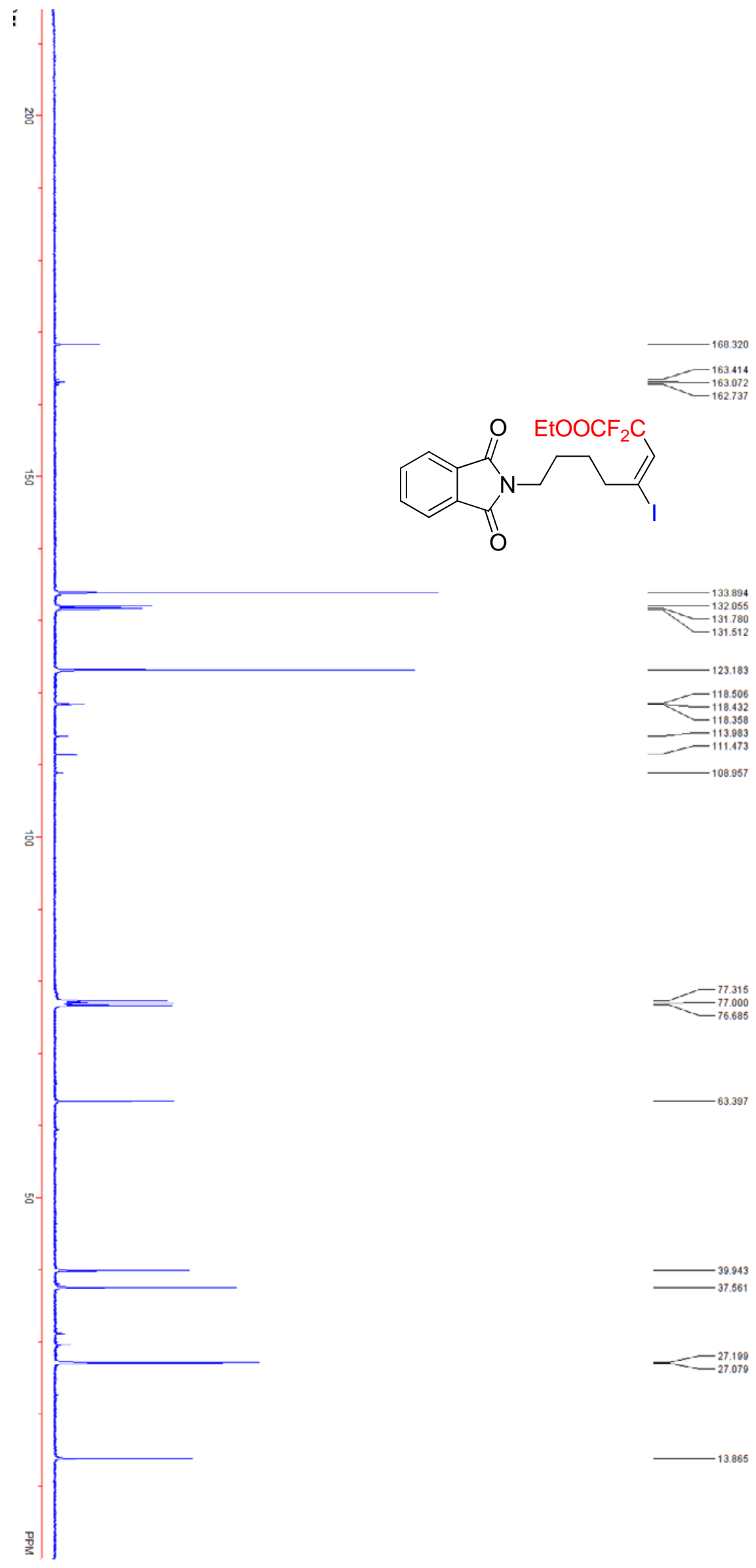




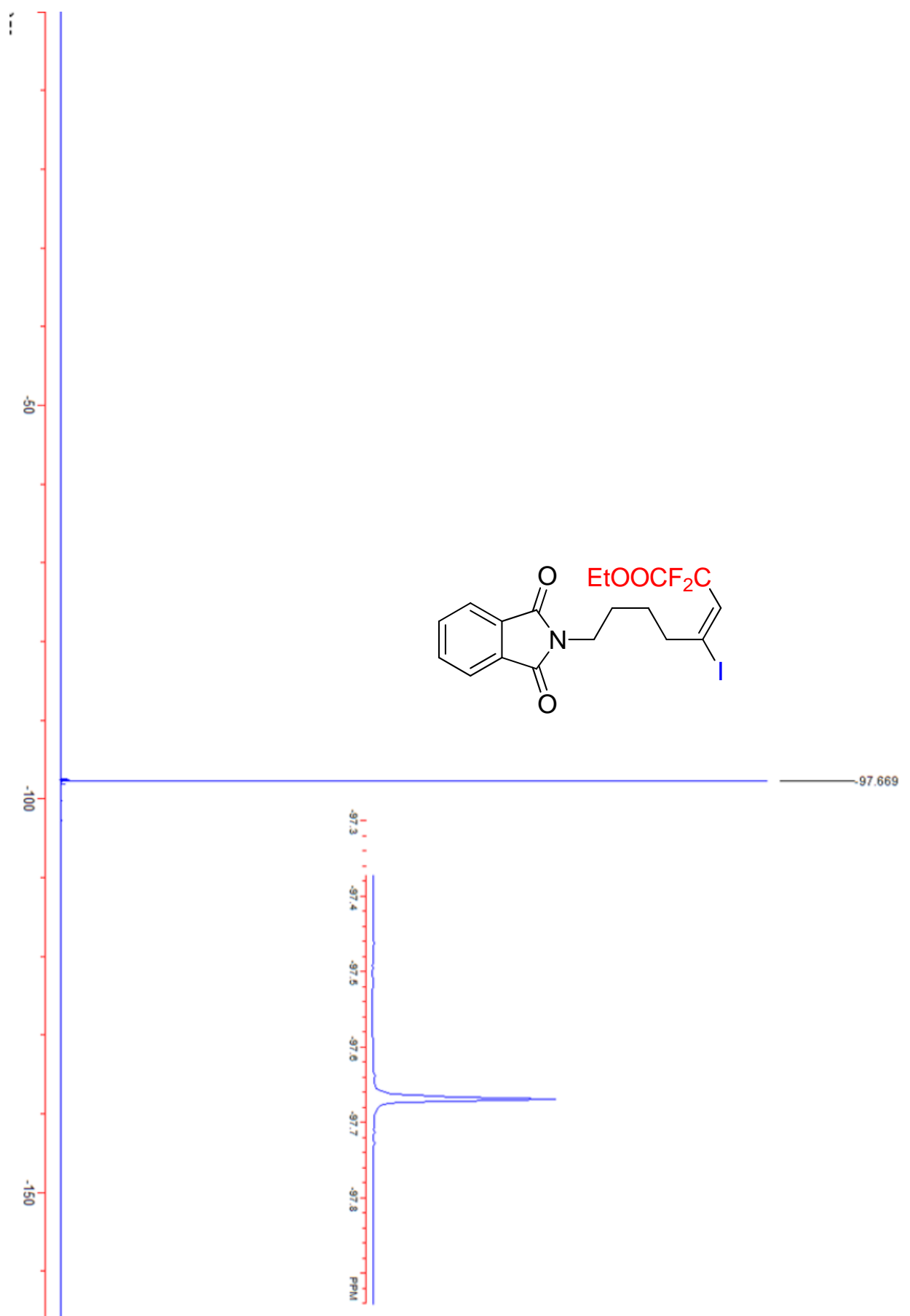

$\mid$

S63 


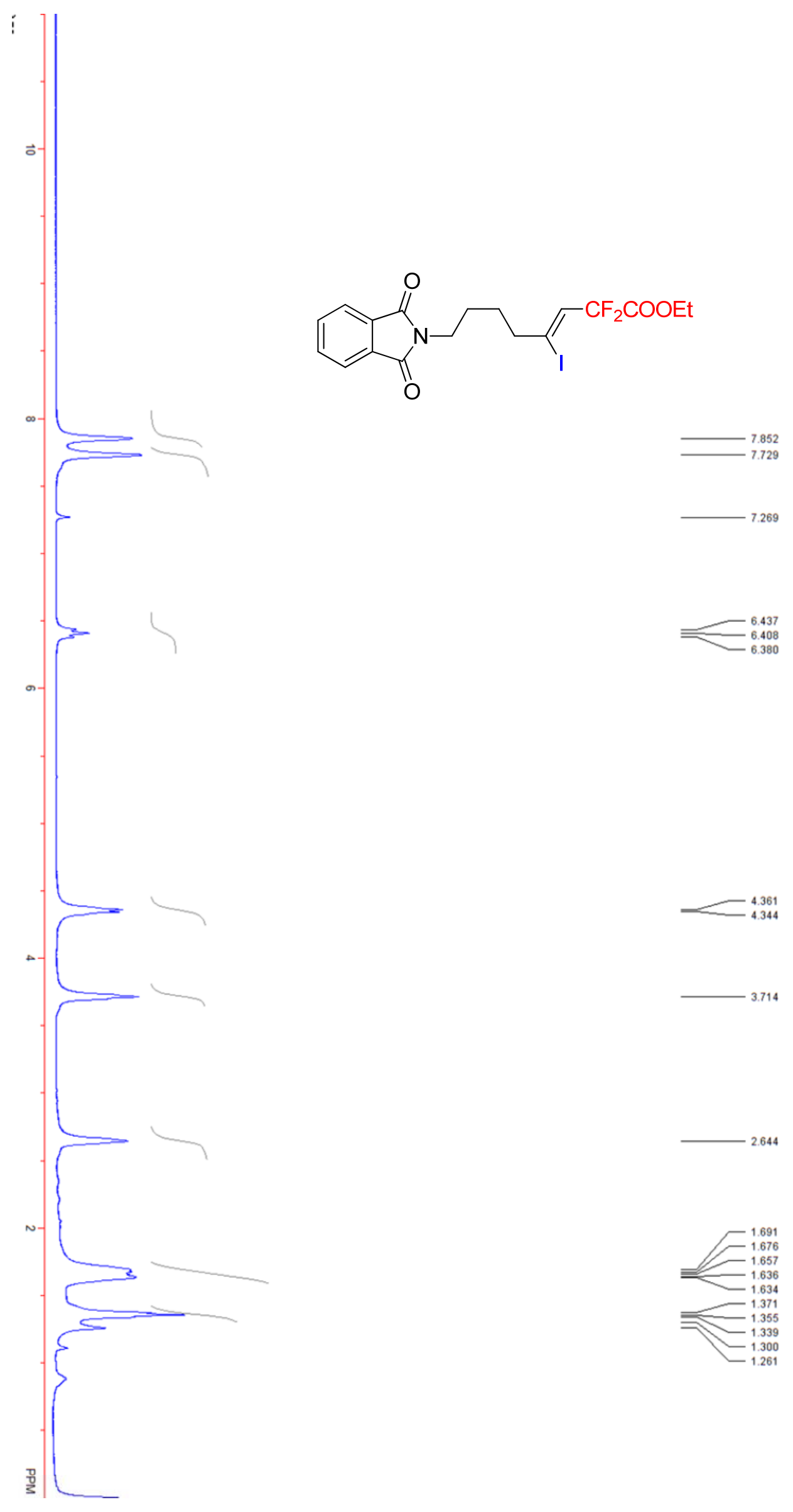



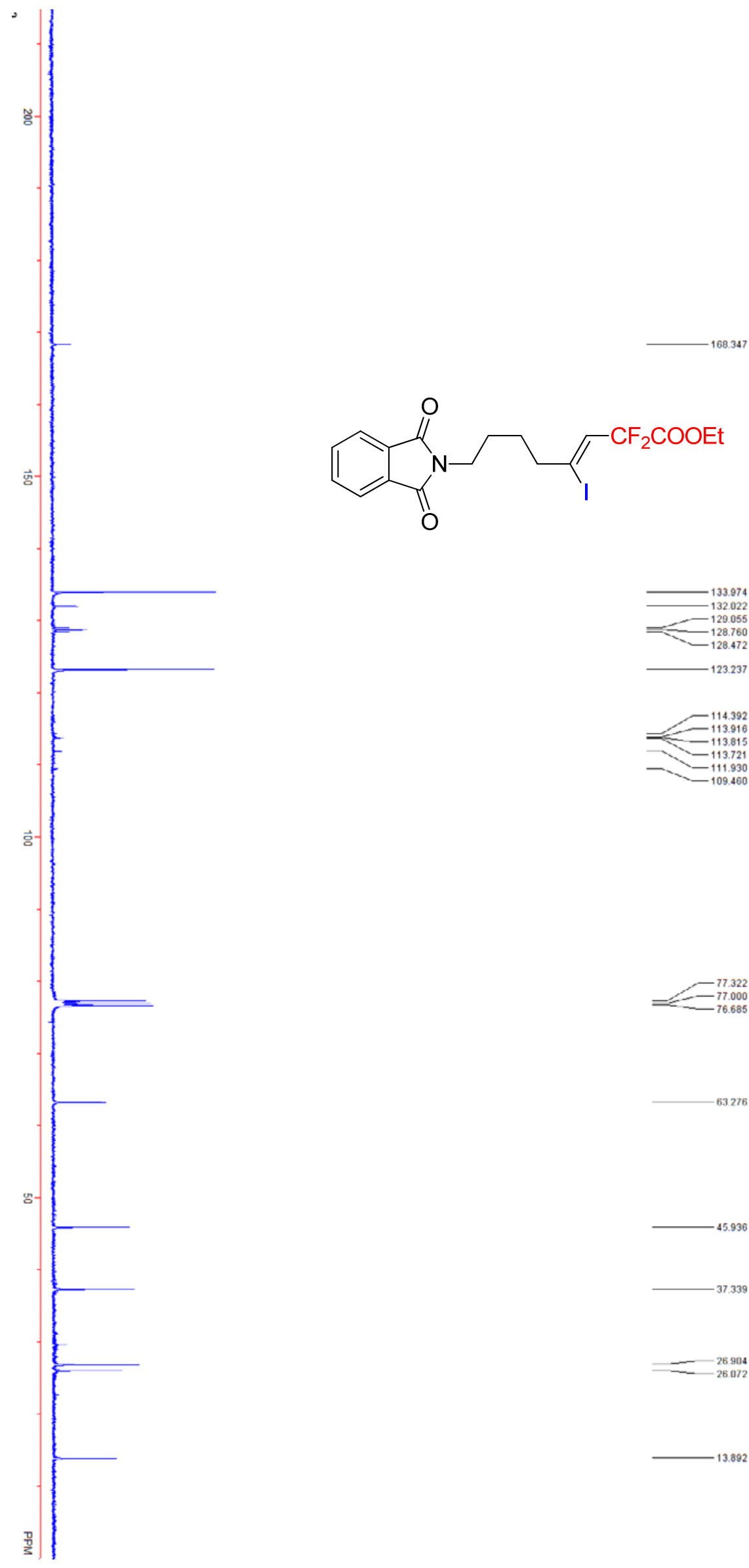

$-13.892$ 


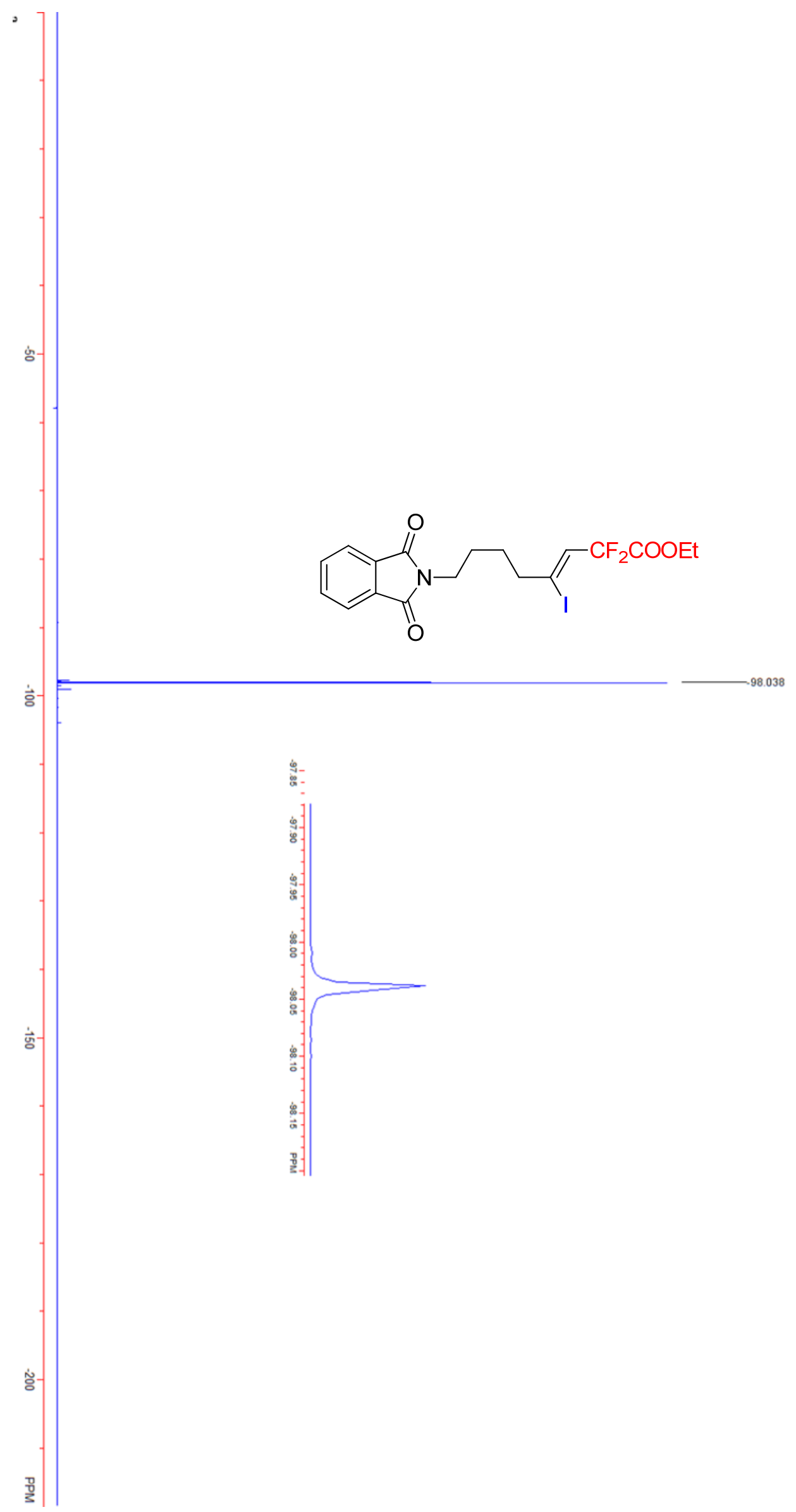




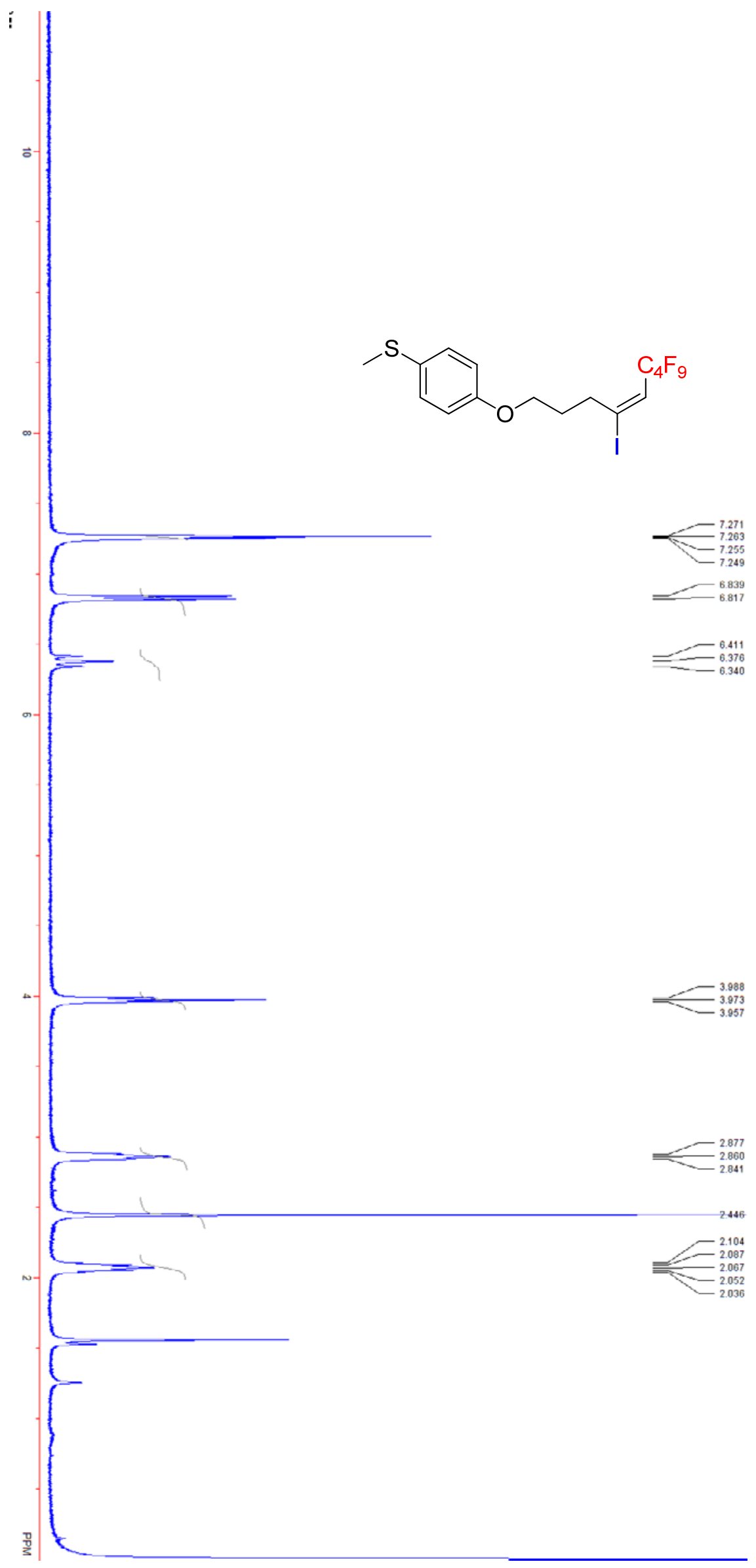

S67 


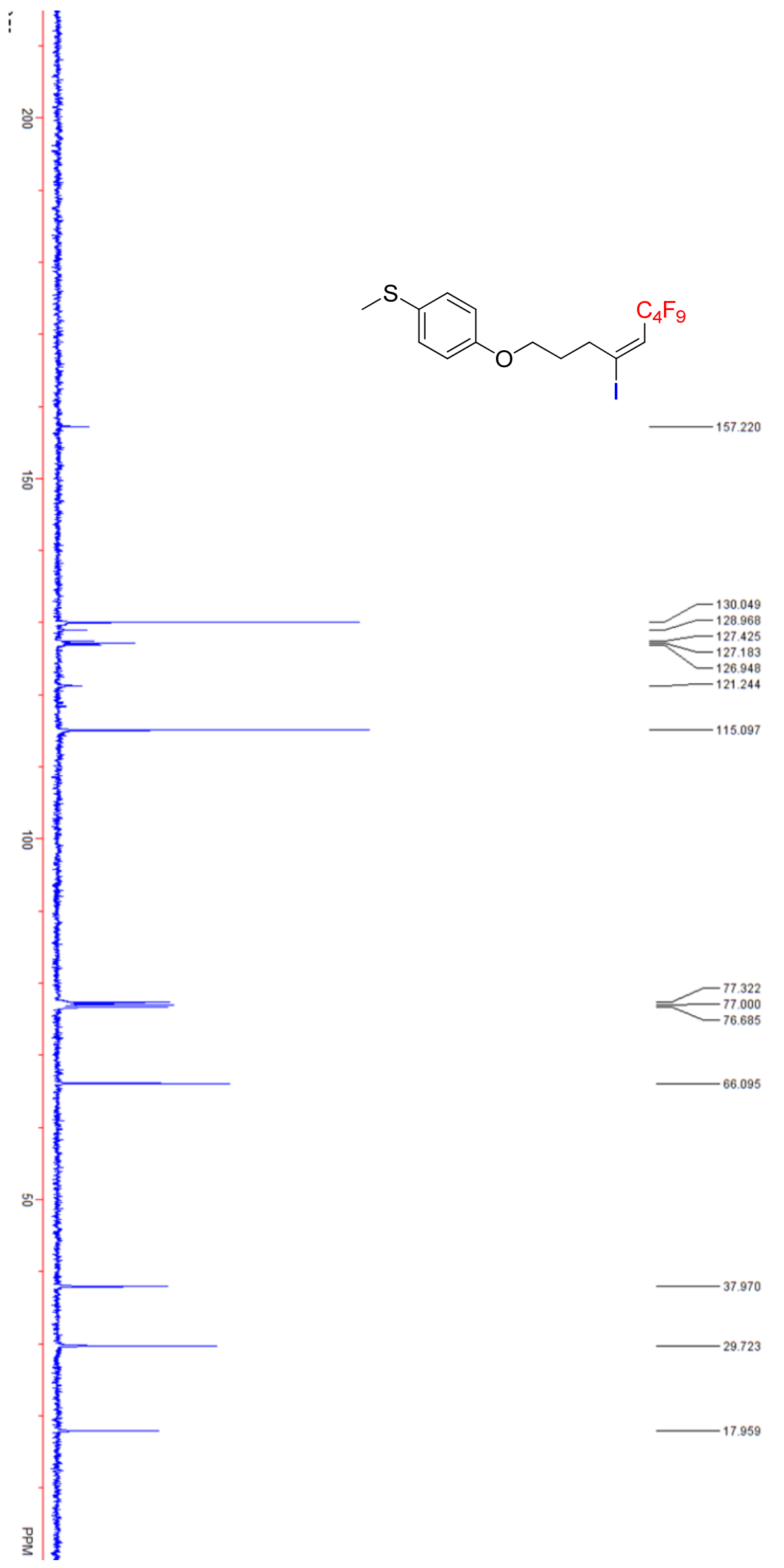

S68 


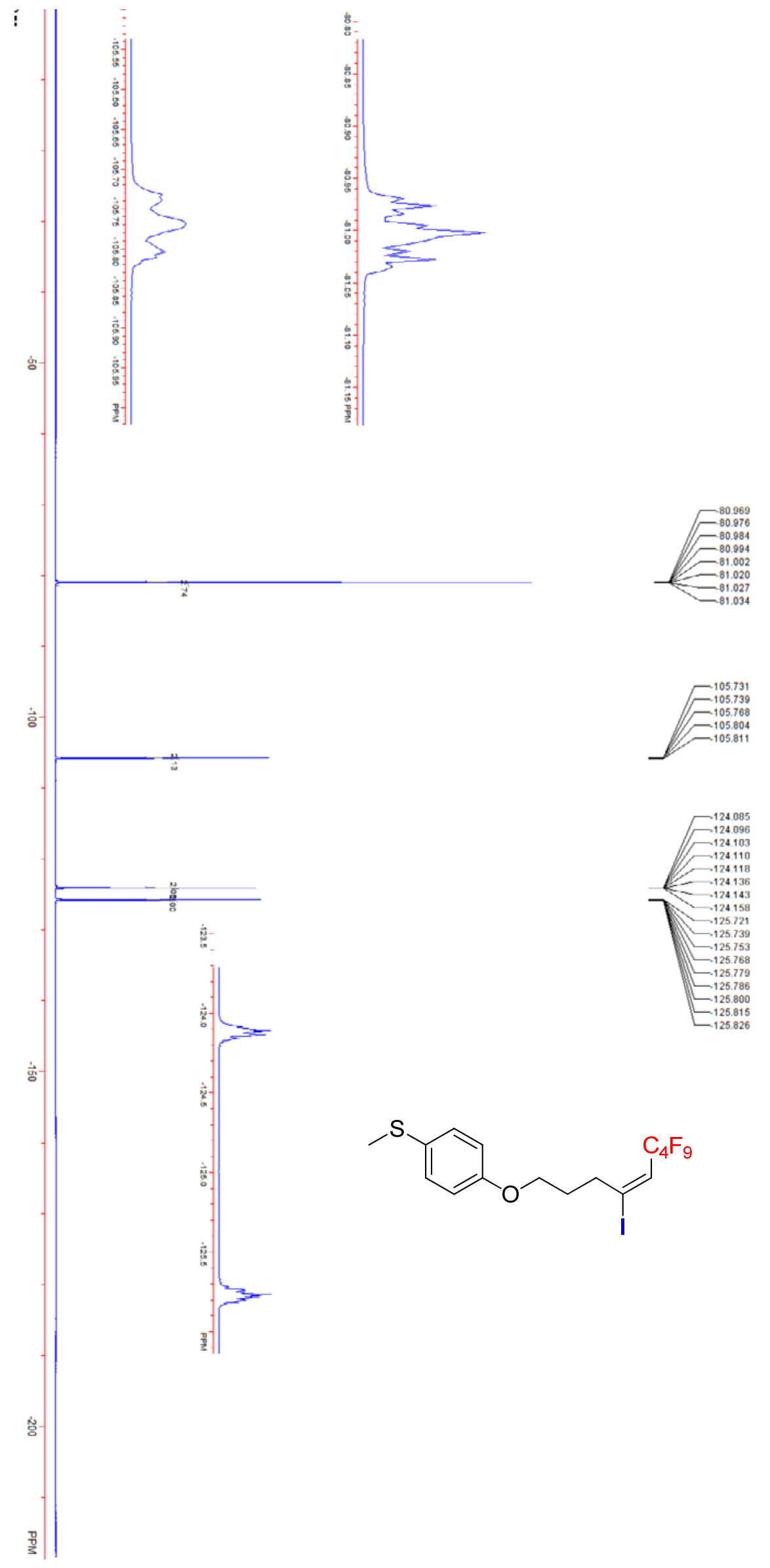




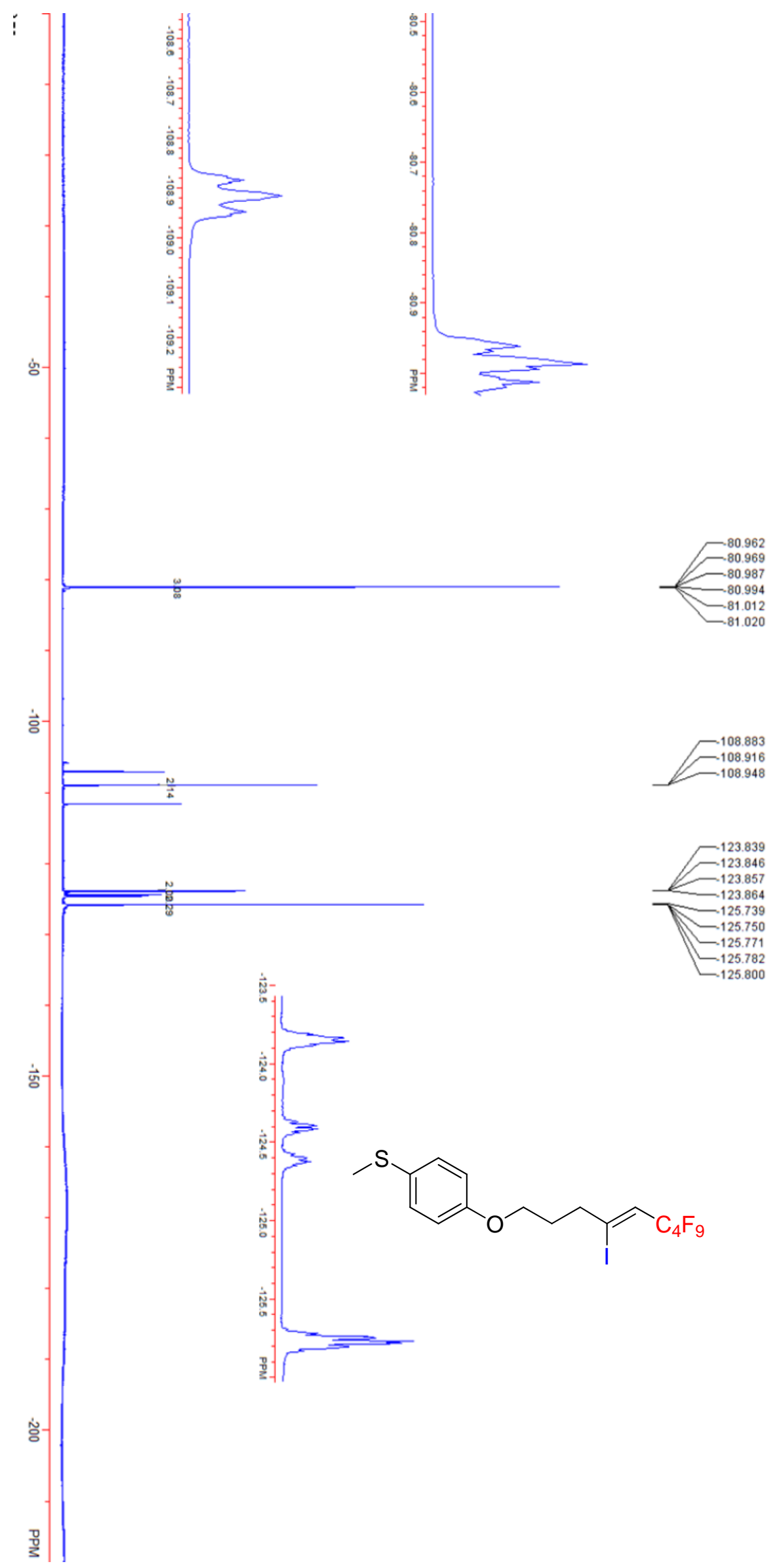




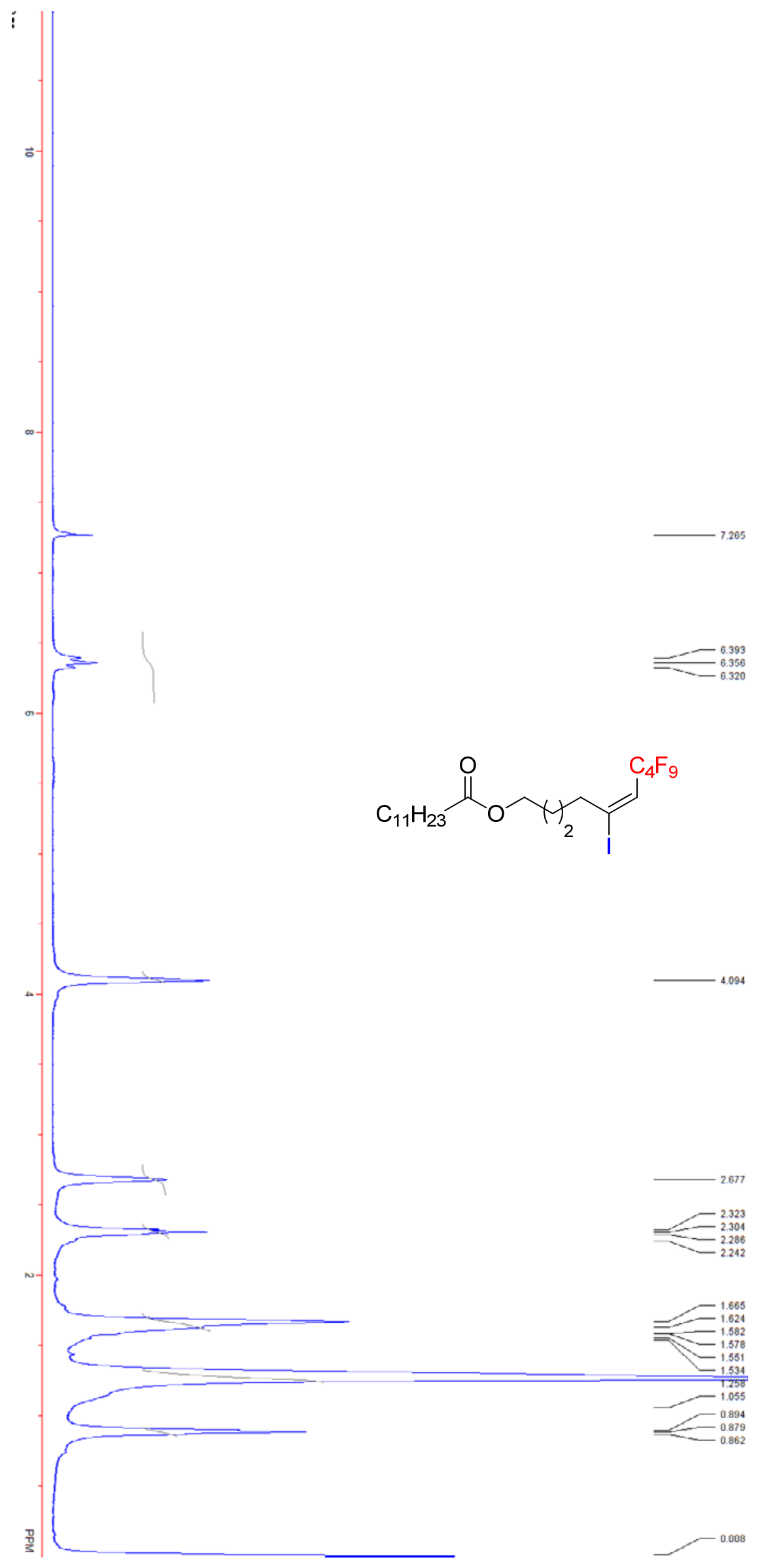




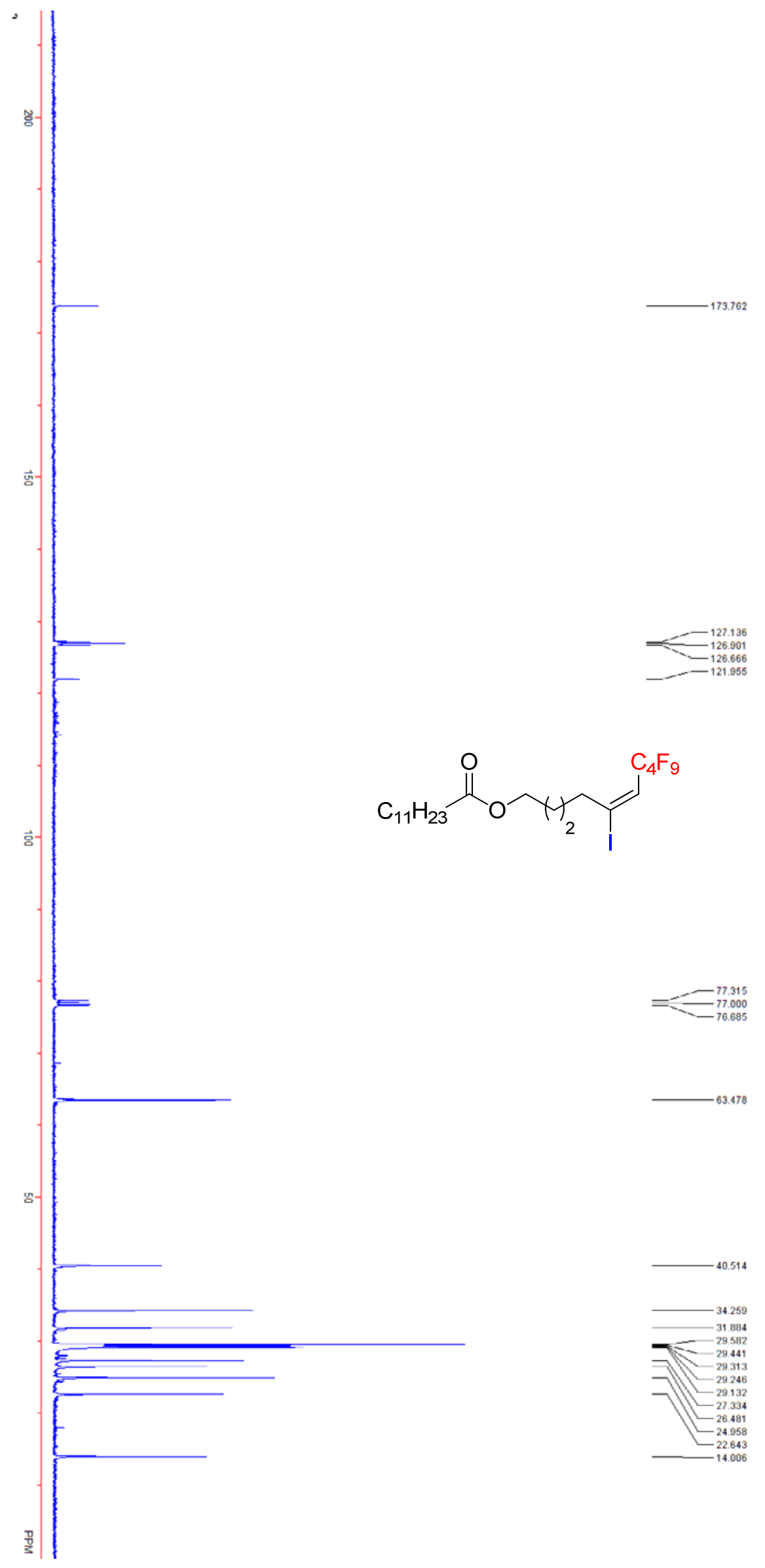




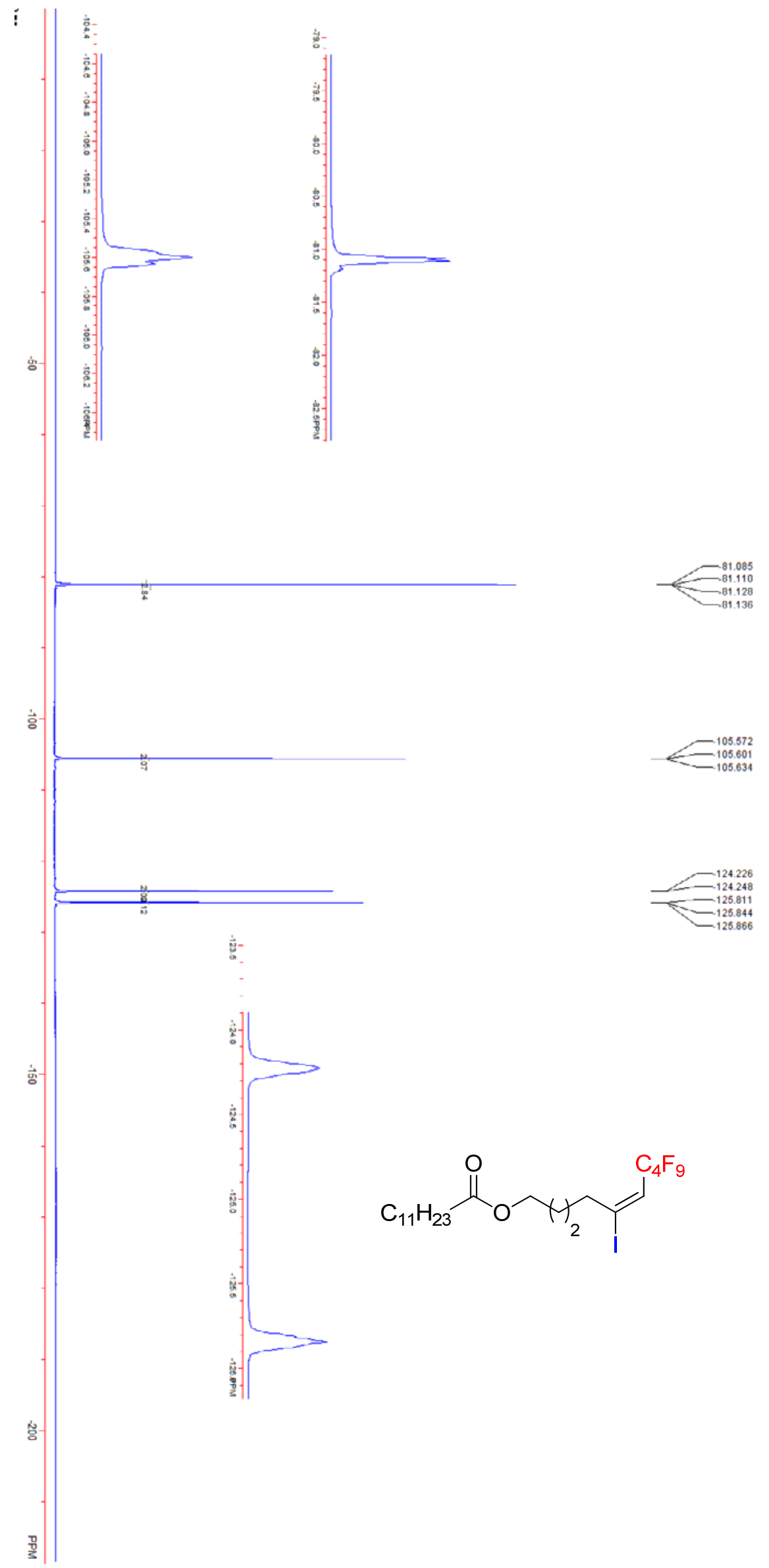




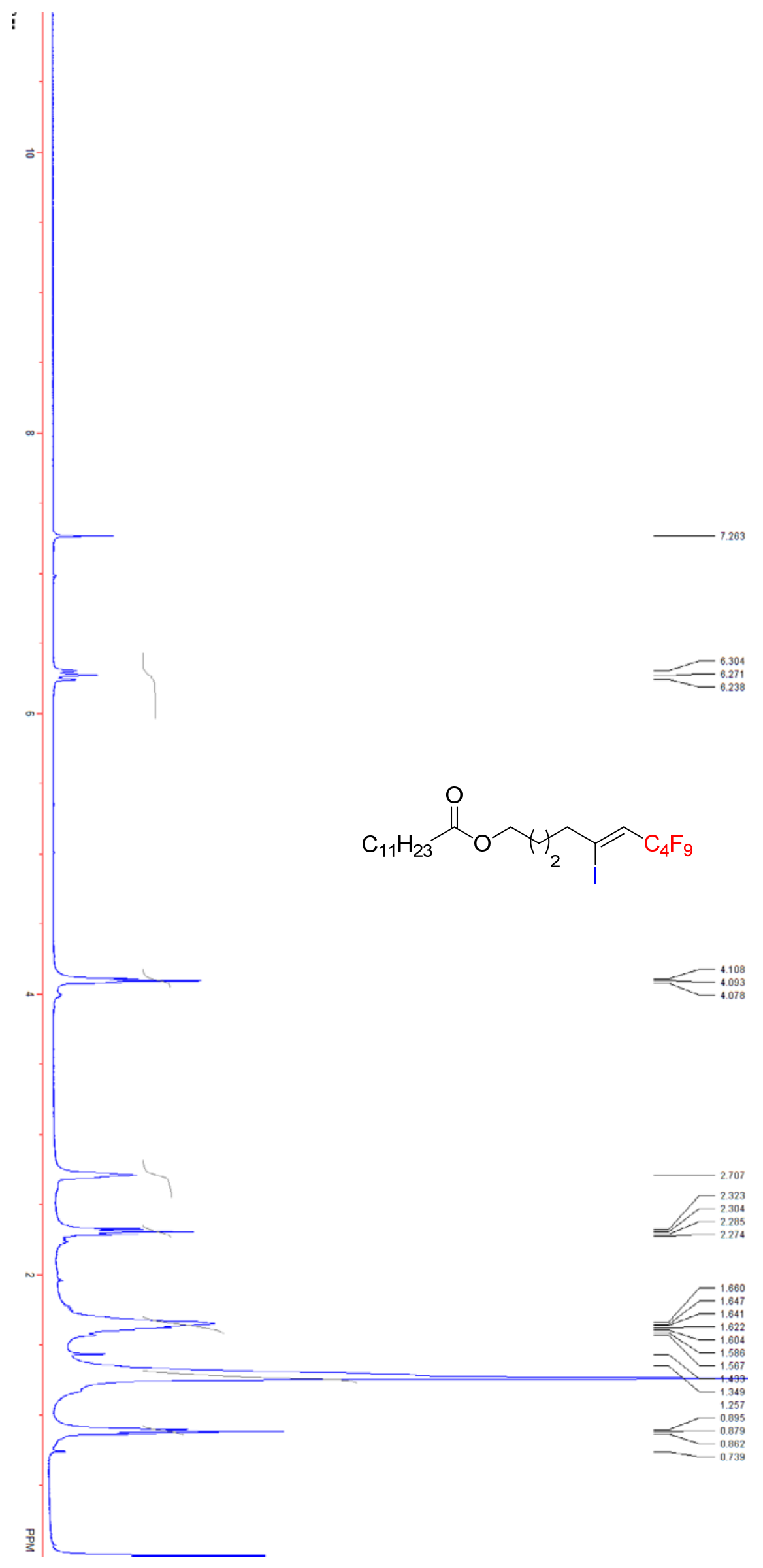



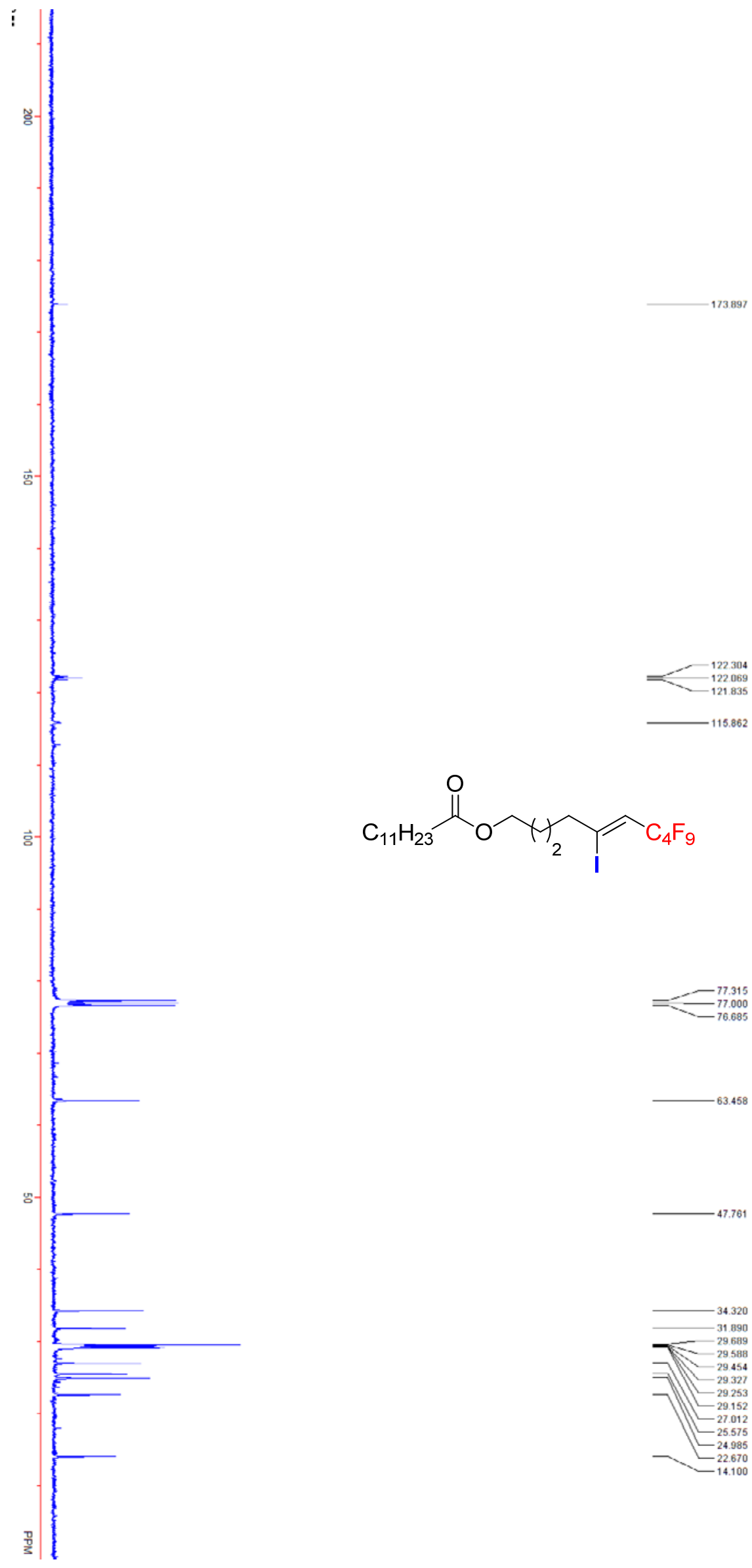

$\mathrm{C}_{11} \mathrm{H}_{23} \overbrace{\mathrm{O}}^{\mathrm{O}} \sim \mathrm{C}_{4} \mathrm{~F}_{9}$
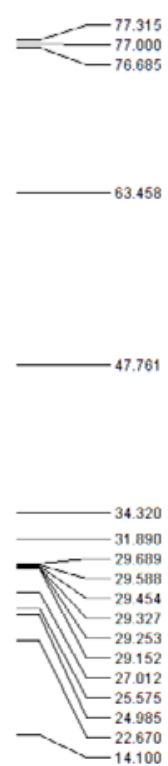


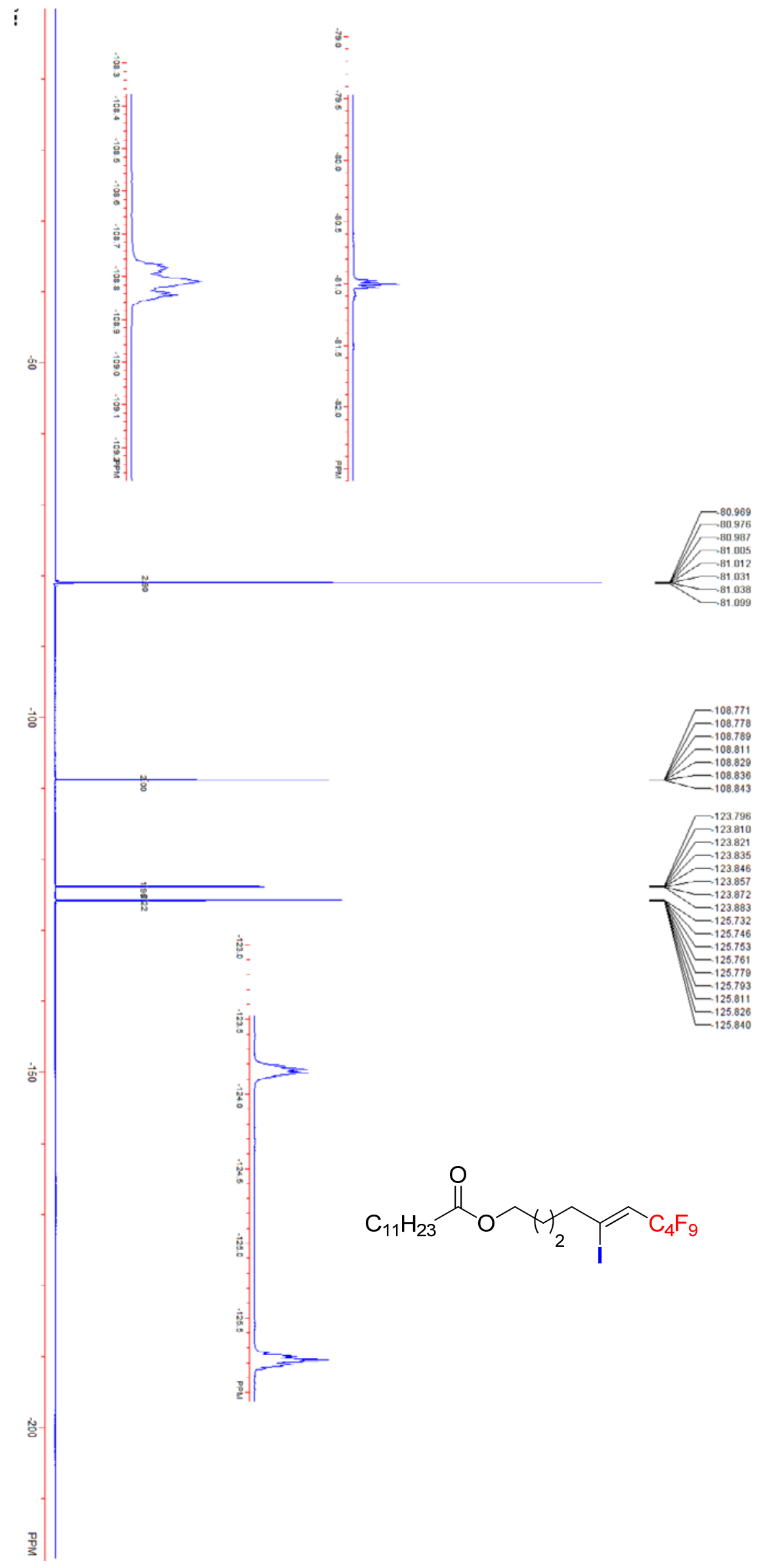




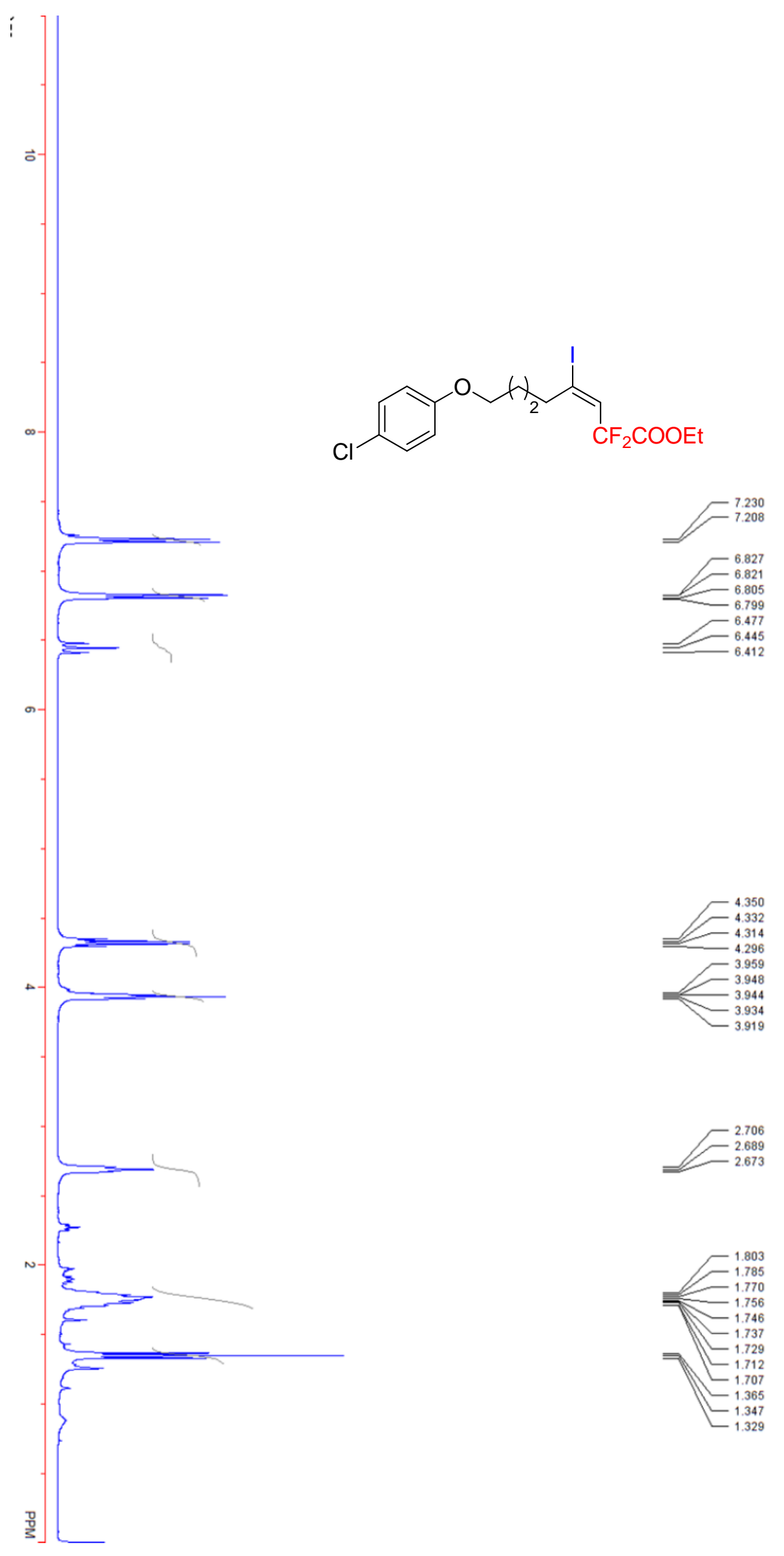

S77 

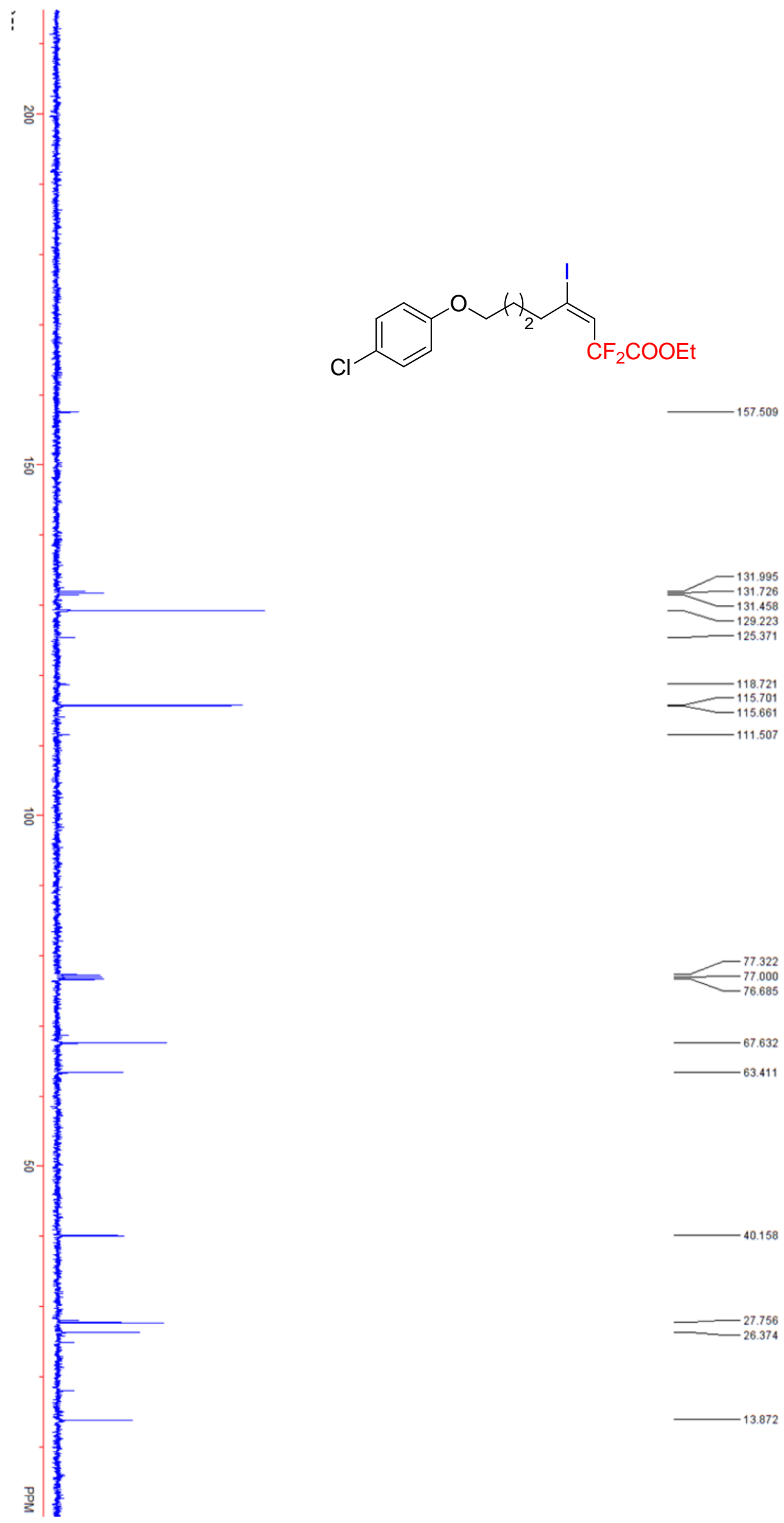

문 


$$
\sqrt{F}
$$




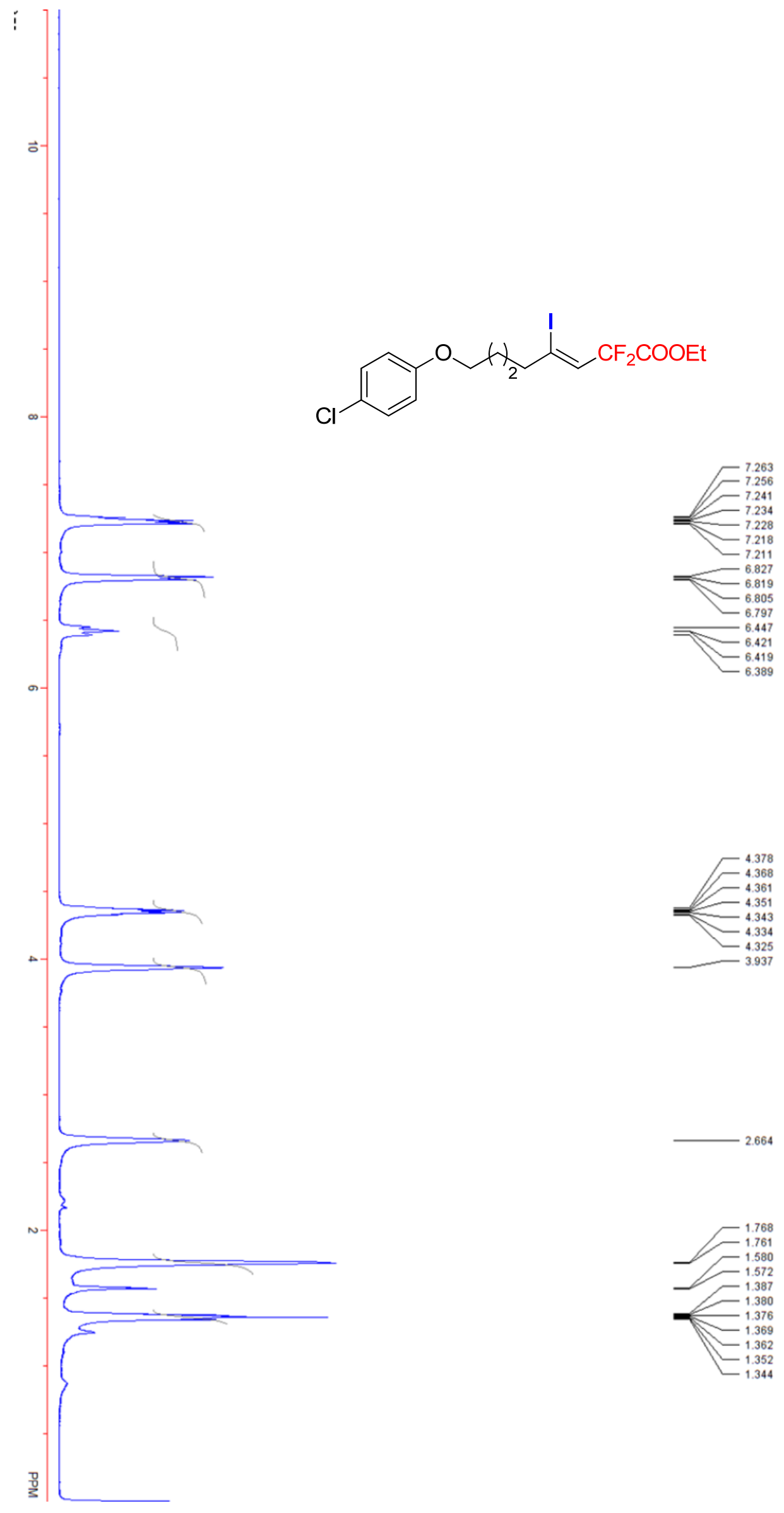




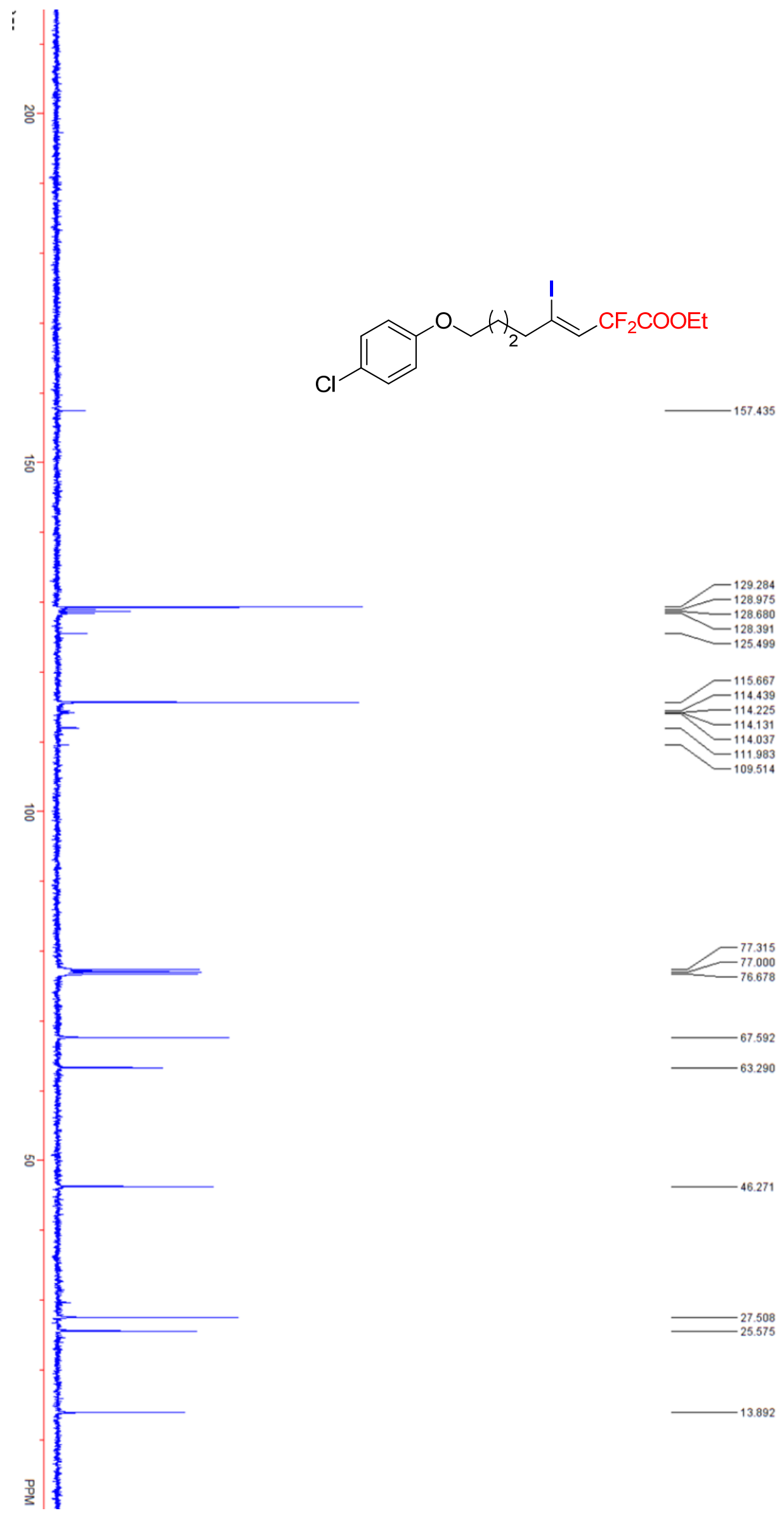




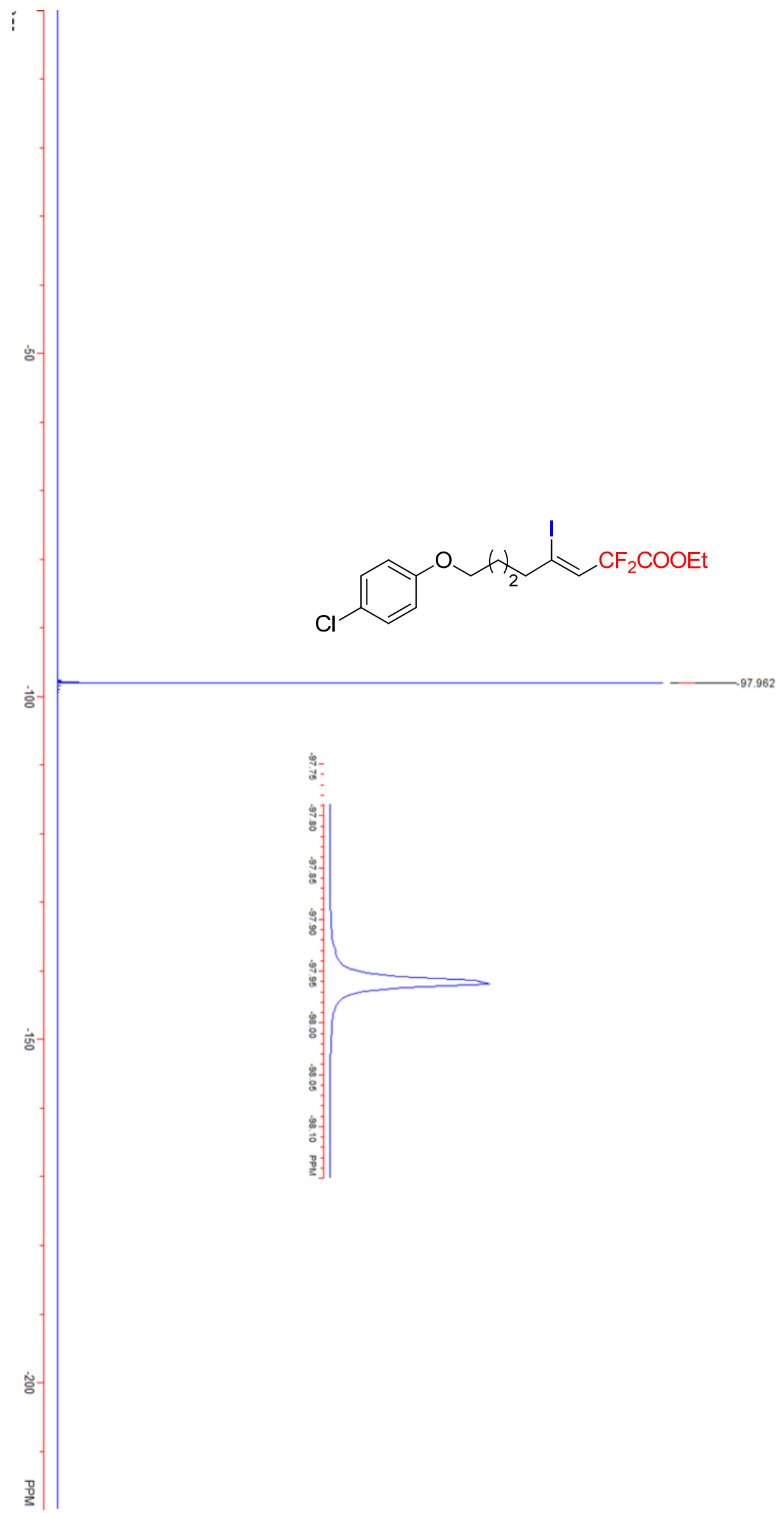




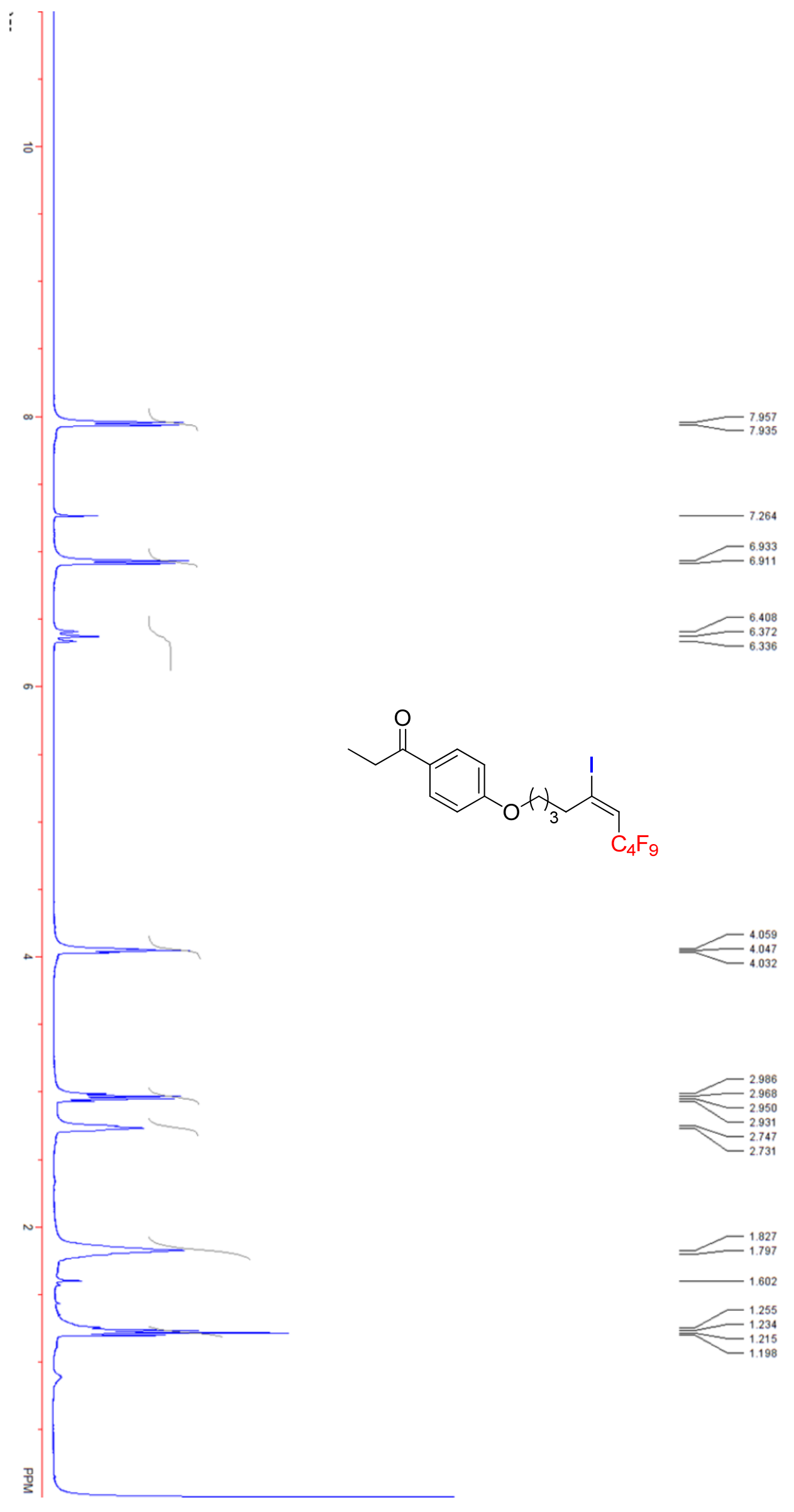

S83 

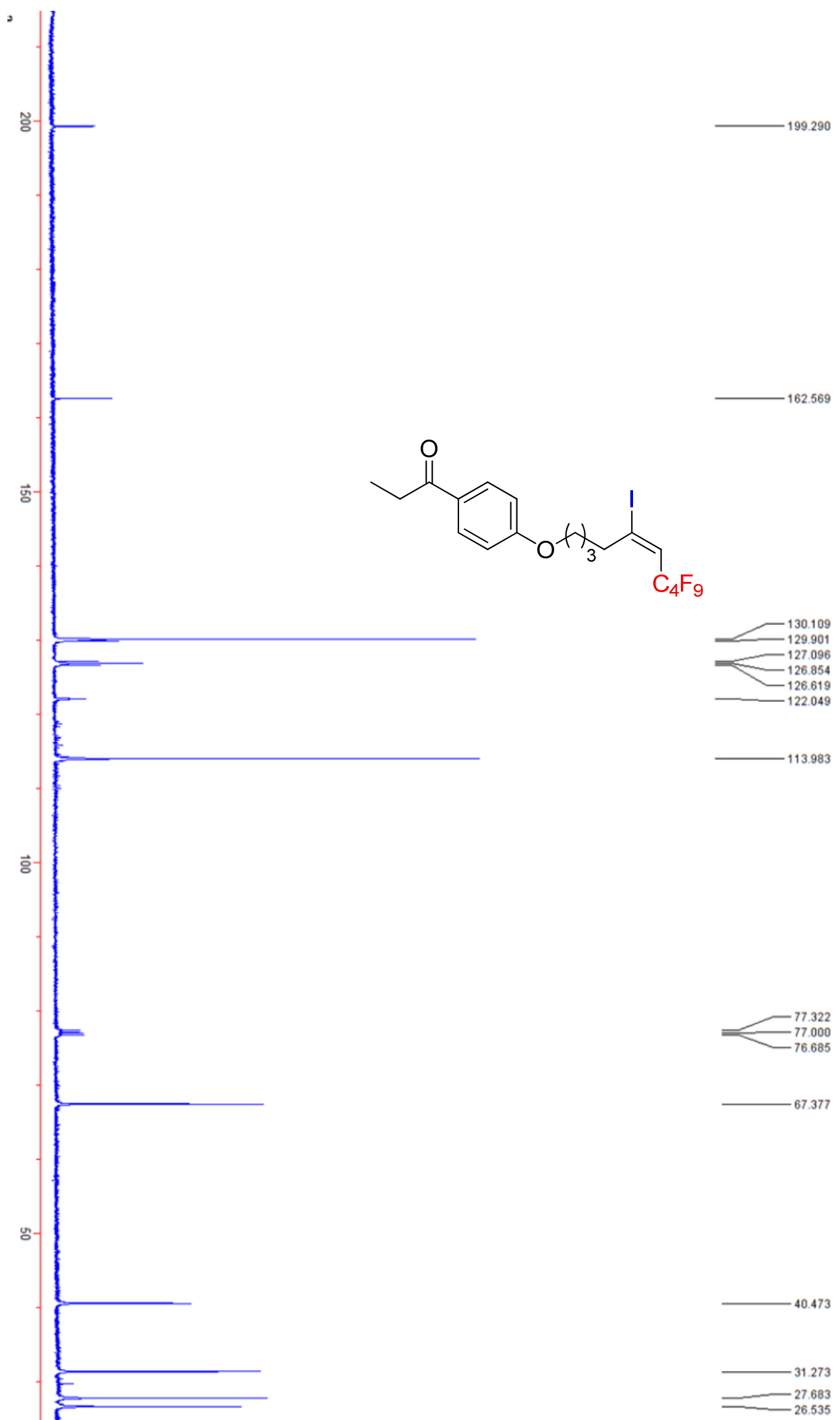

S84 


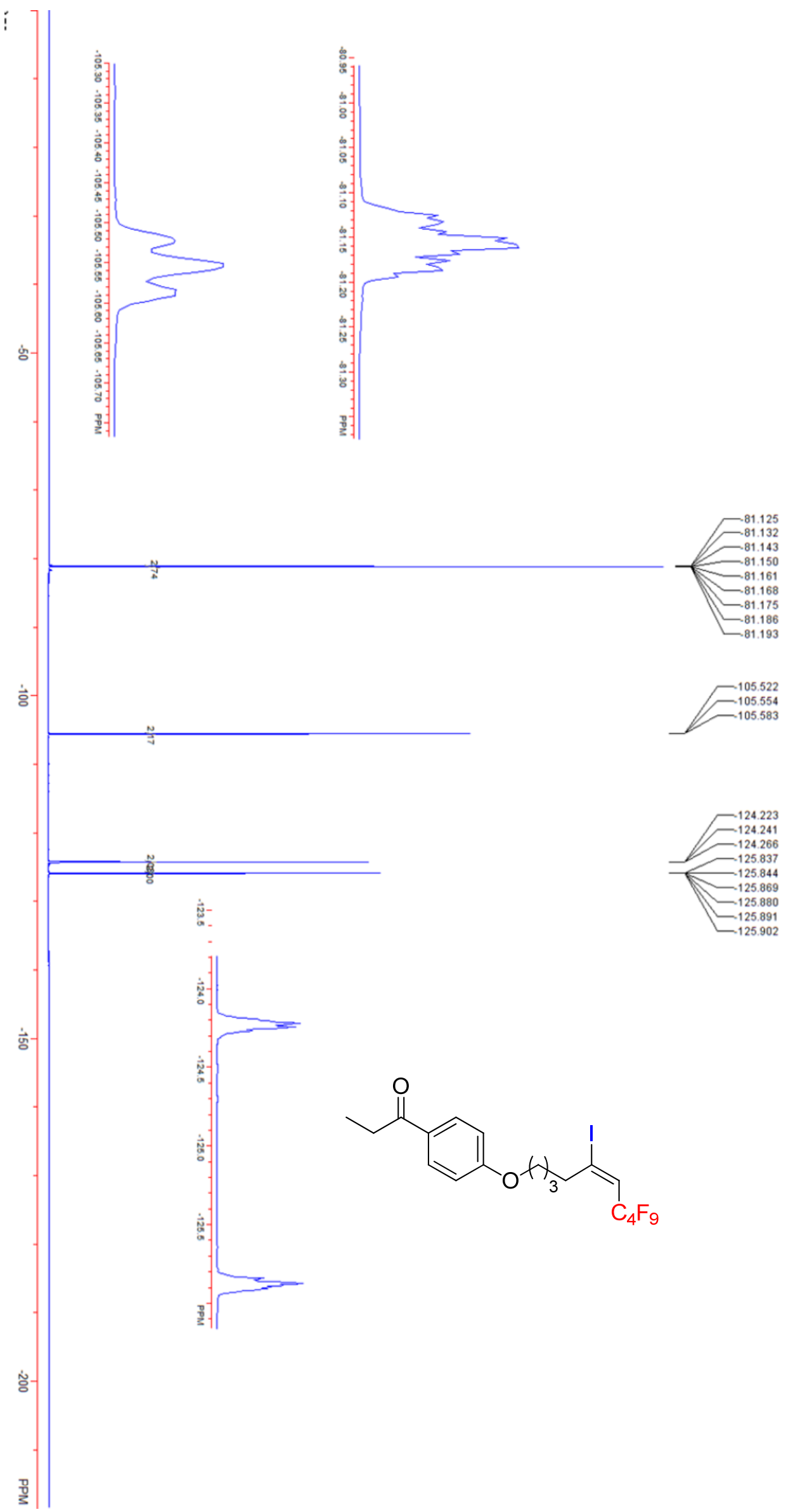



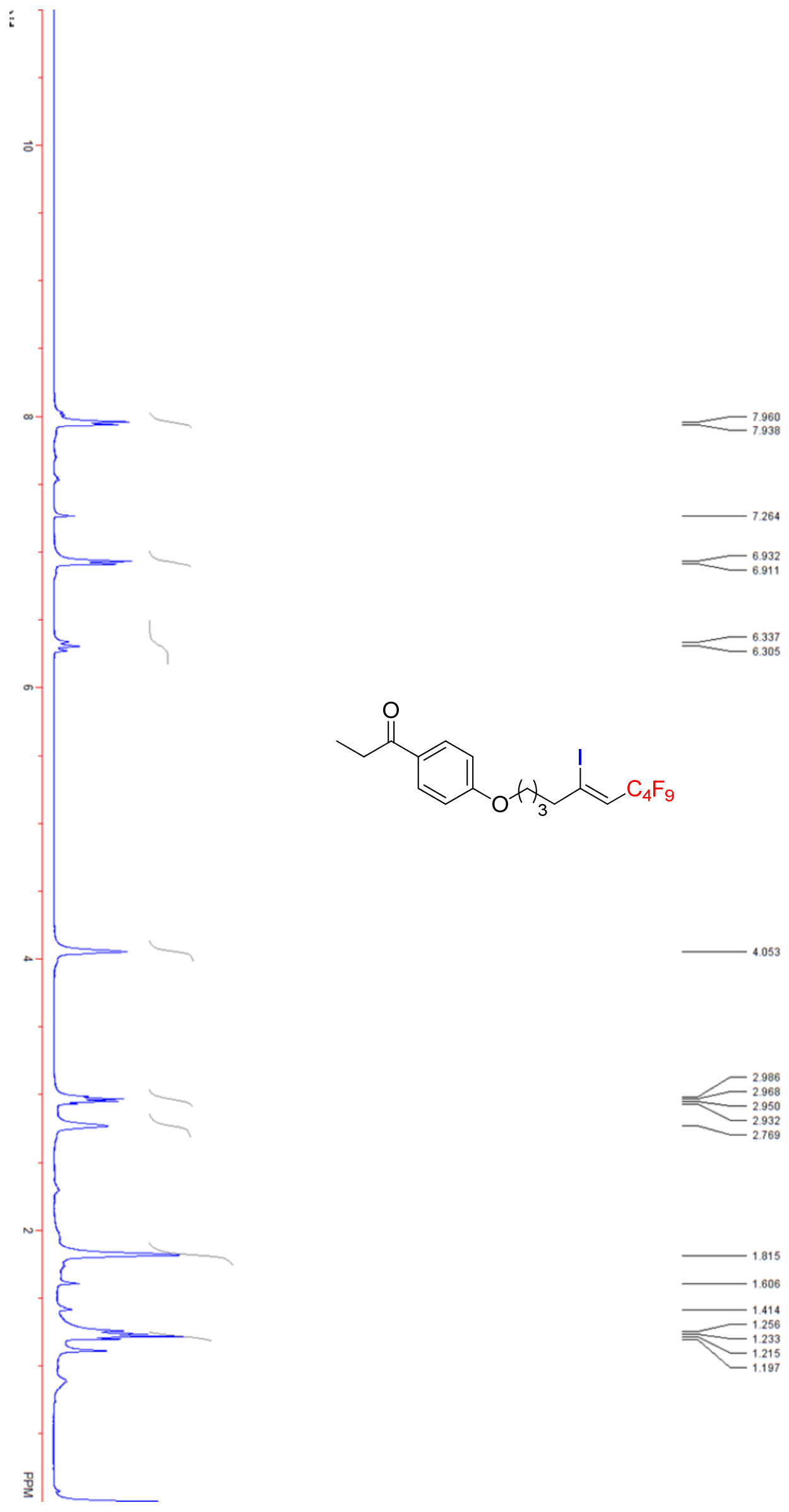

S86 

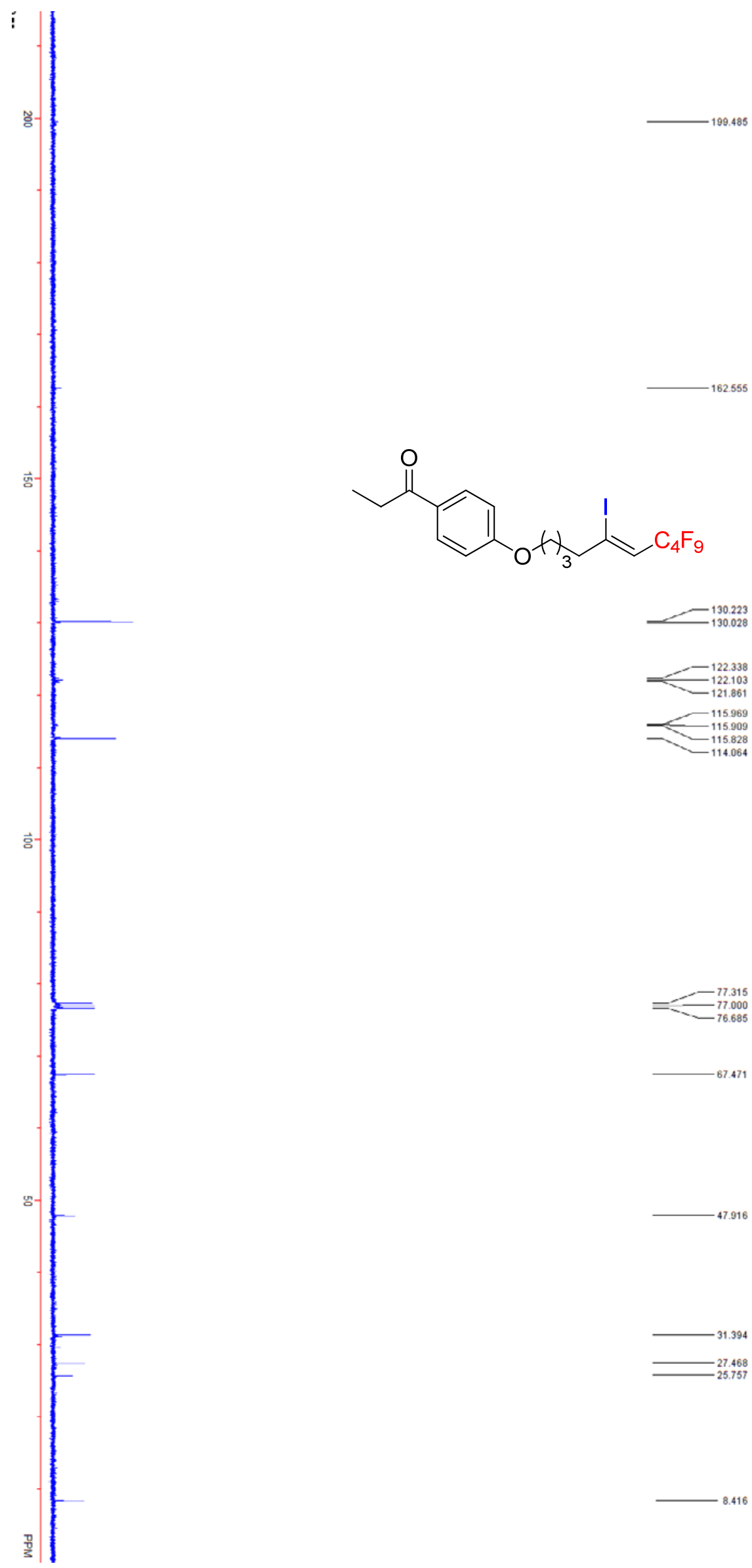

${ }^{130.223}$

${ }_{121.861}^{122.338}$

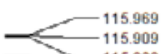

$-{ }^{115.828}$

77.315
77.000
76.685

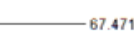

$\begin{array}{r}27.468 \\ -25.757 \\ \hline\end{array}$

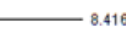




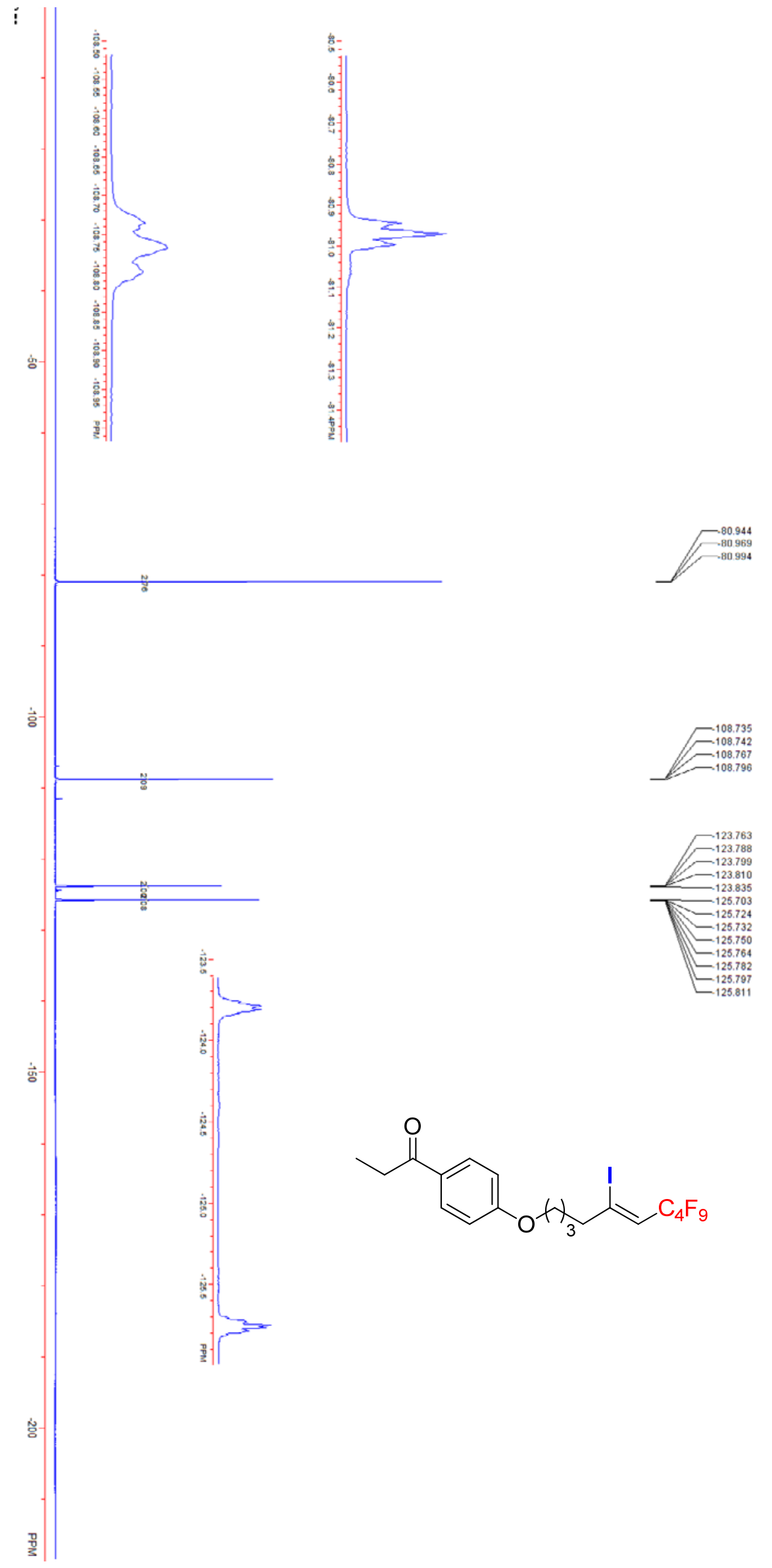




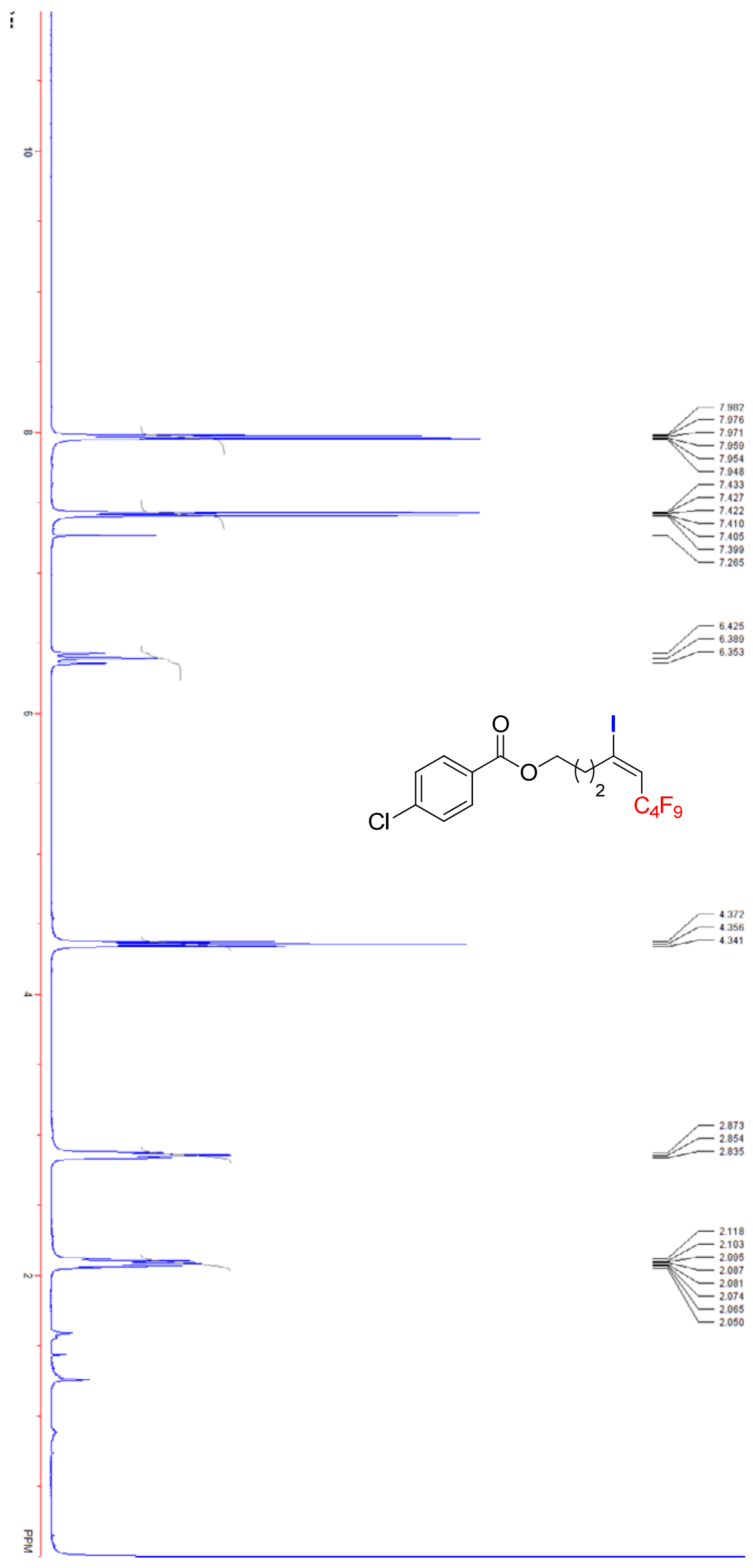



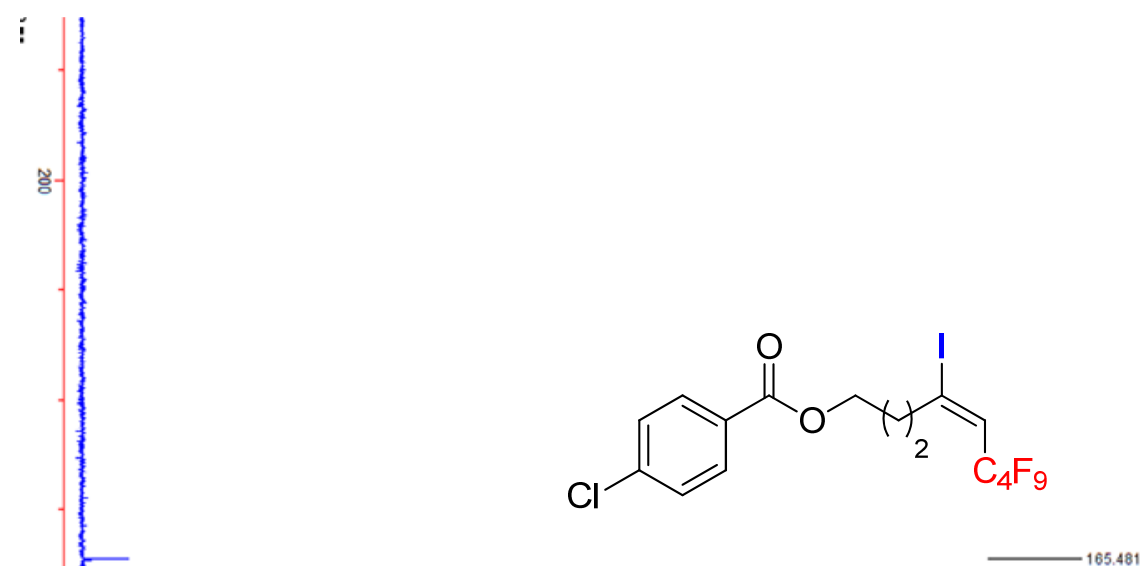

함

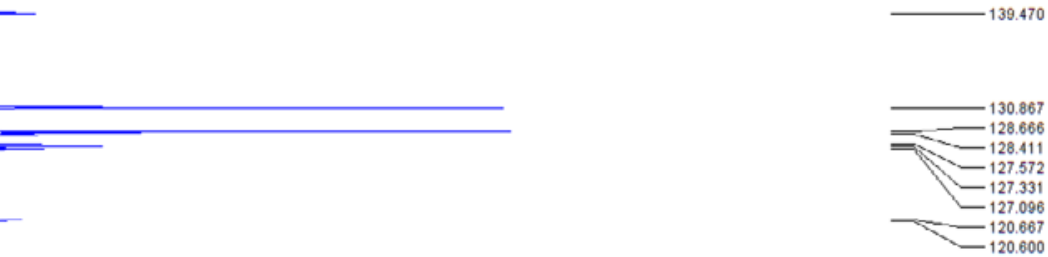

홍

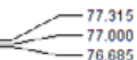

ㄴ.

끈 


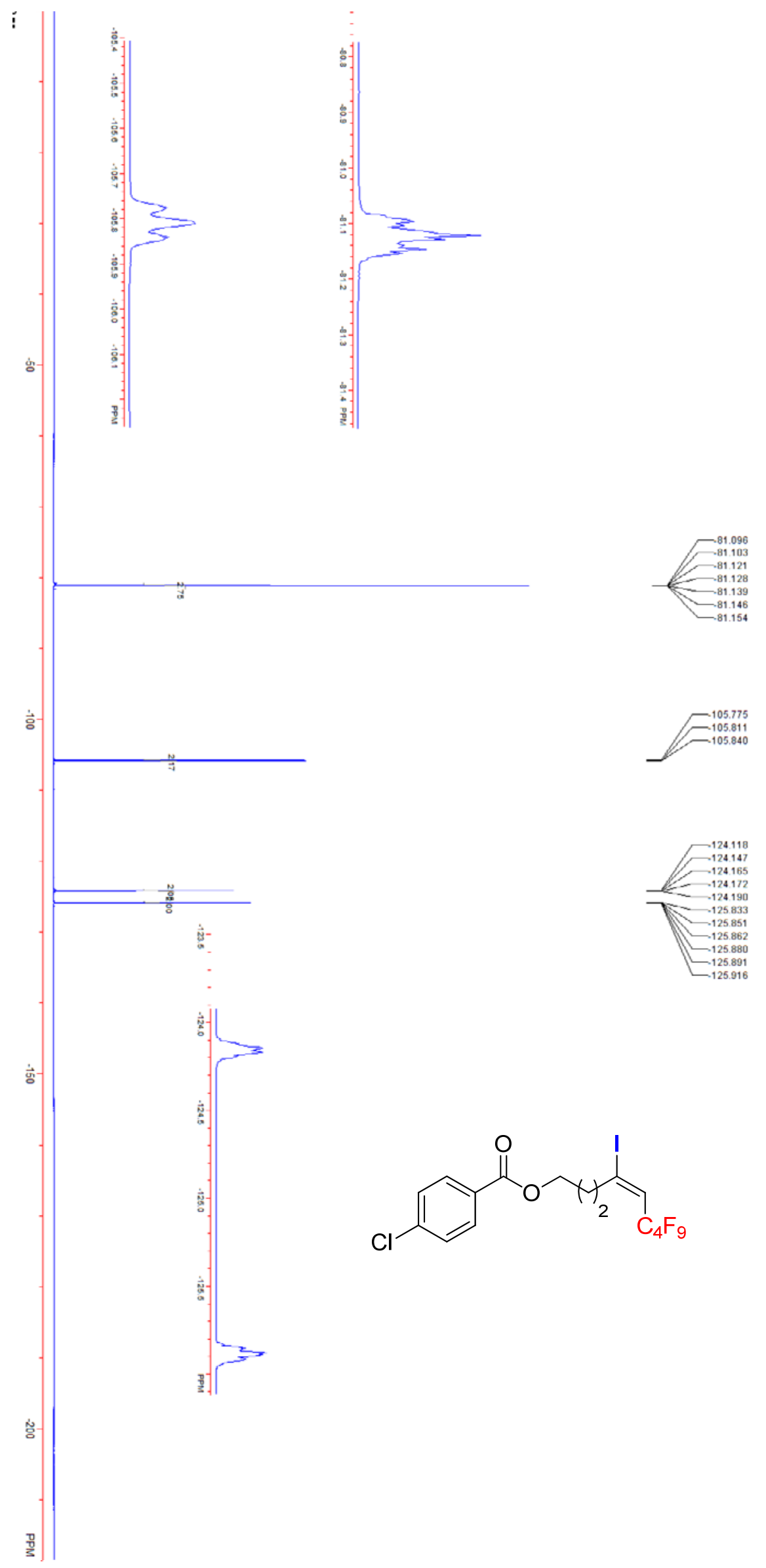




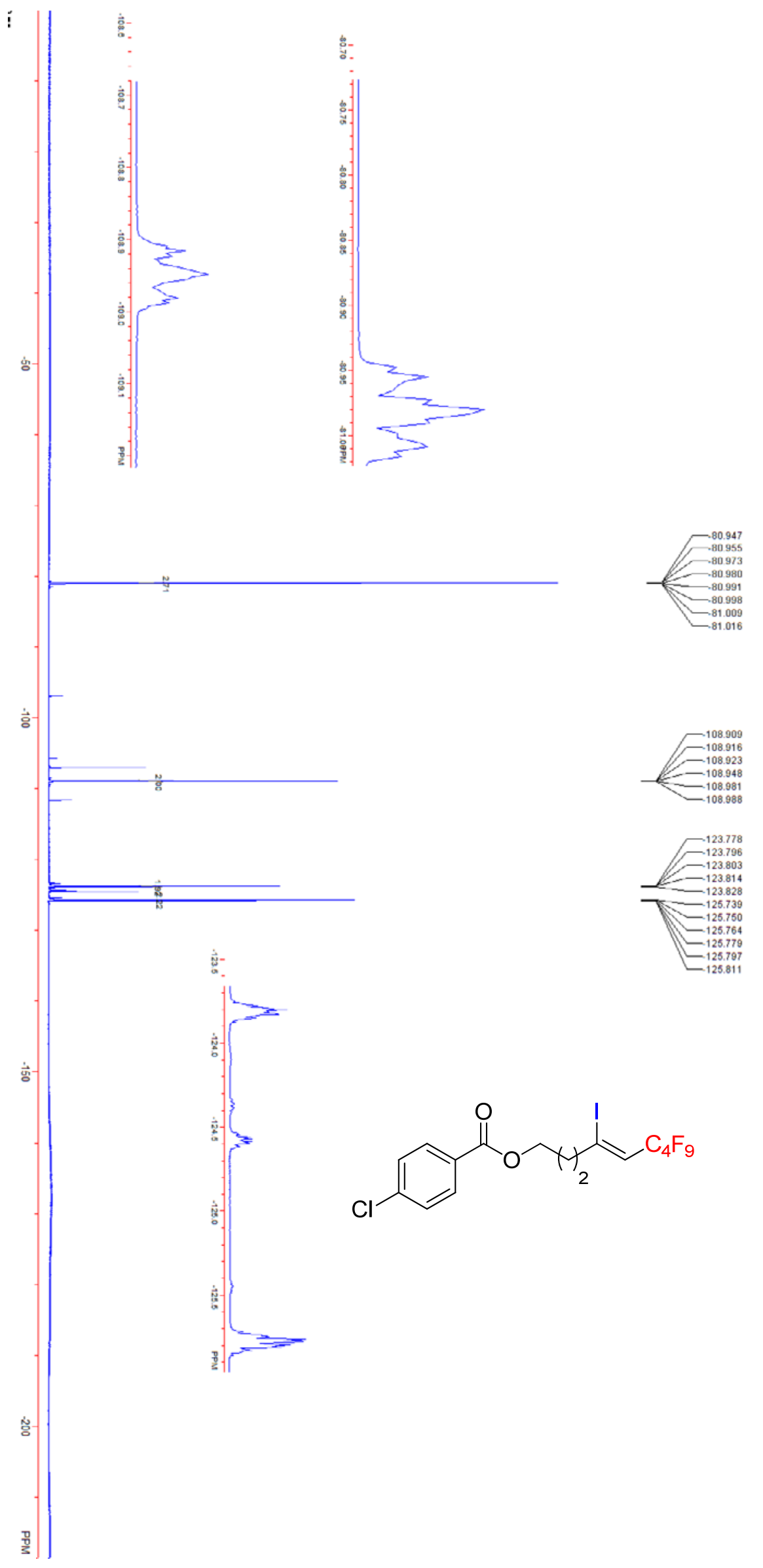




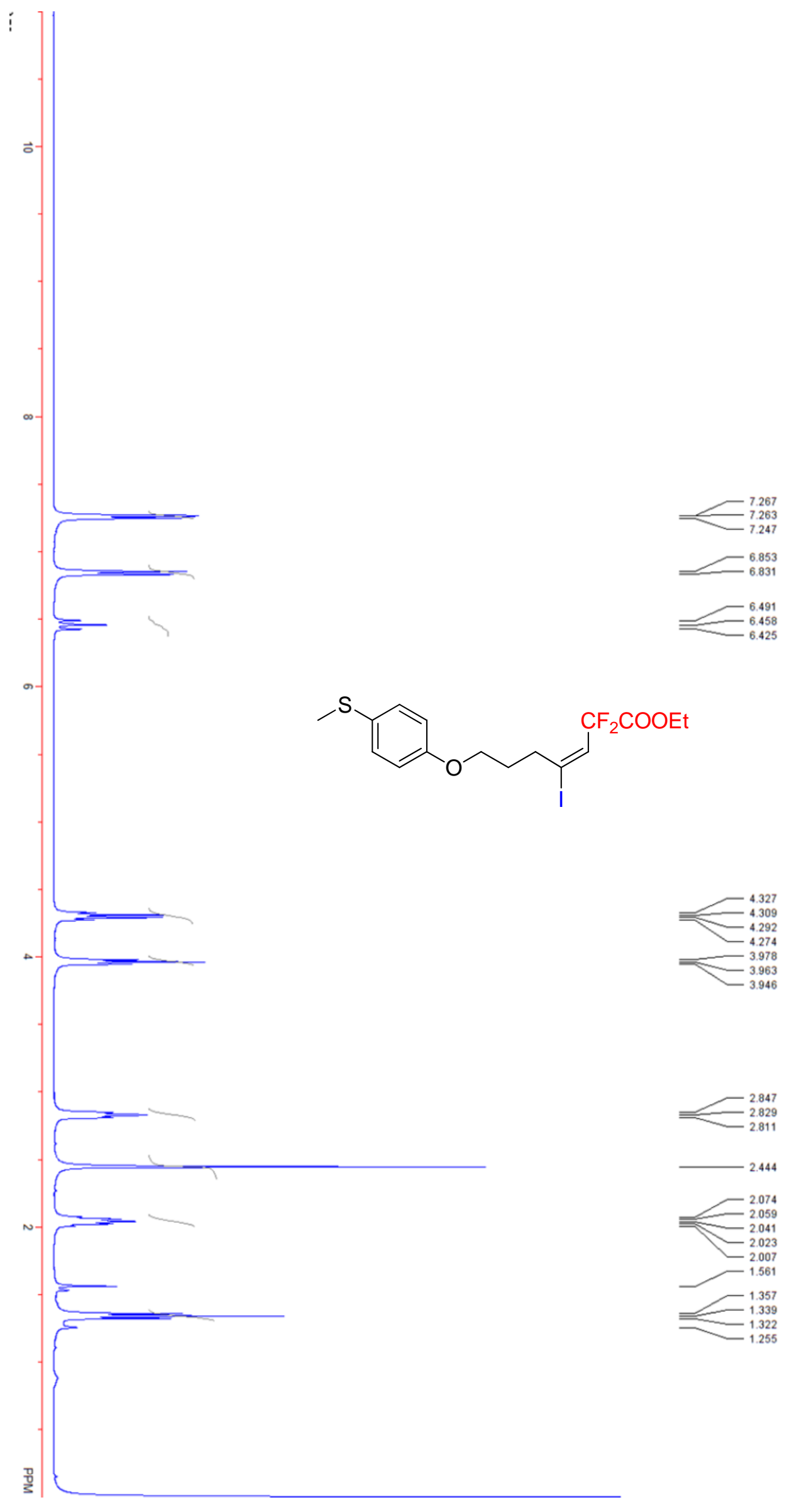

S93 


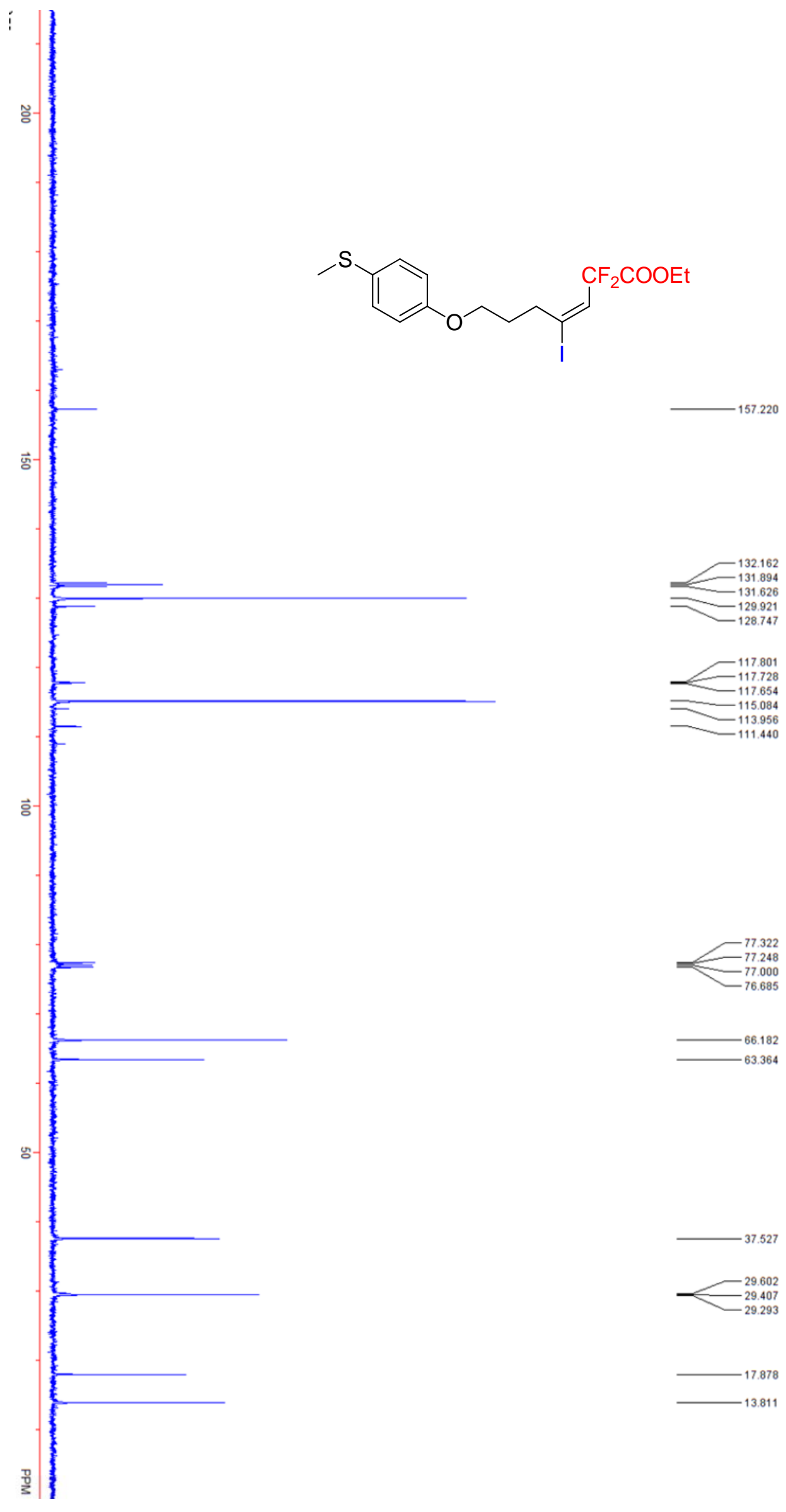

S94 


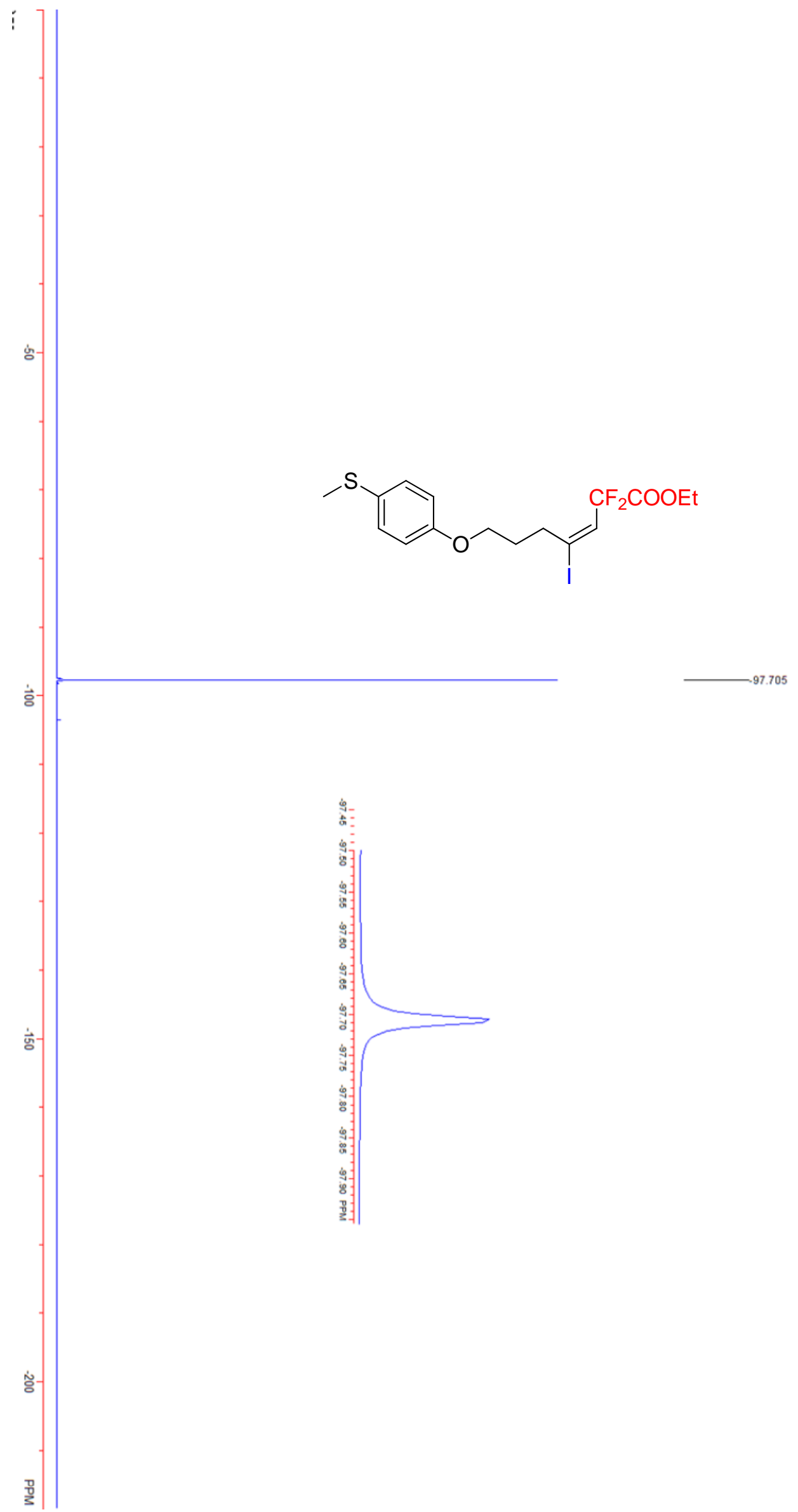




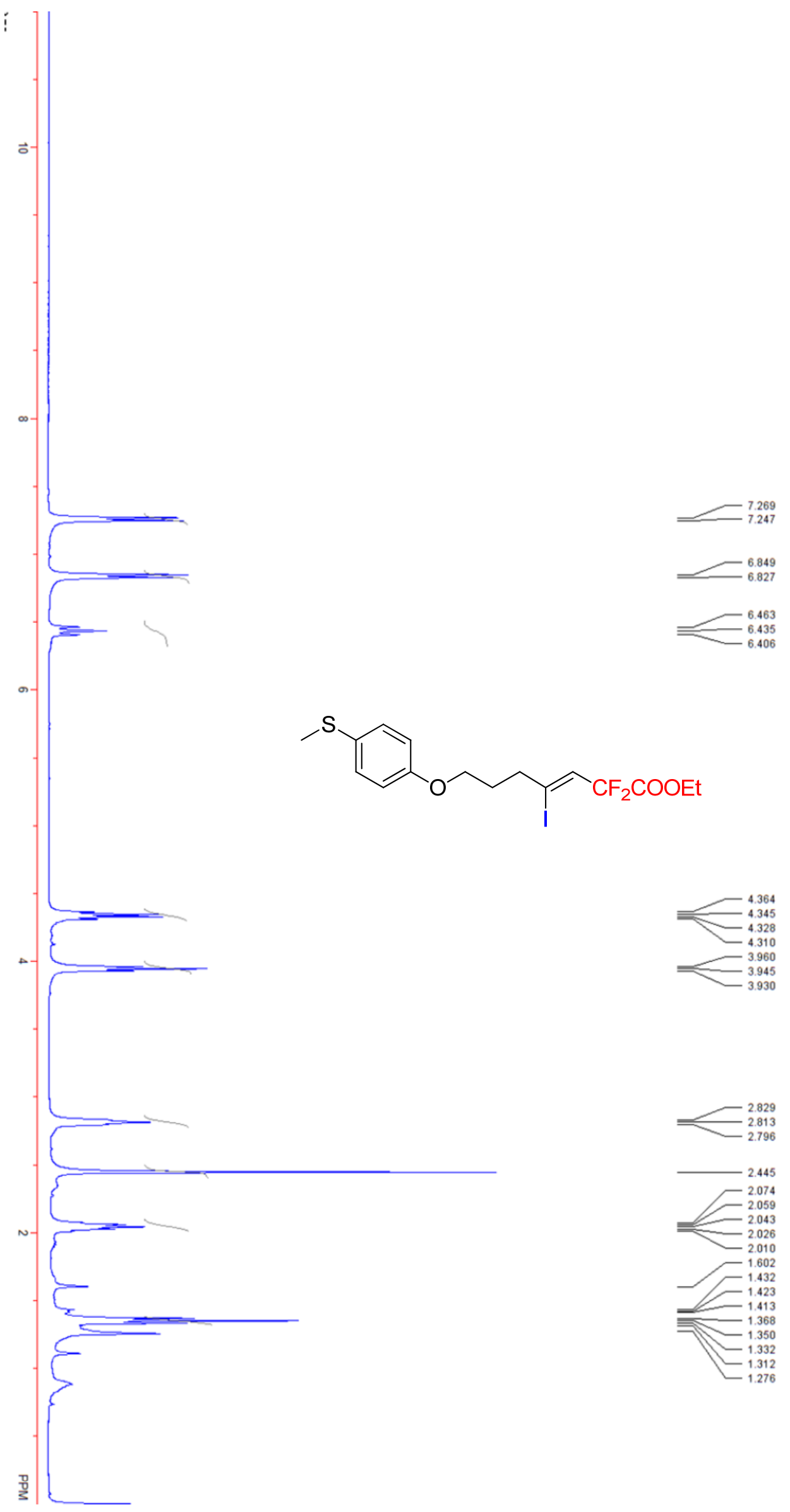



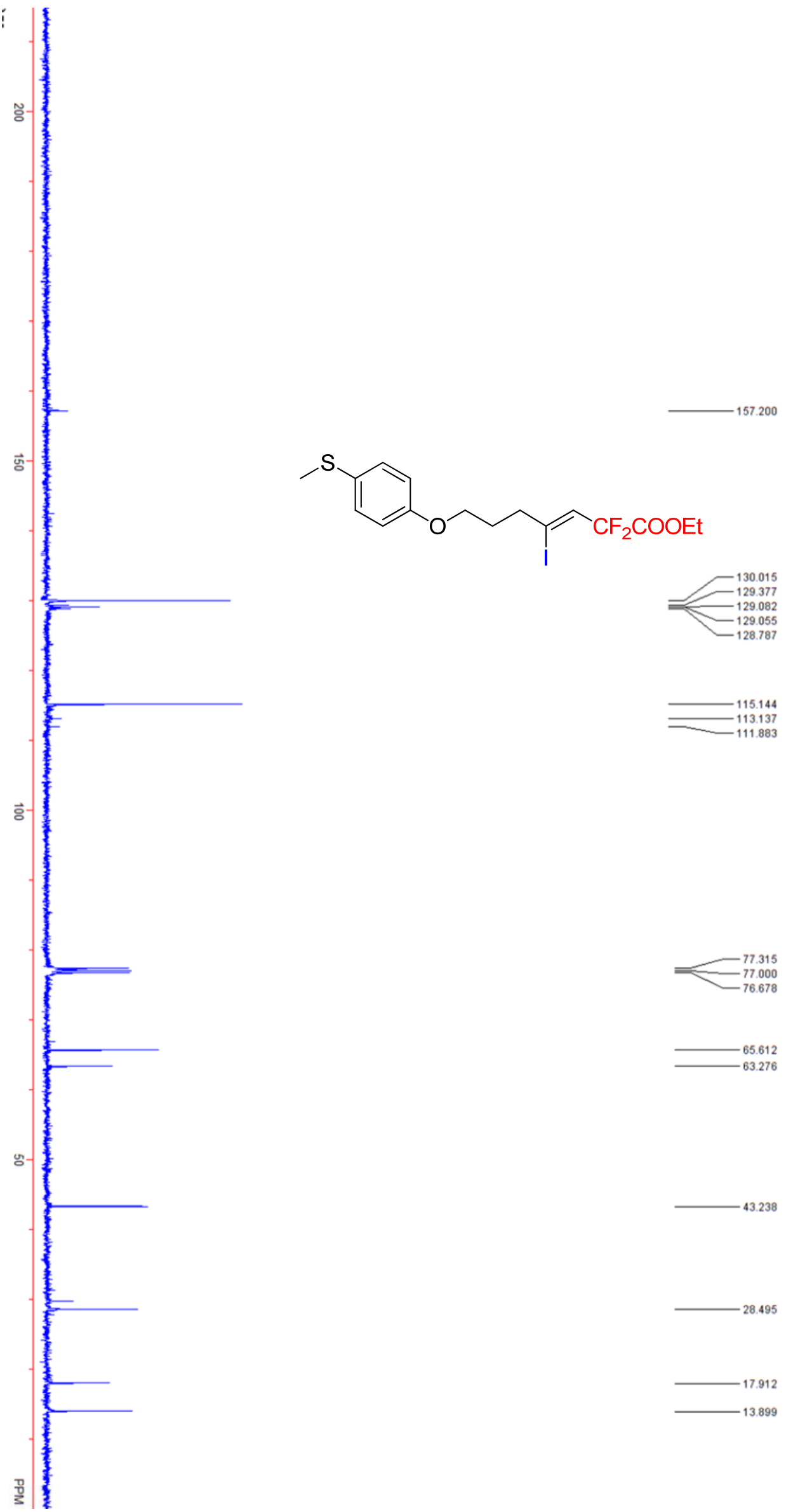


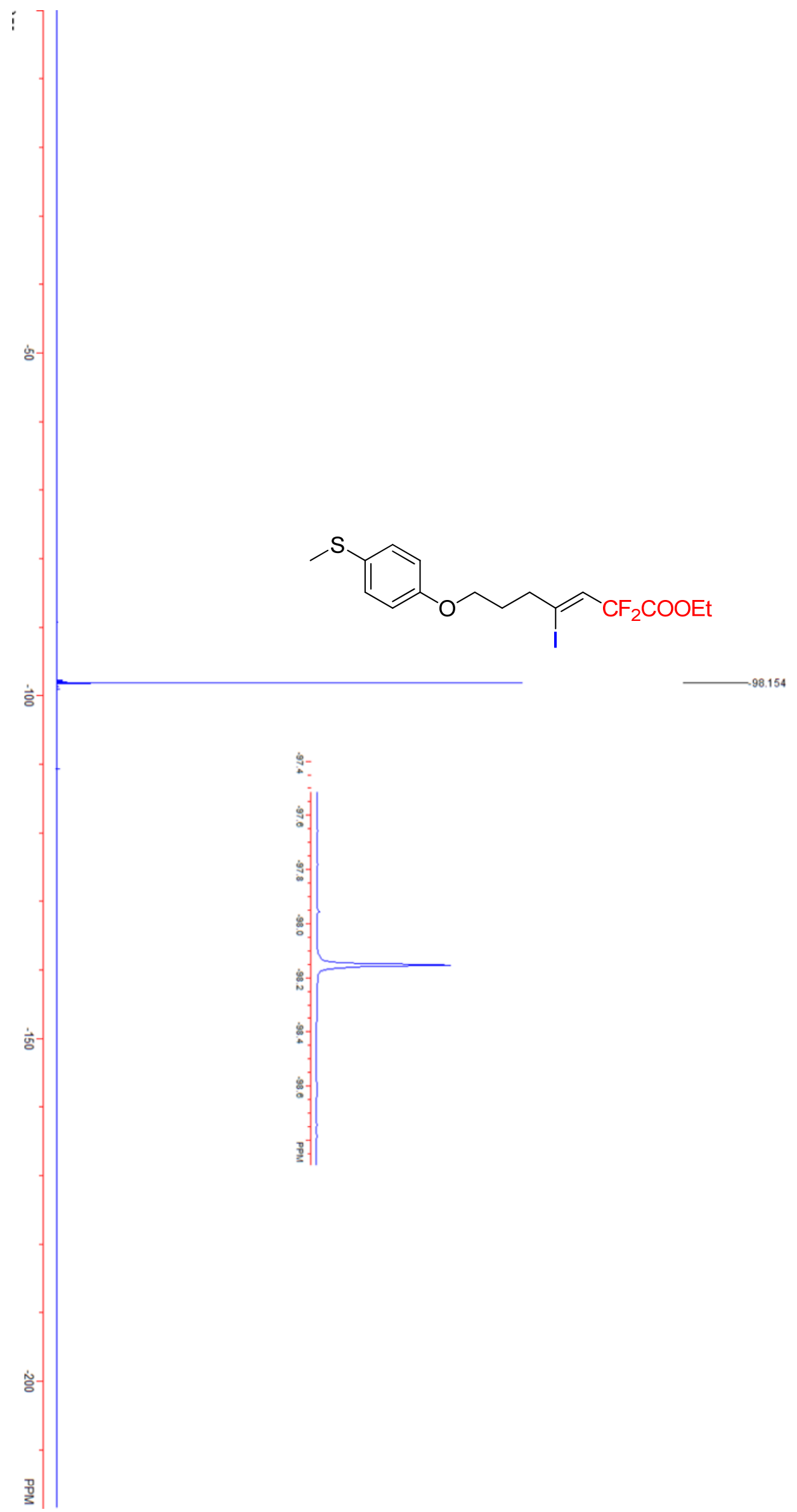



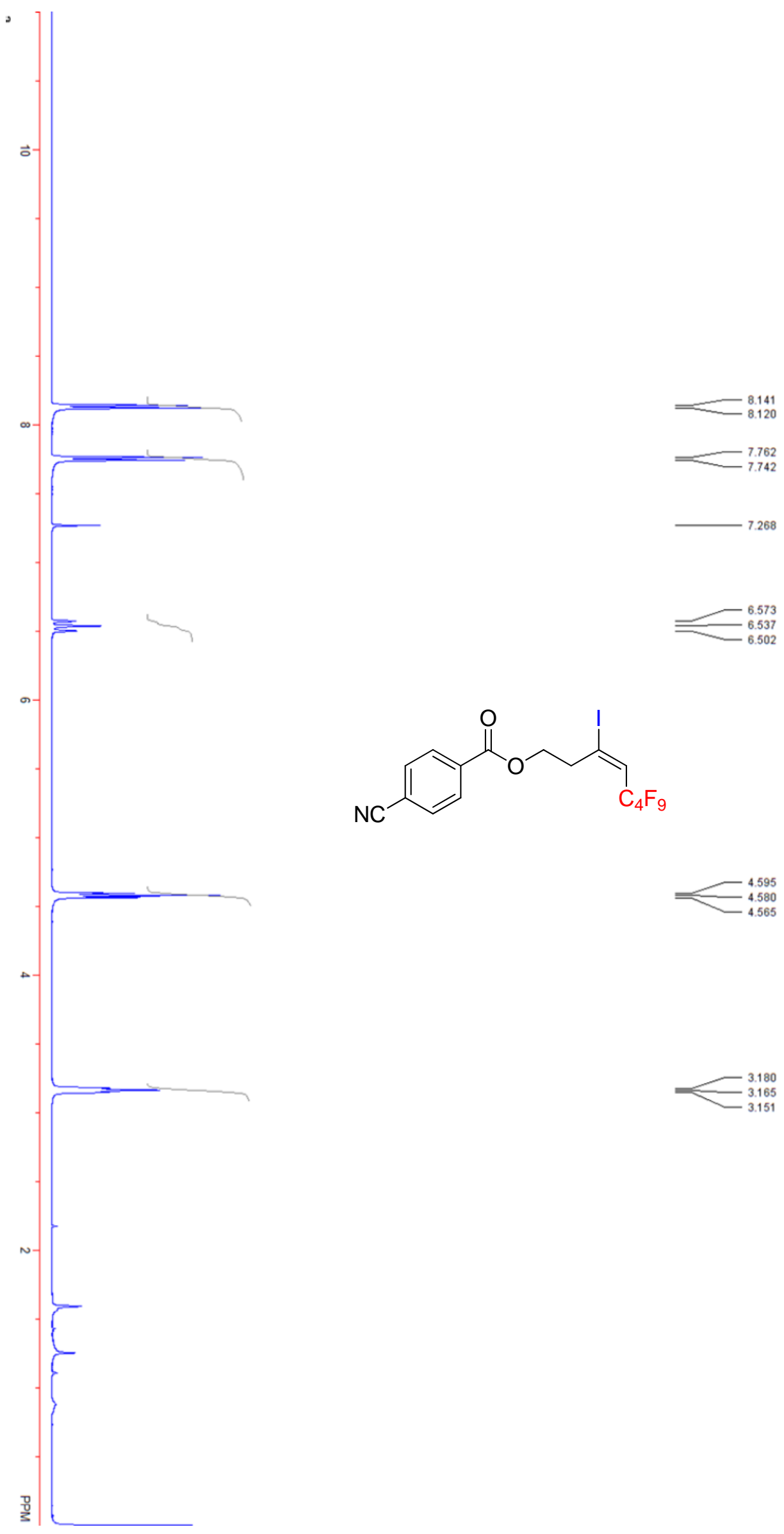<smiles>N#Cc1ccc(C(=O)OCC/C(I)=C\C(F)(F)F)cc1</smiles>
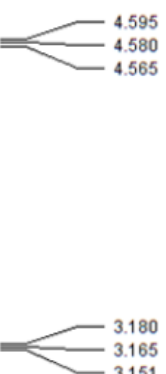

3.165
-3.151 


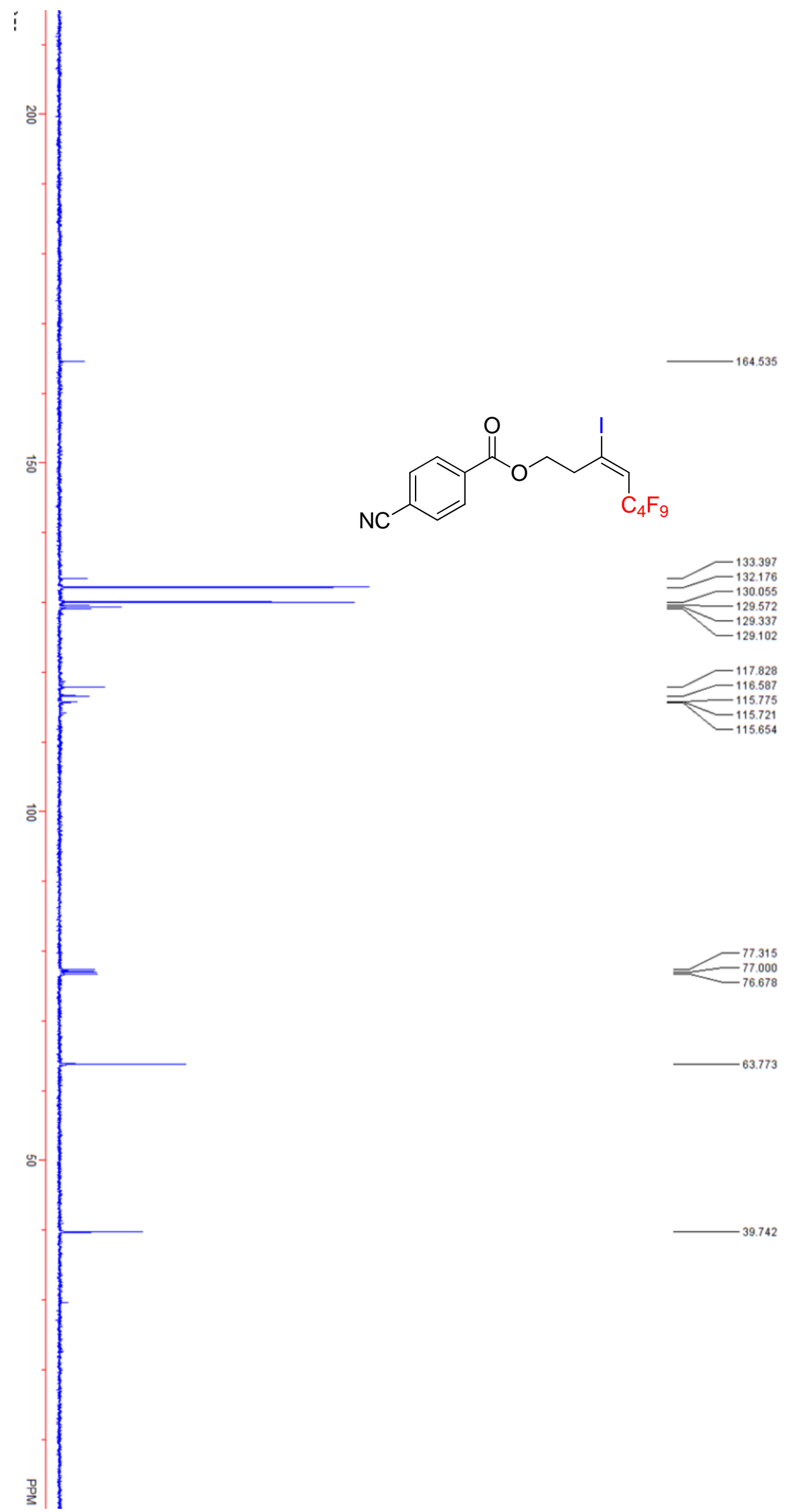




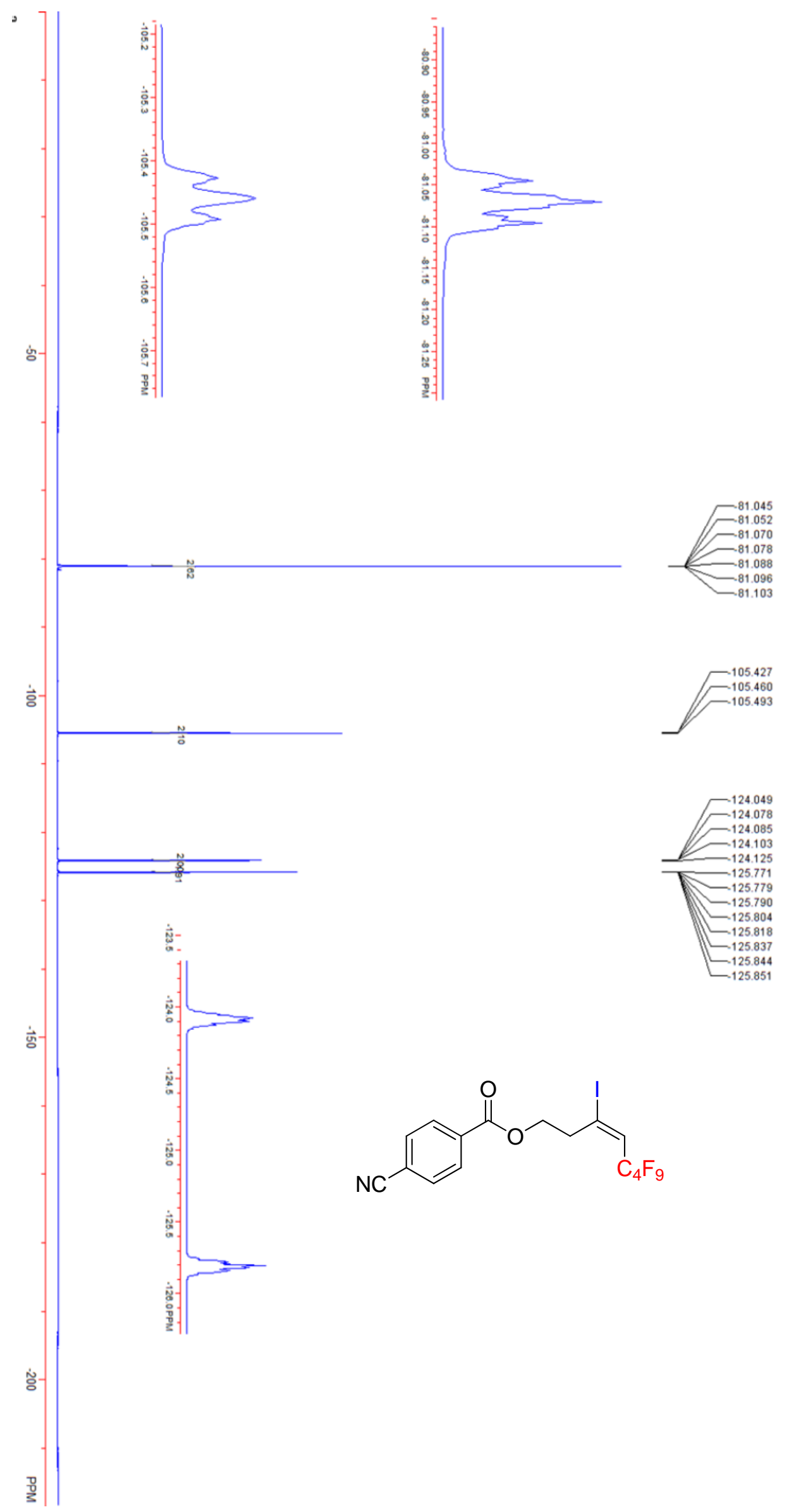




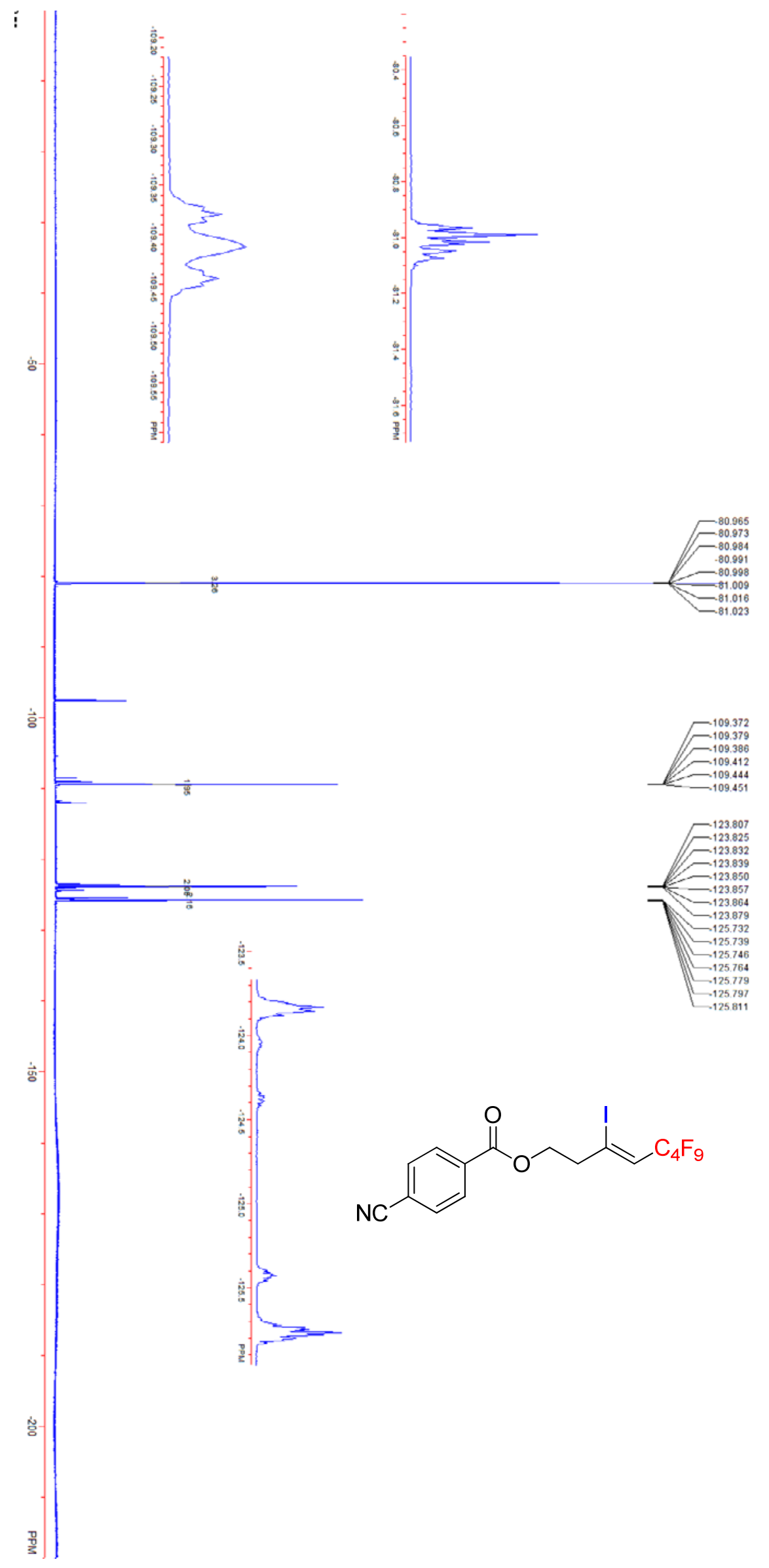




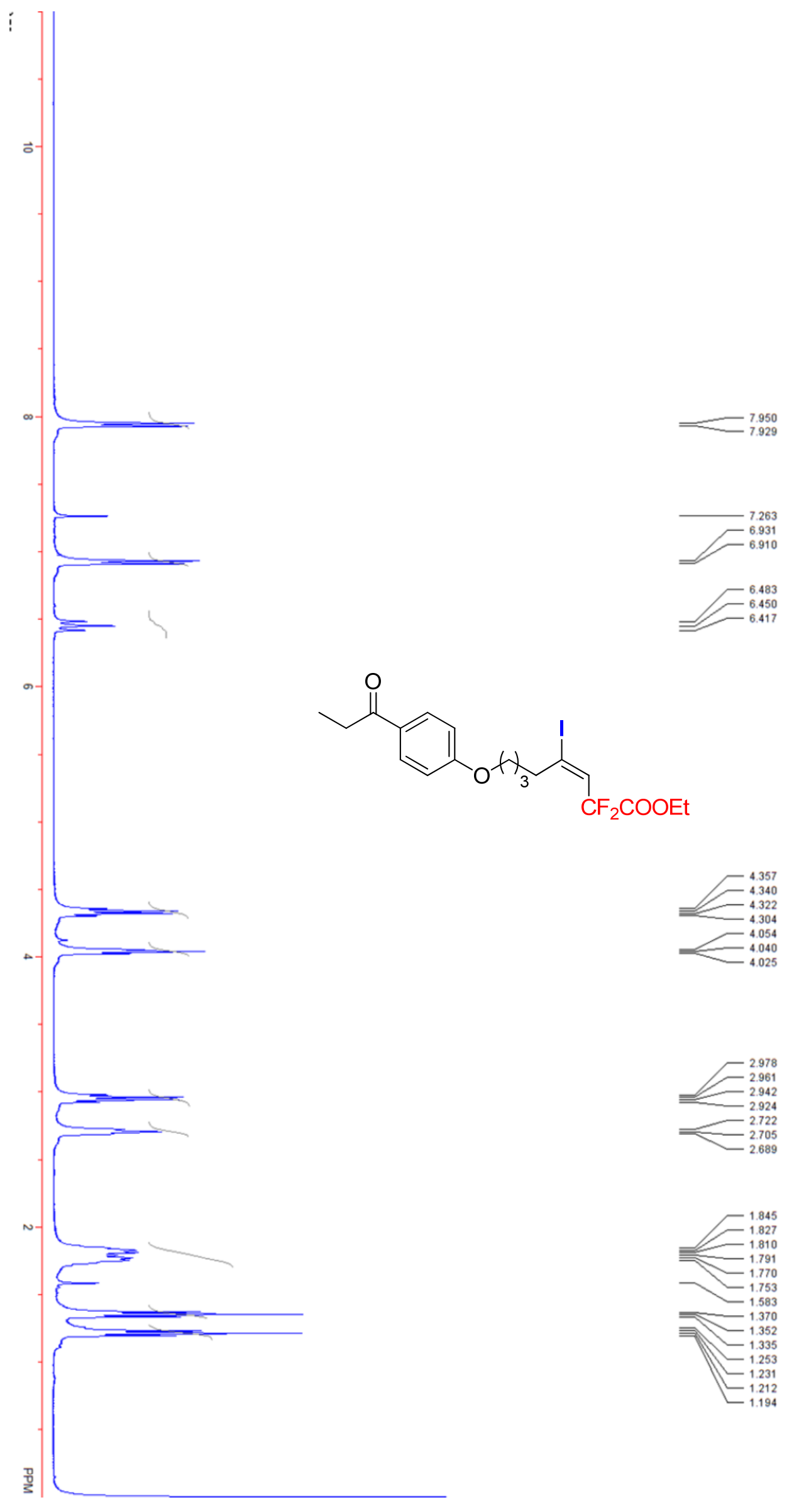

S103 


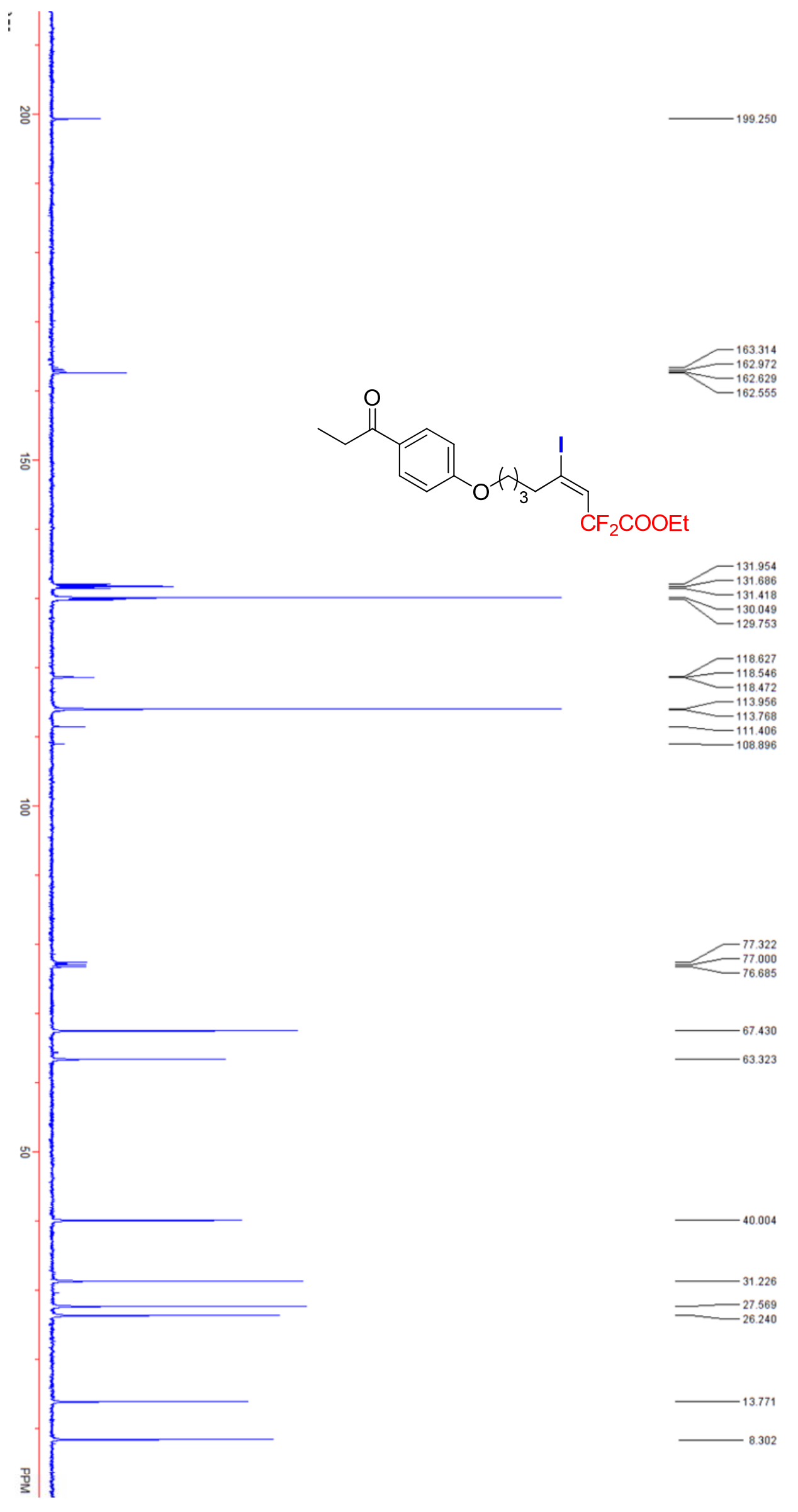

S104 


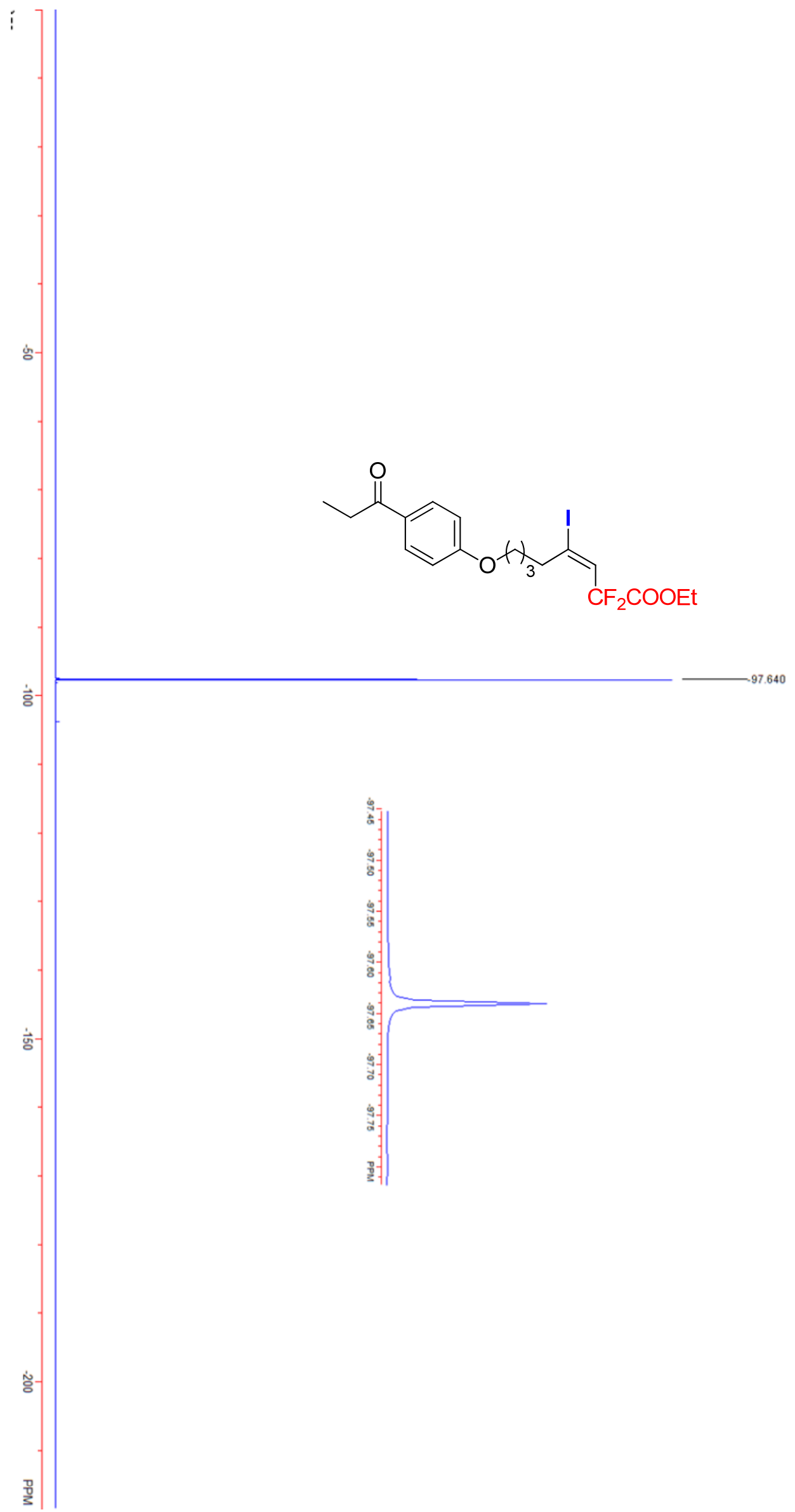




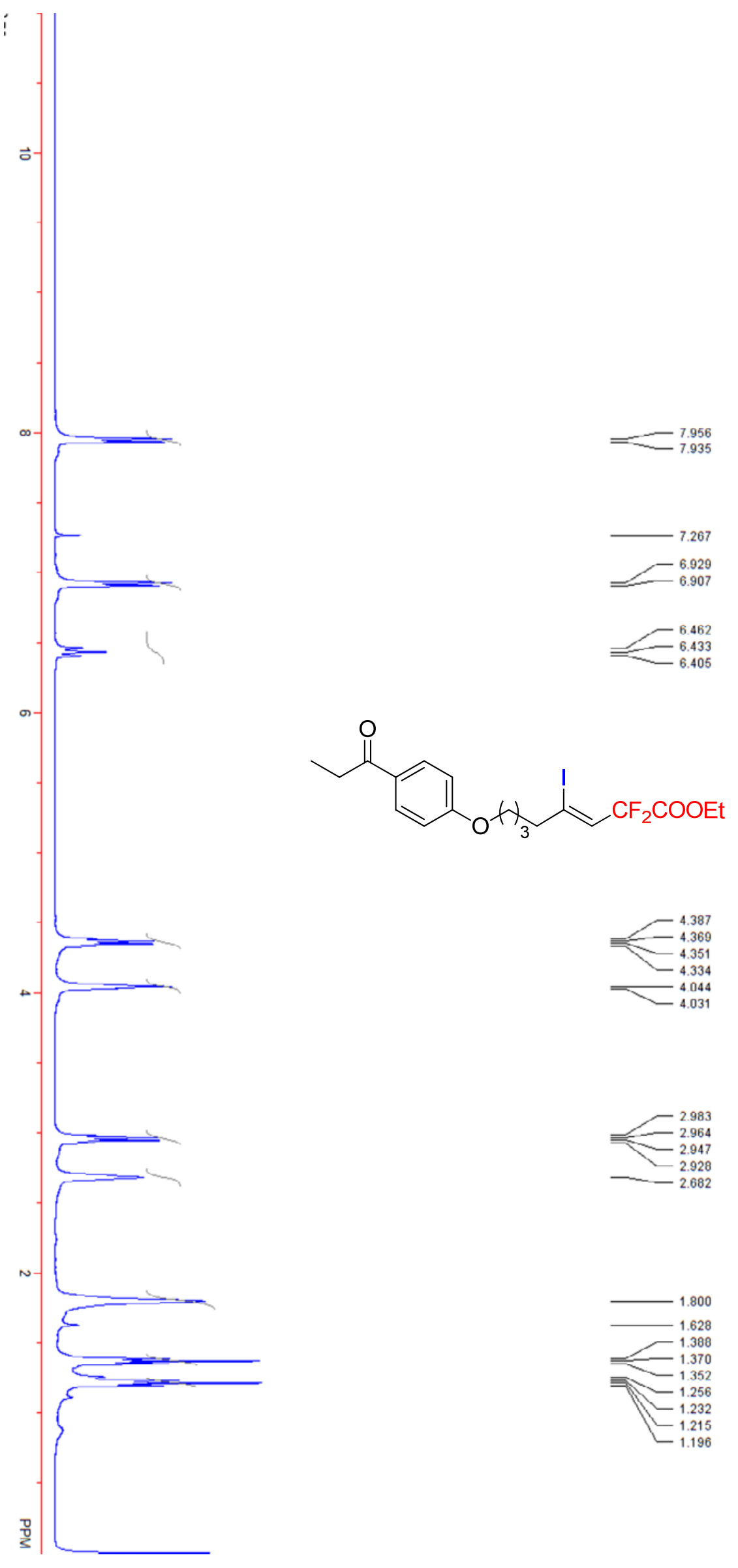




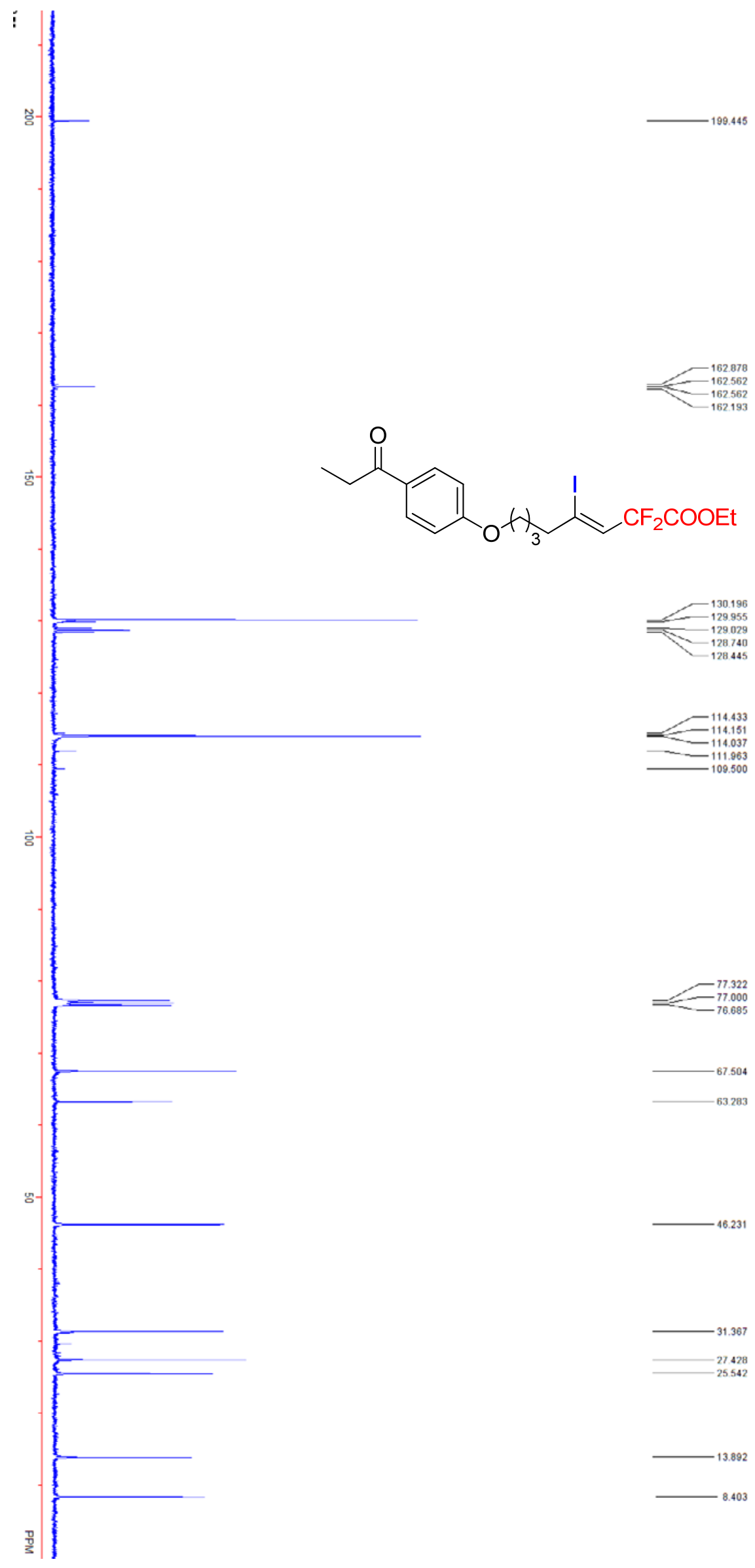




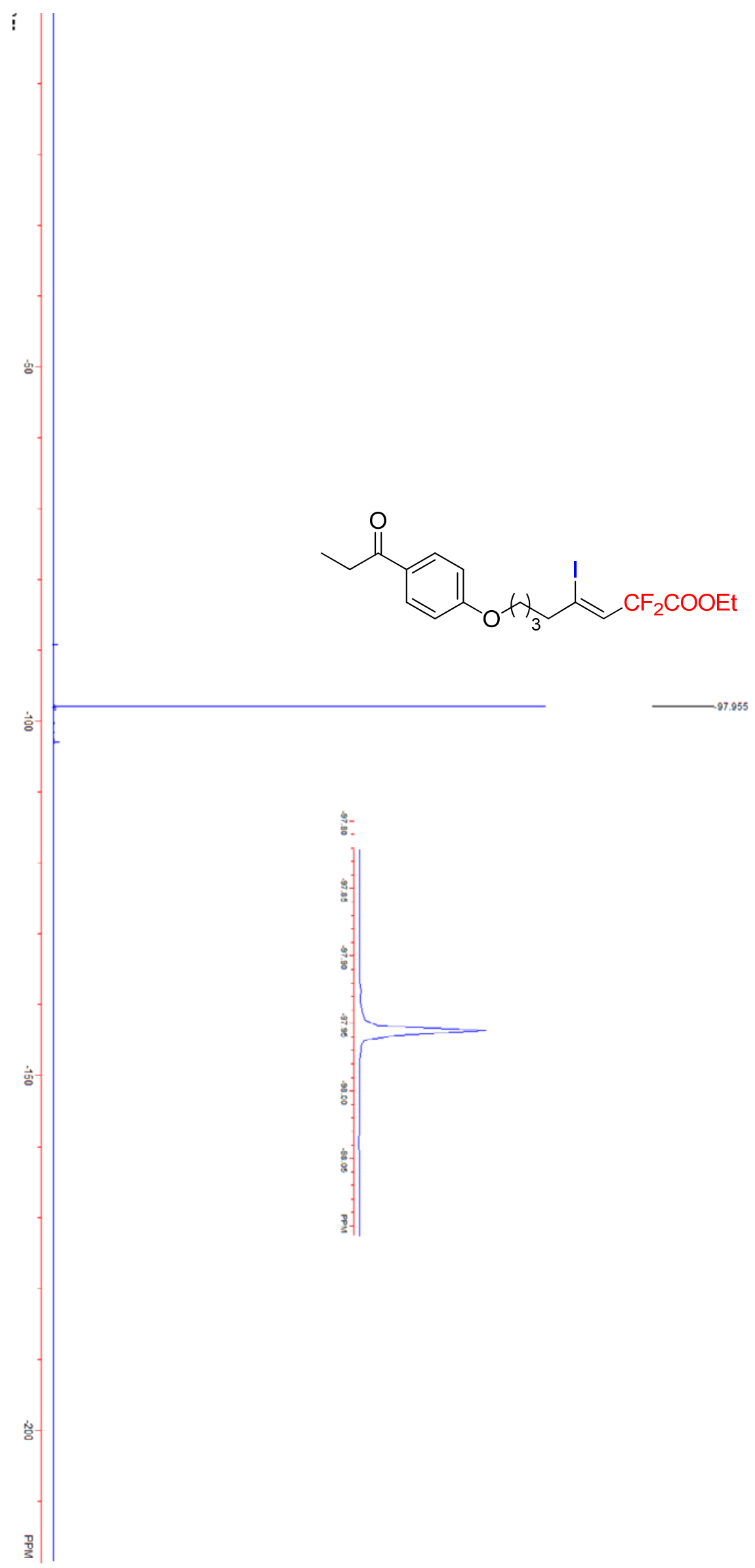



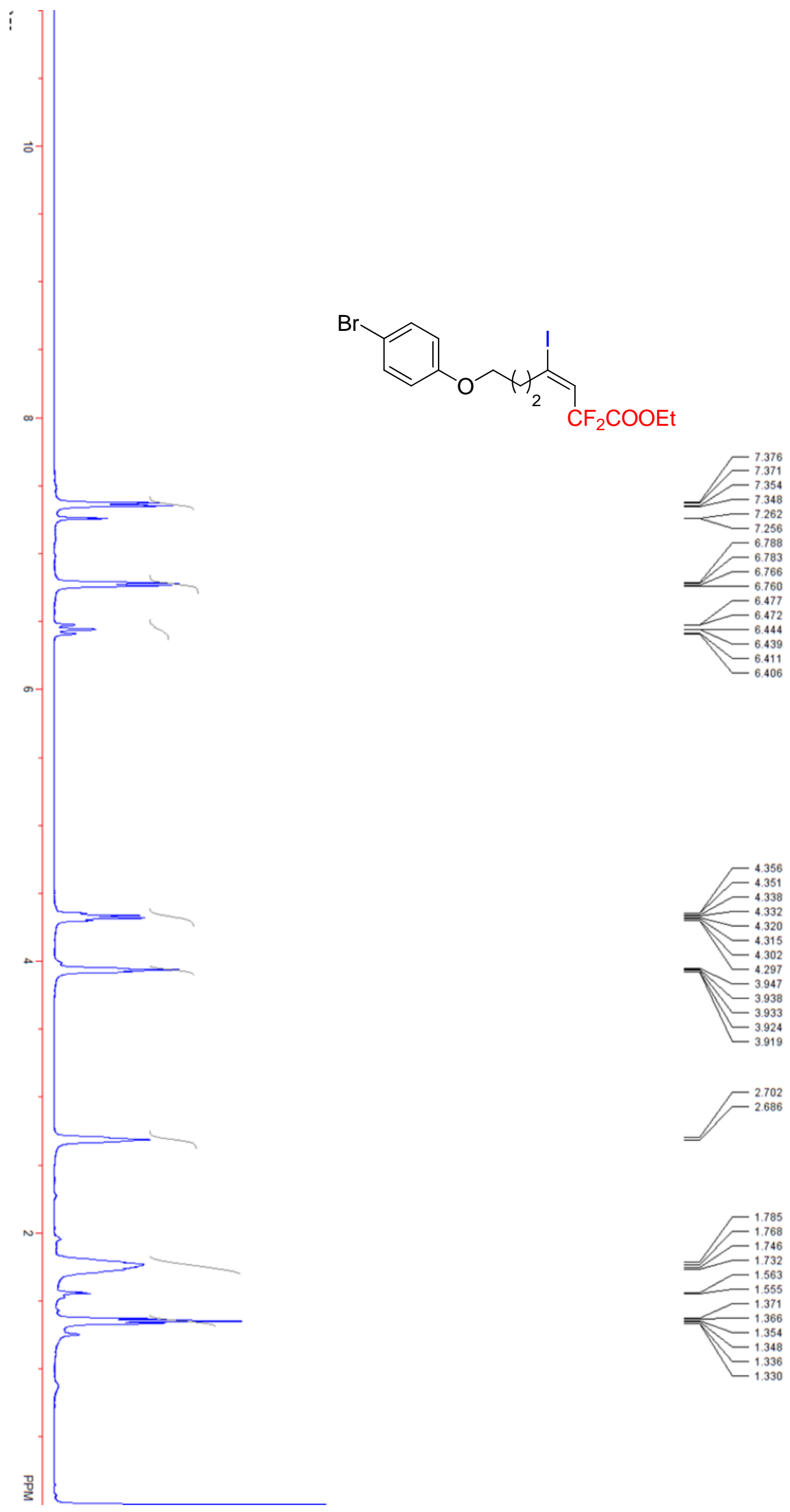

S109 


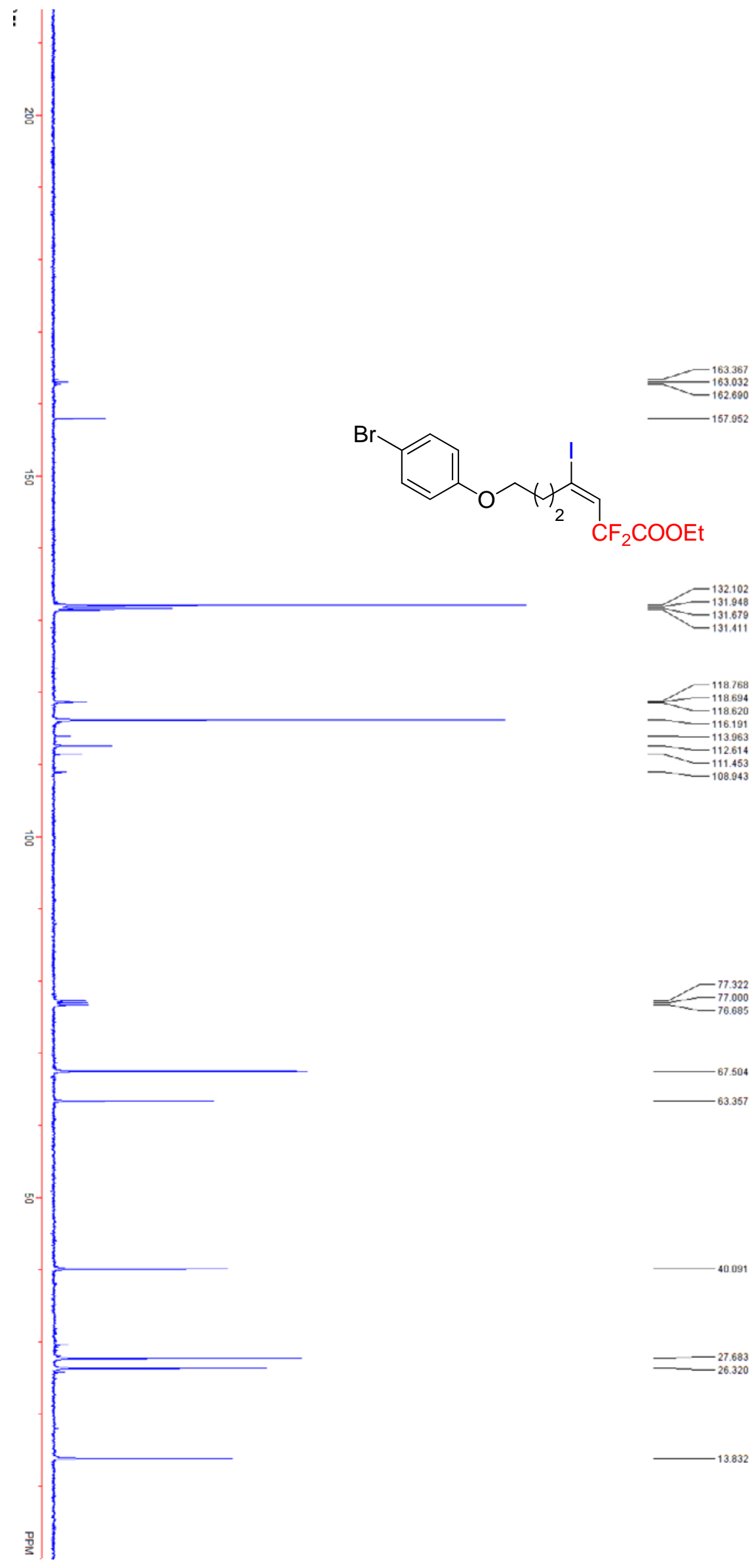




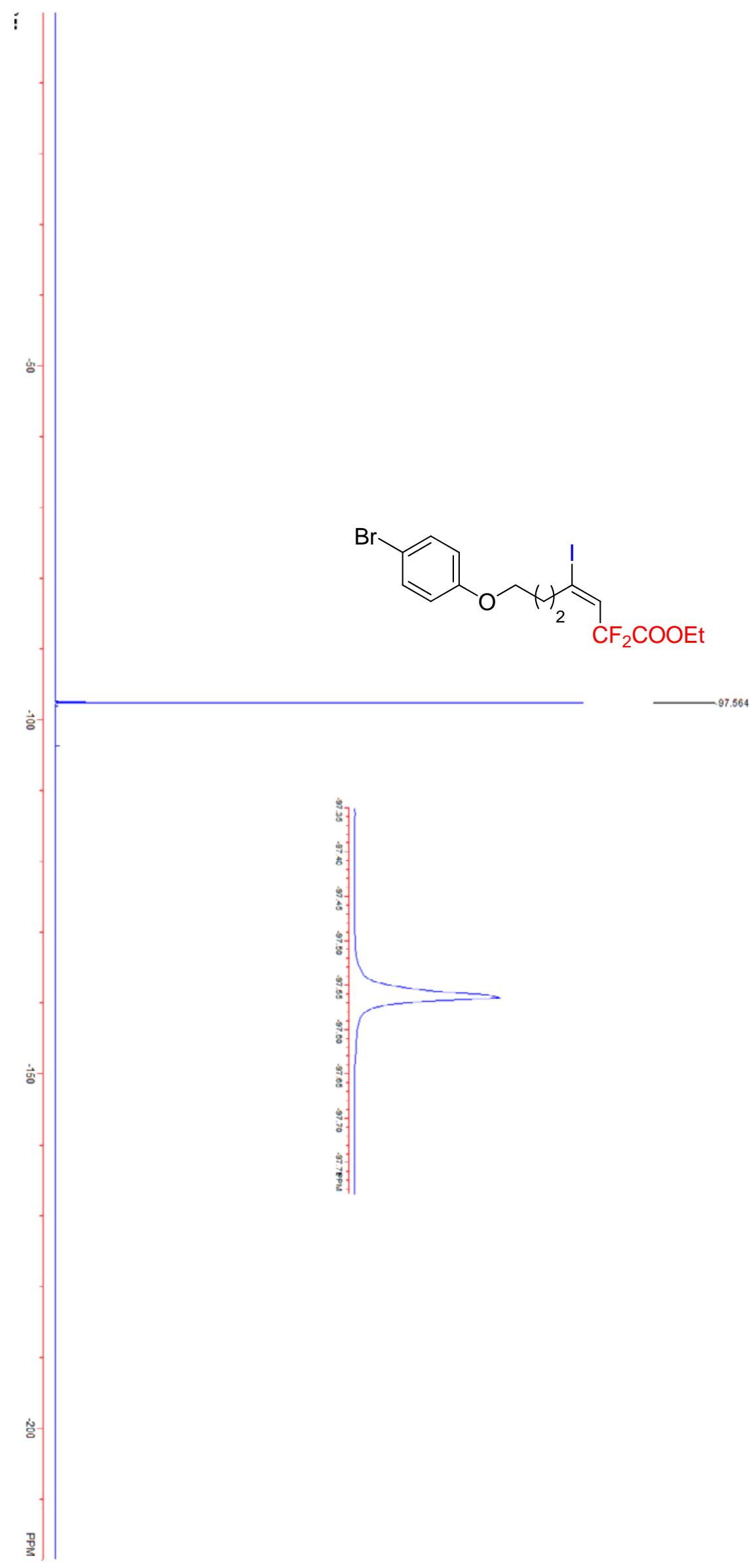




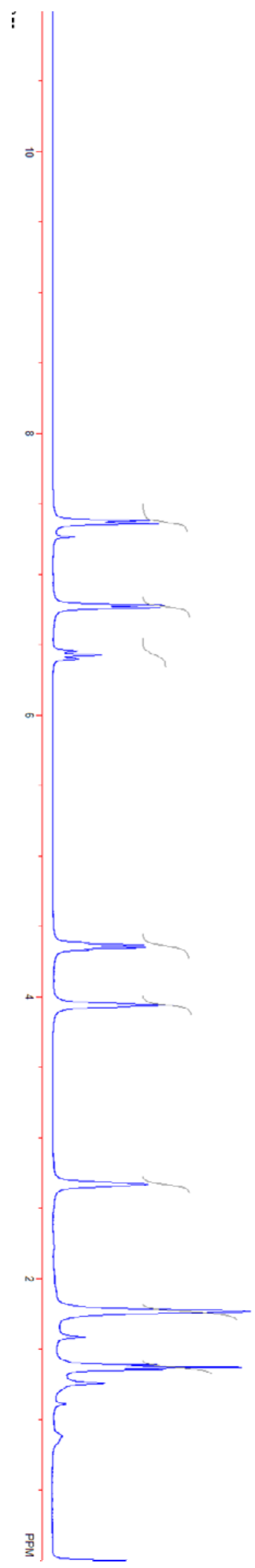

COCF
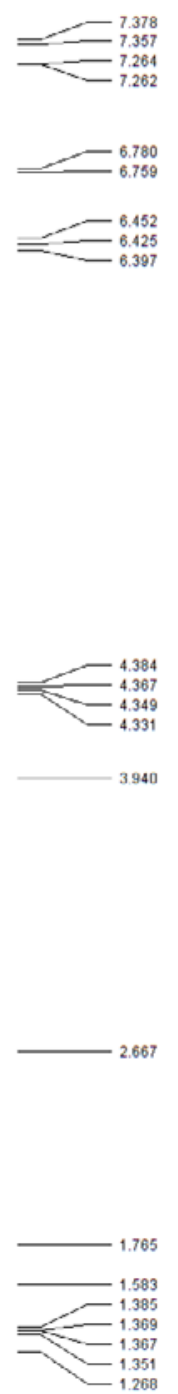

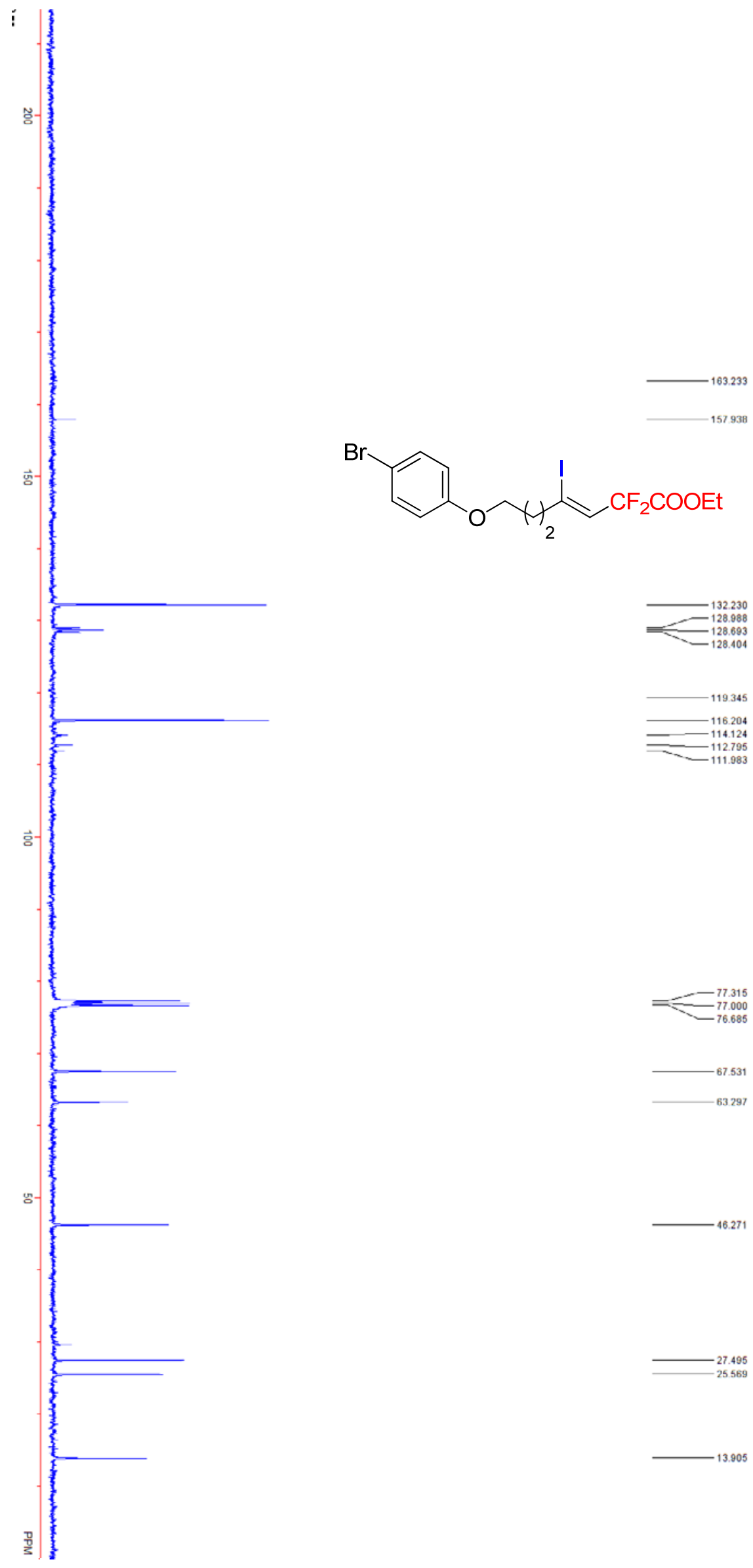


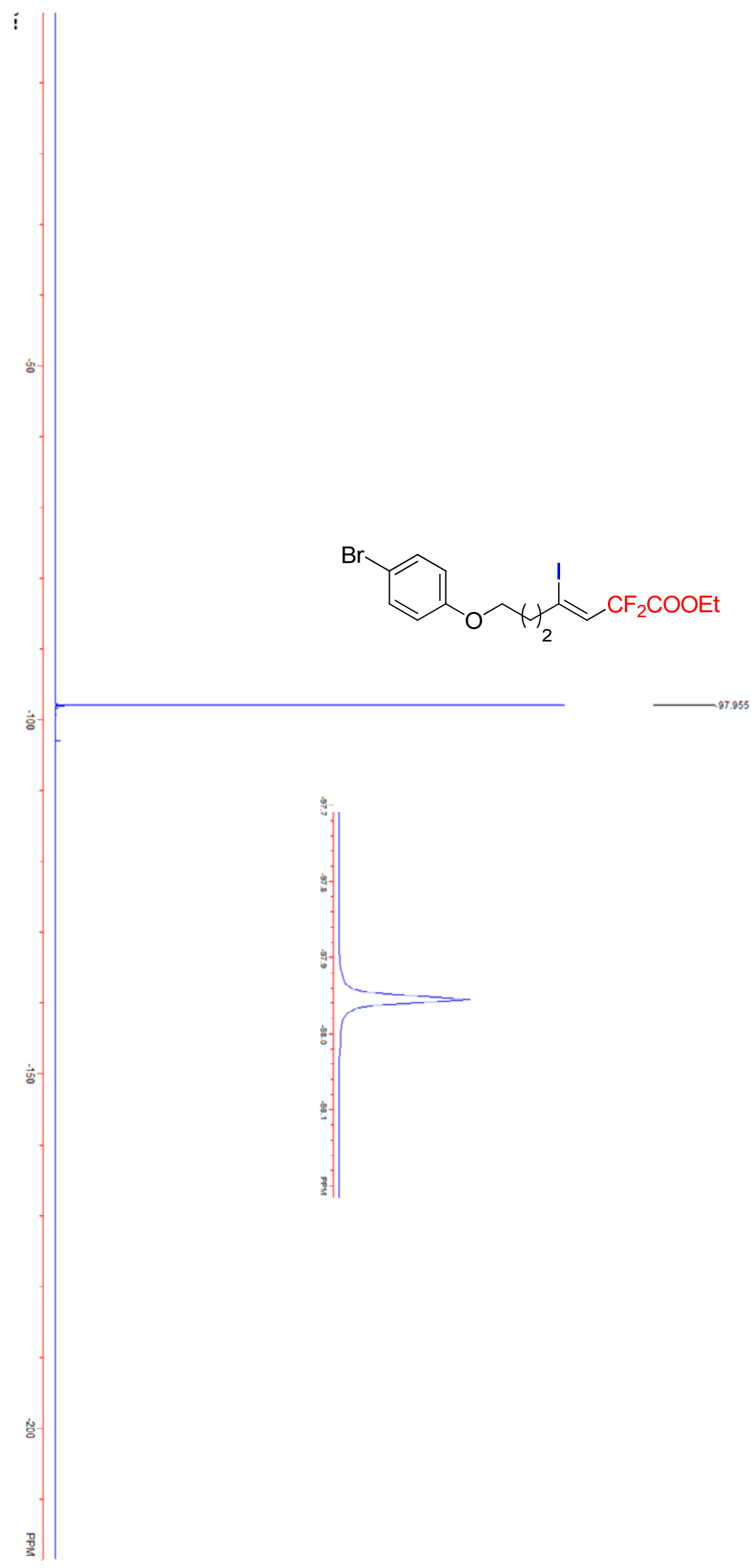




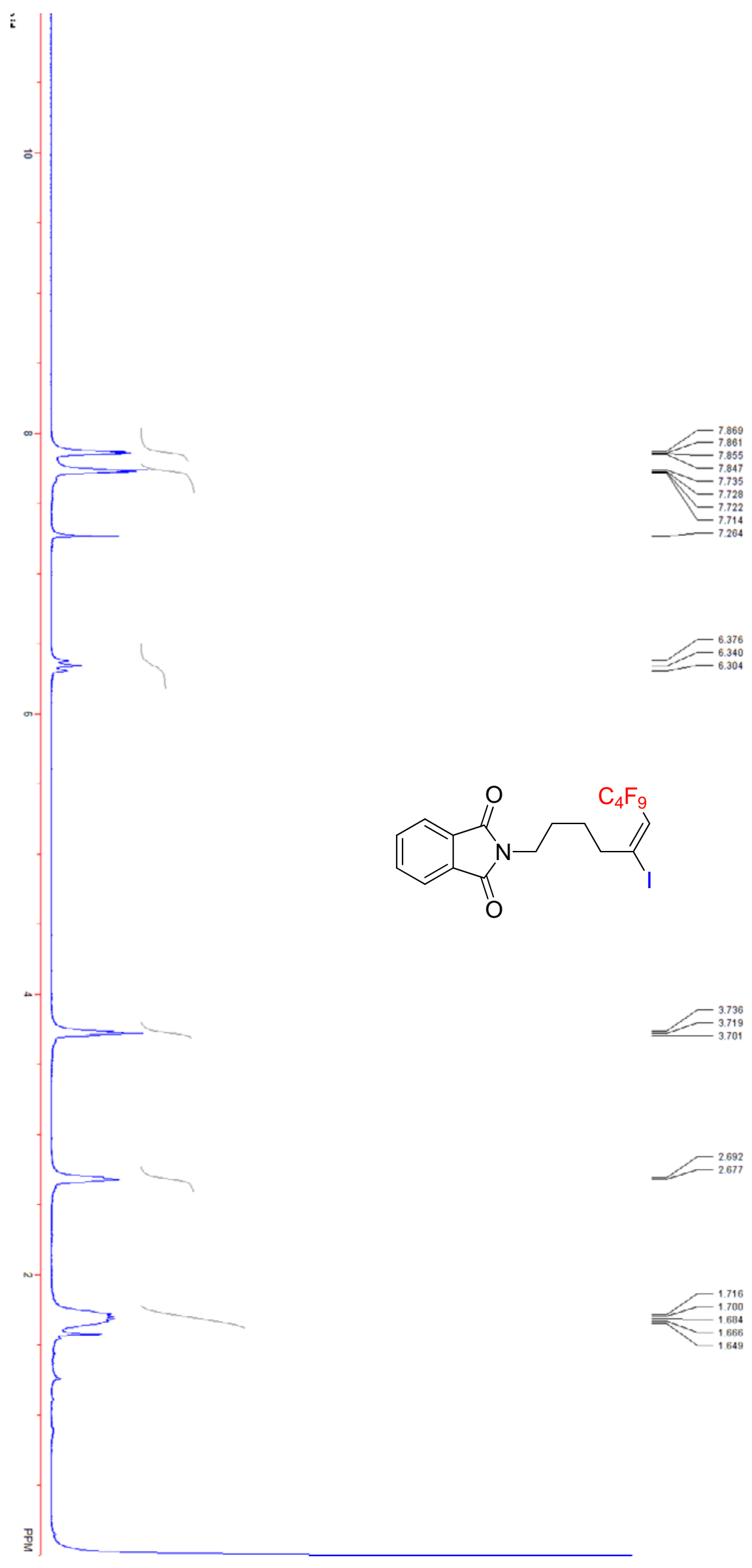



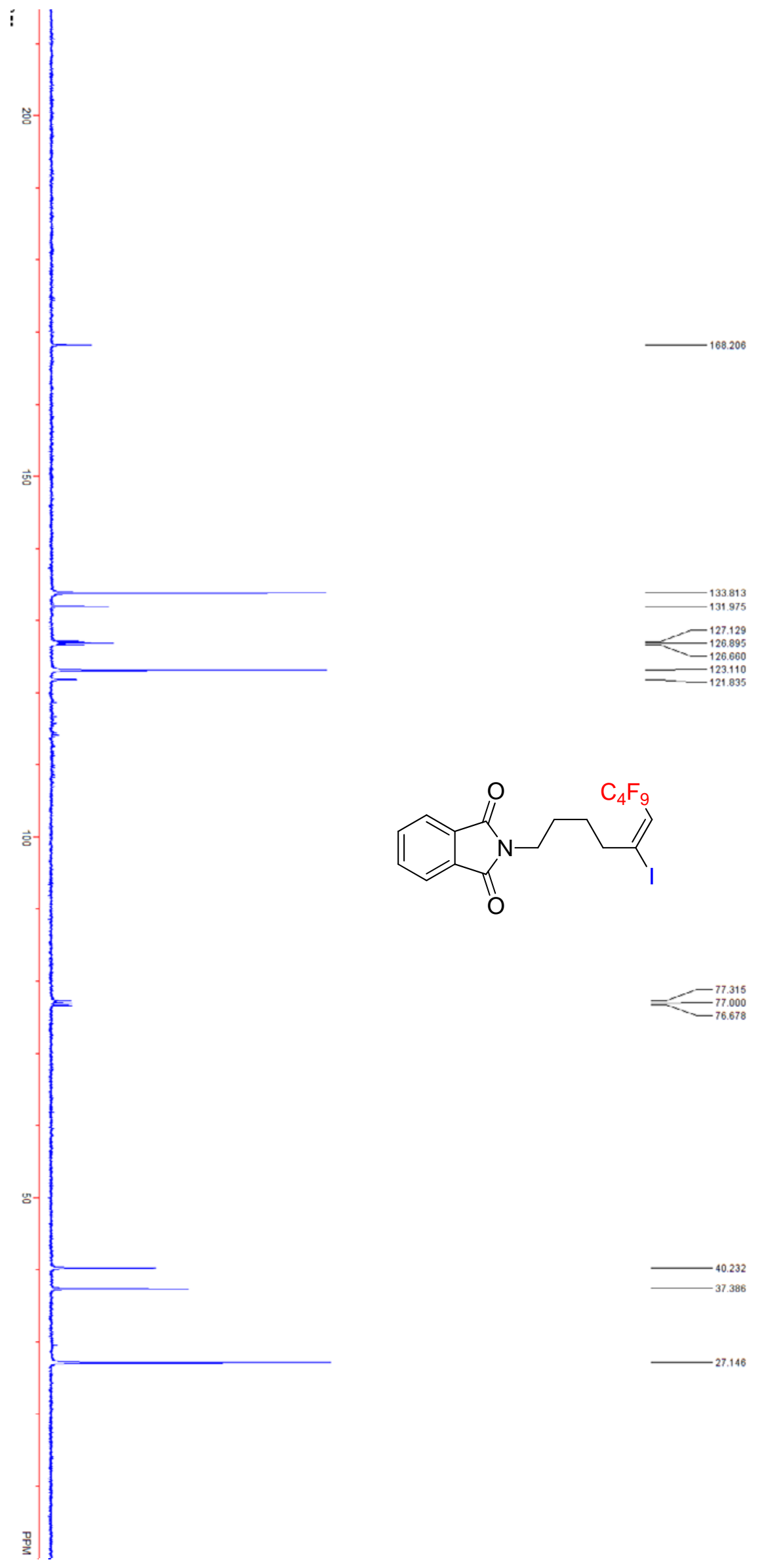


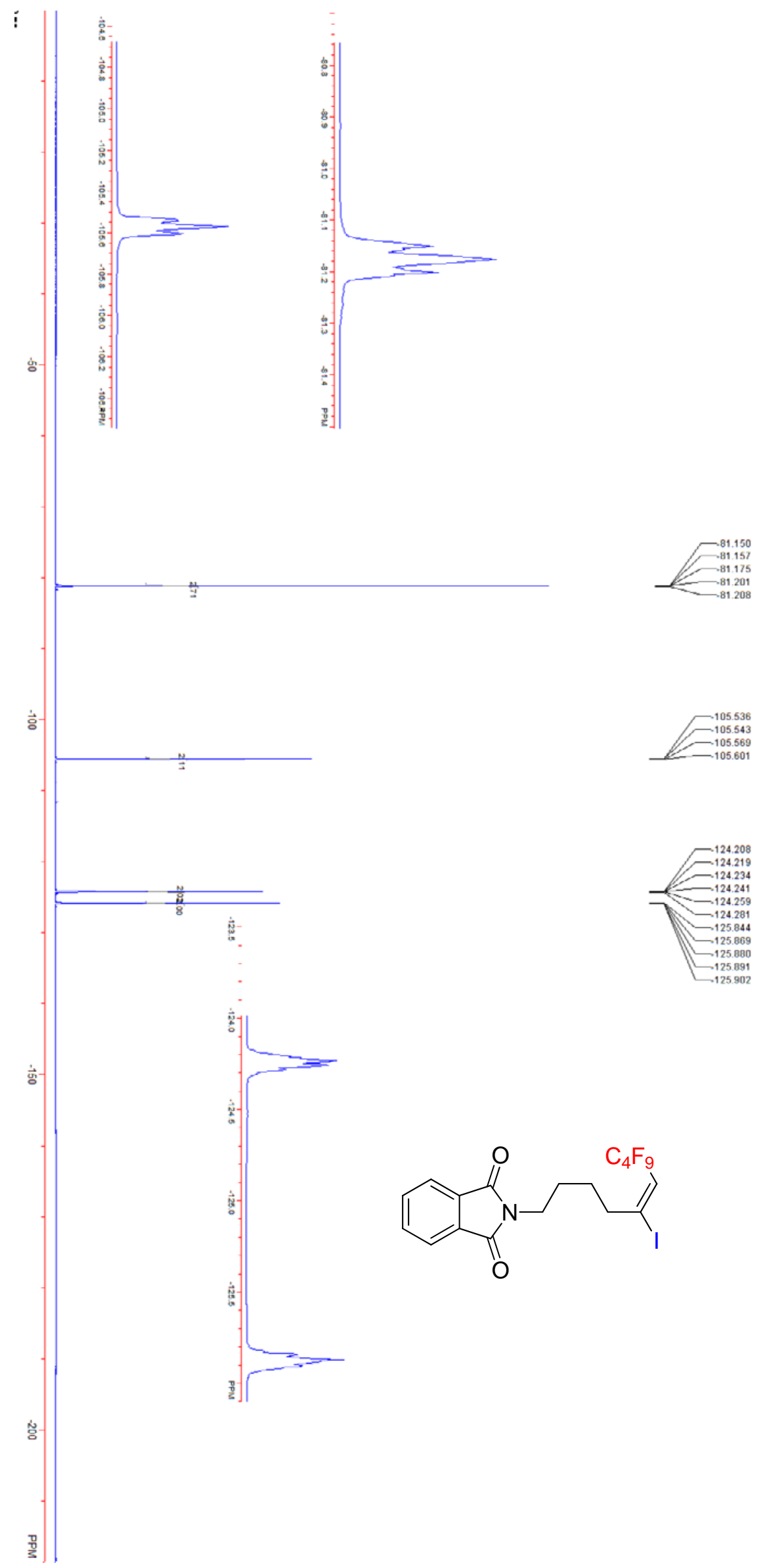




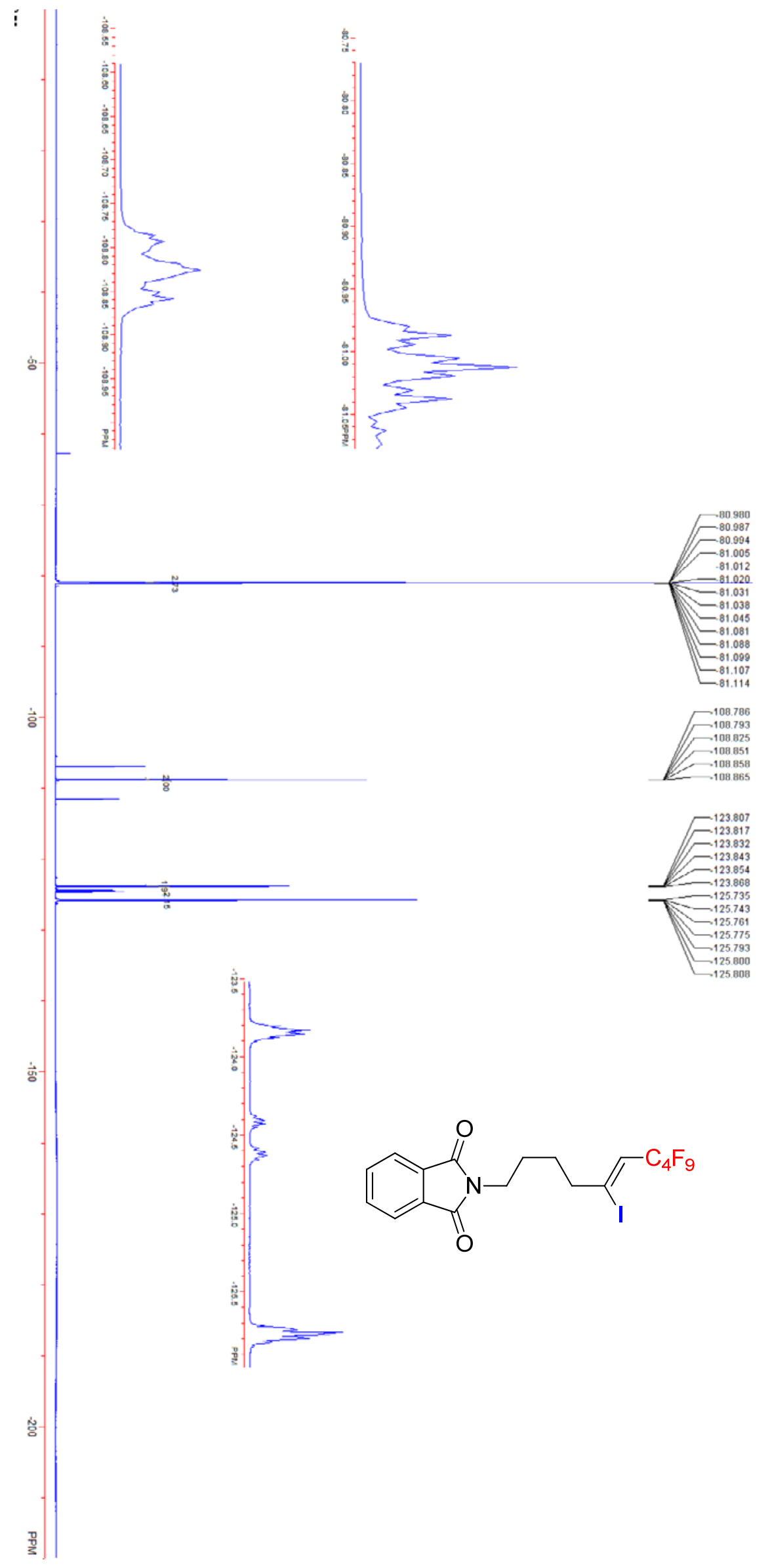




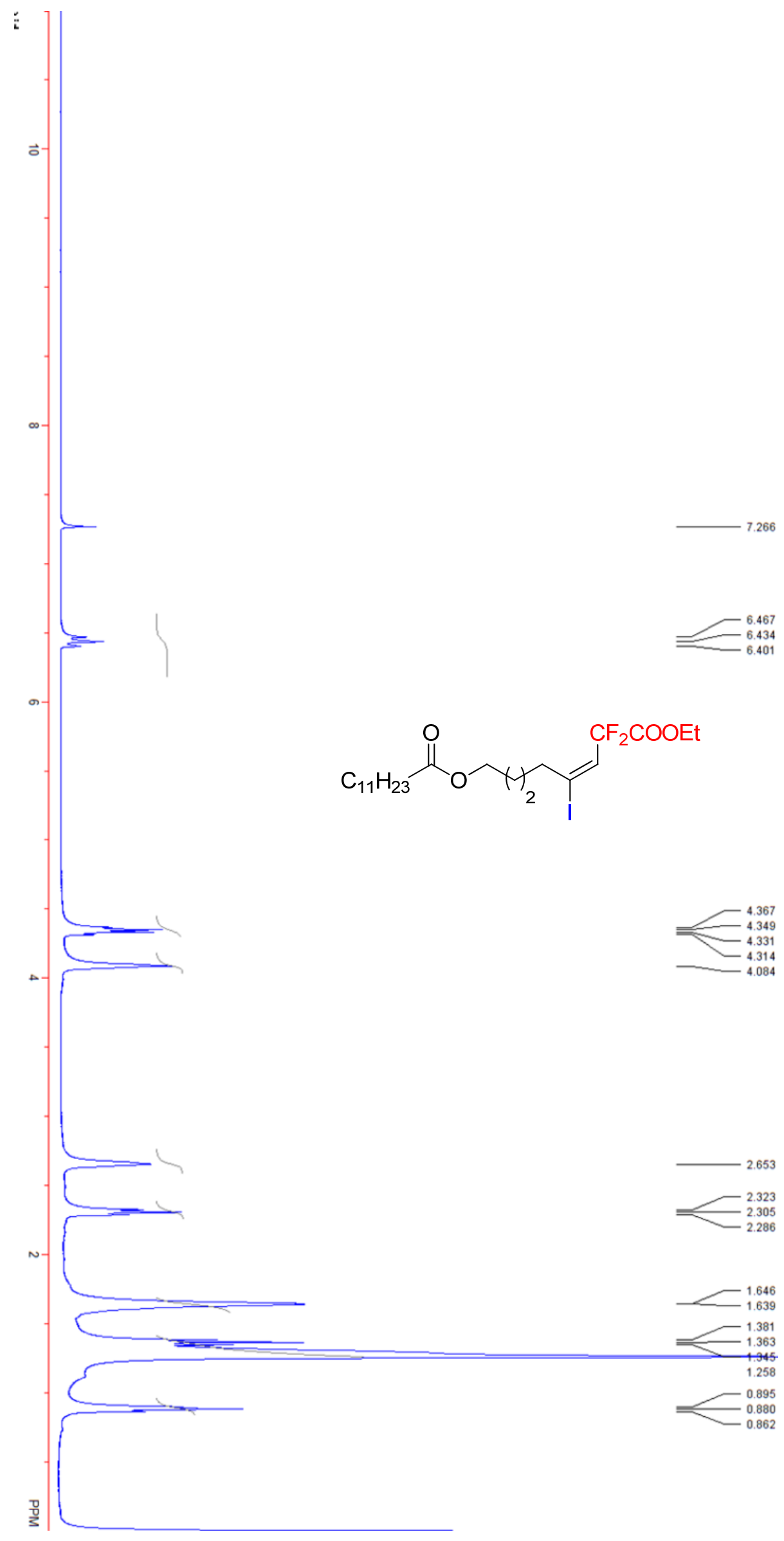




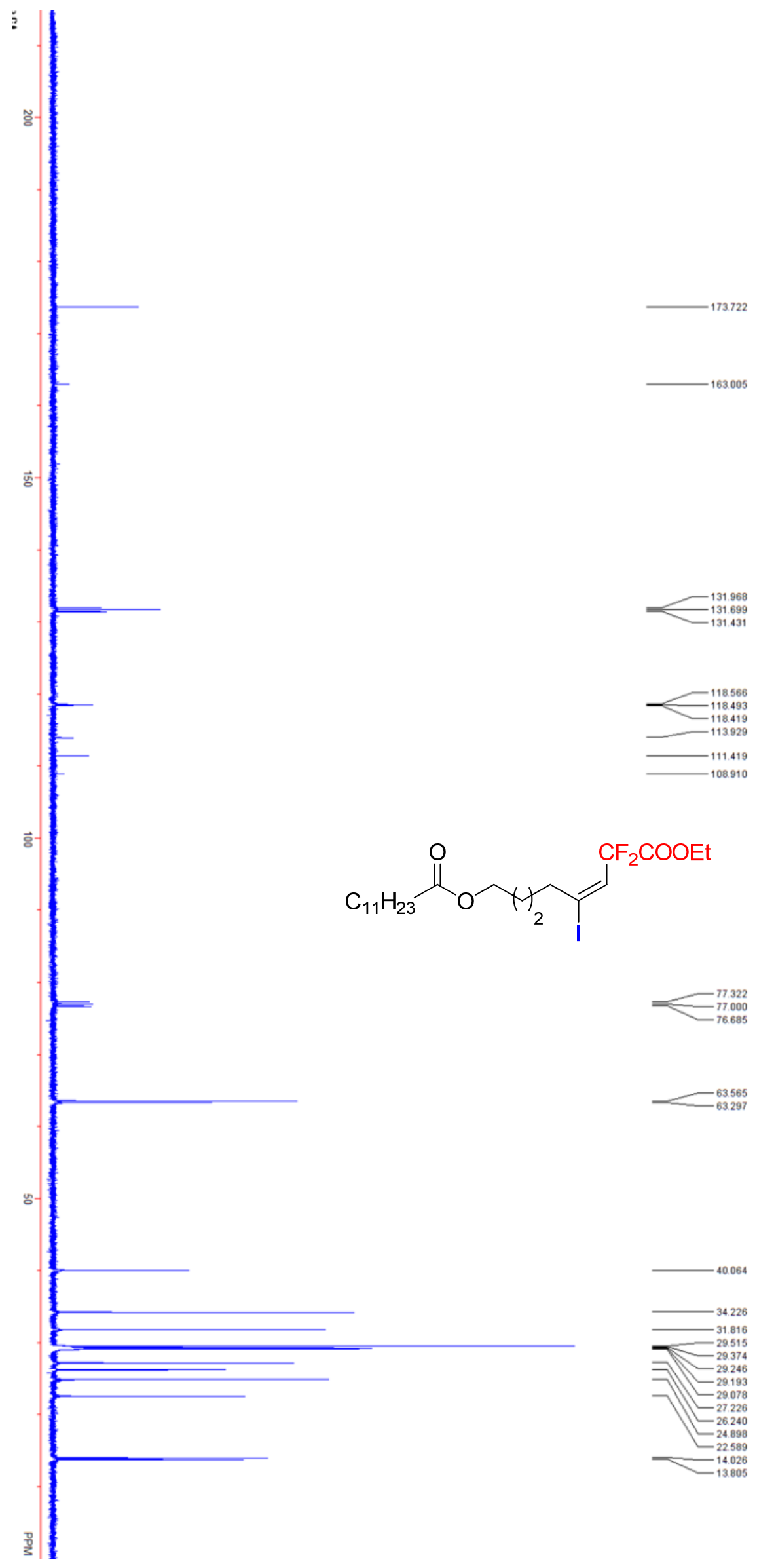




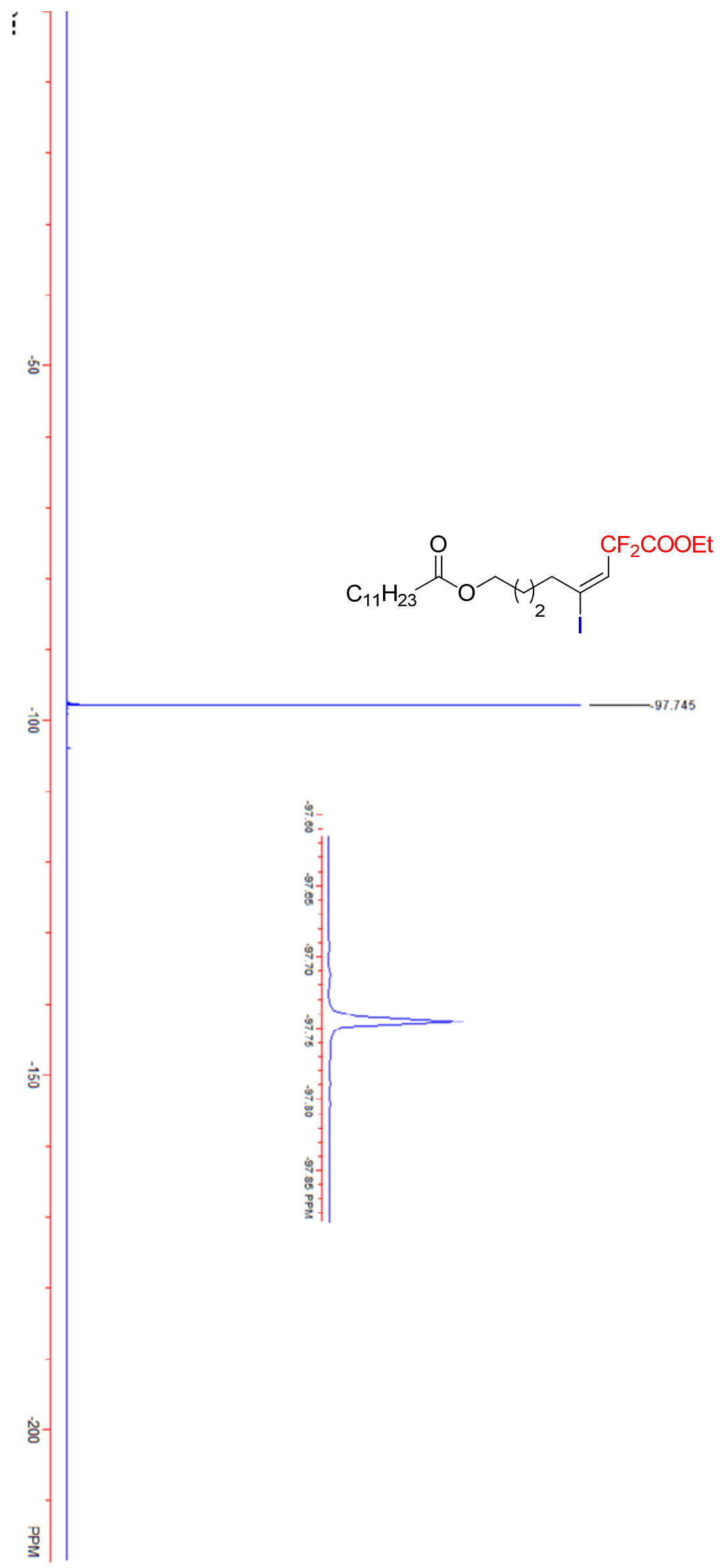




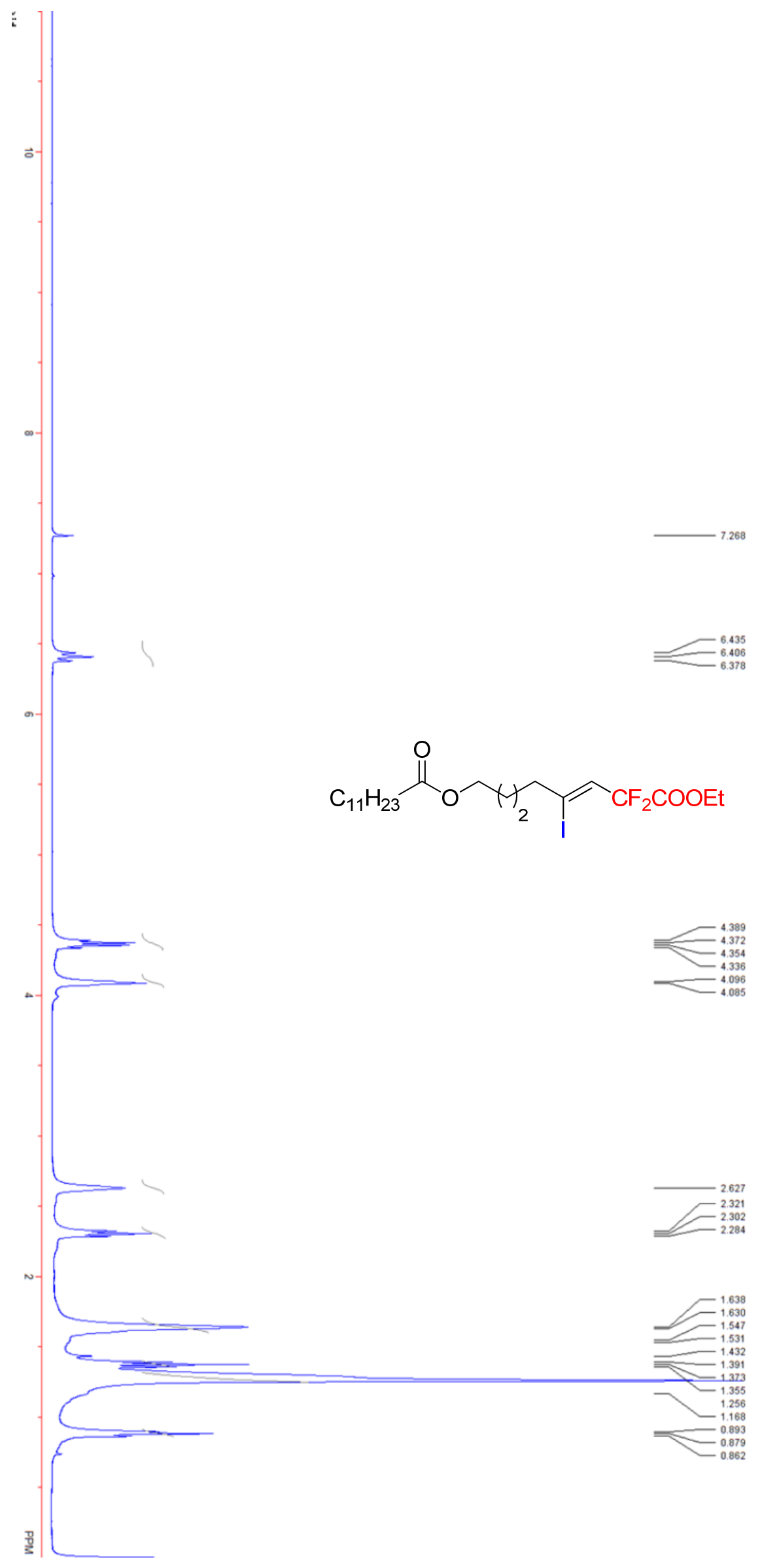



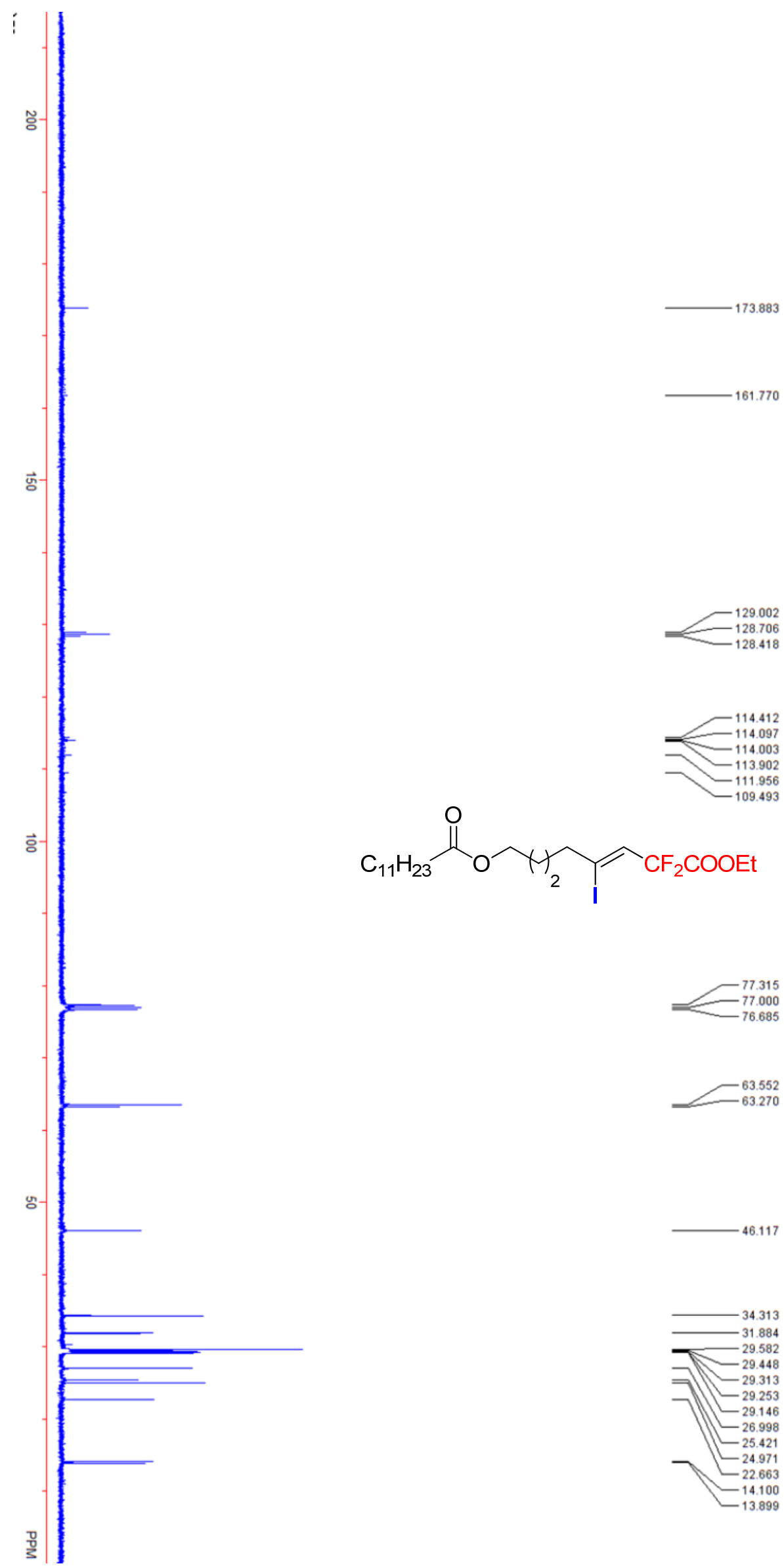


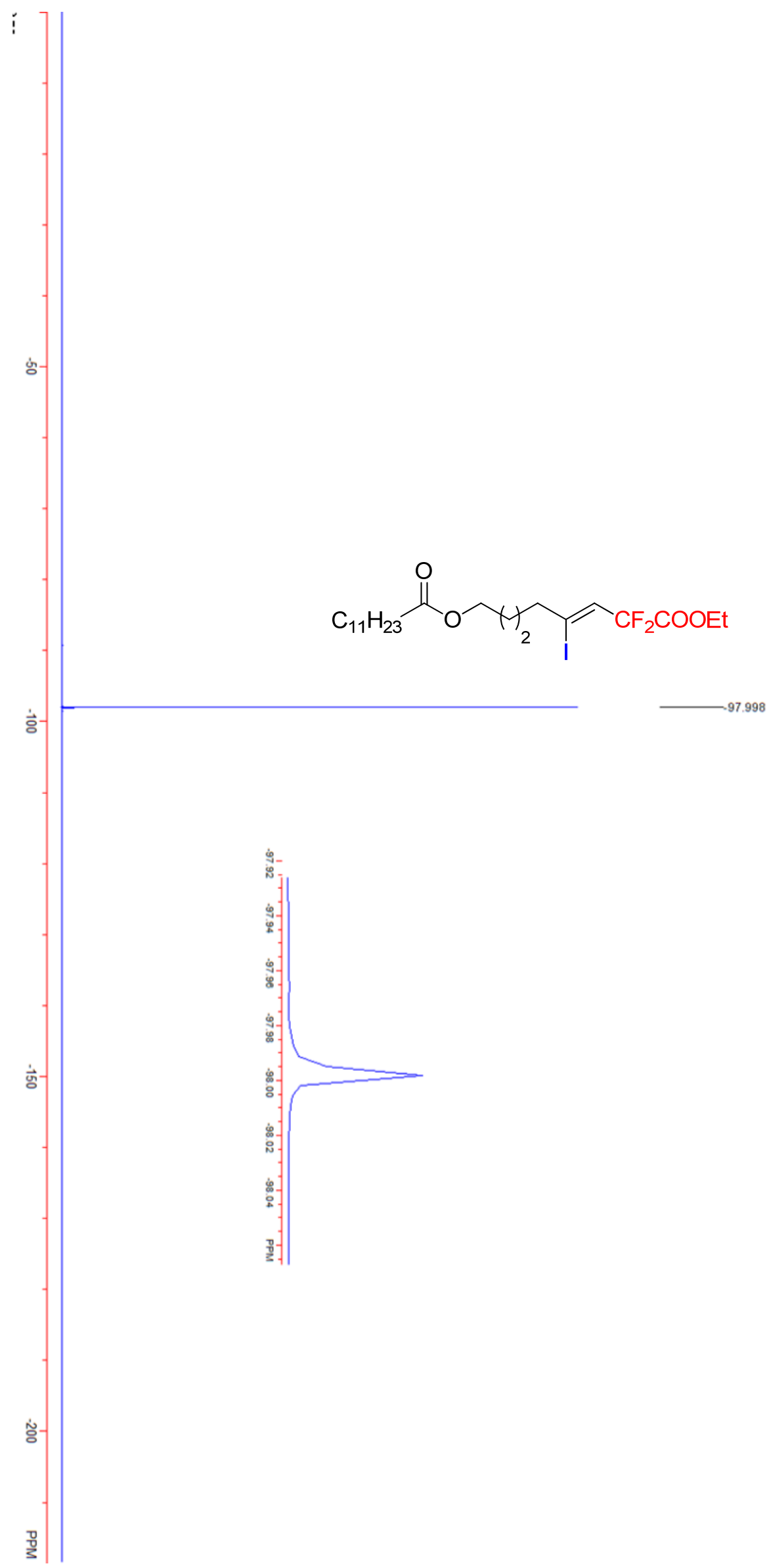




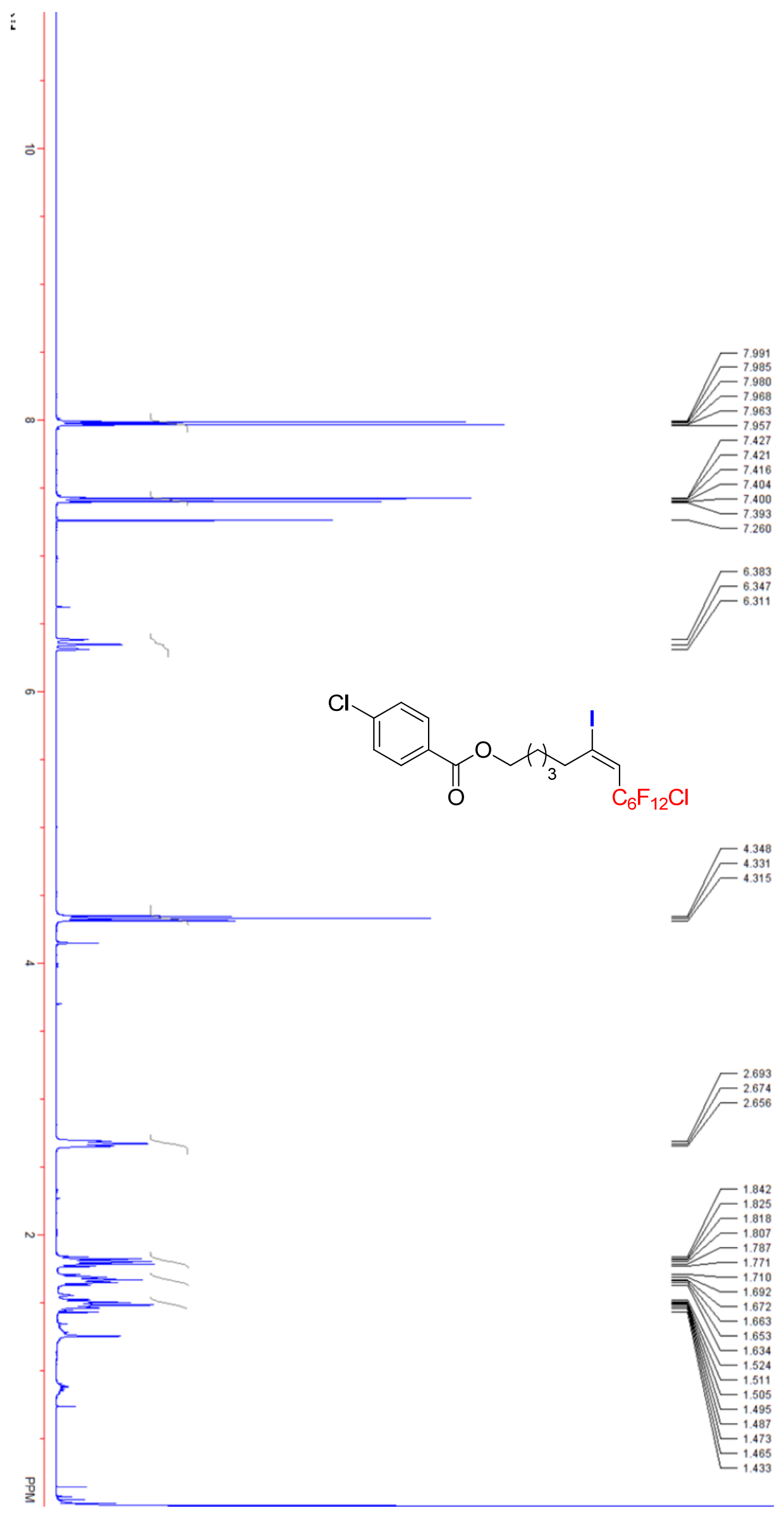




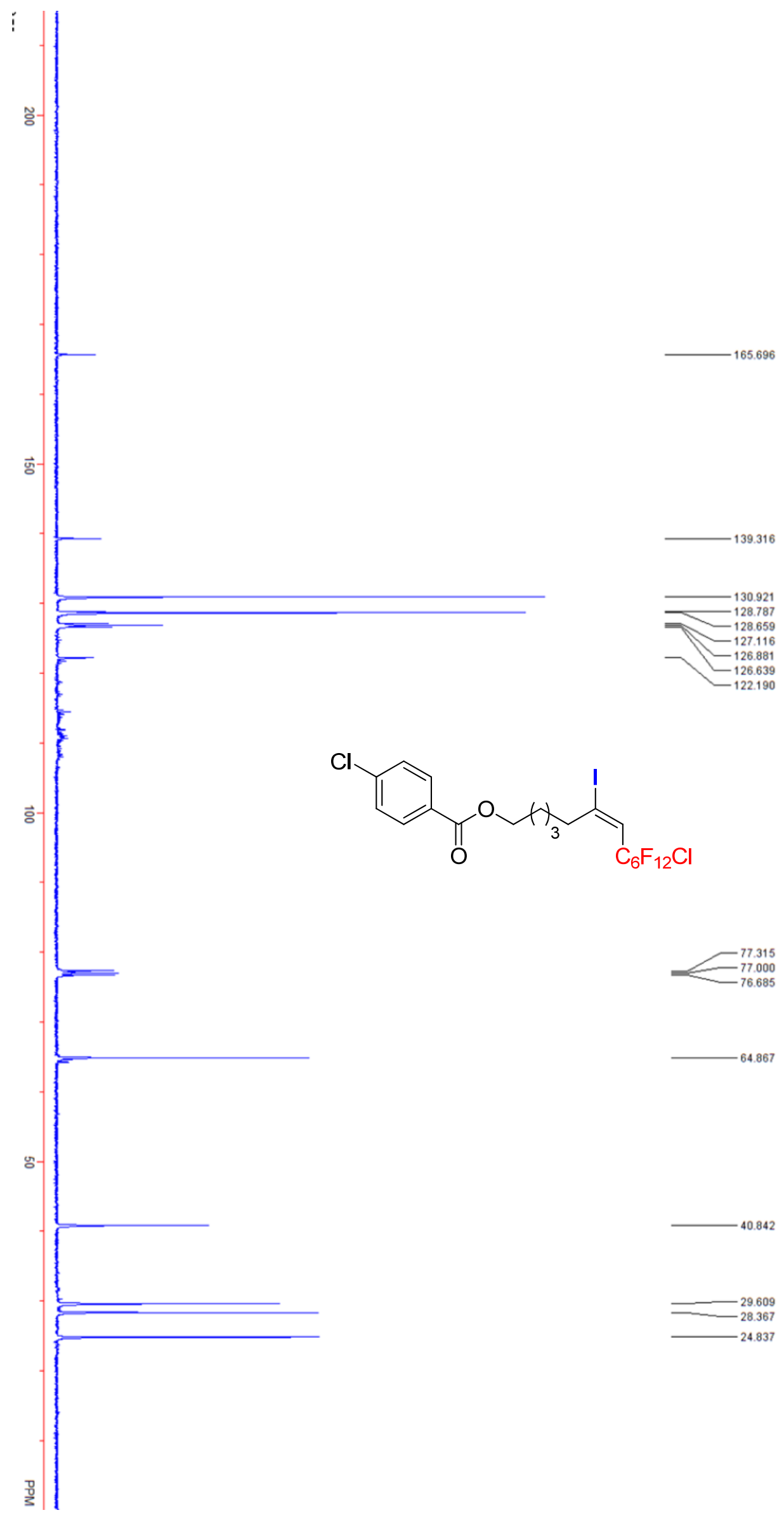




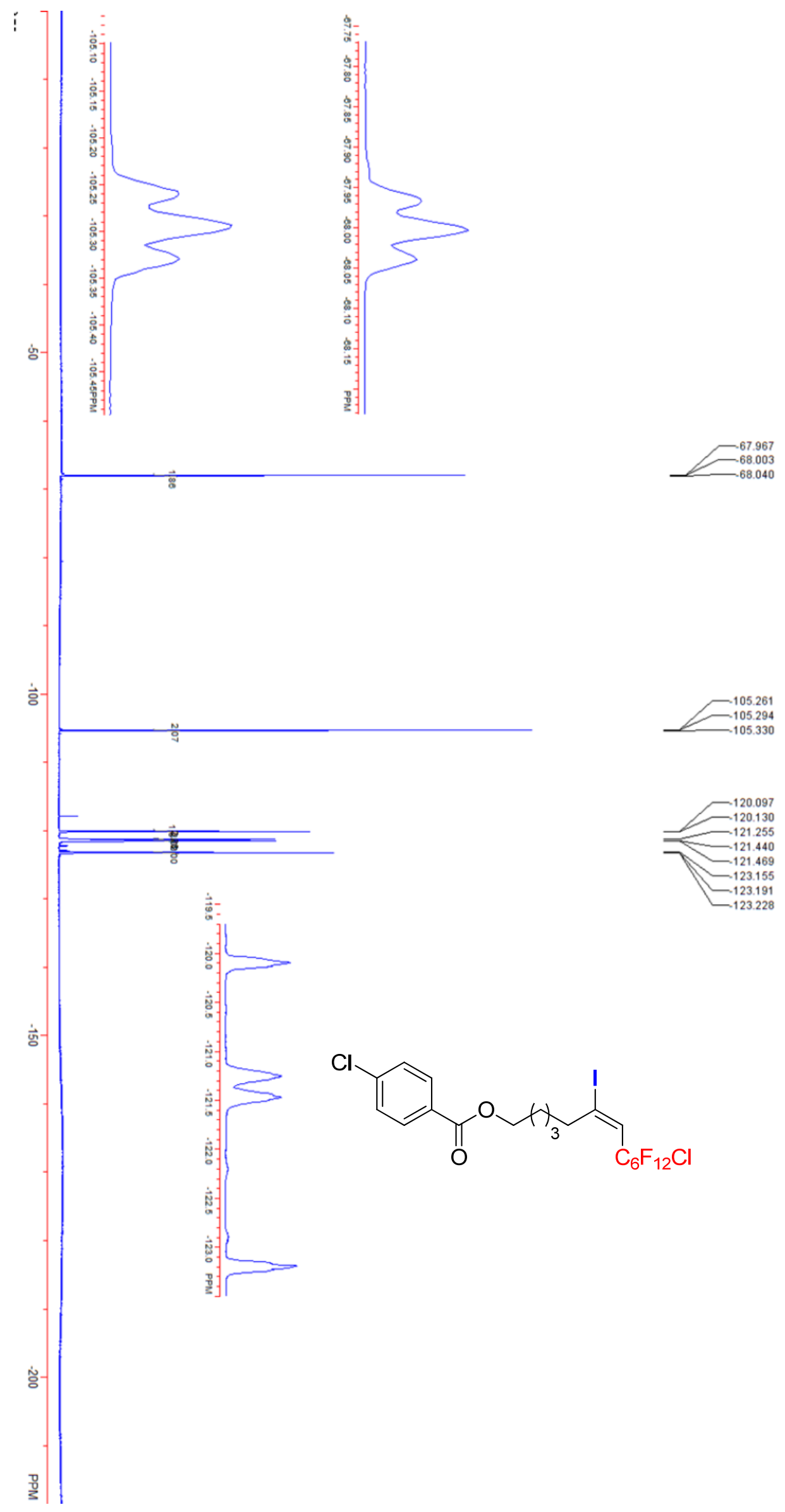




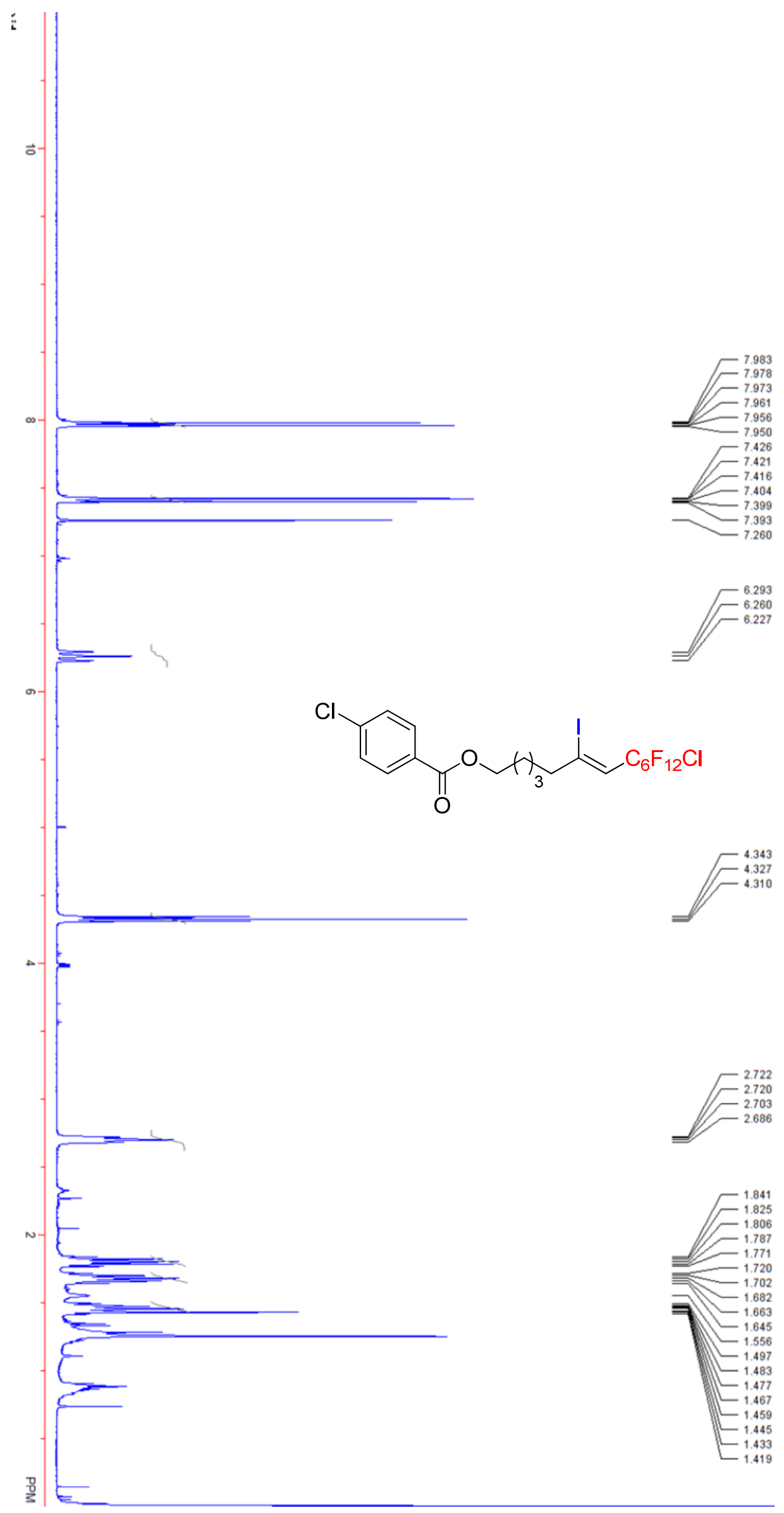



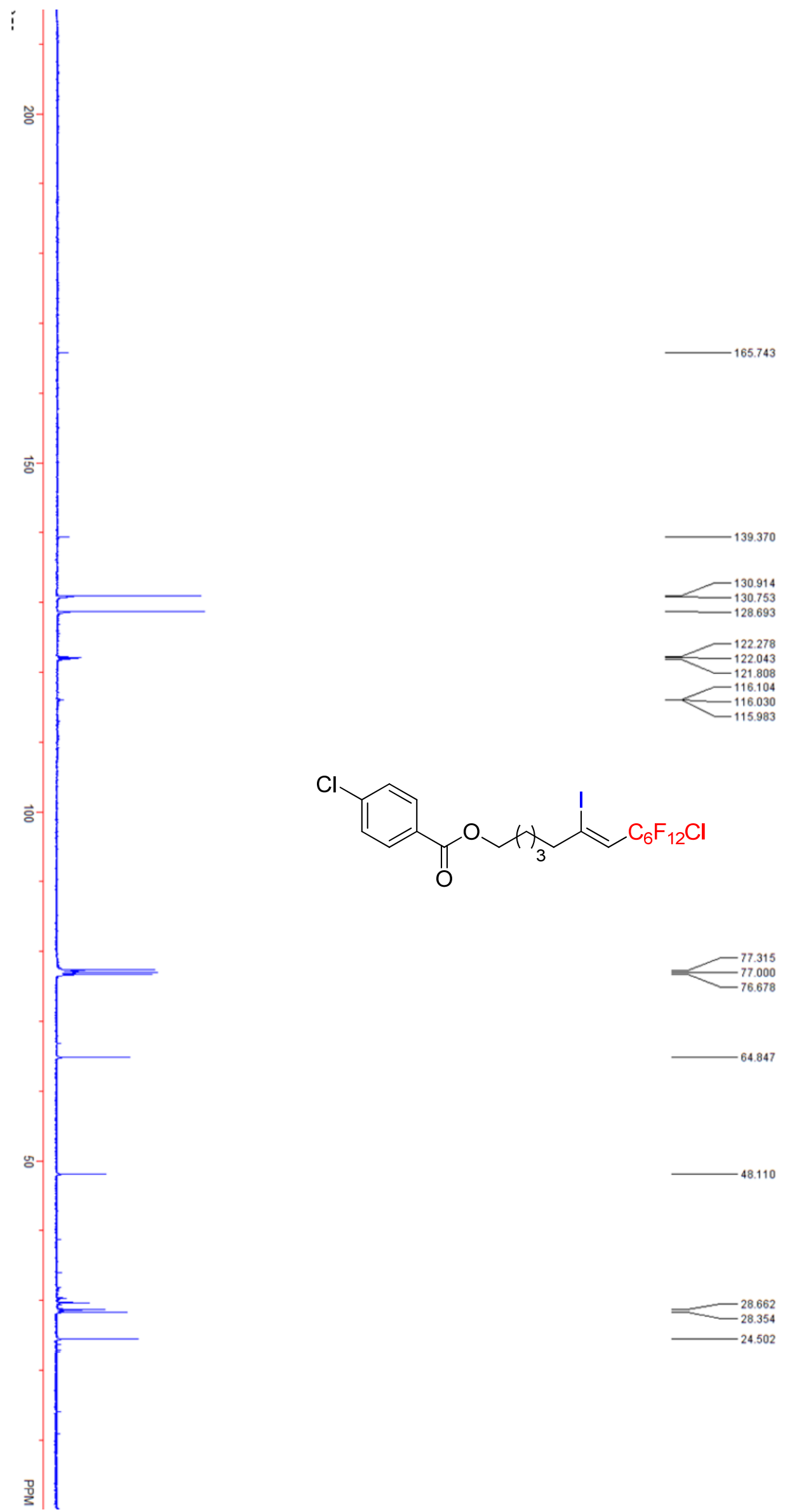<smiles>O=C(OCCCC(I)=C[SeH2+]Cl)c1ccc(Cl)cc1</smiles>

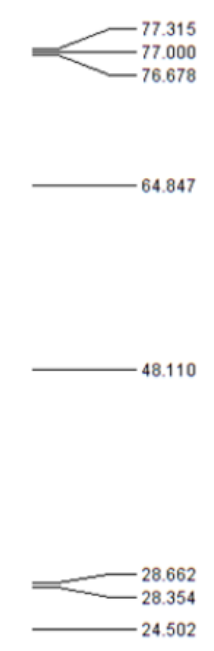




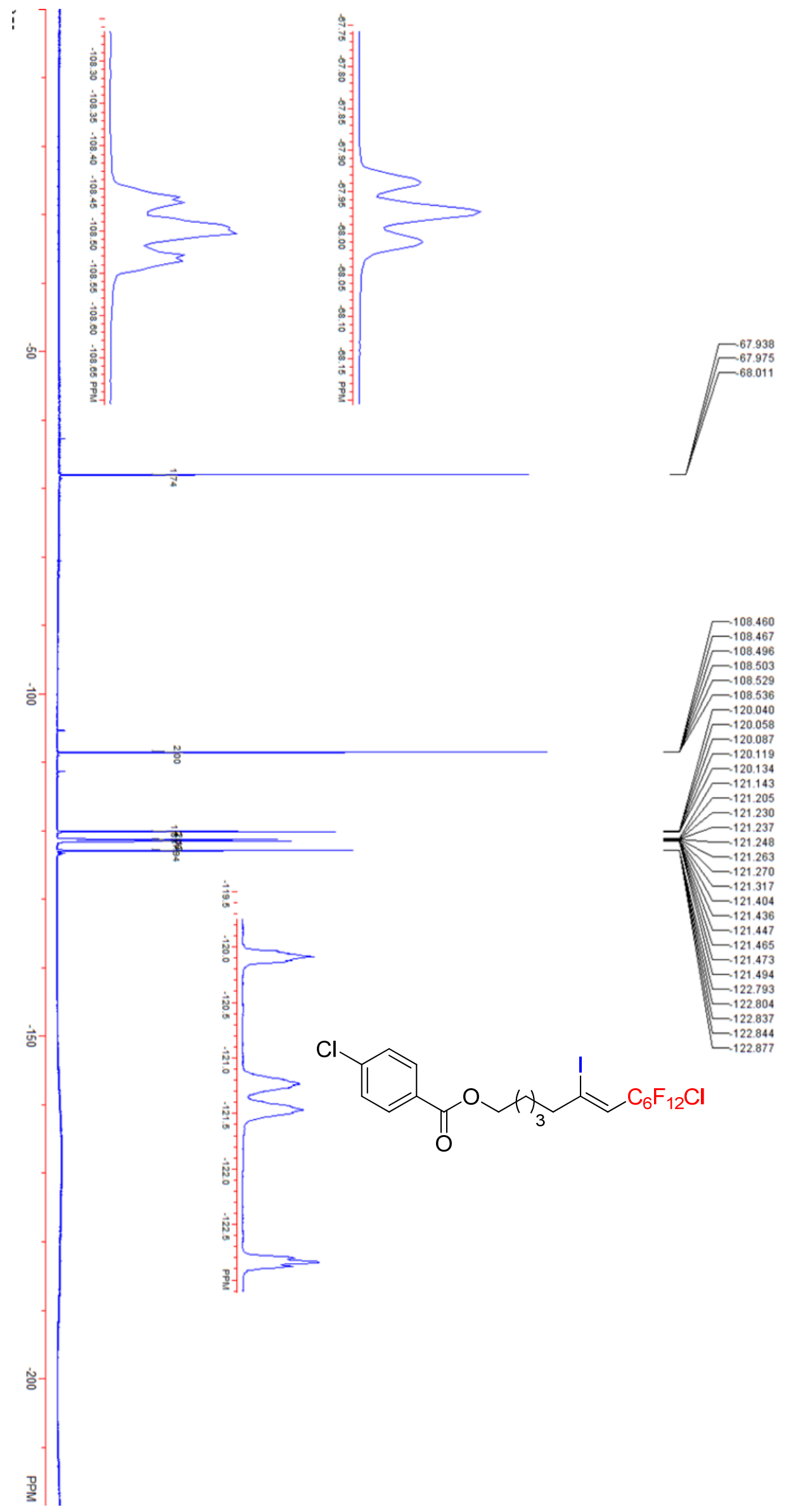



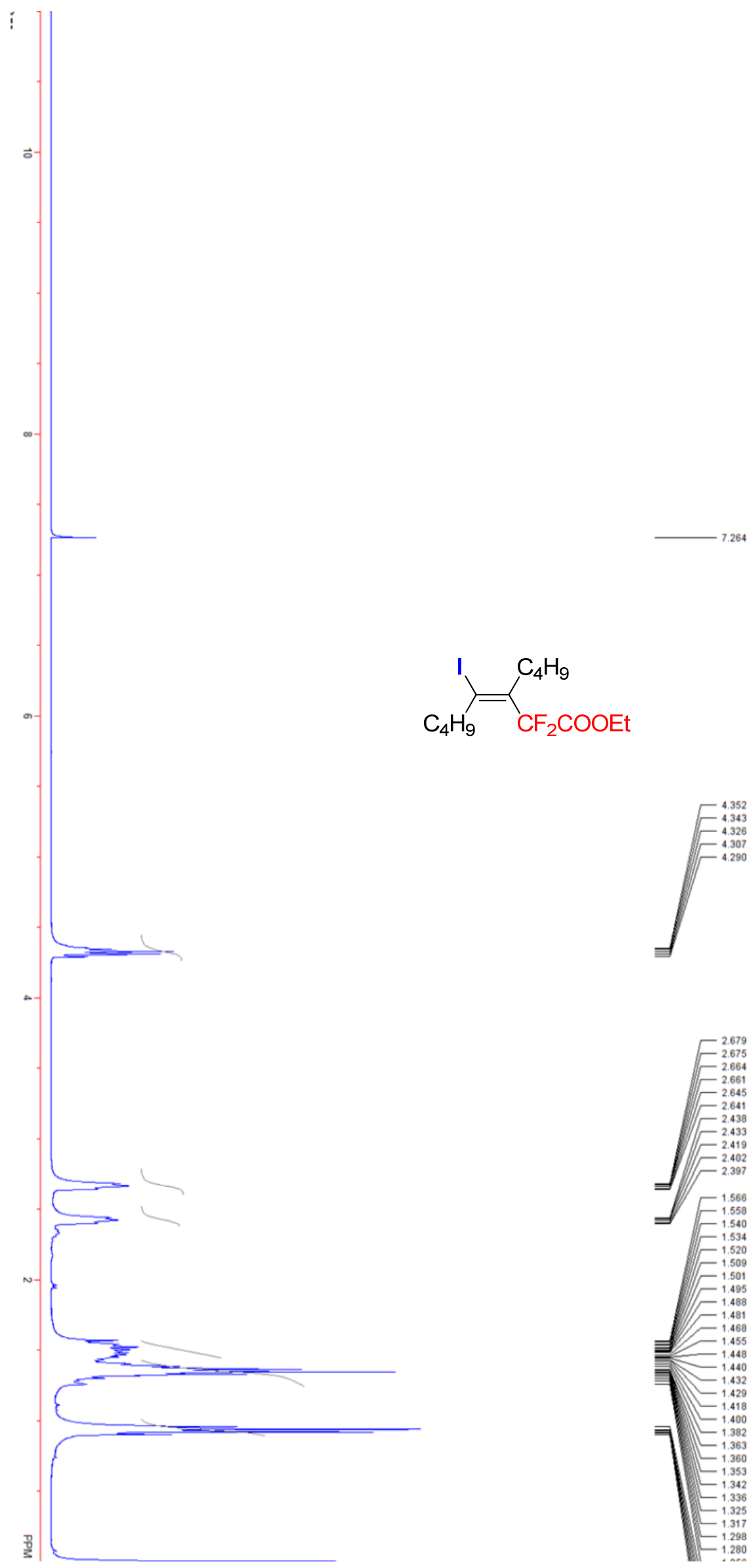

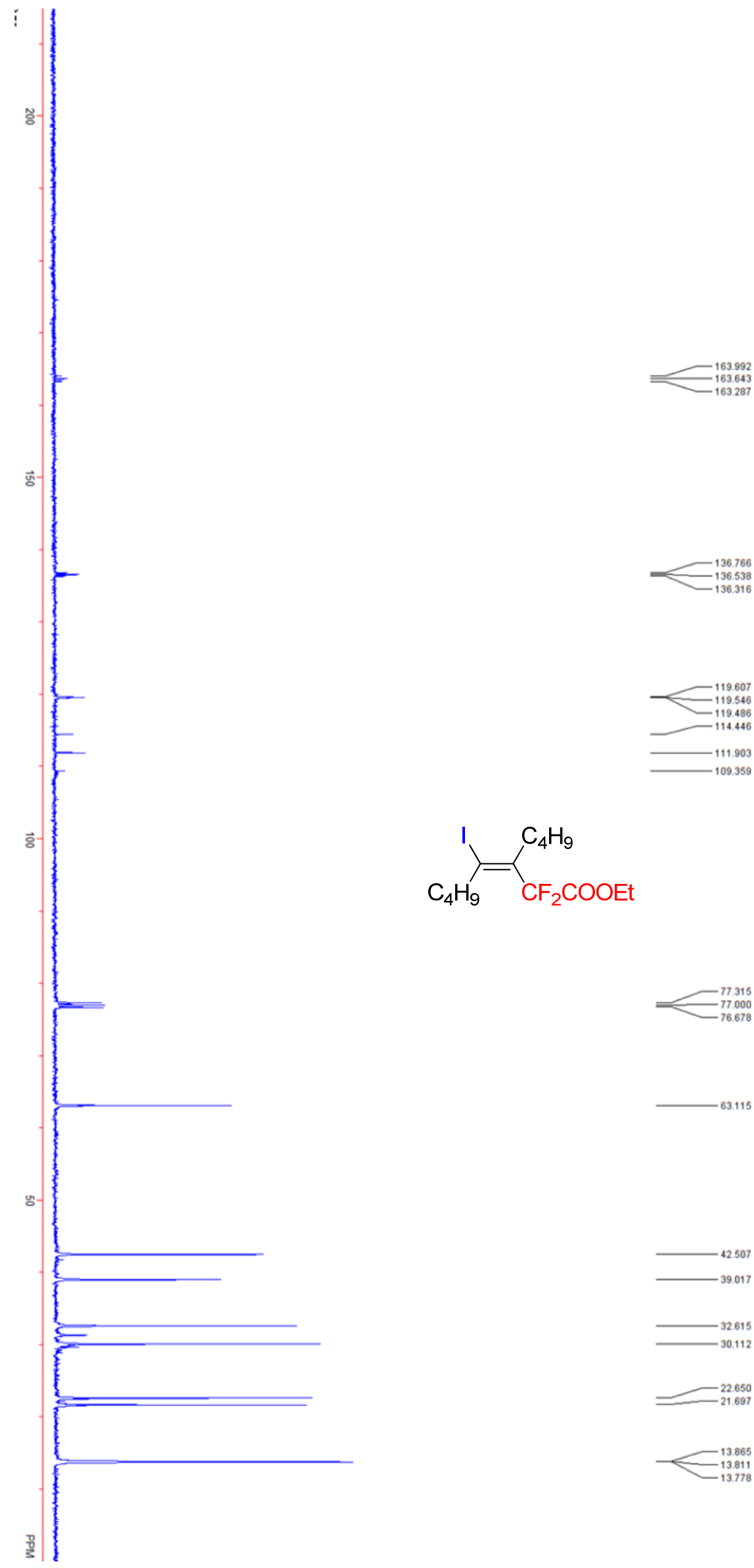


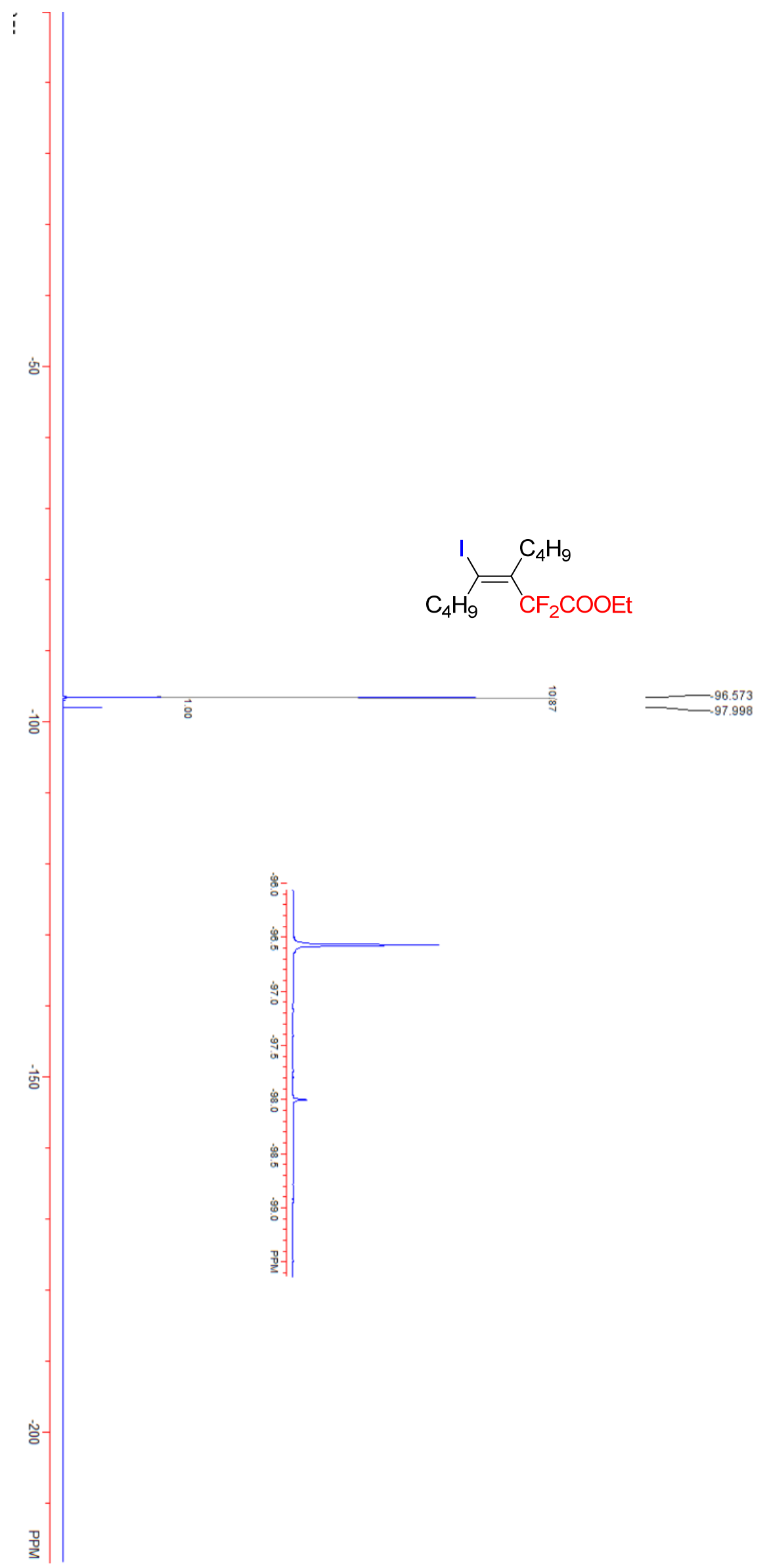




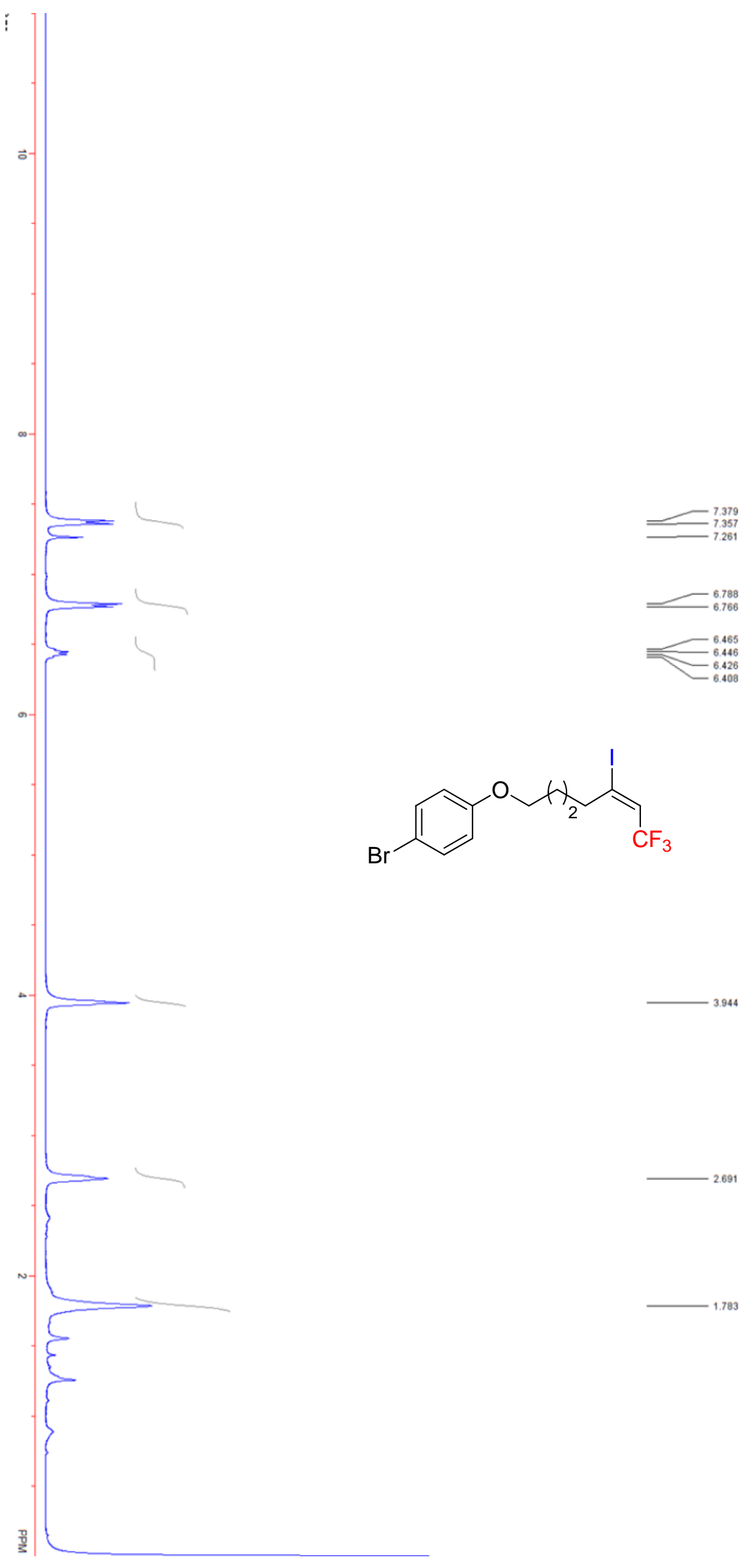



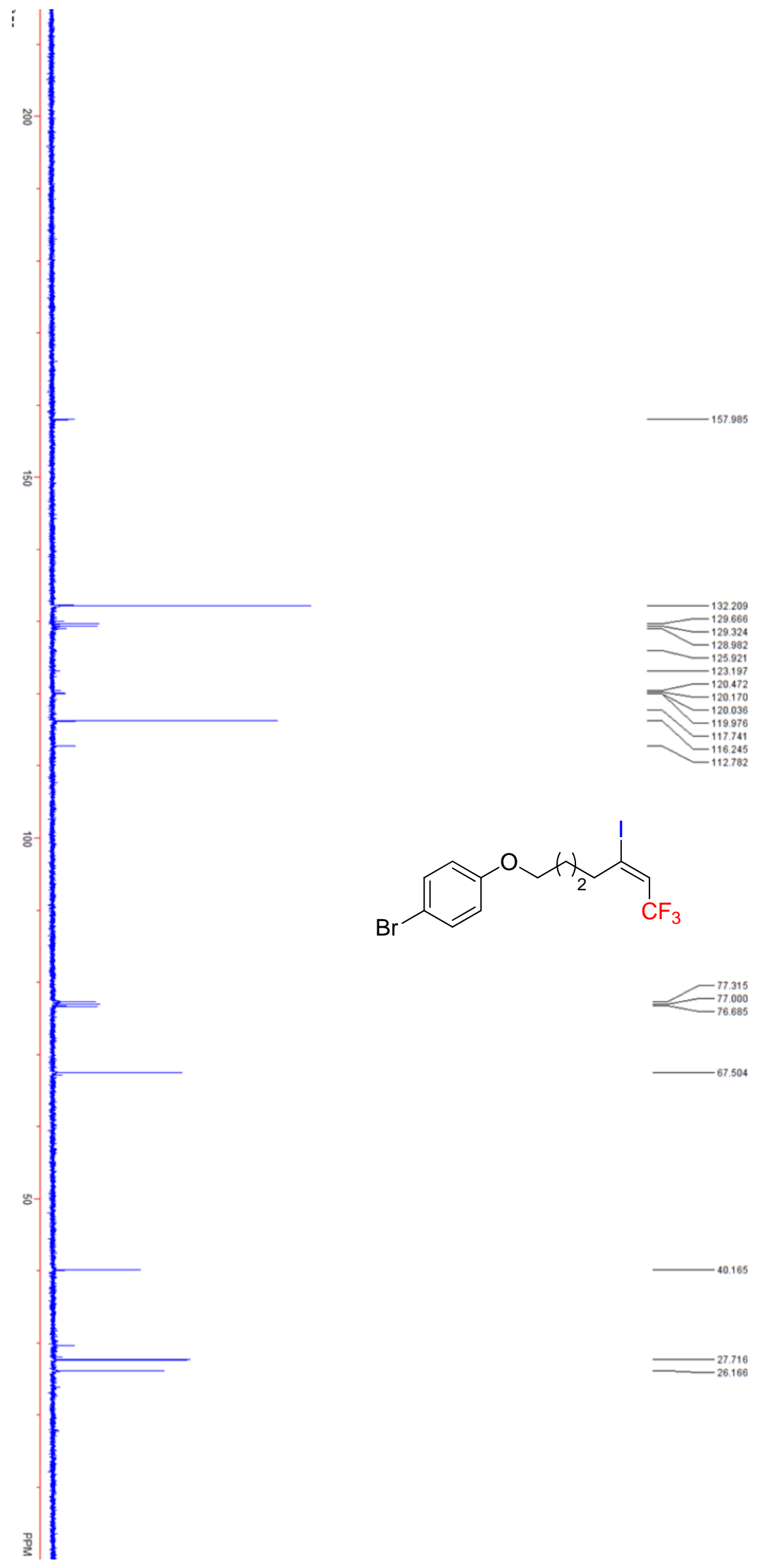


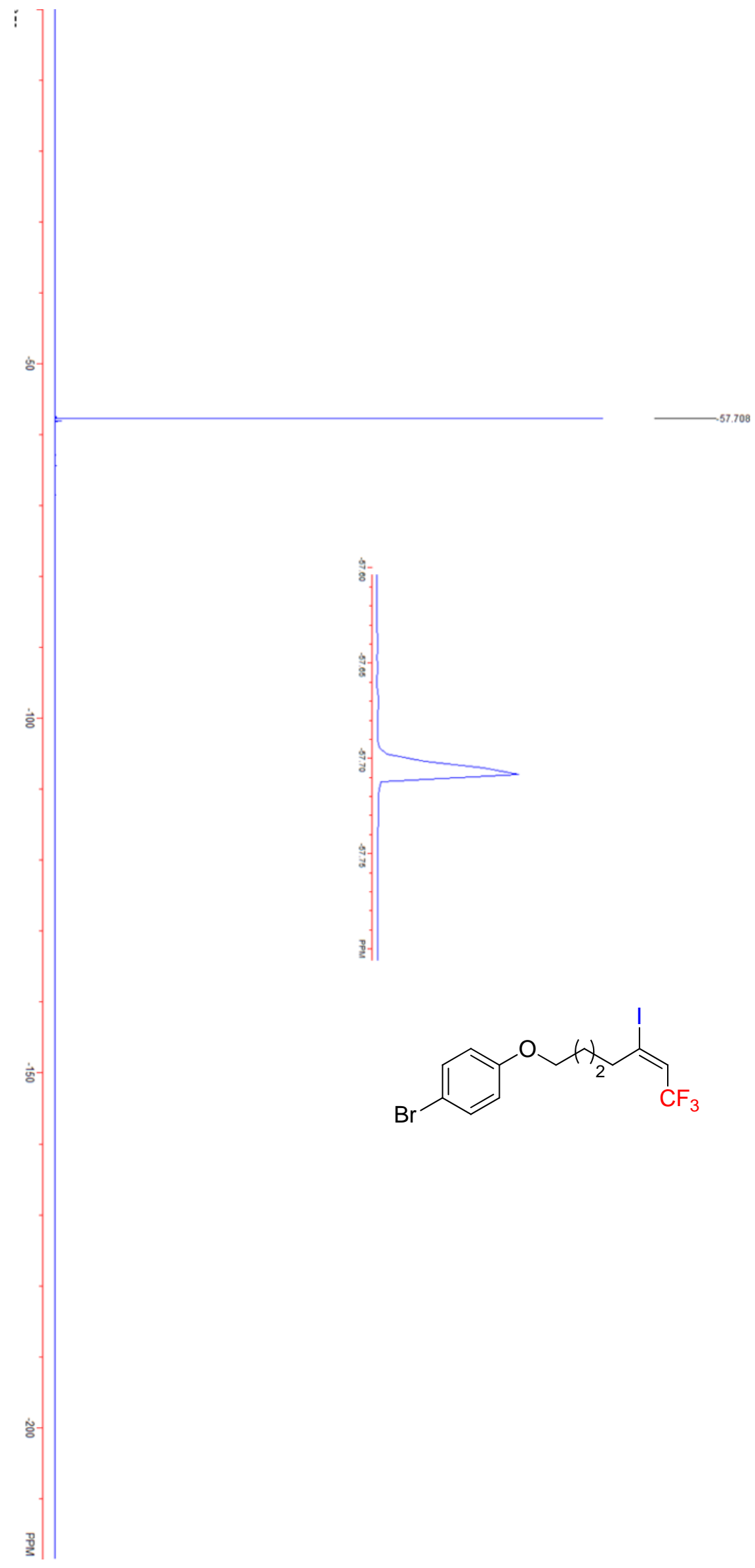




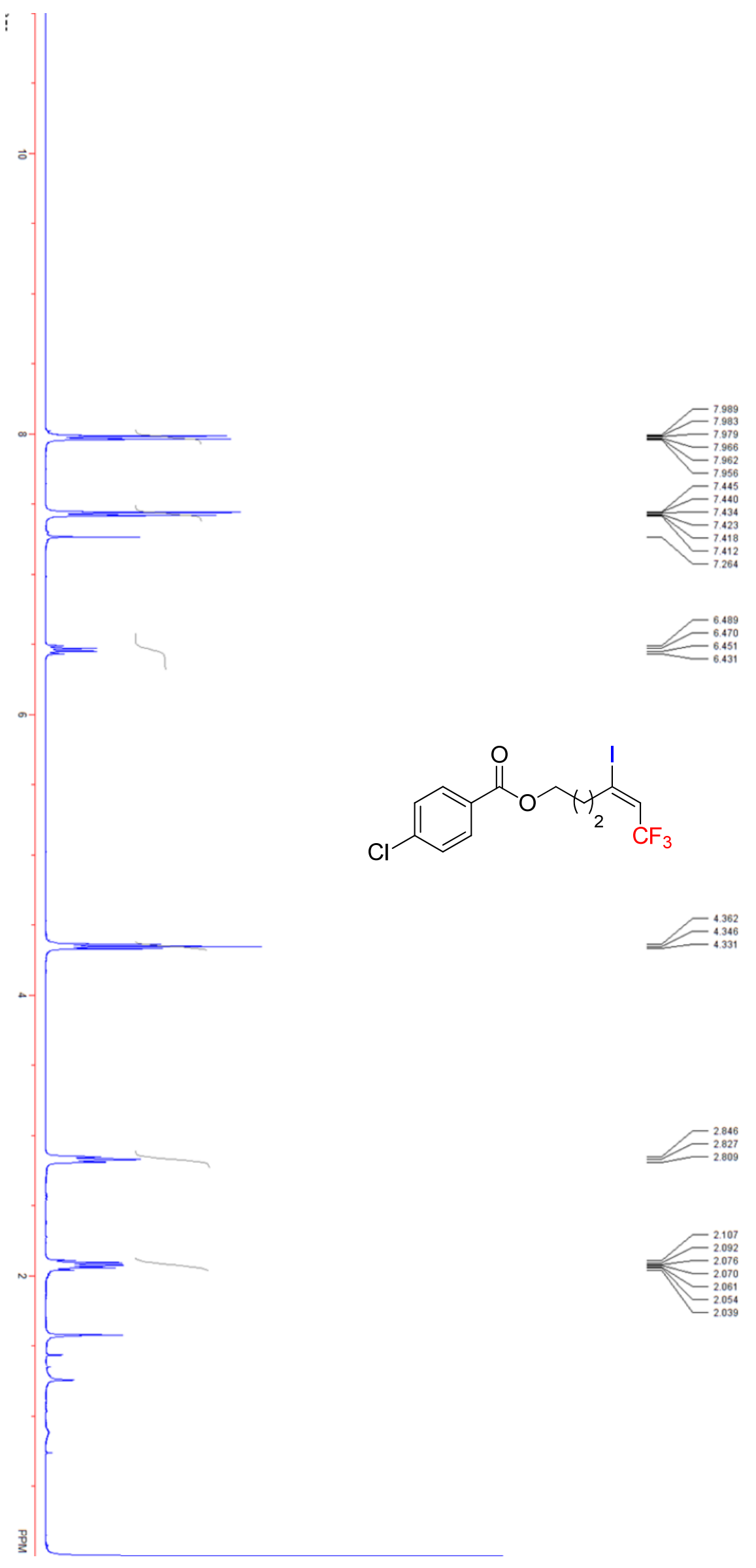




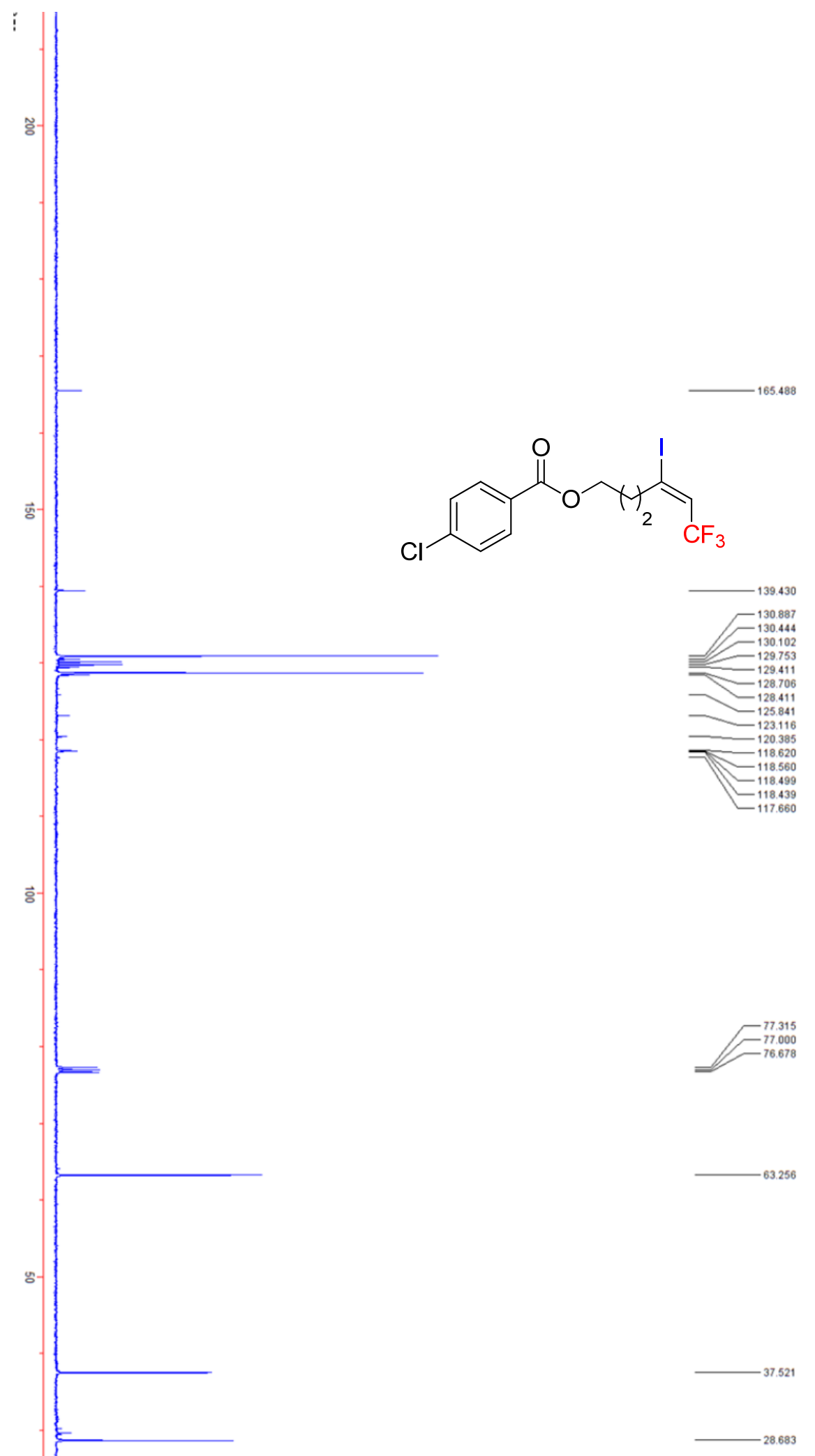




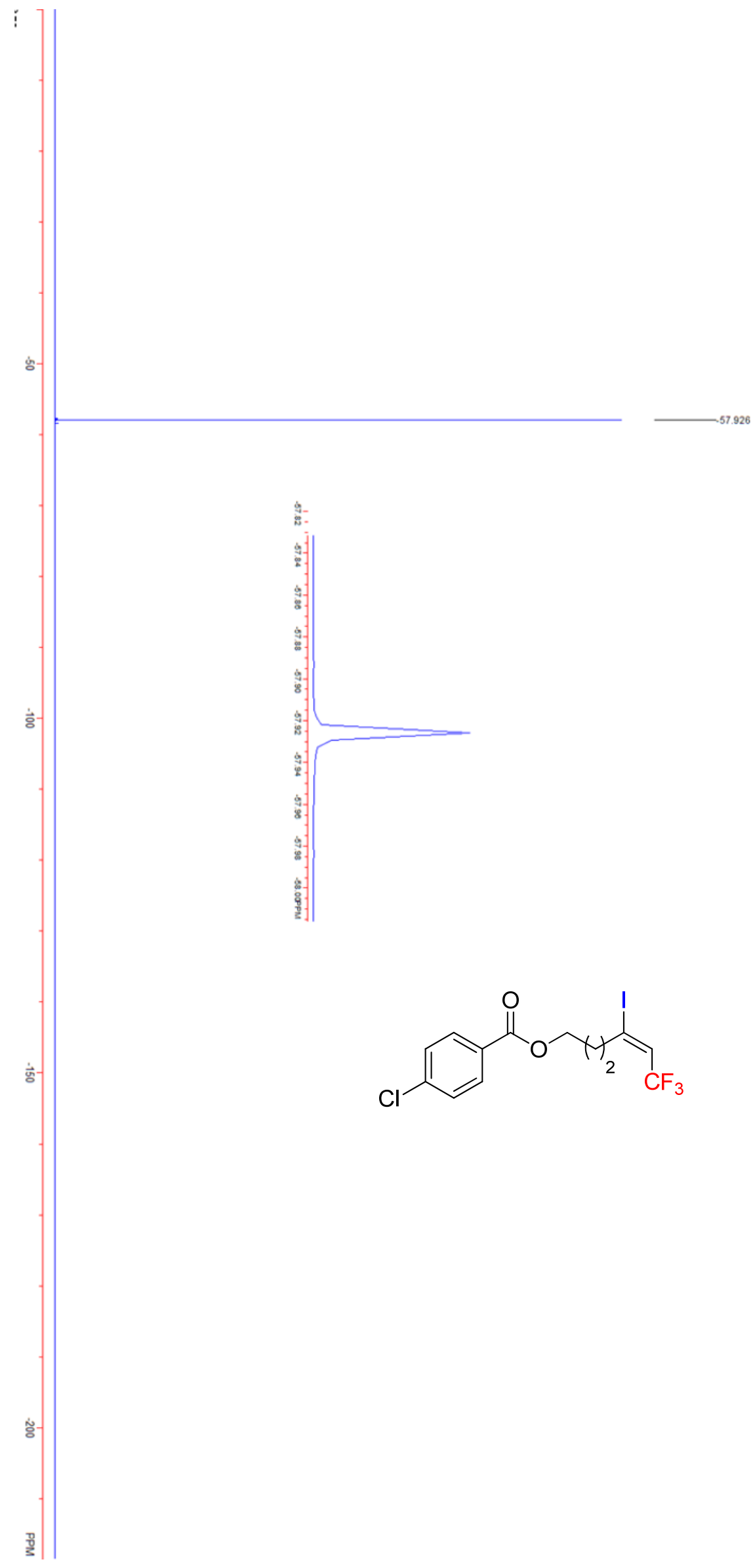



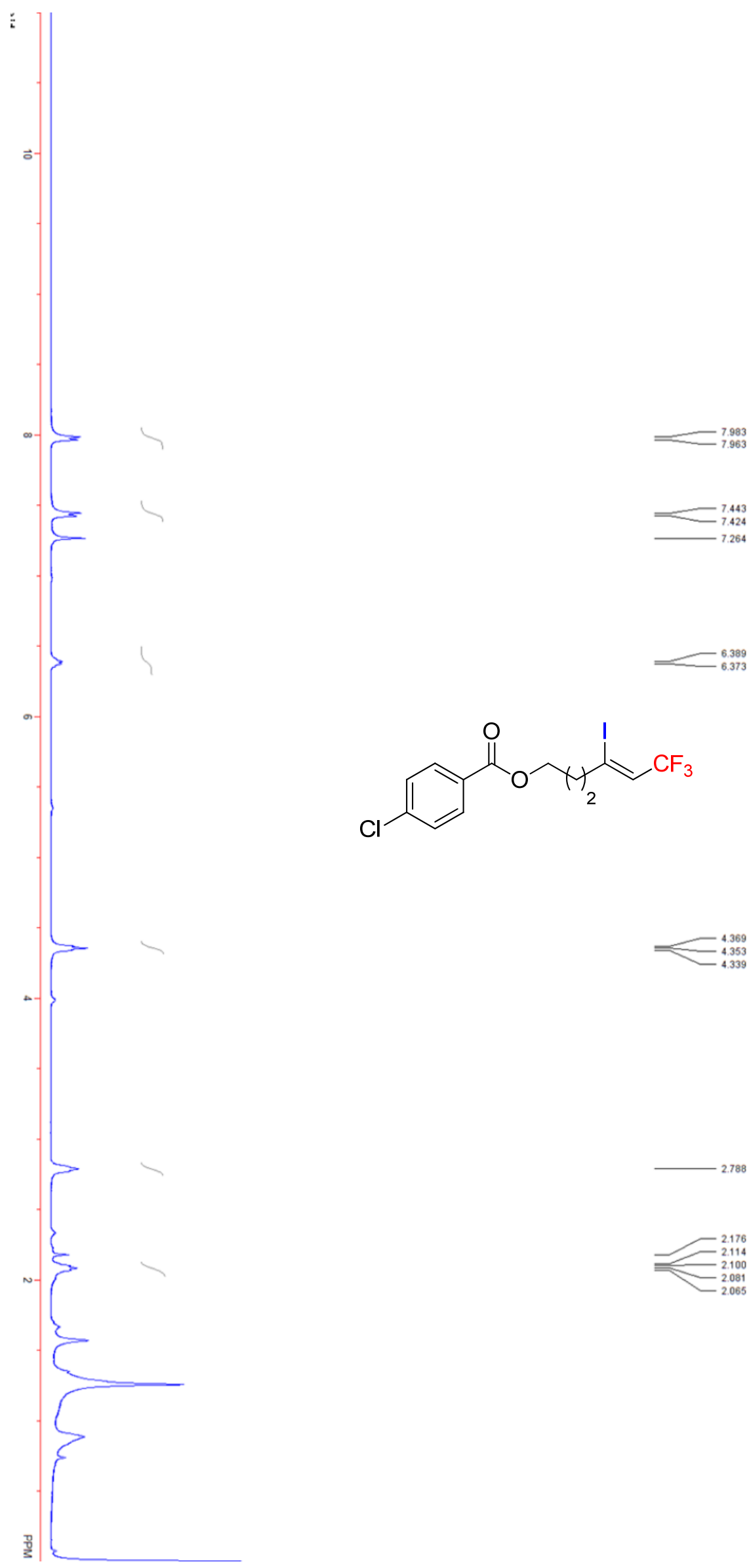<smiles>O=C(OCCNC(I)=CC(F)(F)F)c1ccc(Cl)cc1</smiles>
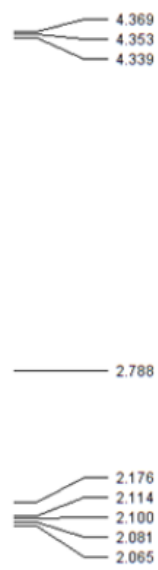

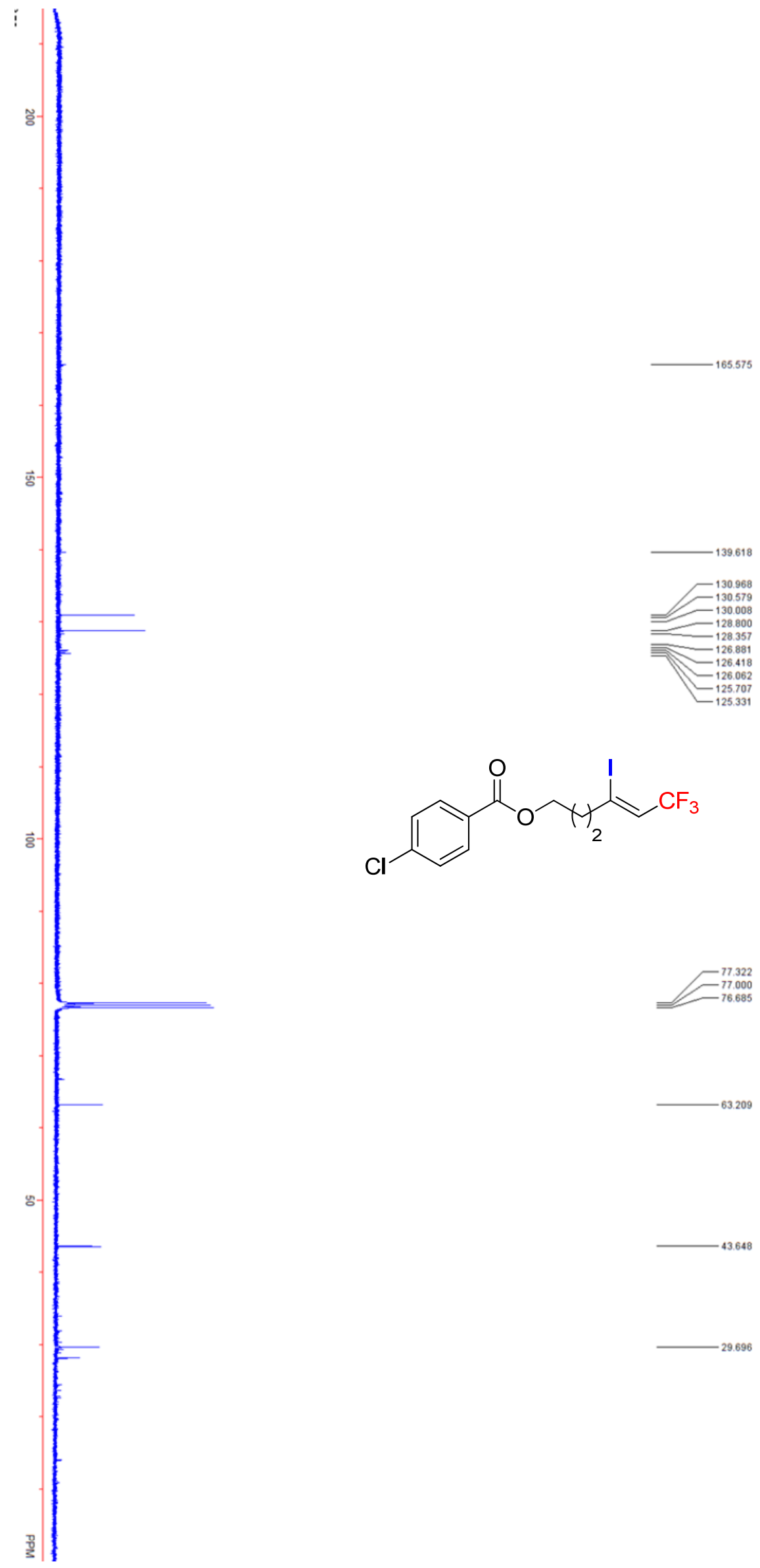<smiles>O=C(OCCNC(I)=CC(F)(F)F)c1ccc(Cl)cc1</smiles>
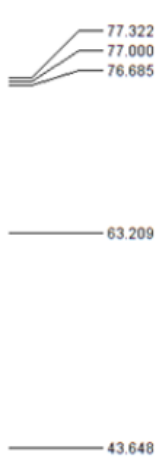


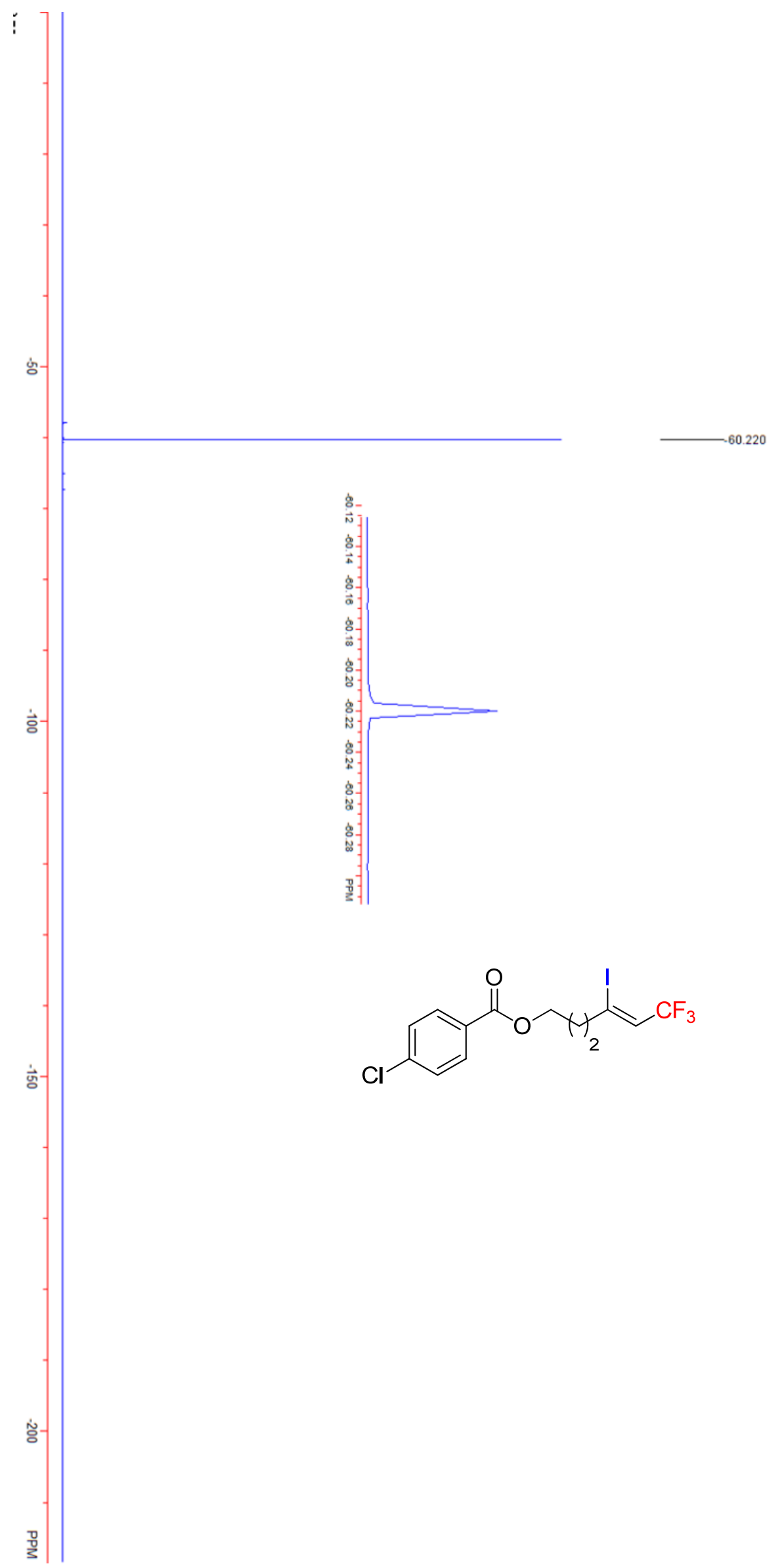




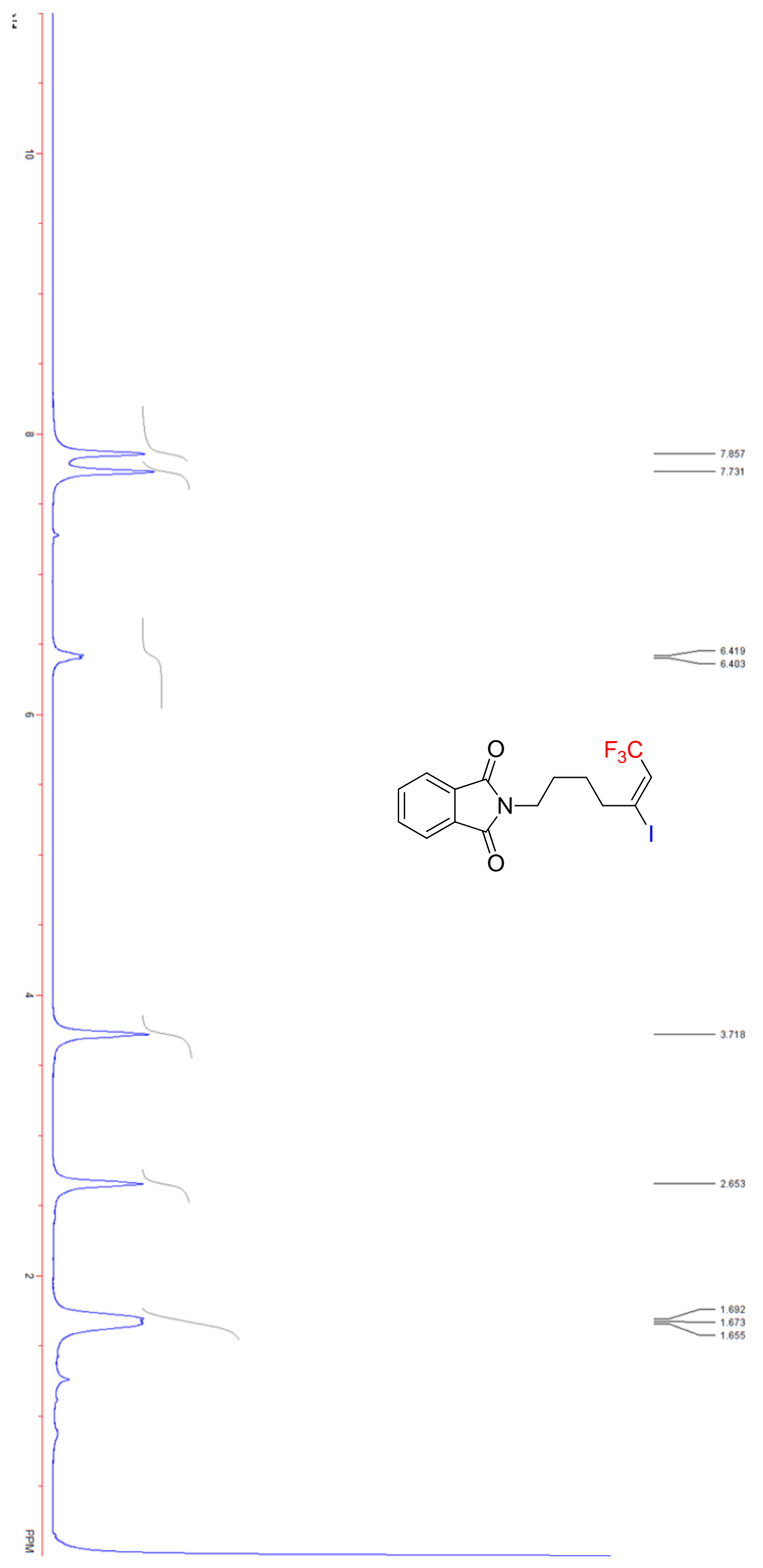




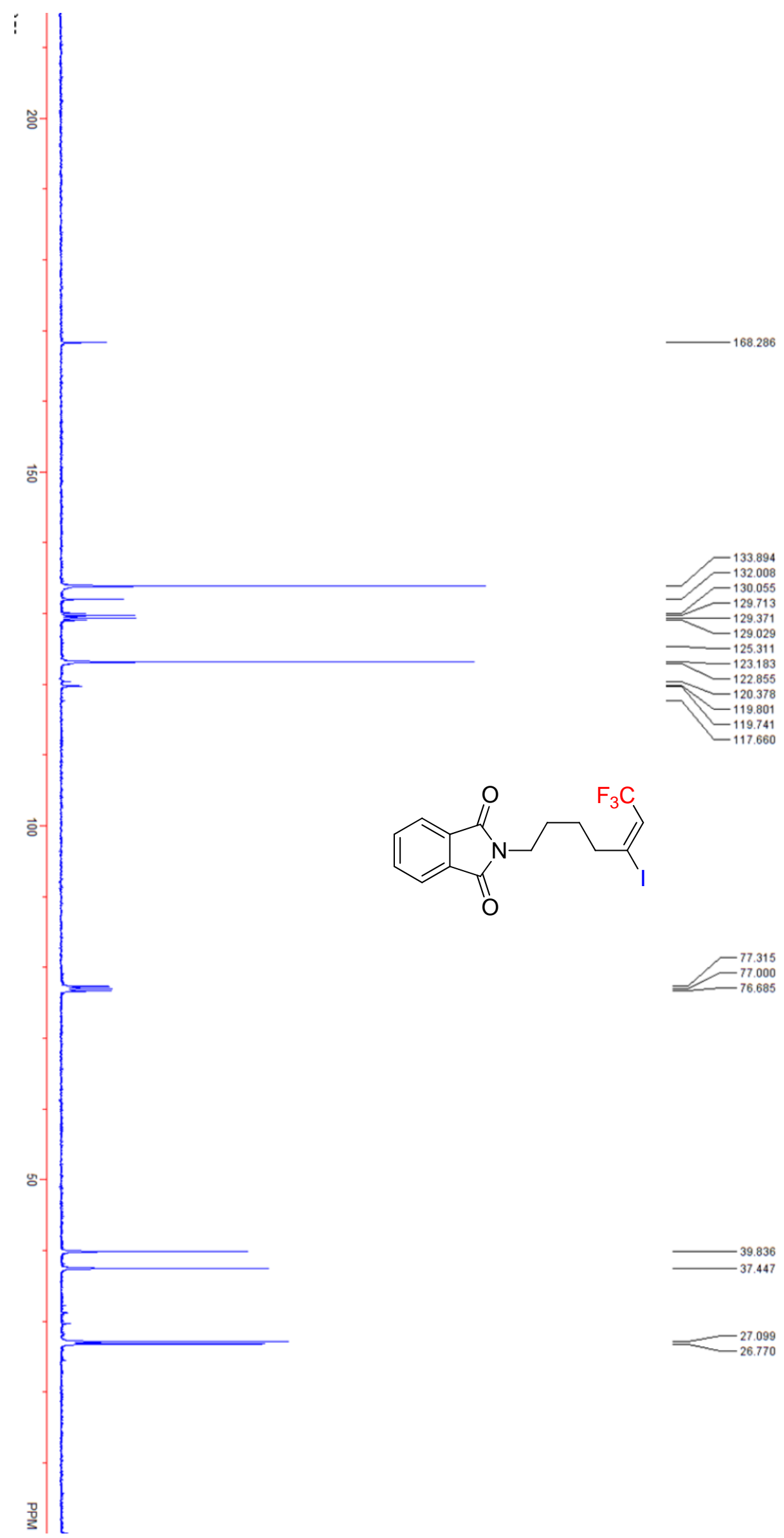




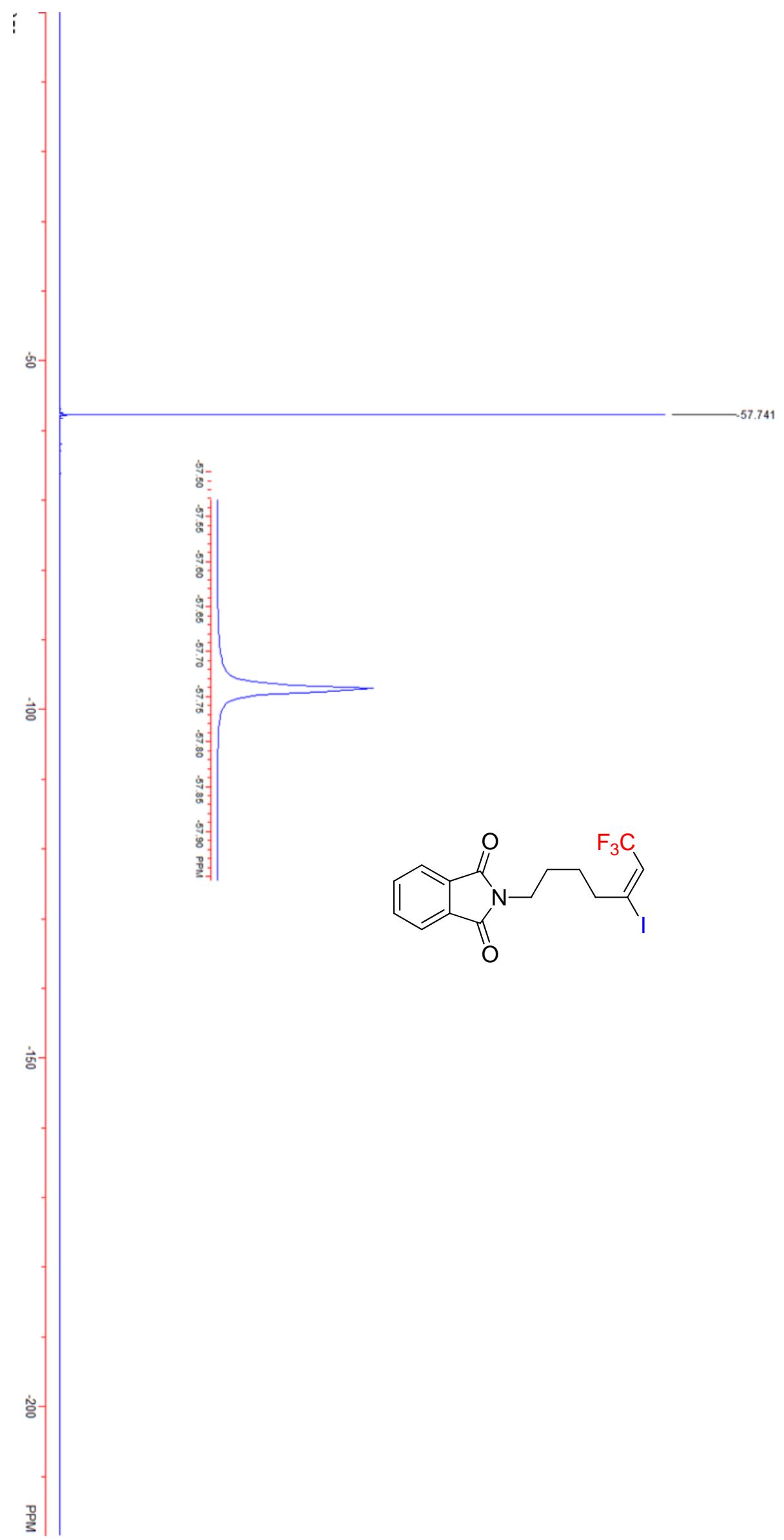




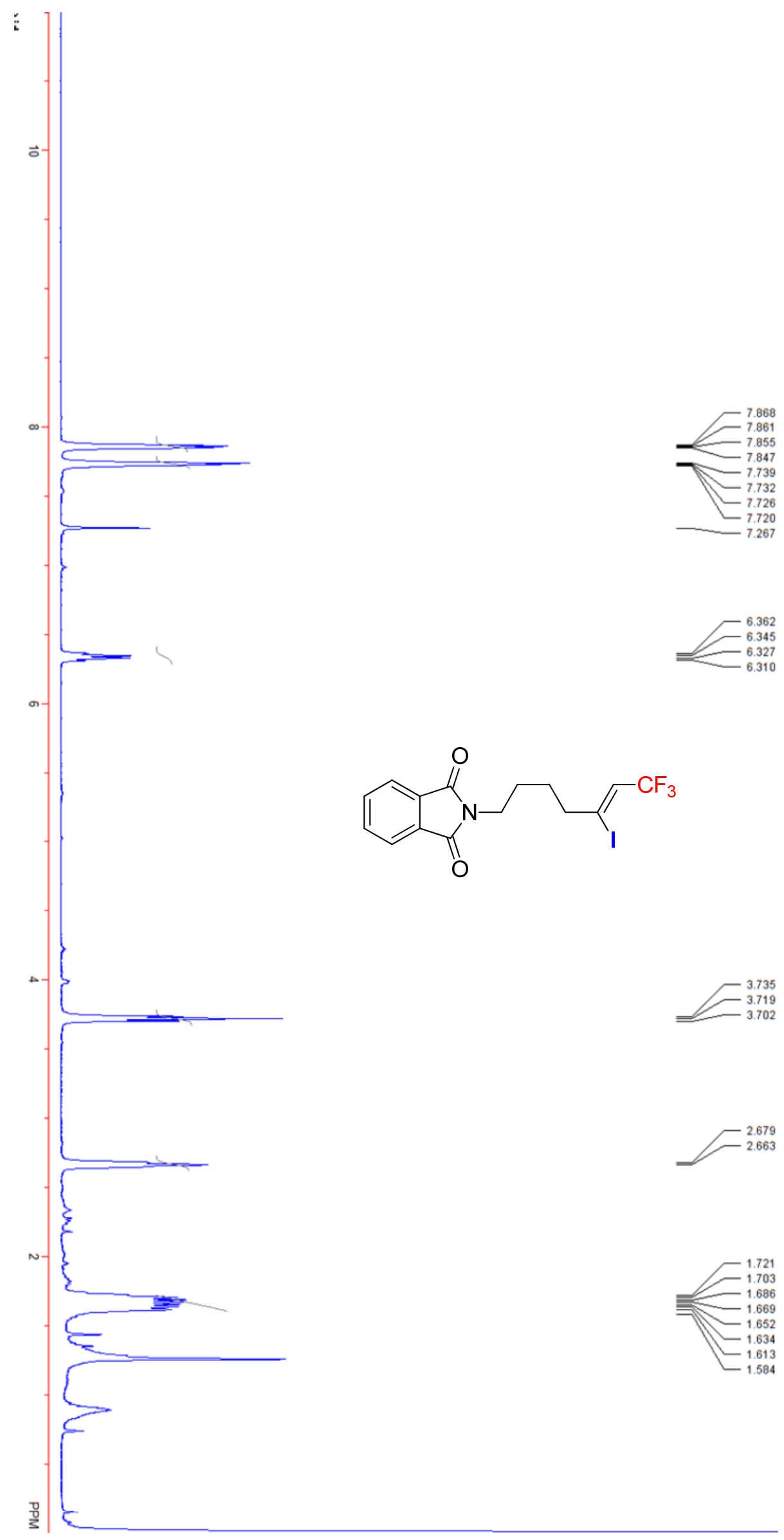




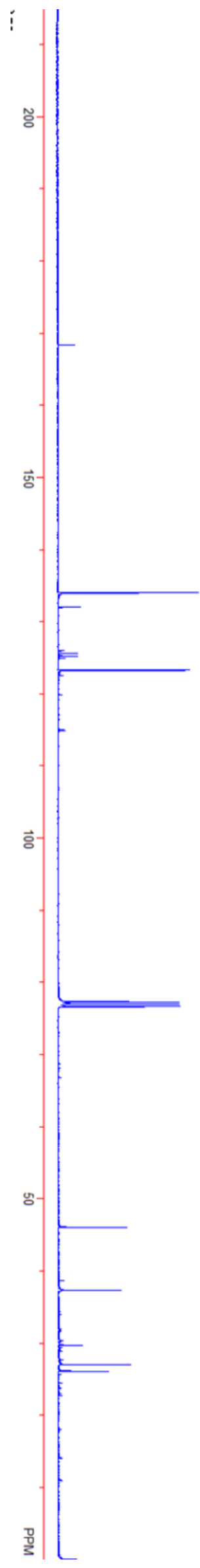




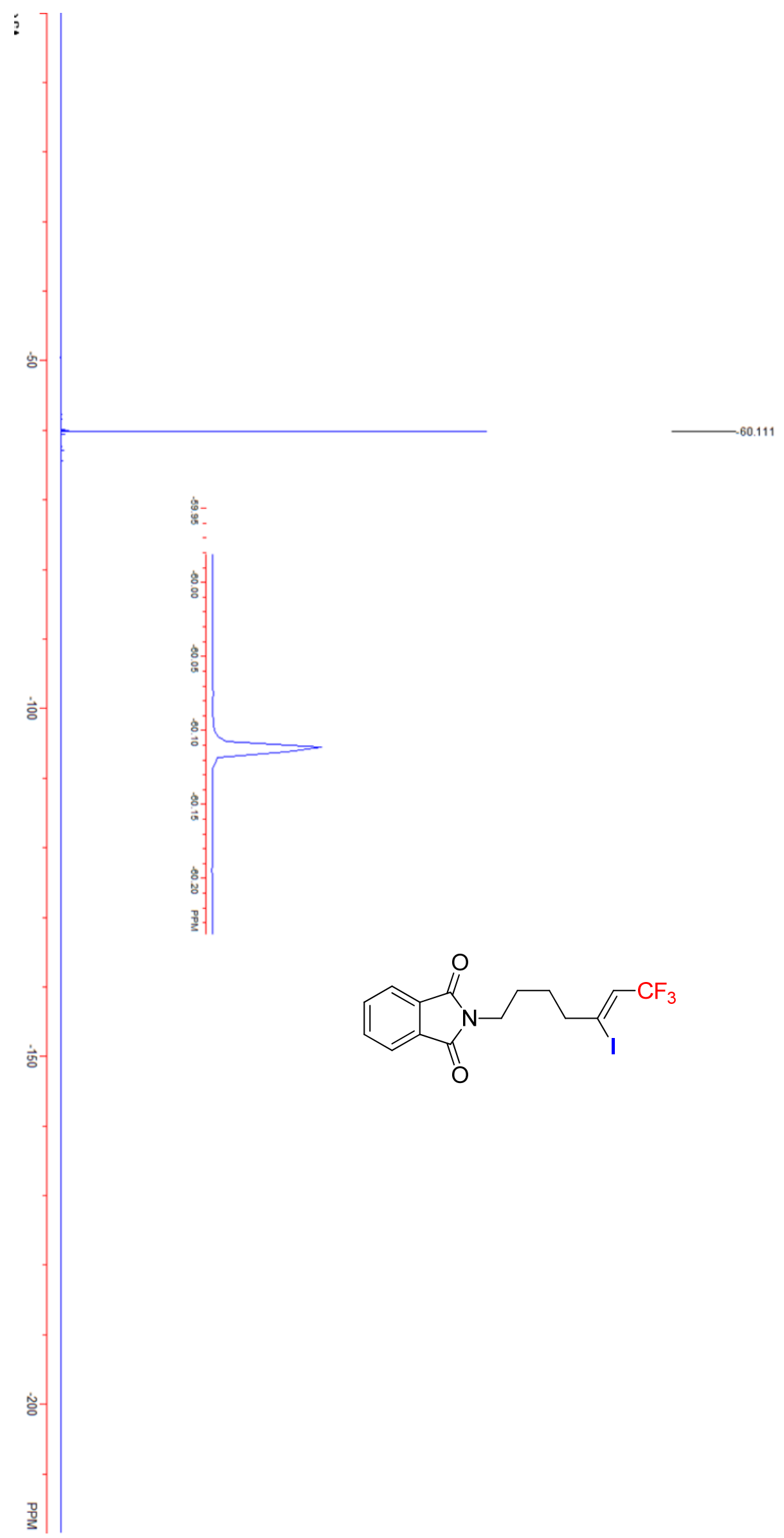




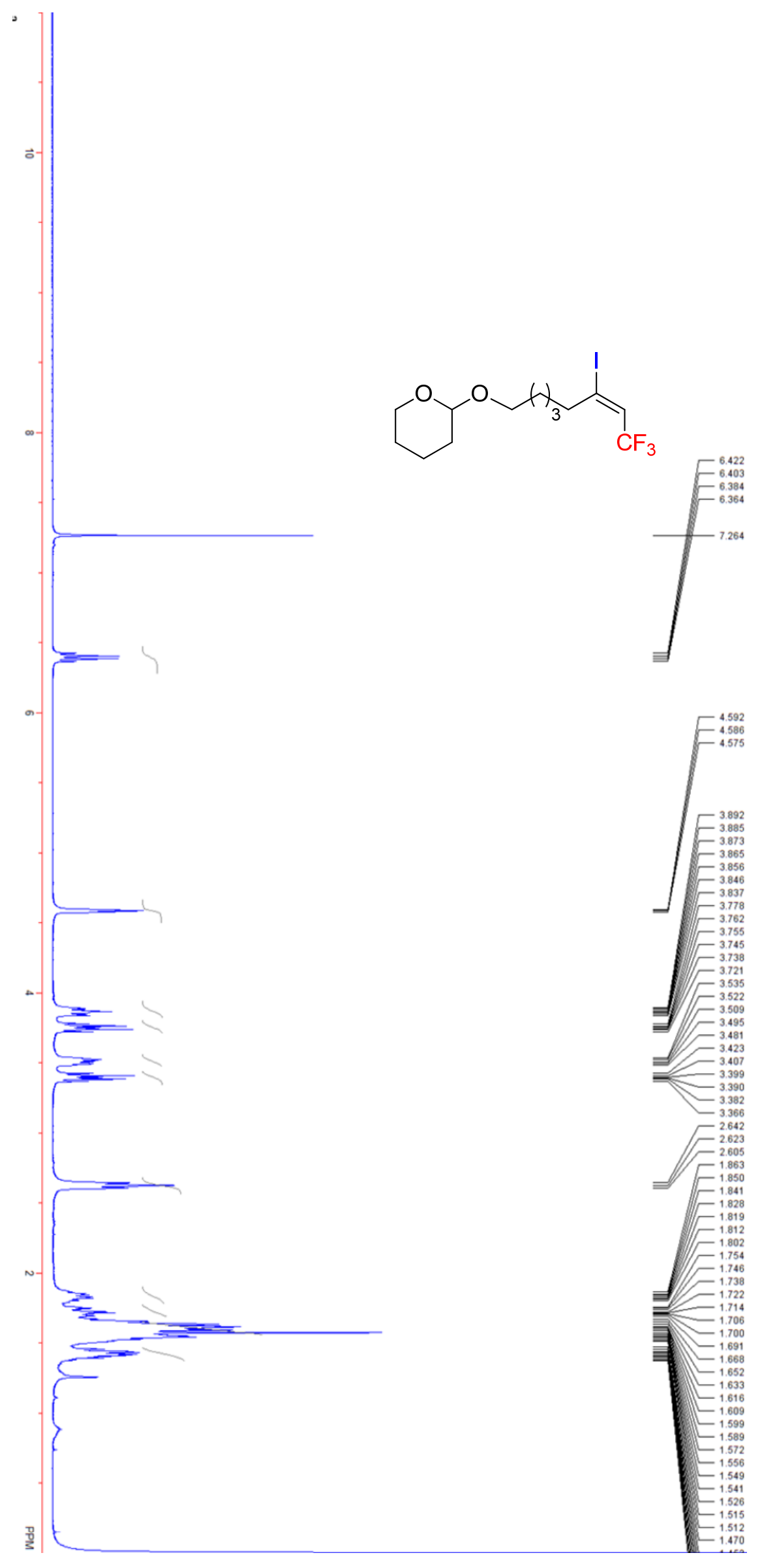



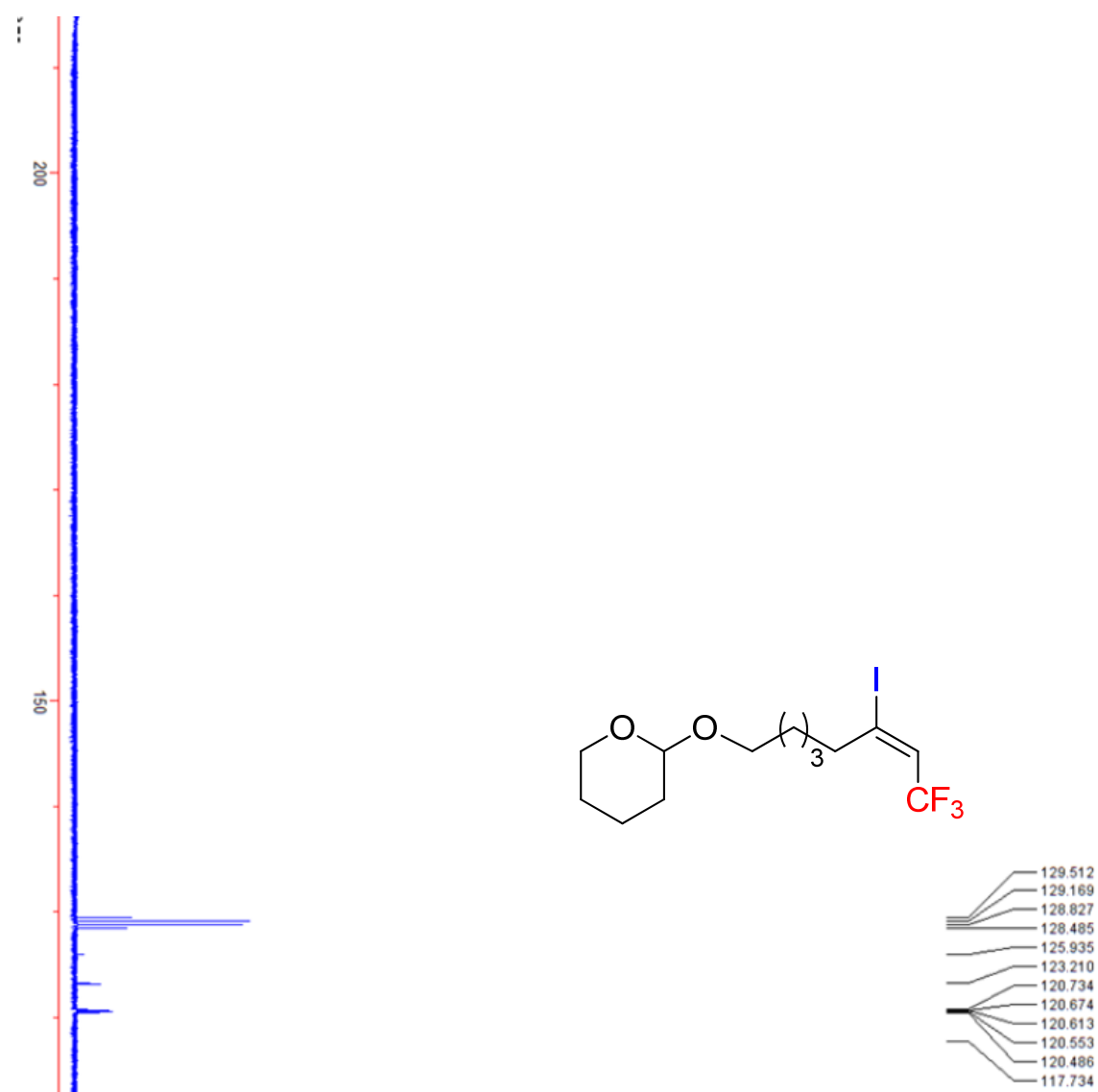

형
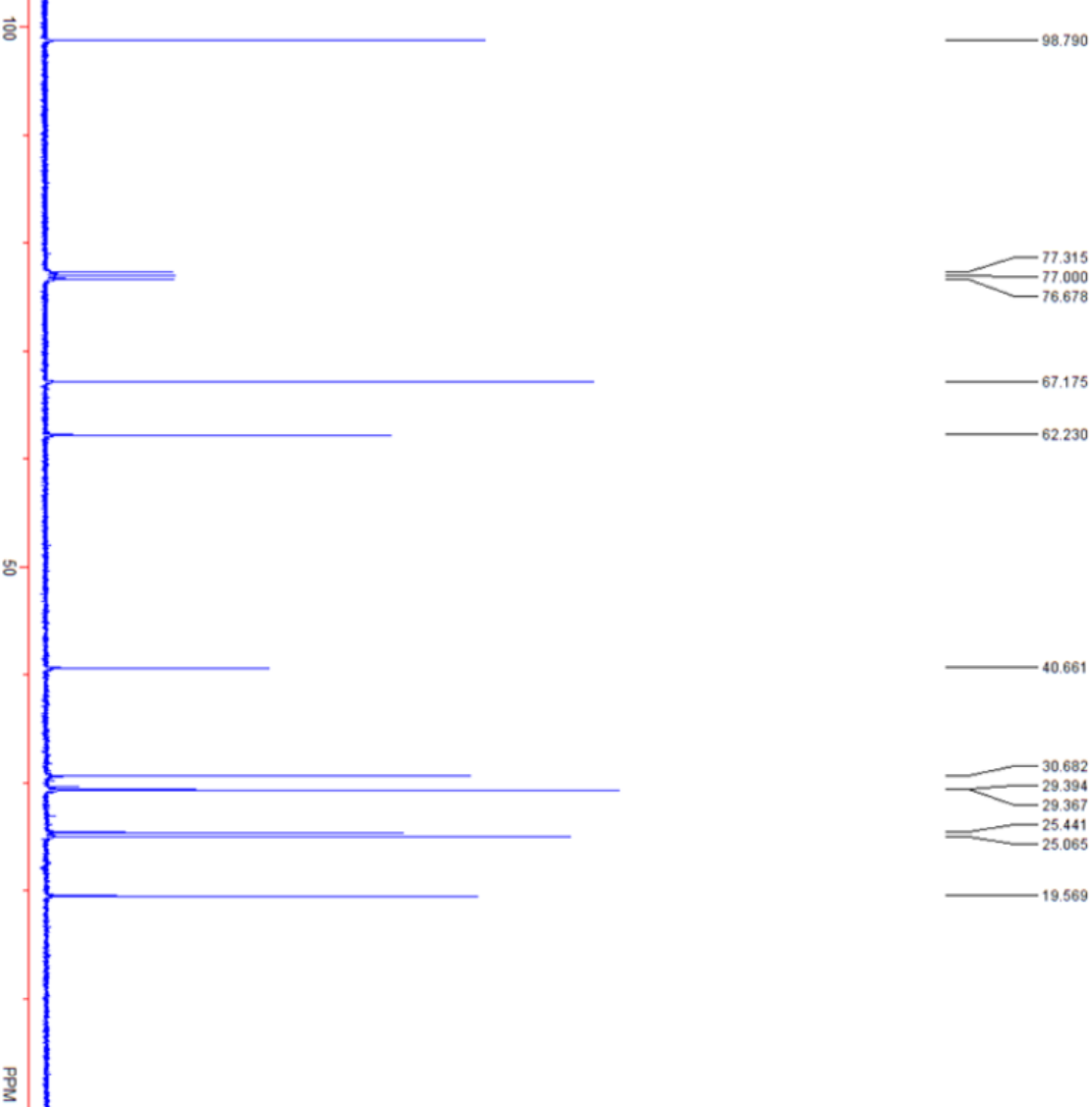


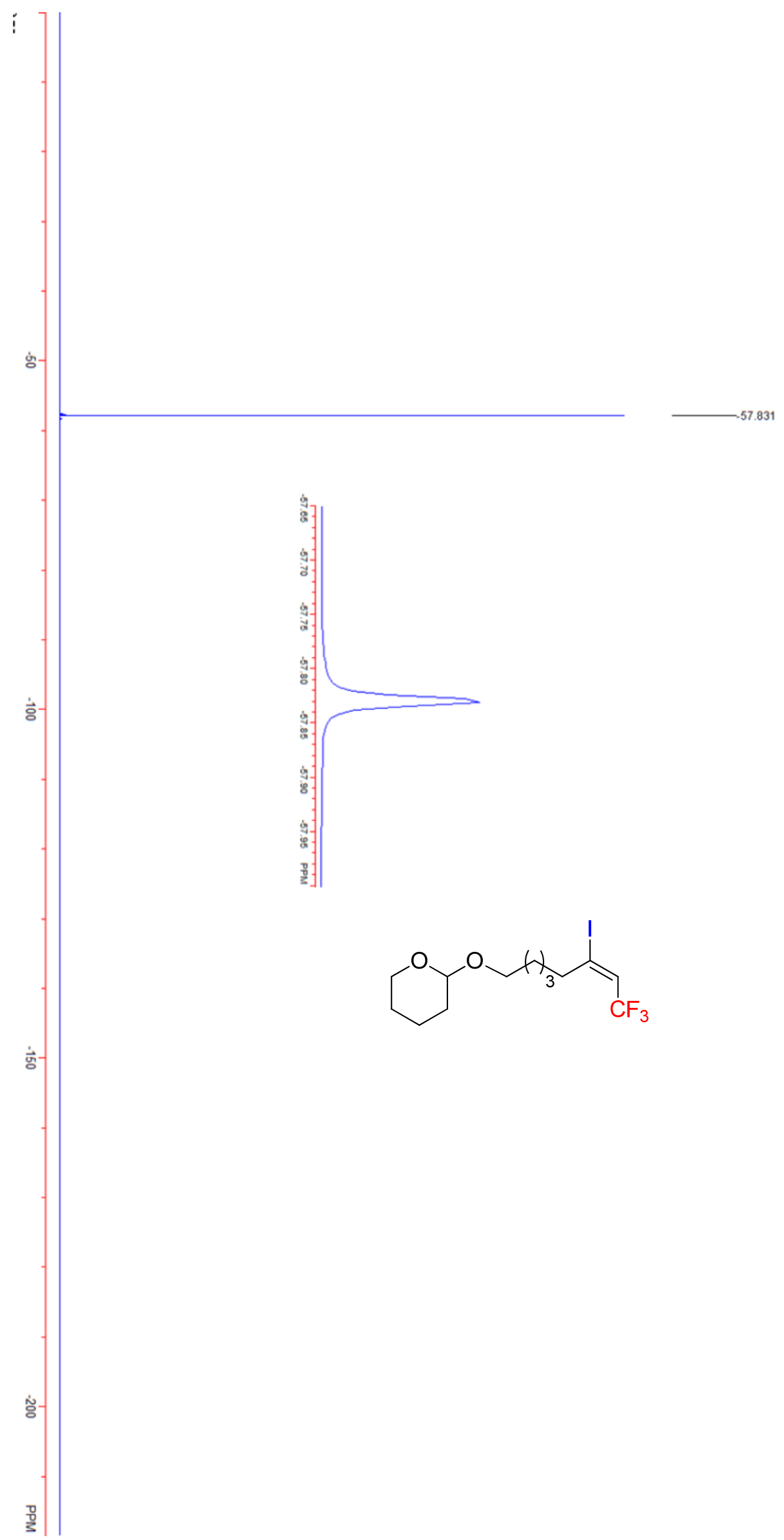




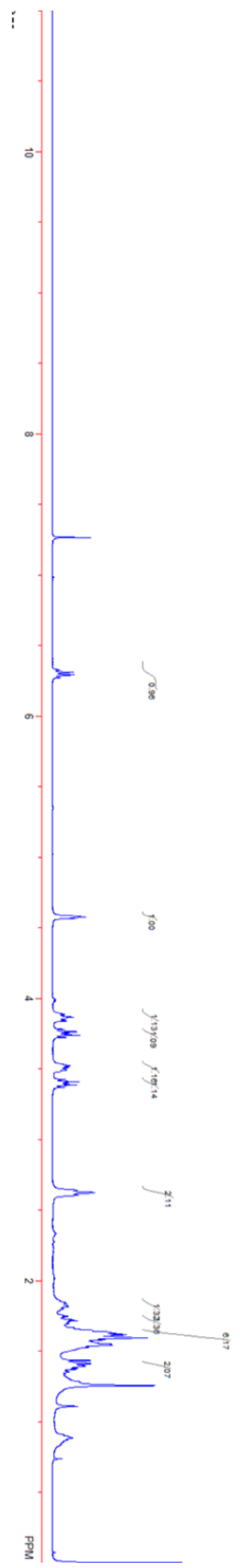

$\mathrm{O}^{\mathrm{O}} \sim \mathrm{H}_{3} \mathrm{CF}$
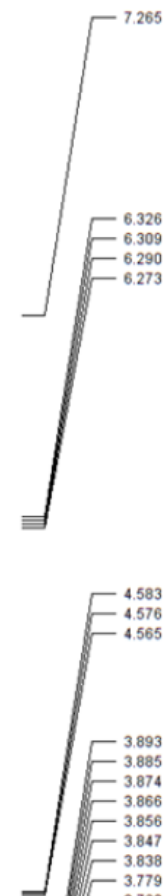

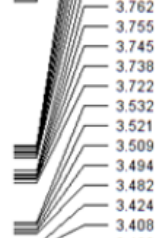

$\leftarrow_{3.368}^{3.392}$

$F_{2.640}^{2.640}$

$F^{1.657}$

$F^{1.892}$

$F_{1.814}^{1.822}$

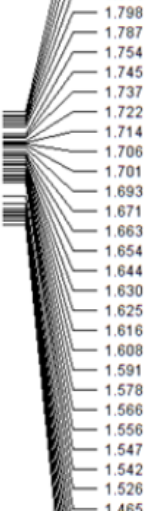



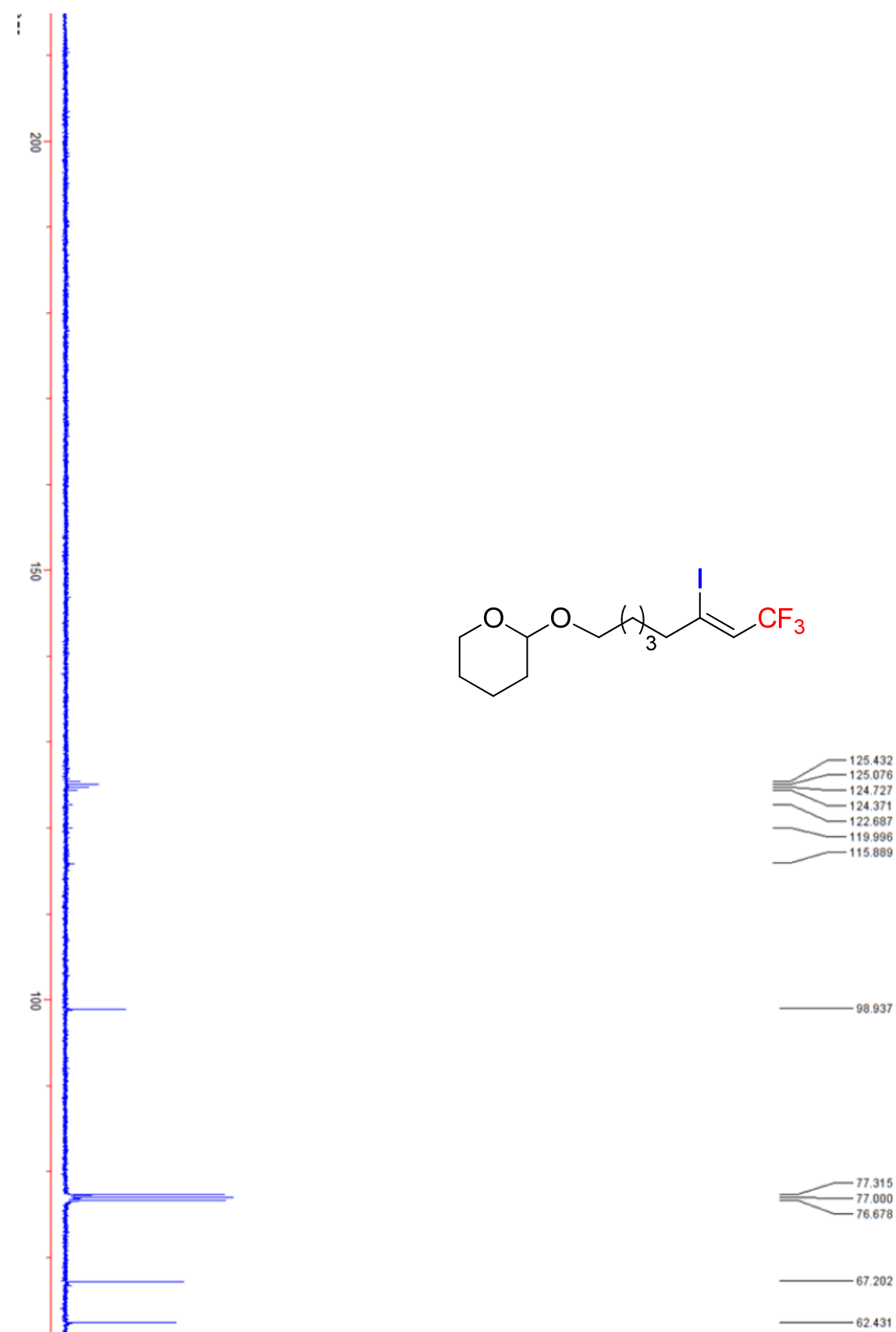

$8-$
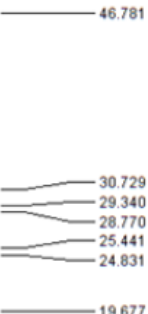

$\frac{2}{3}$ 


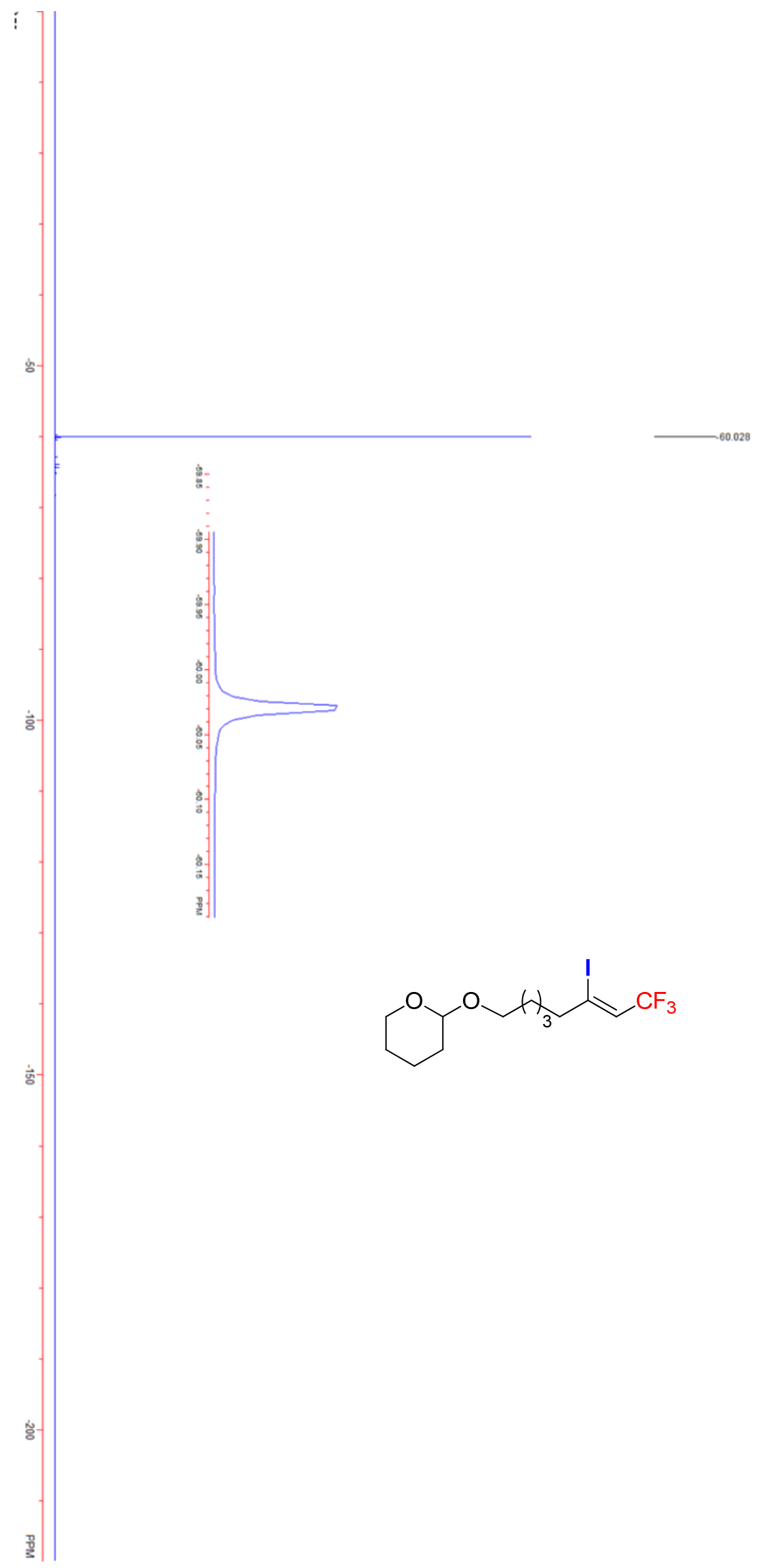




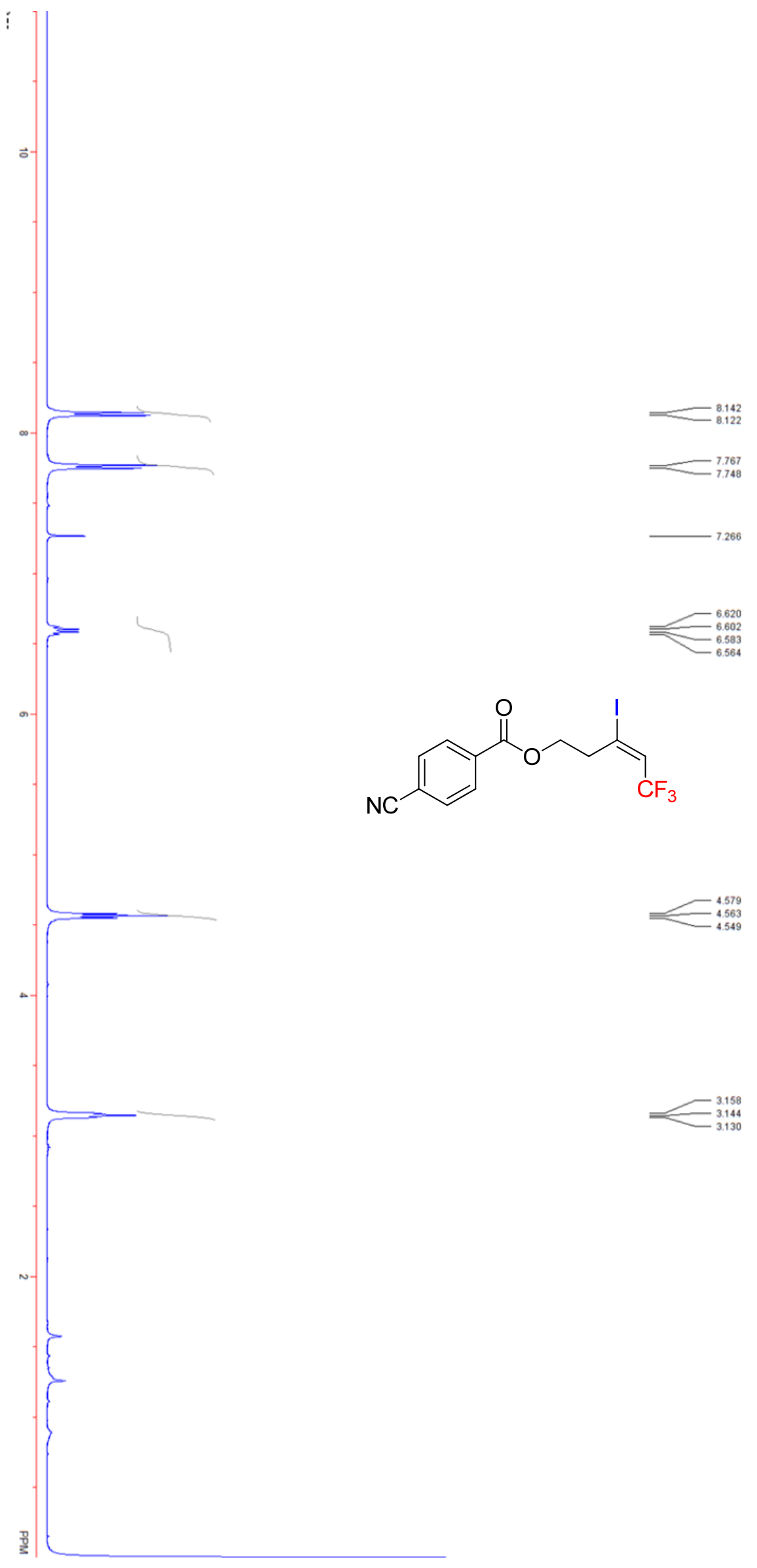




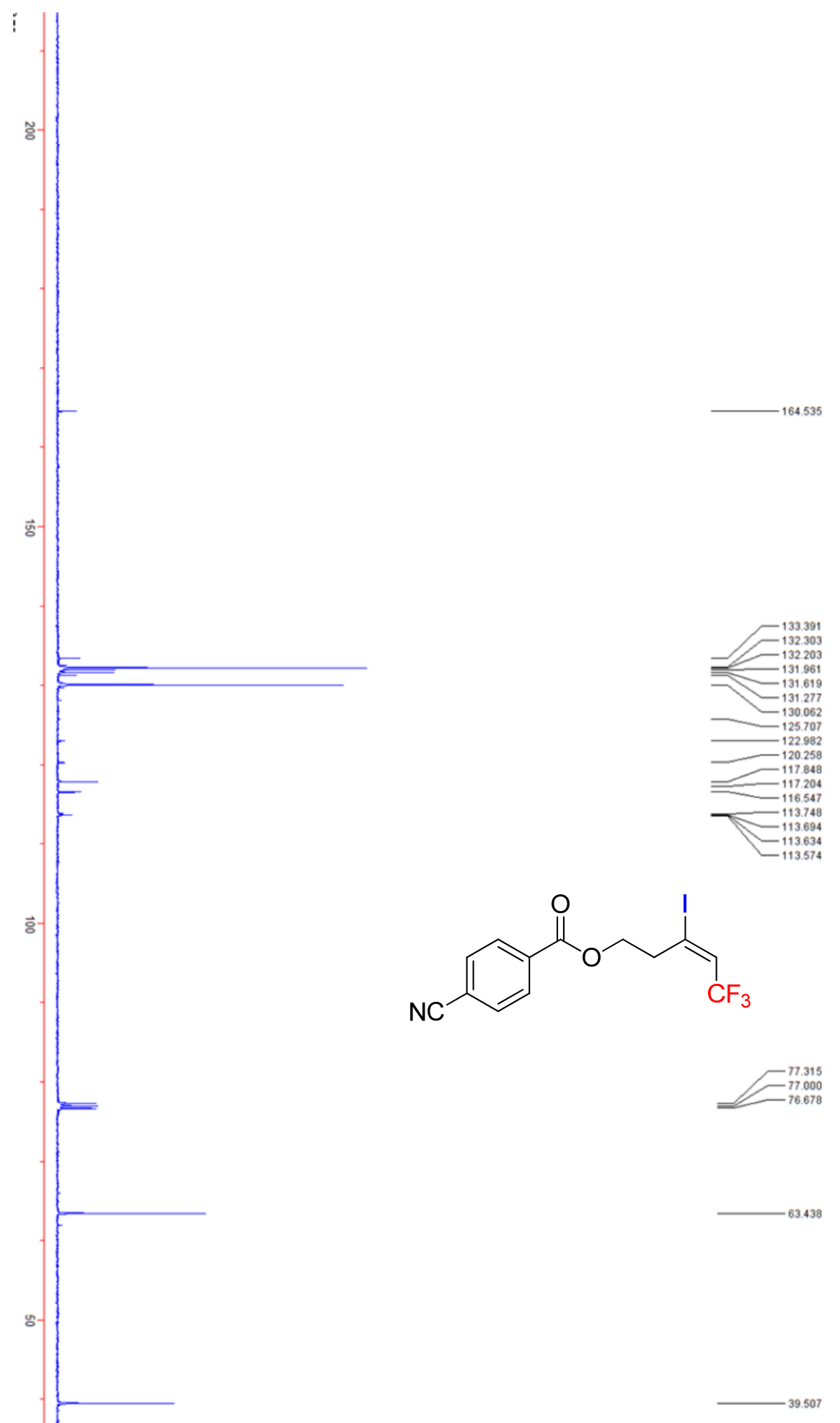




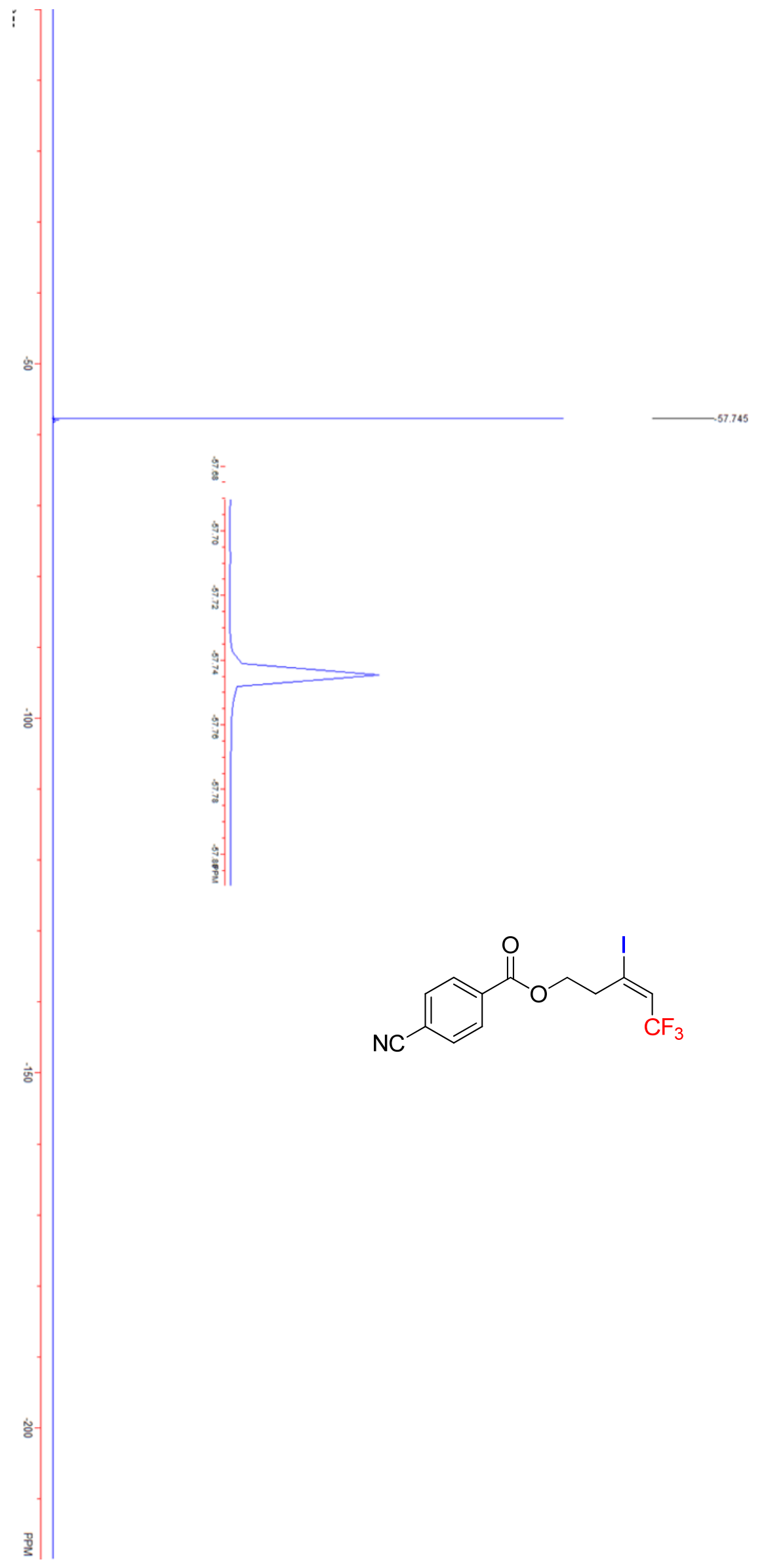




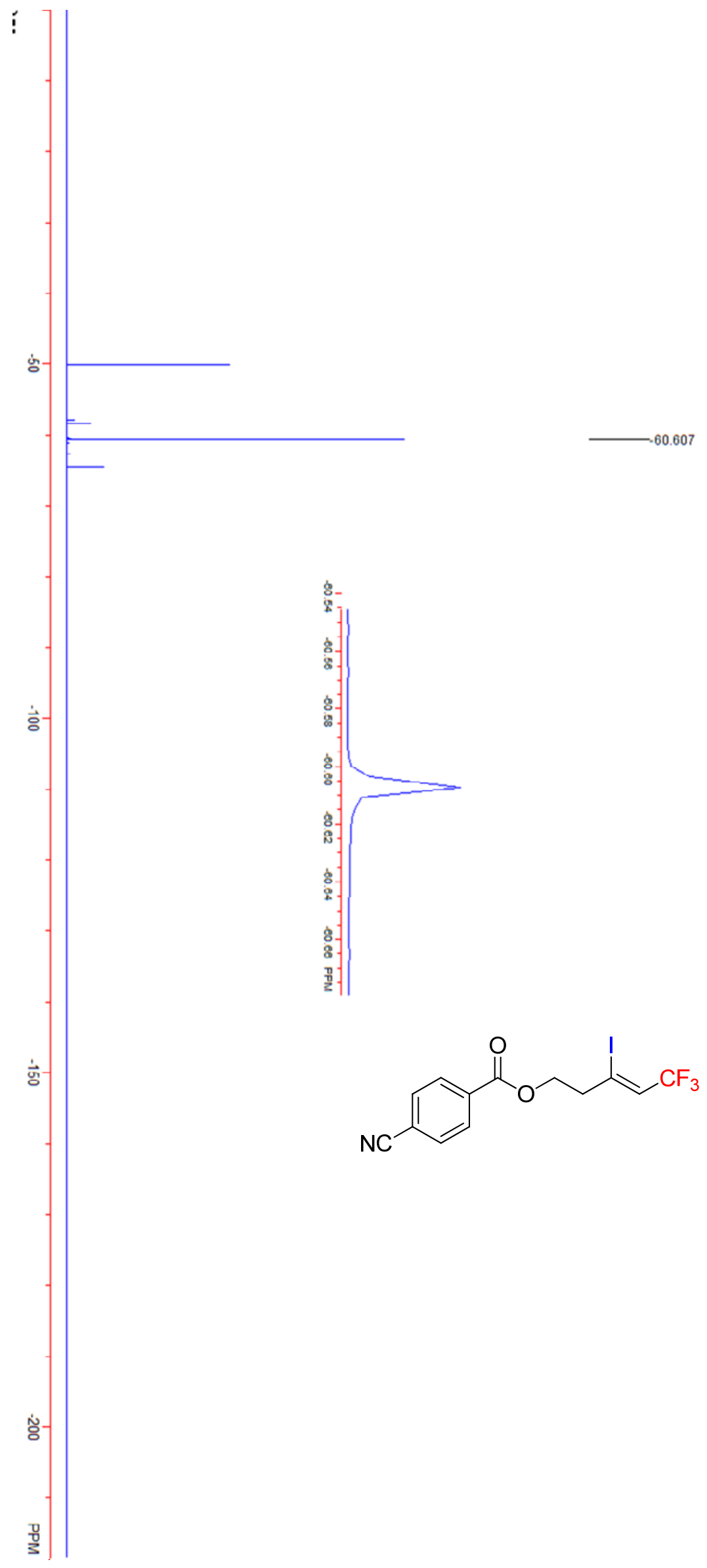




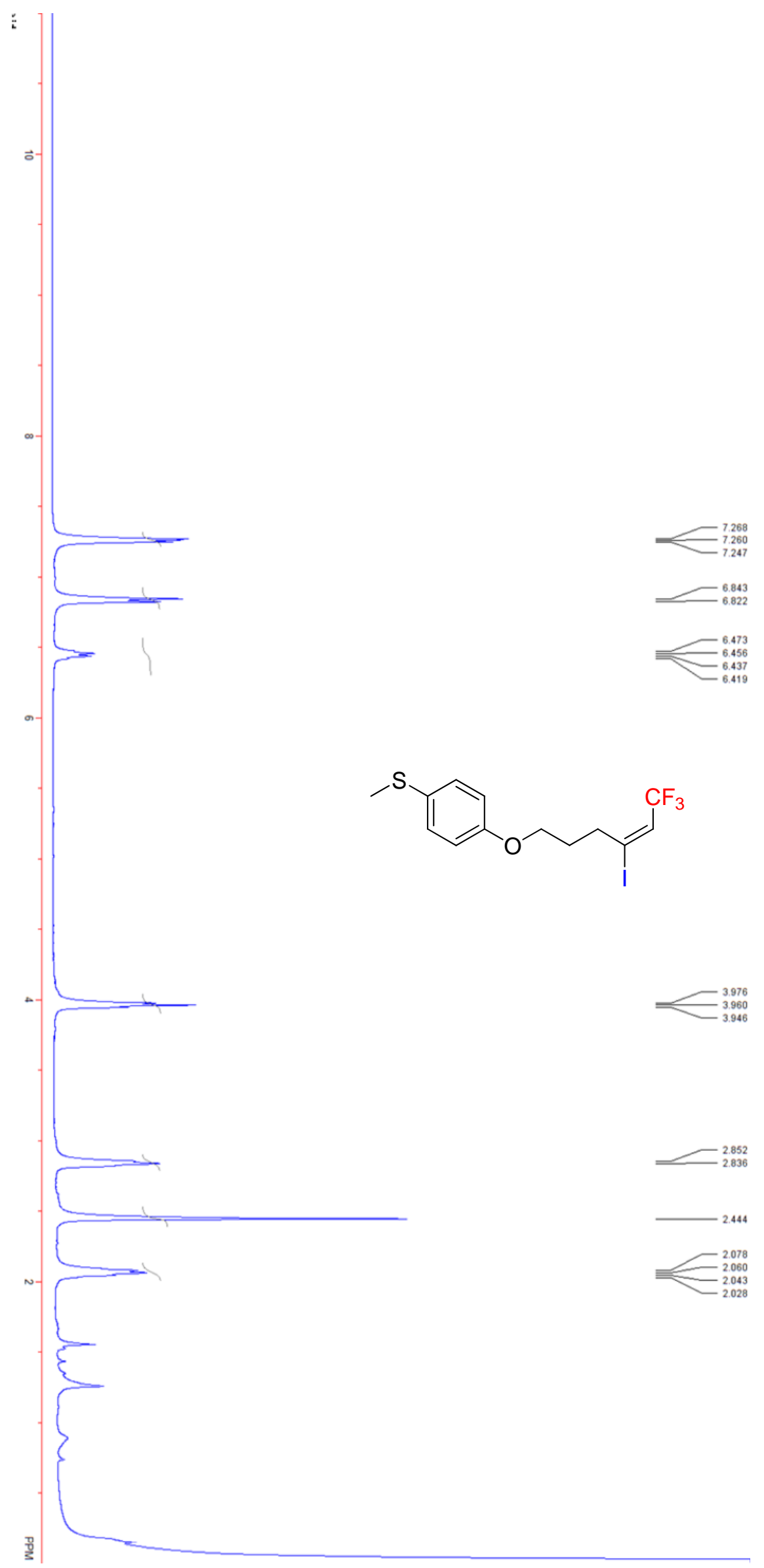




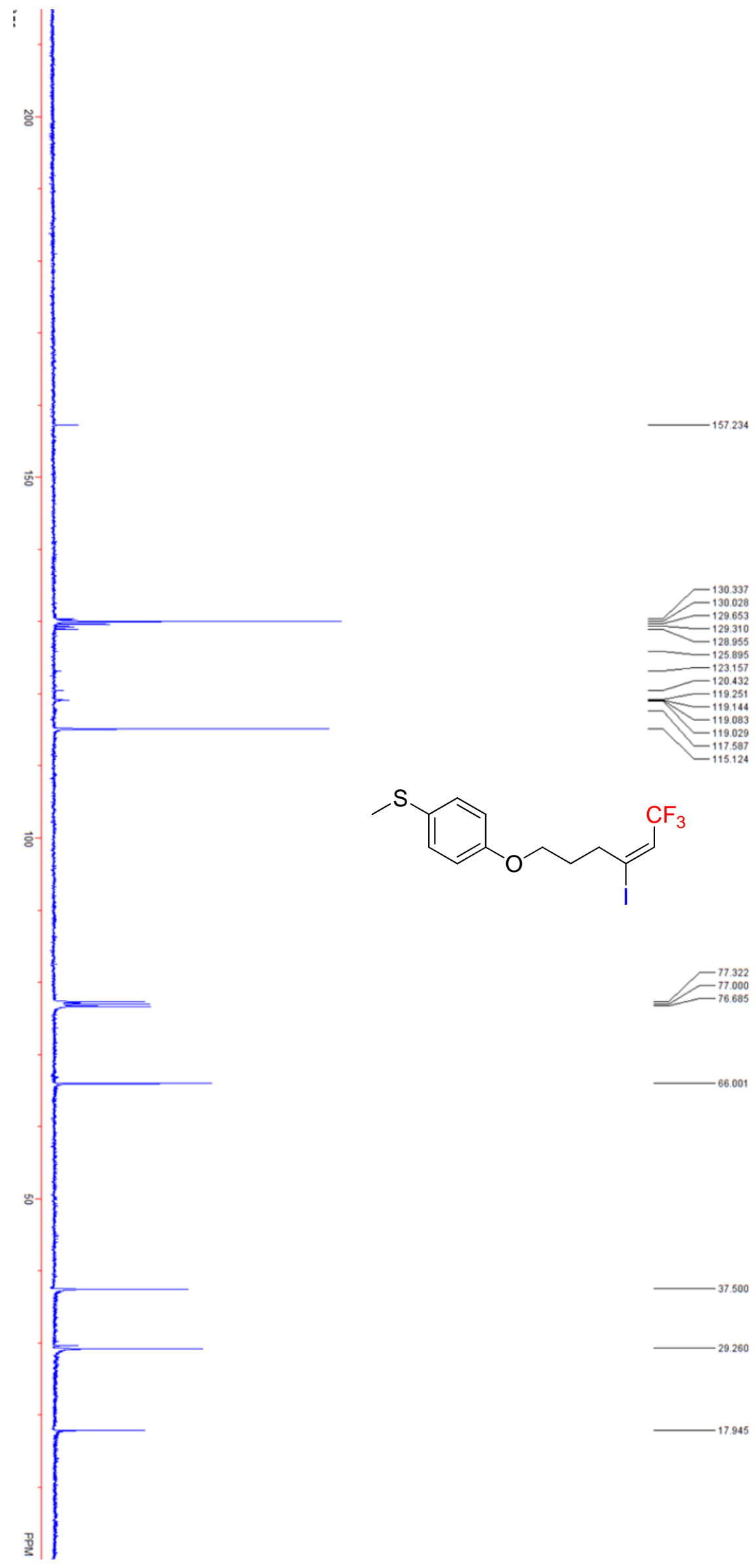




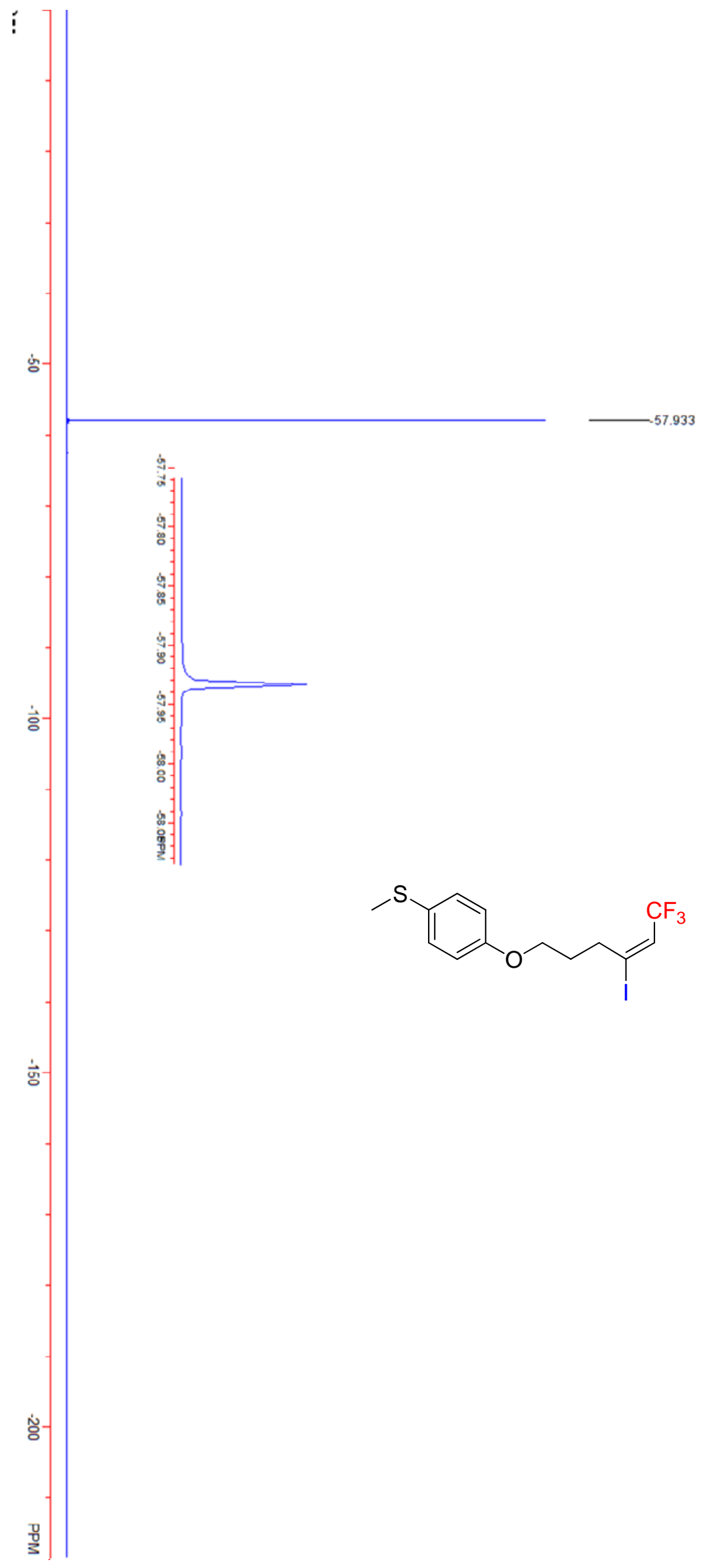




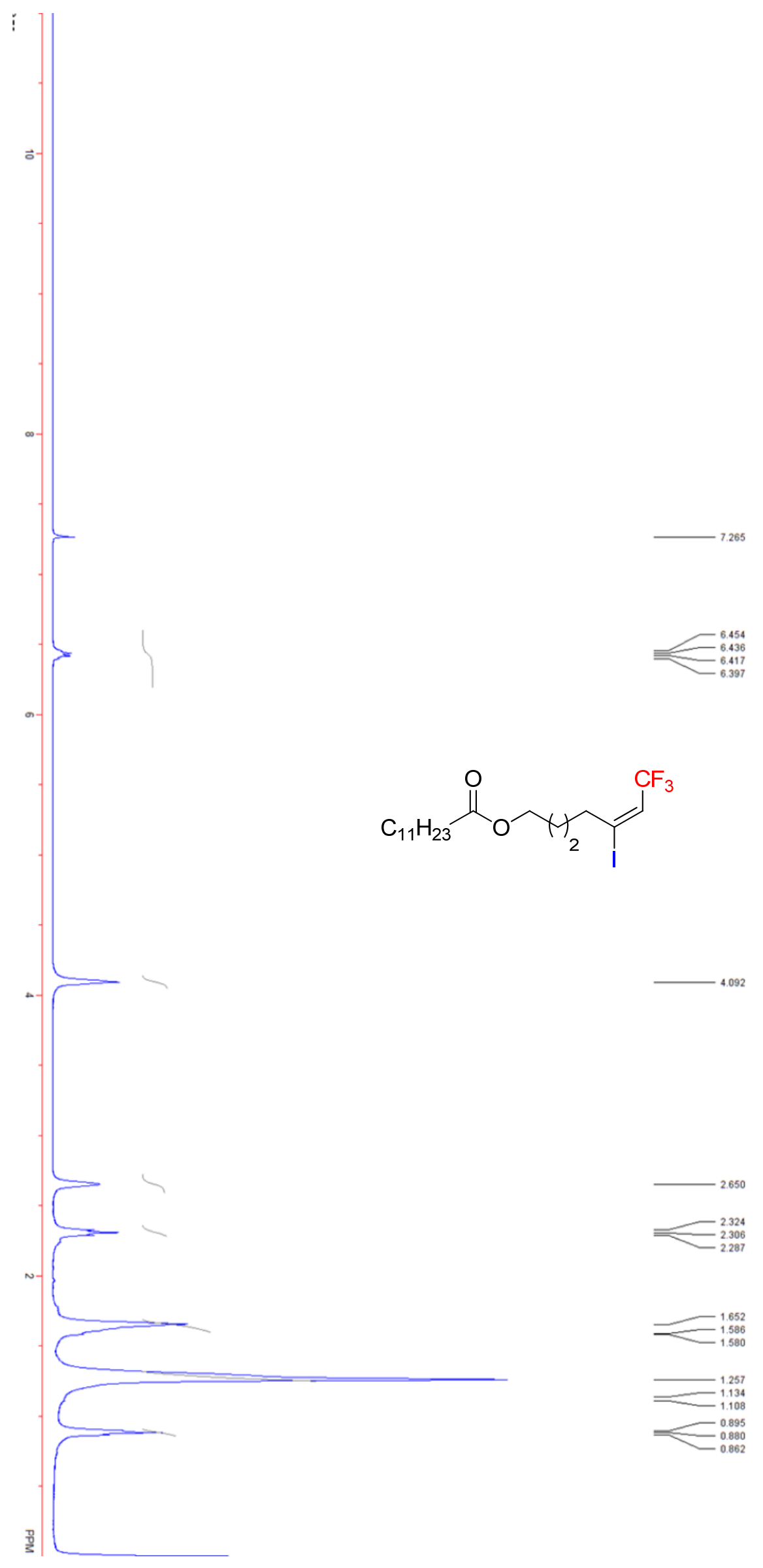




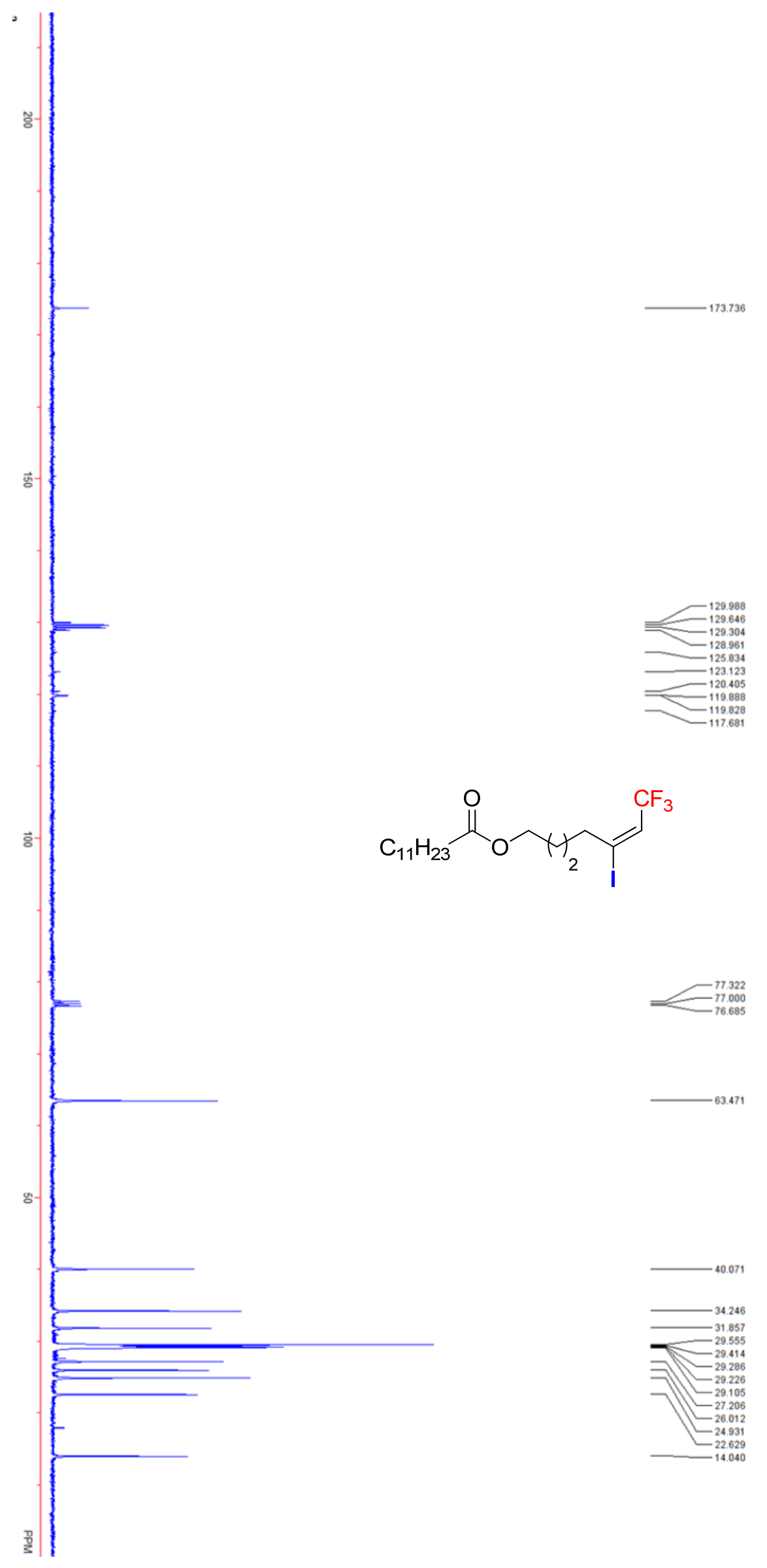




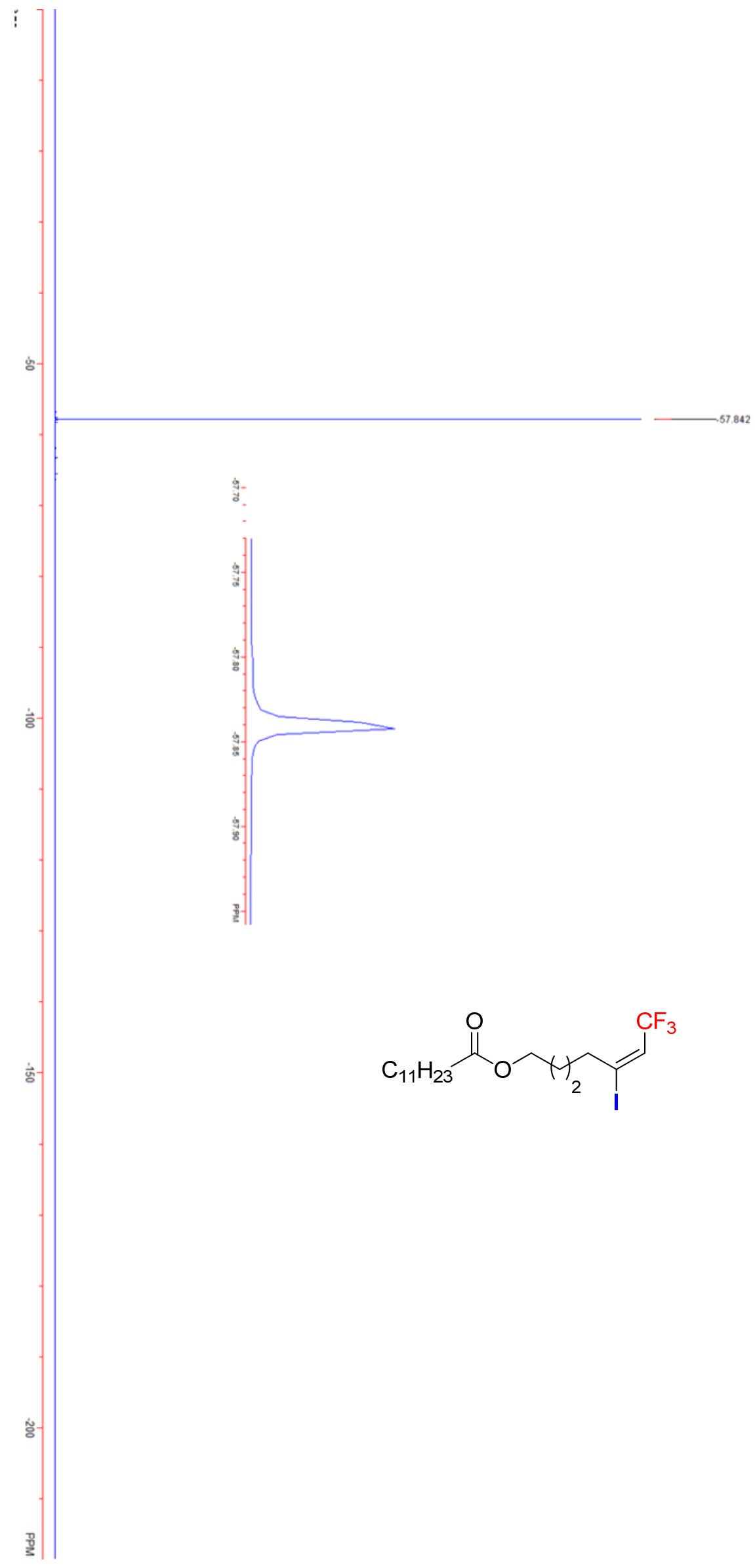




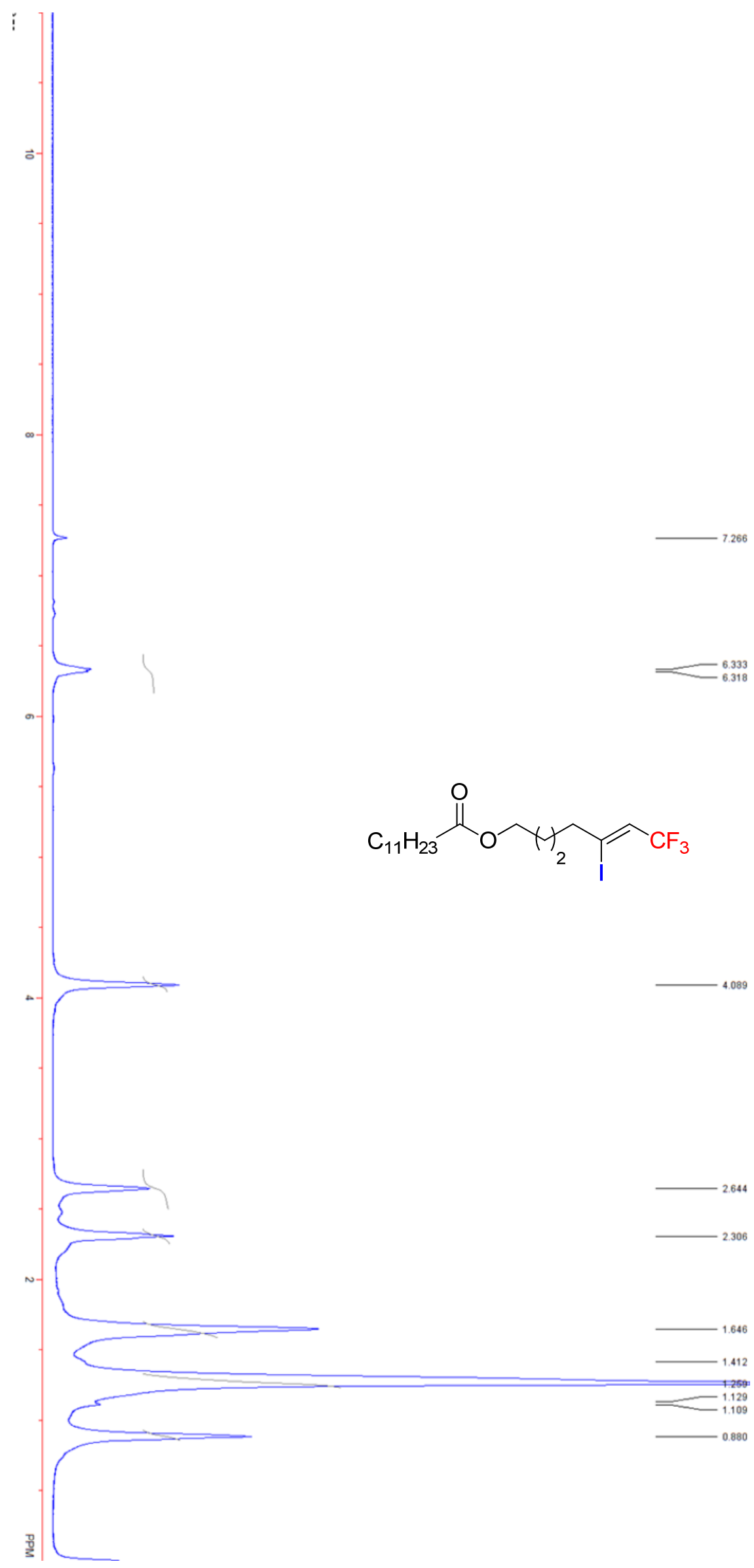




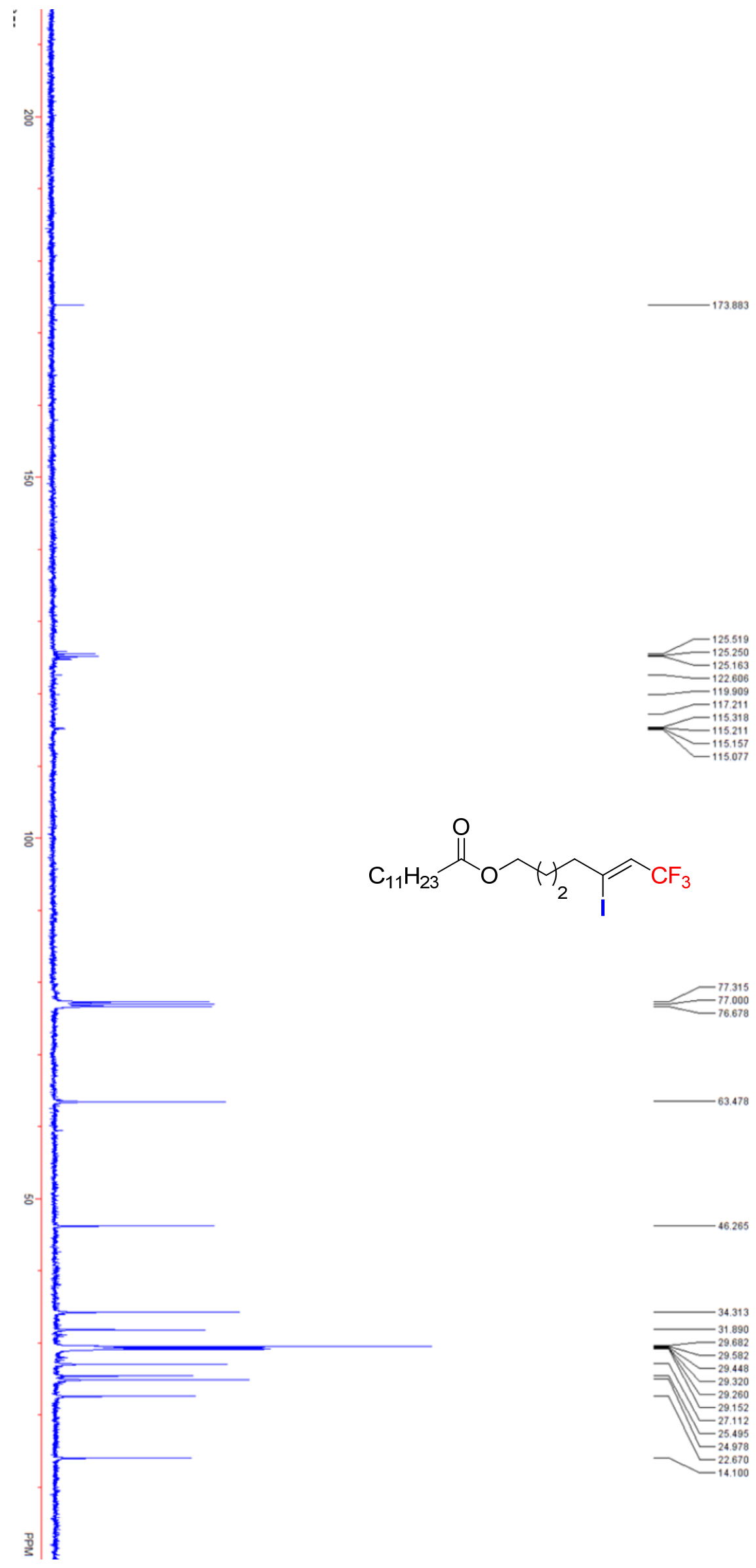




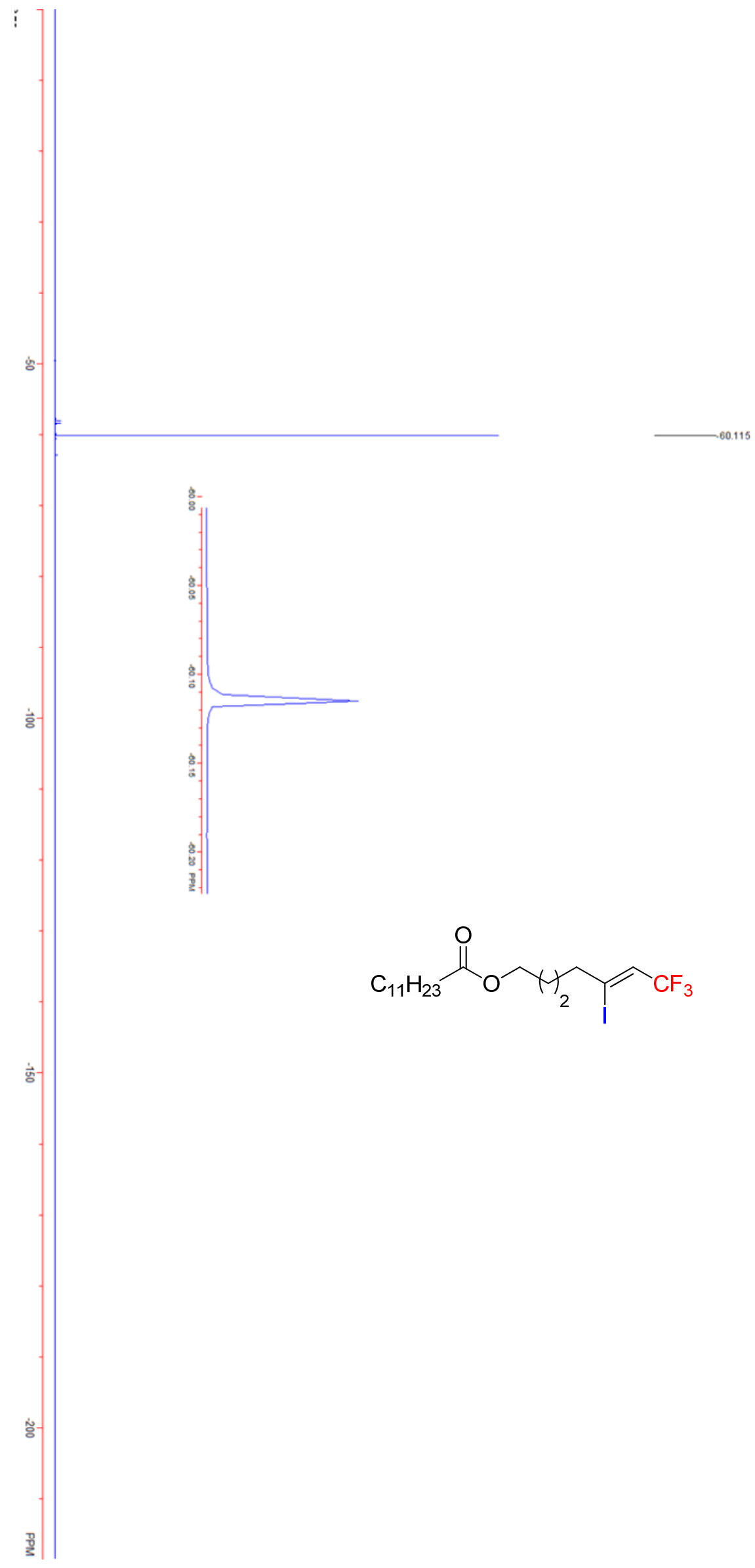




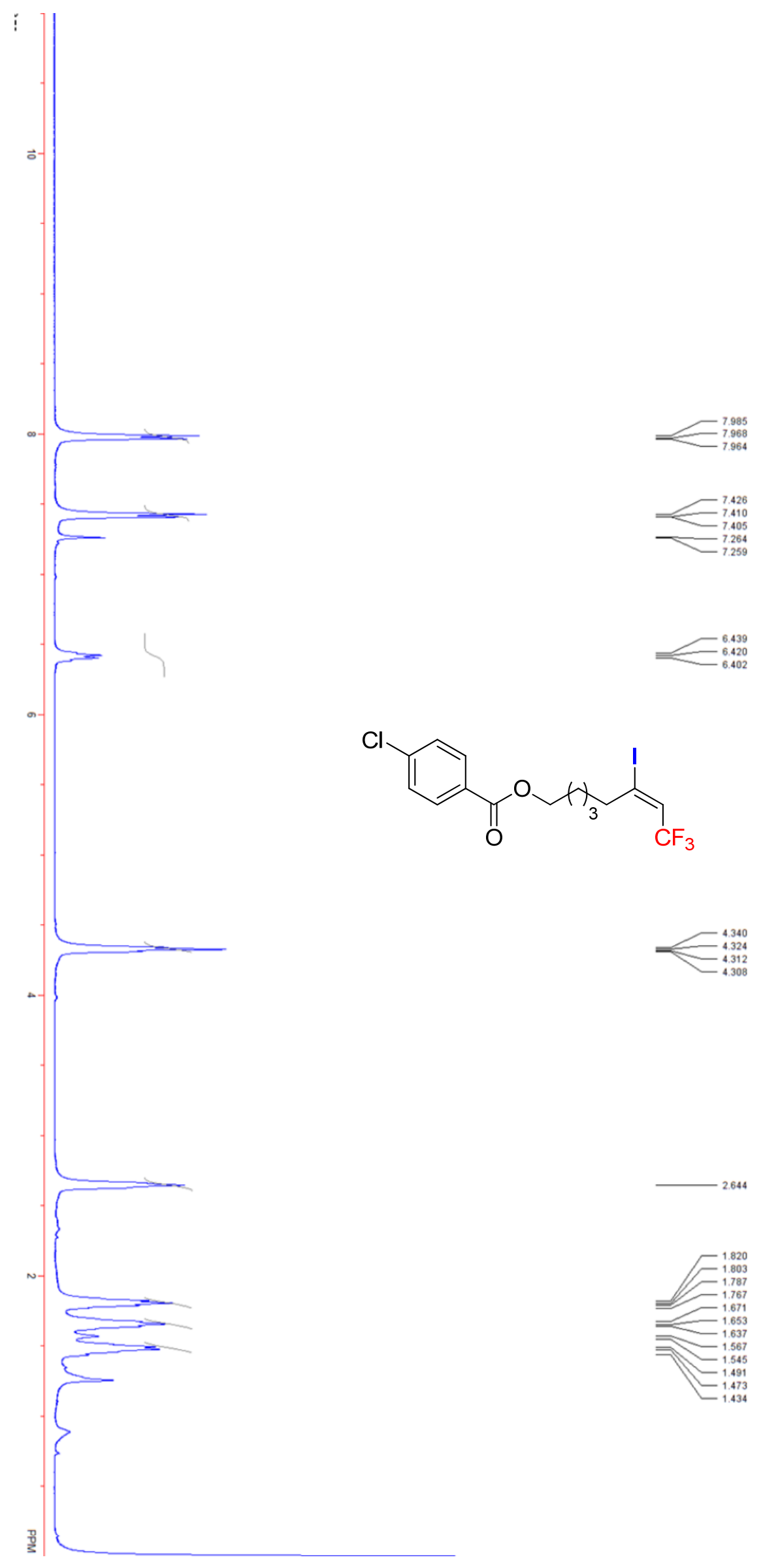



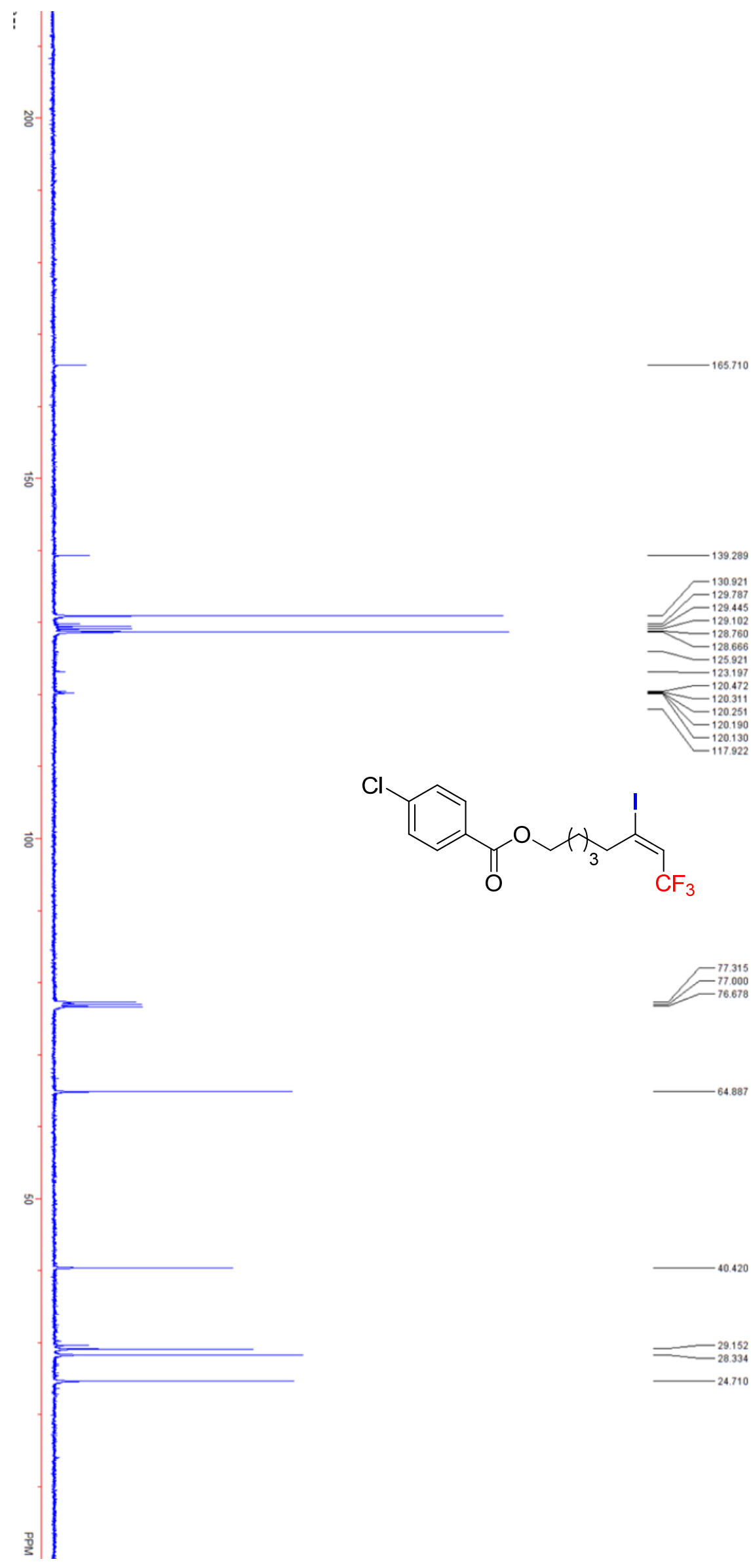


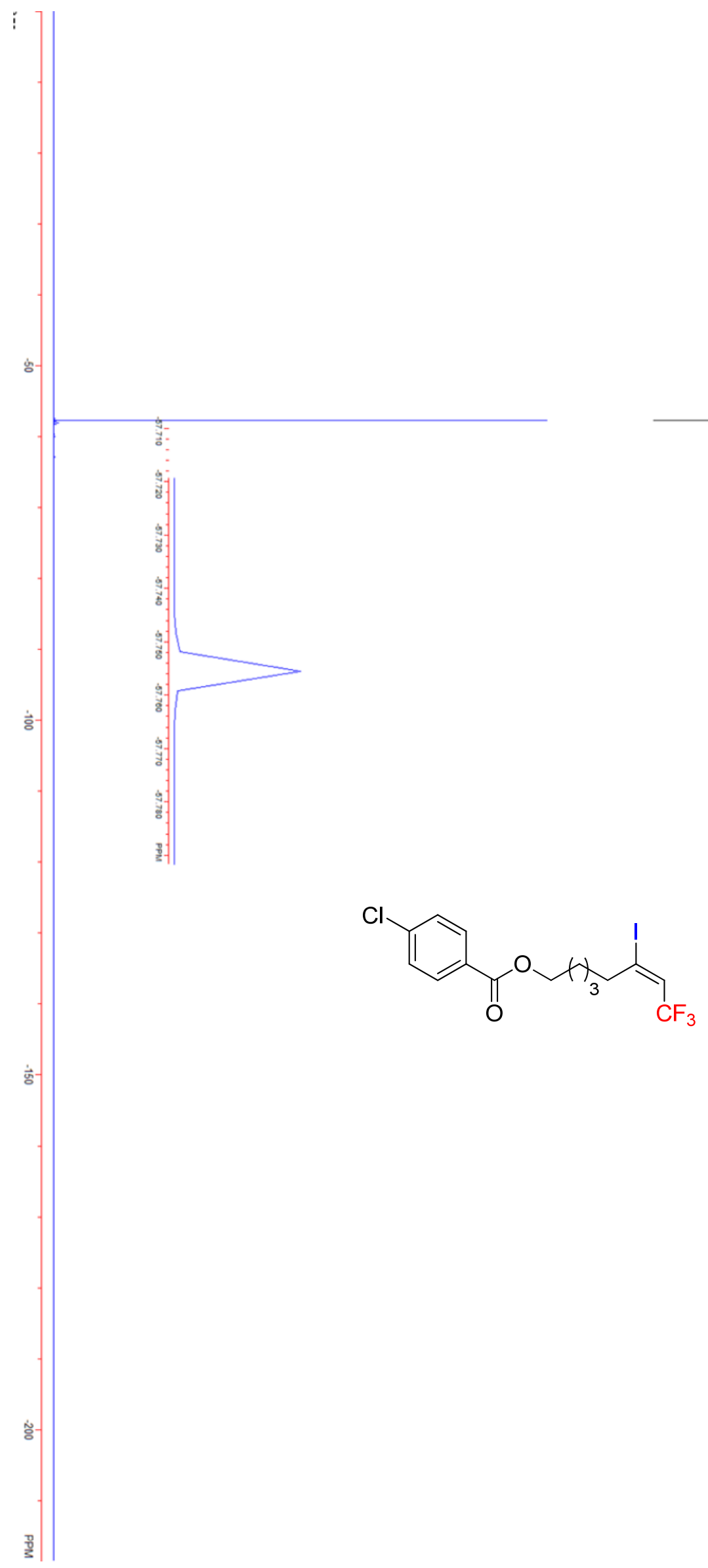



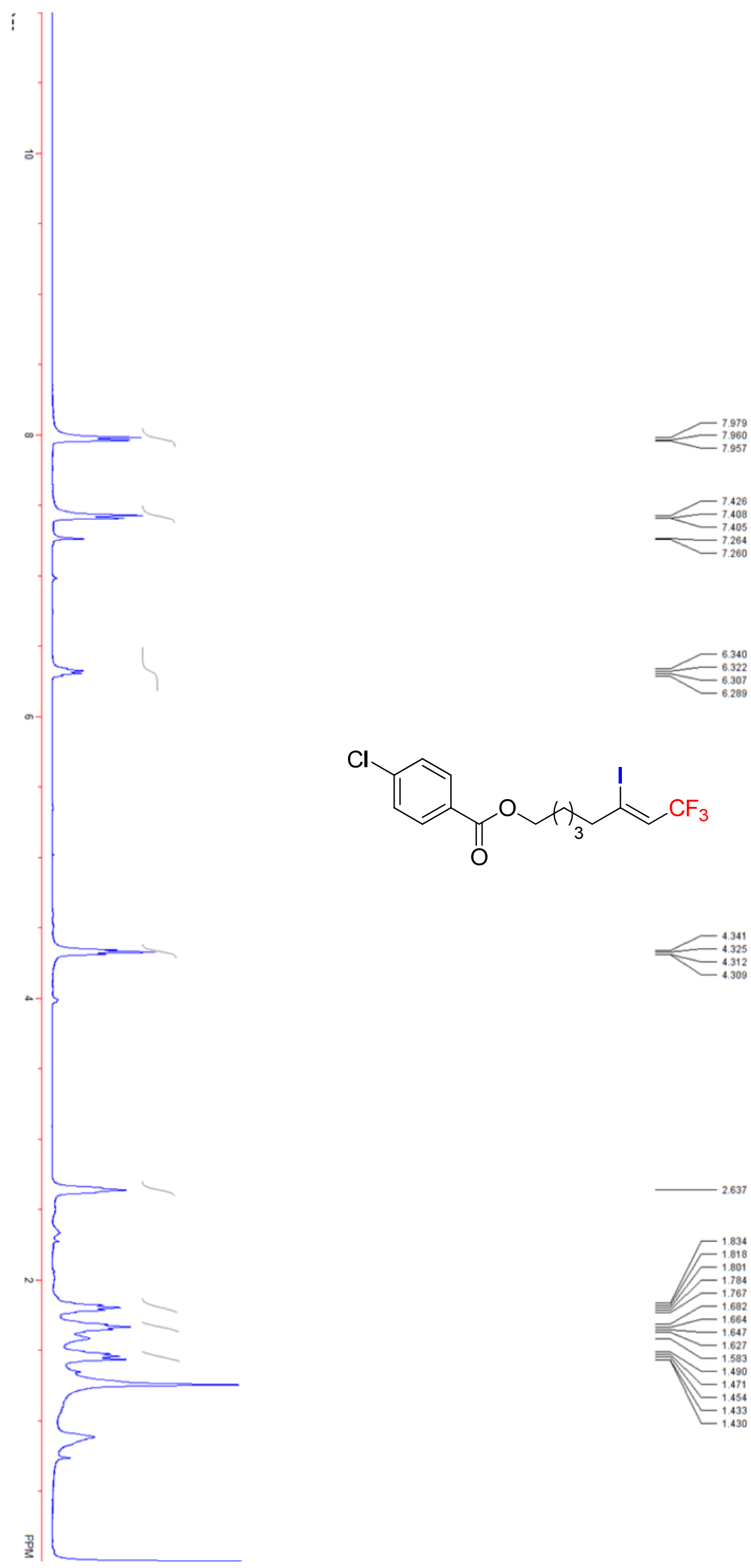

$\mathrm{Cl}$<smiles>O=C(OCCCC(I)=CC(F)(F)F)c1ccc(I)cc1</smiles>

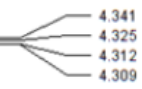

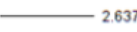

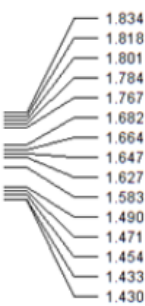



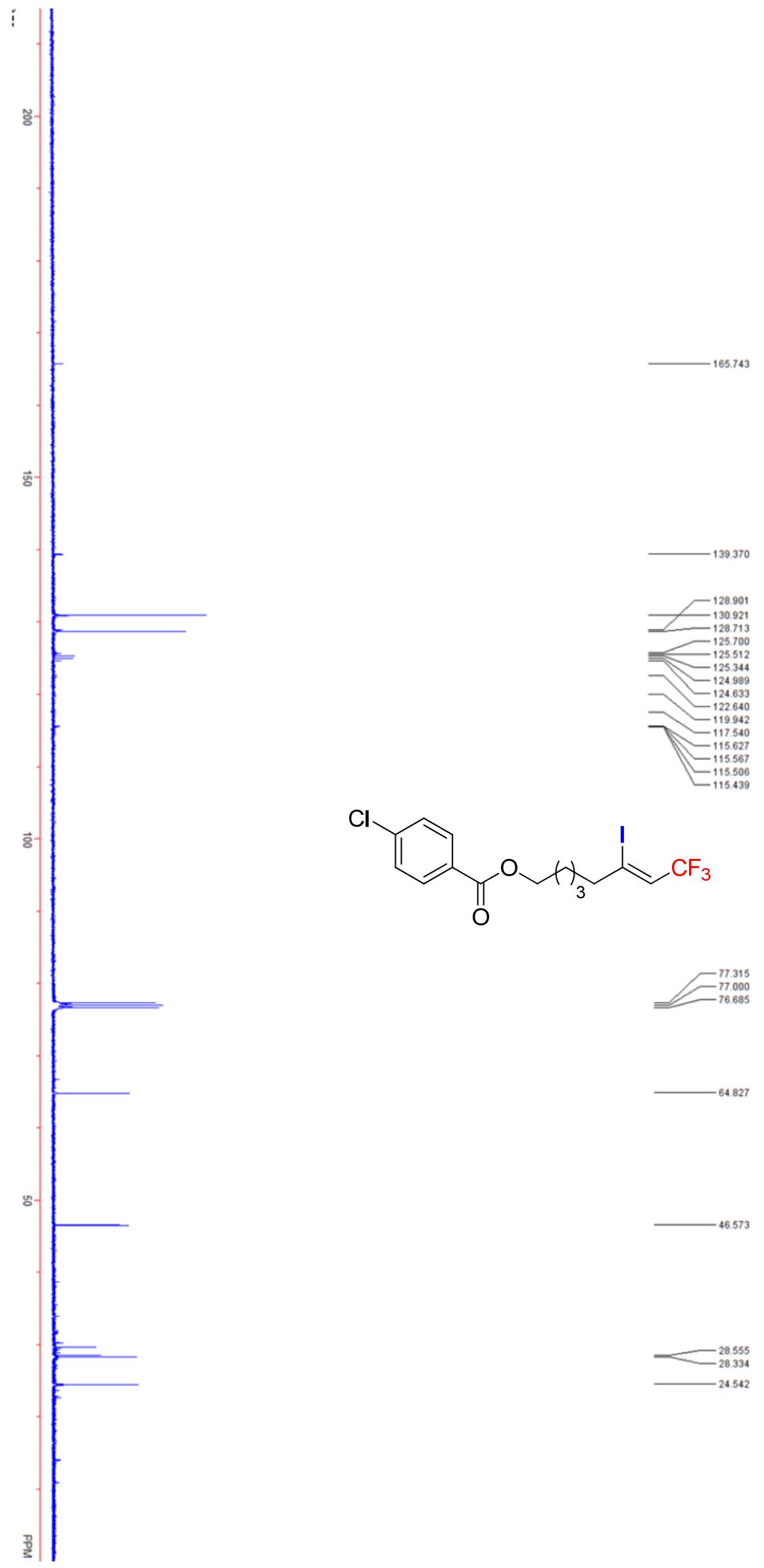


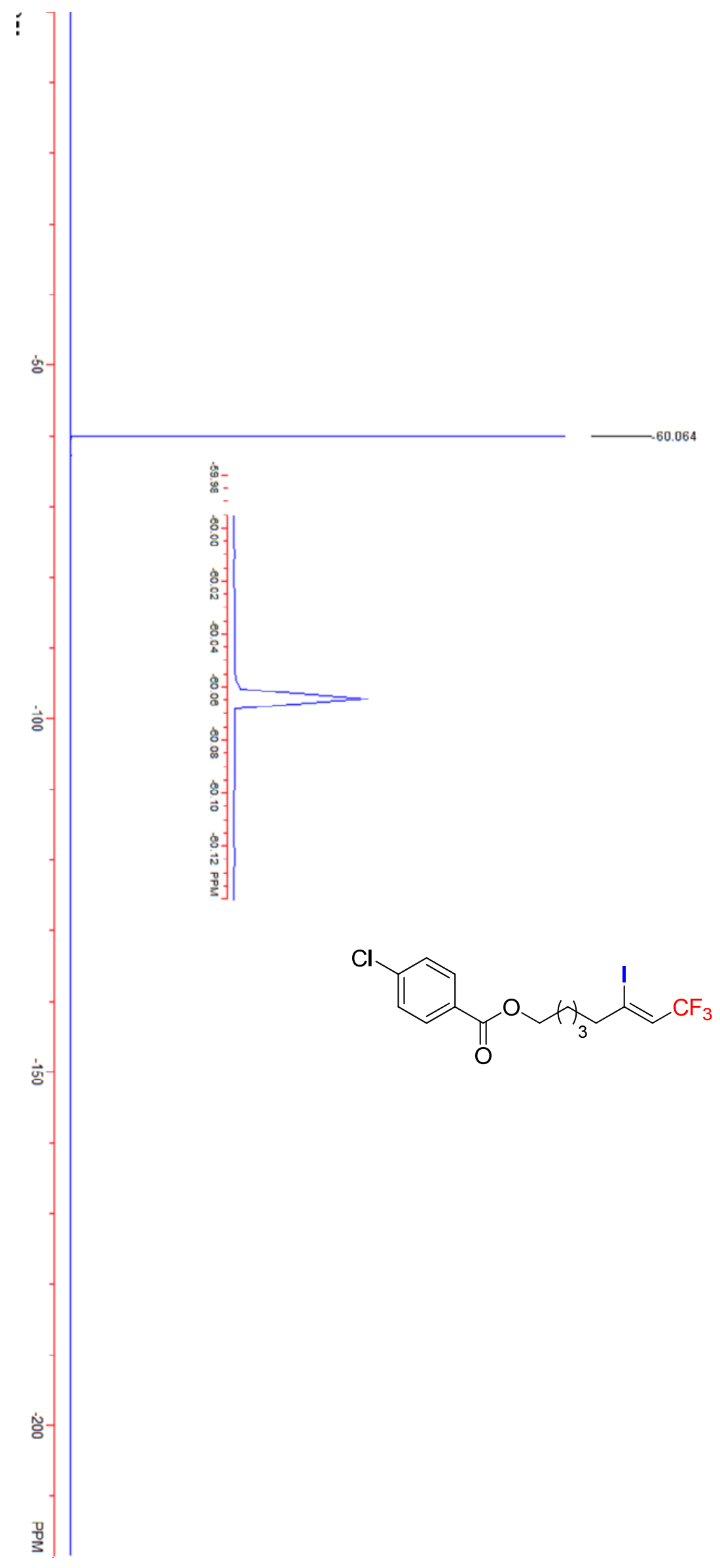



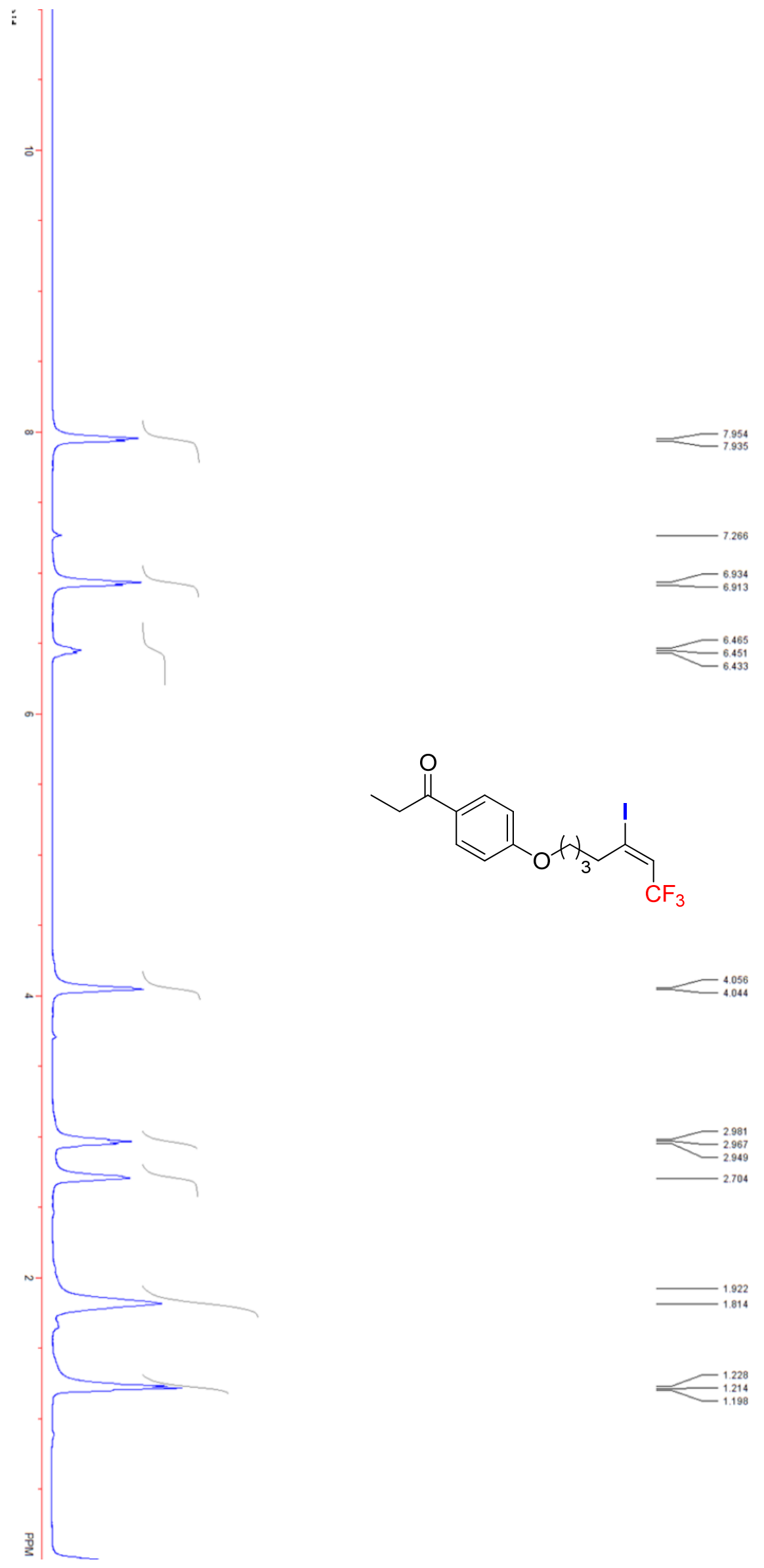

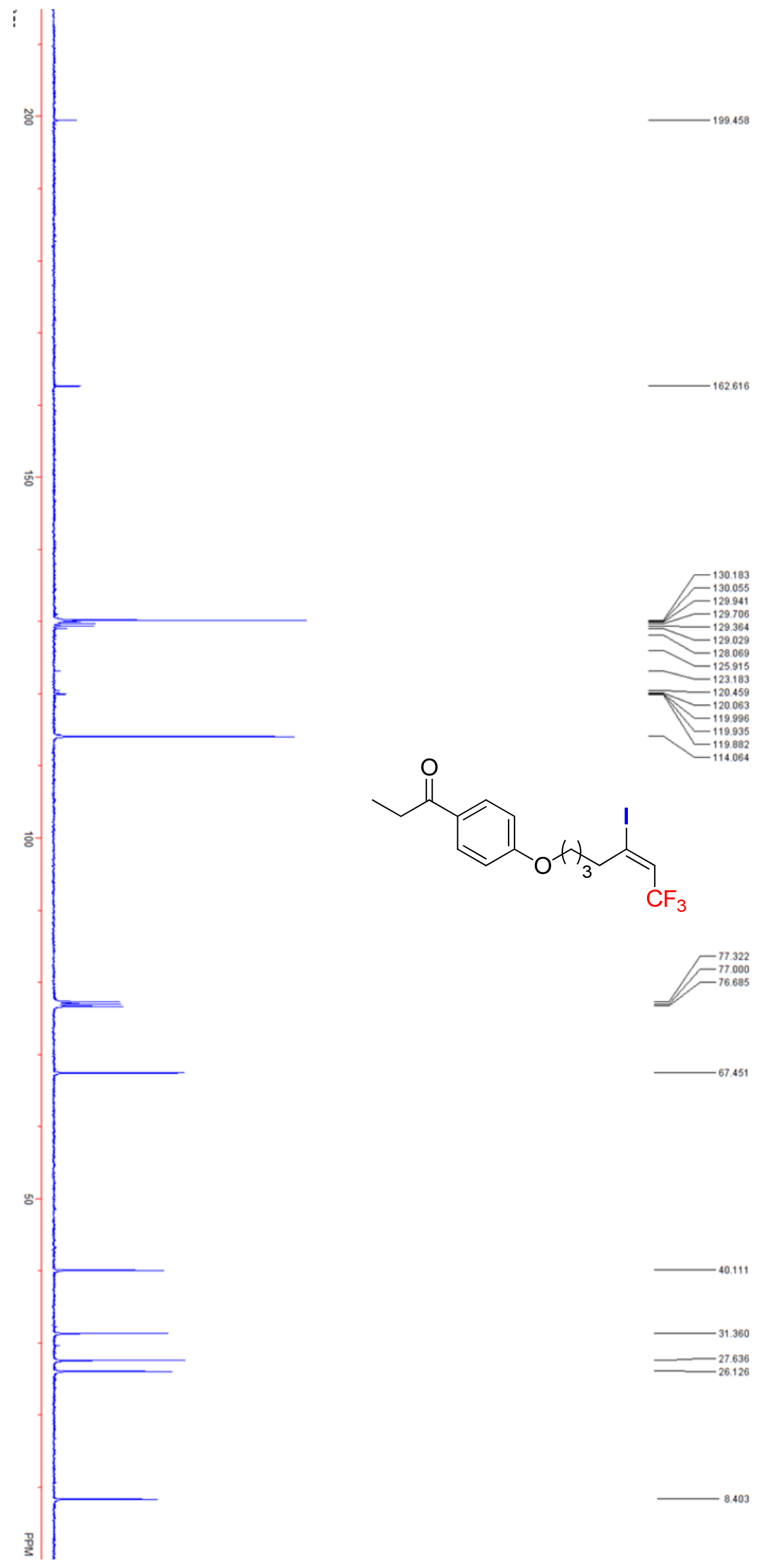


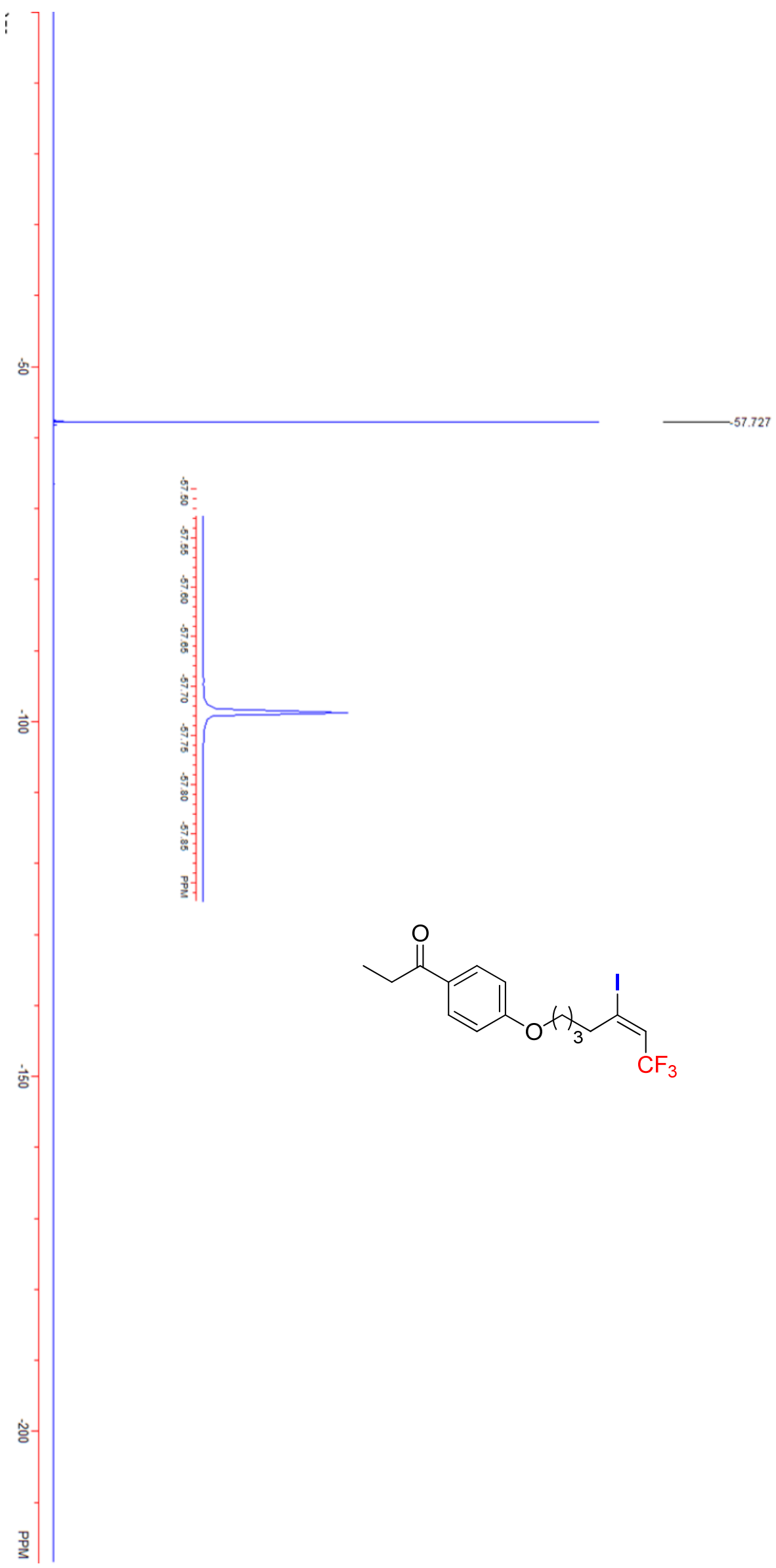




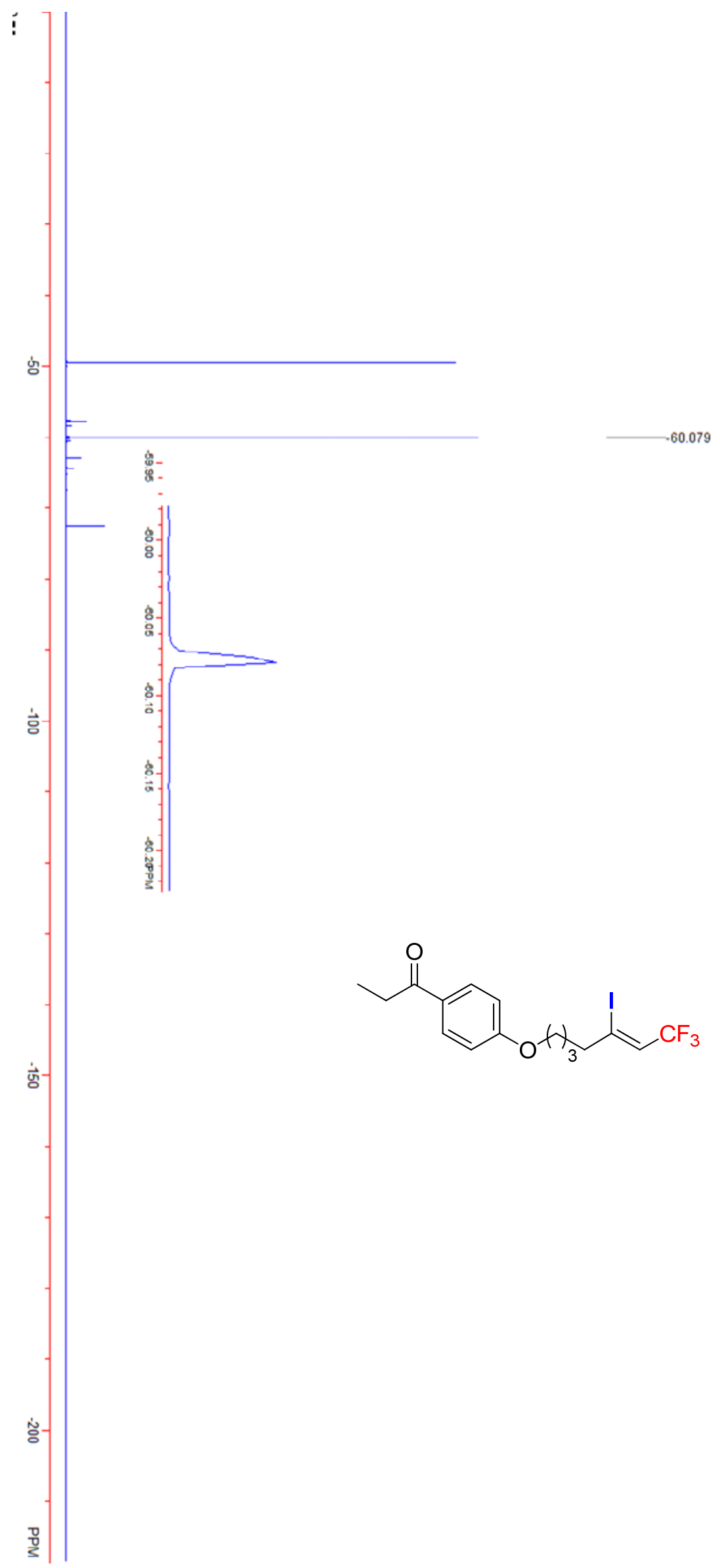



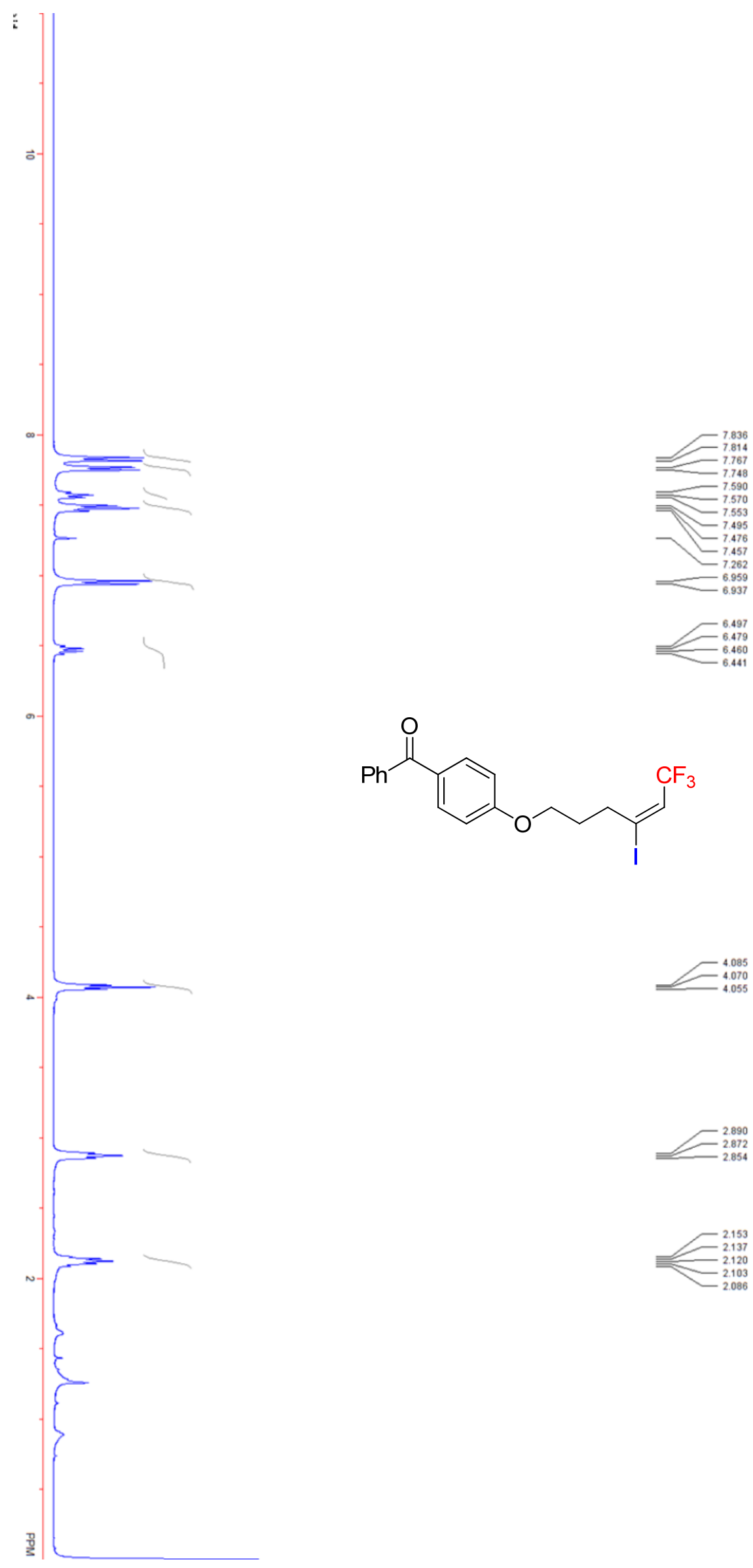<smiles>O=C(c1ccccc1)c1ccc(OCCC/C(I)=C\C(F)(F)F)cc1</smiles>
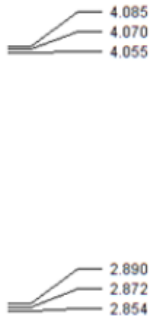


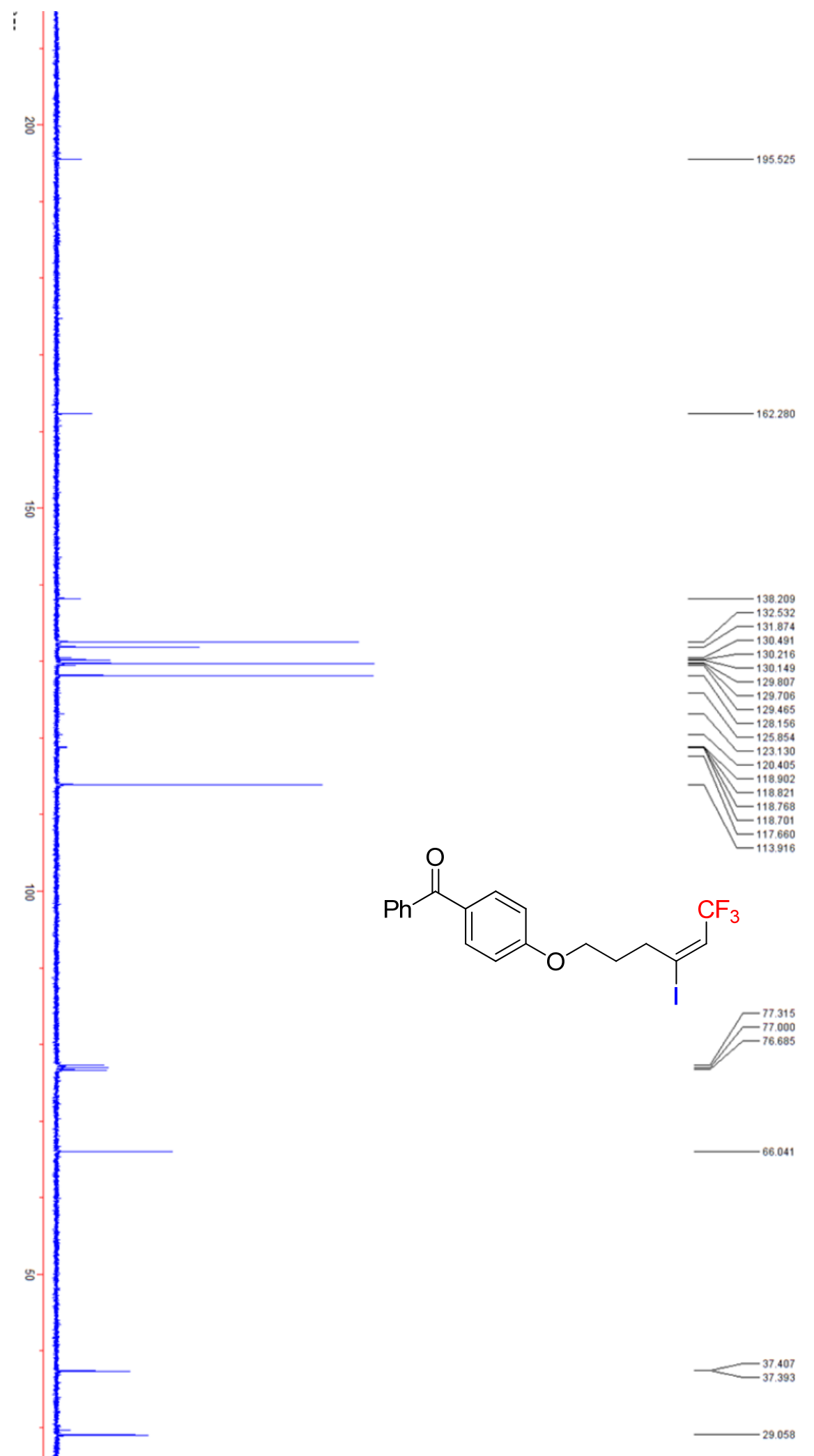




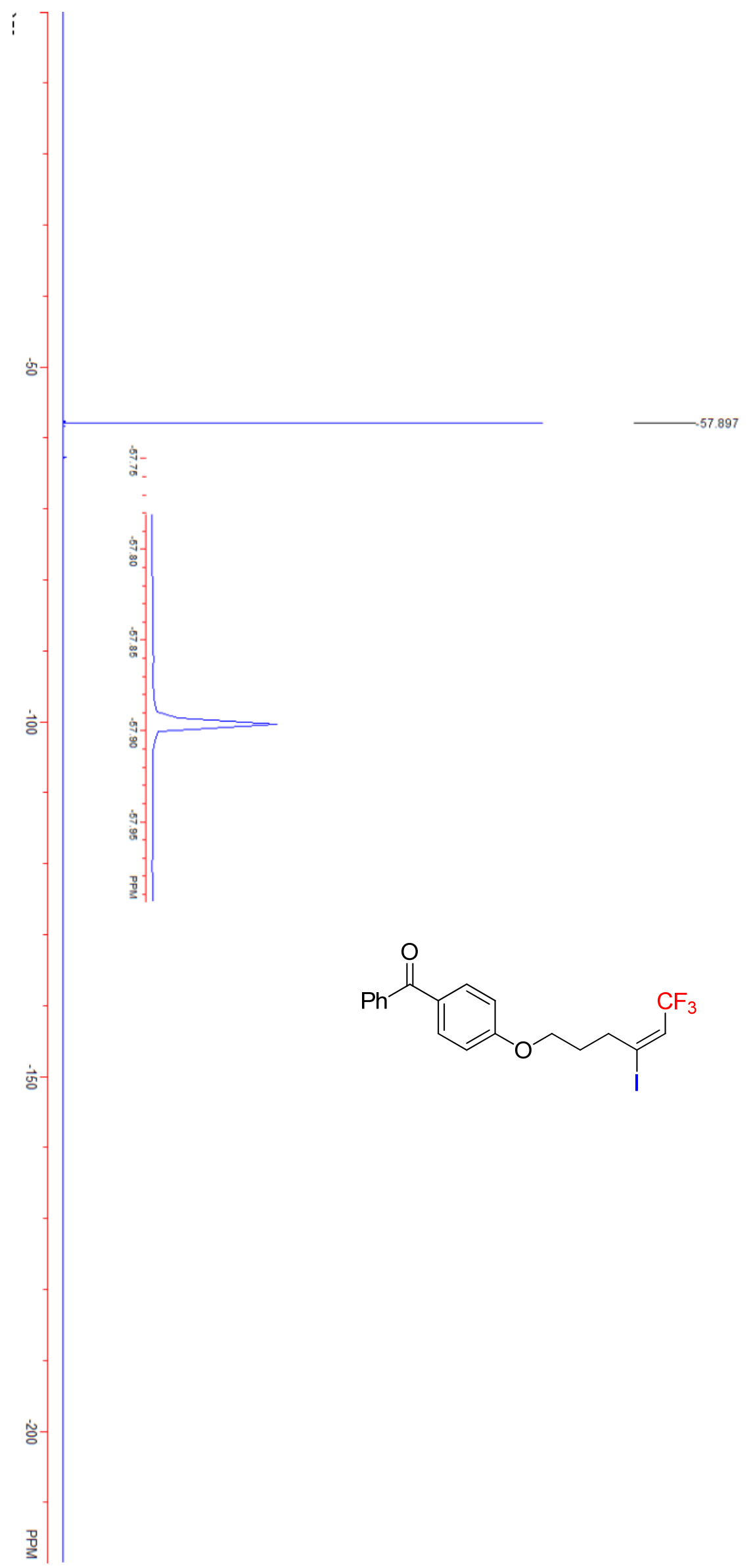




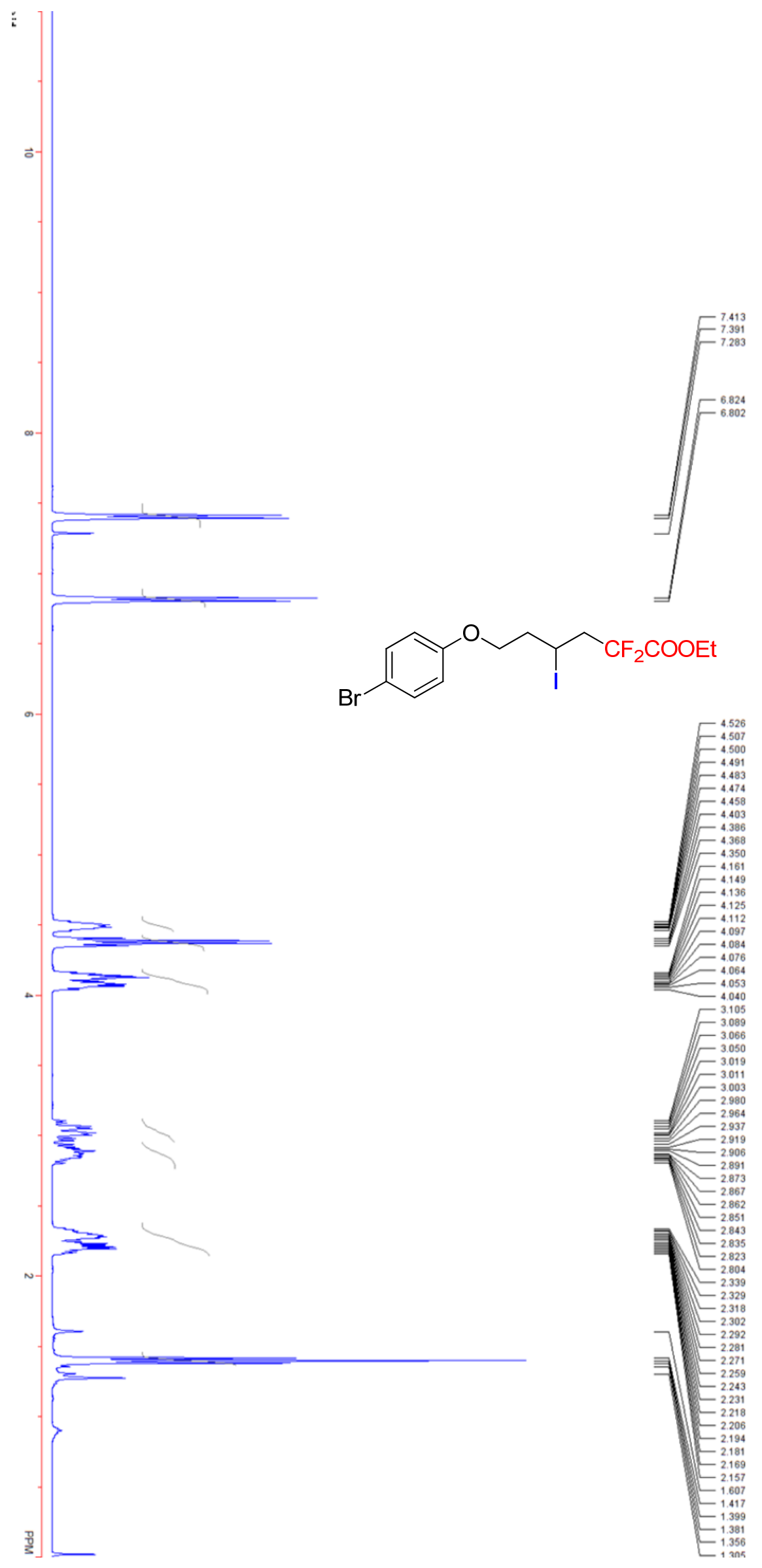




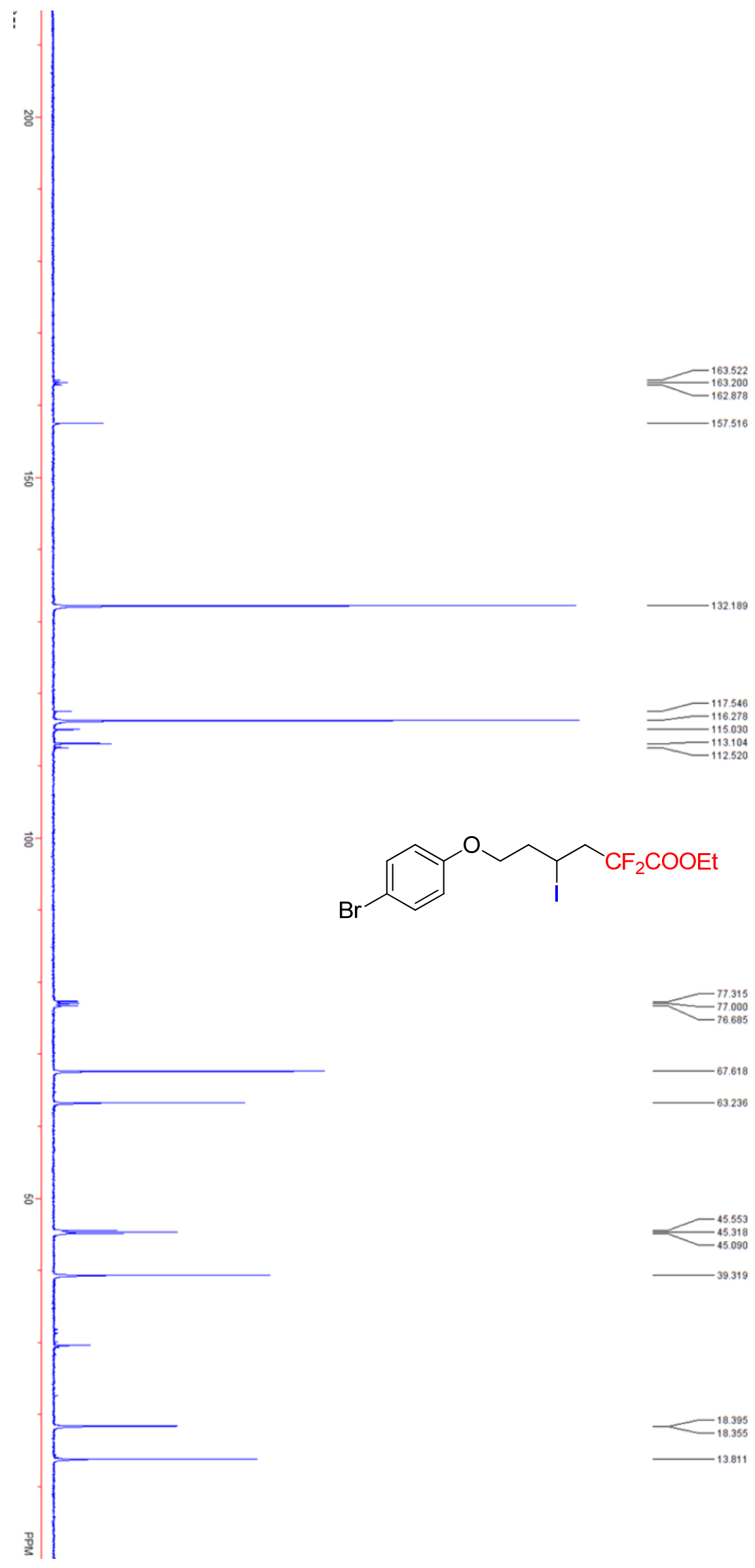




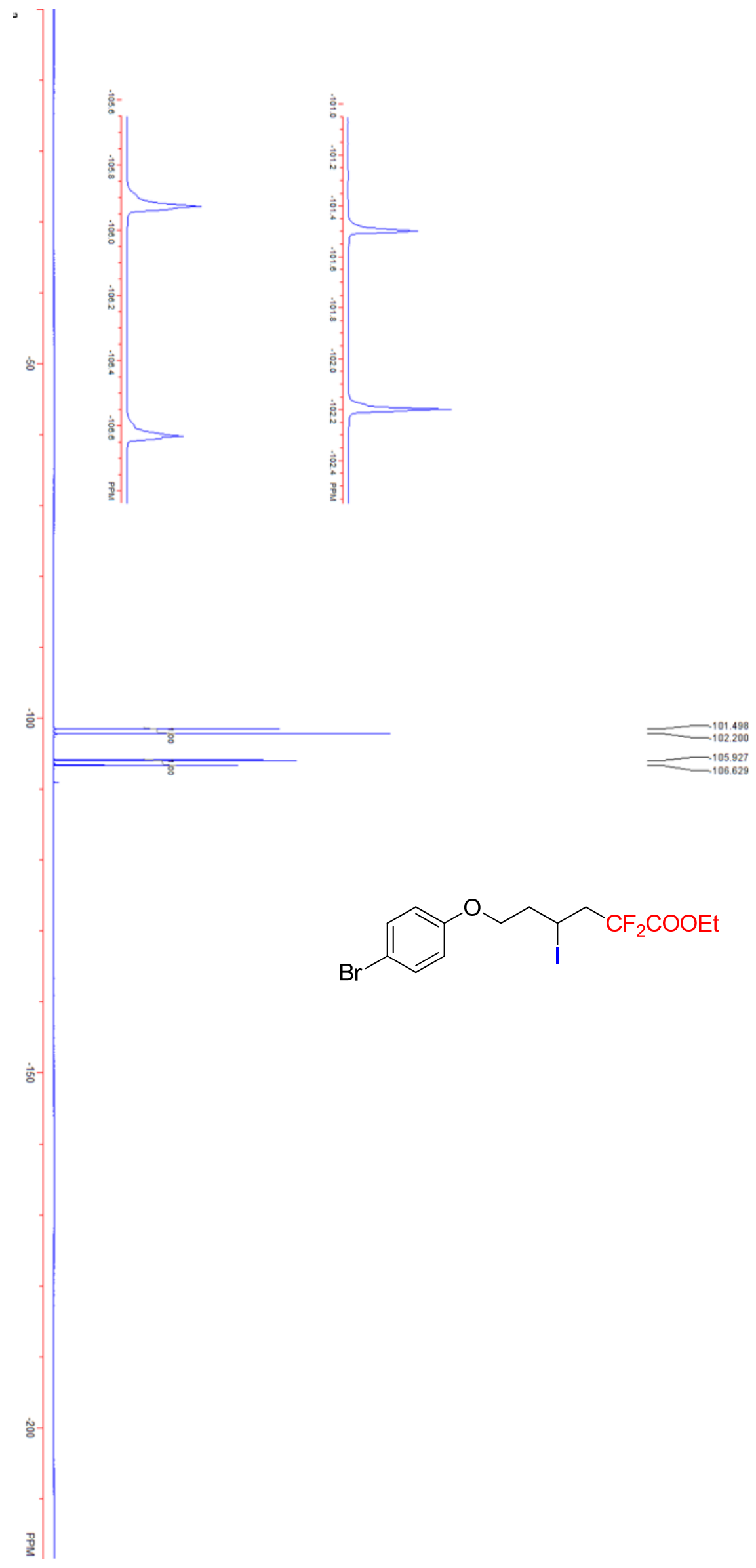




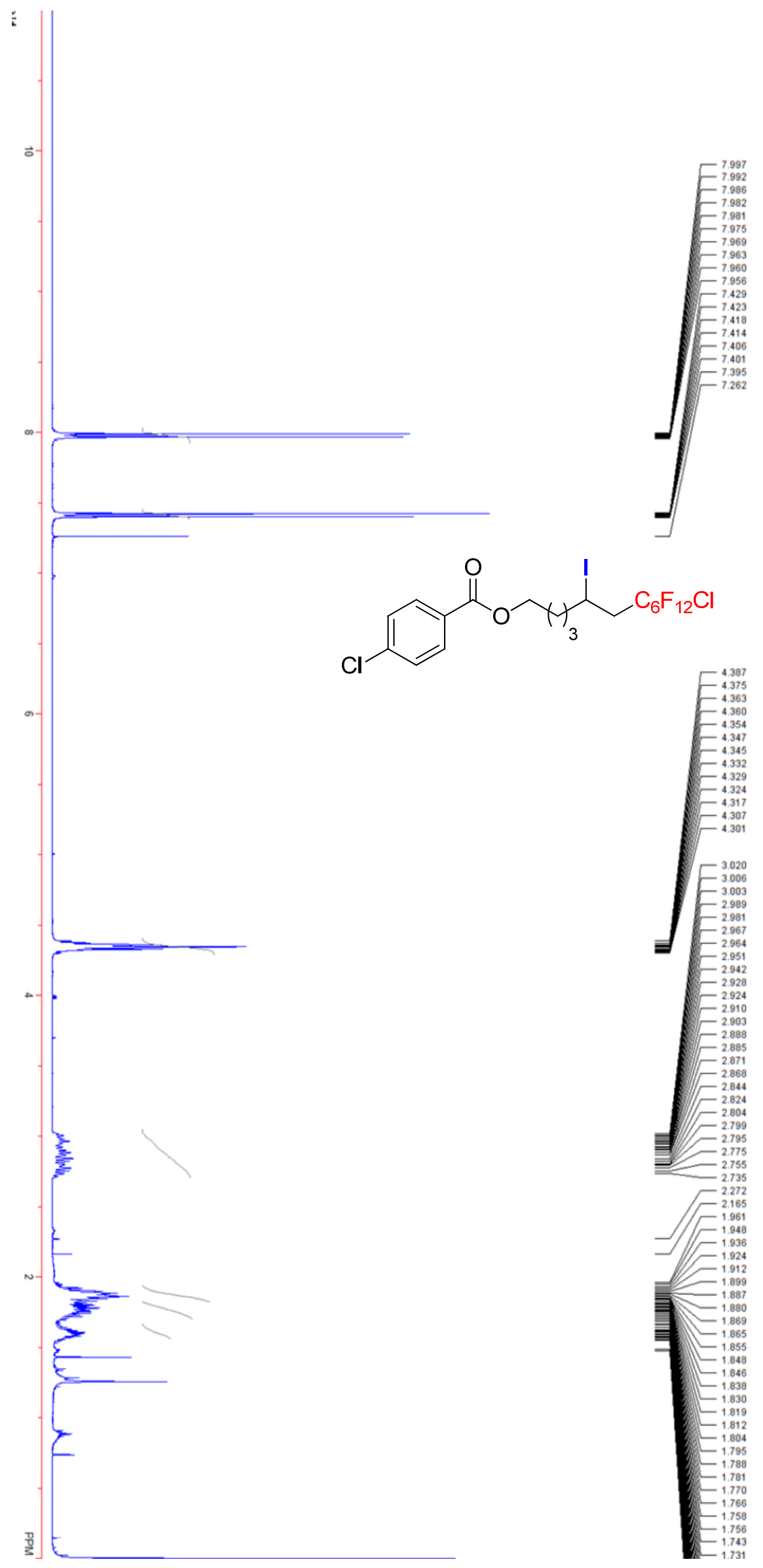




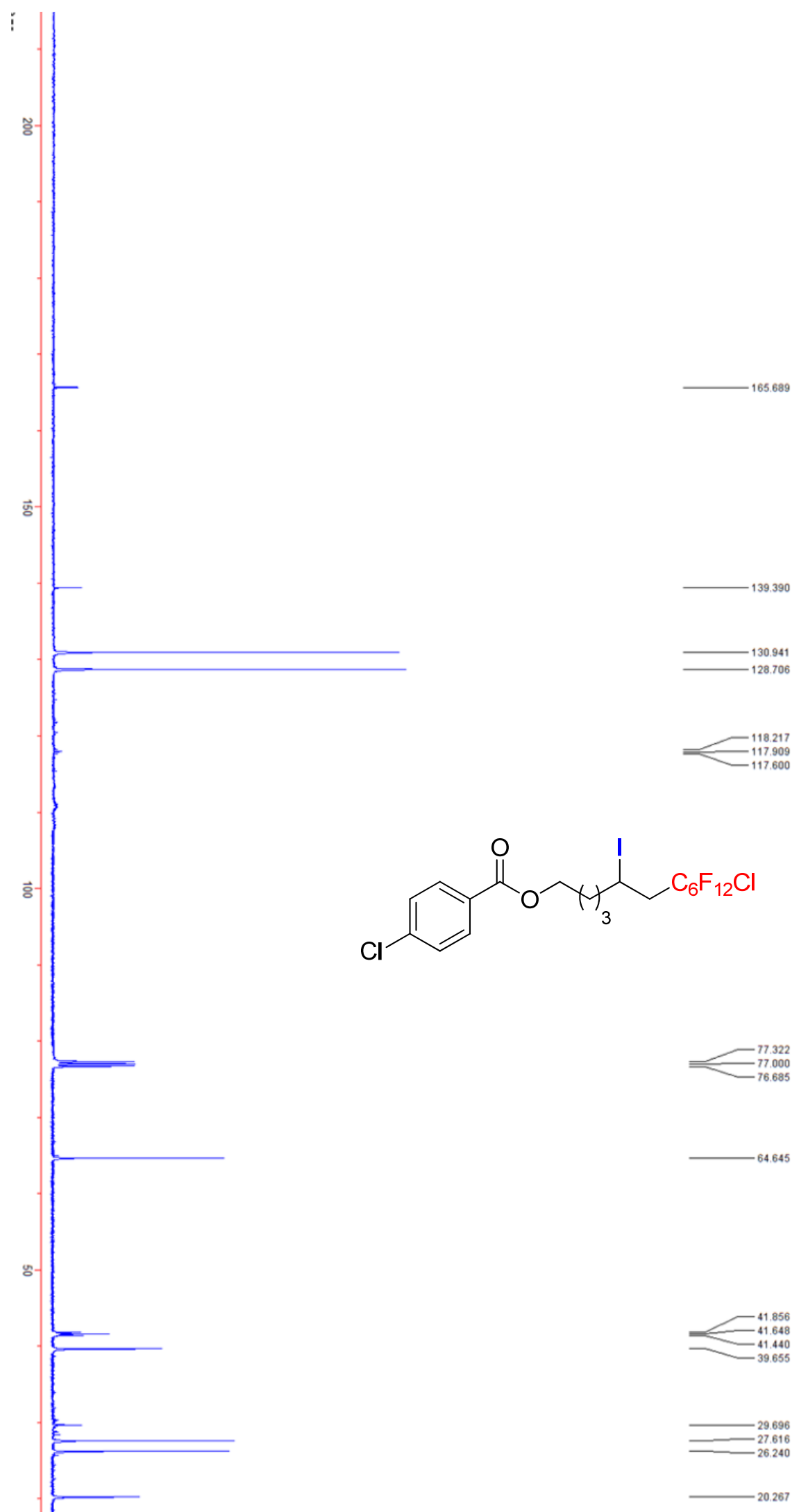




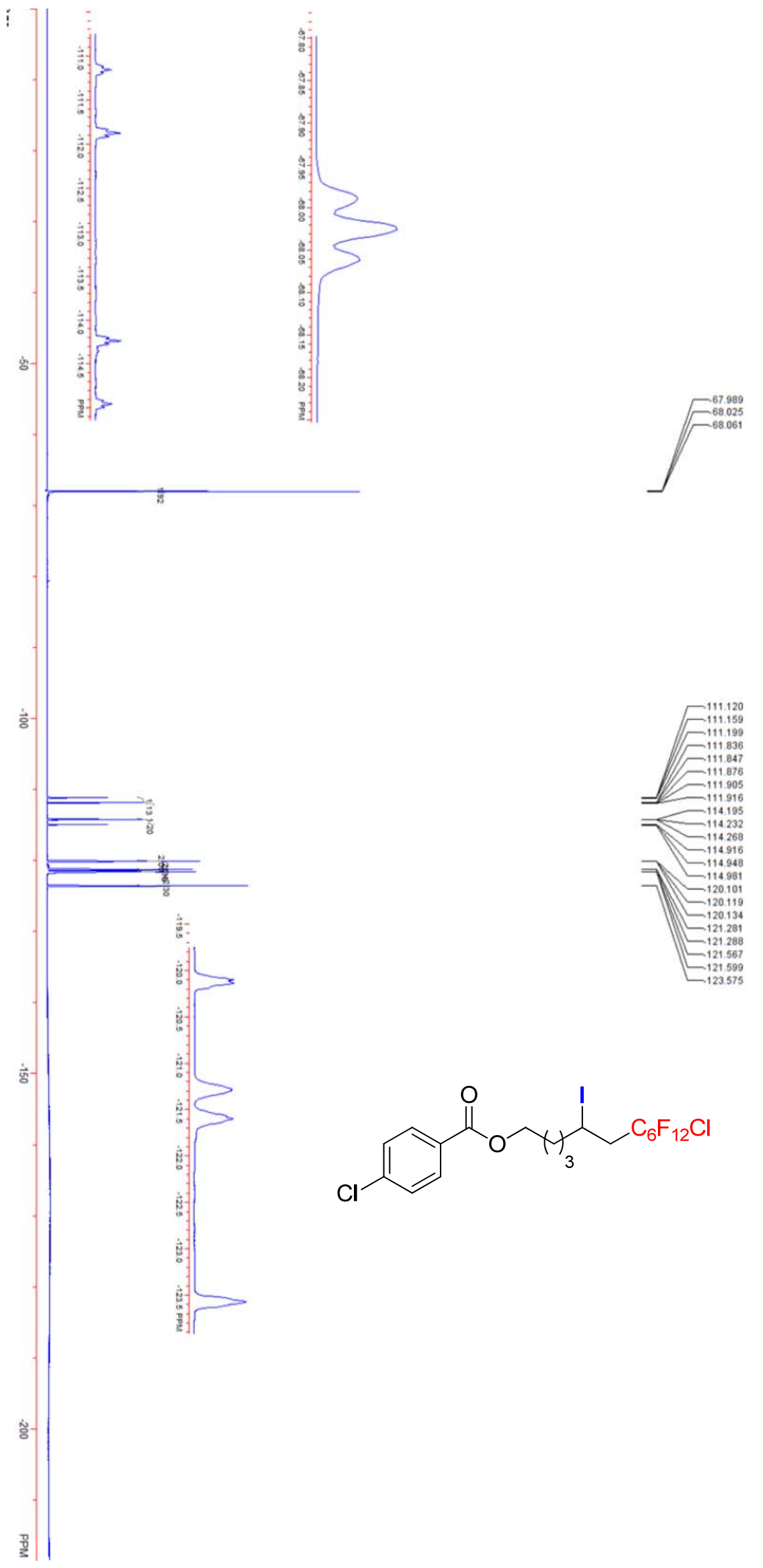




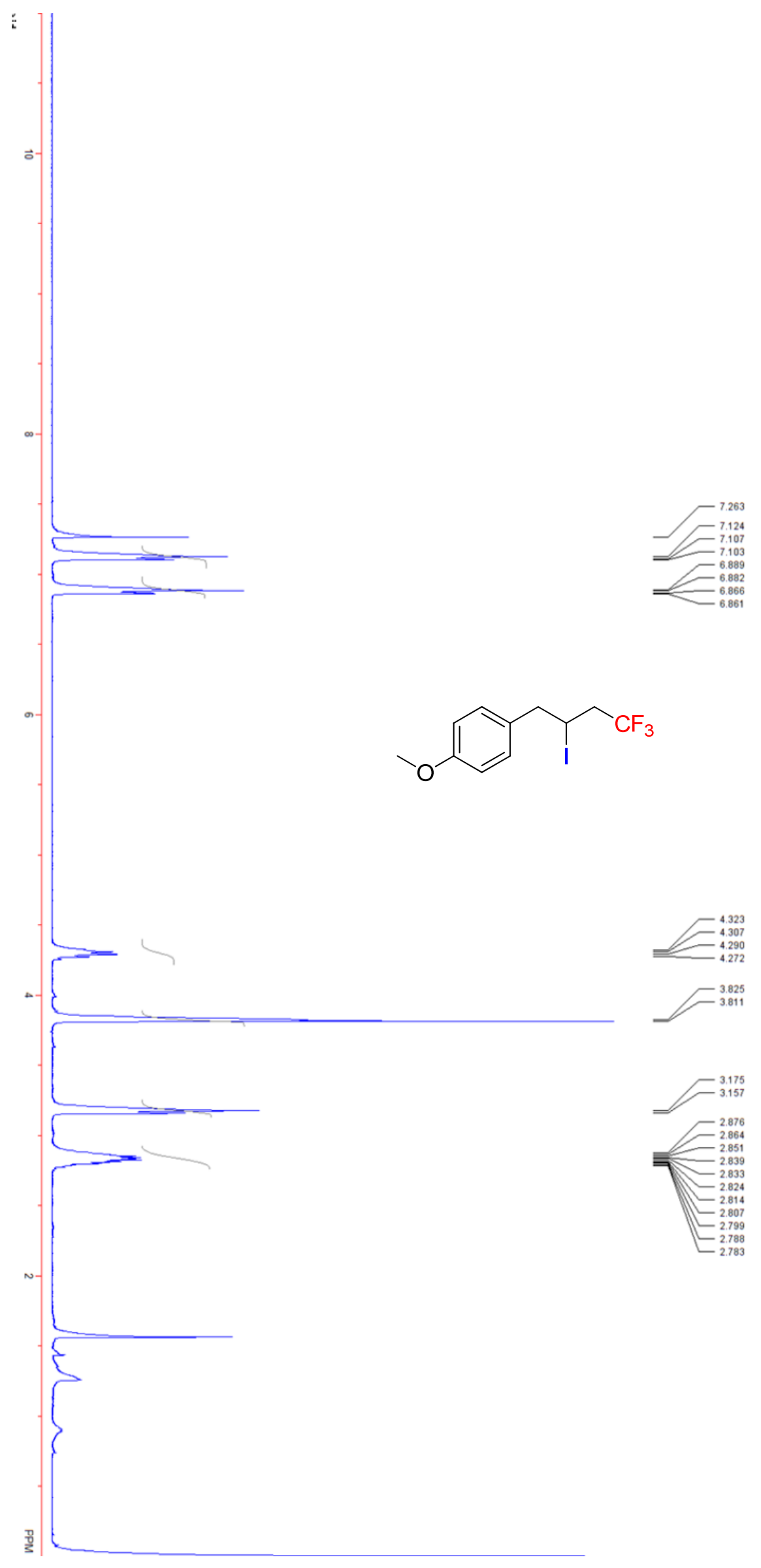




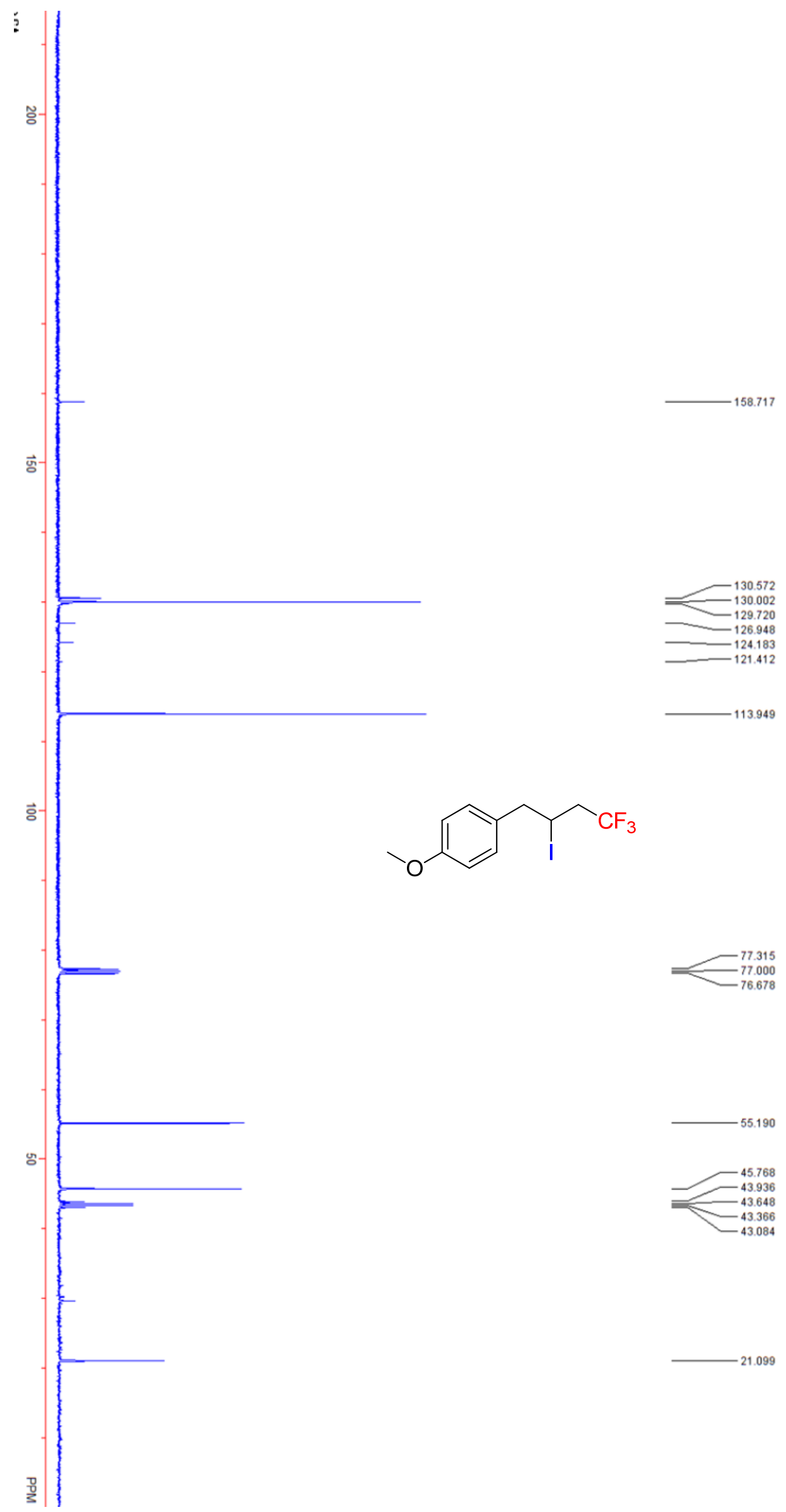




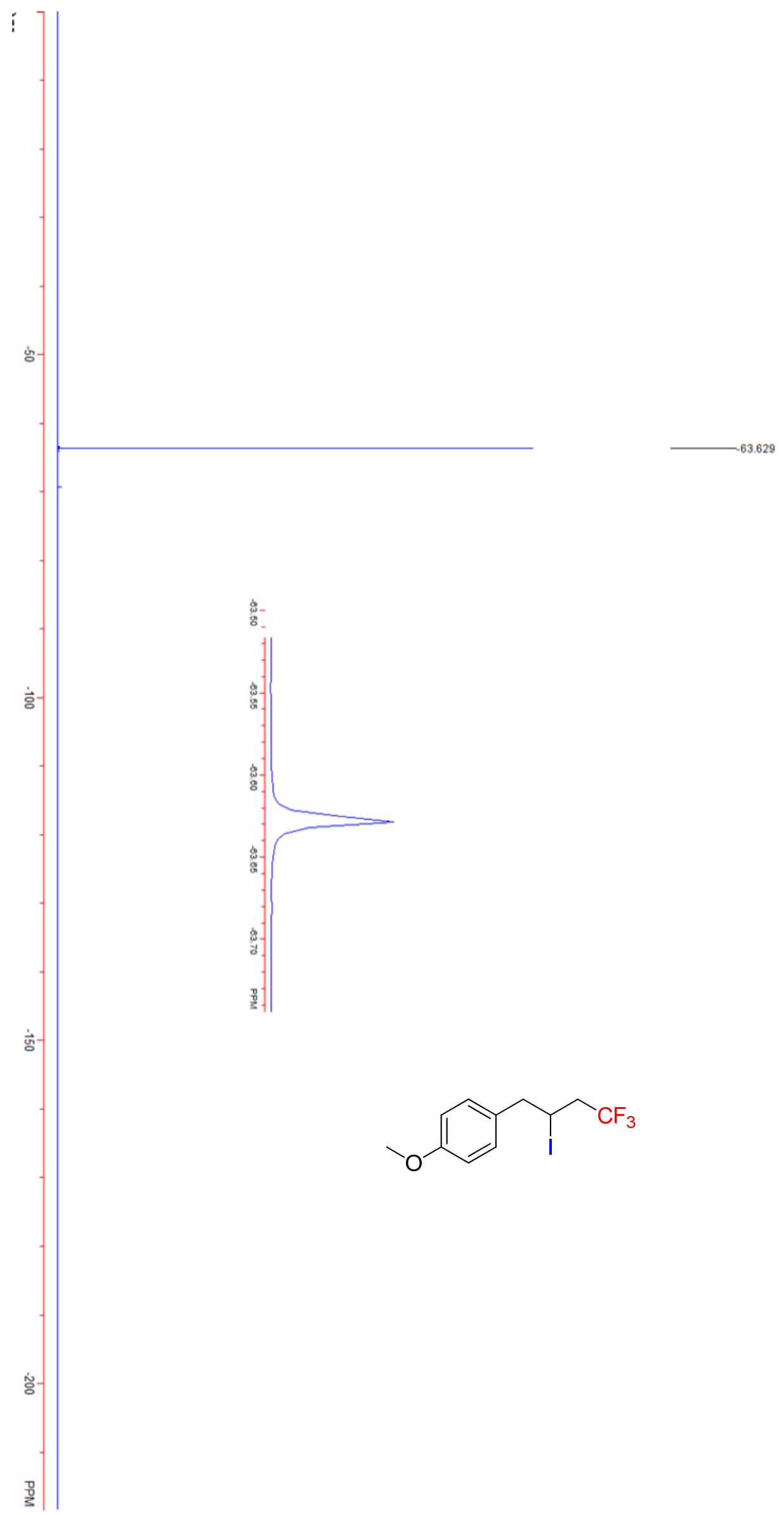




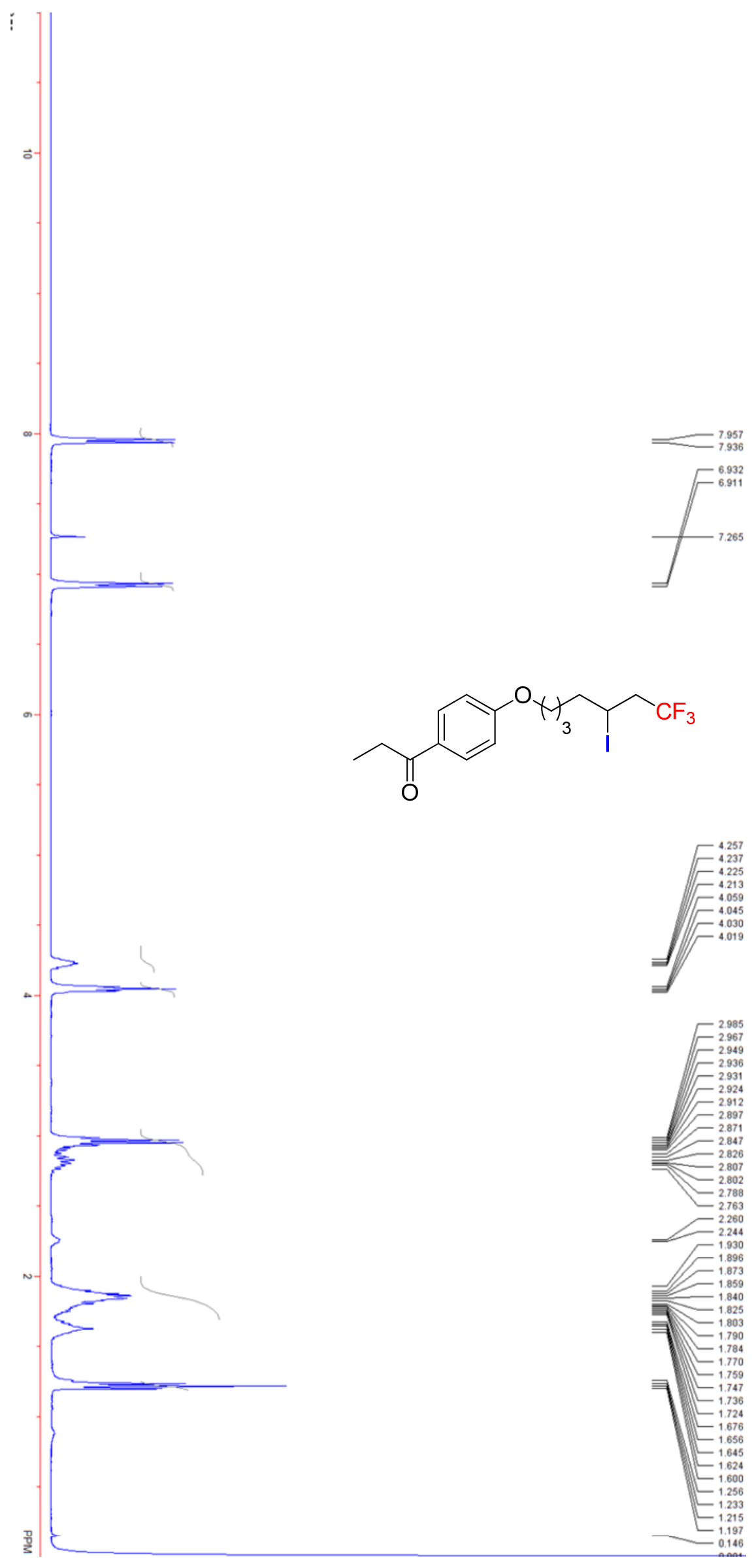



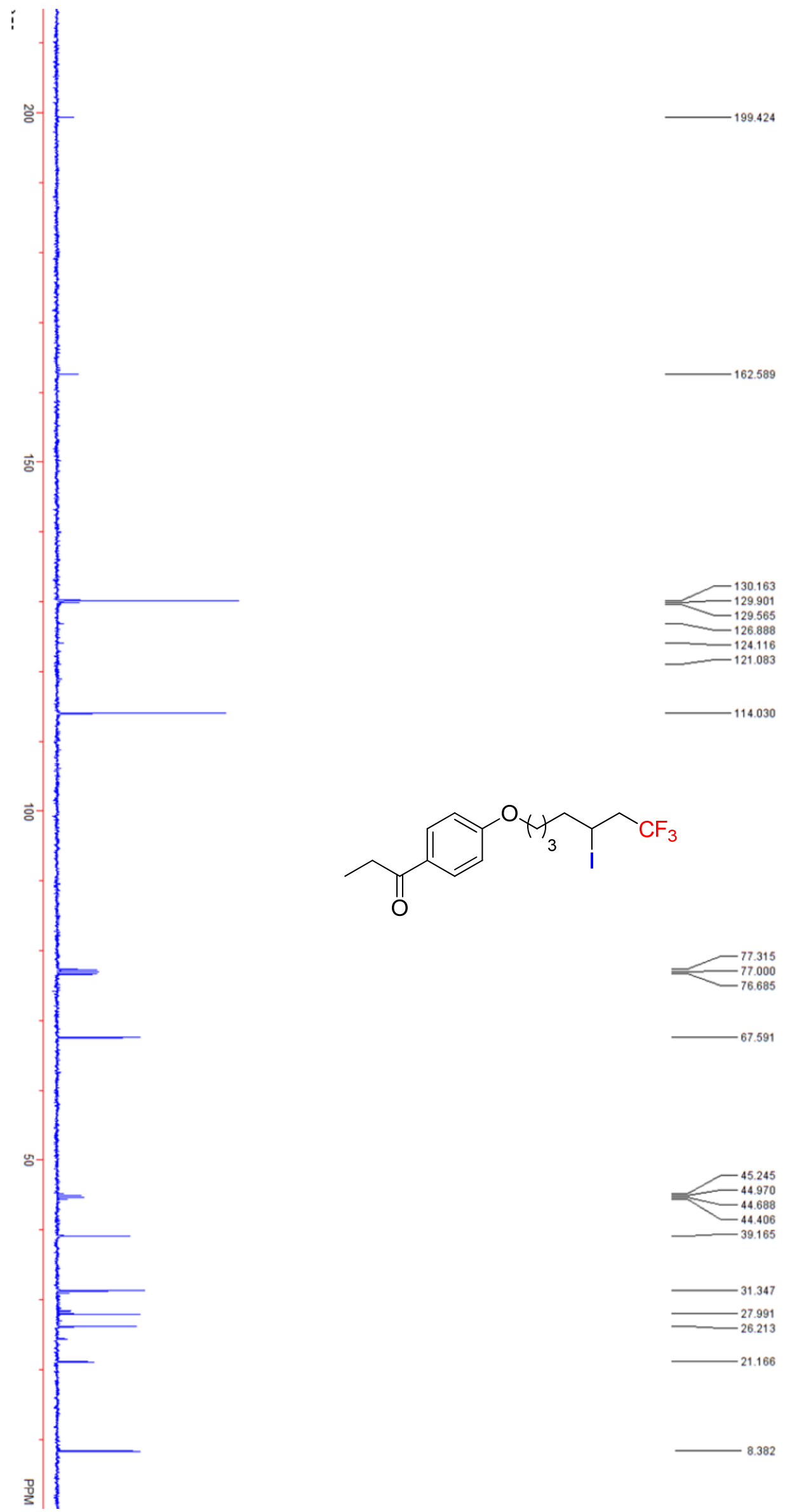


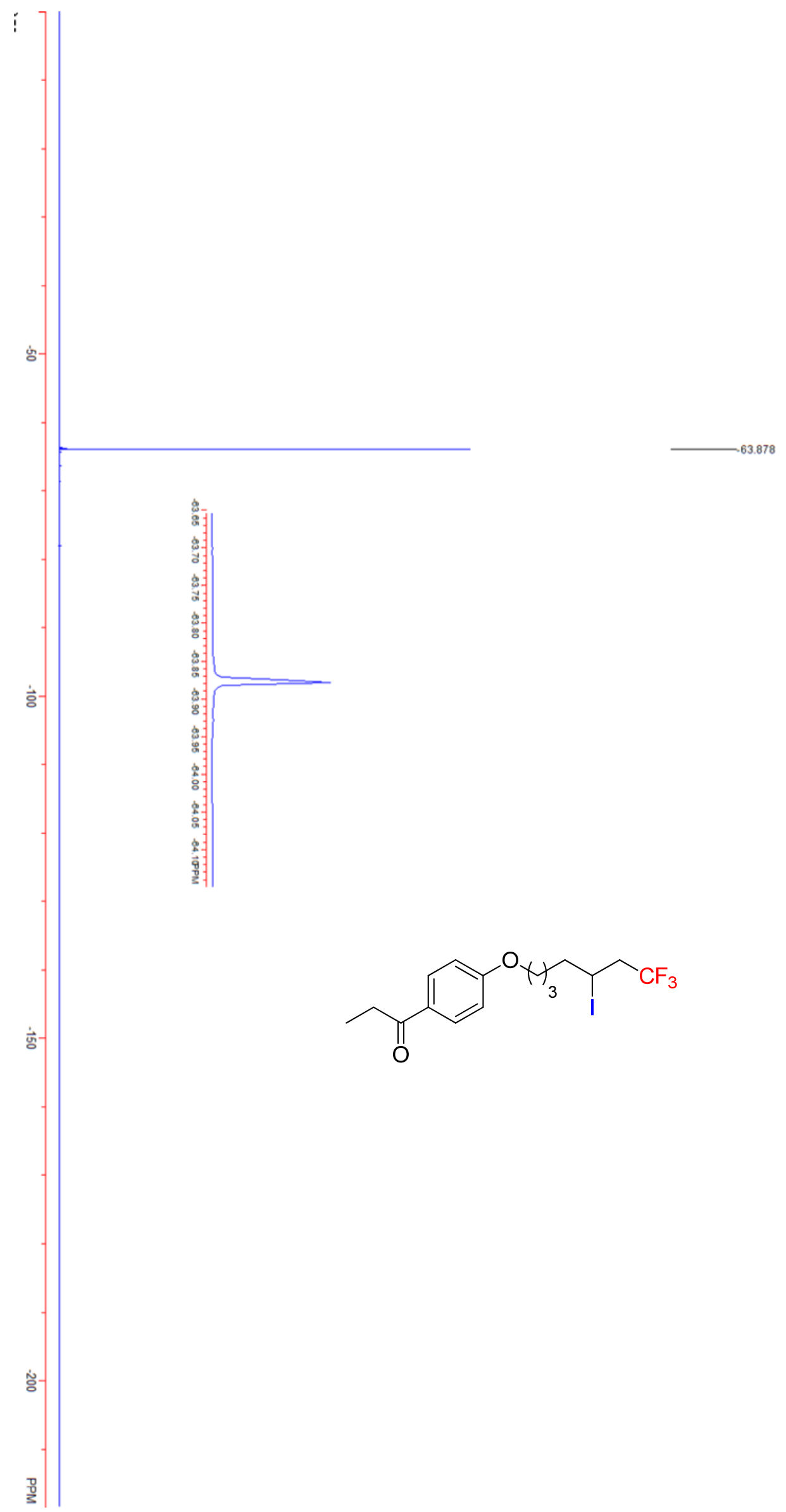




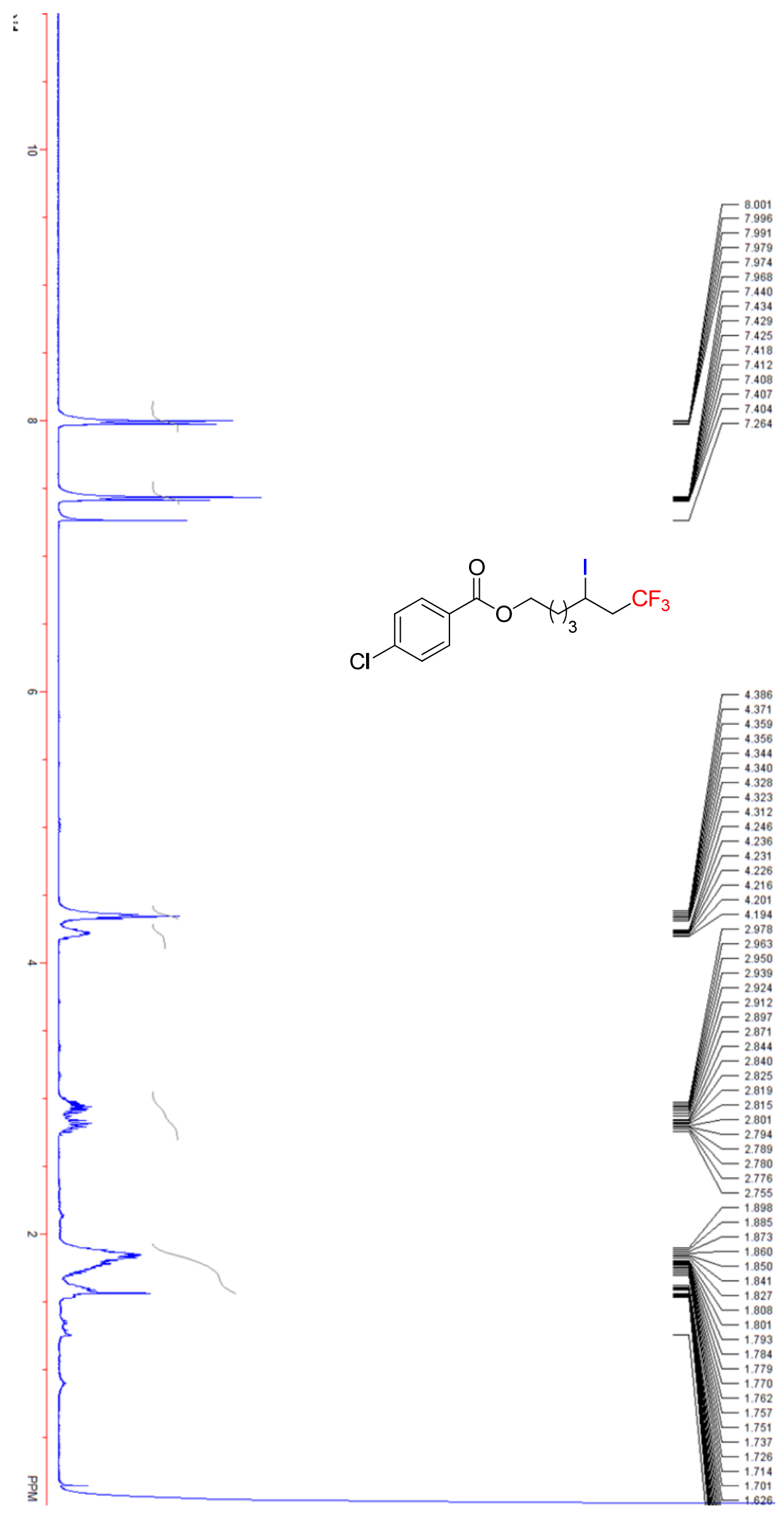




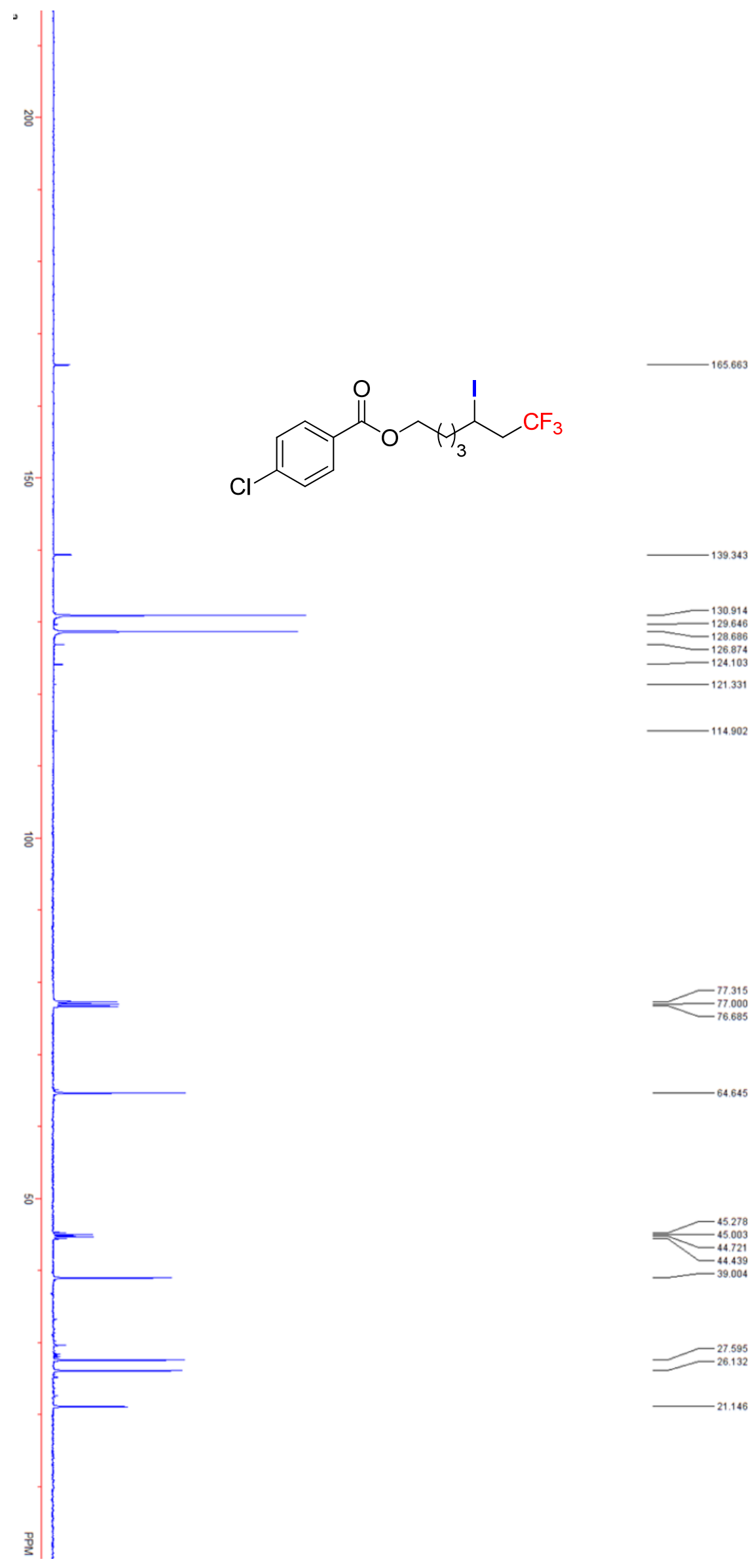




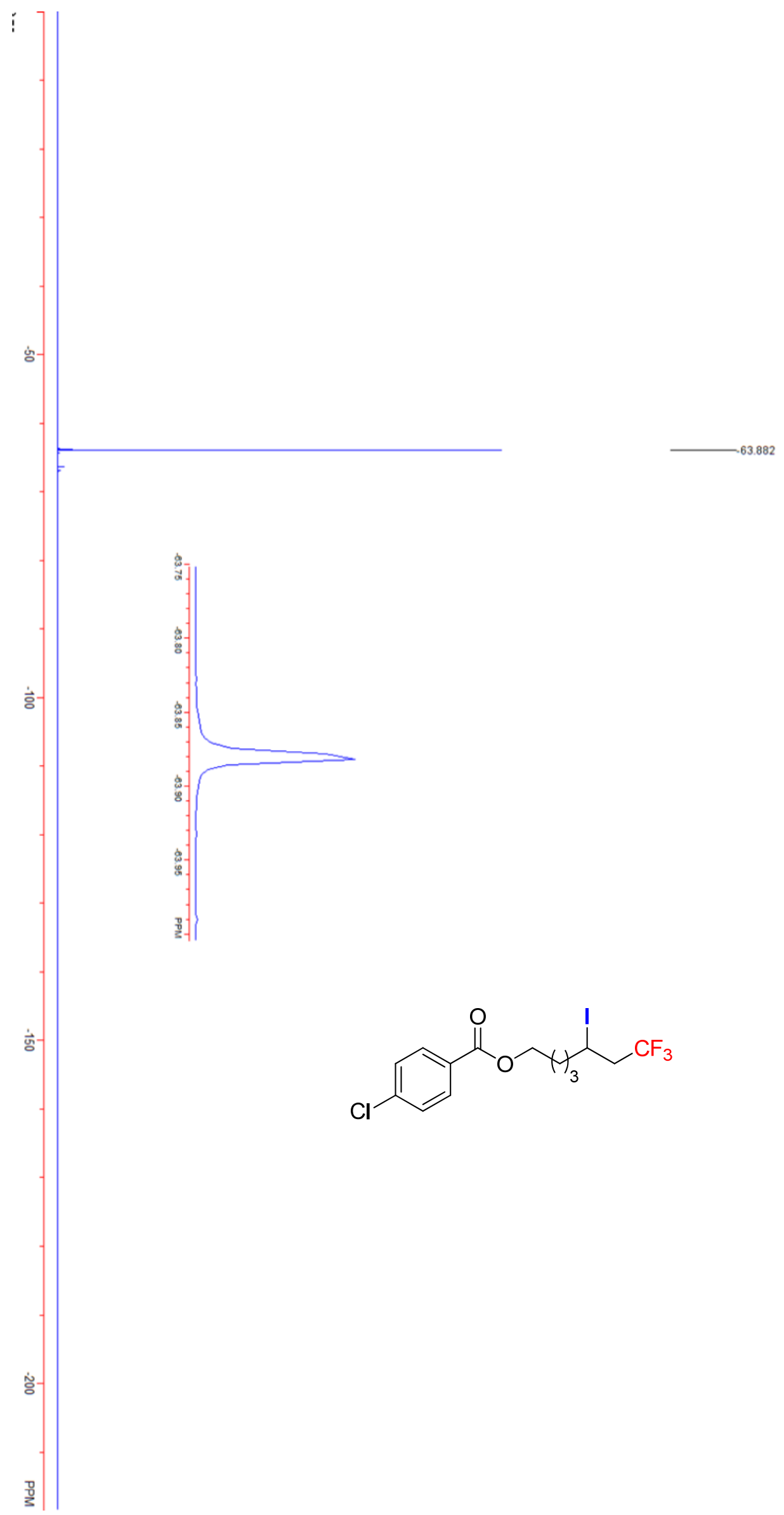




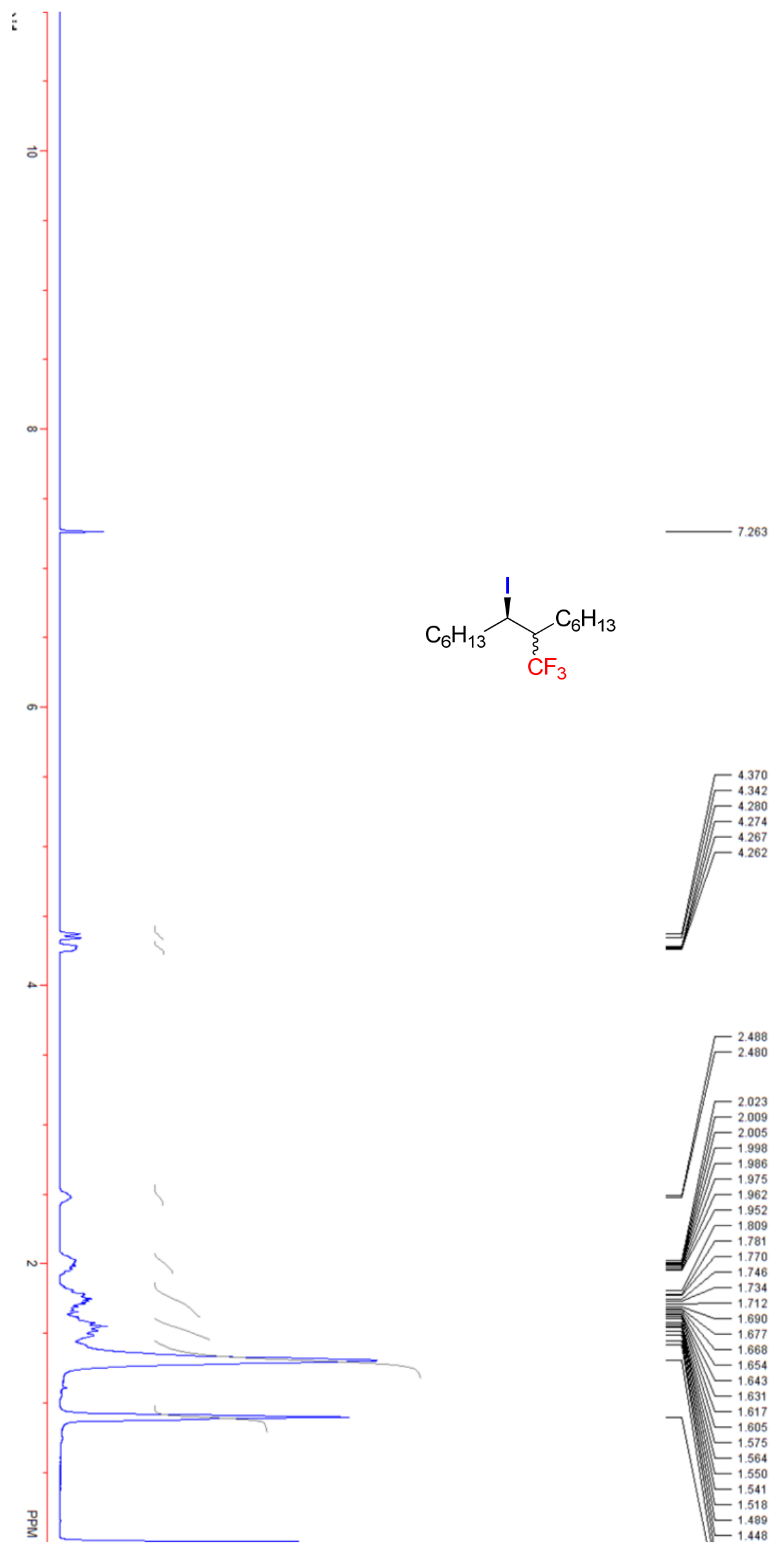



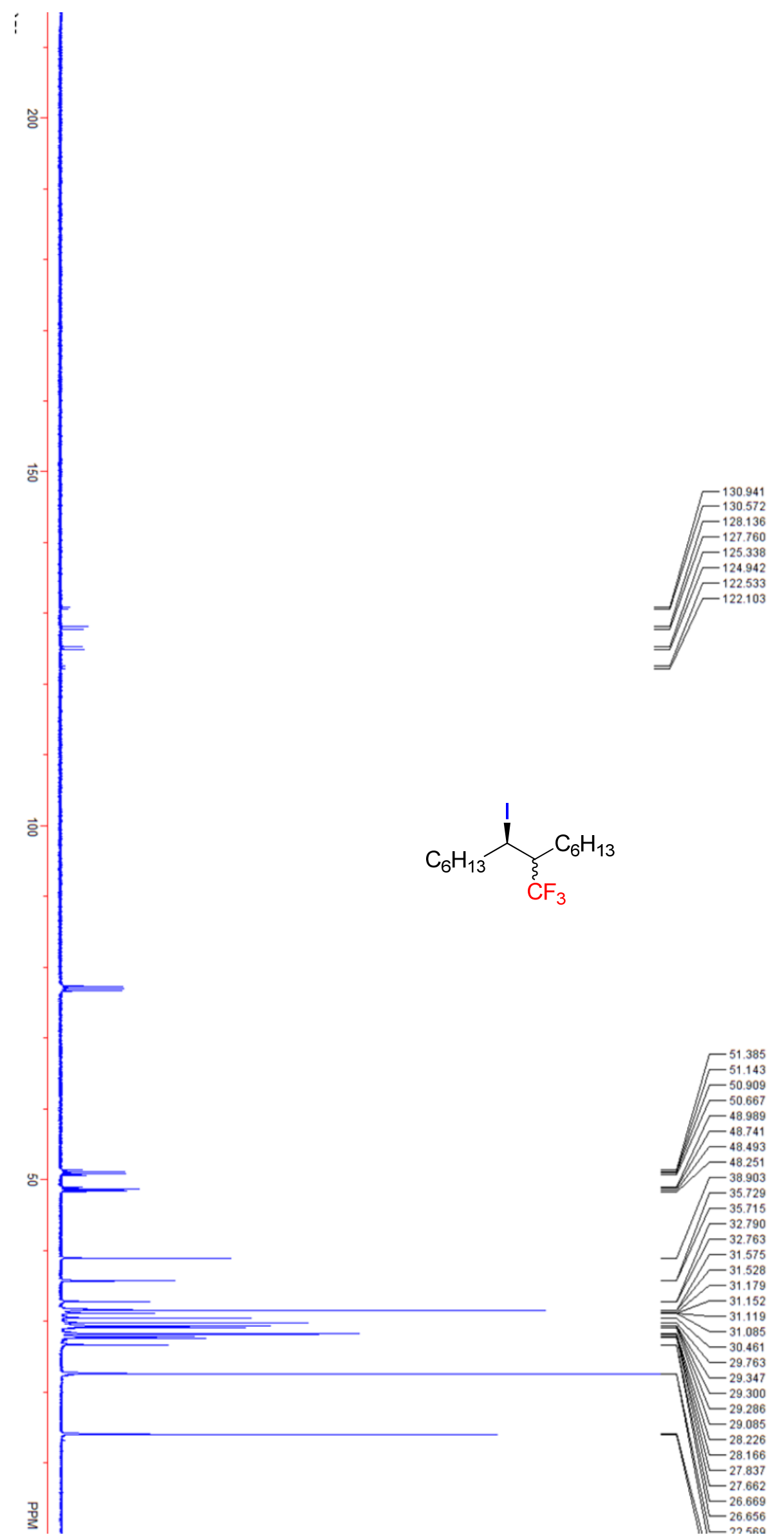


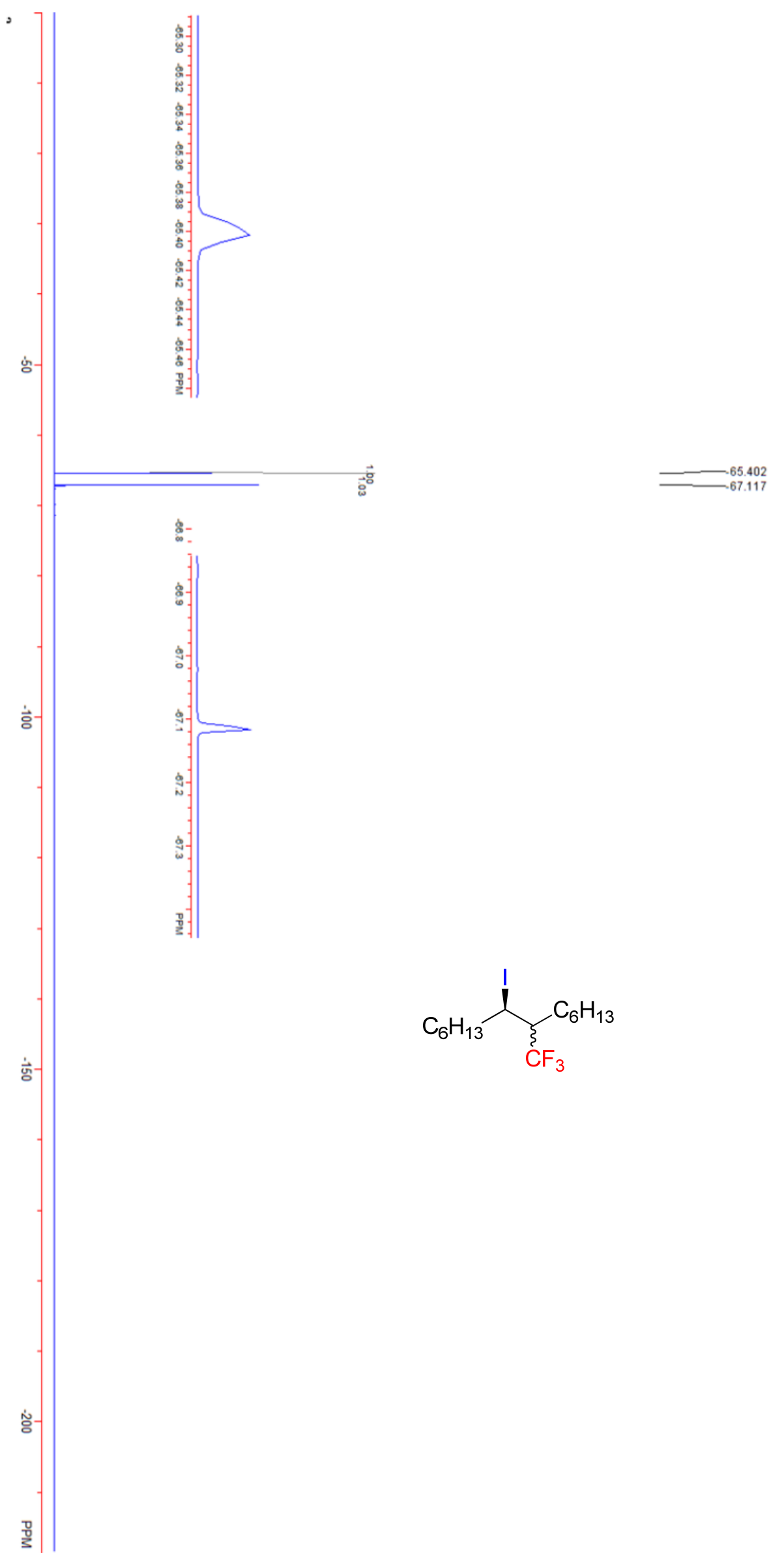




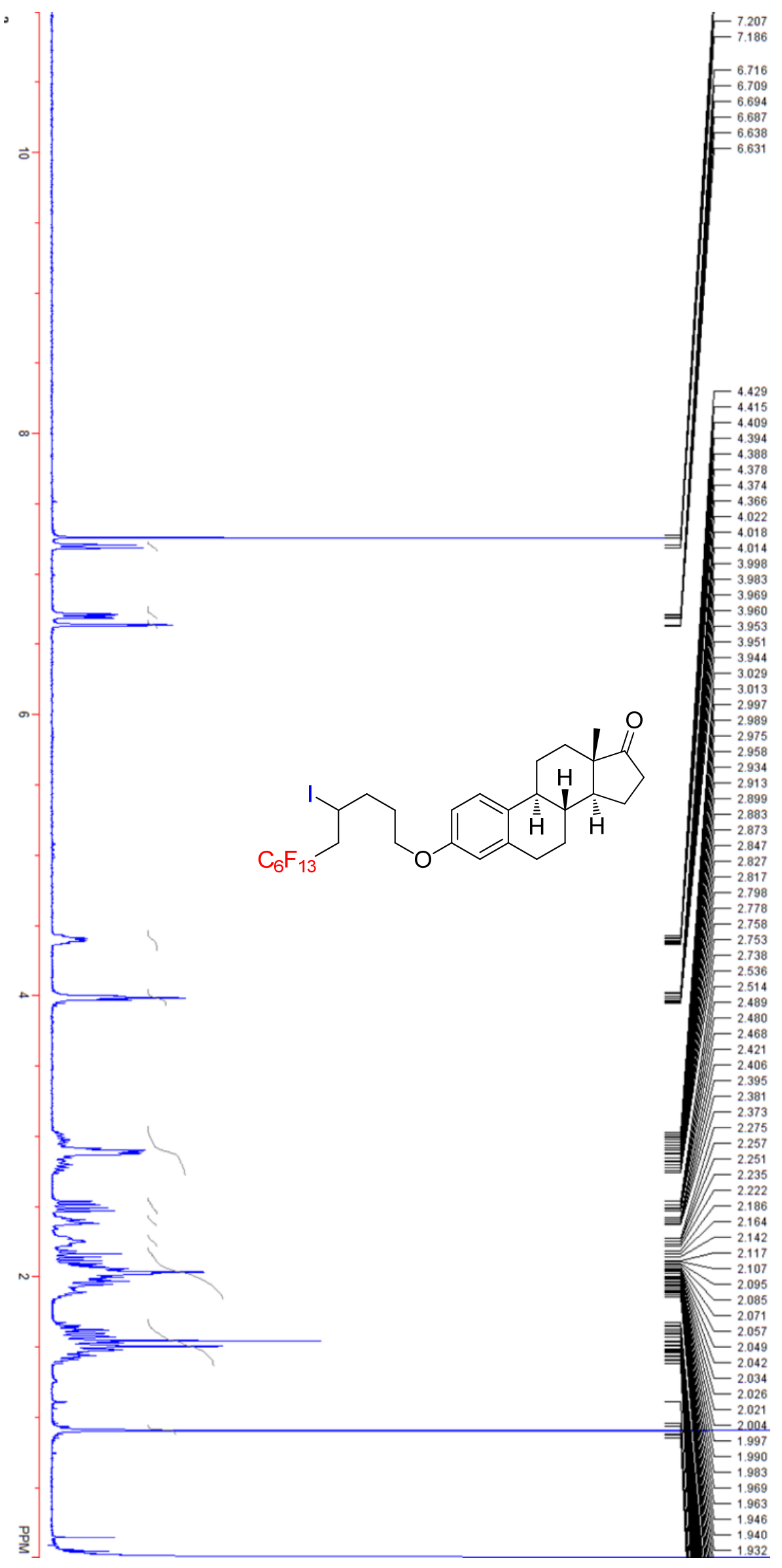

S199 


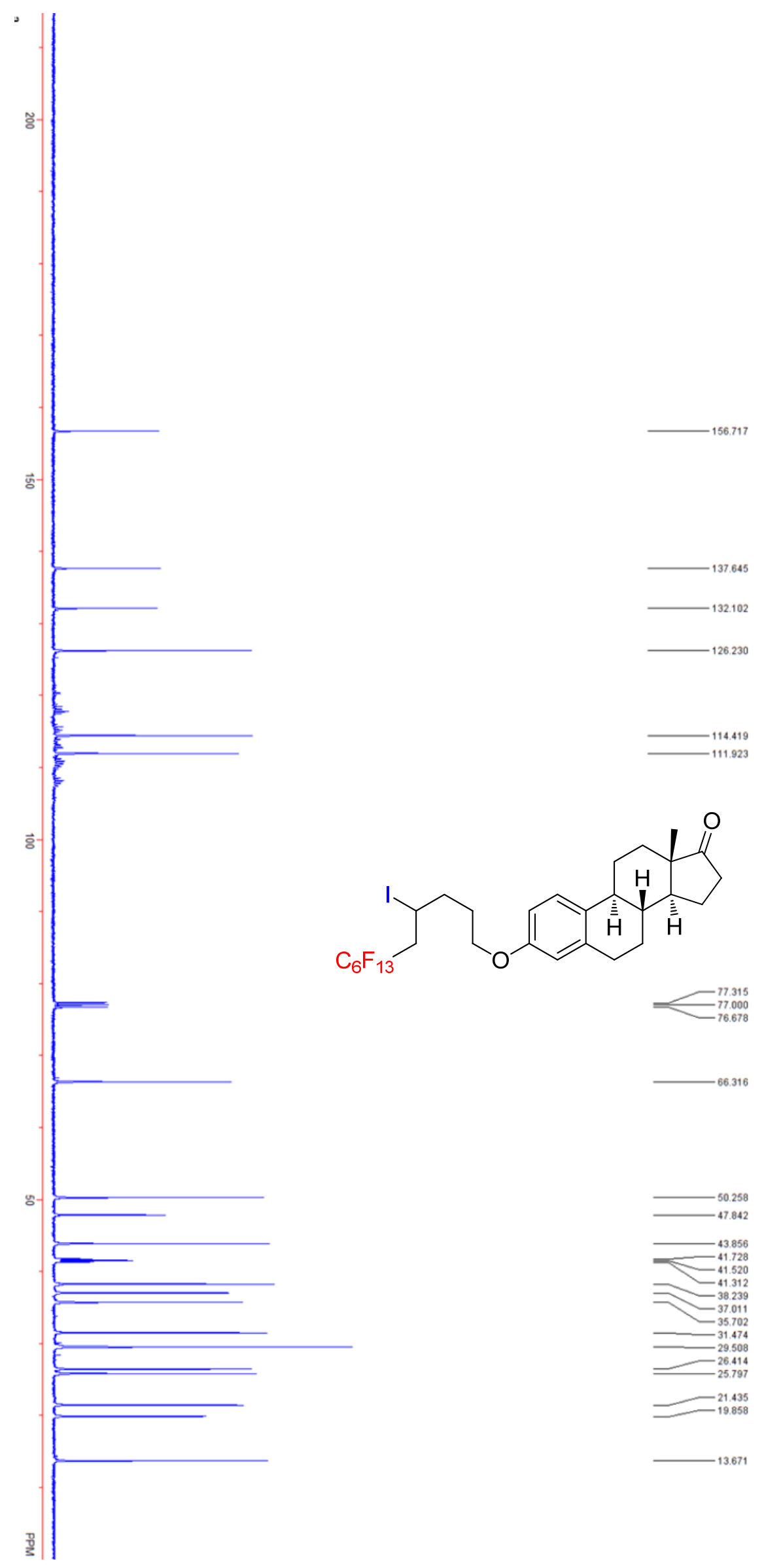




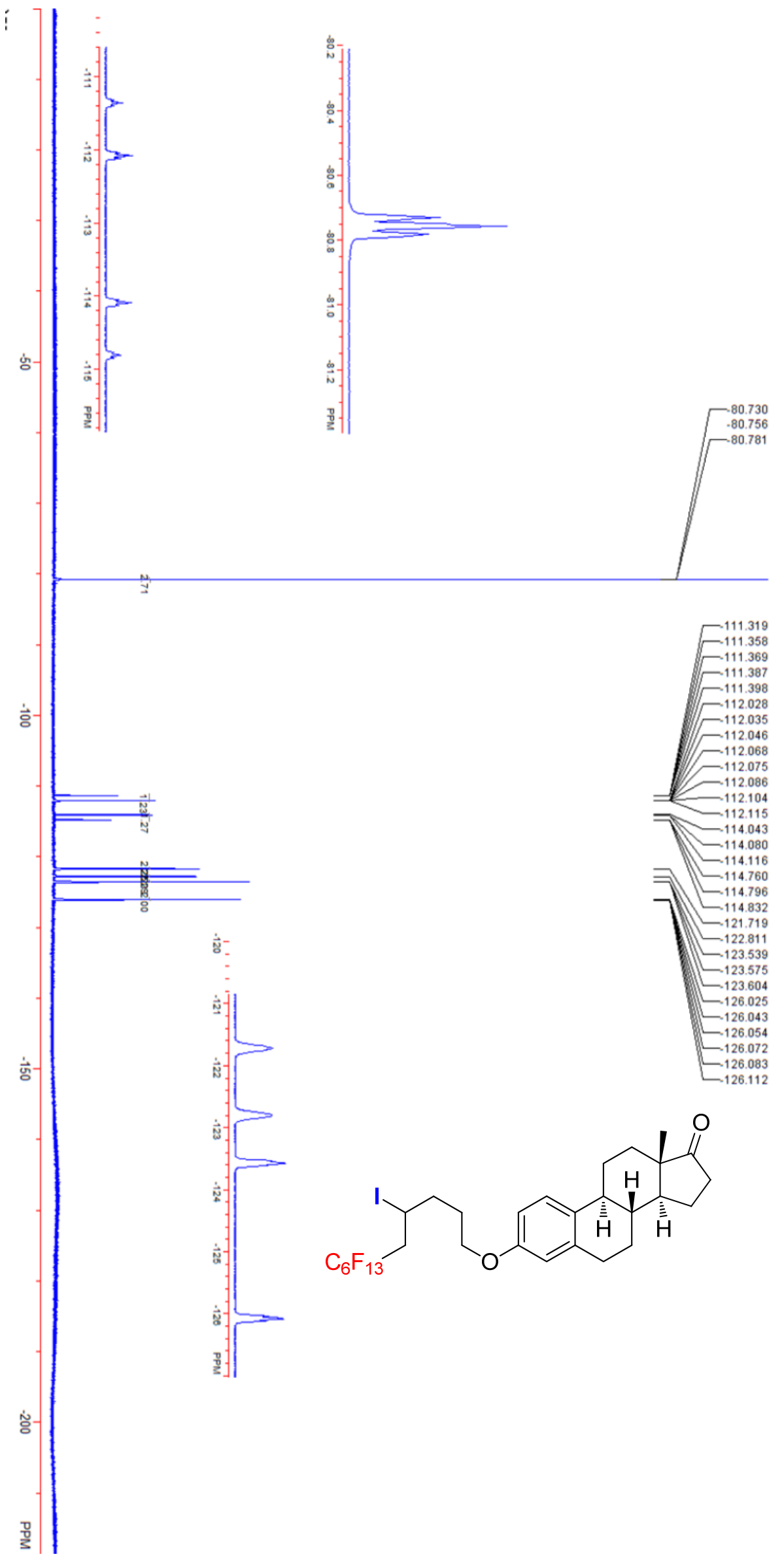




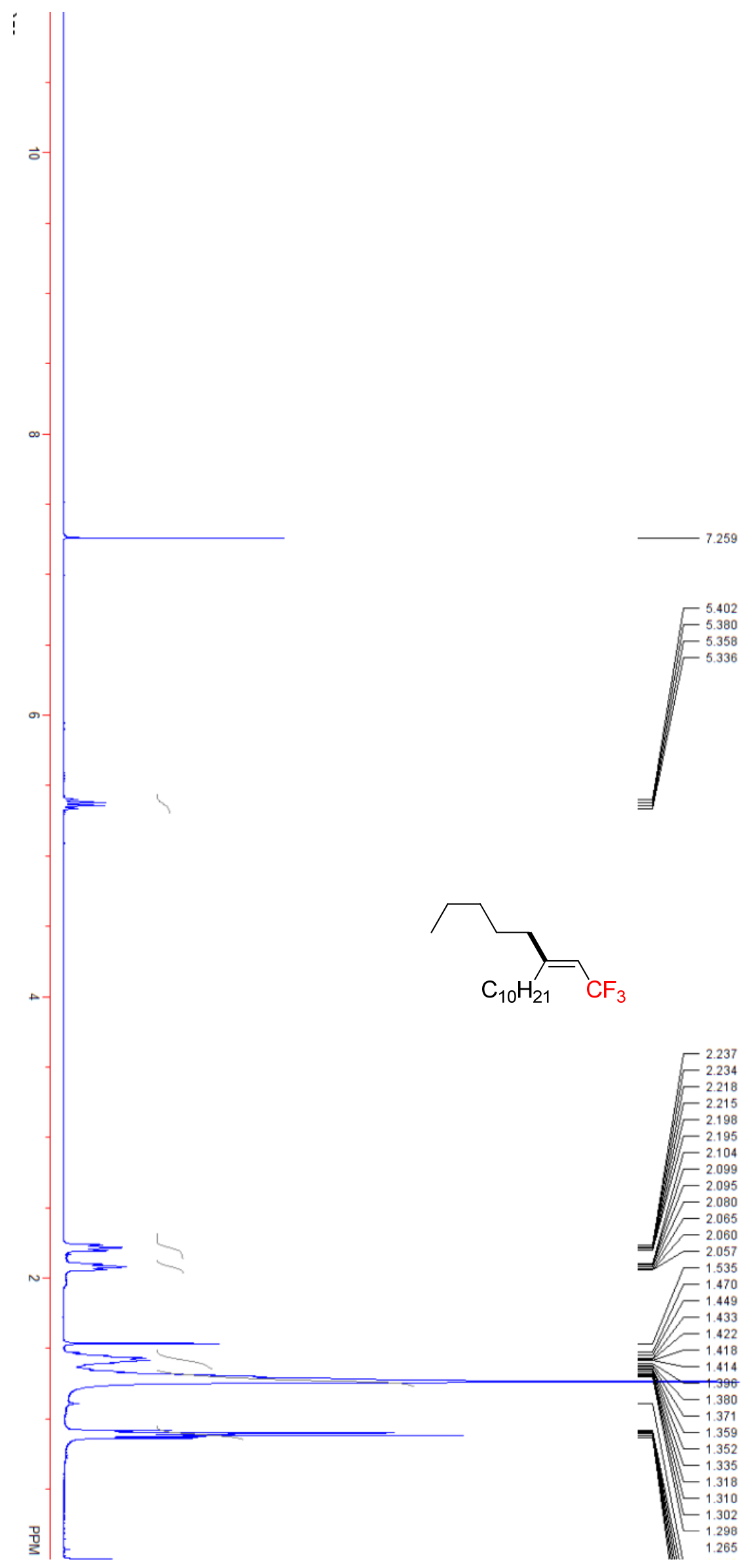




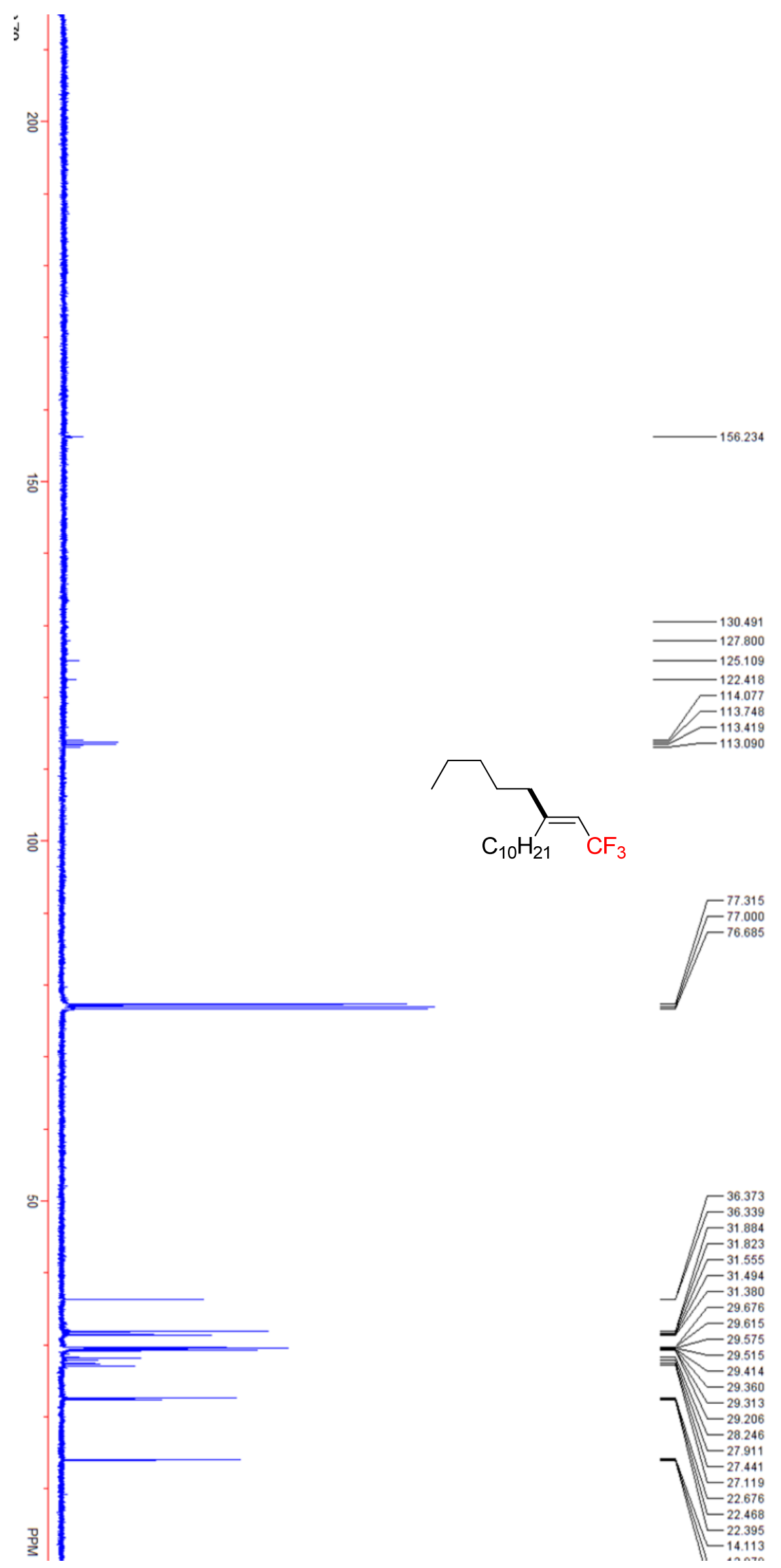




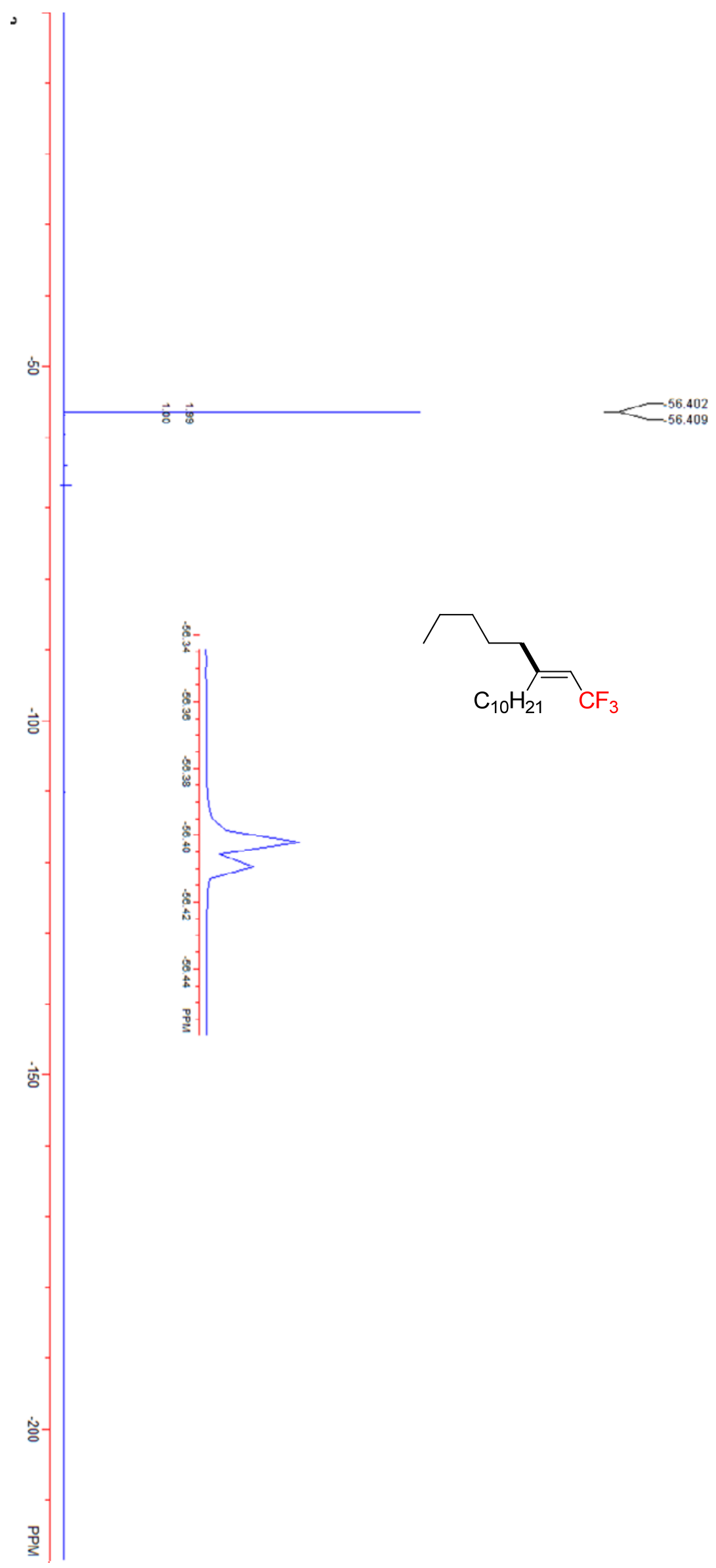




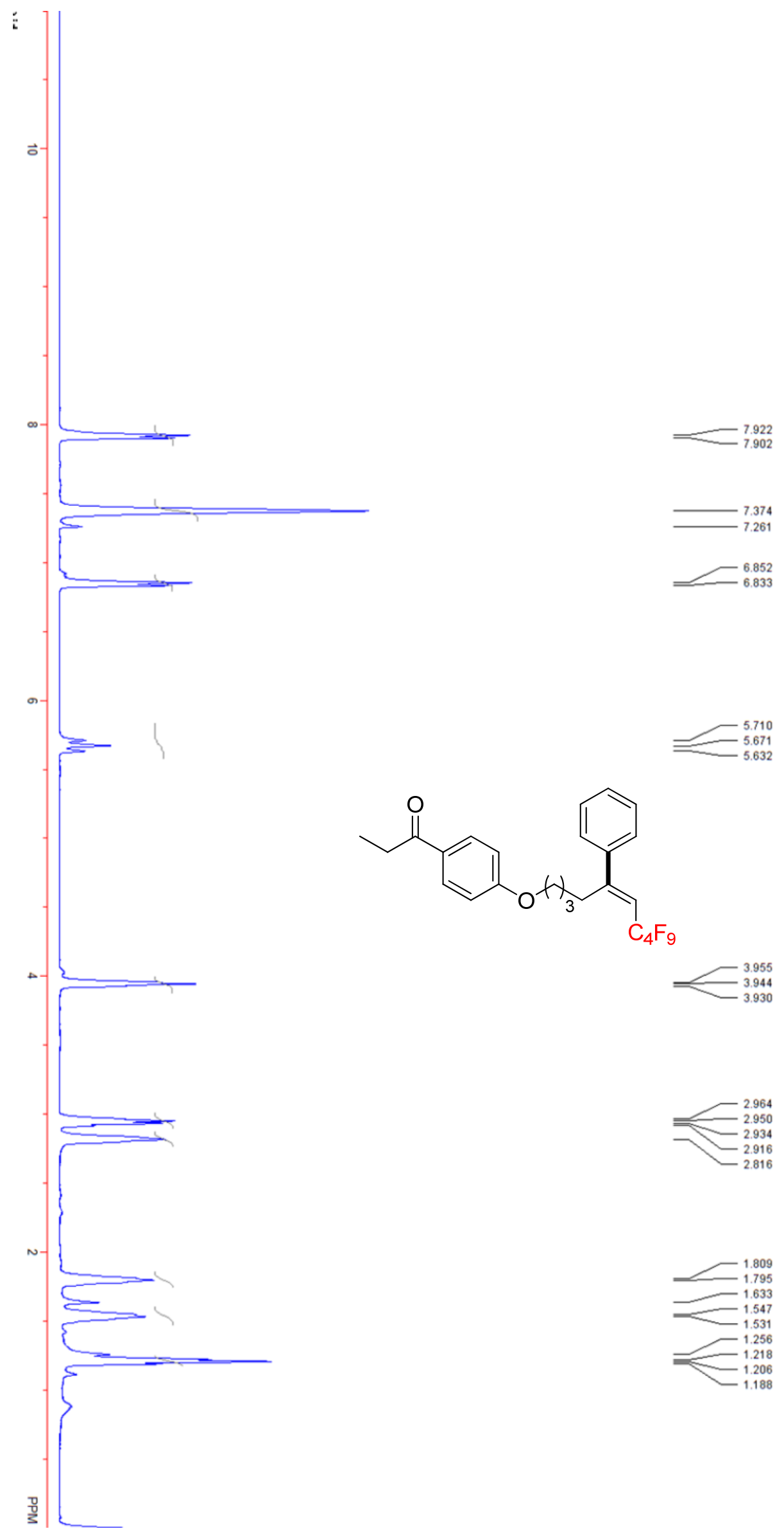



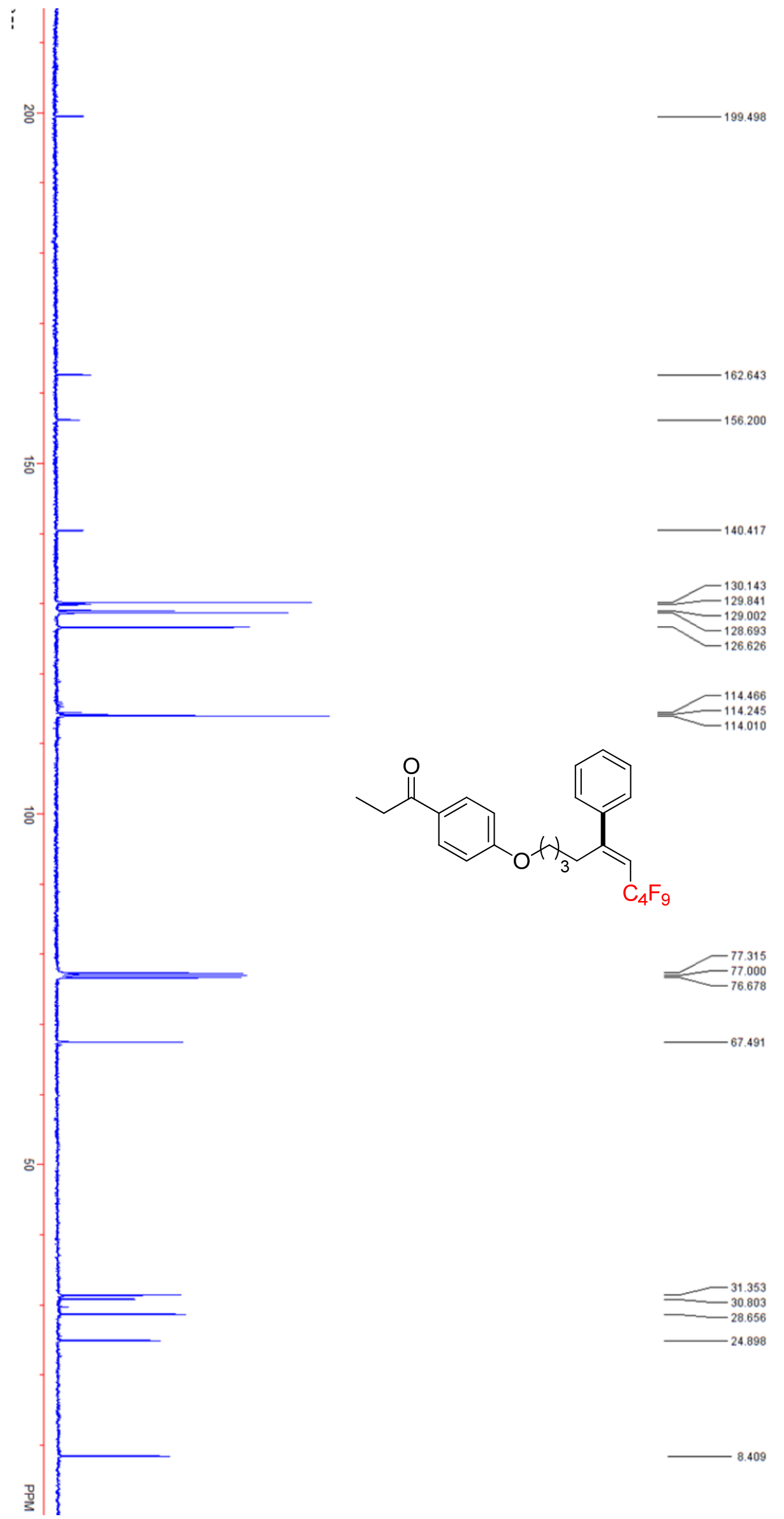


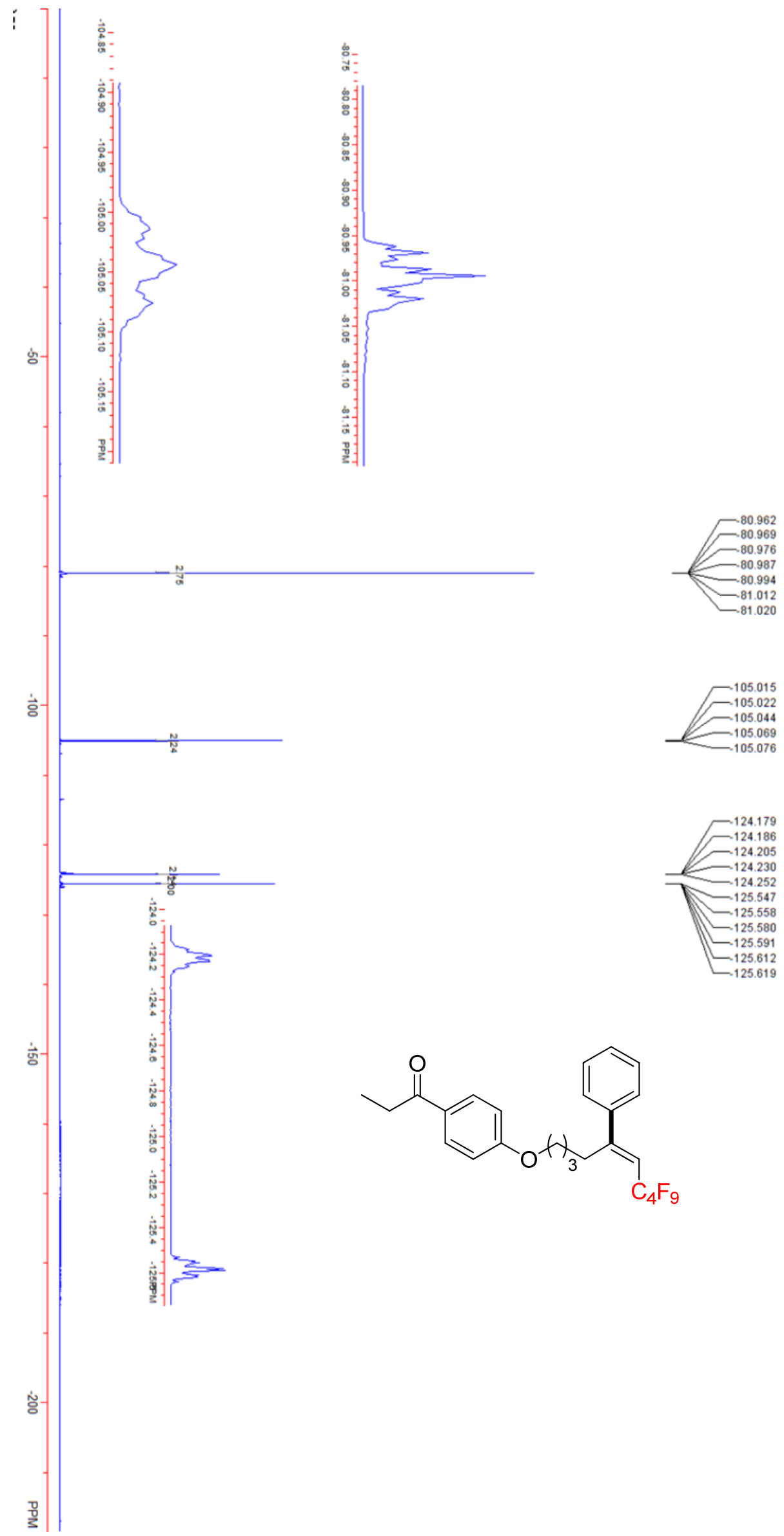




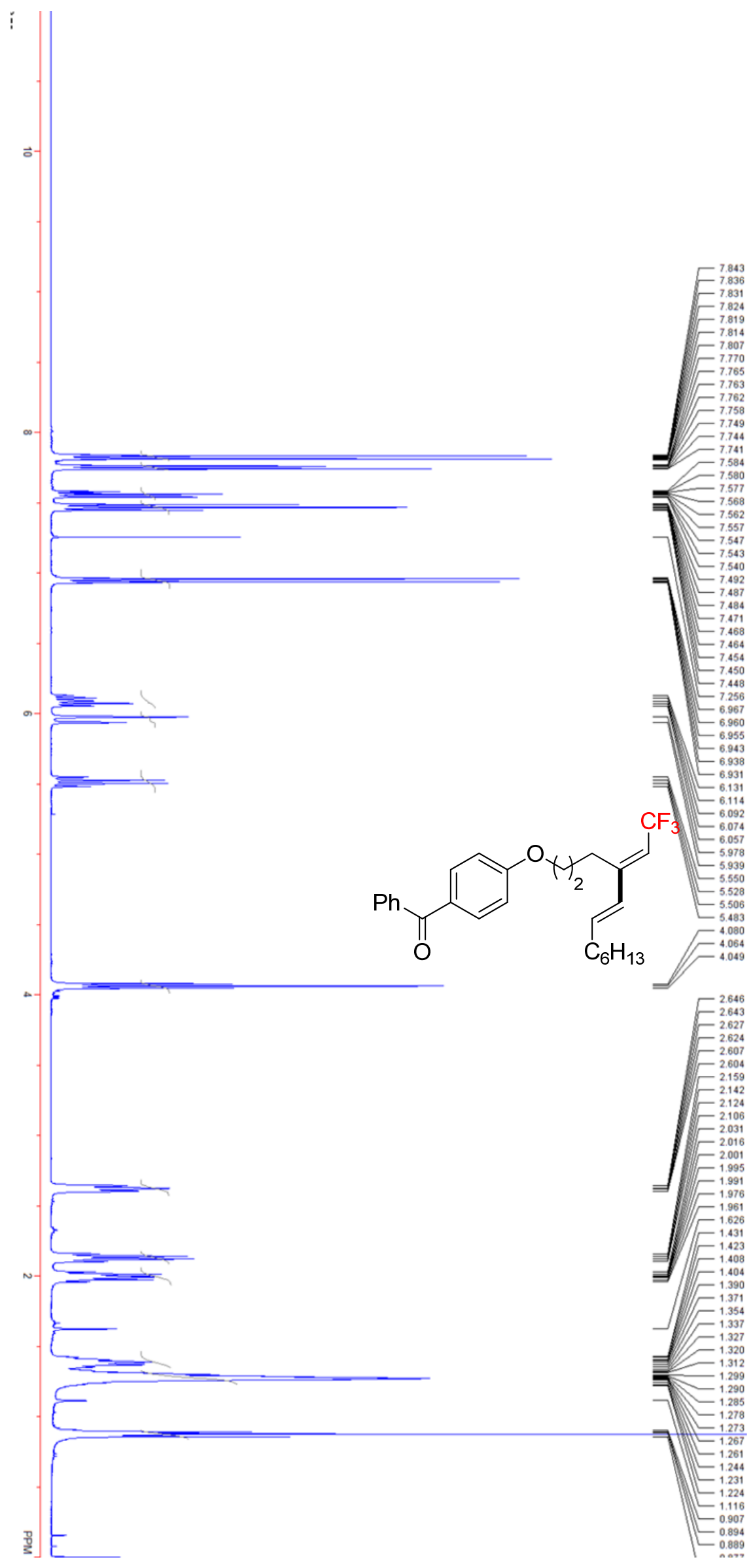




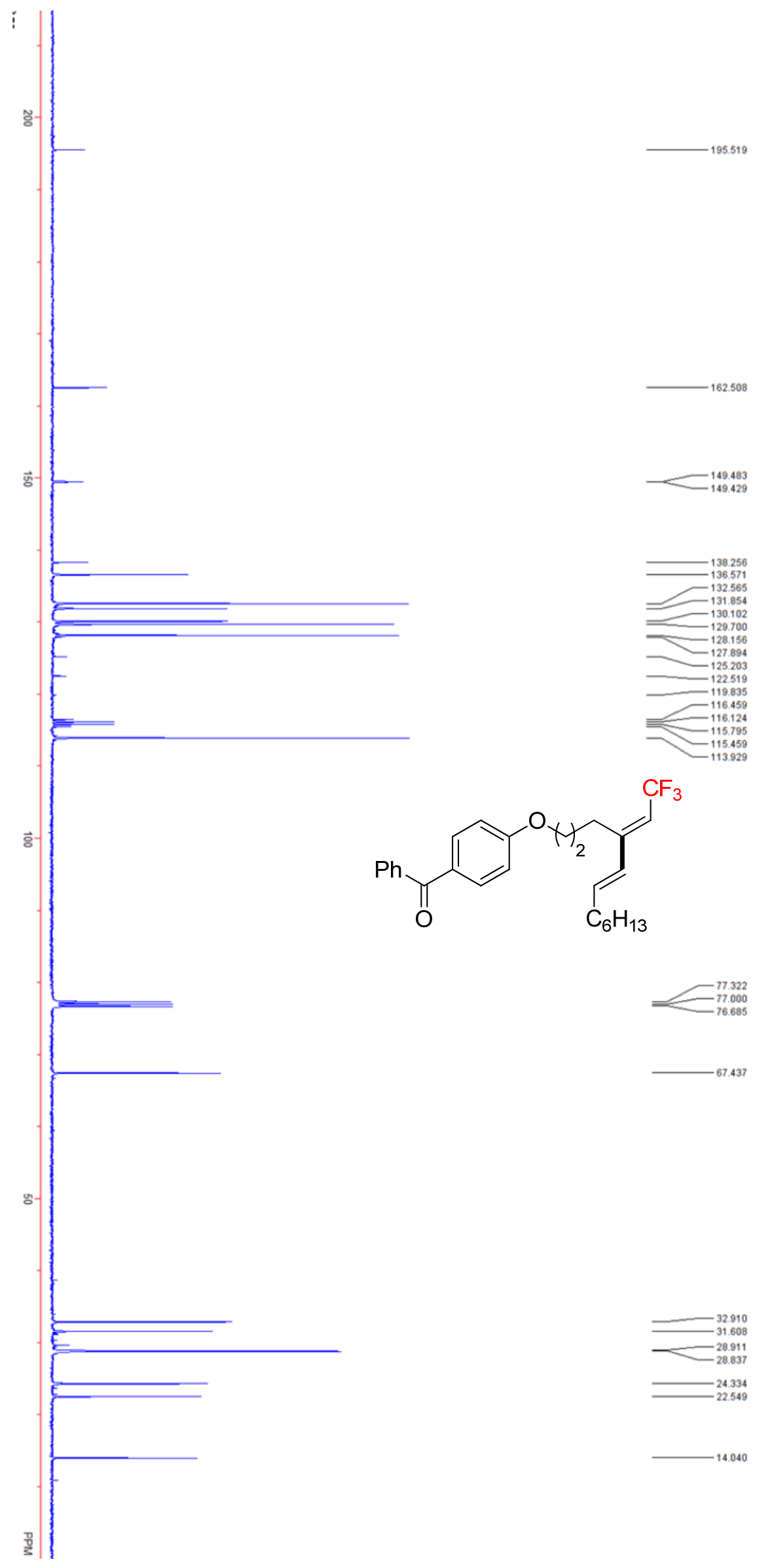




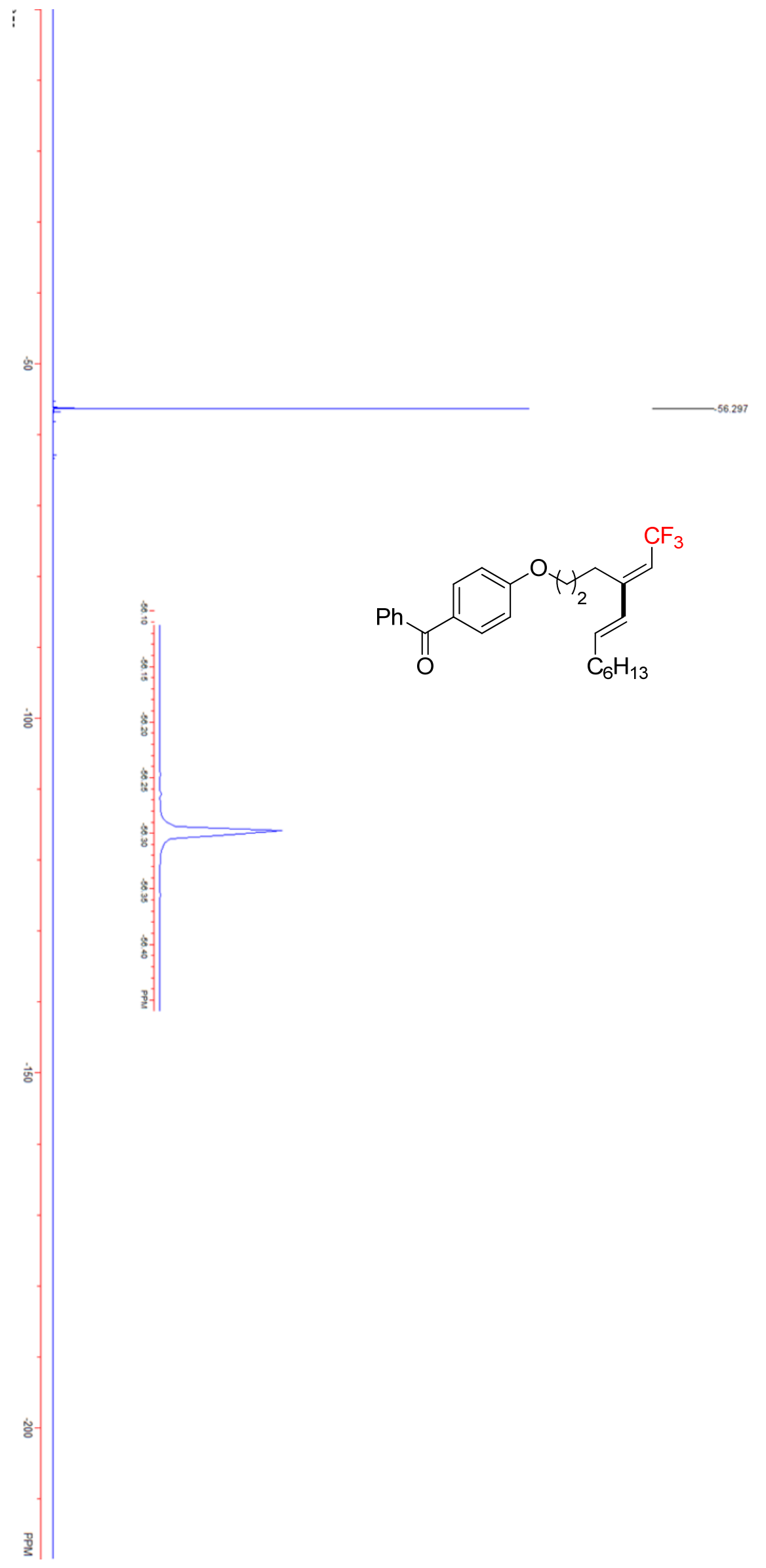




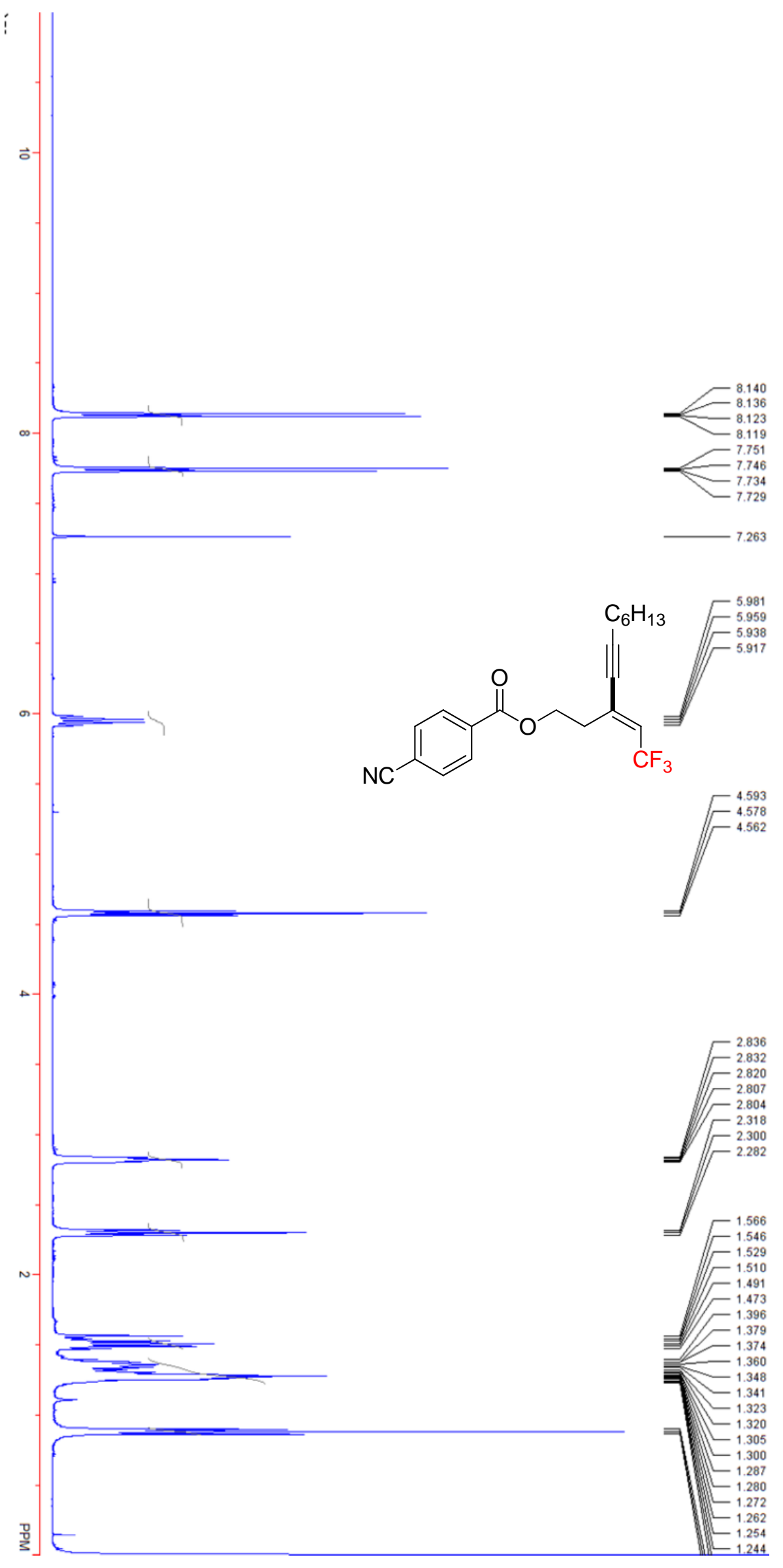



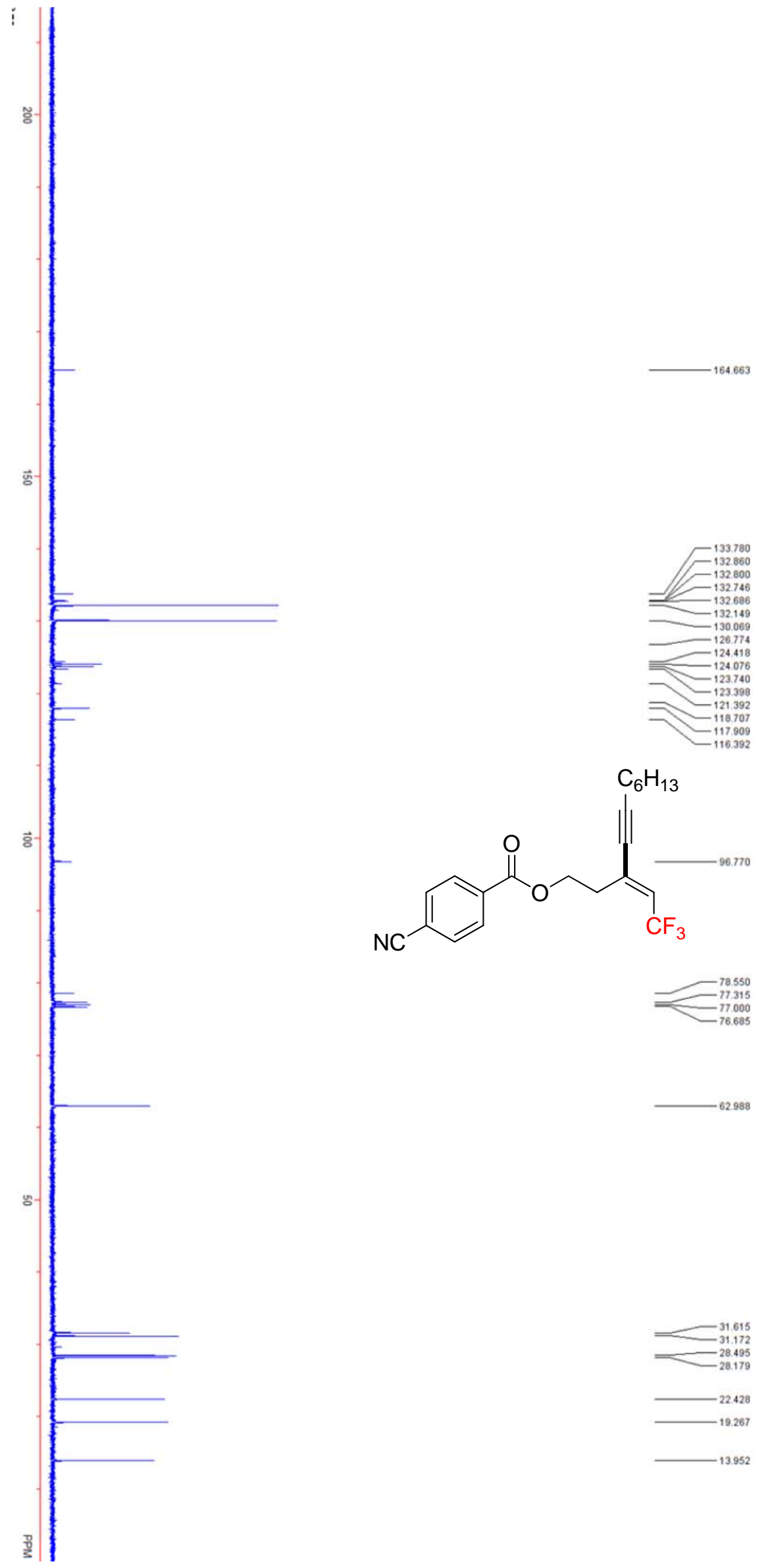


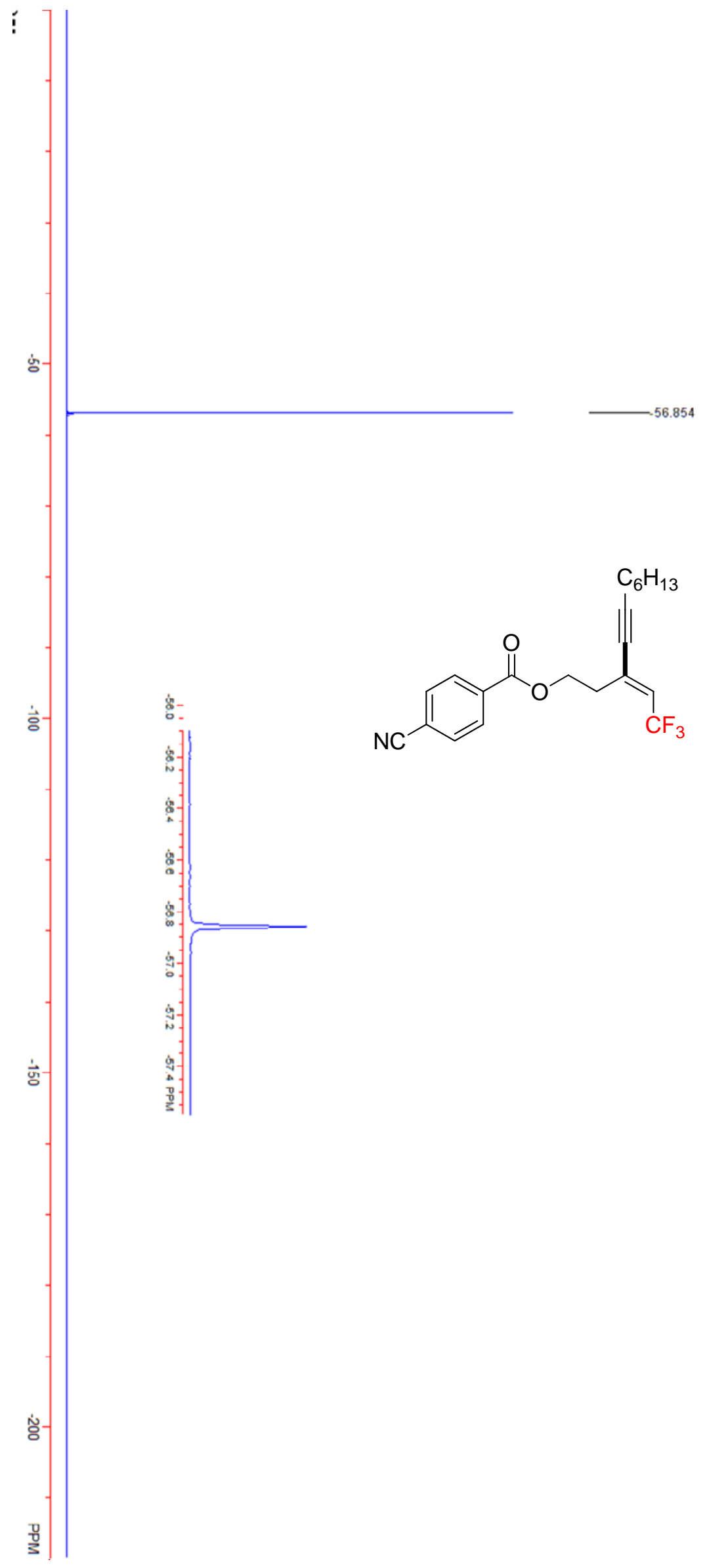




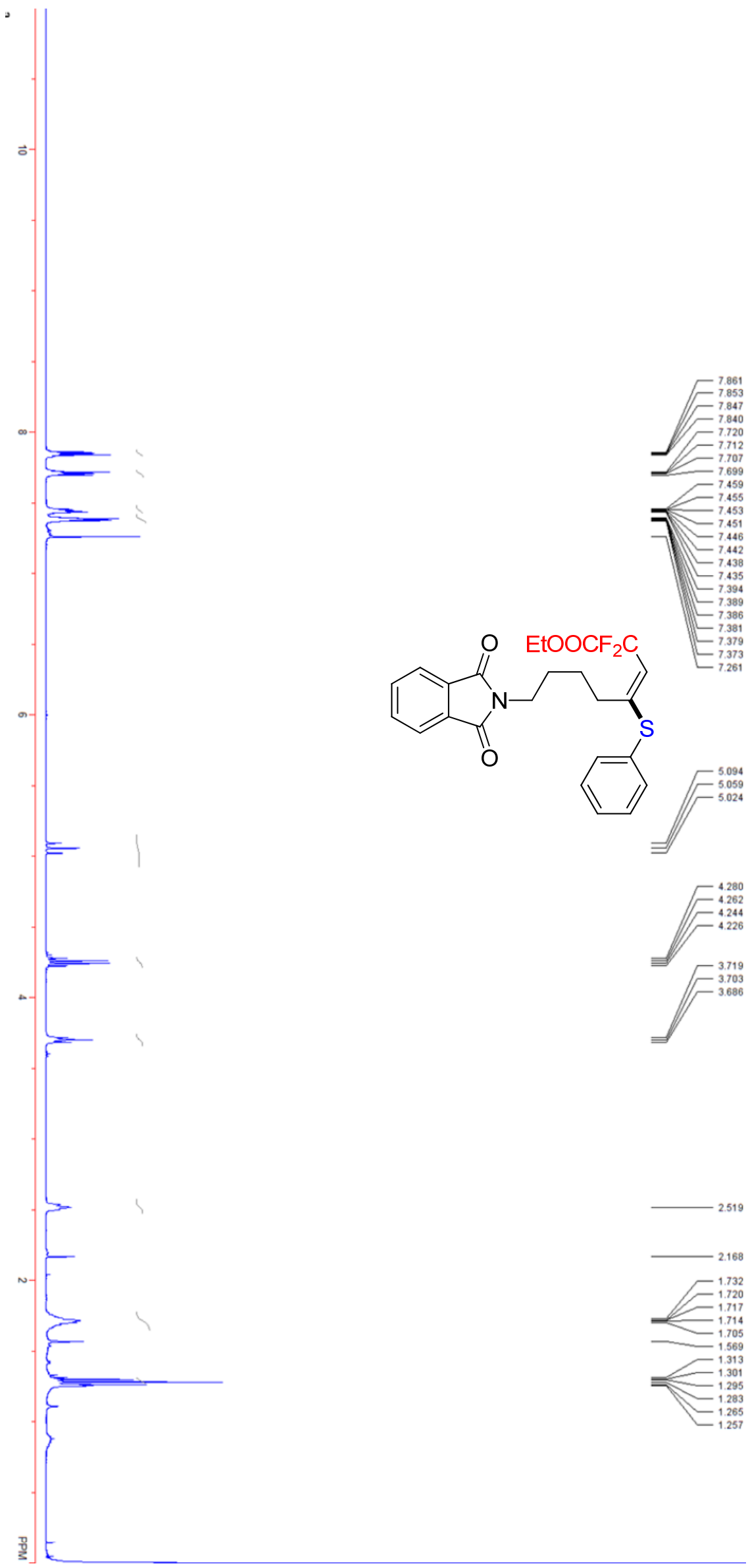

S214 

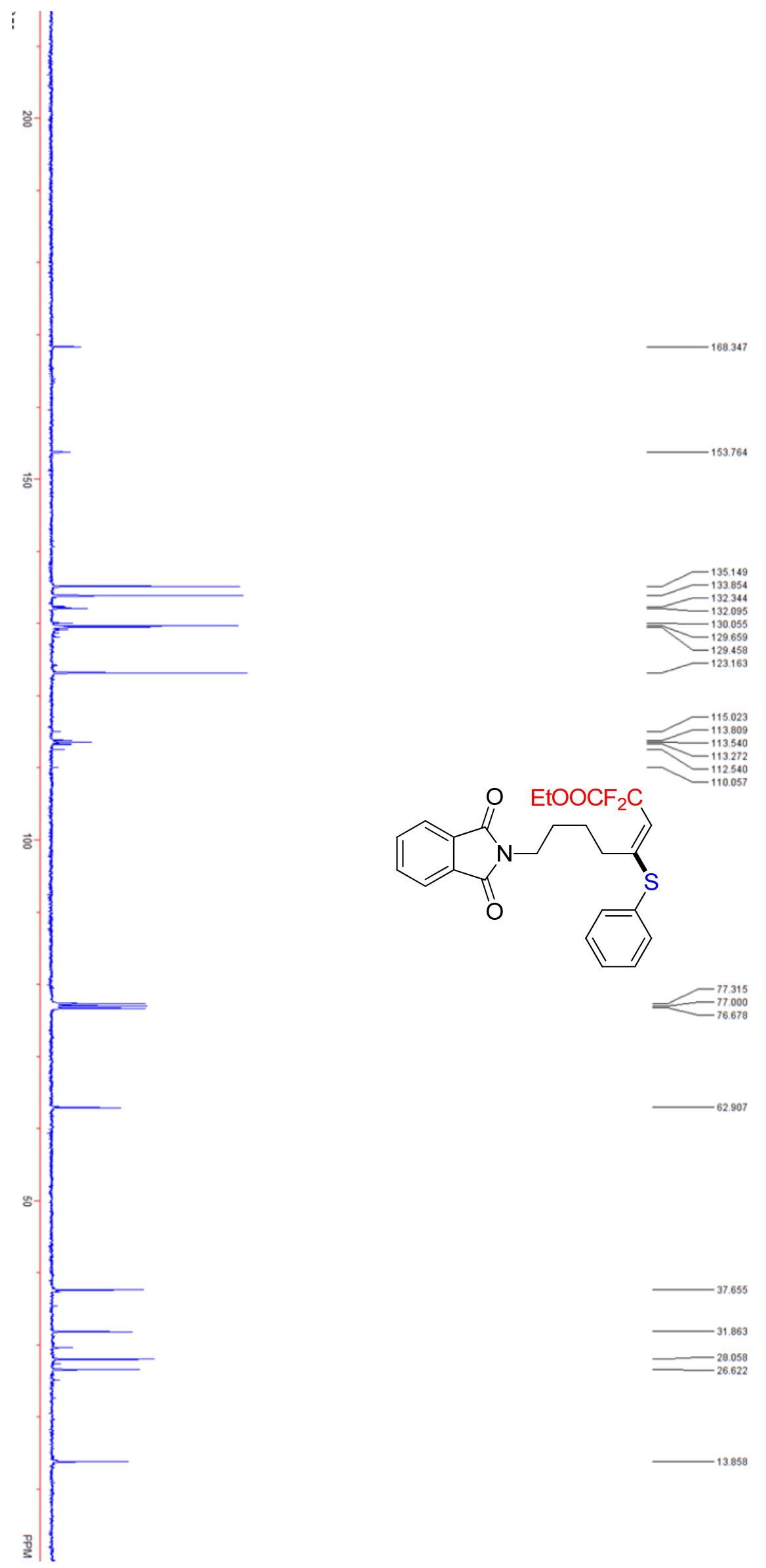


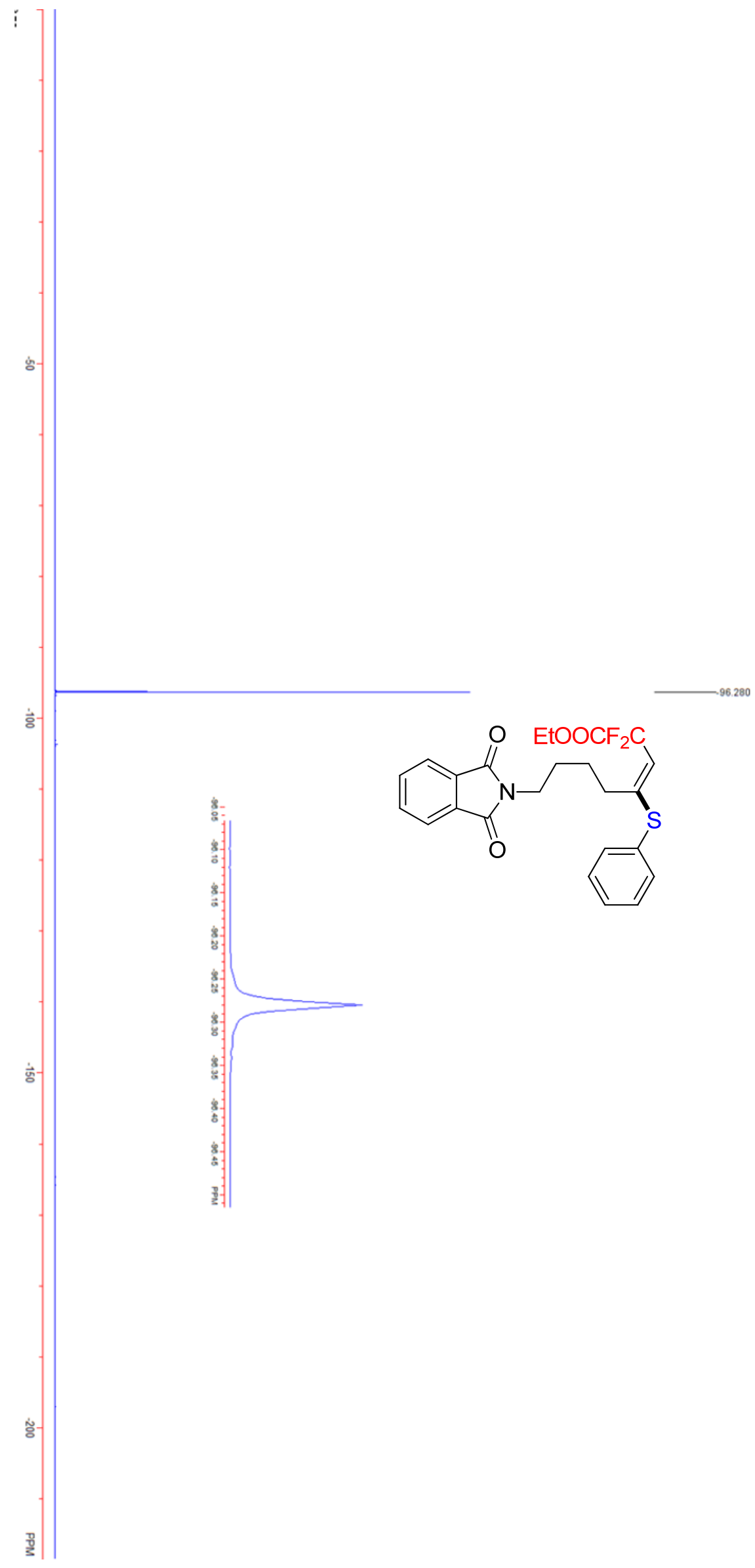




$$
H
$$



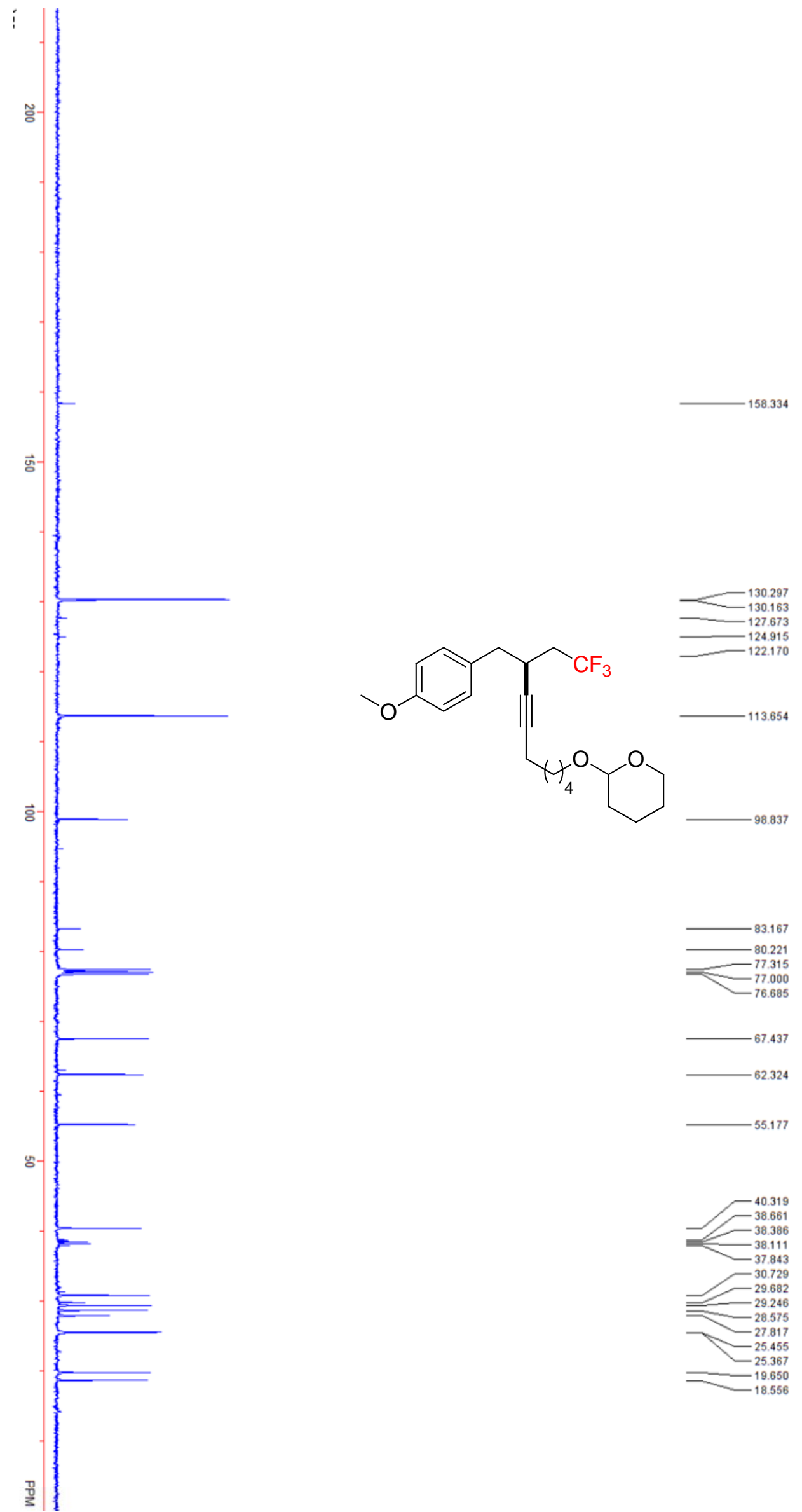


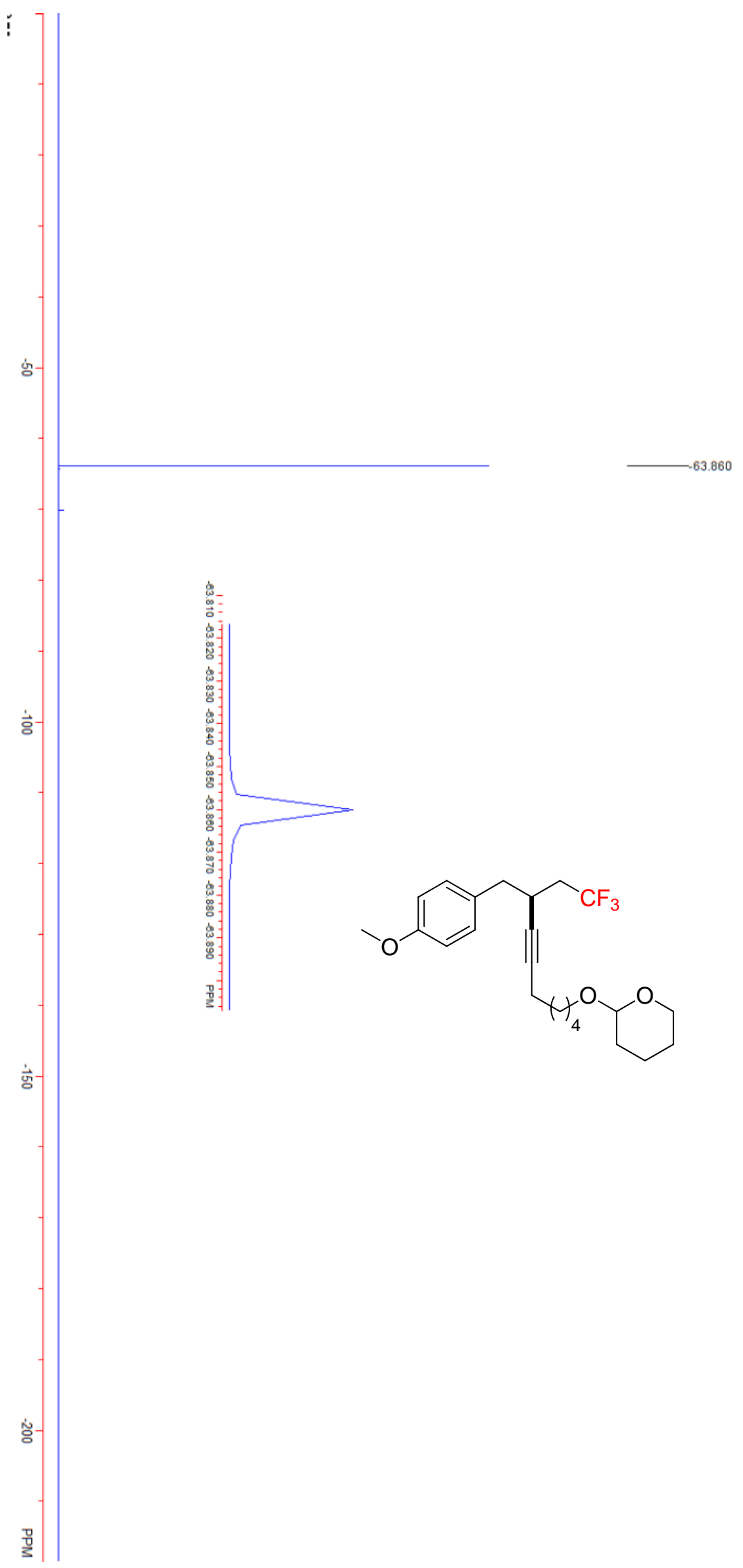




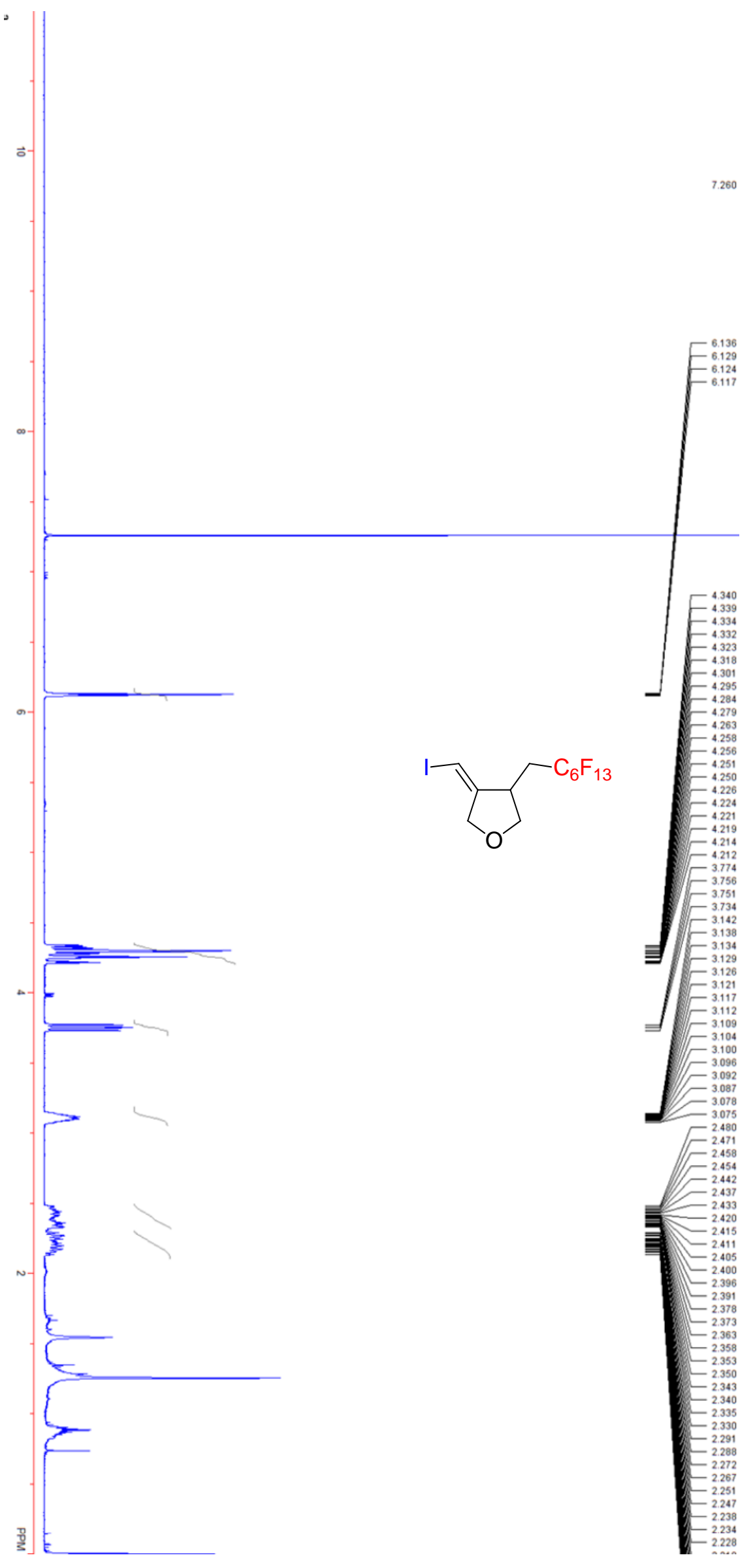




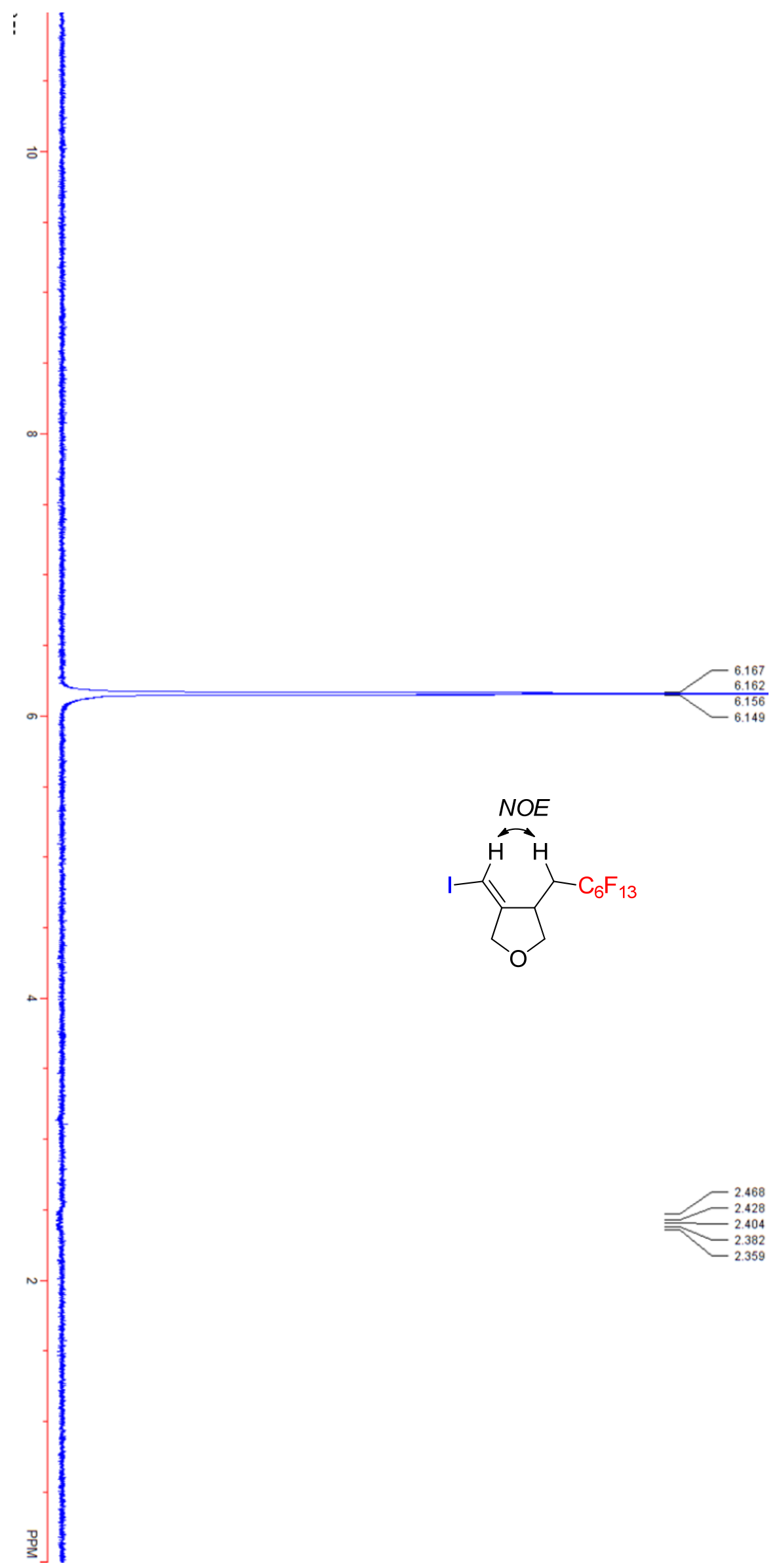



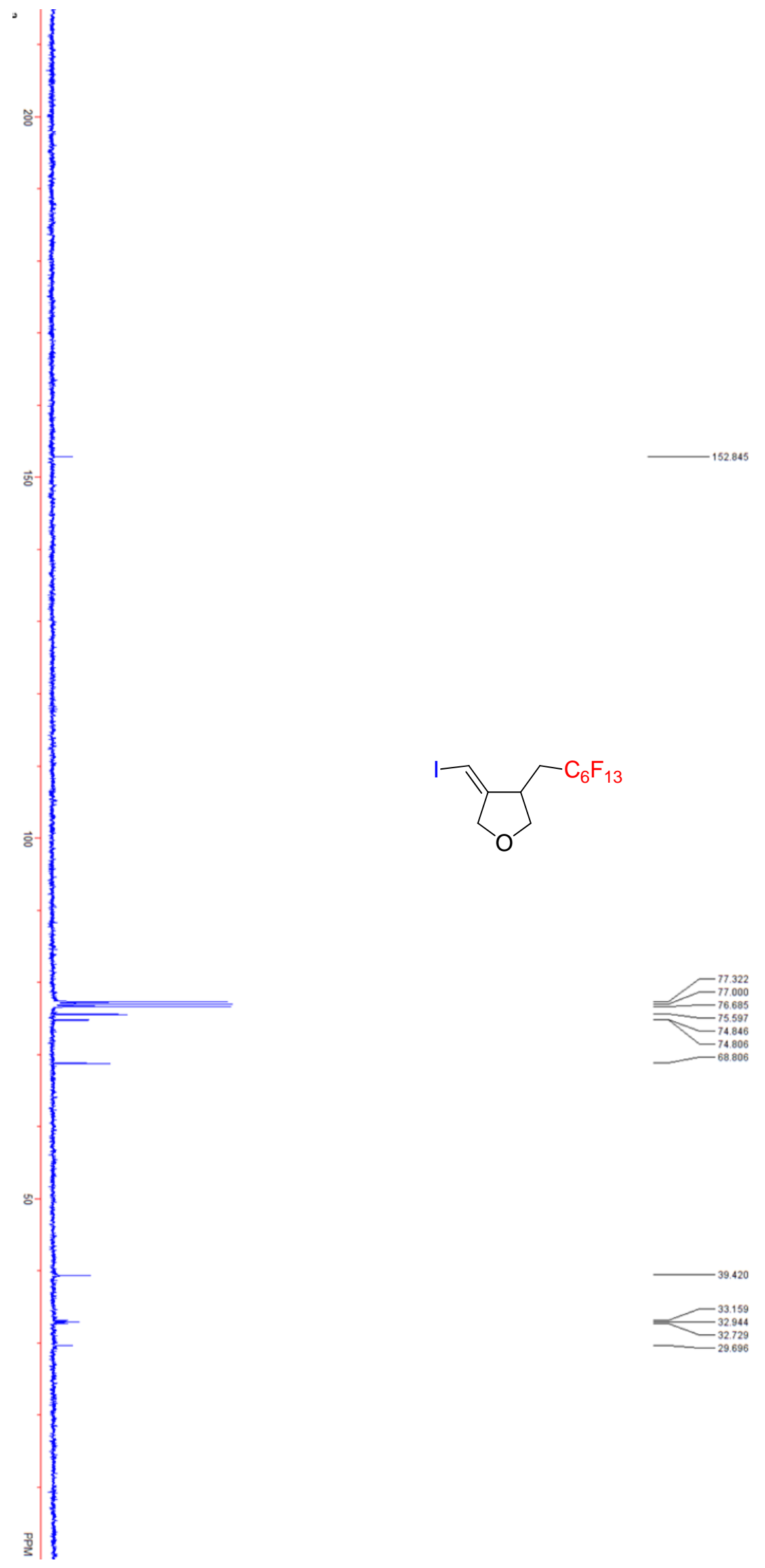


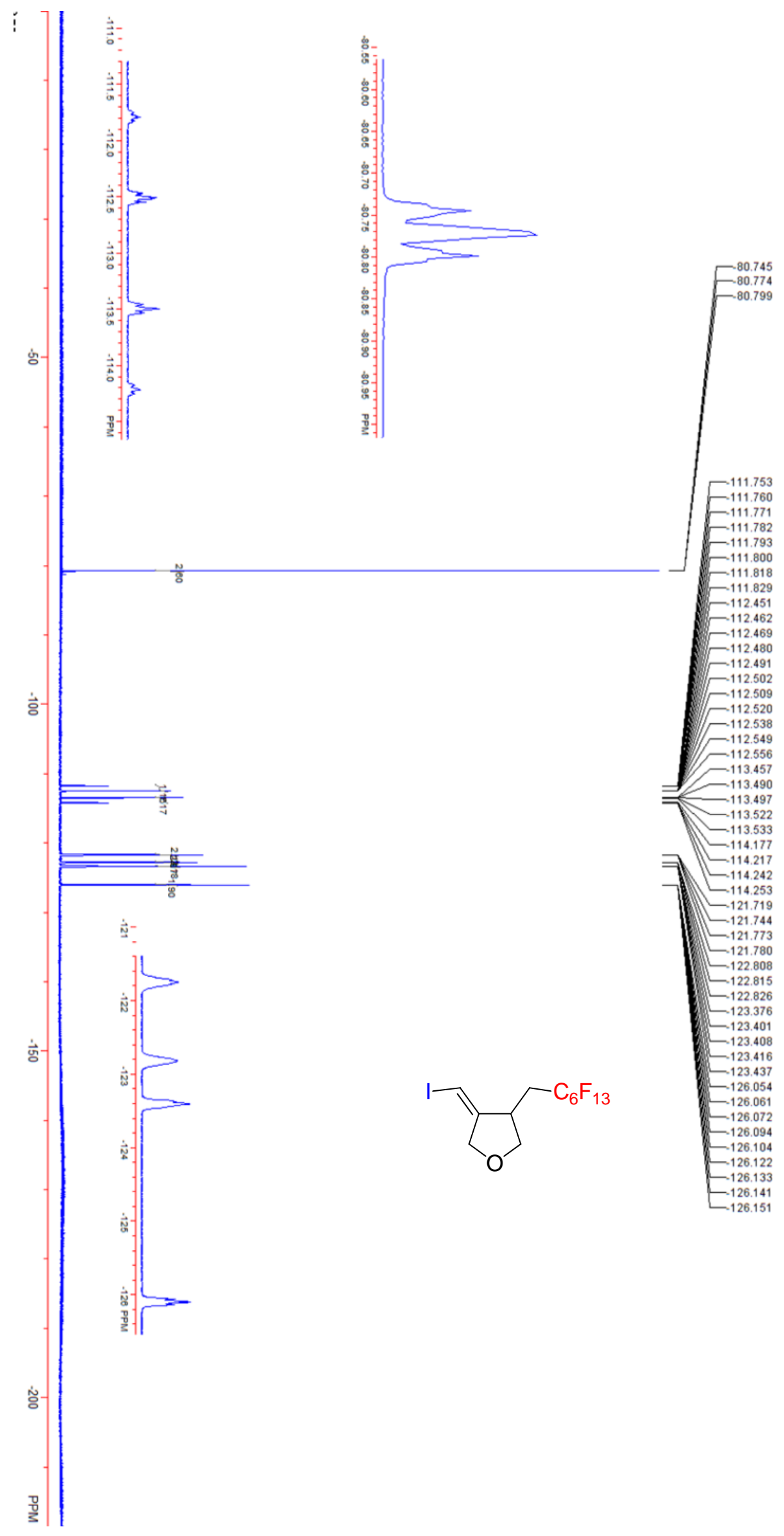



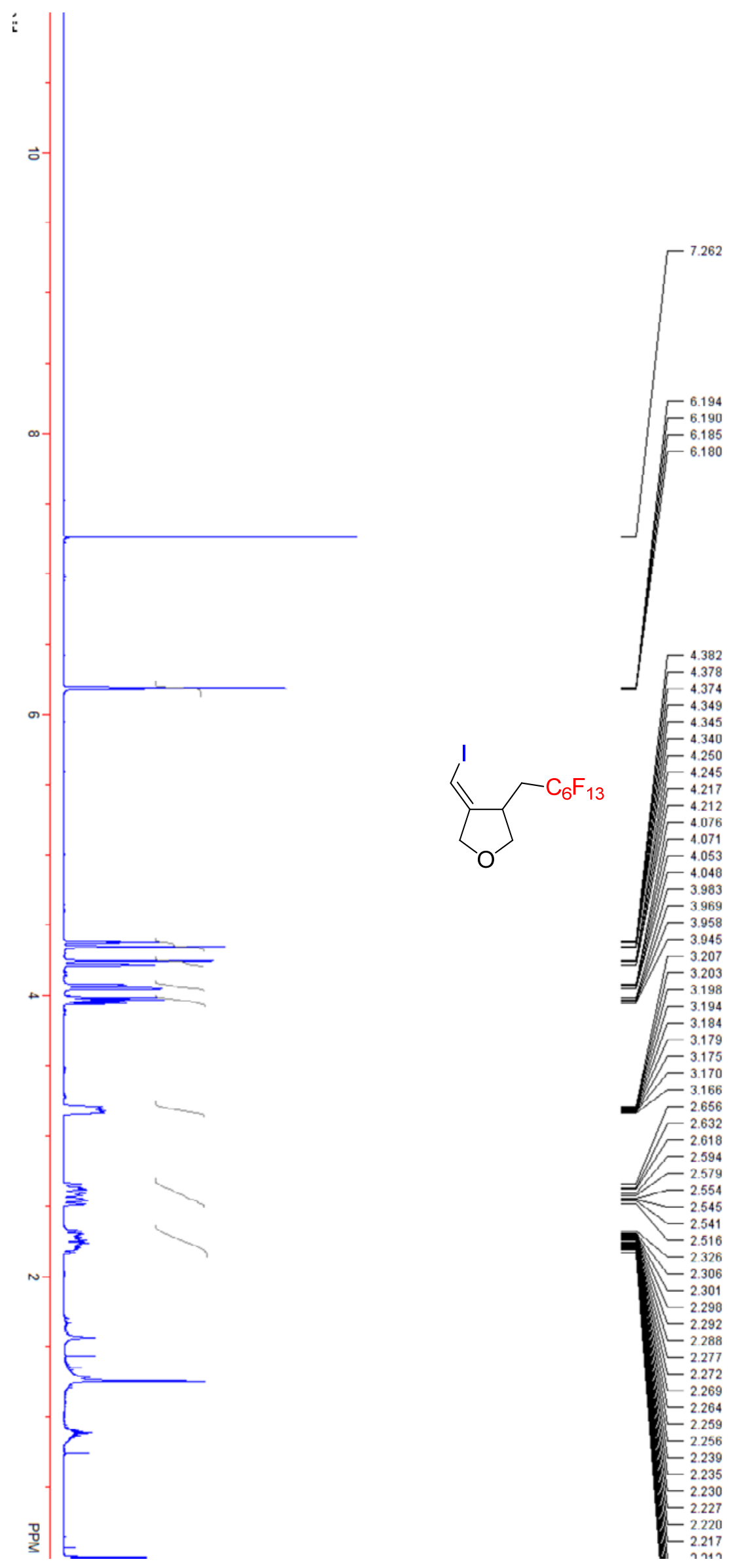


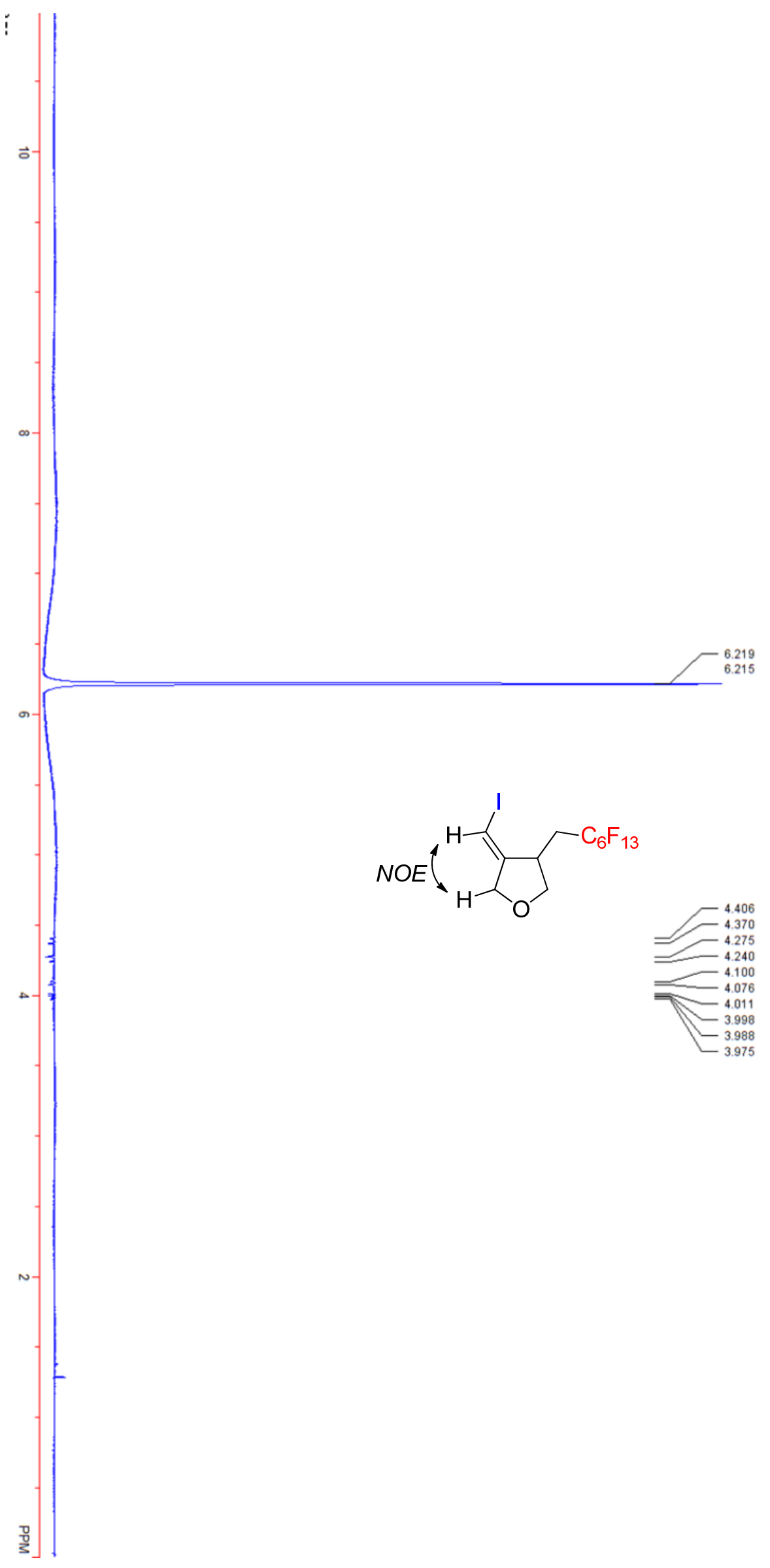



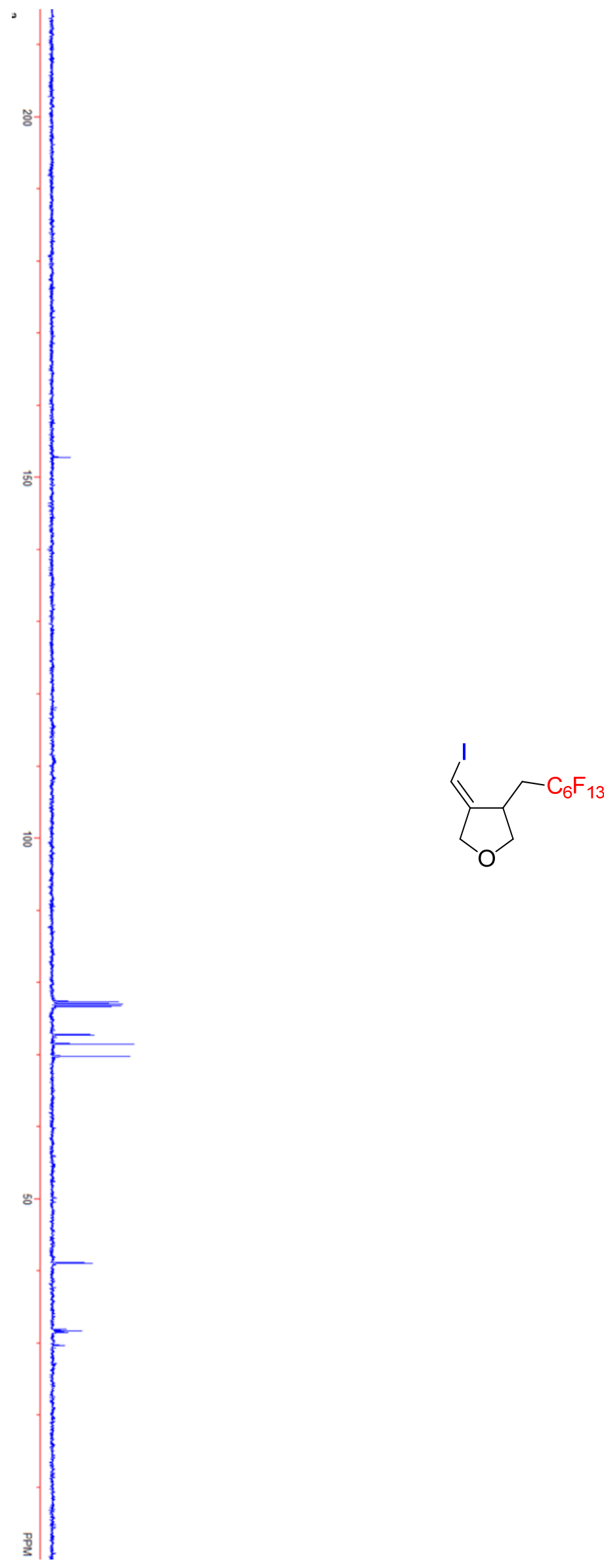


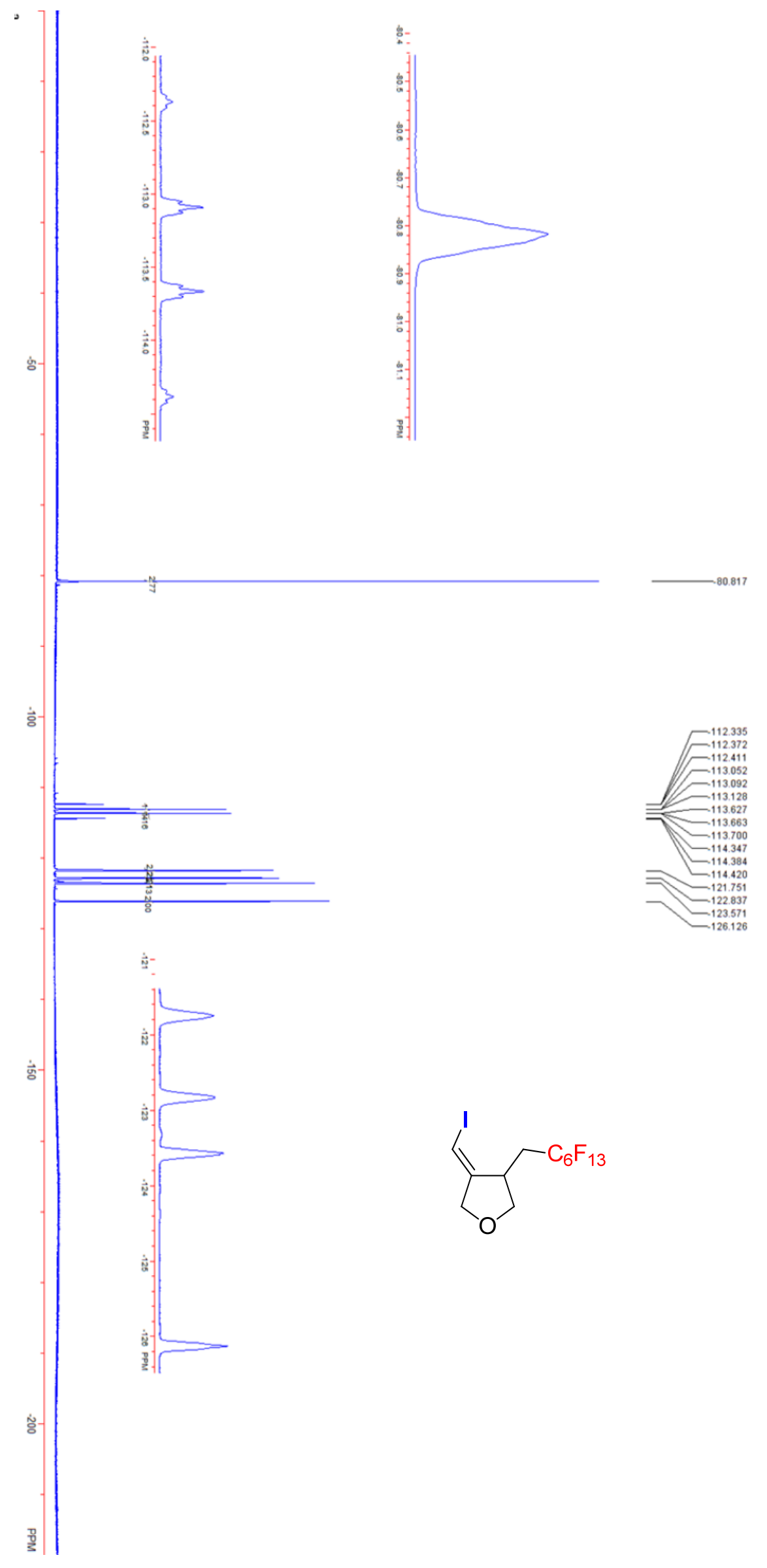




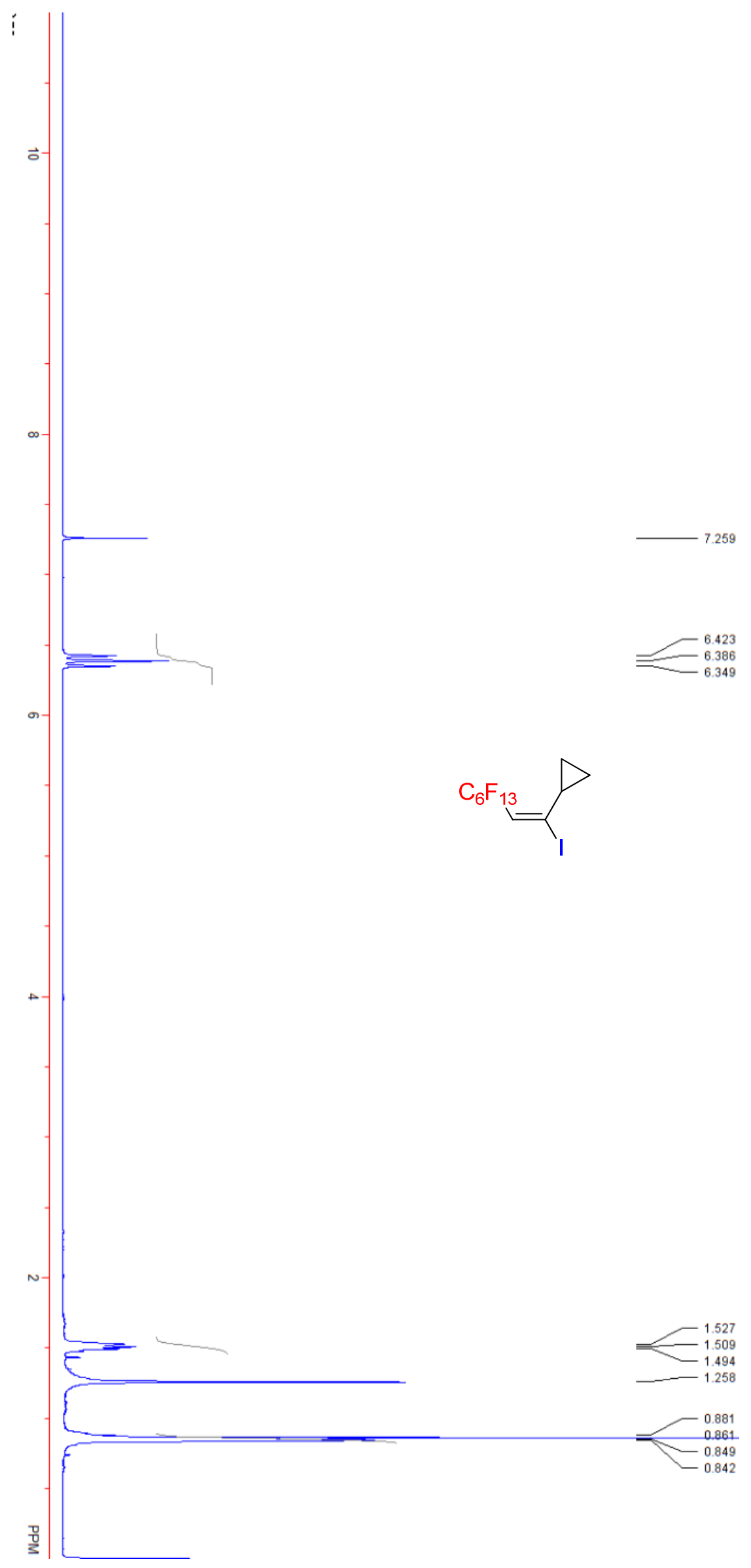



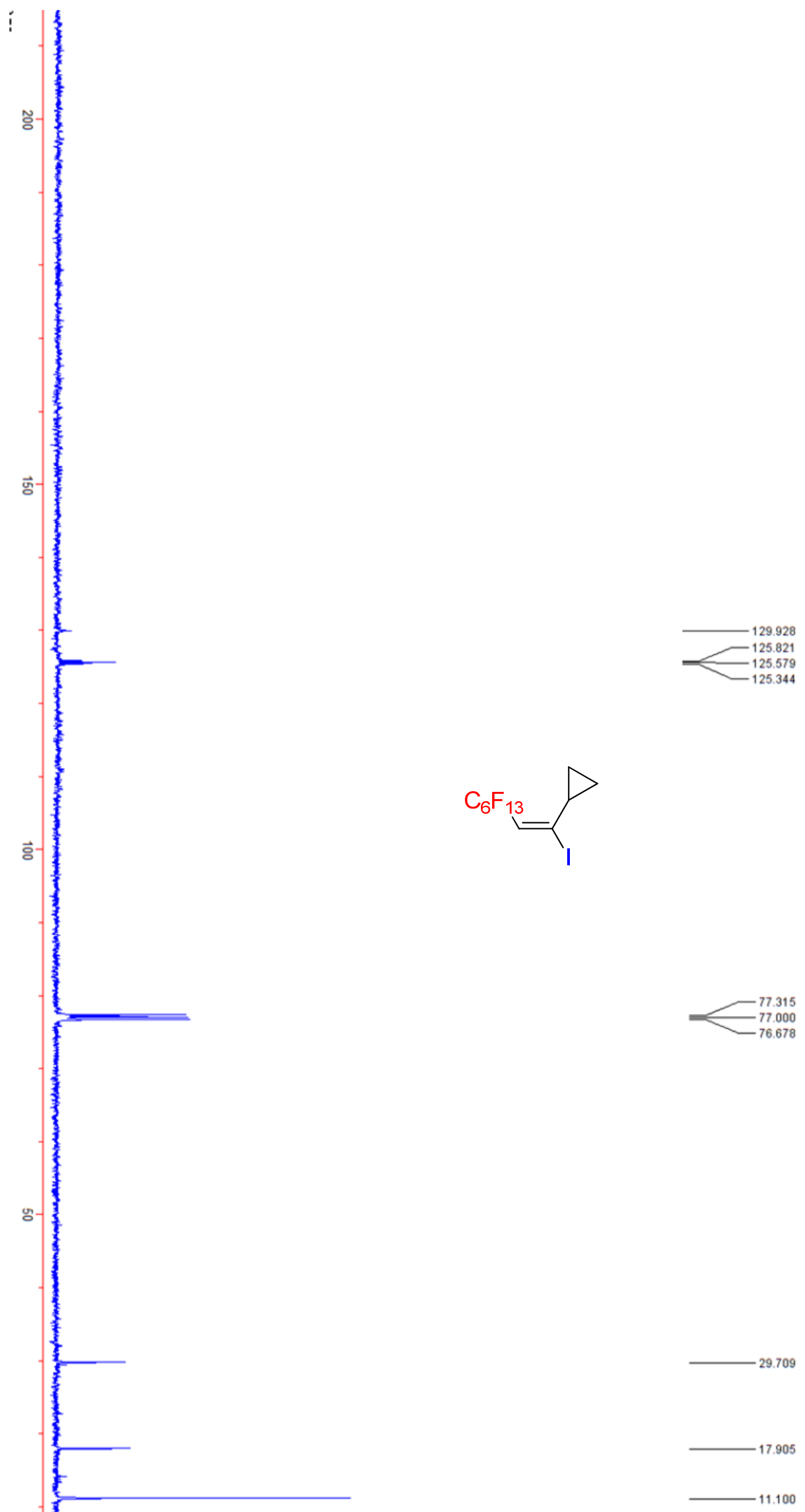

S229 


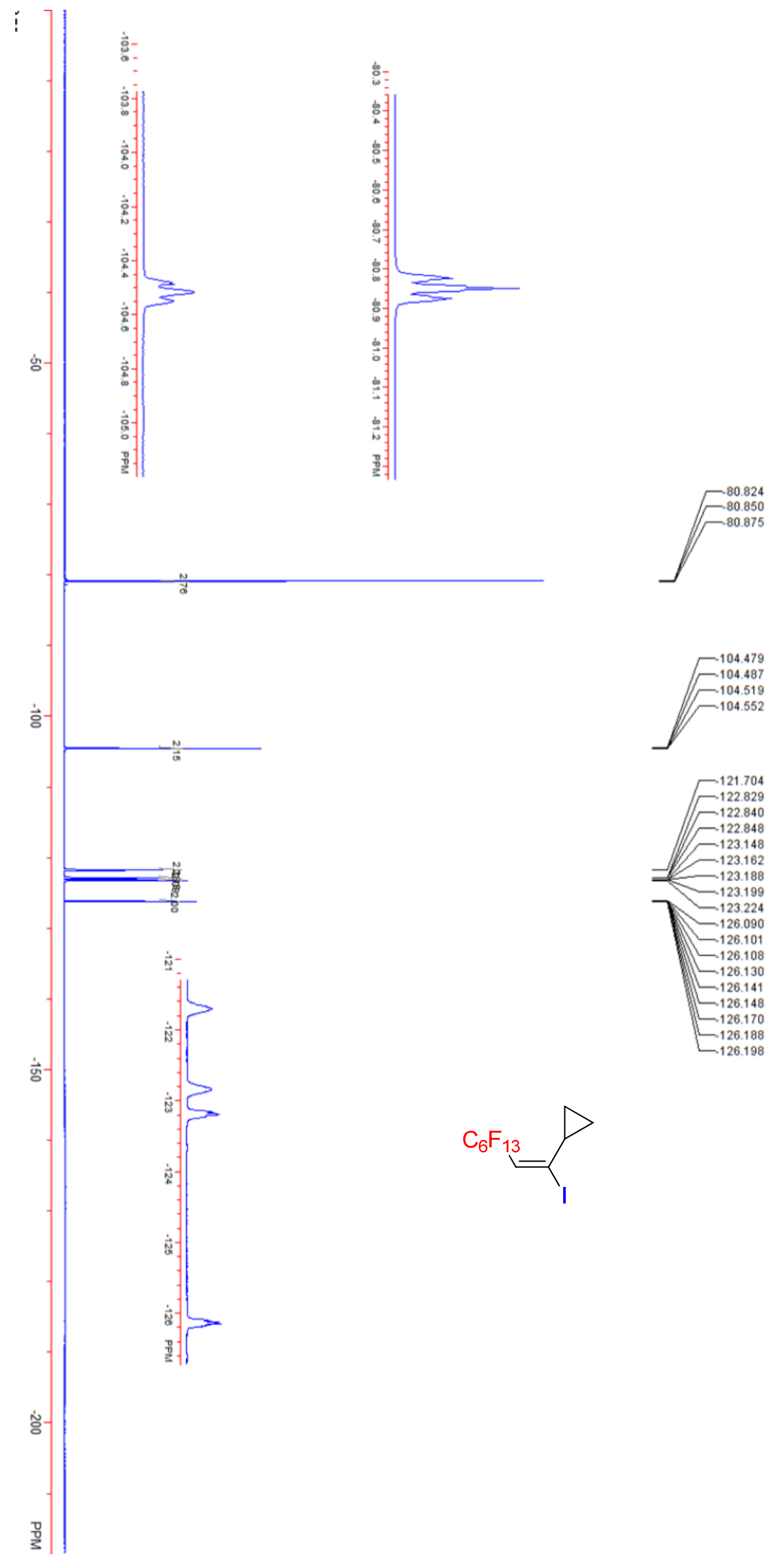




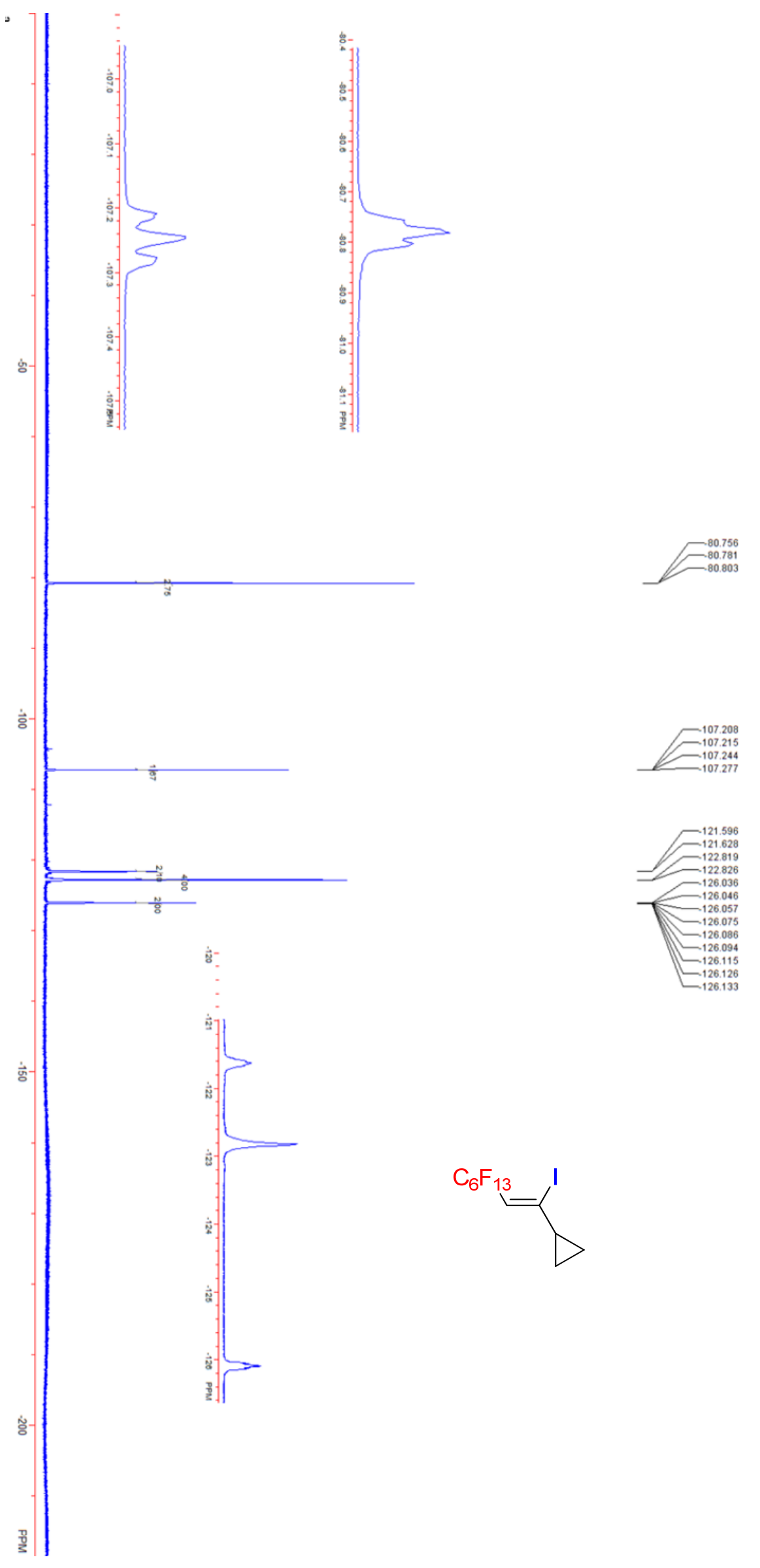




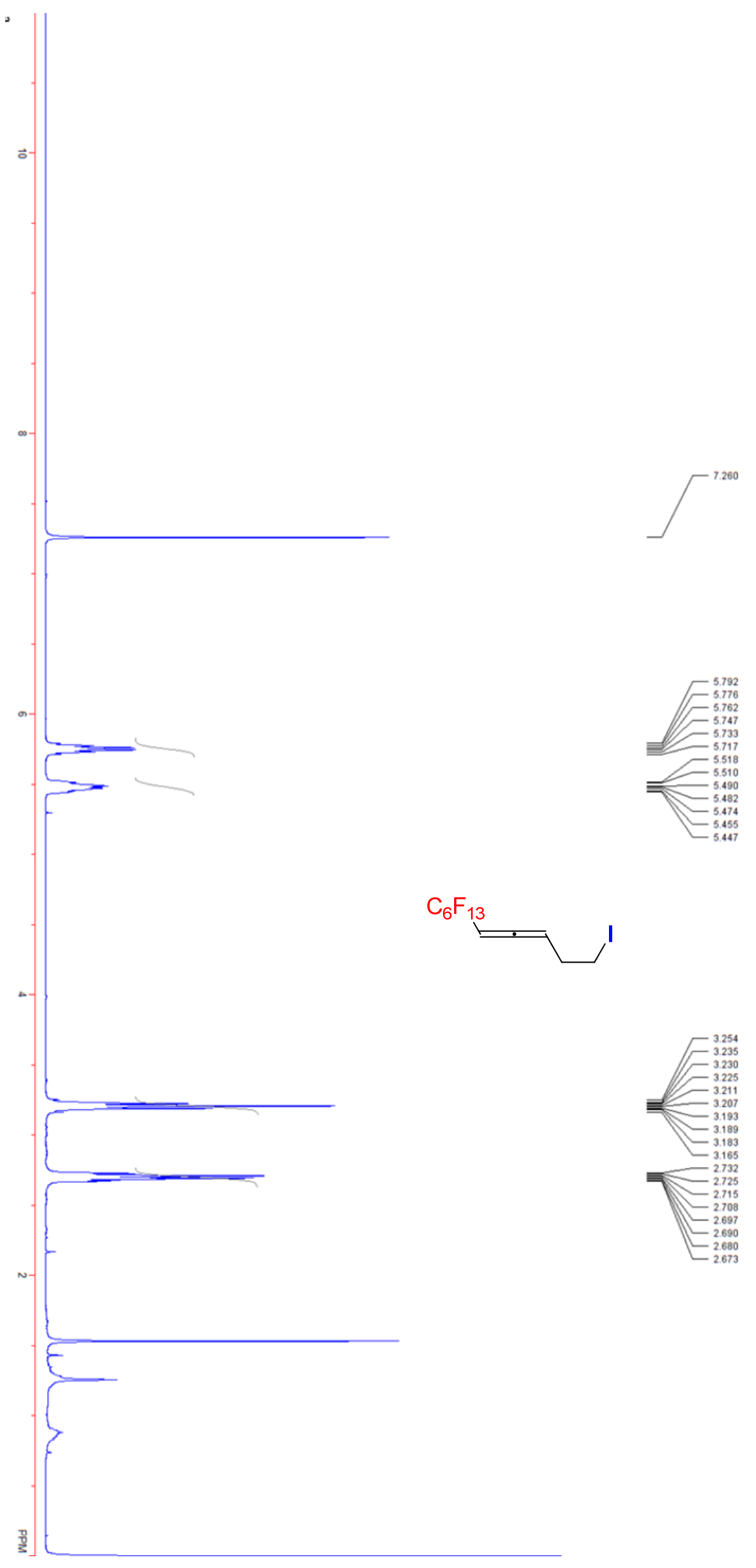



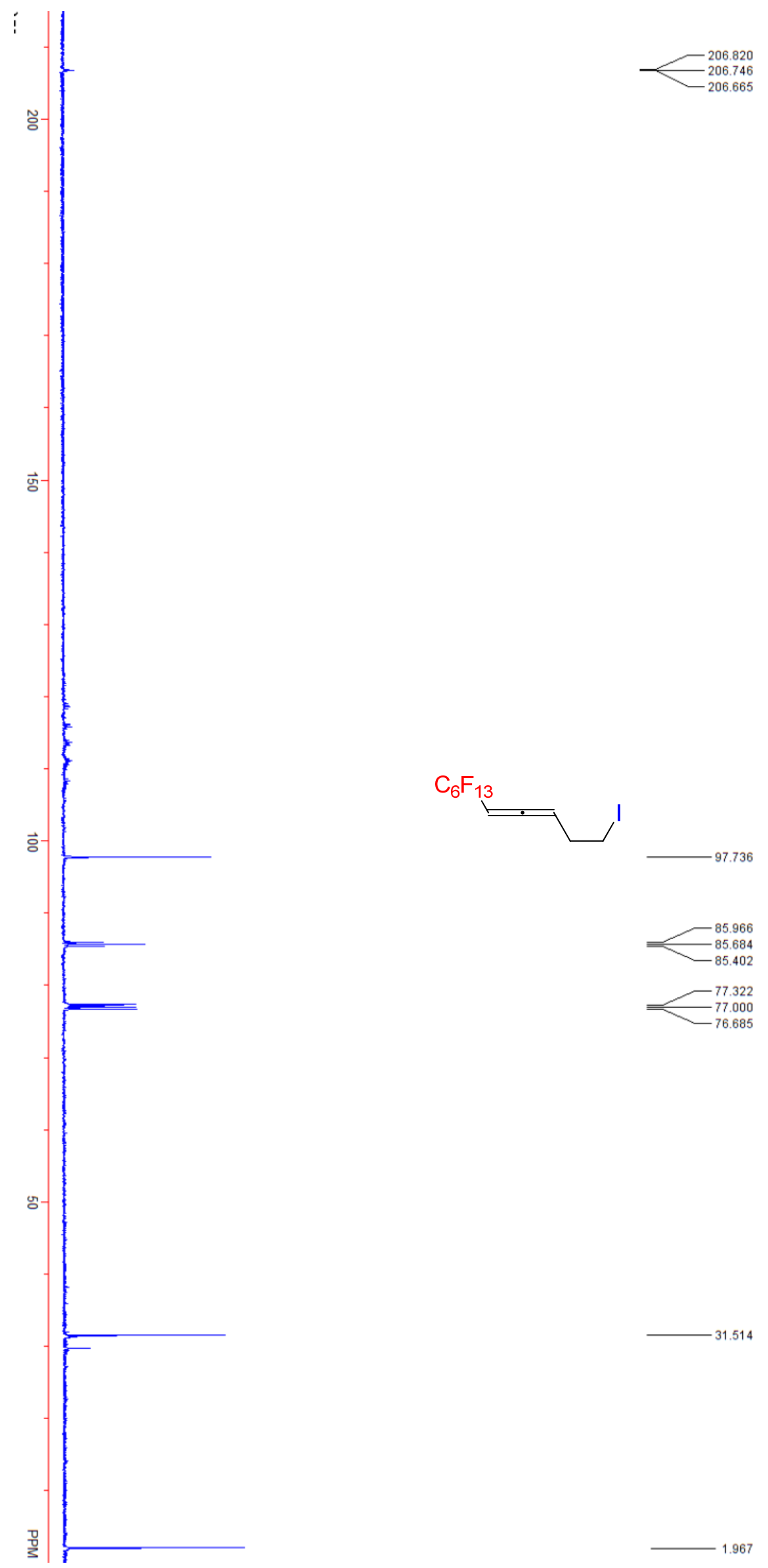


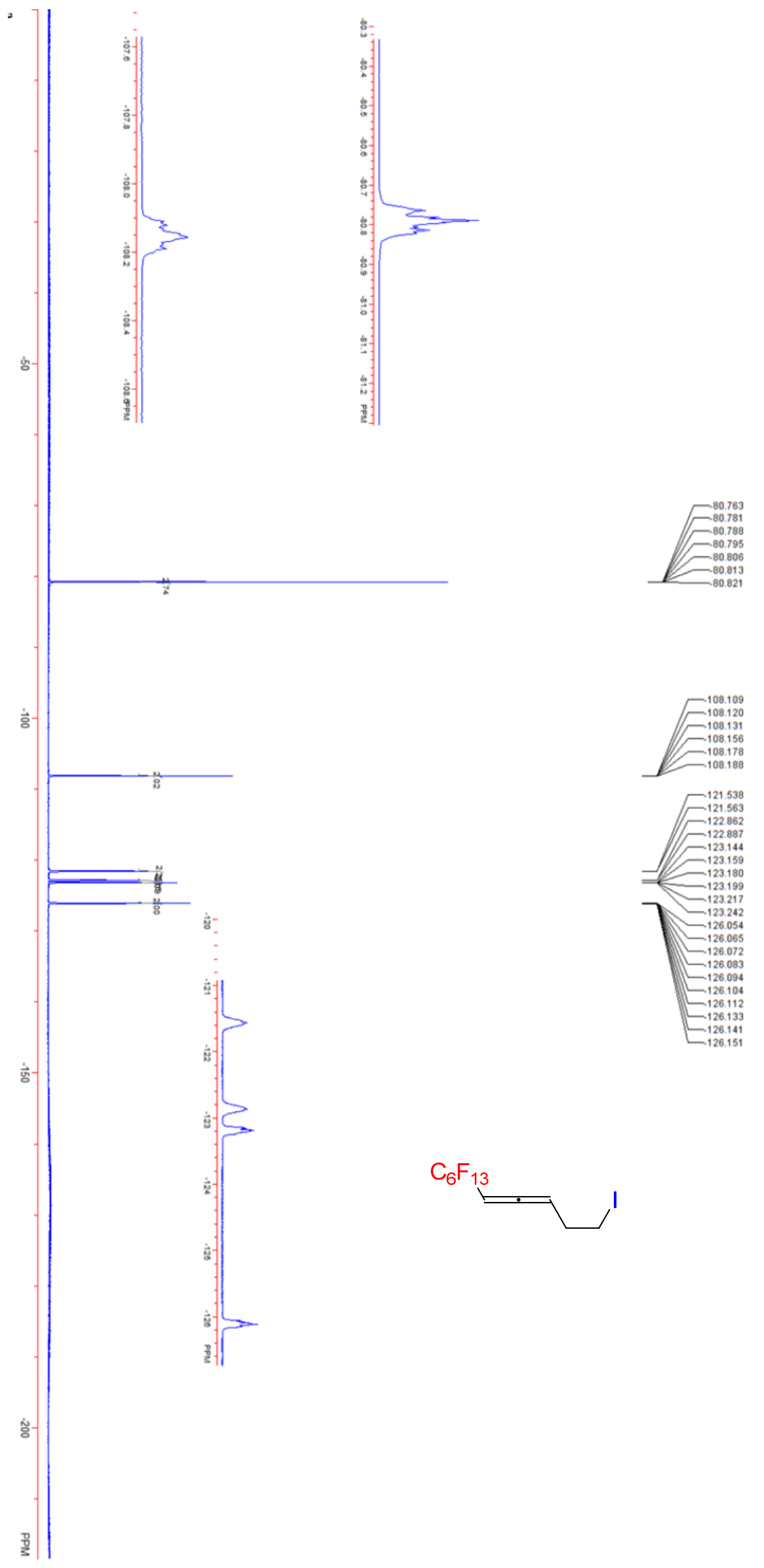

